\title{
Older people's use of mobility aids in the built environment
}

\author{
Allyson Rogers
}

Submitted to Swansea University in fulfilment of the requirements for the Degree of Doctor of Philosophy

December 2020 


\section{DECLARATION}

This work has not previously been accepted in substance for any degree and is not being concurrently submitted in candidature for any degree.

Signed

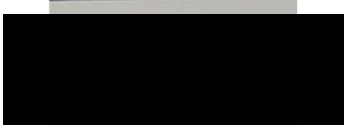

(candidate)

Date

$23 / 12 / 20$

\section{STATEMENT 1}

This thesis is the result of my own investigations, except where otherwise stated. Where correction services have been used, the extent and nature of the correction is clearly marked in a footnote(s).

Other sources are acknowledged by footnotes giving explicit references. A bibliography is appended.

Signed

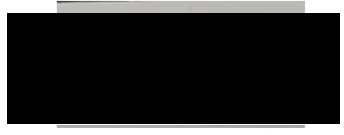
(candidate)

Date $.23 / 12 / 20$

\section{STATEMENT 2}

I hereby give consent for my thesis, if accepted, to be available for photocopying and for inter-library loan, and for the title and summary to be made available to outside organisations.

Signed

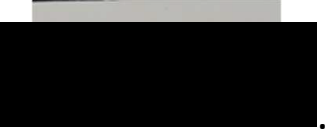

(candidate)

Date

$23 / 12 / 20$ 


\section{Acknowledgments}

I would firstly like to thank the people who gave up their time to participate in this research. The research process was more than just interviewing people, it was getting to know people, listening to their stories, and making their experience matter. I thank them for sharing that experience with me. Every single participant made me laugh, so while there is a very serious message, you were all a pleasure to meet and chat with.

Paul, my husband, whose steadfast support and encouragement has enabled me to complete my studies. We have weathered many illnesses, a global pandemic which, at the time of writing this, is far from over and many other issues through this process, yet he has remained steadfastly my rock, my no.1 fan. You are my inspiration and my foundation.

My children, Richard and Amelia are inspiring and excellent sounding boards as both are also pursuing PhDs in their respective fields. Thank you for encouraging me. You both amaze me with your wit, wisdom, and unfaltering support.

I recently referred to my supervisory team as the dream team because that is what they are. Dr Charles Musselwhite, Dr Gideon Calder and Dr Ashley Frawley. Charles as primary supervisor has, throughout this sometimes-rocky journey, been approachable, encouraging and unwavering. He has allowed me to be happy, angry, anxious and excited, yet kept me focused. Ashley always has the right advice at the right time and has given so many valuable tips and tricks to get the best out of my time and the resources available, including ways to write what I am thinking effectively. She always seems to ask the most penetrating questions which ultimately gets the best out of me. Gideon joined the supervisory team after hearing me present the research at a Swansea University Postgraduate conference. He brings a sense of calm, and coming on as a supervisor in the way he did gave me a great sense of confidence in my work. Thank you all so much, you really have been a pleasure to work with and I feel fortunate to have had the benefit of your experience and professional example. 


\begin{abstract}
The world population is ageing, by both number and share of older people (WHO 2013), leading to ageing issues being increasingly discussed within transport and mobility fields (Murray 2016; Schwanen \& Paez, 2010). As those over 70 are the group most likely to have mobility and accessibility issues, there is a need to look at the wider relationship between mobility and ageing in relation to health and well-being (Musselwhite 2016). The social model of disability and resulting accessibility legislation has heralded a great deal of improvement for the inclusion of those with disabilities, but there seems to be little attention paid to emotional or psychological needs of people with physical impairments (Oliver, 2013), or disability and mobility aid use by older people (Jönson \& Taghizadeh Larsson, 2009; Minkler \& Fadem, 2002; Phillips, Ajrouch, \& Hillcoat-Nallétamby, 2010; Raymond, Grenier, \& Hanley, 2014). From the perspective of mobility aid provision, outcomes are measured in terms of independence for user, without considering the impact of this functionality on mobility aid user or carer, family or social life (Hammel et al, 2013). This study has taken an ethnographic grounded theory approach using observation, sound recording, photography, storytelling and discussion as methods for data collection. People who use mobility aids $(n=11)$ were recruited to give accounts of mobility aid use during daily routine activities, along with the accounts of some of their family members and carers $(n=6)$. Constant comparative analysis through coding and revisiting the field on an iterative basis was employed to describe and begin to understand mobility aid use by older people in the built environment. A complex and interlinking array of experiences and obstacles to inclusion was found for this group of people on individual, relational and infrastructural levels, with themes of identity, relationships and infrastructure and interlinking systems emerging from the data. The findings contribute information regarding a complex relationship between mobility aid use and ageing. The impact of reduced mobility and mobility aid use on identity and relationships is exacerbated by ineffective or inconsistent policies and systems. Social, psychological, emotional and physical costs of unequal access are being met by older mobility aid users and their carers. The implications of these findings are that this area requires more attention from both research and policy and planning arenas to ensure older people who use mobility aids are appropriately accommodated in the built environment. Policy and planning are found to not consistently provide a cohesively supportive environment for older people who use mobility aids to effectively participate socially.
\end{abstract}




\section{CONTENTS}

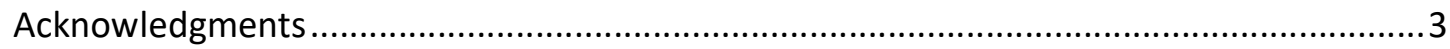

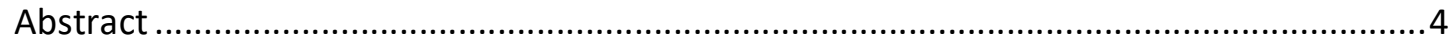

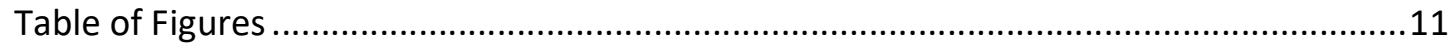

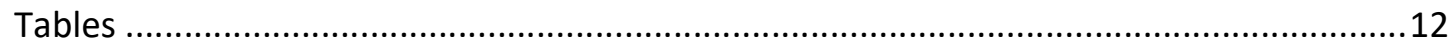

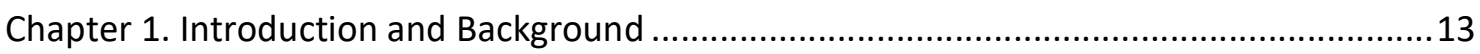

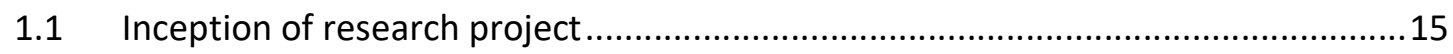

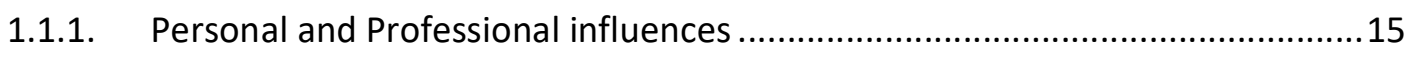

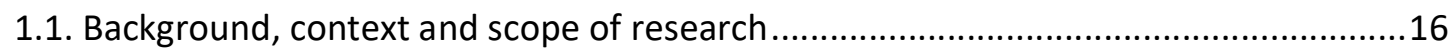

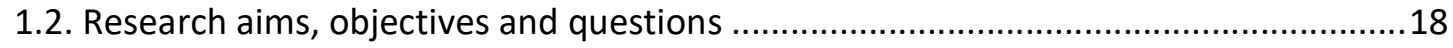

1.2.1. Aims

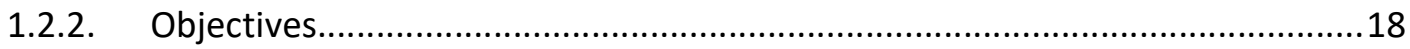

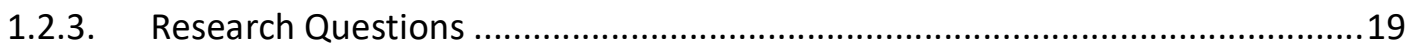

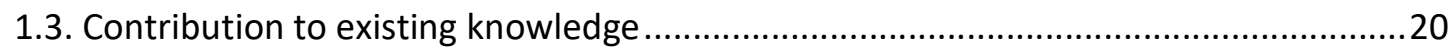

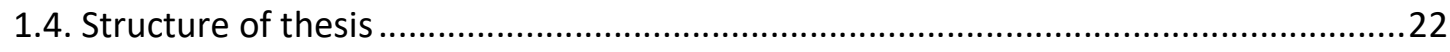

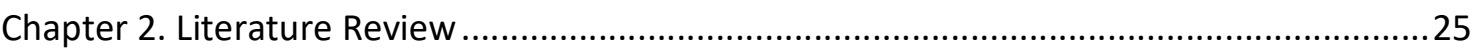

2.1. The scope and purpose of the literature review........................................................26

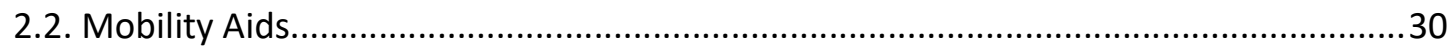

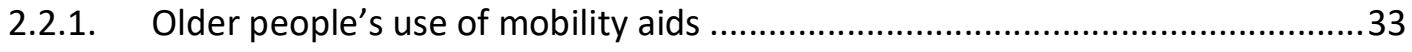

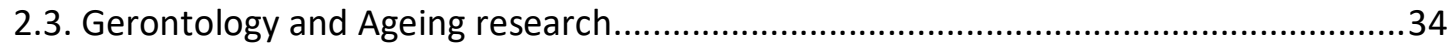

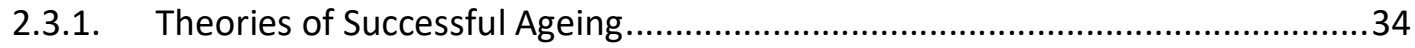

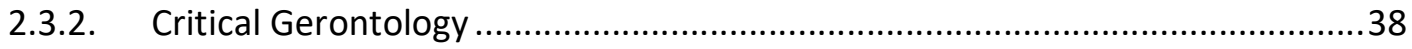

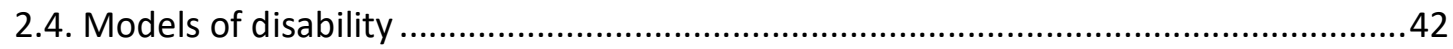

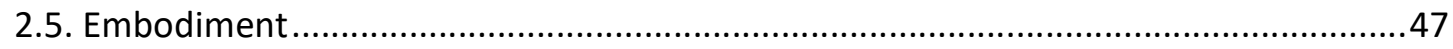

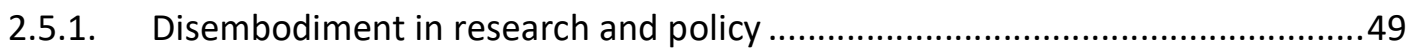

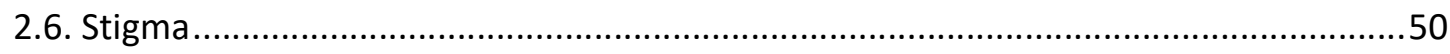

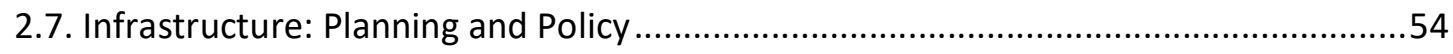

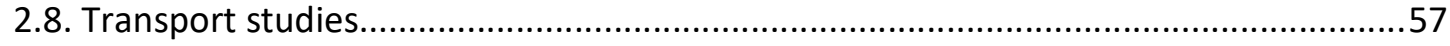

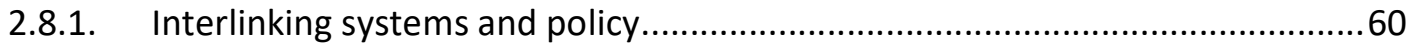

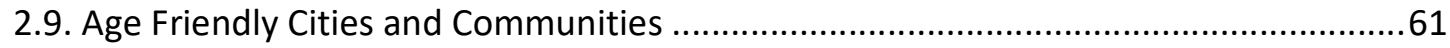

2.9.1. World Health Organisation Age Friendly Cities and Communities...................61

2.9.2. Critiques of the World Health Organisation approach to age friendliness .......65 
2.9.3. The Dublin Declaration on Age-Friendly Cities and Communities in Europe ....66

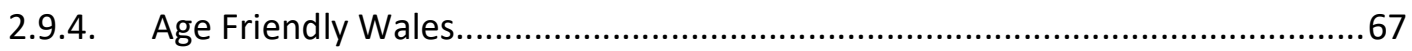

2.9.5. Interpretation and implementation of Ageing Well in Wales plans.................69

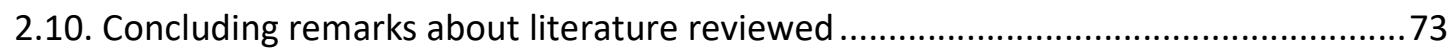

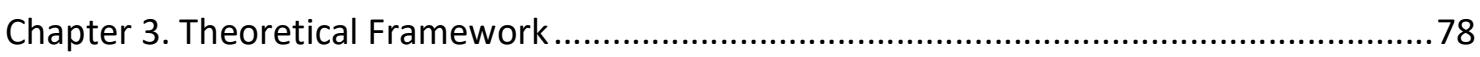

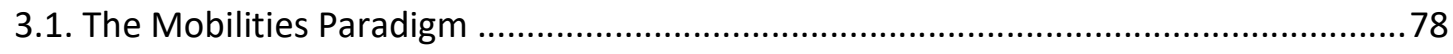

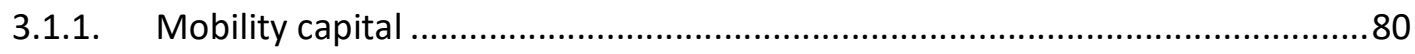

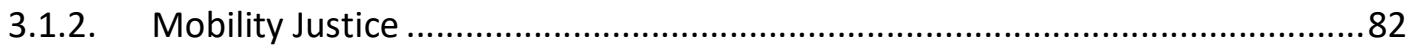

3.1.3. The mobilities paradigm to research older people who use mobility aids .......83

3.2. Interdisciplinary nature of mobility and gerontology studies .................................... 85

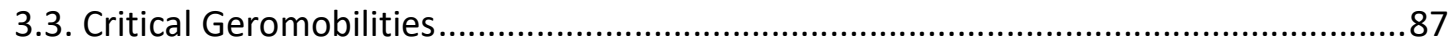

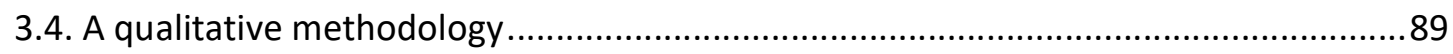

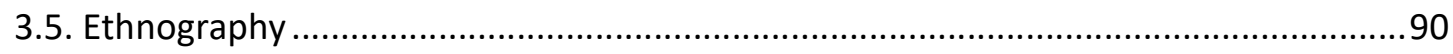

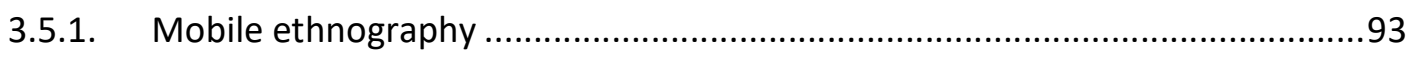

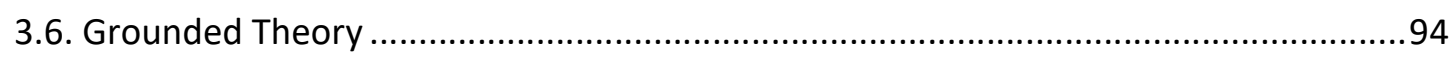

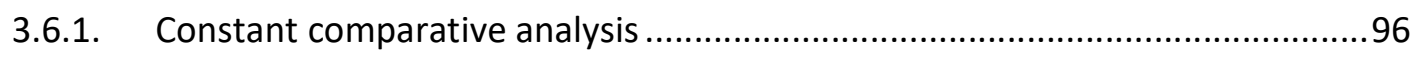

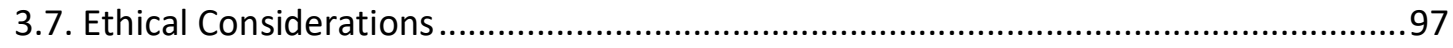

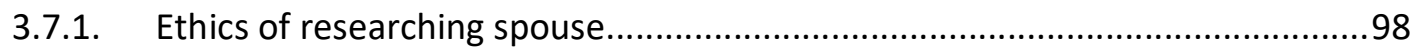

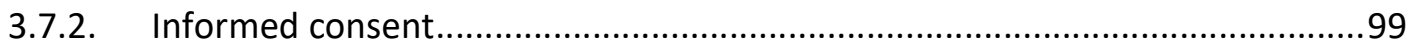

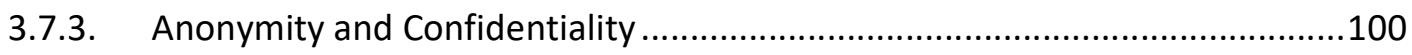

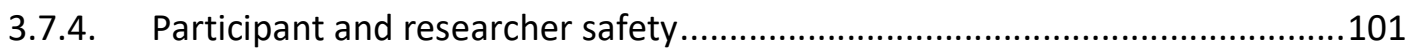

3.7.5. Participant well-being during and after the research.................................. 102

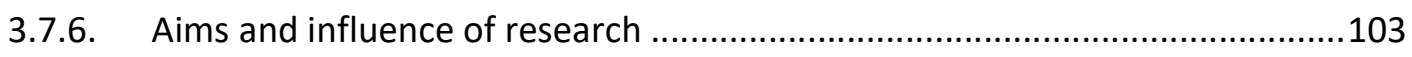

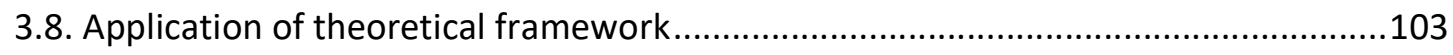

Chapter 4. Methods: Design and Data Collection ...........................................................104

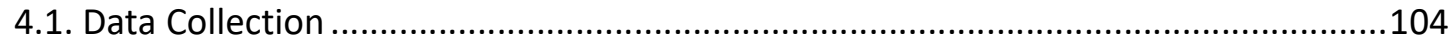

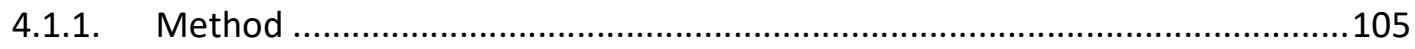

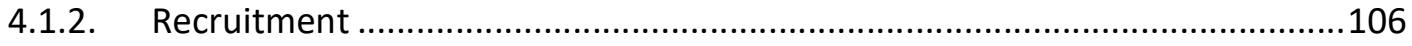

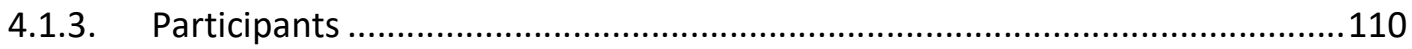

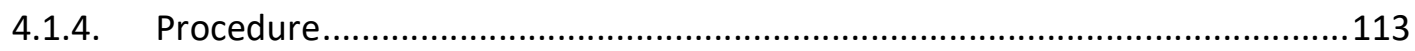

4.1.5. Constant comparative analysis during data collection .................................117

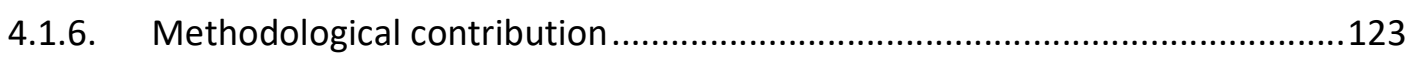

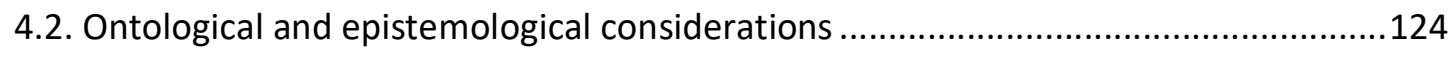




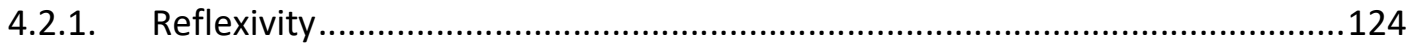

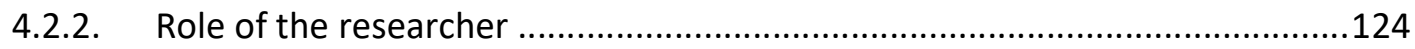

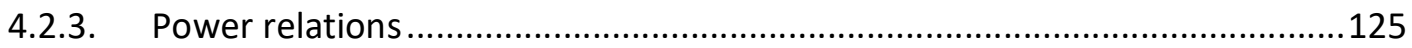

4.2.4. Conclusion of ontological and epistemological considerations .....................128

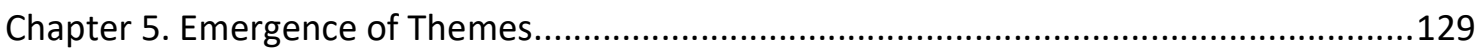

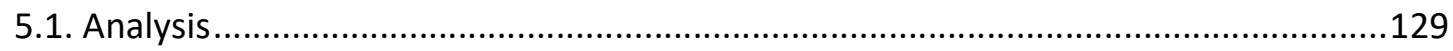

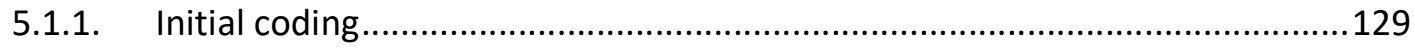

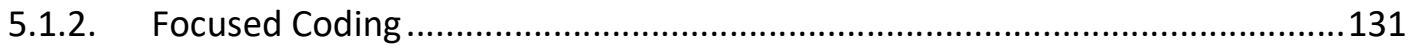

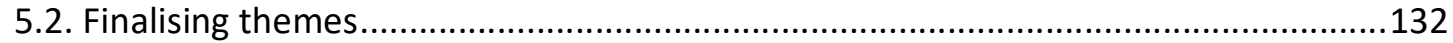

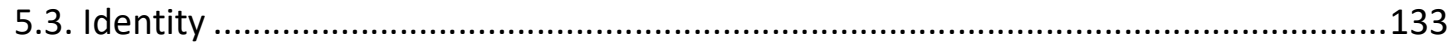

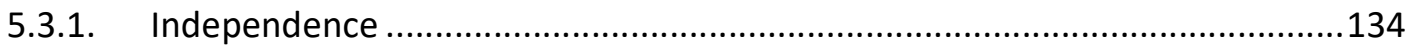

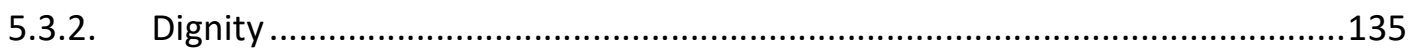

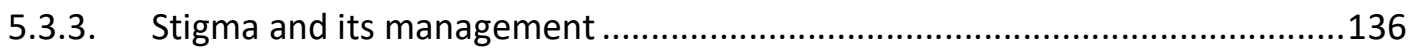

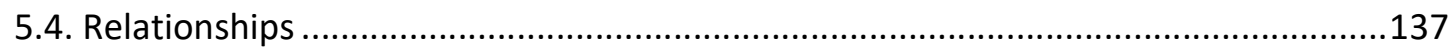

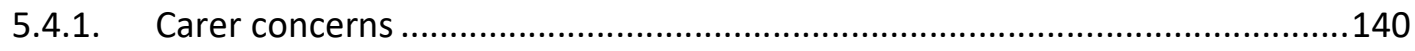

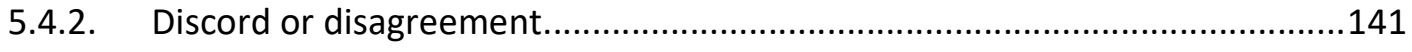

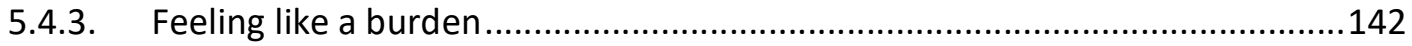

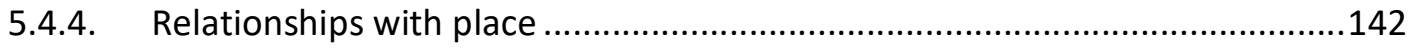

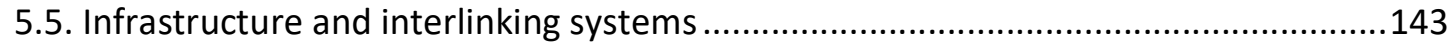

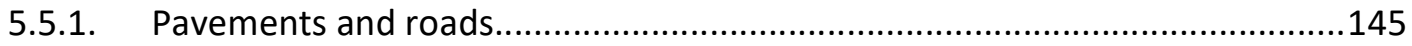

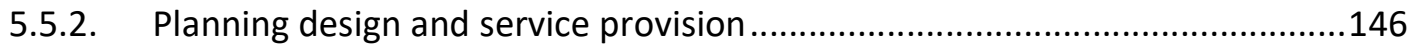

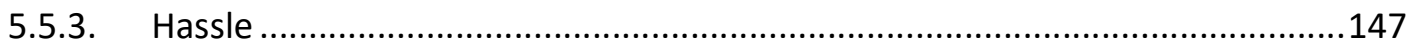

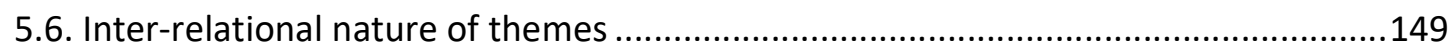

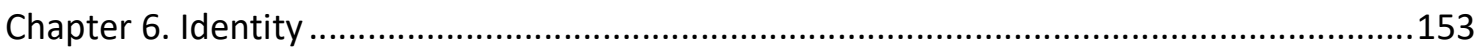

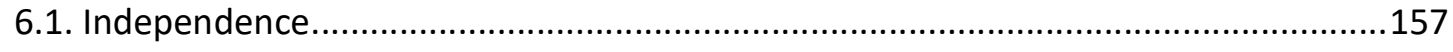

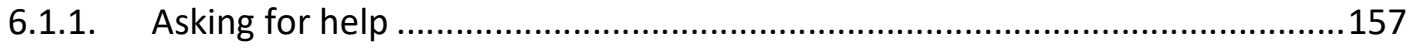

6.1.2. Self-reliance as a signifier of independence ................................................159

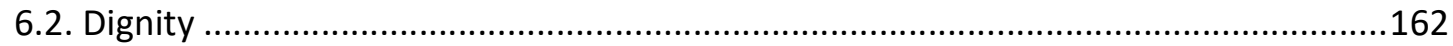

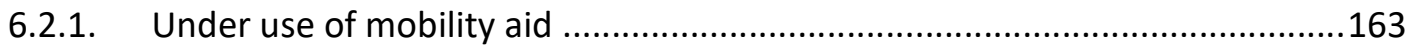

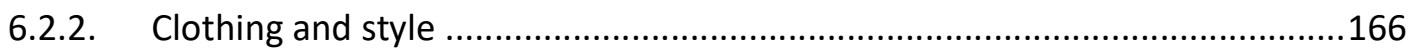

6.2.3. Maintaining identity (manifesting as vanity) .............................................167

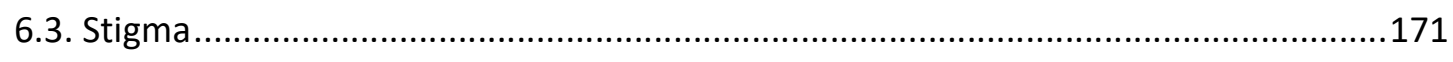

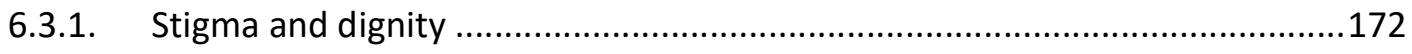




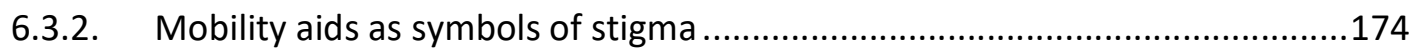

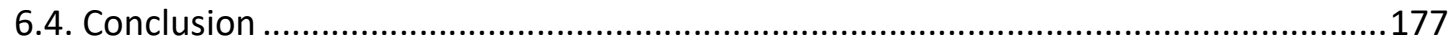

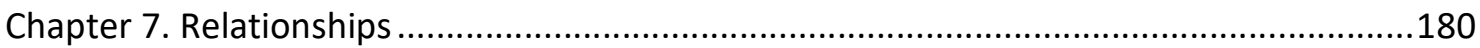

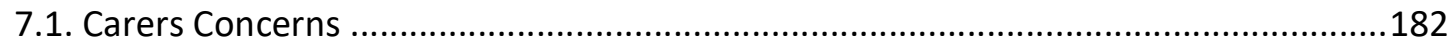

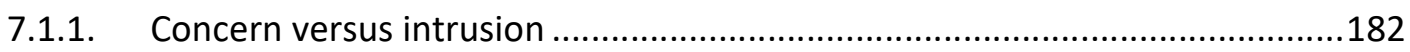

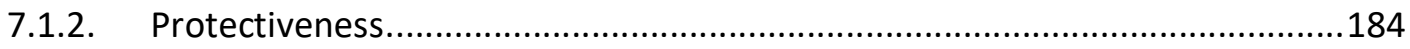

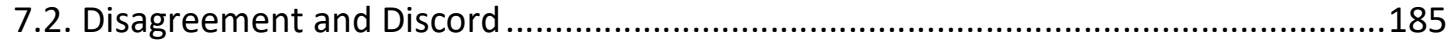

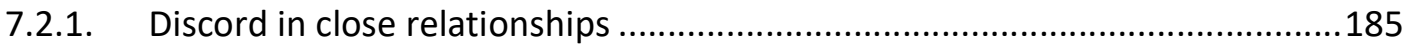

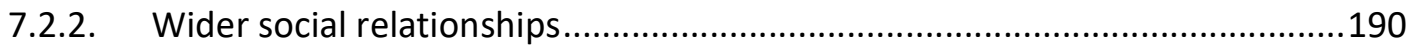

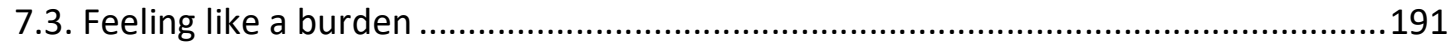

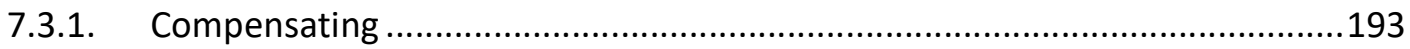

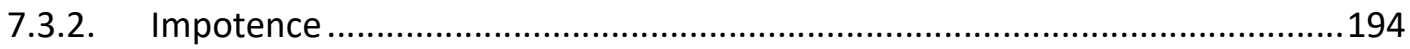

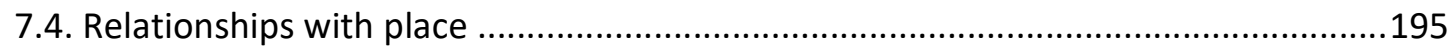

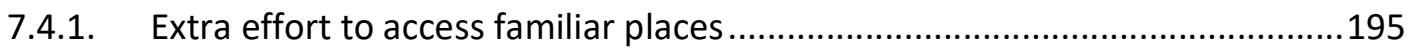

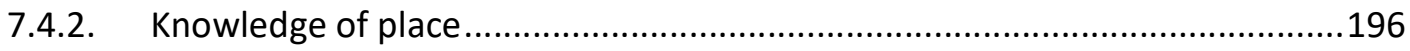

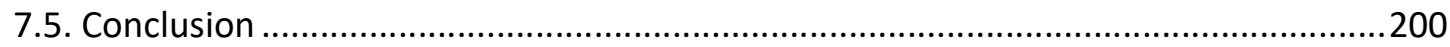

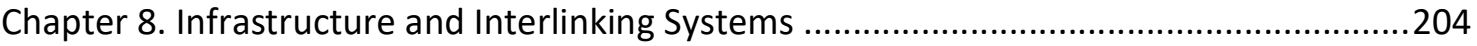

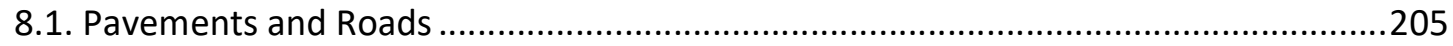

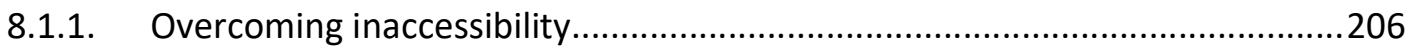

8.1.2. Safety concerns and additional physical cost ..............................................209

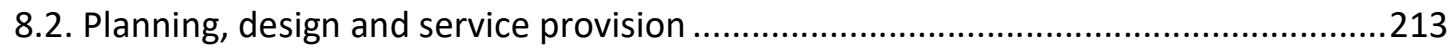

8.2.1. Inadequate provision of parking and access ..............................................214

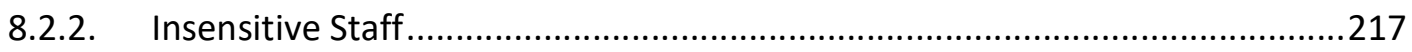

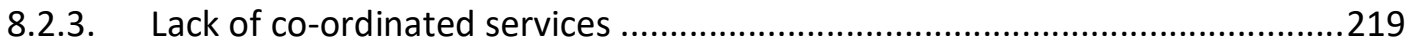

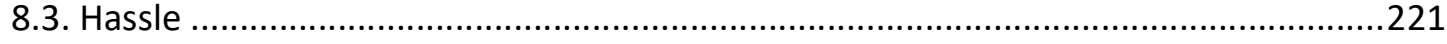

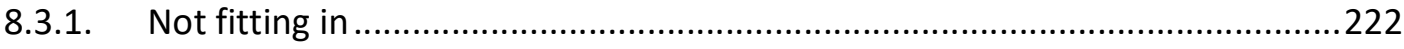

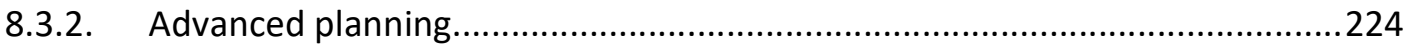

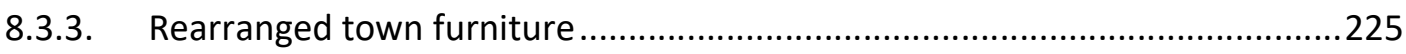

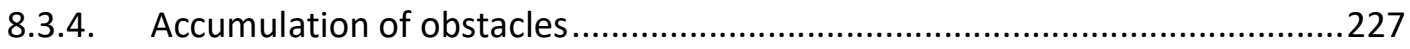

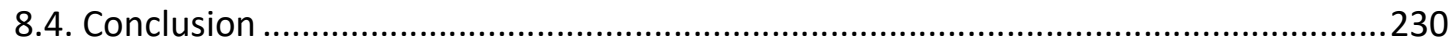

Chapter 9. Outliers and demographic considerations ...................................................233

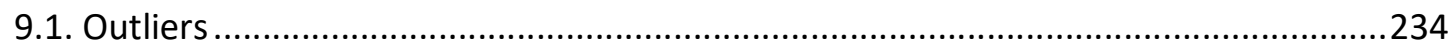

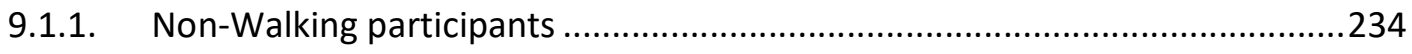




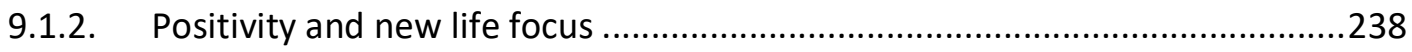

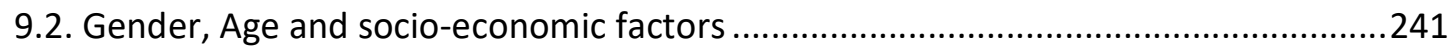

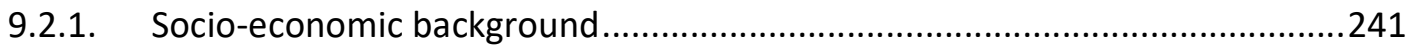

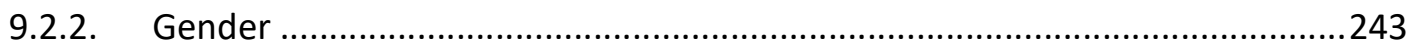

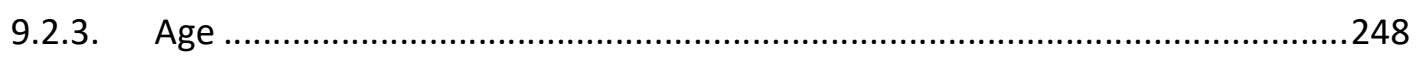

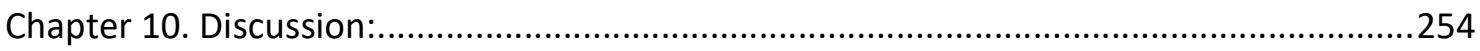

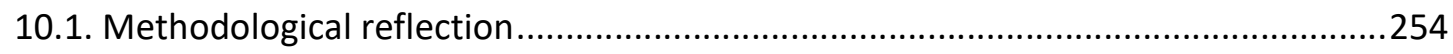

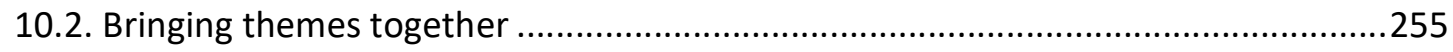

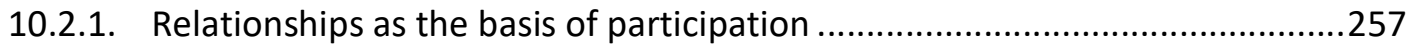

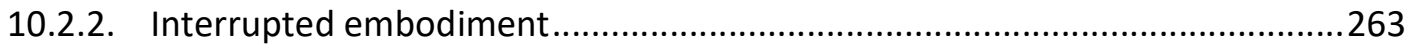

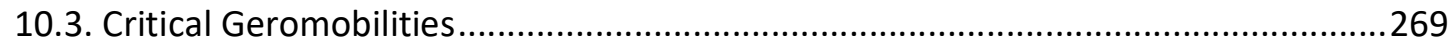

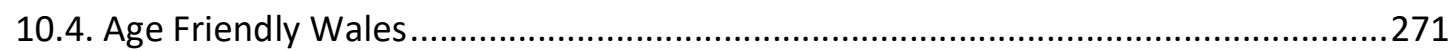

10.4.1. Role of policy and planning in the Feedback loop .....................................277

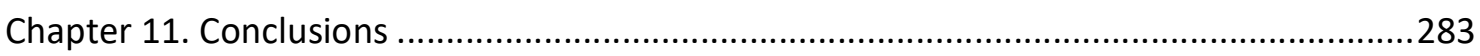

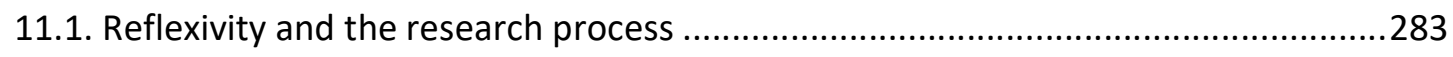

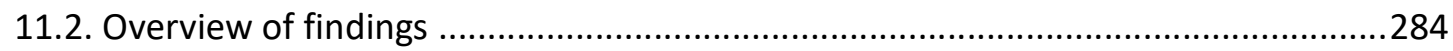

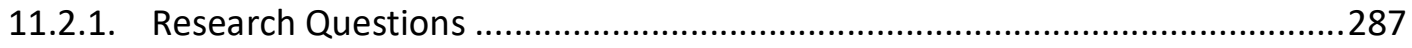

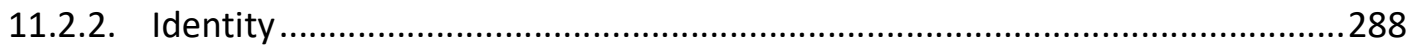

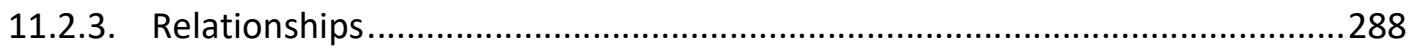

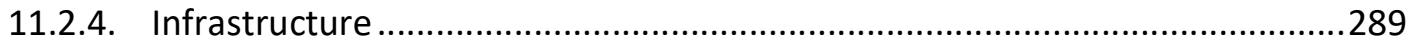

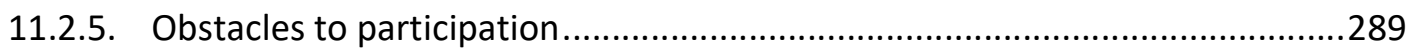

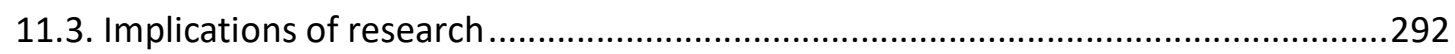

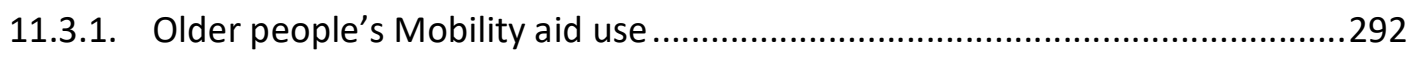

11.3.2. Age Friendly Cities and Communities Initiative .........................................293

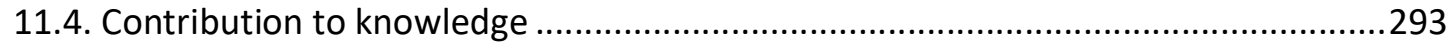

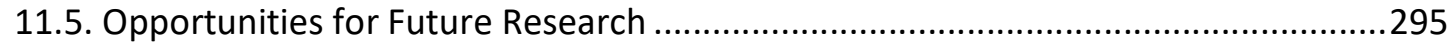

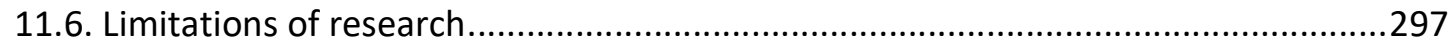

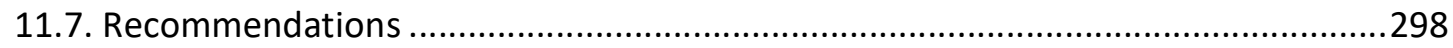

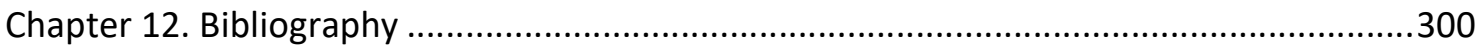

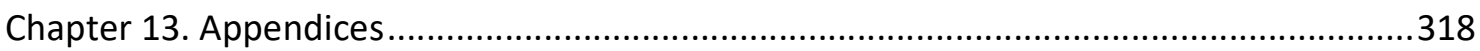

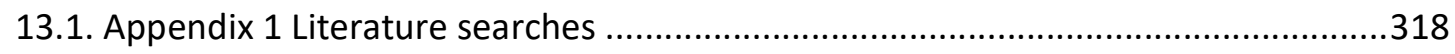

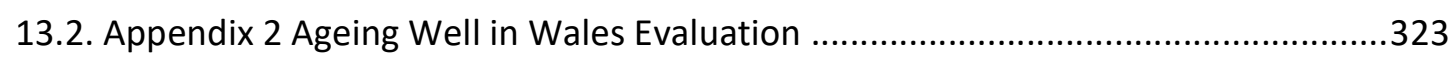

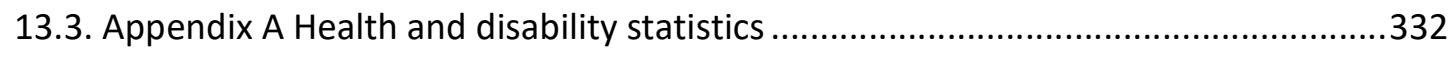




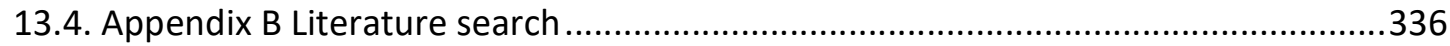

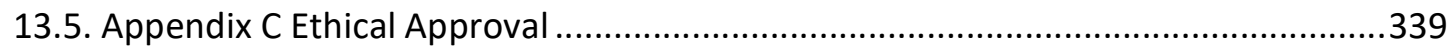

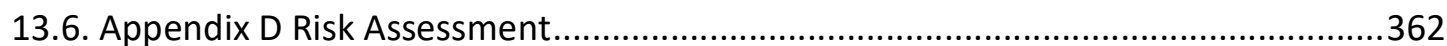

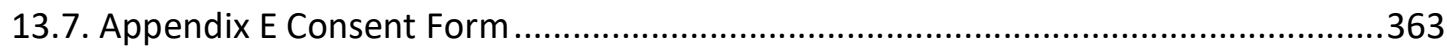

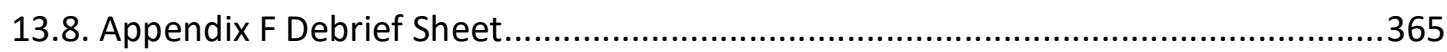

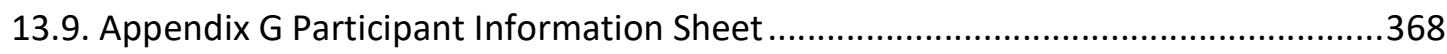

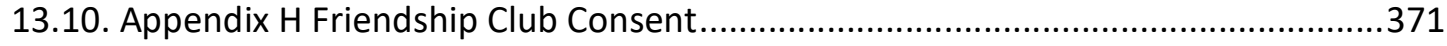

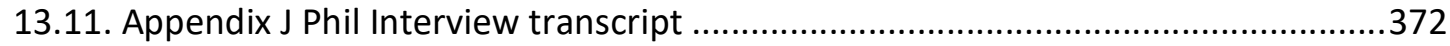




\section{Table of Figures}

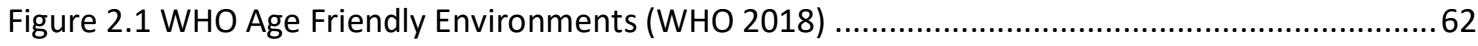

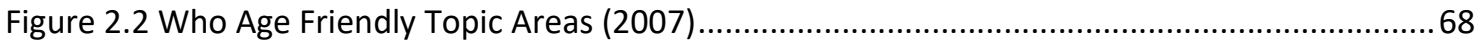

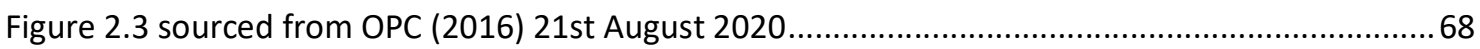

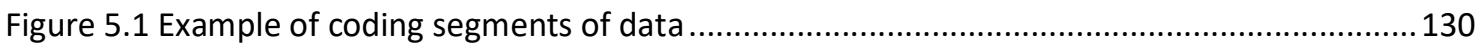

Figure 5.2 Focused coding and resulting themes ........................................................................ 132

Figure 5.3 Coding example within theme of identity ..................................................................134

Figure 5.4 Example of data coded to independence then identity ................................................. 135

Figure 5.5 Segment coded to embarrassment within dignity in the theme of identity .....................135

Figure 5.6 Segment coded to stigma under the theme of identity ................................................. 137

Figure 5.7 Segment coded through interpersonal aspects to relationships.......................................138

Figure 5.8 Segment coded to relationships through carer concerns................................................. 141

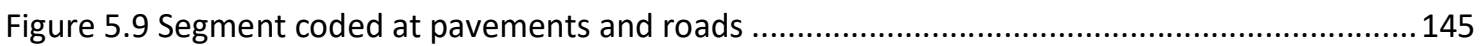

Figure 5.10 Coding via impractical or incompatible aid to planning, design and service provision ... 147

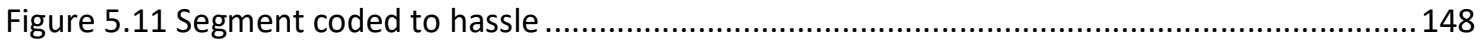

Figure 5.12 Each theme represents a different kind of obstacle set ...............................................149

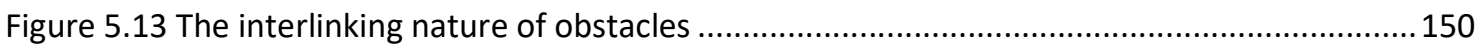

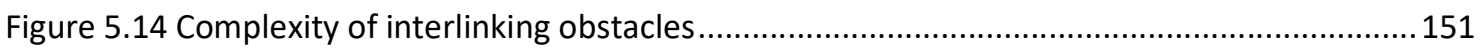

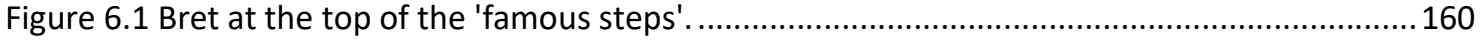

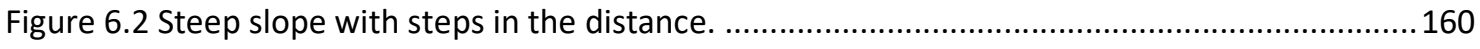

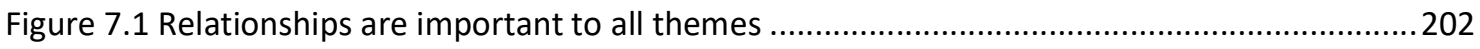

Figure 8.1 Map showing walk from Theresa's front door to garage door ..........................................207

Figure 8.2 Photo of pavement layout outside Theresa's garage door.................................................207

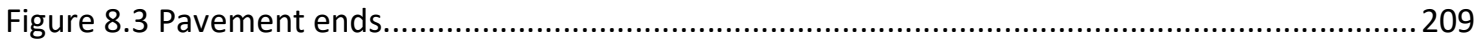

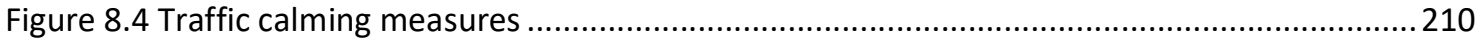

Figure 8.5 Cracked pavement \& Theresa walking on the cracked pavement ..................................212

Figure 8.6 Distance walked by Theresa along cracked pavement as shown in photo.........................212

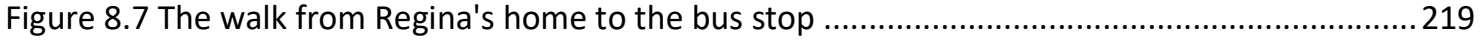

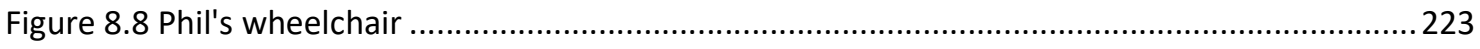

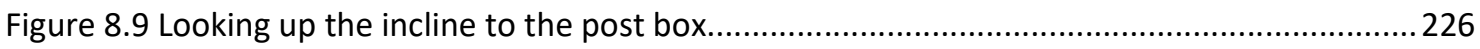

Figure 8.10 Looking down the hill to the ATM that displaced the post box......................................226

Figure 8.11 Pavement terrain after crossing the road from the car park.........................................227 
Figure 8.12 Cable ramp.

Figure 10.1 The intersection of relationships in analysis and results .......................................257

Figure 10.2 Feedback loop of obstacles to mobility aid use ....................................................259

Figure 10.3 Situated reality of feedback loop consequences for individual..................................261

Figure 10.4 Who Age Friendly Topic areas (2007) as per Age Friendly Wales website Nov.2018 .....272

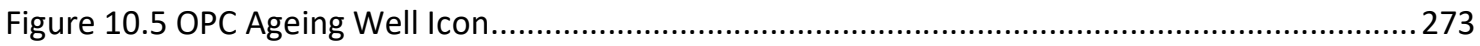

Figure 10.6 WHO Age Friendly Environments Europe Icon ....................................................2 273

Figure 10.7 Current online version of 'Making Wales a nation of age friendly communities' ..........274

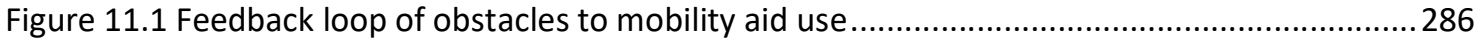

Figure 11.2 Situational reality of older people using mobility aids. ...........................................291

\section{Tables}

Table 4.1 Participation information ................................................................... 111

Table 4.2 Order of participation and notes of interest...........................................118

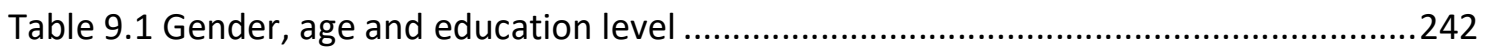

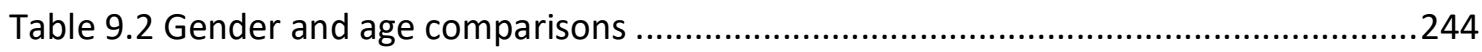

Table 9.3 Age, walking and mobility aid data ....................................................245 


\section{Chapter 1. Introduction and Background}

The increasing ageing population is a phenomenon highlighted and investigated by many disciplines in the social sciences. Transport studies, environmental planning, human geography, political science, sociology and gerontology are just some of the disciplines invested in understanding the impacts of ageing populations on society, place, systems and services (Aguiar \& Macário, 2017; Chaney, 2013; Imrie, 2012; Katz \& Calasanti, 2015; Murray, 2015; Musselwhite, 2010, 2018; Schwanen \& Páez, 2010). The focus on global and local ageing populations has been taken seriously by governments, in line with Healthy Ageing guidance from the World Health Organisation (WHO, 2016, 2018, n.d.). Healthy Ageing is aligned to creating an environment and opportunities to enable olderpeople to be whom and do what they value throughout their lives. The concept refers to functional ability rather than absence of illness, as many older people live with conditions that, with control, have little influence on their wellbeing ${ }^{1}$. Relating to having the necessary capabilities to enable continuation of chosen activities and lifestyles, functional ability includes a person's ability to meet basic needs, make decisions, build and maintain relationships, be mobile and contribute to society (WHO, n.d.). Age Friendly Environments continues to be a priority for the next decade of WHO work (WHO, 2017).

This research project explores the experiences of older people in Wales who use mobility aids, to understand the extent to which the Healthy Ageing and Age Friendly concepts, emerging from the World Health Organisation and subsequently Welsh Government, reach and support the everyday experiences of this population. The premise of functional ability is examined not as an academic or policy concept per se. That is to say that I did not ask people about their experiences of age friendliness, rather I set out to understand how policies intended to provide age friendly environments in Wales are experienced by older people use mobility aids. Are older people who use mobility aids able to function in the terms outlined by those of functional ability, healthy ageing and particularly age friendly

\footnotetext{
${ }^{1}$ Well-being is a contested area and often defined differently by various disciplines and areas of research and policy. Well-being as a term and concept can also be interchangeable with or an aspect of Quality of Life. These debates, definitions and conceptualisations are beyond the scope of this research. I have taken a broad approach to the term well-being as meaning being generally positive about emotional, social, economic and physical state.
} 
aims? As Age Friendly Communities is the guidance related to community and environmental factors, this is the area of Health Ageing that most impacts upon mundane, routine matters of daily life.

To establish research involving mobility aids, it is important to define the meaning and use of the mobility aid. However, mobility aids are difficult to define, not least because reference to them is not always consistent. Sometimes the terms mobility device, assistive device, or assistive technology (AT) can be used instead of or interchangeably with the term mobility aid. Assistive technology also incorporates 'aids for daily living' such as toilet seats, special water taps and epilepsy monitors, meaning that mobility aids can be assistive technology, but not all assistive technology is a mobility aid. Some sources describe mobility aids as devices that aid walking or improve the mobility of someone with a mobility impairment (Disabled World, 2018; Leonard, 2017). A United States Government website also directly defines mobility aids as items to help a person walk or move when disabled or injured (Medicine Plus, 2016). The term mobility aid is a more elusive term to define within health criteria in the United Kingdom (UK). The UK National Health Service (NHS) website provides information about walking aids, mobility scooters and wheelchairs (NHS, 2018). Literature in this arena mostly refers to aids as assistive technology, but is often concerned with aids for daily living as well as, or rather than mobility aids. Mobility aid is the term used throughout the thesis, for consistency, clarity and suitability. As will be discussed in greater detail in the methods section (Chapter 4) of the thesis, the inclusion criteria for mobility aids within this study was any item that aids mobility as defined by participants.

At the beginning of the research process, the focus was on the ability to be mobile with an additional concern for contributing to society. Those who have mobility difficulties are more likely to be socially isolated and unable to continue with social activities or be mobile outside of the home (Aguiar \& Macário, 2017; Mackett, 2018; Musselwhite, 2018; Ormerod et al., 2015). However, as the research evolved it became evident that all of the aspects discussed under functional ability are relevant to the research and inextricably linked to each other. Movement and communication are fundamental to building and maintaining relationships and contributing to society. Inability to move freely by one's own volition can lead to concerns of personal choice and decision-making being removed. Individual, social, psychological, emotional and physical obstacles were found to be interdependent and 
inform each other. My own experience as carer for my chronically ill husband has led to my understanding that while mobility aids are designed to enable mobility, they do not always create social inclusion, or indeed recreate the ability to continue with previous practices. It is to my own understandings and the inception of this research project that we turn next.

\subsection{Inception of research project}

In order to understand the inspiration and inception of this project, it is necessary to offer an account of my professional and personal history. The project is a culmination of disparate but intersecting experiences of both academic and personal life; from understanding the disruption to social communication and movement that mobility aid use can bring combined with an academic, inquisitive mind.

\subsubsection{Personal and Professional influences}

My first academic interest was psychology, having previously worked in human resources environments, coming to degree level study at the age of 30 . For personal and financial reasons, I was unable to complete the degree at that time, so my ambition was shelved. The wish to complete a degree began again when my husband became chronically ill. Studying criminology and psychology with the Open University allowed me to pursue my ambition once again, whilst caring for him. I have a lifelong interest in why some people become criminals and was interested to pursue this path. Whilst completing the degree, I became interested in marginalisation of problem populations. On completion of that degree, I decided to pursue further education studying at Swansea University for a master's degree in social science research methods. The structure of the master's degree course allowed students to pursue their own research interests, and as such I continued to follow the interest I now had in marginalisation and disability, mobility and specifically the use of mobility aids. The assigned supervisor for my dissertation on this project also happened to specialise in older people's transport, which is where I was introduced to the application of my interests to older populations.

A more personal reason for the focus on mobility aids in the built environment is that I am the sole carer for my husband who is a walking stick and wheelchair user, meaning I experience alongside him the changes that occur with mobility aid use. I had noticed over 
three or four years that we had gone from being a couple who, though not endlessly social, went out sometimes, to not even going shopping together because of the difficulty my husband was having with mobility. I did not set out thinking that all mobility aid users would have the same issues as my husband, but it did set me thinking about the differences mobility aid use might make to social activity for older people. Increasingly, my academic interests aligned with the personal, and I became interested in the ways embodiment and disability might be included in the ways ageing and gerontology are researched. Older people are increasingly likely to incur mobility difficulties or a mobility restricting disability (Christensen, Doblhammer, Rau, \& Vaupel, 2009; Mackett, 2018) with the potential requirement to use a mobility aid, making older people who use mobility aids a significant and growing portion of the population of Wales. This study was thus born out of a combination of personal experiences and research knowledge, leading to interest in the ways older people experience using mobility aids, aiming to understand daily practices and routines to explore any barriers to participation in day-to-day routines.

\subsection{Background, context and scope of research}

As a research project within the scope of understanding the meaning and context of an 'Age friendly Wales', it is important to contextualise the concepts of Age friendliness, and wider ageing policy contexts in Wales. Age Friendly Cities (and/or communities) is a World Health Organisation (WHO) initiative within the wider Healthy Ageing Action Plan and has been taken up in many countries and regions. Being the WHO, information on their website is advice rather than policy, however, they provide a framework of information sharing, data collection and best practice. The Dublin Declaration on Age-Friendly Cities and Communities in Europe 2013 was a document signed during the European Union Summit on Active and Healthy Ageing in 2013. The declaration establishes commitments to develop, measure and benchmark age friendly cities and communities (WHO, 2014). Regions sign up to the declaration to work towards and gain age friendly accreditation which acknowledges an environment which values and plans adequately for older people. This is particularly pertinent because of a large proportion of older people with chronic illness and/or disability in Wales. 
The focus for this thesis is on mobility aid use in the built environment with an age range of fifty and above. The age range was chosen because it reflects the age focus of the Assembly Government of Wales 'Age Friendly Wales' criteria. It is difficult to ascertain definitive statistics regarding the population of mobility aid users over 50 in Wales. This is largely because data is collected in terms of working age which spans 16-65 and 65+, however combining knowledge from different sources has helped to conceptualise the size of population concerned. Using the latest population estimate for Wales released by the Office for National Statistics (ONS, 2019) and information compiled on my request by a member of staff at the ONS, (Appendix A) approximately $8 \%^{2}$ of the population of Wales falls into the categories of (i) being over 50 and also (ii) potentially using a mobility aid. Alternatively, 2011 census data (ONS, 2018) indicates $17 \%$ of people in Wales aged 50+ reported a mobility restricting disability. There is a large disparity between the two figures, and as there are not any specific sources of data regarding mobility aid use it is difficult to assess, especially given the additional tendency for some older people to omit mobility aid use or mobility difficulties from their responses. It can be said, though, that a proportion of older people in Wales have day to day activities restricted by a health problem, disability, or reduced mobility. An age friendly Wales must include mobility supportive criteria in order to be fully inclusive and effective. Such criteria should reflect and address the everyday experiences and concerns of older people in our communities.

The project was designed to explore the ways older people feel about using a mobility aid and whether they feel or experience planning, services and the built environment cater for their needs, but without directly asking those questions. Family members and carers views were also sought for two reasons. Firstly, because being socially and emotionally connected to the mobility aid user, they had an insight into any social and emotional changes that may have occurred with a person's uptake of mobility aids. Secondly, particularly when the carer is also a family member as is often the case, they are also affected by the mobility aid. It could be that mobility aid use can be an obstacle even within the carer or familial relationship, or as some literature suggests uptake of a mobility aid by one member of a

\footnotetext{
${ }^{2}$ Inspection of Appendix A will show that I have made a judgement on which health/disabilities listed were likely to require use of a mobility aid.
} 
couple could lead to social isolation of older couples (Giesbrecht, Miller, \& Woodgate, 2015). This could be especially true where there is comorbidity and the complexity of a carer with illness or senescence. A holistic and mundane, everyday sense of daily mobility aid use from the perspective of the user was sought. This study was designed to find out whether there are barriers to inclusion and understand the nature of such barriers, along with how they might be located within the wider context of ageing and within the parameters of everyday routines. Understanding older people's own perspectives of their use of mobility aids being the primary focus.

\subsection{Research aims, objectives and questions}

The research aims, objectives and questions are set out below to provide an overview of the intentions of the research project.

\subsubsection{Aims}

The predominant aim of this research was to explore and describe mobility aid use by older people in Wales within their routine experiences in the built environment, within the context of Age Friendly Wales policies. A further aim was to consider if there are barriers to social inclusion or participation for mobility aid users, and establish the nature of any obstacles.

\subsubsection{Objectives}

The research objectives were as follows:-

- To describe older people's mobility aid use within daily routines and activities,

- To explore the meanings older people attach to mobility aid use,

- To explore any changes to participation that have occurred since each participant began to use their mobility aid,

- To identify and discuss the individual, relational and structural factors involved when older people use mobility aids, including any obstacles and barriers faced,

- To evaluate age friendly policy on national and local authority levels to understand how mobility aid use is assimilated into policy, planning and services. 


\subsubsection{Research Questions}

How do older people relate to mobility aids and how useful are mobility aids in navigating and enabling everyday routines and scenarios? This overarching question includes the following sub-questions:

How do older people relate to their own mobility aids?

How do older people feel about or consider mobility aids in general?

How do older people feel about themselves when needing to use a mobility aid?

How do carers feel about their loved one's use of a mobility aid?

How do older people feel the built environment incorporates the need for mobility aids?

How well do older people feel their mobility aids enable them to continue with their everyday routines and lifestyles?

If there are obstacles to participation as previous literature suggests, what are the obstacles to participation?

How effective are age friendly policies in addressing the needs of older mobility aid users?

Exploring the everyday use and experiences of mobility aid use by this population was chosen for a few reasons. Firstly, because of a high proportion of both older people and those with mobility restricting disabilities in Wales than in other United Kingdom countries according to the 2011 census (ONS, 2018). There are not any reliable sources to indicate how many mobility aid users there are but the mobility restricting disability responses are a good guide (ONS 2011). Secondly, although the mobility needs and wants of older people are often discussed in transport, sociology, gerontology and geography domains (e.g. Aguiar \& Macário, 2017;(Holstein, 2011), hardly any focus is given to everyday, routine movement of older people who use mobility aids. The third reason is that mobility aids are considered mobility enhancing and therefore maintain continued social participation, however the 
social model of disability (e.g. Oliver, 2009; 2013), planning (e.g. Hamraie, 2017) geography (e.g. Gaete-Reyes, 2015) and disability studies literature (Mazurik, Desjardins, de Grosbois, Poldma, \& Gelech, 2014) suggest social and structural obstacles are faced by people with mobility difficulties or those who use mobility aids. A further reason for focussing on mobility aids is that while older people are more likely to live with chronic illness, mobility issues or generally decreased mobility through reasons associated with ageing (Gagliardi, 2007; Giesbrecht et al., 2015; Mackett, 2018; Murray, 2009; Schwanen \& Páez, 2010), there is little focus on mobility aids in any of the official documentation surrounding either Ageing Well in Wales or the World Health Organisation Age Friendly Cities or Communities initiative.

\subsection{Contribution to existing knowledge}

The contribution to existing knowledge in this research is the focus on mobility aids, and the processes involved in older people's experience of using them in the built environment. The diverse array of literature outlined in Chapter 2 pertains to mobility, older people's mobility, and general mobility aid use, including the social, physical, and psychological facets thereof. However, there is scant literature regarding the experience of mobility aid use in terms of any processes required to continue usual routines and practices. Therefore, current literature lacks any outline of the processes involved in older people becoming adept at incorporating mobility aids into their daily lives and routines..

The mobilities paradigm highlights the role of movement as a vital aspect of social life and participation (Elliott, 2010; Peace, Holland, \& Kellaher, 2006; Sheller \& Urry, 2006; Urry, $2000,2007)$, thus indicating the importance of mobility and accessibility in social inclusion and continuation of daily practice. The disabling aspects of environment have been discussed in current literature within urban studies, human geography and disability studies and gerontology (Hamraie, 2017; Oliver, 1984, 2009, 2013; Pfeiffer, 2002) to name a few. The relationship between mobility and reduced social participant or social isolation have also been researched (Barlew, Secrest, Guo, Fell, \& Haban, 2013; Korotchenko \& Hurd Clarke, 2016; Krantz \& Egard, 2016; Lucas, 2011; Mazurik et al., 2014; Milner \& Kelly, 2009; Newton, Ormerod, Burton, Mitchell, \& Ward-Thompson, 2010; Raymond et al., 2014), as have the repercussions of mobility aid use on identity and other psychological and 
psychosocial impacts such as stigma (Peace, 2013; Rowles, 1978; Rowles \& Bernard, 2013). The focus of this study on older people's use of a mobility aids as tool for navigating the built environment enables consideration of the processes and strategies used to navigate local areas, from the mobility aid users own viewpoint, and in relations to age-friendly policies. While many of the issues researched within this study have been comprehensively identified and researched within different disciplines via various methodologies, they have not done so from this perspective or with the aim of understanding mobility processes, and therefor to catalogue accessibility or inaccessibility, obstacles, and the cost of overcoming any obstacles.

Bringing together the disparate disciplinary approaches and aspects of research as ways to study the experience of practicing mobility when using a mobility aid contribute to existing knowledge. Utilising walking interviews within the theoretical backdrop of both mobilities and critical gerontology brings focus to the movement and accessibility afforded to older people using mobility aids as tools to navigate their local environment. These different approaches are intermeshed as ways of recording and understanding experience, brought together in this study to examine the ways practising mobility may be enhanced or inhibited by planning and policy, social and physical structures, attitudes and beliefs. A multidisciplinary approach is employed, in order to begin to document and describe any obstacles that are experienced.

Additionally, in order to assess the experience of mobility aid use in line with WHO Age Friendly Communities guidance, and evaluation of the interpretation and implementation of ageing well and age friendly directives since committing to the Dublin Declaration of Age Friendly Cities, has been undertaken. Literature outlining the plans each local authority has for older people, and the provision for mobility difficulties, in particular mobility aids has been evaluated. From this, the research establishes the needs and wants of a sample of older people who use (or should use) mobility aids, and compares this to policy and planning provision to establish a picture of what obstacles exist and how they may be tackled through planning and policy provision. 


\subsection{Structure of thesis}

The thesis is structured in 11 chapters, commencing with this introduction. Chapter 2 presents a review of literature which is wide ranging. Starting with an explanation of the scope and purpose of such a comprehensive appraisal of disparate areas of research followed by an overview of literature relating mobility aids and their meaning within this research, along with current understandings of their use. Literature regarding both ageing and disability is explored, incorporating models of disability to give context to mobility aid use within understandings of disability and how such understandings apply to ageing and older people. Further expansion of understanding mobility aid use by older people is made utilising the concept of embodiment. Stigma is also examined as ageing, disability and mobility aid use are associated with embodied changes and traits that may invite stigmatisation.

The next section of the review examines literature regarding planning, policy and infrastructure and how these focus on older people, with a specific focus on mobility aid use. Following on from this, the final section provides examination and analysis of the World Health Organisation Age Friendly Cities work, along with The Dublin Declaration on Age Friendly Cities 2013 and Age Friendly Wales upon which Ageing Well in Wales policy frameworks are based. Alongside this is an overview of the way different authorities across Wales interpret and implement the guidelines.

With an understanding of current literature relating older people, disability and mobility aid use by older people, chapter 3 outlines the theoretical framework of the study. Utilising ethnographic grounded theory methods, this qualitative study uses constant comparative analysis to understand accounts and observations. These processes are outlined in this chapter, along with an outline of the theories underpinning my understanding of the concepts involved, the sensitising concepts of the study. While grounded theory is inductive, all researchers have a starting point and established concepts that inform their world view (Charmaz, 2014) . Within the sensitising concepts are theories of critical gerontology and the mobilities paradigm which are then presented in a combined form as critical geromobilities, all of which informed the study and my own understanding of the research and concepts within it. Ethical considerations conclude Chapter 3. 
Chapter 4 details the methods used in research design and data collection, along with ontological and epistemological considerations, with Chapter 5 , summarising the process of analysis and coding, refining and finalising themes, along with an outline of each theme and sub-theme. This chapter also highlights the complexity and interconnectivity of the themes representing obstacles and phenomena involved in mobility aid use by older people. The next three chapters, 6, 7 and 8 go on to explain the findings in greater detail. Chapter 6 explores the theme of Identity, with subthemes of Independence, Dignity and Stigma. Stigma intersects with Relationships, which is the subject of Chapter 7. Subthemes in Chapter 7 moves from intimate to public relationships starting with Carer concerns, moving to Discord or disagreement, and Feeling like a burden ending with the more public aspect of Relationships with place. In line with understanding interaction with place, Chapter 8 Infrastructure and Interlinking systems examines infrastructure, planning and service provision with subthemes of Pavements and roads, planning design and service provision and Hassle, which explore infrastructural processes of inclusion or exclusion of older mobility aid users.

Discussions are carried out over 2 chapters. Chapter 9, 'Outliers and demographic considerations' firstly discusses outlying or unexpected results. These are discussed in the context of the other findings, alongside gender age and socio-economic factors which form the second half of the chapter. Chapter 10 'Discussion: Bringing themes together' discusses the findings as a they relate to policy and practice, outlining relationships as a central mediation process of participation and providing a Feedback loop model of obstacles to mobility aid use for older people. The chapter also discusses how embodiment is interrupted in a specific way by mobility aid use. Notions of interrupted embodiment led to the ways in which construction of a critical Geromobilities perspective could assist in understanding the role of policy and planning in older people's mobility aid use. This understanding could help better disseminate the values and crosscutting context of Age Friendly Wales and Ageing Well in Wales Policy initiatives.

Chapter 11 concludes the presentation of this research thesis with an overview of the findings related back to the research questions, and how obstacles to participation have been described and understood. The chapter goes on to outline the implications of the 
research, contribution to knowledge and opportunities for further research. Limitations of this research are discussed followed by recommendations emerging from the findings. 


\section{Chapter 2. Literature Review}

The literature reviewed here covers a wide variety of subjects and perspectives. Beginning with an overview of the types of subjects examined regarding mobility aid use, the review provides an overview of the pertinent academic debates and status quo. Literature searches including terms of "mobility aids" or "assistive technology" and "ageing" or "older people" (see appendices 1 and B for more details of literature searches) returned results mainly aligning with healthcare and the medical model of disability, which as we will see below (section 2.4), dominates many discussions of mobility aids. This medicalised approach does little to incorporate social or environmental factors in mobility aid use. Mobility aid literature in the healthcare domain focuses on areas of use such as measurement of gait (Brach, Studenski, Perera, VanSwearingen, \& Newman, 2007), falls and stability of walking aid users (Costamagna et al., 2017; Gell, Wallace, Lacroix, Mroz, \& Patel, 2015), or the reliability of tools to measure the concepts of stability or quality of life (Jette \& Haley, 2005; Muir-Hunter \& Montero-Odasso, 2016). From the perspective of occupational therapy and mobility aid provision, outcomes are measured in terms of independence for user but do not tend to consider the impact of mobility aid use on the individual, social or family life or any carer (Hammel et al., 2013). This means that any social, psychological, emotional or physical implications or costs involved in uptake of mobility aids by older people are not well understood.

Establishing current understanding of social, emotional and psychological aspects of older people using mobility aids led to an investigation of models of disability.. Medical or social understandings of mobility aid use required appraisals of social and medical models of disability because of the different ways of understanding and conceptualising disability, and therefore mobility aid use. Assessment of disability and mobility aid use also incurs the need to understand embodiment and any changes to the body involved in or caused by mobility aid use, senescence or disability is examined. Stigma, as a potential outcome of changes to embodiment, ageing, disability or mobility aid use has also been reviewed here. In the latter part of the review, literature examining obstacles presented within infrastructural domains of transport systems and policy are explored. 
The factors involved in older people's use of mobility aids in the built environment encompass many areas and disciplines. From a conflation of terms such as assistive technology, mobility technology and mobility aids within medical and rehabilitation literature, to consideration of practical and social exclusion understanding the daily experience of mobility aid use by those aged 50 or over is complex and multidimensional. Disability and ageing are rarely studied or discussed together (Jönson \& Taghizadeh Larsson, 2009; Minkler \& Fadem, 2002; Phillips et al., 2010; Raymond et al., 2014). Academic accounts explicitly or implicitly portray ageing successfully as a function of non-disabled people which is further compounded by the lack of visible impairments or mobility aids in advertising or media representations. Ageing with a disability has been implicitly demarcated as unsuccessful (Taghizadeh Larsson, 2013), and ageing research and policy tends to overlook disability (Jönson \& Taghizadeh Larsson, 2009; Minkler \& Fadem, 2002; Raymond et al., 2014). Which is assessed within literature examining infrastructural domains policy, planning and transport research. Moreover, an assessment of Age Friendly policy approaches is included in the literature review to provide context to the infrastructure and policy setting in Wales.

\subsection{The scope and purpose of the literature review}

The literature reviewed in this chapter was reviewed predominantly before data collection began to inform the research of existing knowledge. The study employs grounded theory methodology, which invites researchers to avoid seeking to fit social phenomena to existing theory, although there are debates around how this might be achieved. Kathy Charmaz (2014) highlights disputes within grounded theory literature around the process and timing of a literature review because there is a danger of viewing data through such a lens of previous ideas and theorisations, known as 'received theory', which can result in data being forced into pre-existing categories. Grounded theory processes incur a need to review literature continually throughout the data collection process. Some concepts and literature of interest emerged from the data that had not already been studied at the literature review stage. Although it is customary to present all literature encountered in the literature review, I have presented some of the literature pertaining to concepts arising from data throughout the thesis. I chose to present the literature in this way for clarity and transparency in the 
methods and reasoning I have taken in analysing data. Where this occurred, the literature is included within the analysis and relevant chapters. For example, dignity was not an aspect of literature that was studied before data collection and analysis commenced, so does not form part of the literature review chapter. Literature regarding dignity is referred to through the emergence of the sub-theme in Chapter 5 within the analysis of data, thus reflecting the process of grounded theory. To gain a contextual understanding of older people's mobility aid use, and the current knowledge therein, literature searches were conducted using the search phrases "mobility aids" and "older people" with substitutions for these phrases such as assistive devices or assistive technology and older adults or ageing (and aging) which returned disappointingly few results. Applying selection criteria of focusing on qualitative data that included mobility aid users lived experience in outdoor environments, results were further lowered (see appendix 1). As research progressed and themes began to emerge, searches were conducted in these ways to begin with, as appendix 1 indicates, with searches such as "mobility aids" + "older people" + independence, the same confounding factor that the inclusion of the term mobility aid either brought about the inclusion of medical and rehabilitation literature, which were excluded if they did not represent user experience outdoors, and they rarely, if ever did. Alternatively, inclusion of the term mobility aid drastically reduced results.

The term mobility aid was imperative as this thesis focuses on the impact of mobility aid use, but as described above, drastically reduced search results to the point that often searches did not produce relevant information. Therefore, searches would be conducted in other ways. It sometimes involved focusing on items that the first stage of the literature review highlighted, following reference trails from document to document by finding a relevant article or chapter, then following up on references within that piece, or work that had cited it. Other times literature was found by seeking understanding of the focus of the particular theme (such as planning and design) then seeking some relevance to older people and/or mobility aid use. Another method of searching literature was to identify existing themes in literature relevant to older people's navigation of the built environment. An example of this would be older people's well-being within transport and planning literature to seek out any mention of mobility difficulties and specifically mobility aid use. Within the 'Infrastructure and interlinking systems' theme particularly, but also both other themes, 
themes partly emerged because of literature I had already read and in this way, my analysis and reference to literature was cyclical.

A wider contextual approach to the multidisciplinary a multi-disciplinary nature of this research project, the literature searched for this project was wide ranging. At the start of the project, general literature relating to both theories of ageing and critical gerontology were searched, alongside the mobilities paradigm. Gerontological theories, and particularly critical gerontology are relevant to the understanding of everyday experience of older people and ageing, while the mobilities paradigm. The mobilities paradigm examines the role of movement in lived context. While transport studies or planning contexts study modes of movement in the built environment, the mobilities paradigm offers the ability to understand the role of movement in maintaining social life (Urry, 2007). Having searched literature regarding critical gerontology and the mobilities turn, searches moved on to ideas of disability and embodiment, both of which were highlighted by both mobilities and ageing literature. In turn, disability literature and embodiment led to the notion of stigma which was searched before the study commenced. Infrastructure, planning and policy and transport studies were searched to establish current knowledge of the built environment and provision for older people, especially those who use mobility aids.

Researching a project which has an experiential focus and multi-disciplinary nature meant that the scope of the literature relating to it was broad. The research project was aimed at describing and understanding the impact of mobility aid use on individual and social activity of older people in Wales as part of a wider Ageing Well in Wales initiative. Population ageing is a well-documented phenomenon that is increasingly discussed within transport and mobility fields (Aguiar \& Macário, 2017; Kaiser, 2009; Musselwhite, 2015, 2018; Musselwhite \& Haddad, 2018; Nordbakke \& Schwanen, 2014; Schwanen \& Ziegler, 2011). Chronic illness and senescence, the natural process of deterioration with age, both affect people increasingly with age (Giesbrecht et al., 2015; Jepsson Grassman \& Whitaker, 2013; Murray, 2009) so older people are more likely than their younger counterparts to experience mobility difficulties requiring the use of mobility aids, be it occasional through to everyday or continual use. The resulting potential for reduced mobility is linked to social isolation, loneliness, and negative health and well-being consequences (Aguiar \& Macário, 2017; Mackett, 2018; Musselwhite, 2018; Ormerod et al., 2015). Although around 17\% of 
the population of Wales are fifty or over with some kind of activity restricting disability, this crucial area has been consistently under-researched.

Literature pertaining to mobility aids was explored with a wide remit regarding the term mobility aid, and incurred the confounding issue of mobility aids being termed assistive technology by some disciplines and sources. The term mobility aid was preferable to this research because not all assistive technology aids mobility as outlined in chapter 2 . Mobility aids are used by those with physical disabilities, chronic illness or senescence which is sometimes referred to as age related disability. Within this research senescence refers to any age-related disability that incurs changes to behaviour, movement, practices or causes pain but is not necessarily thought of by the participant to be an illness or medical concern. That is the participant considers it to be an aspect of ageing to be expected. While senescence is only likely to occur in older people, disability and chronic illness can occur at any age. Disability literature incorporates chronic illness in that it discusses embodied mobility differences, but generally falls short of including age related mobility difficulties and disability, or older people with disabilities. Likewise, literature on ageing tends not to discuss disability. Literature rarely discusses disability and ageing together even though it is entirely possible to be both older and disabled. Failing to address both ageing and disability together fails to address the impact of disability within the growing ageing population. Moreover, embodied changes that come with senescence utilise the same kind of mobility aids in general as those with physical disabilities caused by other phenomena and illnesses. Embodied changes that accompany chronic illness, ageing and disability also have psychological and emotional impacts.

Reviews of disability literature were undertaken to understand the physical, social, psychological and emotional aspects of disability within society and social structures. These reviews led to the social and medical models of disability. Both models are explored within this review to understand the impact these models have had on individual and societal understanding of disability and impairment. The wider implications of disability with marginalisation and stigma are also considered within the scope of the review. While the research design utilises grounded theory and therefore makes no hypotheses about findings, disability studies literature does indicate physical, emotional and social obstacles to participation. Studies of disability also refer to stigma which forms a fundamental part of the 
literature review to enable understanding or existing social attitudes to mobility aid use by users and non-users.

While the research design utilises grounded theory and therefore makes no hypotheses about findings, disability studies literature does indicate physical, emotional and social obstacles. Mobility and mobility inequality are examined to interrogate the parameters and current knowledge of access to physical movement and social activity. Both areas are concerned with the ways people can move around and access important elements of social life which are implicated in well-being. Understanding movement and the ability to be mobile also incurs the use of transport and planning. Transport studies, planning and the design of the built environment are therefore relevant areas of literature that were examined. Reduced accessibility, due to inadequate planning, policy or social support may create, either consciously or subconsciously, a psychological interpretation that people who cannot access places and social arenas are intentionally excluded. All of these aspects, that is mobility, social inclusion, planning and the built environment, accessibility and addressing pejorative behaviour are addressed as part of the Ageing Well in Wales and Age Friendly Cities and Communities evaluation. To begin with, it is vital to define mobility aids and their use by older people both in terms of this study and wider contexts.

\subsection{Mobility Aids}

Mobility aids are tools of navigation for people with mobility difficulties and are vital to enabling access to social activity routines for many. As indicated within the introduction to this research (Chapter 1 ), the definition of mobility aids can be elusive, and crosses over with assistive technology (AT). Currently, literature regarding mobility aids tends to stem from healthcare domains such as occupational therapy and rehabilitation (Hammel et al., 2013; Meyers, Anderson, Miller, Shipp, \& Hoenig, 2002; Sonenblum, Sprigle, \& Lopez, 2012). Clinical insights into mobility aid use for specific illnesses (Foley, Prax, Crowell, \& Boone, 1996; Kader, Jonasson, Iwarsson, Odin, \& Nilsson, 2018) and medical specialisms such as ageing (Campbell, Reinken, Allan, \& Martinez, 1981; Navaratnarajah \& Jackson, 2017) are also prevalent, however these sources rarely includes social aspects of mobility aid use as will be shown below. Literature which explores social aspects or routine experience of mobility aid use tends to focus on wheelchair use. These sources are also explored, to 
establish current knowledge of mobility aid use in social context. The final section regarding mobility aid literature examines literature regarding older people's use of mobility aids.

Literature regarding day-to-day experience of all types of mobility aid or assistive technologies, is difficult to find. One pertinent article regarding older people's preferences for the design of streets discusses mobility aids. Whilst seeking to understand how the design and maintenance of streets relates to the mobility and independence of older people, this study reported 51 per cent of participants with some kind of mobility, vision or hearing difficulty, connecting mobility aid or assistive technology use with mobility and independence (Newton et al., 2010). As will be shown in the Chapters 5 and 6, independence is an important factor in older people's experience of mobility aid use. lezzoni (2003) utilised qualitative methodology to understand how people cope 'when walking fails' due to chronic illness, incorporating the experiences of people using a variety of mobility aids. Both of these articles highlight the importance of walking and mobility for remaining independent and socially active (Taghizadeh Larsson, 2013). Many studies examine the importance of independence and social activity while exploring the use of one type of mobility aid in particular contexts, often wheelchairs. There is a range of literature regarding wheelchair use and its impact on identity and social life, ranging from a focus on quality of life to wheelchair design, productivity and daily living to the stress incurred by wheelchair users (Barlew et al., 2013; Bromley \& Matthews, 2007; Bromley, Matthews, \& Thomas, 2007; Korotchenko \& Hurd Clarke, 2016; Krantz \& Egard, 2016). The views of wheelchair users within academic accounts confirm that using a wheelchair tends to disrupt life and equality of social interaction (Gaete-Reyes, 2015; Korotchenko \& Hurd Clarke, 2016; Krantz and Egard, 2016). Presence of a wheelchair singles users out as different and many studies point to the notion that disability is the reference point against which normality is valued. Conceptualisations of normal bodies automatically bring conceptions of deviance and vice versa (Barnes, 2002; Davis, 2017; Hansen \& Philo, 2007; Imrie, 2000; Krantz \& Egard, 2016; Pfeiffer, 2002). An examination of the lifestyle of active wheelchair users provides an example of interpersonal relationships being changed by wheelchair use. Using semistructured interviews of eleven participants between the ages of 25 and 52, the study found that the prop of a wheelchair seems to signify the user as an 'open person', somebody who can be addressed at will and who is also representative of all other wheelchair users 
(Mazurik et al., 2014). These sentiments are echoed in Chapters 6 and 7 whereby use of different kinds of mobility aid could affect both identity and social activity.

Research has shown that people who use mobility aids or live with disability often feel socially and practically excluded by the planned environment and by society (Barlew et al., 2013; Korotchenko \& Hurd Clarke, 2016; Oliver, 2013). Contextualisation of obstacles or interpretation of the reasons for or experience of social exclusion have been less forthcoming (Hansen \& Philo, 2007; Mazurik et al., 2014). A study in a comprehensive study of 120 wheelchair users by Bromley et al investigating city centre accessibility concluded that not only are physical obstacles problematic, but that disadvantage can also exist within the real or imagined environment of the self. That is to say there is a connection between accessibility, the individual's perception of accessibility, identity and confidence in navigating the built environment when using a wheelchair (Bromley et al., 2007). Negative meanings associated with wheelchair use alongside ableism as a network of beliefs can negatively affect self-esteem. Use of a mobility device can single the user out as being different further amplifying negative feelings meaning social connotations can be as disenabling as the physical environment. An unaccommodating physical environment compounds social aspects of mobility aid use in a feedback loop (Korotchenko \& Hurd Clarke, 2016).

Research considering the inclusivity of shopping environments features in research of wheelchair use, as shopping is considered both a necessary and social activity. Shopping malls and town centres are explored as sites of inclusion or exclusion so researchers seek to uncover the nature of any obstacles to inclusion (Bromley et al., 2007; Imrie, 2012; Mazurik et al., 2014; Williams, Hubbard, Clark, \& Berkeley, 2001). Interestingly, a study of the accessibility of retail environments using interviews regarding the food shopping experiences of wheelchair users in South Wales uncovered not only some of the aspects within the shopping spaces that might disadvantage them, but also that none of the 120 participants used public transport as it effectively excluded them (Bromley \& Matthews 2007). While the planning of retail environments and city centres is often researched by local authorities, this is carried out without substantive reference to disability or the needs of disabled people (see also the section 2.10 on Age Friendly Cities and Communities). This interconnection of physical accessibility, perception of self, others and environment, and 
infrastructural provision is reflected the feedback loop of obstacles to mobility aid use by older people presented in Chapter 10.

\subsubsection{Older people's use of mobility aids}

Older people are increasingly likely to be affected by mobility and accessibility difficulties because of the increased possibility of chronic disease or age-related disability (Giesbrecht et al., 2015; Murray, 2009; Schwanen \& Páez, 2010).The current study aims to describe and understand the impact of any resulting mobility aid use on individual and social activity, from an experiential perspective. Current literature exposes lack of mobility in older populations as being linked to social isolation and reduced health and well-being which can lead to loneliness, decline and even death (Aguiar \& Macário, 2017; Musselwhite, 2018; WHO, 2018). The term social isolation is not always clarified in literature, but generally refers to the objective state of having minimal contact with other people as opposed to the subjective state of loneliness (Wenger, Davies, Shahtahmasebi, \& Scott, 1996). Despite the importance of social and psychological aspects of well-being related to mobility aid use by older people, much of the literature remains medically focused.

Literature often relates to measurement of gait, falls and stability of older walking aid users (Brach et al., 2007; Costamagna et al., 2017; Muir-Hunter \& Montero-Odasso, 2016). Nonetheless some literature regarding psychological or social factors of mobility aid use by older people is available. One study exploring psychosocial factors of mobility aid use and how these might impact the ability to age successfully found some reduction in social participation among 96 participants aged between 45 and 97 . Although the design of this

study was mostly quantitative, using scales of self-reported psychosocial factors along with activity and participation information, interview data was included to gain a more in depth understanding of why some people do not use mobility aids (Martins, Pinheiro, Farias, \& Jutai, 2016). The psychosocial remit of the study and recruitment of participants with a wide variety of mobility devices is valuable, however heavy reliance on scales can overlook day to day use and routines that affect lifestyles and may have an impact on social activity. Other studies have also sought understanding of the goals and needs of mobility aid users, finding that uptake of a mobility device can depend on satisfaction and compatibility with the device with the device. Imposition of generic devices without consideration of lifestyle or 
preference can hinder social interactions and negatively impact self-esteem and independence (Boerema, Van Velsen, Vollenbroek-Hutten, \& Hermens, 2017; Martins et al., 2016).

Korotchenko and Hurd Clarke's (2016) work to understand the experience of ageing with a wheelchair recruited only powered wheelchair users. Design of space was found to be unaccommodating and thus a physical obstacle. Heavy doors, cluttered aisles, height of shelves and narrow checkouts were all signalled as problematic. Negative meanings associated with wheelchair use, as disability is cast with diminished status as a human, are also highlighted in a study of the embodied experience, practical and cultural considerations of ageing using a power wheelchair in Canada. Mobility devices were identified as a signifier of loss of independence and reduced perception of wellbeing. The qualitative interview methodology of this study also allowed for contemplation of the unsettling nature of living with a chronic illness which can, in and of itself, undermine the structures and knowledge of everyday life (Korotchenko \& Hurd Clarke, 2016). Research to understand the experience of powered wheelchair use, then, has indicated a complexity of physical and psychological factors informing the social status of users. Mobility is potentially facilitated not only by mobility aids, but also other environmental, individual and social factors.

\subsection{Gerontology and Ageing research}

From its inception as the scientific study of old age as declared by Elie Metchnikoff in 1903 through to contemporary theories of ageing, gerontology has extended beyond disciplinary boundaries (Martin \& Gillen, 2014). Through more than a century of research into ageing, various theories have been proposed and contested. This section examines the academic theories of ageing, from the emergence of theories of successful ageing to more recent notions of critical gerontology. Such theories of ageing form an important backdrop to the lives of older people because they inform policy, healthcare and planning approaches.

\subsubsection{Theories of Successful Ageing}

Ageing successfully, as discussed within this chapter, has been an enduring concept within social gerontology from Havighurst, (1961) declaring both his activity theory and Cumming 
and Henry's (1961 cited in Havighurst, ibid) theories to both be theories of successful ageing. Critical gerontology has critiqued successful ageing as based on value assumptions and studies ageing in isolation from other life events. The societal outlook that results from successful ageing and active participation discourses also fails to address intersecting factors such as gender, race, and lifelong or acquired disability. In other words, critical gerontology questions the validity of successful ageing outlooks (e.g., Holstein \& Minkler, 2007, Katz, 2015; Raymond et al., 2014). This successful and/or active ageing focus within policy frameworks will be returned to throughout the themes and results of this research. Critical assessment of the meanings and lived reality of concepts such as being active, what is successful and how equal is access to success, movement or activity are questioned Theories of successful ageing are distinct from theories of the process of ageing because, by definition they make assumptions and offer accounts about value, that is, success and happiness. Disengagement theory and active ageing are contrasting theories of successful ageing. The former suggests that success rests upon mutual withdrawal between an ageing person and society, the latter that maintaining activities and attitudes of middle age as long as possible into older age is favourable for both individual and society (Havighurst 1961). Understood as a normal process, disengagement was proposed by Cumming and Henry in 1961 (Havighurst, 1961; Kastenbaum, 2016). However, the theory was critiqued as justification for removing older people from social situations. Furthermore, rather than older people choosing to withdraw, it has been argued that ageist structures of society forced older people to step away from significant roles and activity (Kastenbaum, 2016). Activity theory puts forward the opposite view that maintaining or finding substitutes for the activities of middle age as long as possible into later life provides for success in ageing, which Havighurst (1961) himself asserted was the view favoured by most gerontologists. Research interest following the activity theory/disengagement theory controversy has generally concluded that older people who remain active rather than disengaged tend to demonstrate higher morale (Kastenbaum, 2016). Successful ageing and activity theory have since been central concepts to ageing policy and research, often with the terms successful ageing and active ageing incorrectly used as interchangeable terms (Foster \& Walker, 2015; Raymond et al., 2014). More recent manifestations of successful ageing are likely to emanate from Rowe \& Kahn's (1987) conceptualisation of successful ageing which states 
that previous research and focus on ageing and age-related losses of function ignored the heterogeneity of ageing. By incorporating elements such as diet, exercise, psychosocial factors and personal habits as part of evaluating the ageing process, disease and disability could be anticipated, physical and cognitive function maintained, and social engagement continued. Thus, the distinction was made between usual ageing in which extrinsic factors contribute to ageing processes, or successful ageing, in which extrinsic factors are either neutral or positive in the ageing process (Rowe \& Kahn, 1987).

Beyond the distinction between usual and successful ageing, the paper recommends that gerontological research should seek to understand transitions in later life. Transitions that maintain function or regain function through physical or psychological adaption and with equal importance, transitions from usual ageing into disease and impairment (Rowe \& Kahn, 1987). Rowe and Kahn's (ibid) paper has been influential in many fields of research from geriatrics and gerontology to nursing, psychology and political science. The concept of successful ageing has become widespread and is used widely in gerontology, scientific, medical and research and public discourse (Bülow \& Söderqvist, 2014; Johnson \& Mutchler, 2014; Martin et al., 2015). The concept of successful ageing transformed approaches to ageing in research arenas and medicine. The resulting shift to preventative medicine and individual responsibility for ageing successfully has drawn many debates and criticisms (Baltes \& Carstensen, 1996; Bowling \& Dieppe, 2005; Bülow \& Söderqvist, 2014; Katz \& Calasanti, 2015; Martin et al., 2015; Minkler, 1996; Minkler \& Fadem, 2002) as notions of ageing processes could be managed through behaviour took hold.

One of the debates arising in the biomedical arena is the difficulty of establishing differences between normal (or usual) processes of ageing, processes of disease or other non-ageing process that nonetheless may occur whilst ageing. Geriatric opposition to successful ageing therefore claimed that if successful ageing could influence the ageing process, there could be no natural death, only manifestations of lifestyle or dietary indiscretions (Bülow \& Söderqvist, 2014). From a Psychological perspective, a model of successful ageing came from Baltes \& Baltes (1990; Bülow \& Söderqvist, 2014; Martin et al., 2015). Whereas Rowe and Kahn's (1987) framework highlighted the role of external factors such as environment and lifestyle, Baltes \& Baltes (1990) envisaged limits to or possibilities for ageing to be influenced by both external elements and internal cognitive factors. Their 
model of selective optimisation with compensation (SOC) is based on the premise that successful ageing equates to successful individual development across the life course, a process which incorporates individual and cultural variations, and psychological and emotional adaptive abilities (Bülow \& Söderqvist, 2014; Martin et al., 2015). The psychological approach to successful ageing, while not a direct criticism of Rowe \& Kahn's approach, incorporates intrinsic as well as extrinsic factors of ageing successfully.

Critics of successful ageing agendas note that the criteria and definitions of ageing they offer result in concepts of success and failure without consideration of factors such as lifestyle, choice and intersecting inequalities, and especially avoidance of disease and disability (Bowling \& Dieppe, 2005; Foster \& Walker, 2015; Katz \& Calasanti, 2015; Minkler \& Fadem, 2002; Stowe \& Cooney, 2015). Rowe and Kahn's model specifies successful ageing as ageing without illness or disease, so those with chronic illness or senescence, or who are ageing with lifelong disability could be construed as failures within this framework. Khan himself noted that the model could have unintentionally labelled the majority of older people as unsuccessful, although this reflected American culture in his view, rather than the concept (Kahn, 2002; Katz \& Calasanti, 2015). This is a highly contentious point particularly pertinent to this thesis, as those who use mobility aids could be considered a failure within the discourses of successful ageing, but also use tools (i.e., mobility aids) which outwardly signify this.

Overall, the use and promotion of active and successful ageing paradigms into everyday policy and practice are deemed to have resulted not only in the disenfranchisement of those with disability and chronic illness (Katz, 2015; Katz \& Calasanti, 2015; van Dyk, 2014) it erodes ideas of earned retirement, replacing them with claims of moral responsibility to remain well rather than becoming a burden on the state (van Dyk, 2014). As will be considered in the next section outlining Critical Gerontology (2.3.2), value assumptions around choice and lifestyles incumbent in the concept of successful ageing have consequences for older people who use mobility aids or have mobility difficulties. Alongside this, other debates that critique empirical and methodological limitations, and lack of attention to intersecting inequalities will also be discussed. 


\subsubsection{Critical Gerontology}

Critical gerontology challenges mainstream social gerontological theories of successful ageing ${ }^{3}$, seeking to uncover methodological value assumptions that influence the way ageing is researched and portrayed (Holstein \& Minkler, 2007, Katz, 2015). Contemporary mainstream outlooks of successful and active ageing emerge from WHO (2002) definition which indicates a practice of participation and optimisation of health opportunities to enhance quality of life as people age. 'Active' in this context is defined as continuing participation socially, civically, economically, culturally and spiritually (Foster \& Walker, 2015). Ideas of active ageing continue to challenge deficit models of ageing, with general agreement within contemporary neoliberal outlooks that ageing comes with an expectation to age successfully. Neoliberalism converts non-economic domains into economic ones. More than economic policy and free market ideology, neoliberalism is accompanied by deregulation and dismantling of public industries and services and reduction of taxation. Considerations of tax burden are offset onto the individual with discourses of selfresponsibility (Brown, 2016; Martinson \& Berridge, 2015; Moulaert \& Biggs, 2013; van Dyk, 2014).

Translations of neoliberalism into discourses and expectations of ageing through the successful and active ageing outlook are perceived within political circles as part of a winwin situation. Taxpayers and governments win via economic and social contributions from older people, and older people feel better about themselves because they continue to contribute and participate (Raymond et al., 2014; van Dyk, 2014). Consequences of neoliberal outlooks are felt in everyday human experiences though, because the normative reasoning and value judgements aligned with business merits transforms everyday actions and conduct into configurations of humans as capital, and each activity is valued in terms of investment and return (Brown, 2016). Neoliberalism is popular in politics because it offers the ability to govern with relative abdication as responsibility for individual success or failure lies with the individual as they invest self into opportunities available and live up to societal expectations. The discourse and expectation of successful and active ageing that

\footnotetext{
${ }^{3}$ Successful ageing and active ageing are different concepts however they are often used interchangeably, as noted on page 30. There are also related concepts of 'vital ageing', 'productive ageing' (Martin et al., 2015), 'positive ageing' and 'optimal ageing'(Bülow \& Söderqvist, 2014) used in the literature.
} 
aligns so neatly with neoliberalism is sharply contested through critical gerontology, an approach rooted in the political and economic climate of Western societies during the 1970s and 1980s (Phillipson, 2008; van Dyk, 2014).

Critical gerontology considers the systemic reproduction of life chances throughout the life course, with the resulting impacts on ageing for individuals and society. The role of state and systems is therefore considered with examination of structural forces that affect how older people are viewed and how they view themselves (Katz \& Calasanti, 2015; Lucas 2011; Musselwhite, 2018). Different life courses and social factors are enabled by interactions between people and constructions of society. Differences in access to goods and services throughout life bring into focus the power of cultural dimensions and socio-political outlooks highlighting a complex interplay between freedom to choose a life path and systemic life constraints such as culture, gender and class. These differences influence the life course, and thus the ageing process (Luborsky \& Sankar, 1993; Raymond et al., 2014) Alternative life opportunities create disparities in access to healthcare, employment, goods, environmental and psychological factors and thus implications for ageing (Holstein \& Minkler, 2007; Phillipson, 2008; Raymond et al, 2014).

Governance, policy and practice, informing and informed by social expectations rest upon successful and active ageing expectations and ideals based on an ageist foundation (van Dyk, 2014; Katz \& Calasanti, 2015; Martinson \& Berridge, 2015). The resulting values and policies fail to address physical barriers that are faced by those who live with disease and disability, therefore those older people who use mobility aids. Issues around accessibility and mobility, although sometimes present in policy discourse (as discussed in section 2.9 and appendix 2), are inadequate in interpretation and implementation to meet the needs of older people who use mobility aids. A policy and planning culture which does not envisage older people with mobility aids or mobility difficulties disregards or neglects the barriers faced by this group. The mutual interdependence of governance and policy with social attitude and expectation means structural barriers that remain in place for older mobility aid users lead to (and can be led by) the emergence of cultural and social barriers. policies that do not accommodate mobility and accessibility difficulties inform stigma, the ability to remain independent, have social impacts on mobility aid users and their carers/family members, and the other related themes that will be discussed throughout this research. Older mobility 
aid users thus face disabling structural, cultural and social barriers which must be overcome through physical, psychological, and relational processes at the cost of the individual and their loved ones.

There are value judgements underlying the successful and active ageing approach which result in a failure to address power differentials within social strata. Ageing cannot be studied in isolation from other life stages, societal forces or phenomena, therefore successful and active ageing approaches are set on foundations which do not account for gender, culture, life opportunities, poverty through the life course or access to healthcare (Holstein \& Minkler 2007, Katz 2015, Luborsky and Sankar 1993, Raymond et al, 2014, van Dyk 2014). A specific issue raised regarding successful ageing is the wide array of interpretations made of the construct without a widely accepted or applied definition (Timonen, 2016) The resulting outlook has brought about a culture which does not value older people for the wisdom, tradition and memory that ageing brings. When ageing is not valued as a natural process and becomes distorted by political and economic ideology, the poverty and disability that is often associated with ageing also fail to be recognised; individuals are blamed for failing to participate in their own empowerment with no account for the practical or social factors that may have been involved (Katz, 2015). While differences in race and gender are celebrated within anti-discrimination legislation and discourses, difference in age is homogenised by imposing the values and of one age group (middle-age) onto another (older people), a subtle form of ageism which has been termed 'ageimperialism' (van Dyk, 2014).

A main critique of the successful ageing paradigm within critical gerontology has been failure to incorporate notions of choice, lifestyle or intersecting inequalities. Choice is potentially a primary function and asset of neoliberalism and the inherent individualism involved in successful ageing. however, from early twentieth century sociologists such as Simmel and Weber, through to the more modern traditions of Bourdieu and Giddens, critical sociologists have questioned the notion of choice within constraints of opportunity and social inequality. Women, poor or working-class people or minority group members have greater constraints on choice (Katz and Calasanti, 2015). With concepts of choice, agency and lifestyle are unavailable in successful ageing dialogues on which much policy is based, responsibility for success becomes individualised, yet no consideration is made for access to the that which 
facilitates success (Katz \& Calasanti, 2015; Martinson \& Berridge, 2015). Moreover, seeking to eliminate or delay ageing processes through diet, exercise, healthcare and non-exposure to risk factors of decline also implies a preference to retain the values and status of middle age and middle-class. Choice and agency are only applicable within specific parameters then.

Rowe and Kahn's (1997) model of successful ageing was based on the notion of avoiding disease and disability, which stigmatises and marginalises those ageing with chronic illness or disability (Minkler \& Fadem, 2007; Taghizadeh Larsson 2013). Timonen (2017) critiques a lack engagement with disease and disability of policy measures relating to active ageing. With the main emphasis on maximising self-care and shifting public cost to privatised care services, there seems to be a hope that disability and disease will not draw on the public purse in large quantity. Rather, older adults as independent actors will consume good and care services which will be subject to age discrimination legislation. Thus, as Timonen (ibid) highlights, "where dependency begins, policy ends"(p.15). Individuals who succumb to factors associated with ageing such as disability and poverty are blamed for their vulnerabilities and failing to participate in their own empowerment with no accommodation of any social or physical factors that may be involved (Brown, 2016, Katz, 2015; Williams, Higgs \& Katz 2012). Older people are likely to be less mobile than their younger counterparts, yet there are rarely any signs of physical inability or mobility aids in active ageing research or policy agendas (Taghizadeh Larsson, 2013). Being able bodied or the extent of embodied mobility is a further influence on freedom of choice and a social barrier. The absence of research into mobility aid use or reduced mobility within ageing research or successful ageing discourses increases the likelihood of exclusion. Without representation or understanding of needs and requirements it is difficult to assimilate those needs and requirements of older people with mobility difficulties or who need mobility aids into planning and policy. From the critical gerontology perspective, people who are ageing and using mobility aids thus face intersectional social barriers of systemic ageism and from the viewpoint of this study the added potential barrier of ableism of environments. To understand mobility aid use, disability and the role of the environment in disabling individuals, the areas of literature examined next are of models of disability. 


\subsection{Models of disability}

For this research about the experience of older people's use of mobility aid use in the environment, models of disability are a useful tool to underline the role of environments in disabling older people. This very brief outline of the medical model of disability is given to provide context to the social model of disability and the wider discourses of disability that may be applicable to attitudes, policy and practice within the research. The prominence of disability in contemporary politics has grown since the United Nations Declaration on the Rights of Disabled Persons (1975, RES 3447) with most political parties in the UK providing manifesto commitments to promote equality, or hitherto eliminate inequality (Chaney, 2013). The social model of disability was developed in the early 1980 s, evolving from the Union of the Physically Impaired Against Segregation (UPIAS) document The Fundamental Principles of Disability (UPIAS 1976), as part of the emergence of disability rights groups and disability studies (Barnes, 2002; Finkelstein, 2001; Oliver, 2009, 2013; Peace, 2013; Shakespeare, 2013; Tregaskis, 2002). Developed as a tool to aid understanding of the social and structural barriers faced by those with impairments, this model of disability has been prominent in enabling legislation to require accessibility for public buildings and to outlaw discrimination (Oliver, 2013). The coupling of social movement and politicised conceptions brought about significant social change including de-institutionalisation, landmark laws, retro-fitting of accessible spaces, recognition of marginalisation and disenfranchisement of disabled people, and redesign of the urban landscape (Tremain, 2006).

The resulting change in approach to disability allowed for re -classification and conceptualisation of disability as differently abled, which developed new socio-political conceptions and countered medicalised approaches. Medical approaches, often referred to as the medical model of disability, tend to situate disability within the individual and assigns negative values to biomedical anomalies (Beaudry, 2016; Hansen \& Philo, 2007 ). The medical model of disability is the term used to describe medical categorisations and discourses rather than a model that is subscribed to. From this perspective disability is regarded as a personal tragedy localised in the individual whereby the focus of medical intervention is categorisation and subsequent rectification of flaws. Ability is defined in terms of norms and disability as disruption of or inability to meet these norms (Paterson \& 
Hughes, 2000; Tregaskis, 2002). Biomedicine provides the parameters for normal and acceptable function because medical notions provide a pre-social, fixed sense of biological normality or abnormality of bodies and minds in society. The binary outline of normal and abnormal gives rise to acceptance or rejection in society with assumptions of negative value associated with physical anomaly of impairment in biomedical terms (Beaudry, 2016; Hansen \& Philo, 2007; Oliver, 2009; Paterson \& Hughes, 2000; Thomas, 2014) . Herein lies the basis of a major concept under debate between the medical (sometimes referred to as individual) model and social model of disability; the difference between disability and impairment (Beaudry, 2016; Finkelstein, 2001; Harris, 2000; Oliver, 1996, 2009; Owens, 2015; Thomas, 2014; Tregaskis, 2002).

Harris (2000) a bioethicist, questions the coherence of a social model of disability, stating that within critiques of medical models of disability lies the crux that disability causes social exclusion and discrimination, which can be worse than the physical or mental impairment. Harris suggests that the harms of ostracism and hostility in social environments caused by impairments are negative aspects of disability, but not created by the medical model of disability. However, social model advocates suggest that the social oppression and exclusion associated with abnormality emanates from social structures rooted in the medicalisation of disability in the nineteenth century whereby people with impairments were regarded as unable to meet the demands of a 'normal' life (Beaudry 2016; Paterson \& Hughes, 2000). The argument is situated around sometimes complex definitions and conceptualisations of impairment or disability and whether they exist internally or externally to the individual. Medical approaches frame both impairment and disability as within the body and therefore internal to the individual. Questions arise around the role of these biomedical categorisations and discourses in framing impairment and/or disability, which are held responsible for individual and social attitudes. The social model of disability questions the normalisation or expectation of certain types of embodiment purported by medical approaches as both creating and endorsing the social and physical environment that excludes and ostracises those who have impairments. In the social model, impairment is located within the person while disability exists in the environment (Beaudry, 2016; Hughes, 2010; Oliver, 1984, 1996; Owens, 2015; Shakespeare, 2013; Smith \& Easterlow, 2005; Thomas, 2014). 
The social model has been in influential in the UK, enabling disabled people to become organised collectively as a political force, which has resulted in changing not only legislation but also discourses around disability and promoting disability as a civil rights issue (Barnes, 2002; Oliver, 1996; Shakespeare, 2013). The Disability Discrimination Act 1995 required services to make 'reasonable adjustment' for disability, followed by the Disability Discrimination Act 2005 which extended the legal duty of public services to promote disability equality and publish disability equality schemes (Hughes, 2010). The subsequent Equalities Act 2010 brings together and strengthens equality law across different strands of inequality with protected characteristics which include disability along with gender race and age. Disability legislation has also been supported by Human Rights Act 1995 which supports the key principles of equality, respect and dignity (Hughes, 2010). These social and political changes have influenced workplace and accessibility policies, providing more emphasis on social and infrastructural processes to enable those with impairment access to participation. The social model of disability has informed academic, political and lay understandings that while impairment exists within the person, the disability lays in social and infrastructural conditions and attitudes.

Critics of the social model suggest viewing disability as a social and structural issue, places the problem outside of the individual. With impairments located in the body and disability occurring in the environment, this approach can lack contextualisation of obstacles or interpretation of how obstacles affect social experience in a lived world. Disability becomes a universal concept rather than the experience of individuals with differing conditions and impairments (Beaudry 2016, Hansen \& Philo, 2001, Mazurik et al, 2014; Shakespeare, 2013). Vic Finkelstein and Mike Oliver are considered founders of disability studies (Paterson \& Hughes, 2000). Indeed, in his book 'Understanding Disability' Oliver (2009) confirms that he is the person who invented the term social model of disability, although not the ideas behind it (Oliver, 2009: p43). While they do not necessarily agree on all aspects of the social theory of disability, they do not disregard the effects of impairments (Oliver, 2009; 2013), affirming that the social model was not intended to be substituted for personal experience. The social model is instead focused on the collective experience of disablement, to incorporate the shared experience of those with different impairments who face the same social and structural obstacles (Oliver 2009). 
Opposing this notion of collective experience, Shakespeare (2013) highlights the contention that the group of heterosexual white men, many of whom had spinal injuries, who authored the social model represent a narrow experience of disability. Another concern with the social model lies in its strong rejection of medical approaches to disability. The neglect of impairment as being an important factor in peoples' life experience is a major weakness of the social model. Medical approaches to individual impairment reflect embodied experience; rejection of medical approaches and solutions to impairments implies that impairment is not problematic. Moreover, the social model framework of collective disablement renders personal issues and embodiment almost irrelevant (Beaudry, 2016; Hansen \& Philo, 2007; Shakespeare, 2004, 2013). Extending the criticism that the social model of disability fails to incorporate individual experience, sociology, social geography, and social workers use the social model of disability to argue that the built environment causes or reinforces exclusion but do not focus on social elements or relationships (Mazurik et al, 2014). This research takes a renewed approach to the social model by acknowledging the role relationships play in all aspects of life and the interlinking nature of relationships, embodiment, infrastructure and disability, as discussed in chapter 5 and throughout the thesis.

A major strength of the social model has been the progress made in politicising disability and creating a clear agenda for social change (Shakespeare, 2013). Despite this, a lack of policy salience and intersectionality have tended to produce a political policy discourse of disabled people as a reduced category (Chaney, 2013). Oliver (2013) suggests the destabilisation of the social model through criticism of failure to incorporate individual accounts or impairment has allowed a focus on difference to return, thus the focus of economic and social policy remain with or return to impairment and difference. Policy justifications are made around severity of impairment (based on medical frameworks) building stratifications of deserving or undeserving "whilst steadfastly ignoring the barriers we still face" (Oliver, 2013 p.1026). (Hughes 2015) reflects Oliver's (2013) conclusion on policy justifications, affirming that discourses of deserving put disabled people in a position of assumed guilt whereby they have to prove their innocence by way of their entitlement to claim welfare support. 
While the United Kingdom Government discourse of disability has been formulated around anti-discrimination, civil and political rights, legal protection and equality of opportunity, issues of policy salience and intersectionality inform notions of inclusion or exclusion (Chaney, 2013).Using a combination of content and critical discourse analysis, Chaney (ibid) sought to interpret key political texts, manifestos and policy on equality and social welfare for disabled people to determine an issue's salience, that is, how the issue is framed (constructed) within politics and political discourse. The analysis found that since 1979, political parties have increased policy salience in favour of disabled people. Inter and intra party differences, however, make for confusing policy interpretation and voting decisions for the public. For example, while Scottish Labour in the 2010 UK election pledged universal non means-tested provision of care, UK Labour promised free care for those with the greatest care needs. Even within the Labour Party, then, approaches to policy for disabled people differs in terms of means testing for welfare provision and evaluation of care needs (Chaney, 2013). In addition to the variety of interpretations of political discourse and legislation in place to enable inclusion for disabled people, translation into policy into practice is also even more problematic.

Literature regarding disability and also ageing is often punctuated with accounts of unknowledgeable staff within access systems, inconsistent application of policy and inability to access legal address. Policy and legislation complex and multi-faceted, erstwhile only useful if all stakeholders understand them, which is not always the case (Benson \& Keyes, 2014). Reasonable adjustment is a contentious and complex aspect of disability legislation (Brown et al., 2014; Butler, 2016; Hansen \& Philo, 2007; Reeve, 2014). Legally required to accommodate disability, Hansen \& Philo (2007) suggest reasonable adjustment is code for minimum adjustment, leading to disability features always seem to be an add on, not integrated into plans in the first instance (e.g., Hansen \& Philo). This makes accessibility an additional process for people with disabilities, in stark contrast to those with able bodies who can turn up at venues without consideration of negotiation with potentially ill-informed staff to access leisure facilities (Brown et al., 2014). Meeting requirements of reasonable adjustments according to the Equality Act 2010 could be argued to result in 'spatial apartheid' (Reeve, 2014), segregating disabled people (and those with pushchairs) who must access rear entrances. Removal of a physical barrier thus creating a psycho-emotional 
one.

Overall, the social model of disability has facilitated mobilisation of disability as a political and social movement (Barnes, 2002; Oliver, 2009; 2013; Shakespeare, 2013). The resulting legislation and policy outcomes have improved the social status of people living with disability; however, legislation is complex and often difficult to access (Butler, 2016). The situation is extremely convoluted, with many kinds of disability and different forms of exclusion, be they physical, mental or emotional. The over-riding message from the social model of disability is that impairment exists within a body, but disability involves more than embodiment. Disability emanates from the social environment so disabilities can be lessened or eliminated by changing environments, removing barriers, and shifting perspectives and attitudes concerning the abilities of disabled people. This would lead to participation as citizens on an equal basis with others.

For this research into the experience of older people's use of mobility aid use in the environment, the social model of disability is a useful tool to underline the role environments may play in disabling older people. To enable understanding of the boundaries of the body, how mobility aids, ageing and illness might change bodies and attitudes to them, we now examine embodiment.

\subsection{Embodiment}

Whether through illness, ageing issues or both, disability and mobility aid use are highly connected to embodiment. Embodiment is used here in the everyday sense of referring to the processes and functioning of human bodies, but also in the sense utilised by sociological traditions emerging from French phenomenologist Maurice Merleau-Ponty (Engman, 2019; Finlay, 2003). Old age is understood in relation to its bodily manifestations and the emergence of geriatrics as a branch of medicine transformed the 'old body' into an object of medical expertise (Tulle-Winton, 2000). Disablement is also centred around embodiment. Discourses of disablement through social and environmental structures, as promoted by the social model of disability often omit discussion of the body, yet it is the body, the person, that is present in negotiations of social exclusion, environment and oppression (Paterson \& Hughes, 2000). For those with diminished embodied capacity, independence and wellbeing 
can also become diminished in a linked downward spiral (Schwanen \& Ziegler, 2011). Despite the embodied nature of relating to the environment and others, Öberg (1996) criticises the absence of bodies in gerontology, suggesting the discipline is based on and perpetuates the body/mind dualism. Mobility practices require embodied movement which incorporate navigation of complex subject positions, choices and perceptions of responsibility (Doughty \& Murray, 2014).

Scholarly accounts of embodiment have historically assumed the body to be a fixed and biological entity although embodiment is more recently being considered as mutable and changed according to changes of culture, affected by political and economic movements such as capitalism or war (Csordas, 2004; Hastings \& Thomas, 2005). Historical attitudes, and approaches to embodiment, whether it be scholarly, political or popular thought, have shaped the current status quo with historical notions embedded in cultural understandings. Nations, politics, history within them and resulting social attitudes are social constructions made up of human actions. Actions are the result of often routine, banal and unthinking choices, but these choices shape culture (Hastings \& Thomas, 2005). Turner (1994) outlines the way in which late capitalism has emphasised the body as a site of personal identity which is no longer only the product of narrowly regimented social structures such as family, class and education. Individual consumerism produced by capitalism allows individuals to produce identities through purchase of goods and services. The consequences for embodiment have been both positive and negative. Gains of embodied empowerment and emancipation with pushback against traditional patriarchal, sexual and political forms of domination are set against the valorisation of youth, slimness and fitness (Turner, 1994). The politics of personal identity focuses on ideas of embodied being because the body is the infrastructure through which identity and personhood manifest (Turner, 1994). Embodied experiences are therefore situated in the contexts of time and space, current and historical social attitudes.

From the perspective of much recent work on embodiment, the world is navigated via the body; thus, selfhood is enacted, and the world is understood through the vehicle of the body (e.g., Turner, 1994). For $20^{\text {th }}$ century phenomenologists such as Merleau-Ponty, the body is not just a part of being and identity but is necessary for interaction with the environment (Merleau-Ponty, 1968). Bodies are both social and biological, affected by social 
and biological processes. This way of conceiving embodiment has been highly influential across different spheres of philosophy and sociology, and has particular relevance in studying health and illness, because each of these involves assumptions about 'normal' bodily functioning which seem importantly affected by the relationship between biological and social factors (Carel, 2008). In his evaluation of the social model of disability, Shakespeare (2004) highlights the inherent experience of incapacity, vulnerability and dependency that accompany the embodied realities of disability for many. Sustaining a strong sense of self is difficult for anyone, but particularly for one who has been born, or who becomes, impaired (Shakespeare 2004). Changes to the body must therefore result in changes to identity, with illness and disability disrupting the essence of self (Finlay 2003). Navigating the world and enacting selfhood via the vehicle of the body, then, means that the body and identity are both affected by and constitutive of social relations (Shilling, 2005).

The ways people interact with each other, within environments that envelop these behaviours can highlight how issues of personal identity, embodiment and sociability can make the environment more or less enabling (Peace 2013). There is a well-documented link between identity and place both being considered significant in both social environment and urban planning arenas (Cheshmehzangi, 2014; Peace et al., 2006; Spinney, 2008; Twigg \& Martin, 2015). Marketing and retail, for example, consider the social construction of place and identity an area of interest to investigate social processes (Johnstone, 2012). In addition to the literature regarding the symbiotic formation of identity between people and place, there is a proliferation of literature linking health to place whereby factors such as crime, social isolation and urban design are explored as social determinants of health (SDOH) related to place (Sunderland, Bristed, Gudes, Boddy, \& Da Silva, 2012).

\subsubsection{Disembodiment in research and policy}

The representation of bodies in research and policy dialogue can also lead to notions of normal or ideal bodies. The social model of disability, sociology and gerontology have all been criticised for failing to adequately represent bodies in their disciplines. Often, the allegation is that this reflects an assumption that the mind takes precedence over the body in explaining human life and relations. In his critique of gerontological absent body, Oberg 
(1996) suggests that a historical separation of mind and body is a stubborn legacy we as academics are failing to relinquish. Shilling (2005) echoes the sentiment, reminding readers that "the mind is located within, and is inextricably linked to, the body". 'Losing' the body has had consequences for ageing and disability, not least that the contemporary body is one that is subjected to a medicalised gaze. With no other focus on either ageing or impaired bodies other than biomedical one, these bodies are construed as in need of a cure, to be fixed as if a faulty machine (Paterson \& Hughes, 2000). Medicalisation of bodies constructs, or at the very least infers, malfunction. Deemed by scientific measurement of dependency and loss of function results in bodies becoming the responsibility of others; agencies of government or families (Tulle Winton, 2000), which removes responsibility for self.

Alongside this, the successful ageing approach that seems to have propagated ideas of continuing middle age into later years (e.g., Havighurst 1961), removes the visibility and notion of older bodies. With older bodies either invisible or are relegated to the margins of society when they become too old or uncontrollable, old age and ageing becomes further stigmatised (Tulle Winton, 2000). Older people's bodies becoming invisible has consequences for those who are ageing, however. Identity is constantly renegotiated and as people age, can be imposed by the external forces such as ageism, and internal forces absorbed from the social and physical environment to form internalised stereotypes (Peace et al., 2006). Ageism can be defined as discrimination against people of older age, although a broader definition incorporates beliefs about the biological impact of ageing on people of all ages which leads to fear and prejudice throughout the life course (Bytheway, 2005; Gendron, Welleford, Inker, \& White, 2016; Nelson, 2005). Because people can absorb the sense of ageism that is both societal and institutional, we can fear or deny our own ageing (Nelson, 2005). These notions of negative concerns regarding social and individual expectations are deftly described by the concept of stigma, a complex sociological and psychological phenomenon.

\subsection{Stigma}

A sociological term that has relevance to the study of social implications of embodiment, especially when discussing fear and denial of bodily changes, is stigma. The origins of the word stigma lay in Ancient Greek where the term was used to refer to visual 'bodily signs 
designed to expose something unusual and bad about the moral status of the signifier' . Stigmata were burnt or cut into the bodies of slaves in Ancient Greek society, but as Goffman (1963/1990) goes on to explain, the term has come to describe the disgrace itself rather than the bodily evidence of it. Goffman's (1963/1990) book is based on the premise that those with stigmatising characteristics are faced with assimilating into society and 'passing' as acceptable, rather than society accepting disability or the other stigmatising characteristics or traits it discusses. Goffman distinguished between people with stigma or 'normals', clarifying "the normal and the stigmatised are not persons but rather perspectives" (Goffman, 1963/1990 p. 163). People who have stigmatising characteristics are considered not quite human by 'normals', who subsequently compose theories to explain the inferiority of those carrying stigma, which rationalises animosity based on differences. There are 3 types of stigma identified, being embodied, character and race or religion. While different kinds of stigmatising characteristics are considered by Goffman, he proposed that the situations of stigmatised people have enough in common to be classified together for the purpose of analysis.

Parker and Aggleton (2003) assert that Goffman explored stigma devaluing relationships, not people. However, utilising Goffman's conceptualisation of stigma as a discrediting feature or attribute has led to the focus on stigma as being ' $a$ kind of a thing' ( Parker \& Aggleton, 2003 p.14), a culturally constructed value that has consequently been mapped onto people despite Goffman's explanations of stigma as a socially situated phenomenon. Subsequently, research often focused on the stigmatised person rather than the causes of stigmatisation (Parker \& Aggleton, 2003; Tyler \& Slater, 2018). Stigma is more complex than the mere social language Goffman suggests. Social interactions are contextual and informed by larger cultural influences of media and organisations that structure normative expectations and therefore formulate the possibilities for difference. Stigma occurs within wider settings of historical and cultural influence than Goffman credits (Parker \& Aggleton, 2003; Pescosolido, Martin, Lang, \& Olafsdottir, 2008; Tyler \& Slater, 2018).

A complication in the study of stigma is that it can be defined in different ways by different investigators (Bos, Pryor, Reeder, \& Stutterheim, 2013; Link \& Phelan, 2001). For some, stigma is understood as an ideology or behaviour, contrasted with discrimination which is a behaviour. Alternatively stigma can be defined as a combination of cognitive and 
behavioural responses that occur within the context encountered (Campbell \& Deacon, 2006). Bos et al. (2013), affirm that most definitions of stigma are comprised to two fundamental factors, being recognition of difference and devaluation according to that difference. Along with this is the consistent aspect in most accounts of stigma is that it requires social interaction and social context (Bos et al., 2013; Hebl \& Dovidio, 2016). Recent literature acknowledges that stigma reproduces existing social inequalities and is perpetuated by the exercise of social, economic, and political power (Campbell \& Deacon, 2006; Link \& Phelan, 2001).

This study incorporates the concept that stigma occurs in a social and public context, informed by social, political and economic outlook. Public stigma denotes the consensual understanding that an attribute is devalued and leads to social and psychological reactions of people who stigmatise (Bos et al., 2013). Public stigma is central to three other interrelated forms of stigma which are self-stigma, stigma by association and structural stigma (Bos et al., 2013; Reeder \& Pryor, 2008).

Self-stigma reflects the social and psychological impact of stigmatisation, being exposed to harmful social interactions and internalisation of the negative beliefs and feelings associated with the stigmatising characteristic. Self-esteem reduces and psychological distress increases when people are aware of public stigma aimed at them (Bos et al., 2013; Reeder \& Pryor, 2008). Stigma by association reflects Goffman's (1963/1990) courtesy stigma involving those associated with stigmatised individuals. Family members and loved ones of people with a stigmatised characteristic can increase psychological distress and reduce selfesteem in line with the stigmatised individual. Structural stigma refers to the perpetuation and legitimisation of stigmatisation by institutions and systems (Bos et al., 2013). Some authors have acknowledged the role of structural factors in perpetuating stigma (e.g., Link \& Phelan 2001; Parker \& Aggleton, 2003; Tyler \& Slater, 2016). The findings of this research, particularly those outlined in chapter 7 section 4 (7.4) and chapter 8, emphasise the ways in which structures can interact with other social and individual factors to increase the sense of stigma an individual may perceive.

Stigma impacts people in 3 ways: enacted stigma takes the form of treatment by others in the environment, felt stigma is the experience or anticipation of stigmatisation. The third way in which stigma can manifest is through internalised stigma, which is a sense of reduced 
self-worth which accompanies the experiences of enacted or felt stigma (Bos et al). These three ways of being impacted by stigma were found to be intricately interrelated in this study. There were incidences of enacted stigma and self-stigma, as demonstrated in chapter 6 , but more than anything, stigma related to systems and structures. Chapter 8 outlines the various forms of infrastructural challenges faced by older people who used mobility aids. Comments relating to 'Infrastructure and interlinking systems' was second highest-ranking code, second only to mobility aids themselves. Infrastructural factors often formed obstacles to social activity and participation in different ways. As discussed in chapter 5 , infrastructural issues leading to stigma relates to both the environmental infrastructure and services, planning and policy surrounding it.

Contemporary accounts relay stigma as a proponent of power, active in the production of inequality and marginalisation, systemically produced and mediated for economic and political reasons, as a means of dominating certain populations and maintaining social classifications ( Bos et al., 2013; Pescosolido, et al., 2008; Tyler \& Slater, 2016; Parker \& Aggleton, 2003). Public understandings of what is culturally normal and acceptable are produced, reproduced and reinforced by authorities, governments and multiple sources of media. For example, institutional bans in the United States of America (USA) on allowing entry to people living with HIV/AIDS until 2008, or failure to recognise gay marriage are both forms of institutional or structural practices that reinforce stigma (Pryor \& Reeder, 2011). There is also a growing importance within a cultural and economic context of identity as central to experience, which alters the Goffman's positioning of stigma and construction of spoiled and identity. Goffman's conceptualisation of stigma as constructed within social expectations realigns with ideas of identity being constructed and changeable within the parameters of given circumstances and individual perception of identity (Parker \& Aggleton, 2003). That is to say that identity is based on individual, social and structural influences within the personal, cultural, social and political context (Bos et al., 2013; Parker \& Aggleton, 2003; Pryor, Reeder, \& Landau, 1999; Tyler \& Slater, 2018).

Identity is managed using the visual and social information made available by an individual to those around them. Information about abiding characteristics is conveyed through symbols which coincide with other social signs being transmitted. These can include the way somebody dresses, skin colour, wearing a uniform, or a certain hairstyle. Symbols may 
confirm what other signs tell us about someone, like a lapel button displaying club membership. Some symbols claim prestige or desirable status, thus are status symbols, other which draw attention to 'negative' aspects of identity which are termed stigma symbols by Goffman (1963/1990, p.59) , exemplified by the shaved head of a female WWII collaborator. Visibility of a stigma symbol, or the stigmatising characteristic itself is a crucial factor in managing social information and therefore identity management. The consideration is not only of visibility, but "obtrusiveness"(p.66) in the given context. A wheelchair in a boardroom may not be as intrusive as a bad speech impediment according to Goffman (1963/1990). The visibility of mobility aids have been a consideration from the outset of this project because it is known that people can fail to use mobility aids (Boerema et al., 2017; Resnik, Allen, Isenstadt, Wasserman, \& lezzoni, 2009), but it may also be that they are obtrusive to the daily lives, routines and social interactions of those who require their use.

Such ideas of stigmatisation have informed my approach to studying older people who use mobility aids, because the study may entail intersectional considerations of age, disability and possibly gendered use of or attitudes to mobility aid use. There is also a consideration of institutional ableism which is linked to stigma because, as explained above, notions of normal and acceptable are dynamically linked with the processes of stigma, therefore legislation, policy and practice both reflects and directs public awareness and attitudes as well as individual ideas of identity and personal value. As will be examined in the next section, design and infrastructure can reinforce exclusion and stigma because of the inclusion and thus exclusion of certain types of bodies or abilities.

\subsection{Infrastructure: Planning and Policy}

Integration of good design and planning can increase the independence of older people (Newton et al., 2010).

Sociology, social geography and social work are some of the disciplines that utilise the social model of disability to argue the built environment causes or reinforces exclusion but there is little focus on social elements or relationships (Mazurik et al, 2014; Oliver, 2013) . 
Environmental barriers usually refer to structural barriers in the layout of community and buildings. There are ongoing discussions and legislation about universal design, legislation, and accessibility. Barriers can be considered an underlying expression of societal attitude towards disabled people that are played out as exclusionary structures (Peace, 2006). Conformity of space and place is a highly debated issue with research often finding disabled people 'out of place' or opposed to the norm (Burghardt, 2013; Hansen \& Philo, 2007; Serlin, 2012). Hansen \& Philo (2007) propose that space is designed with conformity of nondisabled people in the same way as white people are unmarked as an ethnic category in the west; the category is subliminally assumed as normal and anything outside of this norm is therefore abnormal.

The resulting ableism of environments has been linked to everyday stigmatisation, patronising attitudes and demeaning acceptance of those with different abilities (Hansen \& Philo, 2007; Hughes, 2015; Imrie, 2012). Positioning and location of furniture or size of print; general spatial configuration are aspects of the blueprint of ableist environments, and although Governments spend money and time counting, monitoring and categorising disabled people, they fail to document or report experiences (Hansen \& Philo, 2007). While physical design is essential to ease of access, independence and mobility of people with disabilities, access and movement are structured and designed around conceptions of nonimpaired corporeality, focusing on efficiency of movement that favours mobile or 'normal' bodies. The diverse ways different bodies interact with the environment are not incorporated into design or policy. Design of space and place can potentially create new spaces of disablement if the needs and embodiments of those with disabilities and mobility aids are not canvassed and addressed (Hansen \& Philo, 2007; Imrie, 2012).

Alongside the ableism of environments is a hegemonic discourse of mobility which tends to be enshrined in disability rights laws as fundamental to the liberty of the human body. Reasonable adjustments for disability are required by legislation, which is essentially code for minimum provision. Disability features always seem to be an add on never fully integrated into plans in the first instance. Inclusion for disability thus becomes provisional and usually requires the individual to mimic ableist ways of doing things (Hansen \& Philo, 2007; Imrie 2012). Disabled people have the moral right to mobility, but prioritising and arbitration of competing needs means translation into practical and legal solutions becomes 
problematic (Imrie, 2000, Korotchenko \& Clarke, 2016). The result is a hegemonic discourse of mobility whereby productive bodies become prioritised within a culture that valorises independence and physical ability (Imrie, 2000).

Moreover, architects design with a prescriptive user in mind but appear not to be well conversed in all types of user they are meant to accommodate. Design practices have been accumulated and are unintentionally exclusionary because of the normative templates historically used (Hamraie, 2017). Concepts of normal and average appeared with the birth of statistics and produce modern ideas of embodied average. This average is not neutral however, as it is a "white European, non-disabled, youthful, often masculine" body (Hamraie, 2017, p. 20) . The template of a normal body, then reflects the probability of normal or average within a population, however when these prescriptions of normal are consistently used to create the built/lived environment, they do not define an average person who does not exist, and also exclude difference physically and symbolically (Davis, 2017; Hamraie, 2017).

People with embodied differences or who use mobility aids feel socially excluded, but contextualisation of obstacles or interpretation of the experience of social exclusion in the lived world has been more difficult to ascertain (e.g., Hansen \& Philo, 2007). Shopping routines, as discussed within the mobility aids section are both necessary and social and can be attached to particular social and spatial relations, with emotional experience and convenience playing major roles in choice of outlet. Shopping spaces can be areas of social inclusion, or alternatively can initiate a process of self-exclusion through negative experience (Williams et al., 2001). We will see in the experiences of participants throughout the findings that shopping spaces are integral to the everyday routines of mobility aid users. Obstacles faced by wheelchair users were investigated in a study of shopping accessibility which took place in South Wales (Bromley et al., 2007). Using both quantitative and qualitative methods, the study aimed to examine disadvantage in the spatial environment using face to face interviews with the 120 participants. Interviews included both open and closed questions and were followed up with further in-depth interviews with 30 of the interviewees. 12 participants also took part in two focus groups to discuss the quantitative findings and policy suggestions emanating from the study. The focus of the study was not on older people although $72 \%$ of the sample were 50 years or over. Findings highlighted that 
accessibility in the spatial environment was problematic, taking the form of heavy doors, cluttered aisles, the height of shelves and narrow checkouts. Disadvantage in social and spatial environments can also exist in the real or imagined environment of the self, that is, the individual's perception of accessibility Places that are not accessible may create an interpretation that people who cannot access are intentionally excluded, reinforcing stigma. Hastings and Thomas' (2005) affirm that historical notions of embodied citizenship have shaped current planning ideals which focus upon the ideas of normalised bodies, as discussed within section 2.7 'Embodiment'. Imrie \& Edwards (2007) explore geographer's interest in disability studies, whereby human geographical approaches tend to focus on disability rather than the use of mobility aids but hint at policy and administration inconsistencies failing to incorporate people with mobility difficulties in aspects of community design (Imrie, 2000; Imrie \& Edwards, 2007). The exclusion of impaired bodies in place through planning and policy can thus be translated into expected norms of those places. When impaired bodies do not inhabit a place because they are excluded via infrastructural obstacles, they become unexpected and further excluded by seeming 'out of place', and therefore excluded by social and psychological obstacles. The end result is an exclusion of impaired bodies by infrastructure planning and policy which exacerbates a sense of impaired bodies being out of place and not expected to inhabit or navigate planned environments (Hansen \& Philo; Imrie, 2000, 2012)

\subsection{Transport studies}

As described in the previous sections, dominant discourses of engineering and economics obscure embodied issues of mobility difficulties in the realm of transport and travel (Doughty \& Murray, 2014). Ageing is also very poorly represented in transport research. The focus of mobilities research on older populations has tended to be accessibility and well-being, giving rise to mainly quantitative research methods in terms of transport. Such methods do not access the complexity of meanings, context and aspirational qualities of mobility (Murray, 2015; Musselwhite, 2018; Musselwhite \& Haddad, 2018). A Department for Transport and ONS publication of Transport Statistics Great Britain (2017), published annually, outlines a number of fascinating statistics about travel and transport in Great Britain. The document indicates how many kilometres were travelled by UK citizens (or to 
be more precise, mostly those situated in England), what percentage of those were travelled by car, van, taxi or bus. The reasons for transport are highlighted, such as commuting, leisure, education or shopping. There is a section dedicated to transport and disability (Department of Transport \& ONS, 2017). Some of the statistics published are as follows:-

England: 2015/16

9.9 million older and disabled concessionary travel passes held 970 million older and disabled concessionary bus journeys 98 concessionary bus journeys per pass

9\% of adults reported having a mobility difficulty* in England in 2015

$38 \%$ fewer trips by individuals aged 16+ with mobility difficulties compared to those with no mobility disability

474 trips per person per year were taken by those aged 70+with mobility difficulties - half the number of trips undertaken by those with no mobility disability.

(Department for Transport \& ONS 2017)

As interesting and useful as these statistics are in highlighting the lower amount of travel behaviour of those with mobility difficulties compared to those who do not have mobility difficulties, they do not tell us anything about the reasons for the lower number of trips taken up by people with mobility difficulties, or the embodied experience of transport and travel with mobility difficulties. The reasons for journeys are a welcome inclusion, but again do not explore qualitative, experiential dimensions of transport that may be the key to enabling people with mobility difficulties to travel more.

One study (Velho, Holloway, Symonds, \& Balmer, 2016) incorporating sociological and engineering research highlighted social interactions in the accessibility of public transport for wheelchair users. The welcome and much needed interdisciplinarity gave rise to better understanding of both technical and human factors in wheelchair accessible transport. Interesting insights from both perspectives illuminate the complex and interrelated problems facing both technology and society that come with accessible design. For example, 
the social humiliation felt by wheelchair passengers when the ramp deployment alarm sounds may not have been a consideration within engineering. Conversely, the aggravation of defective ramps not working is more complicated in reality than was considered through design stages. Ramps are designed to stop when an obstacle is sensed. It is not necessarily clear which obstacle is being sensed when a ramp stops working, it could be street furniture, height of pavement or something else. Therefore, the combination of social and engineering approaches supplement each other. Indeed the authors suggest a plural approach to travel accessibility could also include biomechanics and city planners (Velho et al., 2016).

Physical design is essential to ease of access, independence and mobility of people with disabilities. Access and movement are structured and designed around conceptions of nonimpaired corporeality, focusing on efficiency of movement that favours mobile or 'normal' bodies. The diverse ways different impaired bodies interact with the environment are usually not incorporated into design or policy. Conceptions and understandings of the body and movement are deployed within normal social, physiological and psychological cues or behaviour (Hansen \& Philo, 2007; Imrie, 2000, 2012). Providers of transportation services treat disabled people as 'different' 'special' or even 'burdensome' showing difference is used to justify unequal treatment. Even though disabled people have the moral right to mobility, prioritising and arbitration of competing needs becomes problematic in translating needs into practical and legal policies (Chaney, 2013; Imrie, 2000).

Older age is investigated as a discrete category within initiatives and policies which fails to incorporate the variety of relationships people have across age groups and outside of families, thus a cohesive mobility picture of older people is not explored (Murray, 2015). Ageing itself is a lifelong process and people do not just cross a line to become old. However, interventions aimed at mobility improvement for older people tend to remain focused on medical, reductionist models of embodied ability (Musselwhite et al 2015). The perpetuation of focus on medicalised ideas of ability, that is a medical model of disability as discussed in section 2.6.1, means the focus within transport systems tends to remain on accommodating disability or reduced mobility as special cases rather than designing for all abilities. There is discussion within transport research of the link between mobility and health in general being under researched or even neglected (e.g., Aguiar \& Macário, 2017; Musselwhite, 2015). Public policies relating to transport should be developed to promote a 
prosperous environment for all citizens. Urban spaces and mobility within them should be adapted to the population to avoid social discrimination, and create independence and empowerment (Aguiar \& Macário, 2017). This can only occur when all aspects of embodied reality, abilities and social situations are accommodated within planning and policy of mobility.

\subsubsection{Interlinking systems and policy}

Infrastructure is also concerned the provision of health services. As indicated above, transport research and policy consider travel for healthcare purposes to be crucial, but what of the infrastructure of healthcare itself? According to geographies of health inequalities, area-based policy inspired by neoliberal policy agendas inform notions of lower costs than wider structural adjustments. The resulting strategy for wellbeing hinges around prevention and cure, indicating a vision of fit and able bodies and a productive work force (Smith \& Easterlow, 2005). Ideas of a nation of fit bodies echoes Hastings and Thomas (2005) work on the relationship between the design and development of the built environment and creating political inclusivity which also outlines notions of national identity based around fit bodies. Neoliberal values of economic productivity are also at the fore as discussed in sections 2.3.2 Critical Gerontology and 2.4 the Mobilities Paradigm. Once again, the medical model of disability which conceptualises disability as abnormal compared to a theoretical homogeneous norm as discussed in section 2.6. is indicated with notions of prevention for the fit able bodies, and cure for the ill, with those outside of these categories not fitting the model.

A lack of cohesion in addressing the interaction of transport, health and well-being, ageing, disability and embodiment leads to reduced capacity and therefore social isolation, lack of participation and poorer health for ageing populations. If poor accessibility was reduced it would address the potential for false economies and overspending. There are implications for overall taxation spend whereby the cost of care for older populations could be offset with better environments which would decelerate decline and therefore reduce demand for social support and healthcare. With this in mind, we now examine 'Age Friendly Cities and Communities' to explore the ways planning and policy is directed, interpreted and 
implemented for older people in Wales, and specifically how this translates to the experience of older mobility aid users.

\subsection{Age Friendly Cities and Communities}

Making Wales a nation of age friendly communities, originated by the Older People's Commissioner for Wales, is based in the concepts of World Health Organisation (WHO) literature explaining Age Friendly Communities (OPC, n.d., -b, p2). The information outlined herein is to provide background of the policy context and how it is interpreted, implemented and experienced by older people in Wales. Initiation and communication of age friendly cities (or communities) began with the WHO, and the WHO database provides a comprehensive framework of resources and literature to both inform and collate information regarding policies, planning and services for ageing populations. The approaches outlined by the WHO, as will be discussed herein, reflect ideals of equality and promote the value of older people. Within the WHO approach is the Dublin Declaration on Age-Friendly Cities and Communities in Europe 2013, a document by which nations and regions can signify their commitment to becoming age friendly. All local authorities in Wales have signed up to the Dublin Declaration (OPC, n.d.-b). The following sections review literature which outlines the World Health Organisation conceptualisation of agefriendliness and the Dublin Declaration on Age-Friendly Cities and Communities in Europe 2013 to provide context to age friendliness as a concept and the goals of Age Friendly Wales. Following on from this is an outline of Age Friendly Wales to outline the way WHO initiatives and have been interpreted in the context of Wales, and finally in this section is a short evaluation of the ways local authorities have interpreted and implemented the guidelines and directives from both the WHO and the Older People's Commissioner for Wales.

\subsubsection{World Health Organisation Age Friendly Cities and Communities}

The notion of age friendly communities is a fundamental aspect of the Ageing Well in Wales approach, named as an aim in the Welsh Government Ageing Well in Wales framework, with outcomes including recognition of the importance of age friendliness, and development of age friendly communities through community involvement at all ages with support from 
government at both local and national levels. The first phase of the literature review, in line with the inception and proposal of the project as discussed in Chapter 1, was to review and interpret Age Friendly Wales literature.

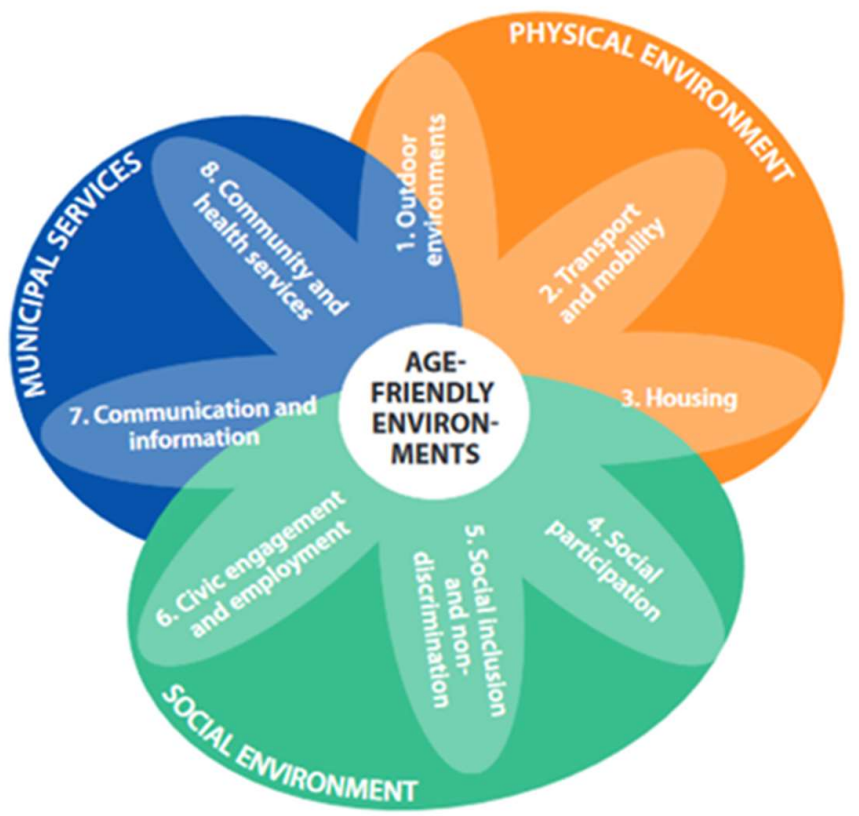

Fiaure 2.1 WHO Aae Friendlv Environments (WHO 2018)

The WHO Age Friendly Communities Guide published in 2007 underpins current age-friendly approaches in Wales. The concepts and research that support the approach, including the rapid increase in ageing populations that coincides with growing urbanisation and the influence of active ageing in the conceptualisation of Age Friendly Cities are outlined in the guide. There is a summary of functional capacity such as muscular strength and cardiovascular output over the life course, which highlights decline in older age, showing that decline can cross a threshold into disability (WHO, 2007). Such decline, according to the guide, can be accelerated or decelerated by changes to both lifestyle and policy measures. 35 cities contributed to the guide, with a bottom-up participatory approach. Information was obtained from older people and caregivers, with most cities also conducting focus groups with service providers from the public, voluntary and commercial sectors.

The guide outlines and explains each of eight domains of age friendliness, why they are important to the experience of older people and gives quotes from people who participated in the research to illustrate the importance of each point. In summarising the approach, it is 
acknowledged that features of the environment reflect the determinants of active ageing in many interconnected ways (WHO, 2007). The advice built upon evidence from research and practice has been continually re-evaluated, crafted from locally and regionally developed tools and practices (WHO, 2007, 2016, 2017, 2018). A more Eurocentric approach, with the intention of creating and supporting more age friendly environments in the context of European health and social policies for ageing populations is found in Age Friendly Europe guides and handbooks such as World Friendly Environments in Europe; Indicators, monitoring and assessment (WHO, 2018) and Creating age-friendly environments in Europe; A tool for local policy-makers and planners (WHO, 2016).

Subsequent iterations of WHO Age Friendly Cities and Age Friendly Environments initiatives, interpretations and implementations are documented on a comprehensive Age Friendly World website (WHO, n.d.) which includes guides, handbooks and incorporates a global network and database of age friendly practices. Age Friendly Environments in Europe: Indicators, monitoring and assessments (WHO, 2018) lists the eight domains of age friendliness:-

1. Outdoor environment.

2. Transport and mobility.

3. Housing.

4. Social participation.

5. Social inclusion and non-discrimination.

6. Civic engagement and employment.

7. Communication and information.

8. Community and health services ${ }^{4}$.

These domains are explained comprehensively within WHO documentation, which indicates that there are porous boundaries between these categories and each domain is multidimensional. For example, engagement in sociocultural activity is likely to be dependent on transport and mobility services. The domains are also organised into three

\footnotetext{
${ }^{4}$ The domains named by WHO contain terms that are contested and potentially open to debate. However, as the initiative is based within the realm of WHO, and the debates are beyond the scope and focus of this study, I have chosen to rely on the WHO definitions and usage of domain names and their consequent application.
} 
clusters which are highlighted by the outer petals of the diagram in figure 2.1, which appears in the handbook. Cluster one incorporates the first three domains as those involved with the physical environment, the second cluster groups together domains 4, 5 and 6 as being pertinent to the social environment and the final two domains make up the third cluster relating to municipal services. The policy domains and clusters provide a framework for governments, local authorities, policy makers and planners to attain a physical and social environment alongside community services that all support age-friendliness (WHO, 2017).

All domains are thus interlinked through the requirement of age-friendliness, and pertinent to the study to some extent, because it is through the physical environment that social aspects of life are accessed.

WHO literature also indicates cross cutting themes that run through the domains and clusters. One such theme is the promotion of physical activity, which the publication outlines is linked to domain 1. outdoor environment and domain 2. transport and mobility, through the necessity to create barrier free environments, strengthening infrastructure of paths, roads and public transport provision and providing safe, accessible parks with places to rest. These aspects provide the ability to move around and access places to take exercise. Domains 3. housing and 4. social participation are involved because physical activity requires housing within reasonable access of key facilities. "20-minute neighbourhoods" (WHO, 2017 p.9) are suggested, where facilities are within a limited zone from older people's housing via walking or public transport. Provision of opportunities for social participation and physical exercise should be within the key facilities, along with supportive environments for social exchange and places to meet. Domain 5: social inclusion and nondiscrimination and domain 6: civic engagement and employment require actions to target individuals who are excluded or vulnerable by promoting activities such as voluntary work, or encouraging neighbourly interaction through community-based initiatives (WHO, 2017).

The cross-cutting, intersecting nature of the domains and clusters outlined in the WHO literature is very important to this research, as my approach to older people using mobility aids explores the ways in which social, emotional and psychological aspects are inextricably linked through experience and lifestyle. I approach this research considering social participation, social inclusion and non-discrimination, along with civic engagement and employment as likely to be linked as aspects of social environment but also enabled by 
access through physical and psychological environments. Inclusivity and participation are not exclusively socially driven by factors of community and friendship, but also by elements of employment and civic participation such as voting or taking part in community groups.

\subsubsection{Critiques of the World Health Organisation approach to age friendliness}

Critical engagement with the WHO conceptualisation of age friendliness questions the identification of domains and classification of their importance. Although the policy outcomes of domains are outlined as interconnected within WHO literature, an ecological perspective conceptualises all domains as social connectivity within a community environment (Menec, Means, Keating, Parkhurst, \& Eales, 2011). This reflects my findings of interconnectivity, identified in all aspects of social participation. Chapters 7 and 8 particularly underline the complex ways in which individuals, policy and infrastructure influence each other, with a model of a feedback loop of obstacles of mobility aid use in Chapter 10 (see figure 10.2). The model outlines the ways policy and planning feeds into notions of stigma and people feeling like a burden which impacts identity and thus inhibits free participation. Inability to move and inhabit spaces freely can reduce older people's ability to shape the identity of place. Older people are often not active in the construction and reconstruction of places because they do not participate as significantly as others socially, economically or politically (Buffel, Phillipson, \& Scharf, 2012). This is supported by literature outlining the significant link between identity and place (Cheshmehzangi, 2014; Peace et al., 2006; Spinney, 2008; Twigg \& Martin, 2015) highlighted above in section 2.5. A universal approach to addressing age-friendliness in widely diverse cities and communities across the globe has also been questioned. Approaches to age friendliness need to reflect the heterogeneity and variation of populations unique to each place (Buffel et al., 2012). Friends, jobs and community groups can only be accessed when the social and physical environment support access. The planning, installation and maintenance of each place is provided and funded by municipal services. Planning age friendly environments requires understanding of the character and context of each place and the people who inhabit it. To gain age-friendly status across Wales, all Local authorities have signed the Dublin Declaration on Age-Friendly Cities and Communities in Europe to signal their commitment to becoming age friendly. 


\subsubsection{The Dublin Declaration on Age-Friendly Cities and Communities in Europe}

The Dublin Declaration on Age-Friendly Cities and Communities in Europe 2013 (WHO, 2014) (herein referred to as the Dublin Declaration or the declaration) is a declaration signed by senior political representatives from across Europe during the European Union Summit on Active and Healthy Ageing in 2013. The values and principles of action within the document are drawn from the WHO policy framework on active ageing which optimises opportunities for health, participation and security to both enhance quality of life and allow people to participate in society according to their own potential for physical social and mental well-being along according to their needs and desires.

Opportunities and challenges are outlined in the second section of the document. These include recognition of the significance of place, stating "where we live, our physical, social and cultural environment greatly impacts upon how we live and age" (WHO, 2014). The built environment and place are recognised as inseparable from personal identity; therefore, cities and communities must be equipped with the means, resources and systems to promote equal opportunity, participation and well-being of all citizens including older people. Planning for economically, environmentally and socially sustainable communities should account for the needs of all and promote the value of older people as a key resource in meeting their own specific needs. Older people should have an active voice in planning and decision-making to ensure age-friendliness. Rather than just accounting for the needs of older people, the declaration promotes the value of older people, stating "older people are a bounty to European Society" (WHO, 2014, p. 3).

The final sections outline the commitments to age friendliness, which means both adhering to and promoting the values, principles and premises of WHO, UN and European Network age friendly programmes. Also collaborating with stakeholders to support the full application of pledges, and communication through international, national, regional and local channels to stimulate and support advances in equal rights and opportunities for older people. The declaration ends with a pledge outlining that where economically feasible and it is their responsibility, signatories will implement specific actions. The actions include promoting general public awareness of the needs of older people highlighting positive social and economic, cultural contributions they make. Central to the tenets of the declaration is 
ensuring a process of citizen-centred engagement, adopting measures to develop inclusive urban spaces and public places, provision of housing within neighbourhoods and communities that are diverse, safe, inclusive and sustainable with attention paid to dignity and autonomy of residents. Establishing public transport systems available and affordable to all is also noted.

\subsubsection{Age Friendly Wales}

The Welsh Assembly Government implementation of WHO age friendly communities is overseen by the Older People's Commissioner for Wales. The Welsh Assembly Government also has a Strategy for Older People 2013-2023 which outlines the desired outcomes timescale of achievement for ensuring the well-being of all older people in Wales. The strategy indicates the policies and initiatives involved which include the Ageing Well in Wales programme overseen by the Older People's Commissioner for Wales, social care implications, the Healthy Ageing Programme, developments of a health checks programme for people aged over 50, carers strategies and tackling pensioner poverty. The Strategy for Older People assimilates the actions and requirements of WHO age friendliness into the existing or developing policy and practice framework within Wales, and like the WHO and Dublin Declaration, outlines the interconnecting nature of policies in the lives of older people, however the message has become diluted. Chapter 2. on environmental resources, for example, indicates that older people have shown concern about pavements and transport, the safety of their environment and the location and suitability of housing, yet does not emphasise the importance of ensuring all policies relating to any of these areas also impact on the others. Each of the 22 local authorities in Wales has committed to becoming age-friendly within the strategies and frameworks of WHO, Europe and Wales goals. 


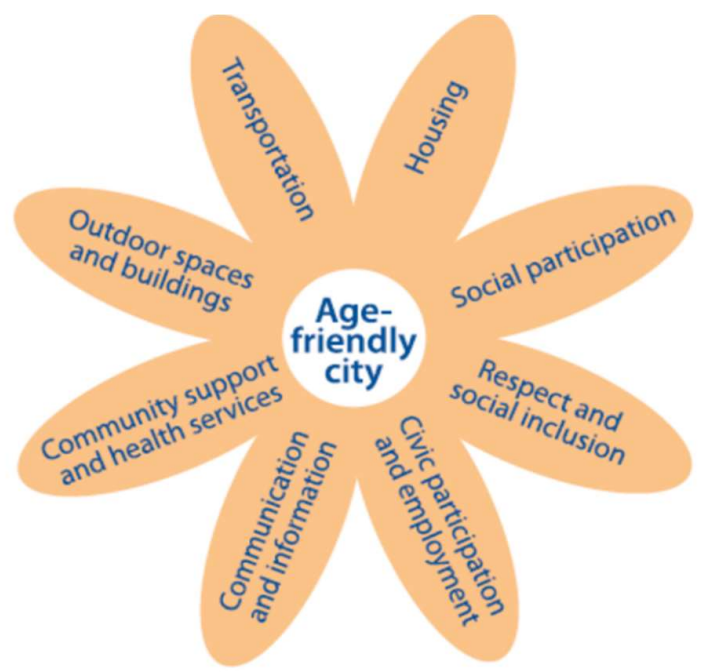

Figure 2.2 Who Age Friendly Topic Areas (2007)

The Ageing Well in Wales website in 2018 (now a legacy website) displayed the diagram for age friendly Wales shown in figure 2.2. It is taken from a WHO (2007) publication which not only depicts the eight domains as separate, but uses the old names. Since then, the site has been updated and the icon changed as illustrated in figure 2.3. The new design reflects one used by Age Friendly Ireland (figure 2.4), which is the first age friendly country. It is a much better icon than the misleading diagram shown at figure 2.2 as it shows the petals, and therefore the domains, as overlapping. This is a very important point within this research project, as it foreshadows findings that different areas of policy and practice need to complement each other and work in unison. Each domain of age-friendliness cannot be treated separately if the aim is to provide a wholly more age friendly environment. The icons give an 'at a glance' notion of the concept, so showing each domain as a separate entity gives the idea that each area of focus is isolated from the others (OPC, 2015).

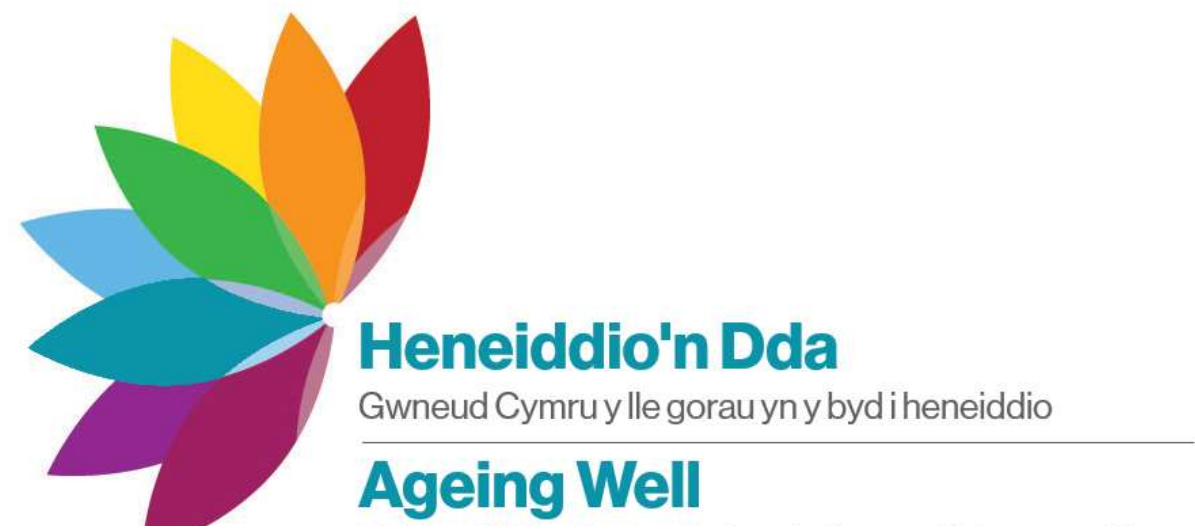

Figure 2.3 sourced from OPC (2016) 21st August 2020 


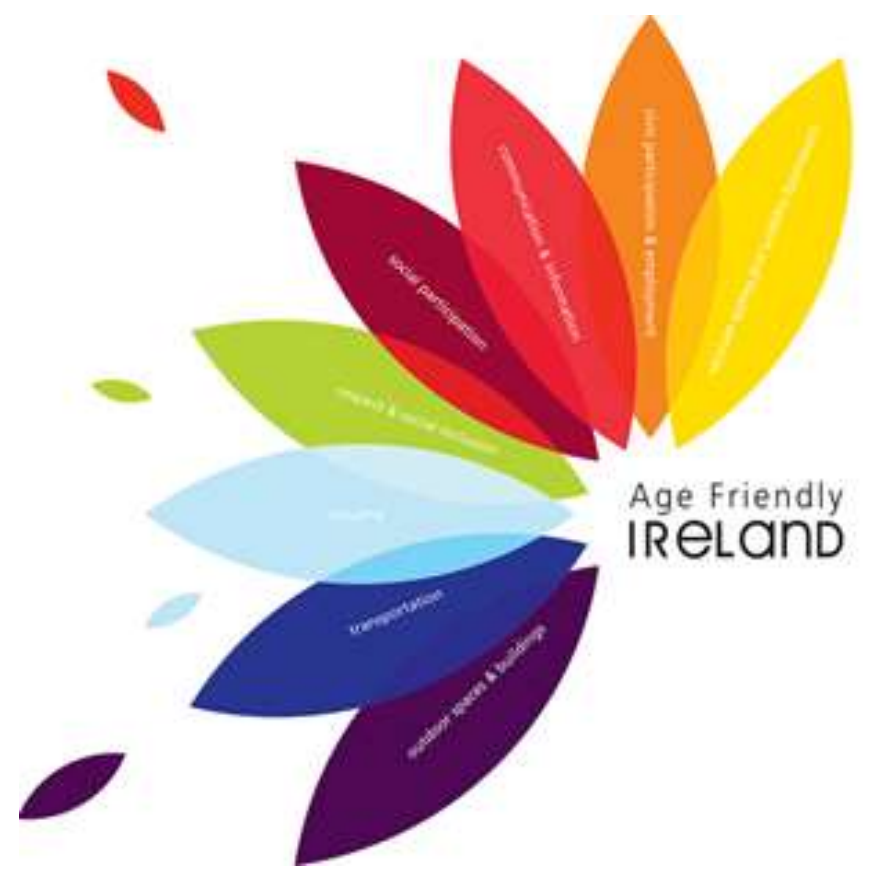

Figure 2.4 Age Friendly Ireland Icon

\subsubsection{Interpretation and implementation of Ageing Well in Wales plans}

Previous research and policy evaluation regarding ageing well approaches suggested a failure to keep pace with changes to policy and strategy. Lyall (2007) noted inconsistencies between different authorities in implements strategies for older people and RémillardBoilard (2018) has reviewed the United Kingdom (UK) network of Age Friendly Communities, suggesting it is of value, however there is no recent evaluation of the interpretation and implementation of individual local authority ageing well plans or age friendly community approaches. Each of the 22 local authority areas ${ }^{5}$ in Wales are listed on a dedicated Ageing Well in Wales website with links to Ageing Well and Older People's Strategy Action Plans. The Ageing Well in Wales website is now a legacy site and no longer updated, which means some of the links no longer work. The updated section of the Older People's Commissioner

\footnotetext{
${ }^{5}$ https://www.ageingwellinwales.com/en/localplans lists the following authorities

Anglesey, Blaenau Gwent, Bridgend, Caerphilly, Cardiff, Carmarthenshire, Ceredigion, Conwy, Denbighshire, Flintshire, Gwynedd, Merthyr Tydfil, Monmouthshire, Neath Port Talbot, Newport, Pembrokeshire, Powys, Rhondda Cynon Taf, Swansea, Torfaen, Vale of Glamorgan, Wrexham.
} 
for Wales site offers greater focus on age friendliness with this as the first item to be seen when opening the 'Ageing Well' tab. Individual authority website links are no longer offered. The evaluation consisted of assessing the approach to age friendliness demonstrated within the documentation of each local authority, and the evaluation is presented in whole as Appendix 2. The assessment began by identifying whether the local authority had produced a specific, stand-alone Ageing Well policy, or if approach is address within another policy strand such as the Strategy for Older People (SfOP) or any other approach that may have been taken. The dates for which the latest plans or updates had been made at the point of the evaluation were also made (and rechecked just before submission of the thesis). The Ageing Well plans or other documentation were then read to understand any explanations of the changing demographic and thus the increased need for more inclusive environments. Alongside this, documents were examined for explanation of World Health Organisation outlines of the eight domains of age friendliness and the Ageing Well Wales five priority themes for age friendliness, which proposes age friendly communities as a priority theme. Explanations of these approaches provide understanding and clarity of the aims and necessity for age friendly environments which give the general public an understanding of how and why age friendliness can and should be achieved. Following on from these aspects, each document was checked for mention of key aspects of age friendliness, and of particular importance for this study, issues of mobility, disability, transport , the built environment, and mobility aids. Ease of use and ease of reading were noted, and finally, a summary was made of the general type of approach taken, any factors that stood out as interesting, useful, or unhelpful.

Different authorities interpret and implement the remit differently as might be expected, with all but one (which does not appear to have a plan published) outlining some kind of action plan with an explanation of local demographics and the five themes of Age Friendly Wales. To understand the current interpretation and implementation I carried out an assessment to understand how the directive has been integrated into planning, policy and practice by the different local government establishments. The response to Age Friendliness within the plans of some local authorities recognises the relationship between poverty, transport and isolation with reference to accessibility both in terms of cost and physical accessibility. Although affordable and accessible transport is a desirable outcome of the 
Strategy for Older People 2013-2023 (Welsh Government, 2013) it is mentioned rarely within local authority plans. Physical accessibility is also a somewhat ambiguous term. None of the Ageing Well in Wales local action plans mention accessibility of transport in terms of mobility, senescence, chronic illness, disability or mobility aids. In fact Ageing Well in Cardiff (The City of Cardiff Council, n.d.) is the only plan to mention mobility aids at all and that is in terms of a 'Smart House' scheme to enable independent living for people 50+. While this is commendable, it does not address access to social events or participation outside of the home. Omitting the need to accommodate mobility aids required by older people who have mobility difficulties seems to be an oversight, not only within local authority documentation, but also at the WHO, European Union and Ageing Well in Wales levels.

Blaenau Gwent (Blaenau Gwent Local Service Board, n.d.), Caerphilly (Caerphilly County Borough, 2015), Pembrokeshire (Pembrokeshire County Council, 2016) and Torfaen (Torfaen Public Service Board, 2015) have published a Strategy for Older People (SfOP) without separate Ageing Well plans but have incorporated the themes of Ageing Well in Wales into the local Action Plan and Strategy for Older People. Anglesey (Isle of Anglesey County Council, 2014) , Carmarthenshire (Carmarthenshire County Council, 2015) and Swansea (City and County of Swansea, 2015) have published joint Strategy for Older People and Ageing Well Action Plans integrating the themes of both initiatives to form inclusive strategies, policies and plans for older people and populations ageing into the future. Merthyr Tydfil County Borough Council and Rhondda Cynon Taf County Borough Council (n. d.) have produced a joint Cwm Taf Ageing Well in Wales Plan. The Ageing Well Plans generally explain the five themes and how they relate to local plans and policies. Most of the action plans also explain the ageing population and demographic in their respective region, giving context to why planning for older people is important. Torfaen Local Authority (2015) notes that people in their fifties can be outraged at being included in a plan for ageing but goes on to explain that the plans are for the future of ageing not just the present ageing population. All Local Authorities have published accessible plans or commitments to making their region age friendly, although the links to these from the Older People's Commissioner for Wales did not always work so some were found through alternative web searches.

Integration and implementation of age-friendliness is carried into the other four Ageing Well themes within some action plans, for example Denbighshire, Bridgend, Flintshire (Flintshire 
County Council, 2016) and Neath Port Talbot ${ }^{6}$ no link. Accessible and affordable transport is often mentioned in the action plans for reducing loneliness and isolation. Gwynedd Council (n.d.), as one example, links lack of transport and housing to loneliness and isolation with a case study reflecting a fictional lady left isolated and lonely in a rural area after her husband dies. With sparse public transport and unable to drive, the lady sells her home so that she can move nearer her children and take up opportunities provided by an Age Well Centre. The action points on the ageing well plan also reflect transport provision for therapeutic purposes but not social opportunities. From interpreting the idea that loneliness and isolation can stem from housing and transport situations, the authority seems to have lost any sense of provision or responsibility for meeting the needs of people becoming isolated and lonely in rural areas. There is no section within the action plan to provide transport or the means to relocate for isolated older people rural areas. The Age Friendly section for the Powys plan outlines only solutions to health and social care issues, with no consideration of social perspectives or connection to isolation and loneliness.

Another aspect of the Ageing Well in Wales plans that could be improved are the data collection resources. For example, Swansea City Council state the inclusion of older people's views: this entails 3 people at LSB Older People Strategy Partnership Group (members of a $50+$ group) 73 respondents online survey, 35 people in 2 forums, 20 people in another forum and 60 people at a drop in event. However, accessibility to these forums for people who use mobility aids or have mobility difficulties is open to question. Could older people access the forums physically, in person or online, and how much publicity was around them. People who are socially isolated would have, by definition, been unable to attend forums and drop in events. Online surveys can only be accessed by those who are aware of them. These issues of accessibility impact upon how well older people's views have actually been represented. The Ageing Well documents for the unitary authority for Carmarthenshire reports public concerns as canvassed by its well-supported 50+ forum which includes street lighting, paving and bus shelters. Solutions provided highlight inadequate funding and the need to influence other organisations such as local businesses.

\footnotetext{
${ }^{6}$ The links to Ageing Well plans were first accessed in August 2019 via (OPC, n.d.-a). There appears to be no longer be any accessible Ageing Well plans or Strategy for Older People for Denbighshire (redrafting plan), Bridgend or Neath Port Talbot.
} 
Overall, the interpretation, assimilation and implementation of ageing well plans are inconsistent and often piecemeal. As previously stated, there is no recent literature regarding the implementation of ageing well plans. While it could be argued the development and implementation of age friendly initiatives has been occurring against a backdrop of increasing austerity, it is also true that in this timeframe Ireland has achieved the status of being the first age friendly country, with similar economic difficulties.

During my assessment of Ageing Well in Wales plans, I was able to meet with and share my assessment with the Older People's Commissioner (OPC) for Wales who asked me to share my findings with the aging well lead in her office. During the conversations between myself and OPC staff, we agreed that proposals to incorporate Ageing Well plans, Age friendly cities/communities work and older people's strategies, with little to no translation into policy and practice is unworkable. There have been too many directives which have provided too many seemingly widespread goals. Better assimilation of goals within directives and policy is already underway within the Older People's Commissioner's office, to give clearer direction of priorities and ways to achieve goals. The need to ensure a crosssection of all types of older peoples' views are canvassed is required to ensure policy and practice reflect the needs and requirements of all older people, not just those able to respond in a way convenient to standard collection methods (Hughes, 2015). People of all abilities, races, religions, levels of income, gender and background should be approached to provide an overall view. While this research addresses older people who use mobility aids, it should be used alongside representation of other groups of older people, or to initiate wider research for a complete picture of the needs of older people across Wales. The design and implementation of planning and policy which addresses the needs of all citizens are vital to ensuring infrastructure in the built environment is accessible to all.

\subsection{Concluding remarks about literature reviewed}

Although the specific study of the daily routines of older mobility aid users has not been a focus of research, there are many disciplines and theories to draw upon for this project. Mobility aids as tools to aid mobility are themselves somewhat elusive in terms of definition and approach, depending on which perspective they are being viewed from, as demonstrated section 2.2. Definitions of mobility aids are generated within healthcare and 
governmental literature indicate that mobility aids are assistive technology, but not all assistive technology aids mobility. Medical, rehabilitation and healthcare approaches to mobility aids tend to focus on fall reduction or improvement on gait. While there is some focus on quality of life for mobility aid user within health literature, this relates to independence in a perfunctory manner. Very few research articles focus on the impact of mobility aid use on daily, mundane activities.

Psychological and emotional facets of disability within society and social structures remain relatively unaddressed in contemporary literature (Oliver, 2013). There are debates between the social and medical models of disability. At the extremes of the debates around social and medical models of disability the social model frames disability as existing in obstacles within the environment, and challenges medical model ideas of biological normality. The medical model disputes that disability can exist externally to the individual. The arguments revolve around the semantics and position of disability, but neither directly address consequences to identity or roles for an individual who lives with disability. The day to day lived aspects of mobility aid use, especially for older people who tend to be omitted from disability discourses, have been lost in these wider academic and semantic debates. This research addresses disability through the notion of mobility capital and in terms of older people, proposes a new approach in Geromobilities, discussed in Chapter 3.

Resources regarding both ageing and disability have been explored to highlight the necessity to include embodiment and bodies in research and policy protocols, methods and dialogue. Without representation of older, impaired bodies, or those using mobility aids as reflected in policy documentation regarding active ageing (e.g. WHO, 2018) bodies that deviate from a prescribed or expected norm become stigmatised (Brown, 2015; Dobbs et al., 2008; Goffman, 1990[1963]; Kusow, 2004; Pryor et al., 1999) or self-stigmatised (Bos et al., 2013; Giles \& Reid, 2005; Reeder \& Pryor, 2008; Werner, Stein-Shvachman, \& Heinik, 2009) through absorbing socially normalised expectations of self. Patterns of stigma can be seen to emanate within politics and economics, leveraging power and producing inequality (Tyler \& Slater, 2016). Mobility aids could be seen to be stigmatising symbols, thus older people who use mobility aids face the stigma of both ageing and a sense of gradually eroding embodied ability into disability, with a symbol to indicate it to the world. The stigmatising symbolisation of mobility aids, whether they bring attention to ageing or disability, or even 
the potential for reduced autonomy is offset against the ability to enable participation. These experiential dilemmas are explored throughout chapter 6 which explores aspects of identity impacted by mobility aid use.

Reiterating the need for embodied representation in research, travel research has found that social isolation is linked to travel accessibility (Aguiar \& Macário, 2017). Mobility inequality is often associated with younger people being favoured over older people. Such age discrimination is systemic and detrimental to individuals and society, based in quantitative reviews of travel needs which do not address social and emotional aspects of travel such as safety and comfort (Aguiar \& Macário, 2017; Mackett, 2018; Musselwhite, 2015, 2017). Moreover, neoliberal approaches to travel provision favour those who are economically productive (Lucas, 2011; Shergold, Lyons, \& Hubers, 2015). A mobilities capital which addresses mobility justice could unlock ways to address such inequalities, a concept which will be returned to in Chapter 3 where we consider a combination of critical gerontology and the mobilities paradigm to provide a theoretical framework for researching mobilities justice for older people. The themes explored in Chapter 8 'Infrastructure and Interlinking Systems' illustrates inequality in access to places and services, and the 'Feedback Loop of Obstacles to Mobility Aid Use' outlined in Chapter 10 demonstrates how inequality can be played out through all of the themes, and therefore all aspects of daily life for older people who use mobility aids.

Investigations of planning and design literature, or to be more precise literature critiquing planning and design, uncovers the ways assumptions about ability and embodied norms are incorporated into plans for built environments. Infrastructure is built around the needs of society, and within that, an average person. Questions arise, however, regarding the existence of 'average' and in the quest to make provision for the many with this blueprint, infrastructure, be it buildings, environments, practices or policies, can delineate what is and is not normal or acceptable (Gaete-Reyes, 2015; Hamraie, 2017; Hughes, 2015; Milner \& Kelly, 2009; Woods \& Watson, 2004). Whether through aesthetic need (Imrie \& Edwards, 2007), ableist design and configuration (Hansen \& Philo, 2007), hegemonic discourses of productive bodies (Imrie, 2000) or inadvertent continuation of historic architectural practices (Hamraie, 2017), place and space is designed with an able body that does not use a mobility aid in mind. A further implication of such ableist design, whereby features and 
functions are constructed with the assumption of use by an average (and therefore able) body, is that when bodies lose ability through ageing and/or chronic illness, the impact sends messages regarding identity, roles and relationships. The complex connectedness of individual, social and infrastructural practices are not accounted for in design of place.

Transport studies uncover similar accounts of assumptions of normality. Bodies that do not fit with these assumptions are expected to assimilate into designs rather than designs accommodating all abilities (Hansen \& Philo 2007; Imrie 2012). Again, this leads to demarcations of included or excluded in travel practices and travel infrastructure. The focus on quantity above quality in transport design and much of the research is continually questioned for the lack of focus on social consequences (Doughty \& Murray, 2014; Lucas, 2011; Musselwhite, 2017; Musselwhite \& Shergold, 2013; Urry, 2000, 2007). In addition, transport research focuses on actual travel with little accommodation of potential travel (Musselwhite \& Haddad, 2018), reflecting the aesthetic needs of people with disabilities or mobility difficulties discussed in section 2.9 Infrastructure: planning and design of the built environment, whereby people might want to travel just for the sake of the journey. Those with mobility aids or mobility difficulties are less able to access this type of travel because it is not considered within planning or provision, therefore at a disadvantage compared to people who are able to access transport easily and travel more freely either by private vehicle or public transport. While there is legislation in place to support rights of accessibility for those with disabilities or mobility difficulties it can focus rather than dissipate accessibility problems. Governmental literature acknowledges travel uptake is lower for those with mobility difficulties. Ageing is also under-represented in transport and travel design and policy, with age treated as a discrete category. Mirroring disability the social, psychological, emotional and affective needs of older people are neglected despite the knowledge that mobility is linked to health and wellbeing.

Literature has been brought together in this chapter to form a picture of the ways older people may experience using mobility aids in the social and built environment. In addition, the nature of policies and governmental structures put in place to support older people has been examined to uncover the source, role and response to ageing well, ageing successfully and age friendly policy initiatives. None of the official or governmental literature involved 
with the ageing concepts mentioned above refer to chronic illness, senescence or mobility aids in their literature.

Each and every aspect of the literature reviewed helps to construct a picture of mobility aid use by older people. From the theories of ageing through models of disability and stigma to design and planning, each section informs aspects of everyday lives for older mobility aid users. It is comprehensive in its reach of discipline and context. 


\section{Chapter 3. Theoretical Framework}

The theoretical framework introduces the methodological basis of a research project, and the standpoint of the researcher. This chapter outlines the qualitative basis of the methods employed to conduct the research with an explanation of the ethnographic grounded theory utilised with an explanation of ethical considerations. The chapter includes some important aspects of specific methodological interest to the study which informed the choice of design and data collection, namely the mobilities paradigm which is particularly pertinent to this study because of the focus on movement held within the approach. Alongside this is the outline of a combination of these approaches as a theoretical backdrop to the research. Ethical considerations are also examined.

The first sections of this chapter provide an overview of my understanding and perspective of mobility to enable a reflexive and transparent account of this research project. Charmaz (2014) identifies the theoretical framework as an opportunity to anchor grounded theory in conceptual underpinnings, and provide a baseline from which the succeeding grounded theory refines or supersedes extant concepts. This overview acknowledges and engages leading theories so that the logic and direction of my own analysis and conclusions can be understood within the context of existing knowledge. The mobilities paradigm underpins an important part of my understanding of social behaviour, and in particular, the ways people with mobility aids or disabilities become socially excluded or isolated. Both critical gerontology as discussed in chapter 2 and the mobilities paradigm examined below are interdisciplinary, which is explored in more detail. The chapter goes on to outline a way of combining critical gerontology and the mobilities paradigm to form critical geromobilities, a framework to understand the movements and obstacles to movement experienced by older people.

\subsection{The Mobilities Paradigm}

The mobilities paradigm forms an important part of my own understanding of social behaviour and therefore of the theoretical approach to this research. This section gives an overview of the literature regarding the mobilities paradigm (or mobilities turn). The term mobilities in this academic usage refers to the conceptualisation and research of the role of 
movement in social life (Urry, 2007 p.17), so often omitted from or overlooked in social science research. Humans are so used to mobilities that most aspects are considered almost natural and certainly taken for granted in the West. Repetitive movements are made possible by diverse but interdependent mobility systems which develop where there is an obligation of movement. A path, electricity, a bridge, mobilisation of an idea in the form of a protest, banks to mobilise money; computers mobilise data and computers themselves become mobilised in the form of laptop computers, tablets and mobile phones (Sheller, 2018; Sheller \& Urry, 2006; Urry, 2007). These are just some of the ways in which human lives are mobilised.

The 'new' mobilities paradigm outlined by Sheller and Urry (2006) abstracted five modes of mobility, being corporeal, imaginative, virtual, communicative and the movement of objects. Corporeal movement refers to actual movement of the body, either in situ or from place to place, similarly the movement of objects is self-explanatory as movement of objects from place to place or in situ if they have moving parts. Communicative movement refers to the sounds, equipment and infrastructure involved in spoken, written, telephone and information technology communication including social media. Imaginative and virtual mobilities are slightly different in that they take on more abstract forms. Cyber methods of virtual travel incur both imaginative and virtual movement, in that the individual both imagines the travel, and can access websites and computer simulations of places to provide an experience of travel without actually travelling. Imaginative mobilities refers to the anticipation of travel and atmosphere of place (Sheller and Urry, 2006). Within the various constructions of actual or potential movements, are combinations of these modes in complex interacting systems which not only provide access to movement, but also direct and constrict movement (Murray, 2015; Sheller, 2018; Urry, 2007).

The examination of different forms of movement over varying scales, from embodied movement to traversing the globe is brought together by the mobilities paradigm. Disparate disciplinary approaches are combined to enable more holistic understandings of mobilities (Cresswell, 2010; Sheller, 2018). Within renewed understandings from the' mobilities paradigm, complex influences and perhaps unexpected repercussions of mobility systems on social relations, equality and power relations have been highlighted (Urry, 2007; Sheller, 2018). Complex social structures do not just emerge from social conditions but are also essential to their 
formation (Murray \& Robertson, 2017; Smith \& Hall, 2016; Urry, 2007). Dominant discourses of mobility are accompanied by systemic practices which set the parameters of understanding and practicing mobility. Spatial, social and political settings influence mobility.

Much work within the mobilities turn has focused on the increasing use of cars and the resulting construction of discourses (Thrift, 2004) and infrastructure around car use (Sheller 2018). Car use provides individual access to freedom, economic success and citizenship valorised by neoliberal agendas, but is also favoured because it is convenient due to infrastructural considerations (Doughty \& Murray, 2014). People lead busy lives that require movement across space to access employment, childcare, education, leisure and shopping, all of which may be served by public transport, but people prefer the convenience and privacy of their own vehicles which have easy access to roads, car park and fuel (Doughty \& Murray, 2014). Infrastructure is not only made up of the physical structures but coconstructed with social structures. The car has been significant in reconstructing civil society, with distinct ways of dwelling, socialising and travelling configured around individual automation (Urry, 2000). This championing of privately-owned vehicles and discourse dominated by reduction of travel time has focused policy and practice on utility needs. Driving a car has both constructed and fulfilled practical, social and aesthetic needs, bit it has meant that those who do not have access to a car can face being unable to meet those needs (Bromley \& Matthews, 2007; Musselwhite, 2018).

\subsubsection{Mobility capital}

In disciplines of both gerontology and transport studies, the term mobility tends to emphasise literal or spatial movement. This definition ignores ideas of motility and potential for movement. Mobility refers to more than just movement, it refers to the ability to move, the potential and choice to move (Kaiser, 2009; Kaufmann, 2016[2002]; Kaufmann, Bergman, \& Joye, 2004). Indeed Kaiser (2009) explores the semantic meaning of the word mobility as potential for movement linked to social, emotional, psychological, and affective circumstances. The nature of investigating mobility and immobility requires attending to motility, the potential for mobility and its subsequent transition into mobility. The level congruence between motility and mobility indicates the level of fluidity of mobility 
practices. As Mobility capital, then, refers to the motility potential people have available that they can transform into mobility, and the benefits this mobility incurs or can be exchanged for such as social, economic or cultural capital (Kaufmann, 2002; Kaufmann et al., 2004). The mobilities paradigm is concerned with the transformation of motility to mobility in line with aspirations and lifestyles, thus with mobility capital (Kaufmann, 2016 [2002]). While motility is identified and performed on an individual level, it is eminently social and formatted by social, cultural, and financial situations and contexts (2002). In order to understand mobility, the context of individual behaviours and decision making is required, with social and embodied experiences along with personal traits giving context to decisions taken within particular environments (Kaiser, 2009). The needs and decisions around travelling and movement at the core of mobilities literature seeks to understand emotional and affective circumstances surrounding the transitions of motility to mobility, with both discretionary and potential travel being legitimate aspects to account for in journey-making (Green, Jones, \& Roberts, 2014; Kaiser, 2009; Musselwhite, 2017; Spinney, 2009).

Access to a car is increasingly viewed as a rural transport solution, but older people do not necessarily find this an accessible or acceptable solution (Shergold, Parkhurst, \& Musselwhite, 2012). Such an approach to rural mobility and motility in rural locations underlines the ways in which states, systems, structures, and attitudes are intertwined to shape, control or enable the mobility of people with an overall entwining of society and infrastructure. Changes in lives, changes in social structures and changes in infrastructure are fundamentally interdependent (Katz \& Calasanti, 2015). Productivity and prosperity are prioritised so those not considered to be contributing economically tend to be omitted from policy considerations (Hughes, 2015). Planning and policy therefore tend to focus on younger people, causing age segregation as older people who no longer work find it more difficult to or cannot access mobility (Musselwhite, 2018).

Much literature on embodied differences echoes the idea of segregation, with conclusions that people who do not meet a prescribed norm, including those who use mobility aids or parents with pushchairs, can feel socially and practically excluded by the planned environment and society (Boyer \& Spinney, 2016). Contextualisation of obstacles to inclusion or interpretation of experience of social exclusion have been less forthcoming (Hansen \& Philo, 2007; Mazurik et al., 2014). Musselwhite and Scott (2019) discuss 
infrastructure, individual perceptions and skills, cultural norms and expectations and social networks as all playing a role in the mobility capital of older people, highlighting the interconnective nature of social exclusion. If the planned environment is not conducive to social inclusion for all older people, it can lead to physical, social, emotional and psychological harm to the older people concerned, but also economic harm to wider society.

\subsubsection{Mobility Justice}

In light of the differing levels of access to mobility, with mobilities both produced by and productive of social relations, the notion of mobility justice provides a platform to examine

the role of social institutions in constituting power. Systems of transport and the governing of mobility and therefore immobility are questioned (Sheller, 2018). Karel Martens (2017) states that the premise of a justice perspective on transportation is fair treatment of persons, which translates to fairness in accessibility to transport. Examinations of uneven mobilities in urban transport, trip making, access to infrastructure, moving and stopping are made to consider fair distribution of accessibility (Adey, Bissell, Hannam, Merriman, \& Sheller, 2014; Sheller, 2018; Verlinghieri \& Schwanen, 2020). The movement of people constantly revises delineations and borders, therefore defining attitudes surrounding what is inside or outside of those borders, both physical and metaphorical. Within these conditions people on the extremes tend to be relegated to the edges of normative mobilities.

Circulations of people can thus be responsible for generating inequalities and politics of power (Smith \& Hall, 2016)

Overshadowed by issues of progress and modernity reflecting dominant engineering and economic discourses (Doughty \& Murray, 2014), and the dominance of speed (Adey, 2010; Vannini, 2014). Those favoured within the accepted transport and engineering frameworks dominant in planning and policy are not too old, not too young, not too slow (Murray, 2017). Among those disadvantaged by these planning and policy frameworks and values are older people (Mackett 2018 Musselwhite, 2018, 2017, 2015, 2010, Newton et al, ), people who have disabilities (Martens, 2018) and those who use mobility aids (Giesbrecht et al; Mazurik et al,2014). 


\subsubsection{The mobilities paradigm to research older people who use mobility aids}

Important aspects of the mobilities perspective utilised in this research project are understanding patterns of mobility that evolve from legislation and policy influences, and the resulting ramifications on equality and power relations. The term 'mobilities' refers to this broad subject of establishing a 'movement-driven' social science in which movement, potential movement and blocked movement are all conceptualised as constitutive of economic, social and political relations (Urry, 2007, p. 43). Challenging conventional transport research assumptions mobilities researchers propose that while structures enhance movement and mobility for some, they reinforce immobility for others (Sheller, 2017; Urry, 2007). Incorporating multidisciplinary approaches to gain holistic knowledge of individuals within the context of their social, economic, and embodied circumstances the mobilities approach provides insight into the social aspects and individual practices of movement that a transport approach does not address (Cresswell, 2010; Sheller, 2017; Urry, 2007).

Transport research and planning seeks to optimise travel and movement patterns, but does not accommodate the specific representations and meanings of human mobility as practised by individuals (Martens, 2012). While transport is thought to offer mobility, there is no consideration of the ability of individuals to attain mobility via the transport options offered (Kaufmann, 2016 [2002]). Transport research does not accommodate examination of motility, mobility or how different groups in society benefit from policy or transport measures (Cresswell, 2010; Martens, 2012). Dominant models of mobility emerge from legislation, policy, and public action, and are ultimately imposed on a whole population. Those who do not fit the dominant model are thus constrained in accessing (e.g., Kaufmann, 2016 [2002]) or will incur an additional workload to access the same services (Boyer \& Spinney, 2016). The Public Transport Accessibility Level, a tool for measuring transport connectivity for Transport for London, for example, assumes a male, non-disabled working age commuter times, spaces and speeds are based on the capacity of this type of body. Anyone who does not fit the model will therefore have to make adaptions to fit the system Middleton and Spinney (2016). 
Mobilities research focuses on the links between transport and social factors. As discussed in the previous sections, mobility capital and mobility justice concepts highlight the role of mobility in a fair and equal society. Aspects such as sustaining social networks, access to community participation, civic engagement and direct or indirect therapeutic spaces are examined through a mobilities perspective (Aguiar \& Macário 2017; Lucas, 2011; Murray, 2015(Lucas, 2011; Newton et al., 2010; Raymond et al., 2014). Although architects, urban planners and engineers attempt to make solutions for mobility in old age, there is an omission of the emotional and affective aspects of potential mobilities leading to inequality of access to services, infrastructure and therefore participation (Kaiser, 2009; Musselwhite, 2017). The focus of travel research on older populations tends to be accessibility or travel for therapeutic purposes giving rise to mainly quantitative research methods which fail to acknowledge the more complex meanings, context and aspirational qualities of mobility (Murray, 2015; Musselwhite, 2018; Musselwhite and Haddad, 2018). Focusing on travel for therapeutic purposes could be said to uphold or compound the medical categorisations of ageing and disability, or at least omit the emotional and affective aspects of potential mobilities (Kaiser, 2009; Musselwhite, 2017). Often research and planning attempts to address mobility solutions for older people but omits consideration of social, cultural and psychological aspects especially when embodied mobility is difficult or impaired (Dumbaugh, 2008; Kaiser, 2009)

Planning and policy that fail to consider mobility in its whole sense lead to health and wellbeing not being linked to the mobility of older people and the subsequent links being neglected in research literature and policy (Aguiar \& Macário 2017; Murray, 2015). Older people can feel imprisoned by space (Rowles, 1978) whereby physical, psychological and cognitive change along with economic deprivation can lead older people to withdraw from the outside world. For example, compared to younger people, older people may find walking or cycling for long periods difficult due to fatigue (Musselwhite, 2018). Mobility needs are more than just the need to run errands, work or attend medical appointments. Traffic and transport psychology, the mobilities paradigm and human geography all emphasise the importance a focusing on people and social context (Musselwhite, 2018). The inability to participate in normal behaviours and relationships available to the majority in society would be considered detrimental to well-being. This affects both quality of life of the 
individual and the cohesion and equity of society. Lack of access and transport are recognised as significant factors in social exclusion, with transport playing a major role in accessing social networks (Lucas, 2011).

Understanding the changing requirements for movement as we age is imperative to enabling social and physical structures, emotional and psychological support. Boyer and Spinney (2016) use the mobilities paradigm to theorise how bodies, identities, objects and spaces are combined to produce mobility. For the purposes of this research, the mobilities paradigm conceptualisation of movement as constitutive of relations permits the investigation of mobility aids (as objects) as facilitators of social participation in wider local environments. From this perspective it could be said that a research project to understand mobility aid use by older people is a project to examine the extent to which mobility aids actually do facilitate social participation, actual mobility and the presence of any mobility inequality. Participation holds a central position in ageing policy and research (Raymond et al., 2014) as discussed in Age Friendly and Ageing Well in Wales literature (e.g., OPC 2015; WHO, 2007; 2014; 2016; 2018). The interpretation and translation of policy into practice is therefore part of the analysis of this thesis. There is a contribution to mobilities research with a rich case study of the ways in which objects, that is mobility aids, combine with bodies, competencies and infrastructure to construct mobility.

\subsection{Interdisciplinary nature of mobility and gerontology studies}

The introduction (Chapter 1 ) to this study highlights the requirement for Age Friendly research to incorporate knowledge and evidence from different disciplines to gain a complete comprehensive view of the impact ageing populations will have on people, places, systems and infrastructures. Mobility and ageing are relevant to many academic disciplines and arenas including psychology, sociology, ecological, social and environmental perspectives (Aguiar \& Macário, 2017; Kaiser, 2009; Musselwhite, 2018; Musselwhite, Holland, \& Walker, 2015; Peace et al., 2006). Chapter 2 demonstrates the wide-ranging disciplines and research arenas involved in the study of ageing and mobility respectively, with gerontology and ageing studies (e.g. Havighurst, 1961; Minkler \& Fadem, 2002; Bowling \& Dieppe, 2005; Katz \& Calasanti, 2015) ageing and disability studies (Taghizadeh Larsson 2013, Oliver, 2009, 2013; Beaudry, 2016; Hansen \& Philo 2007), embodiment (Csordas, 
2004; Hastings \& Thomas, 2005; Tulle-Winton, 2000), politics (Chambers, 2006; Chaney, 2013), 2013) embodiment and identity which incorporates psychology (Finlay, 2003; Shilling, 2005), stigma incorporating sociology, healthcare and wellbeing (Goffman, 1990; Parker \& Aggleton, 2003; Tyler \& Slater, 2018), mobility aids (Costamagna et al., 2017; Muir-Hunter \& Montero-Odasso, 2016) which includes occupational health, nursing and rehabilitation literature, mobility (Dumbaugh 2008, Kaiser, 2009) which links to geography (Imrie, 2000, 2012; Smith \& Hall, 2016; Spinney, Aldred, \& Brown, 2015) planning and design (Cheshmehzangi, 2014; Hamraie, 2017) and transport studies including the study of ageing in transport (Aguiar and Macario, 2017; Doughty \& Murray, 2014; Musselwhite 2018). Each author, article or chapter resourced potentially reflects more than one discipline or outlook. Disciplinary boundaries, though, are not present in participants' experience. The application of discipline, theory and perspective are applied (particularly when using grounded theory methodology) at the point of interpretation. Such application is useful, but it should be as invisible as possible at data collection. Studies of ageing and particularly successful ageing, for example, tend to reflect the discipline of the researcher, with biomedical outlooks, psychosocial views and lay views all informing the nature of what even constitutes successful ageing (Bowling \& Dieppe, 2005). Studies of ageing and movement are multi-faceted and ageing as part of a life experience is not lived in discrete categories. Instead, ageing and movement involves interconnecting and multidirectional concepts and patterns of influence, which are implemented in understanding the world around us (Murray, 2015). Add to this the aspects of embodiment, disability and assistive technology or mobility aids, the many dimensions and complex interconnectivity of academic disciplines involved in this research are clear.

The most important aspect of this research project was to reflect older people's experience of mobility aid use, and how much mobility and social participation changes as a consequence of mobility aid use. As discussed below in sections 3.6 and 3.7, the choice of methodology reflects this intention to allow experience to be the guide, rather than any disciplinary or theoretical assumptions. While I have outlined here a theoretical framework, this examines my academic and personal understanding of the concepts and knowledge involved, rather than providing a conceptual framework on which results were based. In fact, Chapter 4 (4.2.3) highlights that some of the issues I expected were not forthcoming. 
Experience does not fit into a disciplinary category, or a policy category for that matter, so this research utilises the mobilities paradigm and critical gerontology, both of which are interdisciplinary, to understand the movement needs, wishes, requirements and experiences of older people.

\subsection{Critical Geromobilities}

Knowledge of the mobilities paradigm and its focus on movement led to an initial intention to combine the perspective with critical gerontology to form a theoretical framework for studying the mobility of older people. In a similar vein to mobilities, critical gerontology highlights social, political and economic factors within ageing studies. Social lives are not static or immobile, but have largely been studied without exploration of the ways in which mobilities are produced by and productive of social relations and constructions (e.g., Murray \& Robertson, 2017; Smith \& Hall, 2016; Urry, 2007). Disparities in life course and social factors are identified as contributing to inequalities in access to essential life elements such as healthcare, employment, goods, environmental and psychological circumstances all of which have implications for ageing. In short, some people have better access to jobs and healthcare so are better equipped to age with better health and social circumstances (Holstein \& Minkler, 2007; Phillipson, 2008; Raymond et al., 2014).

A critical geromobilities perspective aims to understand the mobility needs and resources of older people. By exploring mobility justice and highlighting ways in which the built environment, policy and planning could better provide for the increasing older population, the mobilities paradigm informs social theorisation of the movement involved in the social lives of older people. Without movement, there is not life according Urry (2007), one of the major proponents of mobilities. Critical gerontology provides a theoretical framework which explores and challenges the successful ageing notions which have been popularised within social gerontology in recent years. There are aspects of gender, culture, race, disability and class not accommodated by the standard approach to successful or active ageing (e.g., Timonen, 2016). Combining critical gerontology and the mobilities paradigm seeks to uncover ontological and methodological assumptions in research and understanding of mobility in social phenomena, particularly focusing on older people. As outlined in chapter 
2, mobility inclusive criteria are essential for not only the health and well-being of older people, but also for national economies and societies, making this a worthy endeavour.

Academic accounts explicitly or implicitly portray ageing successfully as a function of nondisabled people which is further compounded by the lack of visible impairments or mobility aids in advertising or media representation. Ageing with a disability has been implicitly demarcated as unsuccessful (Taghizadeh Larsson, 2013). While ageing research and policy tends to overlook disability (Raymond et al, 2014; Minkler \& Fadem, 2002), disability activism and policy tends to overlook ageing (Jönson \& Taghizadeh Larsson, 2009). With the nature of lifelong disability, senescence and chronic illness all potentially presenting mobility difficulties, there are some crossover points between ageing and disability studies.

A geromobilities theoretical framework was created as an original framework designed to capture and document a mobility capital (as discussed by Musselwhite and Scott, 2019), whereby the ability for older people to mobilise could be understood as a factor of health and well-being. The notion of critical geromobilities was and is conducive to understanding the social abilities of older people through a mobilities capital. Seeking to describe, recognise, measure and evidence a mobilities capital is a necessary endeavour. It is involved primarily with understanding social and physical infrastructure.

Critical geromobilities was thus to be created for the purpose of collecting and analysing data within this $\mathrm{PhD}$ as an original approach to studying the mobility of older people. However, as this PhD is focused on mobility aid use specifically, and the as yet unspecified obstacles to participation faced by older people who use them, it would be difficult to demonstrate and evaluate the contribution of a newly developed framework within a relatively unresearched field. To focus on describing the obstacles to participation for older mobility aid users, which are not well described or understood in existing literature, I decided to reject the framework in favour of ethnographic grounded theory as a more appropriate methodology than using a critical geromobilities tool (as outlined below in section 3.6). Furthermore, being mindful of researching this emotive area with as few preconceptions as possible, grounded theory offered a resource to research phenomena and process with tools to reduce research preconceptions as much as possible (Bryant \& Charmaz, 2007; Charmaz, 2014; Timmermans \& Tavory, 2007). 


\subsection{A qualitative methodology}

Qualitative methodologies offer a variety of methods and approaches to explore social phenomena with an emphasises on process and meaning, investigating situational constraints as well as attitudes and experience. Such diversity of approaches enables an openness and flexibility towards goals and objectives, so that phenomena can be investigated within often complex contexts (Creswell, 2014; Denzin \& Lincoln, 1998; Flick, 2002). Many of the studies of mobility aids referred to within the literature review have utilised either purely qualitative designs to understand mobility aid use (e.g. Bromley \& Matthews, 2007; Frank et al, 2010; Giesbrecht et al, 2015) , or mixed methods (e.g., Velho et al., 2016). Qualitative approaches are needed because understanding the experience of using mobility aids requires qualitative methods to draw out the meanings and contexts of day-to-day use. Mazurik et al's (2016) study of obstacles from the experiential perspective utilised life history interviews alongside a semi structured interview and a visit to mall to give insight into the perceptions and lifestyles of electric wheelchair users, a guide dog user and 3 people with disabilities who did not use aids. Barlew et al. (2013) employed a qualitative existential-phenomenological methodology to examine experiences of stroke survivors, in particular experiences that stood out against the context of participants' daily life. Stroke survivors using wheelchairs felt isolated, frustrated and limited in community participation. Males who were dependent on others were identified as a group who particularly felt powerless and vulnerable, feeling Inferior and less likely to participate outside homes.

The studies outlined above exemplify the bricolage element of qualitative design, in that researchers choose the methods and techniques that they believed would best access the experiences and phenomena being studied. Flexibility of approach allows methods to be adapted to characteristics of viewpoints and practices in the field (Davies \& Hughes, 2014; Flick, 2002), which is important when researching mobility and mobility aids as it is not possible to pre-empt all scenarios, viewpoints, obstacles or the difficulties that may be faced. Experience is the key to social enquiry (Clandinin \& Connelly, 1998) and this research project in particular because of the need to capture lived experience in spatial and social context. This research aims to describe the experience of mobility aid use by older people so that it may be better understood by social science. The methods required to facilitate 
such in-depth descriptions and representations of each older individual's reality of using mobility aids in their lived environments. Representation includes not only which type of mobility aids are used by whom, when and why, but user's perception of wider lives and experiences. The task requires deep understanding of personal experience and pre-existing attitudes incorporating diversity of class, gender, and socioeconomic background.

Qualitative methodology was appropriate for this research topic because of the complexity of contextual factors such as disability, ageing, health status and others which at the point of designing the project were not necessarily known and to capture lived experience in context. As discussed in Chapter 2, many aspects of research, design and policy fail to incorporate the experience of embodied people using the environment. People have bodies as well as minds, but research has historically focused on minds or made assumptions of normative, able bodies. Investigating how older people use mobility aids in their lived environments requires exploration of both movement and thought in situ to holistically capture experience Qualitative research as a field of enquiry incorporates many disciplines, terms, concepts and perspectives. The commonality shared by qualitative research is a naturalistic, interpretive approach which explores the meanings people attach to phenomena. There is an evolution to qualitative research designs that has been referred to as bricolage, whereby methods, techniques and practices are emergent as research is planned and conducted. Choices are pieced together through strategic, pragmatic and selfreflexive processes to enable researchers to access the participants, environment and phenomena being researched (Denzin \& Lincoln, 1998).

\subsection{Ethnography}

Ethnography is an approach to data collection which incorporates experiencing and interpreting the culture and society of those being researched (Flick, 2002; Pink, 2006). Emanating from anthropological studies undertaken at the beginning of the 1900 s to study (often) remote societies, the approach was adopted by the Chicago School of Sociology to study urban settings (Adler \& Adler, 2010; Reeves, Kuper, \& Hodges, 2008). The ethnographic methodology associated most closely with the Chicago School is immersion into the society or culture being observed and use of unstructured in-depth interviewing alongside participant-observation. This methodology requires a balancing of subjectivity and 
objectivity with the researcher being both friendly visitor and detached stranger (Adler \& Adler, 2010). The early 60 s to 70 s saw anthropologists employ a more objective, structured formalistic approach with the most extreme form of objective ethnography being ethnoscience, whereby frames and systems establish a narrow focus on data collection and analysis. Questions regarding legitimising knowledge through scientific notions of credibility emerged (Adler \& Adler, 2010; Flick, 2002; Shaffir, 1999) because social research, particularly through social constructivist or constructionist lenses, reject the idea that a reality independent of subjective or shared viewpoints exists (Flick, 2002). The late 1960s into the 70s and 80s a saw more radical introduction of subjectivity whereby instead of remaining detached, ethnographers were encouraged to supplement observational data with accounts of their own feelings and experiences (Adler \& Adler, 2010).

Ethnographers began to explore and record their own feelings and experiences in the research process leading transparency about the research choices and evaluation. In humanising research, ethnography practitioners could become closer and more integrated with participants. The new way of conducting ethnography provided a more honest account of how the research was carried out (Shaffir, 1999). Instead of methods and disciplinary criteria guiding what is relevant, ethnographic research encourages researchers to navigate settings to comprehend environments and processes using their own understandings. It is a feature of this approach that data is produced from avenues other than, but in addition to interview responses or answers to questions. (Flick, 2002). Ethnography is usually collected alone by one individual researcher (Adler \& Adler, 2010) and a key element of ethnography is the researcher-participant relationship (Kendall \& Thangaraj, 2012). Practising ethnography involves direct engagement with the world and people being studied, observing, listening to and asking questions about people's daily lives (Flick, 2002; Pink, 2006; Reeves et al., 2008); "what they say, what they do, and what they say they do" (Kendal \& Thangaraj, 2012, p. 83). Data is thus a representation of ethnographer's experience as well as observation which, through reflexive practices, is as loyal to the context and intersubjectivities as possible (Pink, 2006). The deeply meaningful descriptive qualities of data gained through ethnographic endeavours enables rich, thorough representations and perceptions of the day to day life of populations (Katz, Holland, \& Peace, 2013). 
Ethnographic methodologies enable social science to move towards description and categorisations that admit the ambiguity and context of the world (Ahmed, 2004). Understanding people, using their bodies and within their environments provides a full and deep insight into the processes of lived experience. Information regarding context helps researchers to interpret information and compare data collected from different situations (Flick, 2002). Ethnography has been used in studies for a variety of disciplines and purposes as a very useful methodology in terms of studying people and behaviour in situ, one of the requirements of this research. Dobbs and colleagues (2008) used an ethnographic approach to investigate experiences of residential care - assisted living ( $R C A L)$ in the setting of longterm care facilities for older people in Maryland, Baltimore. Combining observation with semi structured interviews researchers identified stigma related to age in their exploration of transition into, within and out of care. As you will see in Chapters 5 and 6, the current study has identified stigma in terms of both age and mobility aid use.

In an urban setting in Wales, Smith \& Hall (2016) explored pedestrian circulations of outreach workers in their operational settings. An ethnographic approach offered the ability to position researchers within the setting of events so they could recognise record practices. Researchers observed how action occurs within or between pre-existing boundaries, settings or organisational practices, while also recording the constituting and reconstituting of such boundaries through their practice. Smith and Hall's (2016) study explores both the mobile and situated character of ethnography being used in multi-sited contexts. Multi-sited ethnography enables research to be conducted at sites which have a connection. Rather than a simple comparison of localities, this methodological approach enables the study of people and culture incorporating the role of movement and mobility experiences (Jirón, 2011). 


\subsubsection{To ensure a range of mobility aid experiences and thus movements were}

captured, plans were made to seek participation from different settings and places, making multisite ethnography an optimal choice to collect data. The collection of ethnographic narrative complemented with photographic evidence can track mobility to understand how people move around their environment in both time and space (Jirón, 2011). The method was utilised here to capture and understand the movements of older mobility aid users. Mobile ethnography

Mobile methods have been developing as a consequence of the mobilities paradigm, with politics of embodiment and resulting power relations central to research (Parent, 2016). Dobbs et al (2008) study of transitions into residential care, and Smith and Hall's (2016) investigation of mobile outreach workers, are both mobile research, in that they seek to understand the social implications of movement. The early anthropological studies described in the previous section usually involved a lot of mobility and movement to reach destinations so it could perhaps be said that ethnography has always been mobile. However, a new conceptualisation of mobile ethnography has emerged from the mobilities work devised by John Urry and Mimi Sheller (2006) outlined in Chapter 2 and discussed again later in this chapter, because of the need to attend to movement as a fundamental of social life (Novoa, 2015). Mobile ethnographies can take a variety of forms; being itinerant or siteless when discussing diasporic populations, participation-while-interviewing in which researchers first participate in movement patterns then interviews participants or conducts focus groups to uncover the ways movement and mobilities constitute the patterns of their everyday lives, or 'walking with' people to understand their worldview (Sheller \& Urry, 2006). Walking, or travelling with people in other ways constitutes a form of ethnography that allows what participation in movement while simultaneously immersed 'co-present' with participants (Urry, 2007, p. 40). Mobile ethnography involves moving and analysing movement, attending to practices arising from mobility, the meanings attached to informants' worldviews and feelings about mobility. The result can provide a holistic view of the lives of people and how mobility structures inform, construct and constrict lives. Photographic evidence is often used to supplement data (Novoa, 2015). 
Mobile ethnography was used in this research because it allowed me to be present with participants whilst they traversed their environments, enabling insight into their abilities, difficulties, relationships and the meanings attached to places and things. The journeys we took evoked memories and realisations in participants which I could experience with them or observe, allowed me to experience the terrain, and facilitated a mutual sense of understanding, being, and moving through the places we walked through. A participationwhile-interviewing approach and accompanying photography was therefore employed for this research. Supplementing these data were maps to record the daily movements of mobility aid users, the walk we conducted to compare these movements to previous daily routine movements. A further risk assessment was conducted as part of a safety process, but this also acted as a way of establishing participants' own assessment of their mobility status. Conducting movements and routines with the individuals being researched enables a 'co-presence', a comparable physical presence in which to gather data (Novoa, 2015). Otherwise termed 'on-the-move' interviews, this form of data collection capacitates access to attitudes and knowledge of surrounding environment and is considered an intimate way to connect with landscape and access insights into place and self (Evans \& Jones, 2011).

Walking constitutes a social interaction situated in time and space which offer opportunities to form repeated movements and exchanges, chance encounters and building of relationships. Where we go, with whom and when are shaped by out competencies. Studying movements enables understanding of the consequences of competences interacting with people, policies, practices and environments (Hodgson, 2011). Walking, or otherwise moving around environments with participants allowed for intimate insights into their practices and observations of the ways social and physical structures enabled or excluded participation and activity.

\subsection{Grounded Theory}

Grounded theory was conceptualised during an ethnographic study of Awareness of Dying by Glaser and Strauss (1965 cited in Bryant \& Charmaz, 2007) and later formally introduced in the Discovery of Grounded Theory: Strategies for Qualitative Research (1967, cited in Charmaz, 2014). Whilst exploring circumstances and experiences of dying in hospital, Glaser and Strauss developed systematic, methodological strategies for research which advocated 
developing research grounded in the qualitative data explored and collected. This was in opposition to the practices of other scientific methodologies which seek to deduce testable hypotheses from existing theories (Charmaz, 2014). Attempting to capture the creative and fluid nature of ethnographic research, grounded theory allows focus on process or phenomenon (or both) using successive comparative analyses, inductive-abductive reasoning, memos, coding and theoretical sampling. In this way, grounded theory seeks to uncover events and phenomena situated in social context (Bryant \& Charmaz, 2007; Charmaz, 2014; Timmermans \& Tavory, 2007). A surprising finding during analysis of inductive, emergent ideas leads to abductive reasoning by way of discerning how theory could account for it.

Constructivist Grounded Theory, a contemporary version of grounded theory, was employed for this research because it scrutinises the role of researcher and the research process, acknowledging subjectivity. The social location of both researcher and participants matters because ways of knowing are embedded in these social contexts. Research and research process are thus located in historical, social and political context as well as physical proximity. Data is uncovered through a process of constant comparison and analysis, with a constructivist grounded theory approach discounting any notion of objective external realities, instead understanding social reality to be made up of processes and constructed. Constructivist grounded theory permits designing and fitting methodological strategies to explore the phenomena discovered by the researcher along the way (Charmaz, 2014, 2017). Conducted within the environment in which studied behaviours occurs, grounded theory reflects the experiential lives of participants. The careful coding of words, lines and incidents that have emerged from data sought to view participant understandings of their own world allows for a study that both fits and is relevant to the social world examined (Charmaz, 2014 , 2017). The constructivist grounded theory approach allowed constant assessment of data form participants, alongside my own knowledge and understanding within the lived experiential context of people's daily routine lives.

The focus on process in grounded theory can facilitate because of the relatively unexplored and undefined nature of older people's use of mobility aids in the built environment benefited the current study. It allowed exploration of the ways social attitudes could be framed by legislation, policy and practice, and how identity, relationships and 
environmental infrastructure can work in association to reinforce attitudes, practices and social inclusion or exclusion. Moreover, the relevance of an analytic framework that allows for interest in and attention to social process and social structure as well as any infrastructural dimensions to situations and daily experiences reinforces the relevance of the research (Charmaz, 2014, 2017). While offering a systematic, empirical form of data collection, grounded theory allows exploration of people's own experiences without hypothesising or conjecture (Bryant \& Charmaz, 2007). Grounded theory methodology captures individual experiences within structural context, power dynamics and collective ideologies on which individual behaviours may be reliant, allowing for exploration of both individual and social factors in mobility aid use. The third reason for establishing grounded theory as the methodology for this research is the ability to recognise the uncertain, diverse views, variations in experience and often incomplete nature of knowledge in theoretical interpretation (Bryant \& Charmaz, 2007).

This PhD employed constructivist grounded theory to explore and observe how people's actions, interactions, choice of language, changing embodiment, and especially use of mobility aids as tools of navigating the physical and social environment permeated behaviours, shaped biographies and shaped environments. A major aspect of data collection in this study was the observation of how people interacted with me, other people and the environment. It was important to record incidents of non-interaction as well as interaction, another asset of using this approach. The emphasis within ethnographic grounded theory of studying people in the context of their everyday lives enabled the best opportunity to explore everyday experiences of mobility aid use within mundane, daily routines. The ability to move from site to site, urban or rural, densely populated to less populated, bustling or quieter areas. Such as mobilised ethnography facilitates, allowed for patterns of movement to be observed, followed, discussed and recorded within these different contexts.

\subsubsection{Constant comparative analysis}

Grounded theory methodology employs constant comparative analysis to digest and connect data. Analysis within ethnographic grounded theory takes the form of constant comparative analysis, whereby data is compared with existing data, codes with new codes and back to data until themes and concepts are formed. At the stage of concepts emerging, 
data, codes and concepts are compared with relevant literature and patterns begin to emerge. Once themes and patterns have been identified and noted within one data set, substantiation and support or opposing patterns and themes can be noted in subsequent participants' data. In this way, data is continually analysed, compared within the data set and referred to external pre-existing concepts and theories. Data is compared systematically within and between participants in light of new concepts as they emerge to demonstrate and illustrate relations between categories and later concepts. Codes are compared with codes, codes with categories, categories with concepts, so that comparisons of data, codes, categories and concepts constitute each stage of analysis. Towards the latter stages of analysis, comparative academic literature is referenced and compared. Emergent categories and concepts form the basis of analysis and finally construction of new conceptualisations and theorisations (Charmaz, 2014).

The process of constantly comparing data involves comparing what is said, but also what is not said (Charmaz, 2014), how things are said, how people behave, and the use of memos to record observations of interactions, environments and my thoughts as I took part in discussions and walking interviews. The ability to capture and incorporate what is not said or done within participation fitted well with the nature of previously documented facets of mobility aid use, such as the potential for non-use or stigma. The nature of an ethnographic and mobile methodology in which I could simultaneously discuss, experience and observe participants and they conducted their mobility practices allowed for comparisons of what people said compared to what they did. As Chapter 6 for example will highlight, people may say one thing but do something different. Ethnographic grounded theory methodology allowed data collection to capture aspects of mobility aid users lives that they may not want to discuss, may not have noticed or were embarrassed to mention. With observation and field notes, such aspects could be recorded and analysed. The way this methodology was utilised specifically within this research is documented in Chapter 4 which outlines the analysis and coding process.

\subsection{Ethical Considerations}

Ethical concerns of this research were wide and varied. The universal ethical concerns regarding participation such as power imbalances, anonymity, confidentiality and respect 
were present along with some specific considerations regarding the target research population. Ethical approval for this study was sought and gained from the Swansea

University College of Human and Health Sciences Ethics Committee in June/July 2017 (Appendix C). The application outlined ethnographic walking interviews within a grounded theory methodology, asserting that participation would not be fully substantiated until acquaintance was established with each participant and subsequent research setting.

\subsubsection{Ethics of researching spouse}

There were specific challenges presented by my husband's role in the research. It was important to interview him because of his integral role in my understanding of disability and mobility aid use. Doing so meant I could present a truly reflexive account of mobility aid use and my experience of it as a spouse, a carer and a researcher. To begin with, ethical approval had not included specifics outlining that my husband would be a participant. Following advice from the Chair of the Research Ethics Committee, approval was given on the basis that while the application was not explicit in including him, the interview was not gained under coercion. Phil was and is fully aware of the basis, inspiration, potential outputs and consequences of taking part in the study, not least that as my husband his anonymity and confidentiality cannot be protected in the same way as others, which will be discussed further in the relevant sections herein.

The ethical implications relate wholly to our relationship, and whilst considering his inclusion as a participant, I consulted literature in which ethnographers have catalogued practices regarding self and close relationships. In her article 'Me My*self and I, Personal and Professional Re-Constructions in Ethnographic Research', Eileen Day (2002) outlines her conscious choice to divide the word myself into my*self to explicitly express the construction of self through multiple voices when examining her position in an ethnography involving her sister and her mother. The glimpses of herself as constructed through her family relationships are emphasised rather than hidden to enable a more honest and reflexive voice. This allows her to portray self as researcher, which is also coloured by self as a person, woman, sister, daughter. Ethnographic research is involved in collecting dialogue created between people, a researcher and participants but the practice requires mutual participation. A pre-existing deep relationship does not necessarily negate all power 
imbalances but there will be a known aspect of respect and honour from both sides of the research equation (Owton \& Allen-Collinson, 2014).

Discussion of mobility aid use and walking interview (or discussion) with my husband were undertaken in a formal way, in that time was set aside to conduct these discussions and for me as a researcher to observe his action during a particular hospital visit. As the literature above discerns, it was impossible for me to be researcher but not wife, or wife but not researcher, as there is always an element of both active, not to mention the other roles I play such as mother, daughter and friend. However, when discussing mobility aid use with him, my husband was very articulate and forthright about his opinions, practices, feelings and the obstacles he faces. To the point that I was slightly stunned by the profound language and metaphors he used, such as dropped kerbs being likened to gas masks without which one cannot survive. The walking interview we took together was recorded so analysis could be made of the recording as well as the observations. Unlike other data collection, I have historical knowledge of his mobility aid use, practices, preferences and obstacles faces which will have undoubtedly impacted data collected and interpretation. Phil's initial discussion deliberately took place after many other people had taken part in the study, and his walking interview was the last participation as he was taken very ill during the research process.

\subsubsection{Informed consent}

To ensure participant were fully aware of what taking part in the research involved, an informed consent process, fully informed participants of the process, purpose and potential publication of the research. Part of the research could or would include speaking to myself while walking in the local area so people taking part were advised that being seen with me by those who know of the research could lead to the potential of being identified as taking part in the research, especially those living in my home town. Every participant commented that they did not mind people knowing they were taking part or what their responses were, however, great care has still been taken to anonymise responses. Recording devices were purposely inconspicuous and no note taking occurred during walking interviews, so it was understood that the conversation could potentially be about anything, not necessarily mobility aids. All participants felt this not to be a problem especially as they had been 
assured no comparisons between participants or relationships between people would be discussed in any identifiable way. The people, places and any visual data used would be anonymised, which might include avoiding the mention of occupation or events to ensure individuals were not identifiable. Any private conversation would be stopped if it might be overheard.

All participants were reminded of their right to withdraw at any point without the need to give a reason and without judgement. As some of the participants were neighbours (although not direct neighbours), it was important to ensure that they did not feel coerced into participation. Extra effort was made to ensure people did not feel obliged in any way to take part, or to continue participation for any reason should they start to feel uncomfortable or did not want to continue for any reason. All participants were informed that withdrawal at any point is a normal part of research and totally acceptable. No participants chose to withdraw.

\subsubsection{Anonymity and Confidentiality}

All participants information was anonymised from the point participation as pseudonyms were used for transcription and note taking. Along with participants names, place names have been changed or excluded and any identifying information such as occupation or roles in clubs or organisations have also been excluded. Any visual data has been anonymised, which also ensures individuals were not identifiable. Consent forms and data were kept in locked cabinets and all computerised documentation and analysis has been accessible only through password protected software and documents. It is a different case with my husband, as he is obviously identifiable as my husband. A pseudonym is used for him, but anyone who knows me will know him by association. Phil and I discussed his participation in the study at length, including the consequences that he would not remain anonymous and by virtue of this, his accounts were not confidential. He felt that his account was important to the study, because he inspired the study, because he is a relatively young mobility aid user in terms of the study and because he uses a substantial mobility aid. I felt that his account was important too, for the same reasons as Phil, and because his account gives insight into my perception and standpoint also. I discussed with my supervisors how to handle the situation. Having read some literature regarding researching close relationships, 
we decided the insight was too valuable to lose. Phil understands the implication of being identified as my spouse, but to ignore the relationship would not do the study justice. He completely consents to the waiver of anonymity that this results in.

\subsubsection{Participant and researcher safety}

Safety issues were anticipated and mitigated within the ethical approval process as much as possible. It was important to establish the continual physical safety of both myself as researcher and each participant. As this research was conducted whilst carrying out normal routines and practices, the risks were likely to be those faced in day-to-day life, ergo risks of falls, issues of road safety and similar everyday risks. To ameliorate risk as best as possible and ensure a family member could be contacted in case of an emergency, a risk assessment (appendix D) was carried out before any walking interview occurred. The assessment involved asking each participant what they felt were the risks of walking in the built environment. When the Ethics Committee first insisted, I carry out these risk assessments with each individual, I felt them to be discriminatory against both age and disability because the target population carry out these activities every day. However, having discussed the situation with my supervisors I took the stance that the situation was akin to taking students on a field trip; students are capable of looking after themselves but are still under the overall care of the research institute when on a field trip. There was a secondary benefit to the risk assessments in that they gave a good indication of each participant's attitude towards their mobility aid and embodied ability. Some were perplexed at the need for them though.

To avoid detracting attention from the tasks at hand, conversations taking place whilst in transit were audio recorded. Spaces and places that were either dangerous or uncomfortable for either party would be avoided, but generally places visited would be directed by the participant, driven by their lifestyle, interests and routine. Any physical danger in the environment was mitigated partially by completing a risk assessment immediately before completing the walking aspect of participation. Not only did this assess physical risks, but gave a good indication of each participant's attitude towards their mobility aid and embodied ability. As the researcher I felt a duty to adjust to the needs of 
participants, but at the same time was aware of my own safety. Swansea University researcher safety protocols were always followed.

\subsubsection{Participant well-being during and after the research}

The research respected the needs and wishes of each individual whilst intending to contribute to the development of research and understanding of mobility aid use. The issues discussed were expected to be those brought to the fore by participants themselves, and as such would already be on their minds. This somewhat ameliorated the potential for the research introducing self-doubt. Whilst it was not predicted that the experience would evoke any excessive emotional or psychological reactions, they could not be ruled out. Literature and phone numbers of help groups were carried to advise any distressed participants. It was anticipated that any participant getting distressed could be encouraged to pause or cease participation at any time, however this was not necessary in practice. Telephone numbers were available on the debrief sheet available at the end of participation. The debrief sheet (appendix F) for all participants took the form of thank you leaflets that also outlined contact details for the researcher and supervisor along with helpline and medical advice numbers.

Contentious issues or anything people felt embarrassed about could be talked about in private, as would be explained before any walking interview commenced. Stigma was identified as an issue likely to be discussed, but it was also acknowledged that the stigma of using a mobility tool would only be brought up by the participant who would already feel stigmatised by either using or not using the aid. Furthermore, it was hoped that contentious or confidential issues would be addressed during the initial conversations that were usually held in private. Any discussions held in public were expected to be those that would normally be held in public. The walking interviews were intended to reflect everyday activities so confidential aspects were not expected, and issues that were contentious pertaining to mobility aid use in public were likely to be voiced in public as they would be in normal conversations. It was thought likely that people who use mobility aids in public spaces would be able and willing to articulate issues; those unwilling to discuss issues would also be unlikely to use their mobility aids. However, all eventualities were considered, and 
any potential difficulty was discussed while completing the risk assessment with each participant.

\subsubsection{Aims and influence of research}

It was made clear to participants that the research could not directly influence local decision-making processes, but that the knowledge gained within this process could add to and improve wider understanding of how design needs to accommodate people who use mobility aids.

\subsection{Application of theoretical framework}

The overview of my theoretical understanding of the mobilities paradigm and the potential to combine this with a critical gerontological approach enables insight into later analysis. Acknowledging extant theories enables understanding of my conclusions within the context of current understanding and existing knowledge the basis for my understanding of older people's use of mobility aids rests upon the mobilities paradigm conceptualisation that social life requires movement. The importance of interdisciplinarity with the mobilities paradigm, critical gerontology, and therefore this study has been clearly stated. The qualitative, ethnographic, grounded theory methodology has been explained to highlight the aim of the study to illuminate the experiences of older people who use mobility aids. With the theoretical underpinnings established, the design and data collection will now be outlined. 


\section{Chapter 4. Methods: Design and Data Collection}

The following chapter outlines the specific methodological approach and strategies used to undertake the present study. Each section adheres to the overall aims of the research, which are to explore older people's experience of using mobility aids in their local environments within daily routines and interactions. The chapter begins by outlining the research approach and design. To address aims involved with experience and context, the methodology outlined here refers to a wholly qualitative approach, using exploratory methods to uncover previously undiscovered aspects of experience and context of mobility aid use. drawing upon ethnographic grounded theory. The aims include describing mobility aid use from the perspective of the mobility aid user, and their carers, partners, companions or family members and consideration of any barriers to social inclusion or participation for mobility aid users.

The research question of this study, as outlined in the Introduction (Chapter 1), primarily sets out to describe and understand how older people relate to mobility aids and how useful mobility aids are in navigating everyday scenarios. The overarching question includes examination of how older people relate to their own mobility aids or feel about mobility aids in general, how older people feel about themselves when they need to use a mobility aid and how they feel the built environment, planning and practice incorporate their need for mobility aid use. How carers feel about their partners or loved ones using mobility aids is also included as part of the overall investigation into how well older people feel their mobility aids enable continuation of everyday lives and routines.

\subsection{Data Collection}

The very act of doing research to a high standard is almost always more problematic than anticipated (Davies \& Hughes, 2014, p55).

To seek description and understanding of how older people relate to mobility aids and how useful mobility aids are in navigating and enabling everyday routines and scenarios, within an ethnographic grounded theory methodology, participation needed to take place in the locale of participants. The design of this study is aimed at understanding the routine lives of older people in context as well as research possible can. Data was collected between 
September 2017 and December 2018 in various towns and cities across South Wales. The lived experience, social and built environment context of the research aims for this study required data collection to occur in the setting of participants own lived environments. To understand lived experience, attitude to self and objects or policy and planning, such aspects must be considered in context. It is within context that human behaviour is reflected, identities are situated. Where people are says a lot about who they are (Peace et al, 2013). Social science in general is concerned with people, their relationship with self, others and environments, all of which are accessed by studying experience.

\subsubsection{Method}

The methods used for this research were chosen for the opportunity they presented to understand movement and behaviour within the context of the built environment. Data collection was an adaption of the walking interview. The interviews were conducted about walking and carried out while walking, hence the term walking interview

\subsubsection{Walking interviews}

The walking interview, also known as a go-along interview, is essentially a hybrid of interviewing and participant observation, with the researcher accompanying participants as they go about their daily routines. A key purpose of go-along and walking interviews is to examine a participant's relationship with the environment, as contextual factors are observed and discussed as the walking interview proceeds (Carpiano, 2009; Jones, Bunce, Evans, Gibbs, \& Hein, 2008; Kusenbach, 2003). Using a walking interview technique thus facilitates examination of physical, social and psychological dimensions of movement and behaviour within the context of time and space. It is possible to access participants' knowledge, experiences and memories associated with places, alongside their values and perceptions of physical environments and spatial practices (Carpiano, 2009; Castrodale, 2018; Evans \& Jones, 2011). Within this research, walking interviews allowed for the observation and discussion of mobility aids as facilitators of accessibility and mobility.

Parent (2016), a wheelchair user himself, comments on the invaluable insights a go-along method offers enabling interrogation of mobility justice and differential mobilities. The act of walking, even within academia, is considered a natural ability and is rarely explored explicitly as a source of variance in mobility experiences. Using 'wheeling' interviews as a 
wheelchair user himself, Parent (2016) underlines the value of wheeling interviews as they necessitate negotiation between researcher, participants and the politics of space (Parent, 2016). Researching mobility, using go-along interviews especially mobility aid use, allows evaluation of access and privilege, attitudes and behaviour, which can be examined whilst walking, talking and carrying out daily, mundane routines (Castrodale 2015)

For the purpose of recording and understanding the use of mobility aids by older people there are further advantages of this method of data collection. One advantage is that the environment prompts discussions. Also, it is easier forming of rapport between researcher and participant than it would be in a clinical a setting (Carpiano, 2009; Jones et al, 2008). As both researcher and participant are subject to environmental conditions which, although uncontrollable, provide conditions for researcher-participant relationships to be grounded in cooperation, respect and trust (Castrodale, 2018).

For this research, the term walking interview was used to encompass either walking or using a wheelchair or other mobility aid as part of an everyday routine. It was made clear that a walking interview was not necessary if they did not want to conduct a walking interview, or they do not usually go out, and that walking was an all-encompassing term. If someone would rather have driven because that is what they always do, they were informed that it would be useful for them to drive me around showing me their routine from a driving perspective. No participant chose this option. It was made clear to participants that it was their routine daily movements and routines that were of interest to the study. For the purpose if the research, not walking was informative in its own way, indicating aspects of immobility or reliance on a car, which is discussed in section 9.1.1.

\subsubsection{Recruitment}

Recruitment and data collection commenced in July 2017 after ethical approval had been gained. Participants were over the age of 50 as per the Welsh Government's Strategy for Older People in Wales (Welsh Government, 2013). Men and women were of equal interest to the study and were included if they could provide informed consent and did not have any confounding comorbidity. The aims and objectives of the research seek to describe and understand how older people relate to mobility aids and the environment in which they are used. Within this context mobility aid use and the built environment were being defined by 
the older people of interest to the study, so it felt plausible to the study to also gain definition of mobility aids from those being studied. Moreover, as discussed in Chapter 2, mobility aids constitute more than medical tools for rehabilitation and medical definitions do not capture the experiential realities of social, emotional and psychological well-being so definition was based in the both the requirement for help with movement, and older people's own definitions. Mobility aid could thus mean anything from a walking stick to a walking frame, an aid for vision impairment or a wheelchair.

Use of any physical aid to navigate the environment counted as a mobility aid, as did nonuse of an aid that was prescribed by medical intervention or physical need. The important aspect was the need to use an item for navigation that perceived 'normal' or 'fit' people do not require in normal circumstances. People did not need to be permanent or constant mobility aid users as understanding why people choose not to use their mobility aids on some occasions and not others was also of interest to the study. During recruitment there were discussions regarding what constituted a mobility aid. The criterion was flexible to incorporate any meaning given by those affected by mobility difficulties. One person described her friends and family as her mobility aids, while other people feel uncomfortable relying on people to aid their mobility. Thus, a flexible approach to the meaning of 'mobility aids' provided a more workable approach to understanding the practical, emotional and psychological realities of people with mobility limitations. Carers or family members of those who use mobility aids were also included to provide wider understanding of how mobility aid use might affect family or other interpersonal relationships and impact on people other than the mobility aid user themselves.

Recruitment began through word of mouth and snowballing methods, beginning with my own informal personal networks, to make suggestions about who might meet the inclusion criteria. Individuals were contacted by me in confidence. Each person contacted was also asked to suggest other people. This method turned out to be slightly problematic because people were reticent to suggest others event though it was always made clear that no disclosure would be made from person to person whether participation had occurred. A presentation about the research was given at a local friendship club and ethical approval sought to recruit participants through this route. Some attendees had volunteered to 
participate even at the design stage of the study, before ethical approval had been gained. The committee of the friendship club were asked to sign a consent form (Appendix $\mathrm{H}$ ) for research to be communicated and assisted through the club. This began a research relationship with the club which has continued throughout the research process with periodic presentations and updates. Advertisements were placed with local interest groups, and emails sent to charities, but these methods gained no participants.

A presentation was made to Swansea University Patient Experience and Evaluation in Research (PEER) group, a group of patients and carers, hosted by researchers within the University. Presentations are made to the PEER group at the early stages of research design for feedback on the patient and carer view of the research idea, design and suggested implementation. Presentation was made to both to gain feedback on the research ideas, and also seek ideas for further recruitment. The group were encouraging and felt the research was of great interest and worth to them. There were some good suggestions about following up on the study in terms of influencing policy, approaching people to take part and the notion that those people who are the hardest to find are the ones we most need to hear from. One member of the group suggested asking the local postman of anyone they might know who lives in an isolated place and finds it difficult to get out and about. It was, however, felt to be unethical to approach such a person 'out of the blue' on later consideration. The point that someone who is unable to venture out is the type of person most needed to respond to the research was and is an excellent and pertinent one; reaching those people is difficult.

People began to agree to take part in the study after a second presentation and bowls afternoon at the friendship club. I explained what I meant by mobility aids, that this meant anything that aided mobility, from a walking stick to a wheelchair, rollators (also known as wheeled walkers) and white sticks also count. One lady asked if I was interested in hospital transport cars that were transporting. her husband to and from the hospital for cancer treatment. I would have collected her (and his) views because I felt that if the person felt it was a mobility aid, it is a mobility aid. However, circumstances dictated that they were unable to take part in the end. Some people were nervous regarding what might be involved. There is a balance to be found between passion as a researcher interested in 
understanding lives and routines, and people's mistrust of what they might feel to be nosiness, intrusion, or another official seeking to classify them. If people approached and I sensed them to be unsure, I would explain the process to them, give them a participant information sheet and tell them to take their time, contact me whenever they were ready if they wanted to take part. Many of the people who were recruited from the friendship club were immediately happy to volunteer. One lady was interviewed three times and while we conducted our walking interview, she kept offering to recruit mobility aid users we saw as we walked along!

After connecting with eight participants, I felt that no obstacles to participation were being uncovered. At this point I returned to the local Friendship Club to discuss the research so far and explained that it was difficult to articulate any barriers to participation from the information I was gaining. I told attendees that I wondered if I was assuming there are barriers to participation whereas the reality might be that people to do not want to go out. There was resounding disagreement to that, people do want to go out but there are various reasons they do not or cannot. The encounter re-invigorated my wish to understand the obstacles to participation, and highlighted the complex layered and multi-faceted perceptions people have of their own drives and intentions. It became clear that on a oneto-one basis, people were not necessarily ready or even able to articulate any obstacles, but friends, family and other observers noticed reductions in participation and activity. Another realisation at this point was that many of the participants were over 80 years old, but two were under 60 . As the data was transcribed and analysed using constant comparative analysis, it became evident that there was a good deal of disparity between the accounts of these different cohorts. A specific recruitment drive for people between 60 and 80 was employed. Targeting such a specific age range within what is essentially the wider 'older population' is not an easy thing to achieve, but two more mobility aid users and one carer were identified through university links and wider acquaintances. It was felt at this point that while there was not what might be considered saturation, there were enough sets of data and comparisons to cease data collection. Recruitment throughout the study was difficult beyond it being face to face at the friendship club, family, friends or friends of friends. On reflection, it may have been due to the complex emotional and psychological implications of mobility aid use that people do not feel comfortable discussing, or that 
people do not want to admit their mobility aid use, or even that people do not want to discuss ageing, but recruitment was challenging.

Through the recruitment and data collection stages, it became more apparent to me that my husband would be a participant. As a couple, we almost experience his disability and mobility difficulties as one entity. I find myself speaking in consultations saying, "we have trouble getting out" or "we have been started on a new drug regime", because we both experience it all together. That will not be the case for all couples, but it is for us. His disability and use of mobility aids is a significant inspiration for this research. During the research proposal and initial design stages, he has been a major support and sounding board. As the research proceeded, we both, I think, assumed his experiences would be a foundation of my knowledge and appear in the research, however I did not think of it in formal terms. As I continued interviewing participants, I realised Phil's descriptions and experiences were different to others I was interviewing, and I felt the need to formally interview him, so he became a formal participant. His experience of participation was slightly different to other participants, because he obviously has a more intimate knowledge of me and of the research, but he was able to give rich descriptions of his feelings and experiences as can be seen in appendix J.

The researcher's husband is fully aware of the basis, inspiration, potential outputs and consequences of taking part in the research, most. He is subject to informed consent and right to withdraw protocols in the same way as any other participant. However, as my husband his anonymity and confidentiality cannot be protected in the same way as other participants, which we discussed at length. He fully acknowledges and endorses use of his information as it is inextricable from my own experience which forms the basis of the research.

\subsubsection{Participants}

17 participants were recruited overall with an age ranged from 52 to 89 . While a fairly small set of participants, the data collected contained great depth of experience and social information because of the methods used. Many participants were involved in more than one participation, with discussion, observation and relationship factors with carers all being collected as data. Table 4.1 shows the variety of participants, types of mobility aid they 
used, carer who also took part and their relationships to the mobility aids users. 11 mobility aid users and six carers or family members took part. Eight participants, five mobility aid users and three carers, were recruited via the friendship club relationship. Each of these participants have FC marked next to their names on table 4.1. Another participant is a neighbour I often see when walking my dog, and my husband who both obviously live in my hometown, which is where the friendship club is held. 6 mobility aid users and 3 carers therefore live in the same town and share knowledge of the same environment and facilities.

Table 4.1 Participation information

\begin{tabular}{|c|c|c|c|c|c|c|c|}
\hline Participant & Age & $\begin{array}{l}\text { User } \\
\text { or } \\
\text { Carer }\end{array}$ & $\begin{array}{l}\text { M } \\
\text { or } \\
\mathbf{F}\end{array}$ & Relationship & walked & $\begin{array}{l}\text { No of } \\
\text { visits }\end{array}$ & Mobility aid \\
\hline
\end{tabular}

\begin{tabular}{|c|c|c|c|c|c|c|c|}
\hline Theresa (FC) & 85 & $U$ & $\mathrm{~F}$ & & y & 3 & scooter + stick \\
\hline Ivy & & c & $\mathrm{F}$ & paid/friend & $n$ & 2 & \\
\hline Bret $(\mathrm{H})$ & 83 & u & M & father & y & 2 & walking stick \\
\hline Jenny $(H)$ & 57 & c & $\mathrm{F}$ & daughter & $\mathrm{n}$ & 1 & \\
\hline Regina FC & 89 & $\mathrm{u}$ & $\mathrm{F}$ & mother & y & 2 & white stick + walking stick \\
\hline Gayle FC & 67 & c & $\mathrm{F}$ & daughter & $n$ & 1 & \\
\hline Ron FC & 85 & u & M & father & $n$ & 1 & scooter + stick \\
\hline Mary FC & 52 & c & $\mathrm{F}$ & daughter & $\mathrm{n}$ & 1 & \\
\hline Aileen & 58 & $\mathrm{u}$ & $\mathrm{F}$ & mother & y & 1 & should use white stick + stick \\
\hline Lauren & 27 & c & $\mathrm{F}$ & daughter & y & 1 & \\
\hline Stuart FC & 87 & u & $M$ & $\mathrm{n} / \mathrm{a}$ & $n$ & 1 & walking stick \\
\hline
\end{tabular}




\begin{tabular}{|c|c|c|c|c|c|c|c|}
\hline Deidre FC & 81 & $\mathrm{u}$ & $\mathrm{F}$ & $\mathrm{n} / \mathrm{a}$ & $n$ & 1 & crutches + walking stick \\
\hline Phil (H) & 56 & $\mathrm{u}$ & $\mathrm{M}$ & $\begin{array}{l}\text { researcher's } \\
\text { husband }\end{array}$ & y & 2 & wheelchair + walking stick \\
\hline Will & 73 & $\mathrm{u}$ & $\mathrm{M}$ & $\mathrm{n} / \mathrm{a}$ & y & 2 & walking stick \\
\hline Pam & 78 & $\mathrm{u}$ & $\mathrm{F}$ & wife & y & 2 & walking stick, frame + w/chair \\
\hline Bernard & 77 & C & $\mathrm{M}$ & husband & $y$ & 2 & \\
\hline Dave & 52 & $\mathrm{u}$ & $\mathrm{M}$ & $\mathrm{n} / \mathrm{a}$ & y & 2 & $\begin{array}{l}\text { wheelchair \& crutches } \\
\text { (amputee) }\end{array}$ \\
\hline
\end{tabular}

Participation was not limited to one area, some participants were drawn from places further afield, some from other towns and two from larger cities. Inclusion criteria of being over 50 years old and living in Wales gave a wide scope for recruitment. 6 mobility aid users were male, 5 female giving a good balance of gender. Of the 6 carers, only 1 was male. It is worth noting here, however that 3 of the mobility aid users, Bret, Stuart and Will, had or continue to have caring responsibilities in addition to their own mobility aid use.

There was some variance in the way interviews were conducted. Three people were interviewed without conducting walking interviews, all of whom were over 80 . One was quite mobile and willing to complete the walking interview, but I could not locate her again (despite her membership of the friendship club and living in my hometown!). The other two - Stuart and Ron - were happy to discuss their experiences but not to walk, nor divulge why they did not want to walk. They do both drive and have access to cars however, so perhaps they felt they did not have a walking routine to share. It could have been that they were too busy to meet me on another occasion. It was Ron's daughter who did not want to return for a second interview, and she was very shy, which could have been the reason. Stuart is a neighbour of mine and I know he does not walk anywhere. Most other people conducted two interviews, the initial interview at home or in a café, discussing their day-to-day experience of mobility aid use, their history of gaining the mobility aid and what illness or mobility problems they have. 


\subsubsection{Procedure}

Once participants had been identified, informed consent was sought (see appendix E). The consent form explained research aims and methods in line with the participant information sheet (appendix G). This first contact was designed to determine interest in and confirm eligibility for participation in the study, and to allow participants time to absorb the information before agreeing to take part. An explanation of the purpose of the study and methods used would be followed by the assertion that the study would be conducted over as many visits as they wished it to be. It could be carried out in one visit, whereby we could have an initial chat followed by them showing me around the town as they would usually go about their day. Alternatively, we could have an initial chat, and then I could return on another date to go for a walk with them.

During the initial contact, participants were briefed and given a Participant Information Sheet (Appendix $\mathrm{G}$ ), which gives a more detailed explanation of what taking part in the study involved. This outlined what participants might expect from taking part, the possible benefits and any possible risks. The right to withdraw, data protection, confidentiality and anonymity were explained at this point, and we went through the consent form together before participation commenced. It was ensured that potential participants understood what participation would mean, and at this point a return date would be arranged for participation to take place. The potential participant was given time to decide, but many wanted to continue immediately, happy to take part when advised fully of participation details. Before commencing participation, the participant information sheet was again discussed to ensure understanding by the participant. Once both myself and participant were happy that informed consent had been gained, participation commenced at the time and place decided by us both.

Initial discussions and interviews took place either in participants' homes, or for some people in public places such as cafés. One participant invited me to meet at a hospital, so I could witness the process of fitting a prosthetic limb. Each participant decided and informed me where they would like to meet. Some felt more comfortable in their home environment, others were happier to meet on neutral ground. Before each walking interview could proceed, a risk assessment was completed as per conditions of ethical approval. The risk 
assessment (Appendix D) was carried out by the interviewer and consisted of questions around what kind of risks were presented by walking in the environment and how they would be mitigated. Some participants were offended by the risk assessment because the activities to be carried out were carried out every day without a risk assessment. Some participants were happy to discuss the risks, and for some, the assessment highlighted aspects of mobility aid use that they had not previously considered.

As an example, the map in figure 4.1 illustrates the walk I took with Aileen and Lauren. Each coloured line represents a walk with a rest in between. The green dotted line indicates where Aileen previously walked but can no longer manage. The purple line denotes the starting point of our walk up to a shop in which we spent some time walking around browsing (and sat down on occasions) until our first rest for a cup of coffee. The red line denotes movement after that coffee until another rest inside a department store. The solid line coloured yellow denotes the final part of the walk at which point Aileen needed to stop and send Lauren off to collect the rest of her shopping. The green dotted line highlights a typical route Aileen would have taken before her mobility became so compromised, populated from her description of previous shopping trips. 


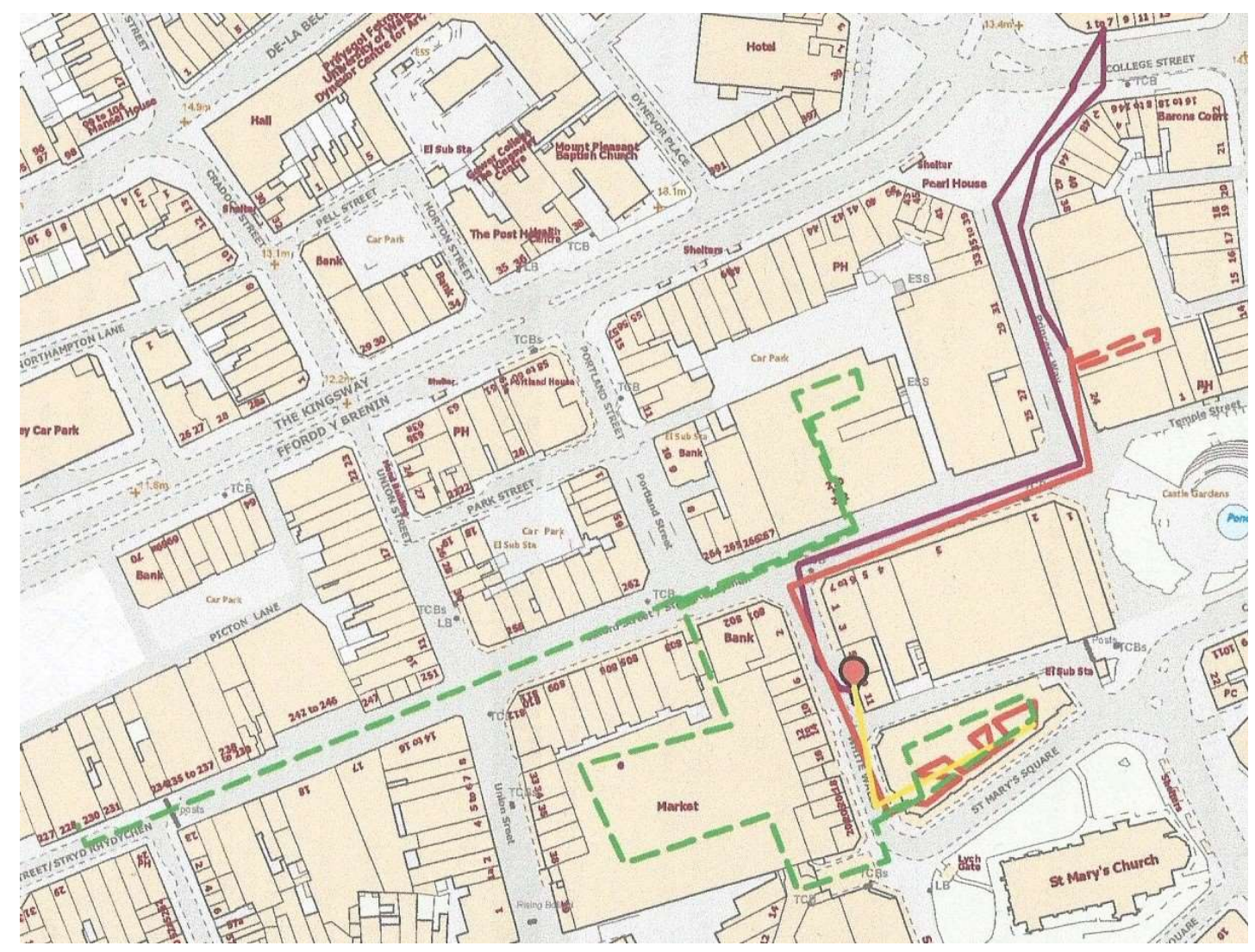

Figure 4.1 Aileen's Map.

Interviews/discussions set out to establish how people felt about their mobility and mobility aid, any changes to their mobility or indeed any changes to the environment that may have affected participants positively or negatively. There was no set interview schedule, only a couple of prompts. To begin with, individuals were asked whether routines and activities had changed since they had begun use a mobility aid, for example, "Have you noticed any changes in where you go since you started using a mobility aid?" . Later in the study, the question became more subtle. This was partly because the original question was often met with a further question, and partly because all participants reported some kind of change to their routines. People were aware from recruitment, consent and risk assessment procedures that the study was investigating mobility aids so usually asked what I wanted to know. The response would be worded "I would like to know about your normal daily routine, where you go and what you do. Just do what you normally do, and I will tag along." If the person did not want to carry out a walking interview, the questions would explore the number and method of their outings. We would go on to discuss changes to their outings and modes of transport since their mobility had changed and how they felt about the 
changes. It was rare I needed to prompt anyone with discussions centred around their mobility aid use, but also all sorts of other daily life. This allowed people to feel comfortable in their conversations and discussions, not feeling as if anything was off limits. My knowledge of mobility aid use also may have alleviated any stigma or embarrassment that may have been felt, allowing participants to potentially be open and honest about their experiences without feeling judged.

A combination of walking interviews and discussions were carried out, depending on the schedule, wishes and physical abilities of each participant. Discussions were led mostly by participants, with prompts given when a feature from previous interviews became apparent or something of interest to the study was highlighted. Each participation was different as each was led by the participant. Sometimes participants asked me what others taking part had said, so I would give a generic summary. Some people were visited only once and discussed their mobility without walking, some were visited more than once, and some conducted the whole participation in one session in a public place.

The constant comparative analysis aspect of the methodology led to participants being occasionally prompted with further questions if a subject touched on by a previous participant was introduced. I also tried to remain as impartial as possible and not lead participants. It is, however, difficult to prompt and not lead. While notes in the ethical approval application referred to asking individuals if places made them feel excluded, this was often avoided during the study as it felt too leading and more accurate to record feelings of exclusion if they were reported through events and conversation as they occurred naturally. As walk along interviews were conducted, feelings, memories and thoughts were evoked in participants. These were not always necessarily relevant to mobility aid use, but often indicated personal histories, identities and viewpoints, facilitated by the rapport we were building in our interactions and the environment, which reminded participants of events or people. Often, this did not require prompting and happened naturally as part of a conversation between two people, that is myself and the participant. If individuals did not know what to do or say at the start of a walking interview, they were asked to discuss, describe and talk about locations and routines as part of a tour of their town/village. Actions and interactions with third parties were observed but not sound 
recorded to respect the privacy of members of the public who were not taking part in the study.

Discussions with participants were sound recorded through the whole process, from arrival at the meeting to the time I left, as we walked and whilst at cafes and in shops. Each participant wore a Dictaphone with a microphone pinned discreetly to their clothing which was switched to record throughout, and I carried a mobile phone which also recorded proceedings. I took photos of difficult terrain, places a participant discussed as good or bad, or infrastructure that we had difficulty with, and also noted observations mentally, then in note form at the earliest convenience. Each participation was transcribed and analysed as soon as possible after the meeting to ensure accuracy and capture the events and feelings of the encounter. Five recordings were sent to an external transcription service, all of which were checked for accuracy and deemed correct. The checking process involved listening to the tapes and reading the transcription concurrently. Notes, comments and photos of these cases were processed as soon as possible in the same way as those transcribed personally. New data was compared with existing data before embarking on more data collection as per constant comparative analysis (Charmaz, 2017). On one occasion this was not possible because two interviews were booked on day after the other, but usually data was transcribed and analysed before continuing to the next participant in line with constant comparative analysis. To begin with, consideration was made of saturation techniques or concluding data collection at 40 participants, however neither technique was employed. On reaching 14 participants, a rich and varied data pool had been acquired, and as discussed in the recruitment section a difference in experience was evident between participants over 80 and those under 60 . The focus of research became to understand these age differences by recruiting participants between 60 and 80 to gain in-depth views of their experiences with mobility aids to enable better in-depth understanding of the age range and age-related differences in using mobility aids.

\subsubsection{Constant comparative analysis during data collection}

In line with the process of comparing and coding occurring throughout the data collection process, the order in which interviews were conducted may have influenced analysis. I have a good knowledge of the space inhabited by the participants, although I have lived in the 
town 6 years and am not a lifelong resident like many of the participants. The shared knowledge and camaraderie that may have been created by my familiarity may have helped conversation to flow, but also may have influenced responses. Table 4.2 shows the order in which interviews were conducted and any differences in the context and dynamics of interviews. The number of participants from my hometown was not an intentional product of the research design, rather more of the recruitment process. Participants were recruited through local connections and a local friendship club at which I gave three presentations, the first in June 2017 to establish a relationship and gain insight into the routines and lifestyles of attendees, the second later that summer, and a third in November 2017.

The participants who live in my hometown were eager to be positive and paint a picture of having few to no personal mobility issues. Bret, Stuart, Theresa, Regina and Deidre are also all over 80 years old, this too was a consideration. Aileen, the second person to take part in the study, was in her late fifties and lived in a city, a contrast to all the other participants in this first half of data collection. The location of her home and participation were different in place and type of environment, but also, she was much more willing to be open and honest about the stigma, pain and struggle she faces living with disability and chronic illness. The differences in both age and location were acknowledged as participants were compared and contrasted.

Table 4.2 Order of participation and notes of interest

\begin{tabular}{|c|c|c|c|}
\hline Participant & Date & Dynamics of interview & Notes of interest \\
\hline Bret $(H)$ & 15-Sep-17 & walked same day & \\
\hline Stuart $(H)(F)$ & 03-Oct-17 & at home, no walk & Uses car even for local journeys \\
\hline $\begin{array}{l}\text { Aileen \& } \\
\text { Lauren }\end{array}$ & 07-Oct-17 & walked same day in dyad & Walked in city centre familiar to Aileen and Lauren \\
\hline Theresa $(H)(F)$ & 08-Oct-17 & $\begin{array}{l}\text { at home then walked } \\
\text { same day }\end{array}$ & \\
\hline $\begin{array}{l}\text { Theresa } \\
\text { scooter }(H)(F)\end{array}$ & 18-Oct-17 & $\mathrm{n} / \mathrm{a}$ & $\begin{array}{l}\text { Theresa invited me to be present while a mobility } \\
\text { scooter salesman visited }\end{array}$ \\
\hline Jenny & 20-Oct-17 & carer discussion & $\begin{array}{l}\text { Bret's daughter/carer - Interviewed separately to } \\
\text { Bret }\end{array}$ \\
\hline $\begin{array}{l}\text { Theresa }(H)(F) \\
\text { \& Ivy }\end{array}$ & 22-Nov-17 & carer discussion in dyad & $\begin{array}{l}\text { Ivy is Theresa's paid carer. She had worked all } \\
\text { night, so this was a short interview and continued } \\
\text { at a later date }\end{array}$ \\
\hline $\begin{array}{l}\text { Theresa }(H)(F) \\
\text { \& Ivy }\end{array}$ & 02-Dec-17 & carer discussion in dyad & $\begin{array}{l}\text { Continuation of previous discussion. Both took } \\
\text { place in Theresa's home. }\end{array}$ \\
\hline
\end{tabular}




\begin{tabular}{|c|c|c|c|}
\hline $\begin{array}{l}\text { Regina(H) (F) } \\
\text { \& Gayle (H) (F) }\end{array}$ & 25-Jan-18 & carer discussion in dyad & Regina was interviewed in her own home \\
\hline Deidre $(H)(F)$ & 26-Jan-18 & no walk & Could not locate Diedre again \\
\hline Regina $(H)(F)$ & 27-Mar-18 & Walked without carer & Walked to a local luncheon club \\
\hline $\begin{array}{l}\operatorname{Ron}(H)(F) \& \\
\operatorname{Mary}(H)(F)\end{array}$ & 28-Mar-18 & $\begin{array}{l}\text { no walk - Discussion in } \\
\text { dyad and Mary alone }\end{array}$ & $\begin{array}{l}\text { Ron and Mary attended a friendship club session at } \\
\text { which I gave a presentation about my study. They } \\
\text { agreed to be interviewed there but did not want to } \\
\text { make another date. }\end{array}$ \\
\hline Phil (H) & 22-May-18 & later date & Walking interview was delayed by illness \\
\hline Will & 11-Oct-18 & later date & $\begin{array}{l}\text { Will was interviewed at restaurant and intended to } \\
\text { carry out a walking interview, but he does not } \\
\text { often walk anywhere, and the weather was } \\
\text { inhibitive }\end{array}$ \\
\hline $\begin{array}{l}\text { Pam \& } \\
\text { Bernard }\end{array}$ & $16-$ Oct-18 & later date & Interview at home \\
\hline Will & 02-Nov-18 & walk in store & $\begin{array}{l}\text { We met at a homeware store and spoke over a } \\
\text { coffee before walking around the store. }\end{array}$ \\
\hline $\begin{array}{l}\text { Pam \& } \\
\text { Bernard }\end{array}$ & 13-Nov-18 & Walk & $\begin{array}{l}\text { Walk around hometown with excellent knowledge } \\
\text { of accessibility and problems that arise }\end{array}$ \\
\hline Dave & 10-Dec-18 & walk same day & $\begin{array}{l}\text { Dave allowed access to a fitting for his prosthetic } \\
\text { limb at hospital, a shop around a supermarket and } \\
\text { then a visit to a city centre. }\end{array}$ \\
\hline Phil $(H)$ & 16-Mar-19 & walk at hospital & $\begin{array}{l}\text { There was a long delay in Phil conducting a walking } \\
\text { interview because he was hospitalised with sepsis } \\
\text { in June } 2018 \text {. }\end{array}$ \\
\hline
\end{tabular}

(H) denotes a hometown participant. (F) denotes a friendship club contact.

After eight interviews (i.e., after Deidre's interview), despite the construction of 112 codes in NVivo, it became quite apparent that for some reason, I was not gaining the information that I felt was there, at least not from the mobility aid users. I knew from previous literature that there were both obstacles and issues of isolation and reduced participation. Data in the form of my observations, feedback from relatives and carers and other information indicated that there were mobility difficulties. For example, some non-verbal data came from the recording of Bret's walking interview; despite suggesting he did not really have any problems, the recording captured the sound of a heavy, foot-dragging limp. His daughter Jenny also hinted that Bret glossed over any difficulties. I became quite despondent and exasperated at this point, trying to work out if it was my research design, data collection method, my manner that was failing to capture the data that I knew was there. Not only was I not capturing obstacles, but I also felt I was not capturing anything of note at all. The 
following memo indicates the kind of questions I asked myself to try and understand what the problem might be.

$26^{\text {th }}$ Jan 2018

Obstacles to what? Obstacles to participation or obstacles to social activity? Obstacles to citizenship or obstacles to normality? Or do these things all mean the same?

Obstacles to participation in what?

Perhaps I could convene a group to answer some of these questions. Do people think they have to overcome obstacles when using their mobility aids and if so what kind of obstacles. Physical, metaphorical, psychological?

Following on from these musings and consulting grounded theory literature to understand what could be done I found a statement from Charmaz (2014, p132) that "your observations and ideas matter". I concluded that my sense of obstacles being a factor in the lives of the people I had interviewed was correct, but I had not been able to access that information. I decided to return to the friendship club to ask people their thoughts on obstacles to participation in a group setting as a way to move forward. As a group, the friendship club had given consent to participate and were sometimes accessed to interrogate findings and new ideas, which was always voted on at the time of access.

I returned to the local Friendship Club to discuss the research so far. As I entered the hall in which the club is located for this meeting, I met a father and daughter as they struggled to get the father through the front door of the hall with the man's rollator. The doors are heavily weighted with an automatic closer, which makes manoeuvring a mobility aid and oneself through it very difficult. After helping them inside, they commented on how much they still get around, but that is probably because the daughter both encourages and accompanies her father, who also still drives. I mentally noted that driving seems to be a major factor for this dyad. Conversing with them further, this father and daughter agreed to take part in my study, but only on that day. Some of their experience is therefore captured as data. The encounter re-invigorated my wish to understand the obstacles to participation, and highlighted the complex layered and multi-faceted perceptions people have of their 
own drives and intentions. This mobility aid user and carer continued to discuss their mobility and did not mention the difficulty they have getting into the hall, and maybe they do not consider it a problem, but I was reminded that they did not express difficulties, only solutions. It became clear that on a one-to-one basis, people were not necessarily ready or even able to articulate any obstacles, but friends, family and other observers noticed reductions in participation and activity. It could be that people become used to less activity after they start to use a mobility aid, but my experience with my husband, coupled with the findings of this study which at this point I found it difficult to articulate, pointed otherwise.

At the meeting, I explained that it was difficult to articulate any barriers to participation from the information I was gaining through my research. I told attendees that I wondered if I was assuming there are barriers to participation whereas the reality might be that there are no barriers, or perhaps people with mobility aids do not want to go out. There was resounding disagreement to that, people do want to go out but there are various reasons they do not or cannot. One carer told me that her mother has a theory that when a partner dies, it removes the person's drive to go out and do things so much. Attendees at the club also mentioned the importance of driving and access to a car is for social activity. People who do not drive and have mobility issues cannot get to the social events and clubs planned for older people. The transport that used to be provided is no longer offered and there are no bus stops nearby. Another lady spoke about how her mother had lost confidence after catching a bus and the driver had driven off before she had managed to get to a seat. We spoke a little about loss of confidence and how that might affect social participation. I also mentioned that some people seemed to be notice stigma while others didn't. It was confirmed by attendees that there are definitely obstacles to social activity and participation for those with mobility aids. , so work continued to understand the physical, psychological, emotional or a combination of barriers that preclude participation in the social life people want within ageing.

The overall response was yes, there are definitely difficulties incurred with mobility aid use, ageing and mobility difficulties. Unless one has access to a car, navigation of the town can be very difficult, and it is only people who are mobile or who have access to cars that attend the club. With this response I regrouped and realised that reluctance to admit difficulty for 
some respondents was part of the wider accessibility, ageing and disability experience. As Charmaz (2014) states when continuing to explain the process of coding, respondents may repress or deny facts, but the role of the researcher is to understand and analyse these actions through participants eyes and logic in order to bring new insights. Reluctance of participants to discuss access difficulties or changes in ability was coded separately to reflect the phenomenon in the analysis.

The first group of participants contained two people aged between 50 and 60, and six people over the age of 80 . Comparisons of those in their fifties to their older counterparts indicated some differences between these two groups which were quite marked. Aileen and Phil, the two younger older people were both willing to share and perhaps even forthright with their accounts of social, psychological and physical obstacles. Deidre, at 81, was willing to discuss some of these obstacles, but otherwise, no older people really talked about inability or obstacles. Regina, the oldest participant was happy to discuss her body winding down like a machine, but again did not discuss an inability to cope or obstacles to social participation. When discussing reduced social activity, she described it as being through choice rather than necessity. The difficulty in bringing any discussion of obstacles to participation seemed to exist within the over 80 age group but it was difficult to discern whether that was an age issue, in that people reach a certain age and stop wanting to go out, or a cohort effect, in that the participants over 80 had been impacted by a social, economic or historic context that shaped their outlook on ageing and mobility in a slightly different way to the contexts affecting those under 60. To address the age gap in participants and give a better understanding of mobility aid use across the age range, more participants between the ages of 50 and 80 were sought. Even with the additional participants, the study could not conclusively identify an age at which attitudes change or if a cohort effect impacts upon attitudes, but it could give a better indication of age differences which could be investigated in future research. Chapter 9 discusses differences between 'younger' older people and 'older' older people in the ways ageing, reduced mobility and reduced participation are accepted and acted upon by these different age groups. 
Will, Pam and Bernard, and Dave were recruited to bridge the age gap in the data. Again, these participants were introduced through university contacts or my own links in the community. Dave at 52 was the youngest participant and also slightly different to other participants because he is an amputee, having had his leg amputated following an illness. Dave was the most active of participants. Will was 73 and lives with Parkinson's disease, using a walking stick to navigate the environment, but by his own admission should use other mobility aids. Pam at 77 also lives with Parkinson's disease and her husband Bernard 78 , took part to help inform study of carer/family impact of mobility aid use. The data collected from these participants was very useful in understanding a wider range of ages from different places and also different mobility aids.

\subsubsection{Methodological contribution}

There is a contribution to knowledge within this methodological approach, whereby walking interviews within a theoretical backdrop of mobilities and critical gerontology can examine hidden and unspoken aspects of inequality in the mobility of older people for reasons of stigma, negative self-assessment and potentially other aspects of mobility that are yet to be discovered. Perhaps because this research is concerned with mobility aid use and ageing, thus participants face two stigmatising characteristics, I found that participants were not always forthcoming with negative comments about the environment. Walking interviews facilitated a good opportunity to build rapport with participants and gain profound insights into their behaviours, attitudes, and abilities within the context of the built environment. The resulting research project contributes to the adaptability and application of this method, highlighting the effectiveness of walking interviews in interrogating issues around accessibility and mobility justice as I set out to do, but also in examining unspoken or hidden aspects of mobility connected to identity, dignity, and stigma. The focus on daily routines and participant experience allowed the research to be led more by participants and their practices, therefore enabling access to personal habits within systems and services. Constraints faced by individuals, and the personal cost of mobility aid use in terms of identity, social life, and effort to gain mobility became identifiable. 


\subsection{Ontological and epistemological considerations}

The above disclosure of my thinking and difficulties through the research process are an important part of grounded theory, or indeed any qualitative research practice. The ontological and epistemological considerations that may have influenced me or the research practices herein are outlined below.

\subsubsection{Reflexivity}

A process of reflexivity is an aspect of qualitative research, and particularly attention is paid to the influence of the researcher in ethnographic research (Creswell, 2014; Denzin \& Lincoln, 1998; Flick, 2002). Thus, an explanation of previous views, training and prejudices are declared, but also acknowledges how the researcher's background may influence the direction and interpretation of the study (Creswell, 2014). Even those of us who believe we do not carry prejudices are influenced by culture, training, our discipline's norms and values, and the preconceptions carried in language. This had been particularly highlighted in disability studies where it is demonstrated that the language used to classify and categorise people and things can subconsciously influence attitudes. For example, Pfeiffer (2000) explains the role of language in pre-loading studies with false dichotomies of good/bad, true/false, or justice/injustice. The world is not as well defined as this language can portray.

\subsubsection{Role of the researcher}

While personal reflexivity or disclosure is not necessarily considered relevant to methodology, it is an important consideration here for two reasons. First, because ethnography and ethnographic grounded theory are based on the premise of researcher reflexivity and an understanding that the researcher is part of the research. The second reason is that the foundation of this research idea was in both personal and academic understandings. Having both academic and personal interests in and understanding of the nuances of mobility aid use, I became increasingly interested in the ways embodiment and disability have been included or omitted from ageing research. Cherry's (1995) reflections on research processes describe how academic theorising grows from reflection on current social questions built on disciplinary and personal foundations; from these researchers build intellectually and scientifically appropriate assumptions, frameworks, methodologies and 
analyses. Thus, the selections of appropriate methodology include personal opinion along with academically trained insights. As the researcher I cannot be divorced from the research, the choices about research methodology or interpretations of findings. The researcher and research process will always affect that which is researched; not just in terms of results, but the behaviour of people, the environment, the surroundings are all impacted by the presence of the researcher. Transparent and honest reflection with and about self as throughout the process allows for understanding and evaluation of the researcher presence (Star, 2007).

The inception of this PhD project came from separate but inextricable experiences in my life as both a social scientist and the wife of a chronically ill mobility aid user. From these standpoints intellectually and scientifically appropriate frameworks and methodologies were chosen to explore and investigate the social phenomena in question, that is, older people's use of mobility aids in the built environment. It is a point worth noting that traditional ethnographers may suggest I could not conduct research from an emic standpoint within the category of older mobility aid users. I am (at the point of submitting this $\mathrm{PhD}$ ) over 50 , but I do not require the use of a mobility aid and thus could not be truly immersed in their worldview. However, as discussed throughout the work, I am married to and care for a man over 50 who uses a substantial mobility device. This puts me somewhere along the emic/etic continuum. Moreover, it is true to say that this population has no 'environment' per se, nor is there a separate culture. Instead, we are all members of the same environment and culture, albeit some at different stages and abilities than others, and mobility aid users require additional tools to navigate the environment. I see it as no different to wearing running shoes for running or using a car for a longer journey; a mobility aid is a tool for a job.

\subsubsection{Power relations}

An aspect of this research process is the ethnographic process of asking people to reflect on their own personal experience. From my point of view as the researcher, the interview process manifested as two parties of equal status conversing to exploring an issue. Conversation as a method of collecting data allows flexibility, better equality in relationship and allows participants to bring up topics important to them (Clandinin \& Connelly, 1998). 
Although I have academic knowledge, each participant knows their own experience. However, it is not necessarily true that the participants felt that way. I made every attempt to make people feel comfortable, and that the research was nothing more than a chat about their experiences, but for a participant it is always going to feel more important because it is not a usual occurrence to have a 'chat' with someone and have them walk around with you. It is also in some ways not true that we were equals. As the researcher, I have influenced what was discussed during interview. Each participant asked at the beginning of a discussion "what do you want me to do?". I always responded that I wanted them to continue with their normal daily routines, take me where they usually go when they go out, but obviously, they do not usually have a stranger tagging along. The resulting walk would therefore have included elements of each participant being aware of my presence, being more aware of their actions, where they were going and why. It was evident on some occasions that participants were exercising some forms of impression management, and this is discussed through the themes and sub-themes of identity, dignity and stigma, also interweaved into relationships and infrastructure via feeling like a burden and hassle throughout the research.

Among the difficult tasks within the process of interpreting and presenting data is framing multi-dimensional real-life events in two dimensions (Fontana \& Frey, 1998). Data from the perspective of both researcher and participant within temporal and situational contexts are presented in analysis, results and discussion without necessarily being robustly acknowledged (Clandinin \& Connelly, 1998). This means there are likely to have been assumptions and feelings on both sides of the participant researcher relationship that were not overtly discussed but present in gestures, body language and, as discussed in the results, forms of data other than interview transcriptions such as the recording of a limp (Chapter 6). My discussion, representation and analysis of older people's mobility aid use is interspersed with my pre-existing knowledge and understanding as the wife and carer of a mobility aid user. I am a part of this experience and knowledge, I interpreted the data into a salient set of results, continually aware that I was making choices regarding how the data was analysed and portrayed. 
My discussions with participants, and necessarily the data collected, would have been influenced by my first (or at least second) hand knowledge of mobility aid use. My husband lives with rheumatoid arthritis, has chronic heart disease and has suffered at least two heart attacks, fractured two vertebrae in his spine almost ten years ago, lives with diabetes and chronic pain syndrome. The list of illnesses is long, especially considering his age of 58 . He uses a powered wheelchair and a walking stick (he is able to walk a few steps). I am well versed in chronic illness and the use of mobility aids both inside the home and in the built environment. This led me to often prompt or continue conversations because of my previous knowledge. The pre-existing knowledge I hold will undoubtedly have shaped this research in a way that a researcher without this knowledge would not have done. This is particularly evident is Chapter 7 section 1 and 2 which examine carer concerns, and disagreement and discord, respectively. I experience the concern and contentions myself, and have personally reflected on the confounding aspects of mobility aid use that affect emotions, behaviour and identity. I could utilise my knowledge to initiate knowledgeable interactions, compare similarities and differences between my role as a carer and as a researcher in these interactions.

Discussion of mobility aid use and walking interview (or discussion) with my husband were undertaken in a formal way, in that time was set aside to conduct these discussions and for me as a researcher to observe his action during a particular hospital visit. As the literature above discerns, it was impossible for me to be researcher but not wife, or wife but not researcher, as there is always an element of both active, not to mention the other roles I play such as mother, daughter and friend. However, when discussing mobility aid use with him, my husband was very articulate and forthright about his opinions, practices, feelings and the obstacles he faces. To the point that I was slightly stunned by the profound language and metaphors he used, such as dropped kerbs being likened to gas masks without which one cannot survive. The walking interview we took together was recorded so analysis could be made of the recording as well as the observations. Unlike other data collection, I have historical knowledge of his mobility aid use, practices, preferences and obstacles faces which will have undoubtedly impacted data collected and interpretation. Phil's initial discussion deliberately took place after many other people had taken part in the study, and 
his walking interview was the last participation as he was taken very ill during the research process.

\subsubsection{Conclusion of ontological and epistemological considerations}

Inclusion of reflexivity and evaluation of my role as researcher, along with the power relations and negotiations incurred within that role is intended to make the research decisions and direction as transparent as possible. Reflexivity requires self-conscious awareness about our position as a researcher, on multiple levels, personal, methodological, theoretical, epistemological and political. The process of reflexively reviewing one's own role in the research therefore aims to question inherent assumptions in the research process (Engward \& Davis). The information within these sections outlines the awareness I had of my own position, knowledge, understandings and opinions when embarking upon this study. Where appropriate, my role or thoughts are referred to within the analysis of data. 


\section{Chapter 5. Emergence of Themes}

The principal aim of this research project was to understand mobility aid use in the everyday lives of older people, seeking to uncover any obstacles to participation that may exist. Consultation of previous literature regarding both ageing and disability led to reflection that obstacles would be structural and practical in nature. Psychological and social factors, such as changing roles within relationships and stigma were also considered possible, partially because of literature such as Erving Goffman's Stigma ([1963]1990) but also from personal experience. With my husband, I have experienced the inadequate provision of dropped kerbs and lack of understanding from service providers that wheelchairs do not necessarily manoeuvre into spaces that have been set up for them. Together we have felt dismay, exhaustion and mistreatment because of his use of mobility aids. Two manual wheelchair users (my husband's is a powered wheelchair) participated both of whom had very different medical and ability backgrounds. I expected less obstacles for those with less obtrusive aids. The themes that emerged highlighted obstacles for all different kinds of mobility aids and different mobility aid users.

\subsection{Analysis}

Methods of analysis and the process by which themes became apparent through layers of coding are outlined in this chapter, with explanations and examples of the process of constructing codes from initial data, focusing codes through to abstracting themes. Description and explanation of data collection, challenges and how these were overcome and the different variables that may have affected people's responses or their own interactions with mobility aids. The second half of the chapter introduces the themes emanating from the study, including how they were extracted from the data and how the themes relate to each other.

\subsubsection{Initial coding}

Constant comparative methods entail comparing data within and between participants from the start of data collection processes, continuing to make comparisons as categories begin to emerge, comparing codes and categories with pre-existing concepts and theories. In this way, data is continually analysed and compared (Charmaz, 2014). The coding process 
consists of taking segments of information in the form of textual language from interview or discussion data and defining them. This first stage of coding is a way of naming and categorising data so that it can be both understood and compared. A second phase of coding enables synthesis and organisation of ideas whilst informing the significant issues and ideas that occur throughout the data (Charmaz, 2017). It is important to point out here that constructing codes is essentially naming sections of data, and in doing so we are taking language that occurred in a natural setting and potentially problematising it so that it may fit better into an academic schema (Charmaz, 2017). The nature of language directing, and pre-loading values and judgements was outlined in the reflexivity section in Chapter 3 , and Charmaz (2017) emphasises the need to consider personal views and hidden assumptions of language in constructing codes, defining significance and describing processes. The following sections and Chapter 5 outline the stages and processes of theme emergence through analysis, and I have been as reflexive and transparent as possible throughout this work about my own background and assumptions. It is always possible that I am not aware of the ways in which I have influenced the research.

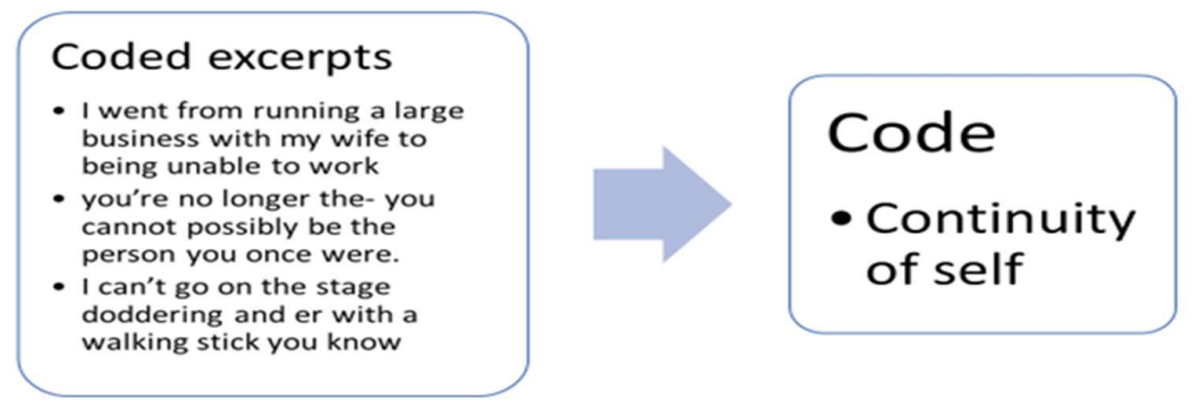

Figure 5.1 Example of coding segments of data

Applying the methods described, data was coded as soon as possible after a discussion or walking interview had taken place using NVivo software. The software was used as it is a way to store and compare data easily. Through the process of comparing and contrasting, regrouping and differentiating variables, a coherent chain of evidence was sought to ultimately form a concept or theory. On some occasions, there would only be one segment of data at one code, and some codes contained many segments of data. I did not necessarily value those with many segments over those with fewer, however. Evaluation consisted also of how the information evolved and how much the concept or occurrence impacted upon 
mobility aid use. Figure 5.1 shows some segments of data and how they were coded as 'continuity of self', a term that seemed to capture a shar sentiment of wanting to continue life as it had always been despite any embodied changes, mobility aid use or onset of chronic illness.

\subsubsection{Focused Coding}

Through coding practices, initial codes such as 'bad planning or design' and 'inconsiderate others' were recoded into more focused codes such as 'interpersonal aspects' (of mobility aid use) or interlinking systems. The initial codes were maintained as child codes of the wider ranging parent codes, so 'inconsiderate others' was held as a child code of 'interpersonal aspects' for example. As Charmaz (2017) within grounded theory methodology and Pfeiffer (2000) within disability studies both point out, language can be personal, directed and sometimes inadequate. The language of my codes is a shorthand for the phenomena I am trying to capture within each code and subsequently the resulting themes. When all data had been collected and coding completed in the first stage, there were 126 codes, even with the establishment of some focused coding. Synthesising and fusing together codes with similar meanings, abstracting codes with significant social, physical and psychological factors and highlighting similarities and differences allowed codes to be condensed and organised. Successful utilisation of grounded theory methodology involves using constant comparative methods and engagement of the researcher (Charmaz, 2017). As codes and categories were scrutinised, analysis became more theoretical. Interrogation of existing concepts and theories is reliant upon my own interaction with such concepts, disciplines and fields relevant to the emerging content. In this case, my pre-existing knowledge of psychology, sociology and disability studies along with ageing, and the multidisciplinary nature of ageing studies and the mobilities paradigm allowed for a wide-ranging source of information, concepts and theoretical understandings. Figure 5.2 illustrates the coding of segments of data through initial and focused coding to final themes. 


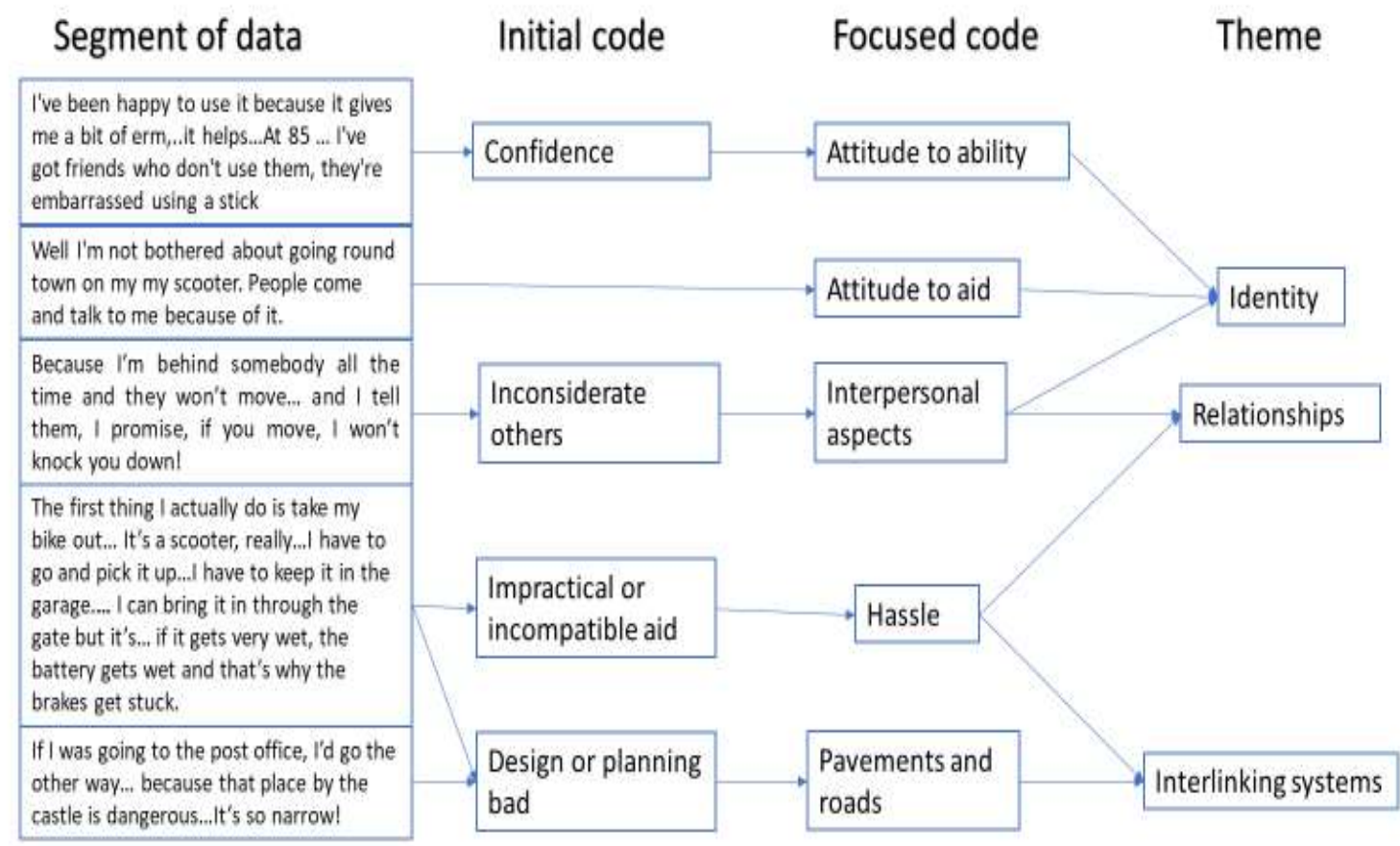

Figure 5.2 Focused coding and resulting themes

\subsection{Finalising themes}

Overall, it is true to say that obstacles were experienced as part of daily life, and as such it is difficult to disentangle one type of obstacle from another. Obstacles were physical, social and psychological; individual, relational and infrastructural, but not as succinct as that description makes it seem. Stigma, for example, is felt within the individual, but requires relationships for it to exist, and can be reinforced by social structures or infrastructure. It is thus important to express here that these themes and sub-themes are intricately intermeshed, some aspects of one theme or sub-theme could sit easily in others. Decisions to place a sub-theme under one theme rather than another have grounding which will be explained as necessary, as will overlapping and interlinking of concepts and themes which will be referred to throughout the remainder of the work where it is relevant to the presentation of analysis, discussion and conclusions. The obstacles that emerged from the data collected were a complex and interconnected mix of factors which fell into three distinct but inter-related themes. The themes of identity, relationships and infrastructure can be understood as separate domains of living and experiencing self in the world; 
individual or internal, interactional or relational and environmental or the world external to self. Although they are presented here as separate themes, indeed often researched as separate entities, identity, relationships and infrastructural systems are intricately and inextricably interlinked in the lives and experience of humans.

\subsection{Identity}

Identity involves the sense a person has about self and their role in their relationships, families, the wider community and society. Psychological and sociological explanations of identity submit that identity is formed of both internal conceptions of self and the ways these are presented and experienced externally in social settings (Fukuyama, 2018; McLean, Syed, \& Hammack, 2015). The psychological, sociological and emotional dimensions to this study mean that for the purposes of this research, this theme is conceptualised as the way a person perceives themselves and considers they are perceived by others. Self-perception emerged as a very important aspect of mobility aid users lives, as will be explored within the identity theme, which is explained in more depth in Chapter 6. The analysis of data shows how mobility aid use influences identity through the ways in which changed mobility can alter understandings of self and subsequent presentation of self to others. The theme identity emerged through persistent comments relating to subjects such as continuity of self, autonomy, determination to continue with activities despite obstacles or difficulties, matters of self- esteem and references to state of mind before use of mobility aid became necessary. Subthemes identified within the concept of identity were independence, dignity and stigma. These three subthemes are closely related and inform each other. Figure 5.3 indicates some data that were coded to identity.
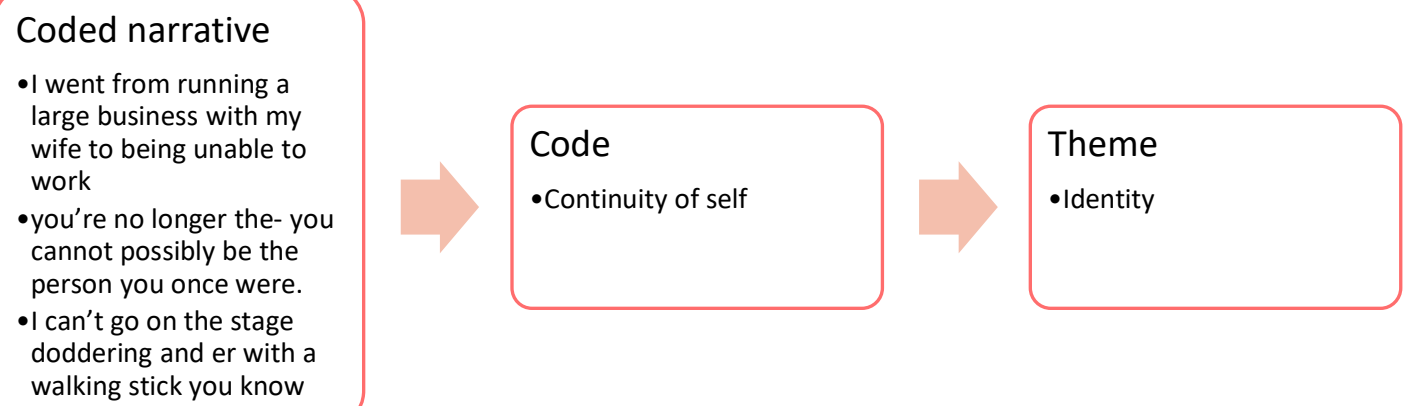
Figure 5.3 Coding example within theme of identity

\subsubsection{Independence}

Independence is the ability to move freely and live without being dependent on any other person for basic requirements (Schwanen \& Ziegler, 2011), although the specific meanings and practices can vary in different research, policy and practice settings (Schwanen, Banister, \& Bowling, 2012; Schwanen \& Ziegler, 2011). In addition, the concept of independent bodily movement is encapsulated within the concept of natural and normal within spatial and societal boundaries (Imrie, 2000, 2016). Korotchenko and Hurd Clarke (2016) highlight the cultural valorisation of independence and physical ability in their study of mobility aid users in Canada. Independence is closely linked to mobility and well-being, in that the loss of independence coincides with reduced well-being (Aguiar \& Macário, 2017; Schwanen \& Ziegler, 2011). Being unable to continue with daily routines independently or becoming inter-dependent means having to ask for help or rely on others to complete an action when it could previously have been carried out alone. Embodied changes during the ageing process or occurrence of chronic illness can result in reduced bodily mobility and therefore increasing dependence or interdependence, but the wish to be independent remains. Inability to continue with daily activities and routines without thought, anonymously, can be felt as detrimental to identity. Independence manifested within interviews and observations as a factor highly important to identity, with many participants either talking about independence or rejecting help. Figure 5.4 demonstrates a segment of data coded to independence in which the participant reveals his need to reinstate independence. His requirement for independence linked strongly to confidence and valuing self for him.

Independence is a socially valued as indicated above, so loss of independence incurs reduced social status and stigma according to social norms and expectations. Losing independence is therefore closely tied to the concepts of stigma and dignity, both of which are imperative to identity. 
and get my independence back which pushed me to do things I would never do before
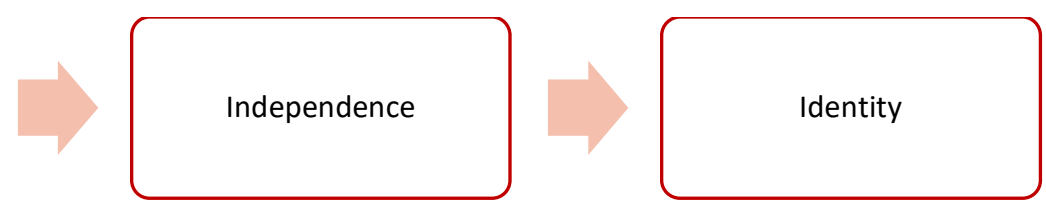

Figure 5.4 Example of data coded to independence then identity

\subsubsection{Dignity}

Dignity was not a subject addressed in early literature reviews, as stigma was the concept, I considered would be relevant. Stigma is particularly relevant to embodied difference, bodies which are not accepted as normal, as outlined in chapter 2. Various terms were used for the working theme, with factors such as embarrassment, vanity and reticence to admit reduced ability all being included. It took a while to incorporate the various manifestations and meanings attached to dignity to finally emerge as an overall theme. At the time of writing, dignity seems obvious, but it was not during the analysis process, probably partly because of the different ways people demonstrated dignity.

Dignity is widely acknowledged as an aspect of humanity and equality. The first article of the Universal Declaration Human Rights (UDHR) states that "All human beings are born free and equal in dignity and rights" (United Nations, 1948), the declaration fails to define dignity (Mann, 1998). Although various conceptualisations and definitions exist within research and literature, the definition of dignity used within this study is that of a universal value, a sense of worth, a part of identity emanating from evaluation of self in comparison to others and societal values. Dignity arises from adequate evaluation of the worth of inner self (Edlund, Lindwall, Post, \& Lindström, 2013; Jacobson, 2009).

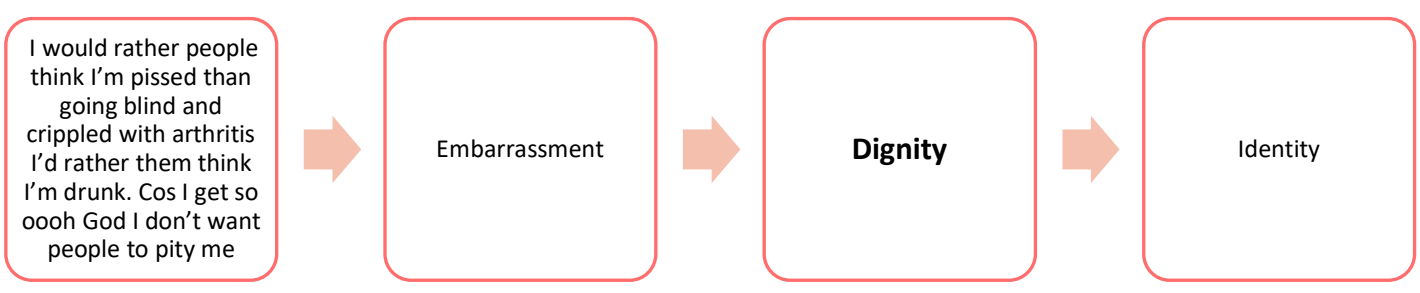

Figure 5.5 Segment coded to embarrassment within dignity in the theme of identity

A universal value, dignity can also be contingent, meaning it can be violated or promoted depending on circumstances (Jacobson, 2009). This aspect of dignity links it to Goffman's (1963/1990) concept of stigma which illustrates how identity is spoiled through stigmatising traits. Dignity can thus be removed within the concept of changed embodiment stigmatising 
trait or symbol such as a mobility aid, as discussed in greater detail in the next sub-theme Stigma and its management. As coding at figure 5.5 illustrates, embarrassment is an aspect of dignity discussed by Goffman (1963/1990) as an aspect of stigma. To become embarrassed equates to loss of dignity which both negatively impact identity. Dignity manifested in different ways within the study, which appeared to correlate with age. People over 80 avoided asking for help, wanted to give the impression they could cope independently and generally wanted to maintain an appearance of better ability than they generally had. There was a tendency to make the best of things and avoid complaining or which appeared to be mechanisms for dignity. Avoiding complaining could also be aligned with avoiding embarrassment as complaining is not a valued activity in polite society. Two people in the under 80 age group were more focused on physical appearance and not wanting to seem older than they were as markers for dignity. In both cases, a sense of dignity reduction could lead to non-use or improper use of mobility aids. Harm could be caused to physical self in the name of dignity when mobility aids were not used or used inconsistently or incorrectly. Some of the codes that appeared under the umbrella of dignity were stoicism, vanity, autonomy and keeping up appearances.

\subsubsection{Stigma and its management}

The third and final subtheme of identity is stigma. 'Stigmatised' and 'normal' are perspectives that are generated in social situations, but managed within personal identity and ego (Goffman, 1963/1990). Goffman uses the term personal identity to describe the more intimate identity people use within personal relationships (rather than encounters with strangers) and ego to mean "felt identity, namely, the subjective sense of his own situation" (p129). These definitions identify that both identity and relationships are integral to stigma and its management. The inter-reliant nature of identity and relationships, and necessity of relationships for societal expectations and stigma to exist mean this sub-theme could have been placed within the relationships theme. However, the situations in which the concept of stigma arose within the study highlighted a removal of dignity or other negative impacts on perspectives of self-identity and related to the management of stigma. 


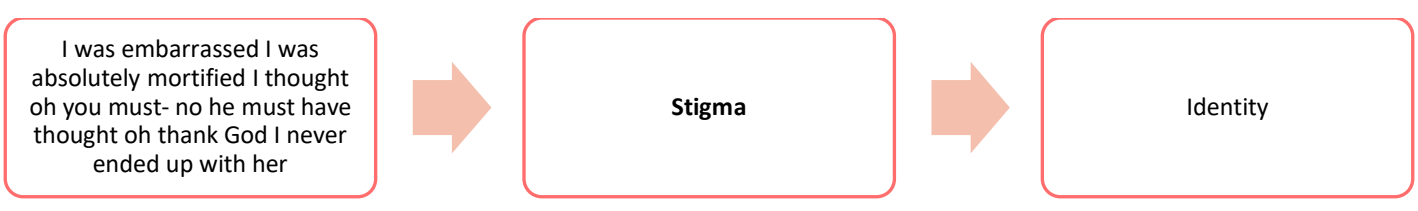

Figure 5.6 Segment coded to stigma under the theme of identity

Stigma emanated from the data in the form of codes such as embarrassment, inconsiderate others and aid as a signal to disability. Mobility aids seem to encompass both a disgrace and the (almost) bodily evidence of being unacceptably different. Within this study the conceptualisation of stigma includes ideas of idealised embodiment and the power relations apparent in practices of stigmatisation as discussed in Chapter 2. Attitudes within culture and societies are perpetuated by nations and politics. Ableism mobilises resentment and constructs those who have different bodies as outside of the norm (Hastings \& Thomas, 2005; Hughes, 2015; Sendi, 2014). Using a mobility aid equates to interrupted embodiment within the concept of stigma, whereby the day-to-day activities one usually carries out are not only physically interrupted, but socially and emotionally through actual or perceived social judgements, categorisations and strata.

As the coded segment in figure 5.6 illustrates, some participants were willing to discuss their embarrassment and feelings of standing out because of using a mobility aid. Other participants talked of their unwillingness to use a mobility aid until they realised their life would become easier or more productive when they began to use one. Sometimes non-use of mobility aid or being unwilling to progress to a more substantial mobility aid signified stigma, as discussed in more depth in chapter 5 . For those who did not use a mobility aid, or refused to progress to a bigger aid, the reason was always linked to not wanting to stand out, or perception of appearance. The stigma sub-theme is situated within identity rather than relationships where it could also be placed because of the way stigma tended to manifest as perceptions of self. Stigma is only possible through relationships; through perception of perception of how others perceive you and how others do react to you.

\subsection{Relationships}

Relationships forms the second main theme of the research findings, with sub-themes of carer concerns, disagreement and discord, burden and relationships with place.

Relationships as a specific focus are not addressed in the literature review, partly because all 
facets of life are regulated and navigated through relationships. As discussed within the literature review, literature pertaining to emerging themes is discussed through that theme. Information, status and acceptance of people is negotiated between individuals consciously and subconsciously, verbally and physically through embodied actions. The term 'Relationships' covers a vast wealth of knowledge, meaning and interpretation, but the definition of relationships herein is any interaction an individual has with another person, place or object. This sense of relationships emerged as a way to conceptualise the interactions mobility aid users had with the world external to self. In that sense relationships as a theme encompasses the use of mobility aids, how older people relate to them, to other people and the built environment. The term relationships then, encompasses not just interpersonal relationships but also relationships with objects and systems.

As demonstrated below, because coding within the constant comparative analysis methodology is a bottom-up type process, the codes first named in this area were factors such as 'considerate others' which became a higher-level code of Interpersonal aspects before the focused code of relationships was identified. Relationships was a focused code that incorporated codes indicating interaction with others in some way as identified in figure 5.7. Some of these codes were 'aid from person', 'bad service', 'considerate others', inconsiderate others' 'pressure to fit with solution' and 'unwanted attention'. The relationships referred to in this section are not all between mobility aid user and other people. The segment in figure 5.7 refers to the daughter of a mobility aid user and her relationships with other rugby fans for example. Within the feeling like a burden sub-theme, the relationships discussed could be with self or home, as highlighted by the example of Regina in Chapter 7. The final sub theme of relationships with place also places the focus of the relaionship on interaction with place and space.

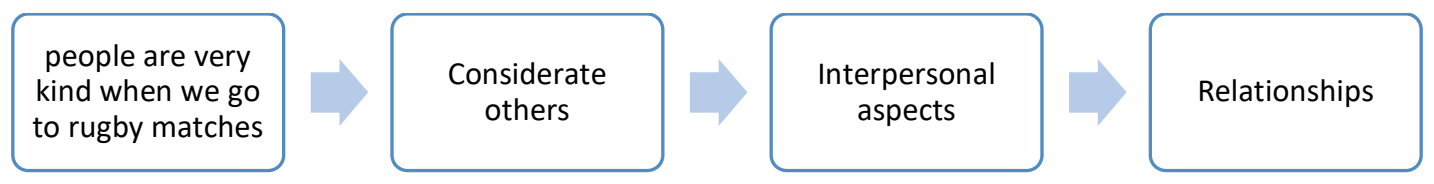

Figure 5.7 Segment coded through interpersonal aspects to relationships 
The field of relational sociology conceptualises social reality as involving interactional social processes which cannot be separated from individual actors (Donati, 2011; Emirbayer, 1997; Hillcoat-Nallétamby \& Phillips, 2011). Framed is this way, issues occurring in society are neither based in individual or collective action, but the articulation of social relations. Meaning, significance and identity all emerge from interpersonal transactions, and it is the study of these transactions that become the unit of study within relational sociology (Donati 2010). From a relational sociology perspective, then, relations and relationships are inherent in, and fundamental to all social interaction. Any context in which humans interact with people and things will involve some type of relationship. Meaning, significance, and identity emerge from the changing roles within interpersonal transactions and the dynamic process becomes the primary unit of analysis (Emirbayer, 1997). The purpose of the relationships theme within this thesis is not to interrogate the relationships themselves, but highlight the important contextual and role played by people, social context, cultural norms, place and objects in mobility aid use by older people.

Relationships are integral to social life and social participation, so in some ways relationships link all themes together. Identity is formed via relationships which occur within infrastructure, an inter-relation which is explored and advanced throughout the thesis . People gain a sense of self and form identities through their interactions with others. This is best explained through social identity theory, in which Tajfel and Turner (Tajfel, 1970; Turner, Brown \& Tajfel, 1979) proposed the notion that individuals construct and understand self and their own identity by aligning themselves with social groups or categories. Turner went on to develop a social identity theory of groups which ultimately became a self-categorisation theory, indication cognitive processes that underpin the links between self and a group. The overarching conceptualisation of social identity theory and social identity theory of groups assumes that group relationships and self are intricately woven together and reciprocal (Abrams, 1998; Abrams, Hogg, \& Marques, 2005). Family, ethnicity, and gender are all types of social identity groups. Relationships are therefore inextricably linked to Identity through the groups one identifies with and therefore the relationships one has (Abrams et al., 2005; Deaux, 2000; Levine, 2012). Data within this study reflected these notions of group and relational identity which informed individual 
identity. As the subthemes discussed below indicate, relationships informed how participants managed their daily routines and lifestyles often to compensate for aspects related to individual identity, as discussed further in chapter 6 and 7.

Participants commented on relationships in many different ways, and the dyads were different in each case, so the intensity of mobility aid user to carer relationship varied greatly. In addition to this, while there were some parent child dyads, one mother and daughter lived together, another did not. Two father-daughter dyads participated and again, one pair lived together, the other did not, and in one of these cases neither considered the daughter to be a carer. With such variation of relationships, I did not consider the specific relationships of participants to be comparable, which is why there is no focus of the dynamics of the dyad relationship within this study.

\subsubsection{Carer concerns}

Carers are an important focus when discussing any kind of changes in embodiment, identity, ability and health. Carers, especially if they are close family members, are able to provide a unique insight in to changes of routine or changes of attitude that people do not always notice in themselves. It is also an important avenue to explore because carer's views and observations are often not explored within research. Carers in this study include husbands, wives, daughters and a friend. Mobily aids inevitably interrupt some of the communication and navigation of environment, impacting on relationships bi-directionally, in that both the individual using the aid has to change their practices, and so do those around them. The term carer is used here to include any person taking care to assist someone who uses a mobility aid and does not necessarily reflect a relationship defined by reliance. Carers often voiced concerns regarding their loved one, which could create quite complex situations. On occasion, a carer would offer an explanation within a dyadic interview that was later refuted by the mobility aid user. In some families an adult child thought the parent was capable of more than they were achieving, but in another family, the daughter wanted her father to do a little less for his own safety. Carers needed to balance maintaining a continuous relationship with the mobility aid user with unique concerns wholly focused on mobility aids or mobility issues. Figure 5.8 highlights a segment coded to carer concerns in which a carer comments on the fact that her mother refuses to use the mobility aids she is prescribed. 
Such concerns, if not managed carefully, could impact upon the roles and identity of the mobility aid user within family structures, as discussed in detail in chapter 7.

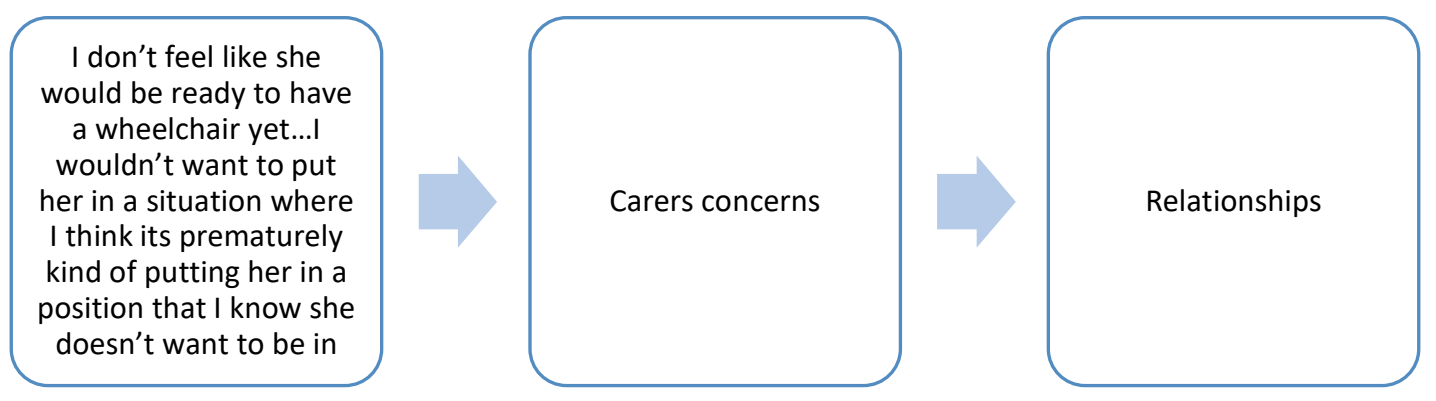

Figure 5.8 Segment coded to relationships through carer concerns

Carers concerns also brought together other codes as a group code, with most items coded under carers concerns, but incorporating other items like 'caring time consuming' and 'caring physically demanding'.

\subsubsection{Discord or disagreement}

Discord and disagreement began as a code within interpersonal relationships, but I felt it to be an important factor in daily routines, management of mobility aid use and of identity and stigma, relating back to the previous theme of identity. Discord or disagreement could arise because of nuanced changes in routine and the changes to identity mobility aid use could bring. From slight differences of opinion or shortness of temper to outright rudeness, discord could impact upon the emotional and psychological well-being of participants, especially when it occurred with strangers or service providers. Such negative interactions could lead to reduced participation in a service or event. While discord could manifest in the interactions with strangers or members of staff involved in service provision it also sometimes occurred within close caring relationships. The differences in opinion were not arguments, but underlying misunderstandings or different ways of wanting to proceed in a situation. There could be differences of approach to the safety and well-being of the mobility aid user, with adult children often wanting to limit the activity of their parent for the sake of safety, linking discord and disagreement to the carer concerns identified in the previous sub-theme.

Factors such as environmental obstacles or stigma are interlinked with discord and disagreement because of limitations on social activities of the mobility restricted party. 
Sometimes, family members or carers underestimate or do not understand the limitations or their loved ones' illness or mobility difficulties. Embarrassment or a sense of dignity might lead to non-use of a mobility aid, leading to carer worry or needing to access more time to support the loved one. Carers were very understanding of their mobility reduced loved one's emotional and psychological as well as physical needs, and their insights were invaluable to understanding mobility aid use. Discord could arise for a variety of reasons, as discussed in more detail in chapter 6 , but sometimes it was because the mobility aid user did not want to feel like a burden.

\subsubsection{Feeling like a burden}

Participants used the term burden to describe the sense of extra load or responsibility their mobility difficulties or mobility aid management placed on carers or loved ones. In some cases, an additional sense of responsibility was mentioned by a carer, but this was not in the context of burden, only of caring. Six participants talked about the notion of burden; Aileen,

Phil, Dave, and Theresa as mobility aid users (or non-user in Aileen's case) discussed aspects of feeling like a burden or holding up proceedings; Jenny as a carer noted that she felt some of her father's behaviour was focused on not being a burden to his family. Through first stages of analysis, burden was situated within 'attitude to mobility aid'. It seemed to be an attitude held by mobility aid users that their mobility aids or mobility difficulties made them a burden on others. As data collection, analysis and interpretation continued through the project I began to understand how much the sense of burden had the potential to impact upon the identity, dignity and relationships of participants. Burden is closely linked to identity because of the inability to remain independent or continue routines at the same speed or in the same way as before mobility issues or aids became a part of their lives. As discussed earlier in this chapter, identity, dignity and independence and a sense of being a burden are interlinked and can be experienced simultaneously or in quick succession. Feeling like a burden is situated within the relationships theme because it is in only possible to feel like a burden through a relationship.

\subsubsection{Relationships with place}

The final section in the relationships theme is that of relationship with place. During the first phase of coding, segments of data which eventually were coded as relationships with place 
were placed under codes 'design good', 'design bad' or 'advanced planning'. Advanced planning referred to the extra planning often involved when travelling with a mobility scooter or wheelchair. These elements could have been situated within the infrastructure sub-theme, but I realised there was also a connection to some of the comments made that I had coded to 'continuity of self' in which participants recounted their memories of visiting places they could no longer visit, or places that reminded them of deceased loved ones. I noticed a thread connecting all of these ideas, in that they are all concerned with the relationships people have with places. Places are meaningful to people, familiar (or unfamiliar), where memories are made and identities formed (Peace, 2013).

People have relationships with place, be they familiar or unfamiliar places, inclusive or exclusive. The sub-theme of relationship with place is focussed on understanding how people's relationship with places affect the behaviour and identity of individuals who use mobility aids, and ultimately the group 'mobility aid users'. People interact with space and place and with other people in places. People connect to and from memories in places; the policies and regulations on places either include or exclude people. Including or excluding participants by way of inefficient planning, ineffective policies or failure to provide services could impact upon the experience of participation, the ability to enact self, and sometimes result in feeling like a burden. Relationships with place, along with all other aspects of the relationships theme is explore in depth in Chapter 6.

\subsection{Infrastructure and interlinking systems}

Informed by interaction between the individual and other people or the environment, including social and systemic structures, practice and policies through to the built environment, infrastructure and interlinking systems such as housing, health services and transport provision provide the support structure upon which life is played out. The title infrastructure and interlinking systems is a deliberate incorporation of aspects of the built environment that would traditionally be called infrastructure with other supporting networks and structures that enable the movement of people and social activity in line with the mobilities paradigm. Within the mobilities paradigm, infrastructure is supported by an array of networks, policies and practices that are interlinked and inter-reliant. People need a transport system that moves them from their home to places of employment for example, 
but the transport must be affordable, accessible and safe with policies and laws to support these factors. Bus services need to run on time, not be overcrowded, be safe according to legal frameworks, be driven by qualified personnel. Qualified personnel need to be trained, safe vehicles require maintenance and testing. Accessibility for those with mobility aids requires design, planning and testing. Timetables need to account for differences in boarding and leaving vehicles for those with mobility difficulties. There are a multitude of systems and services that are required to support and maintain infrastructures. This theme reflects the infrastructure and the interlinking, complex array of systems, and also the complexity of ways the infrastructure and systems interrelate.

Infrastructure and interlinking systems elicited more comments, with 254 segments of text, than any other subject except mobility aids themselves which contained 343 segments. For comparison, a code I had called 'interpersonal aspects' which ended up as relationships held the third highest rate of references was with 128 segments, and within this were subsections relating to customer service from transport or mobility aid providers and experiences of bad service within a hospital environment which both relate back to infrastructure and interlinking systems. Many of the circumstances and comments made in the study were either about infrastructure and systems or linked to them because of the way the built environment, social activities, healthcare, transport and housing are all reliant on infrastructure in some way. If infrastructure is defined as the basic organisational structures and facilities such as buildings, road and power supplies needed to operate a society or enterprise, then life and social activity within that society is reliant on infrastructure. infrastructure enables the movement of goods, people and money by providing transportation, services and buildings for community activities (Larkin, 2013).

This theme explores how the external environment presents obstacles to mobility aid users, incorporating planning and policy because these regulate all aspects of the built environment in some way. Outdoor environments, social participation and transport and mobility are three of the eight domains or age friendly cities or communities, and potentially not only have to be age friendly in and of themselves, but also work coherently with each other and the remaining domains to provide accessible environments for all. Infrastructure that does not work to provide a cohesive set of structures and services to users fails to accommodate their needs. Even if, for example, housing is adequate and the transport is 
adequate, if they do not work in unison, social needs are still not being met. Participants experience of infrastructure and interlinking systems is examined if greater detail in chapter

8. The policy implications of policy and practice that does not work consistently and cohesively together are examined in chapter 10.

\subsubsection{Pavements and roads}

A major factor in mobilising the environment are the pavements and roads that enable us to walk, drive and otherwise transverse land from one place to another. The sub-theme was formed early in the research as a subject commented on by many participants as being problematic or inaccessible is some way. Nine of the eleven mobility aid users mentioned difficulty with pavements or roads for different reasons, but all had issues navigating them with their mobility aids. Two carers also mentioned how concern for roads or pavements factored in the mobility of their loved ones. Pavements and roads are coded as a joint entity because they are experienced together and each impacts the other inseparably, with speed of traffic and width of pavement being factors in accessibility. This theme also incorporates the code 'precedence of motor vehicles' and 'potential travel', a code used for segments of narrative relating to places people might want to access but did not try to because they felt there would be no way to overcome obstacles. Figure 5.9 demonstrates a segment of text coded to pavement and roads which highlights how badly maintained pavements could impact upon the ability to be mobile.

Look at the paving slabs, half the kerb is up... It's a trip hazard.

\section{Figure 5.9 Segment coded at pavements and roads}

Pavements and roads

Inability to use pavements or roads without difficulty presented obstacles to participation in social activities because attending social events requires mobilising through pavements and roads. Obstacles within this vital element of infrastructure therefore equate to obstacles to participation. Transport and mobility forms one of the domains of age friendly cities, however walking is a mode of transport that tends to be overlooked within the planning and design of infrastructure. As chapter 8 demonstrates in more detail, road transport tends to take precedence over pedestrians. When places become more inaccessible for mobility aid users, they are potentially more dangerous. 
An increased sense of danger felt by either the mobility aid user, or their carer who worries about their safety links this sub-them to the relationships theme. Relationships with place which are changed because people can no longer access places as they used to, sometimes leading to people feeling like a burden because they now require assistance. Discord and disagreement could become evident in the relationships of mobility aid users and their carers or loved ones because of safety and well-being concerns and time-management. Identity is inextricably linked to pavements and roads also, as a sense of independence is lost if mobility aid users cannot traverse environments easily, dignity is especially relevant as many people do not want to ask for help or be seen to need help, and stigma is increased with the use of mobility aids or increased dependence. Discord and disagreement can result from the difficulties produced by ineffectively planned or designed pavements and roads.

\subsubsection{Planning design and service provision}

Planning design and service provision is a focused code made up of codes from varying elements of infrastructure. Codes of 'inconsistency', insufficient services', 'incompatible or impractical aid' 'space to manoeuvre', 'planning good/bad', 'bad experience' and 'inconsiderate others'. Viewed from the perspective of the mobilities paradigm, the three elements of planning, design and service provision need to work in unison to ensure the movement required for social interaction, and thus political, economic and social engagement (Urry, 2007). Indeed, from the mobilities perspective, people who work for local councils from social workers to environmental planners, health authority to transport company staff, even, or maybe especially people planning and enforcing car park regulations, are as much a part of the infrastructure as the hardware such as street lights and paving slabs. The consensus of experiences from participants led to that same conclusion within this study: planning, design and members of staff who are the interface of services that support planning and civic or social services need to accommodate the requirement of services working in unison and cohesion. Experiences recounted and occurring during the study illuminated a less than supportive infrastructure for those with mobility difficulties. From less than friendly bus drivers to museums with large sections inaccessible to people with even slight mobility difficulties, services and design of place could present obstacles to participation. 
I've got a wheelchair I can't use, I can't go out cos I can't get in the car, what do I do die of boredom and starvation cos I can't go shopping and they start going through the bog standard answers - 'well you're just one on a list,

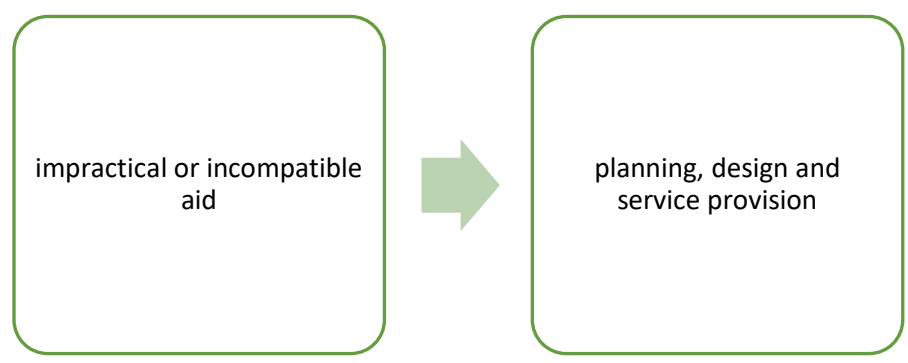

Figure 5.10 Coding via impractical or incompatible aid to planning, design and service provision

obtaining a working wheelchair from the NHS department dedicated to wheelchair provision, despite his inability to live independently without one. This segment has been chosen to demonstrate that a department which exists solely for the purpose of providing aids to enable people with mobility difficulties is entangled within an infrastructure of systematic procedure that does not meet the needs of its service users. The inconsistency in services, failure to understand or consider the needs of people who use mobility aids gave a sense to participants that they were not valued, reinforcing ideas of stigma, reducing independence and dignity, all of which ultimately had a negative effect on identity. The relationships with service providers and staff at the staff at the interface of services like transport and healthcare could often be strained or difficult linking this sub-theme closely to discord and disagreement. Feeling like a burden could be the result of ineffective planning and service provision, which can also feed into carer concerns. It needs little explanation that relationships with place are highly impacted by the nature of design, planning and services available. Each and every aspect of planning, design and service provision could result in the sense that accessing the built environment and thus social participation involved a great deal of hassle.

\subsubsection{Hassle}

The final section of the third and final theme is hassle. Hassle is an all-encompassing term describing the general difficulties in infrastructure and attitudes faced by mobility aid users. The term hassle is used here to describe situations that are inconvenient and irritating and present an obstacle to the flow of daily life. As a sub-theme, hassle discusses the daily interactions with people, objects, public services and even mobility aids themselves that can 
become problematic. Routines that could and should be similar to those for people without a mobility aid were reported as reduced because of the hassle involved in mobility aid use.

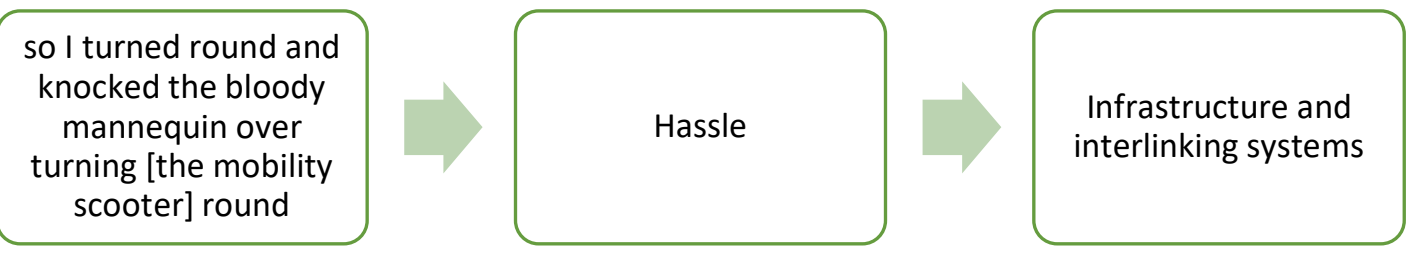

Figure 5.11 Segment coded to hassle

Hassle was not necessarily named by all participants but certainly alluded to by all mobility aid users in some way. 15 of the 17 participants mentioned a hassle of some kind. Figure 5.11 identifies an issue Will had whilst shopping with his wife using his mobility scooter, one of the ways in which hassle could be conceptualised. Hassle encompassed the smaller obstacles, physical, attitudinal, psychological or emotional that mobility aid users were confronted with in succession throughout their daily routines. In some ways, hassle could be said to summarise the study, in that it found no huge obstacle, no participant suggesting they can never get out, but a constant stream of niggles and problems, that put together each and every time one leaves the house, makes a problematic and draining experience. However, some participants did not like to admit to any sense of hassle, even though their accounts and observed experiences demonstrated it. The process involved maintaining dignity and independence in the face of difficulties for the sake of maintaining identities. Relationships with people and place could be affected by hassle, as people navigated obstacles, they also navigated frustration, disappointment or denial of their abilities or needs. Chapter 8 inspects the nature of infrastructure and interlinking systems and services in more detail. The remainder of this chapter explores further the inter-reliant and interrelational nature of the findings. 


\subsection{Inter-relational nature of themes}

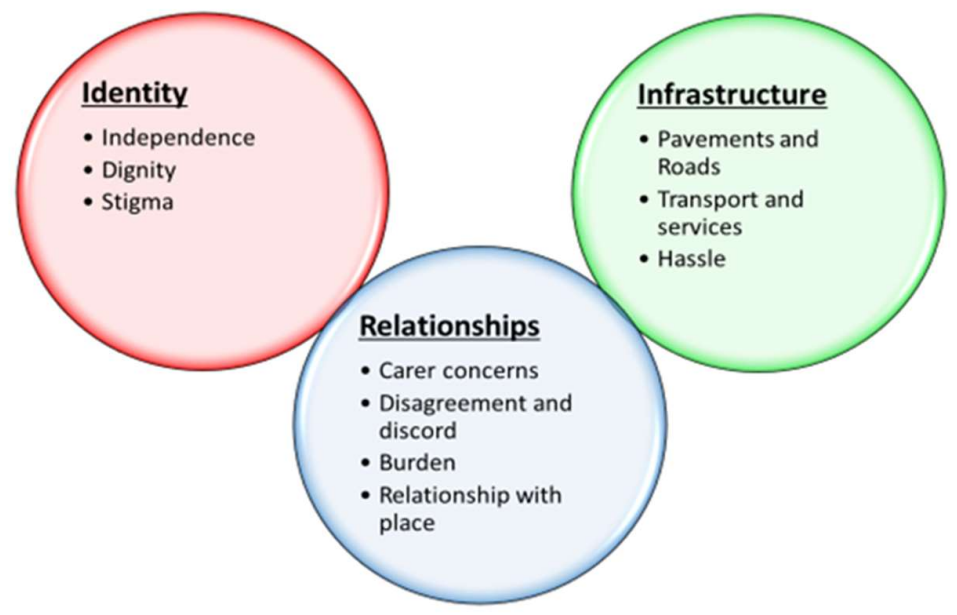

Figure 5.12 Each theme represents a different kind of obstacle set

The themes and sub-themes as discussed so far can be presented as shown in the diagram figure 5.12. In the routine experiences of participant, though, the factors involved in mobility aid use are all inter-reliant and intermesh with each other. Identity, relationships and infrastructure with its interlinking systems and services are all aspects of interactions between individuals and the social world. Each domain in which obstacles exist are not only equally debilitating and obstructive to social participation, but each one impacts upon the others (Figure 5.13). The diagram shows ways in which in which identity, relationships and infrastructure overlap to inform and impact on each other. Placed between each of the main themes are sub-themes that might be called bridging sub-themes. 'Stigma' is framed within the identity theme because it is felt by the mobility aid user and impacts on identity, but a relationship is necessary for stigma to exist, so it could have been placed and analysed as part of the relationships theme. 'Hassle' is part of the in infrastructure and systems theme because it is mostly systemic or design issues that create the hassle referred to. It overlaps heavily with to identity because of the ways in which systemic and design factors can affect identity, dignity and self-esteem. 'Relationships with place' involves a relationship with geographical spaces, but also with the people who inhabit them, or with whom memories have been shared in them. Relationship with space is closely related to 
infrastructure because of the way infrastructure informs the ways spaces and places can be used.

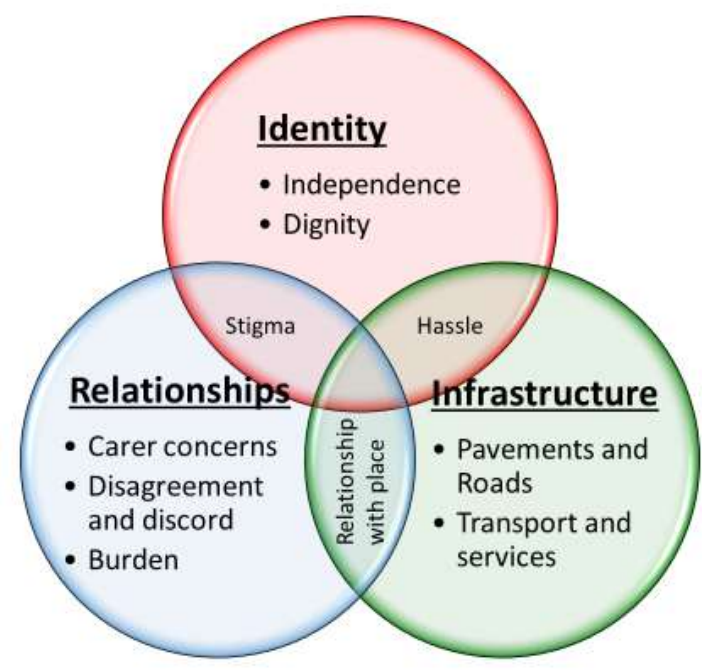

Figure 5.13 The interlinking nature of obstacles

Although themes can be broadly conceptualised as internal and external, obstacles did not necessarily present as clearly internal or external, or distinctly situated in identity or relationships when experienced as part of daily life. Mobility aids, obstacles to participation and daily routines interact and feedback in complex ways, reiterating the ways in which human life and relationships occur in overlapping, continuous, sequential and experiential ways. Figure 5.14 demonstrates some of the ways in which themes and sub themes are cross cutting and interlinking. 


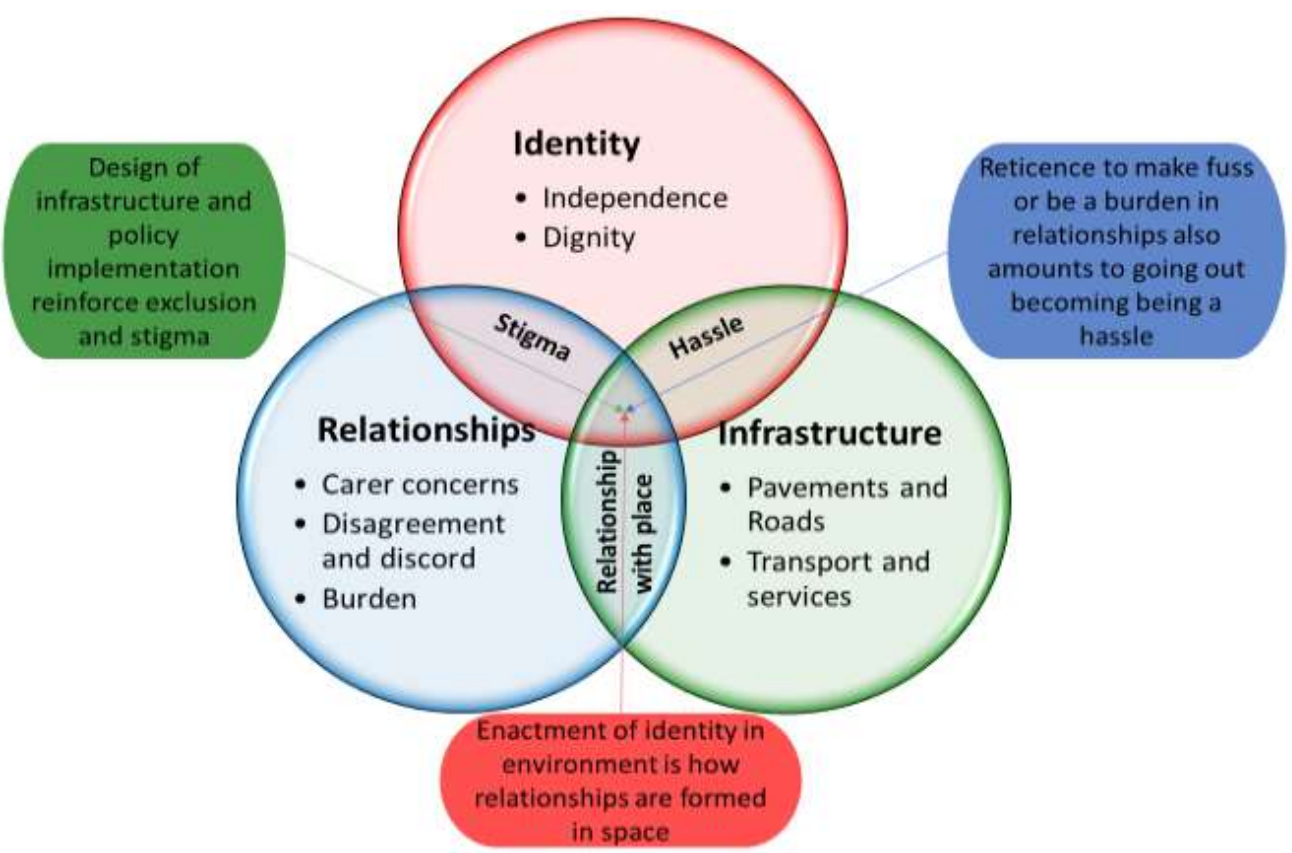

Figure 5.14 Complexity of interlinking obstacles

The diagram presents examples of how these sub-themes might manifest in an alternative theme. Hassle in a relationship could present as a mobility aid user not wanting to be a burden. Stigma can be reinforced by the wording or often omittance of disability or mobility issues which will be discussed in the next chapter. Relationships with place is closely aligned with ability to be oneself in a place, inclusion or exclusion from place. According to the data here, inclusion occurs only with great difficulty and obstacles, changes the way an individual can enact self and perceive self, which feeds back into sense of identity.

Identity and relationships are intrinsic to each other, so changes to identity inevitably impact upon relationships. The infrastructure of the built environment, social structure and interlinking systems are reliant on more distant types of interaction and relationship but are just as influential on the ways people participate, are perceived, perceive self and ultimately identity with self and other in the world. Structures and systems play an inherent role in the ways people are categorised (Burghardt, 2013; Foucault, 1977, 1982; Gilleard \& Higgs, 2002; Hamraie, 2017; Laslett, 1987; Stryker \& Burke, 2000). Research into such structures often fails to address social or individual aspects (Aguiar \& Macário, 2017; Musselwhite, 2015; Oliver, 2013) or conversely research into social structures and phenomena can omit the influence of infrastructure (Cresswell, 2010; Dorow, Roseman, \& Cresswell, 2017; Merriman et al., 2013; Sheller \& Urry, 2006; Urry, 2000, 2007) . This research indicates that individuals 
do not always understand the impact systems and infrastructure might have on their sense of identity. Obstacles apparent in the data were not always voiced by participants as obstacles, especially with aspects such as stoicism or disagreement and discord in relationships that seem like everyday occurrences anyway. They are presented as obstacles where the behaviour or event appears to be created by mobility aid use and would not have occurred if the aid were not present. For example, if arguments ensue because a mobility aid user feels burdensome it is recorded as an obstacle due to mobility aid use, even if the mobility aid user has not made such a connection. These are the events and phenomena that ultimately do affect identity, relationships or participation. 


\section{Chapter 6. Identity}

Identity is a term that is commonly used and varies in theoretical and conceptual interpretation (Stryker \& Burke, 2000). The usage for this theme and conceptualisation is that of identity being the meanings a person attaches to self in the multiple roles they play in differentiated societies. Identity is formed by self-definition in comparison to others according to roles or groups (Stets \& Burke, 2000; Stryker \& Burke, 2000; Wood, Littleton, \& Oates, 2007). Identity theory suggests reflexive evaluation leads to an individual categorising self into a preconditioned social role. Social Identity Theory assigns groups rather than roles, so individuals align themselves with a group rather than a role, positively valuing in-group behaviours and beliefs, rejecting those of out-groups. Ultimately, both theories maintain that an individual takes a reflexive view of self as objective to categorise or classify itself in relation to other social categories or groups (Stets and Burke, 2000). Both approaches also indicate a pre-set social structure in which roles or groups already exist. To

summarise from both theories, identity is formed of reflexive activity to identify with groups and roles. Identities are multiple and flexible and can be used by people to define self in different settings. Personal identity can be described as that perceived as unique to the individual, role identity as the identity role an individual chooses to occupy and social identity the group an individual aligns self with (Carter, 2013). The iteration of embodied \& psychological experience and self-understanding all influence one's relationship with self (Wood et al., 2007), and therefore relationships with others and the environment, reinforcing the complexity of the themes within this research.

Throughout the research, while walking, interviewing and later while analysing, it was difficult to understand whether denial of pain or immobility was due to a need for independence, dignity, fear of stigma or a combination these concepts which are all dependent on each other or closely related. In the mundane but chaotic routines of everyday life, factors of identity and its management are intermeshed and interdependent, making it sometimes difficult to disentangle dignity from independence or stigma. Sometimes all might appear in one utterance or exchange. In the interests of coherency, and remaining true to the original transcripts, every attempt has been made to assign meaning to interviews that were true to the circumstances and context of situations within 
the themes as presented, even though many themes and concepts are cross-cutting, interrelated and complex.

Discussion of self in relation to other automatically iterates some form of identity management, but some people are more conscious of the ways in which they guard and manage their identity than others. Phil articulated the sense of removal of identity he feels with use of a wheelchair, remarking that "it absolutely fundamentally changes your life. The more requirement you have for a bigger mobility aid, the more your life is removed from you.

Phil was a very articulate speaker, he stated very strongly that the greater the reliance on a mobility aid, the more of a negative impact it has on identity. Phil went on to elucidate on why use of a mobility aid fundamentally changed his view of himself, his place in society and how he perceives other see him:

your place in society changes for you yourself because you are now the person in the wheelchair, because people see the wheelchair, they don't see the person they see the wheelchair ... you are not a normal person at all ... you're just not a tenable human being ... if the average person had to walk round and explain to everybody they met that actually, do you know what I may be wearing this pointed hat but I'm actually a normal person.

Aileen also felt mobility aids hugely impacted her identity, to the point that she refuses to use them, despite being blind and in extreme pain with arthritis. The basis of this interview was for Aileen to lead me in a usual activity, and the interaction between Aileen and Lauren also led me to believe that Aileen continues with these activities in her day-to-day life. It seemed imperative to her identity that she continued to carry out shopping activities and without mobility aids. Throughout the whole interview, which comprised of a pre walk interview in a coffee shop, followed immediately by a walking interview, Aileen made with derogatory comments about herself regarding her disability, and how she does not want to identify as disabled even though she knows she is.

I don't want to be classed as disabled although I know I am ... with eyesight and I'm crippled with arthritis ... My left kneecap is shattered ... my right knee is going the same ... I've got arthritis in the base of my spine, the top of my 
spine... it's in my hands ...It's in my feet.

The list of difficulties was reflected in the knobbled and gnarled joints in Aileen's hands and feet which were displayed when she tried on some shoes in a shop. Her legs were slightly bowed, and the difficulty Aileen had walking was plain to see. As we navigated the town, Aileen's daughter Lauren knew when to guide her and when she was alright to walk unaided. Aileen continued after her litany of arthritis sites "ahhh I'm like an old fart and between that and my eyesight ... I think people 'go look at her she's disabled'".

Aileen outlined how she feels use of a mobility aid brings on a different identity status because people talk to your carer or family member as if you no longer exist, saying "I mean it's that classic thing of em does he take sugar? ... It's a classic thing they speak to someone not [me] I'm like excuse me l'm quite intelligent don't you dare don't you dare. I hate it".

Echoing Phil's statements that opened this section, Aileen commented "You see they won't see you, they see that bloody stick first" revealing a shift in identity that renders her feeling almost invisible.

Will does not use a wheelchair also remarked on feeling conscious that use of one will precipitate negative consequences for his identity, not just for his own perception of who he is but also how he feels others will see him.

I'm being perfectly honest with you ... to be out and about you know in a wheelchair in town and ... mates ... and acquaintances ... seeing me in a wheelchair you know ... f feel conscious of somebody who l've, is an old friend.

When asked if mobility aid use impacted his sense of identity, Will outlined the impact of his mobility difficulties and chronic illness on his hobby of being a singer. He gave up this hobby because he felt that he could no longer "go on the stage doddering ... with a walking stick". He does have mixed feelings when evaluating his opinion of mobility aid use. Using his walking stick is necessary for him to get out and about, but can be a nuisance and a sign of ageing:

... yes it can be erm ... a safety net in that respect ... a walking stick erm ... is a good aid because it erm enlightens people to the fact that you've got disability ... and they will erm be a little bit considerate ... it can be a problem 
it can be a nuisance you know getting in and out of the car getting in and out of things ...I'm vain and I don't like to be walking along with my stick because I think it ... ages me

As will be examined further within the themes of dignity and stigma, Will was happy to admit that he is vain, stating "I'm vain and I don't like to be walking along with my stick because I think it makes me... ages me, but that's me as a person". This was part of the reason he did not like using his walking stick nor want to use any other forms of mobility aid such as a Zimmer frame (rollator outdoors) or his mobility scooter which he avoids using (as discussed in the dignity sub-theme).

It is interesting that Will felt comfortable admitting vanity, which is itself stigmatised. The stigma of disability for Will was greater than the stigma of vanity. All in all, Will felt that mobility aid use signals personal illness and problems to others and impacts on his sense of identity. He wants to present himself in a certain way but feels this is not possible when using a mobility aid. At the end of our initial discussion, Will said "lots of others might talk about it aids for disabled people not just... erm how they're designed to help you but how they're designed, their presence affects everything".

Identity is a phenomenon that is spoken about overtly by some, not so openly by others. For those who do not talk about identity, there are sometimes signs or signals that their identity is affected by mobility aid use. Some participants discussed aspects of their daily routines, hinting at the ways in which identity may be impacted by mobility aid use. They did not necessarily speak about their identity, but their words and actions led to conclusions about how they felt about their identity. The failure to mention or lessening the importance of something that highlights issues. During our walking interview, Bret had not divulged what the problem with his knee was, just that it was "a bit painful". He joked that his leg hadn't dropped off when asked if it was getting more painful after about 50 minutes of walking. It is worth reiterating here that Bret chose the route and walks it often, even daily. Bret's daughter commented that she was concerned about his reticence to admit to the gravity of the issue, noting "he won't give me a straight answer and I know he's in absolute agony". It was striking to hear Bret's uneven gait on the recording of our walking interview. Bret limped to the point of dragging his foot which could be heard clearly on the recording, and 
as I was wearing wellies, the footsteps were coming from his heavy boots. It is difficult to define and pinpoint the reasons for not admitting to weakness or ailments, as they could be many, however this example indicates more than one potentiality, and each of these will be discussed in the sub-themes. Independence was a theme throughout the research with participants stressing the importance of being able to continue with activities, duties and roles they felt crucial to maintaining their identity. Admitting a problem directly might have resulted in reduction of Bret's activities. Dignity is also a possibility, intrinsically linked to independence. There could also be an element of feeling burdensome, as considered within the relationships theme. Jenny summed the link between identity and independence when talking about her father Bret: "they don't want to admit to themselves and other people that they can't do something. That's definite with dad because he's so independent." As Jenny suggested, perhaps people do not want to admit to themselves or others that they can no longer carry out tasks they used to handle with ease.

\subsection{Independence}

Independence forms a fundamental part of identity because it enables people to make decisions and enact their lives without asking others to help. Continuation of self, and how others see you, was expressed by participants as being strongly related to the ability to carry out daily mundane routines and tasks without help. Mobility aid use can mean people who have lived independent lives can find themselves having to rely on others.. 'Self-reliance as a signifier of independence' (6.1.2) was a strategy used by some people who indicated the ability to continue with daily routines and activities without the need for help as important. The behaviour was not overtly discussed, but evident in the form of denying any inability or need for help. To begin with, this sub-theme considers 'Asking for help' (6.1.1) as an aspect of mobility aid use which incurs additional effort in maintaining independence and identity.

\subsubsection{Asking for help}

Dave stated that he "never had a problem with how people see me or how they look at me", but his identity status seemed linked to independence and the ability to do things for himself, or never having to ask for help. These factors are deeply intertwined with the themes of independence, burden and relationships with family, friends or the general 
public. The importance of not asking for help became clear when Dave described a recent shopping trip to buy a heavy item "I knew I needed help and that my brain was going "oooh how do I do this?" cos I did want to never ever ask for help".

Dave did eventually ask for help in this situation, but his greatest hurdle seems to be his own perception of how he should cope with his embodied difference and using a mobility aid. Dave pointed out "I've never had anyone pushing me I refuse to do it... when you've been independent all your life, well honestly it ... really irks me when somebody pushes" and went on to ask if I had noticed that he folds the push handles of his wheelchair down (which I had). His daughter has continually asked him to stop doing it, but while he cannot really articulate why, he says he must. He thinks is it so that nobody can just push him “Because I don't want anyone to push me... Don't ask, I do it, don't know... my daughter said that to me, every time "Dad, don't do it". I said I got to". As the quote demonstrates, it is unclear whether Dave believes a random person will push him and take unwanted control, or if it is a statement of 'I can do this without help'. Throughout the walking interview with Dave, I asked him why he acted and behaved in the ways he did, he even asked himself before I got the chance ask, but rarely knew why. He knew that he absolutely wanted to be independent. Dave's interview was certainly peppered with comments regarding his irritation at people constantly offering help, as "it's really it gets very frustrating people asking if you need help all the time".

Conversely, Dave recounts offering to help a younger female with her wheelchair at a wheelchair rugby game, even though he knows/knew she is capable and how irritating he finds it. The person in question asked him what he was doing so he ceased, but he recounted "she was struggling... and it killed me not to help her". The sense of independence and wanting to feel capable seems, then, to be aimed at self rather than directed towards others. It is very much about Dave's appraisal of self and ability to continue with his life as if the amputation had not happened. It seems Dave felt that he needed to put extra effort into maintaining his identity because of the extra attention his disability and mobility aid use brought about, even though he did not make the same judgement of others with mobility aids.

As Dave and I prepared to enter his local Asda superstore, he described how he feels about people always offering help 
this happens every time that I pull up next to the car and stand up then someone says do you want a hand getting that in the car mate... well how do you think I got out, pushed myself round Asda's ...do I look like I'm starving

Although this statement might seem to be aimed at his external identity, having chatted to Dave and discussed events of his life, this sentiment seems to be about two things. It does seem to reflect his need for independence, and just wanting to continue with his life uninterrupted by others. There also seems to be a drive in Dave to live the fullest life possible and almost ignore the amputation. The interview came at a time when Dave was trying to transfer to use of a prosthesis. The progression was not moving as quickly as Dave would have liked because of complications with his skin. There may have been some subconscious frustration playing out in his assertion to be completely self-sufficient. Dave's responses were emphatic in his need to be independent and to demonstrate his independence. As stated previously, despite further probing, he did not know why it was the case, but it was a deep-set requirement.

\subsubsection{Self-reliance as a signifier of independence}

One of the ways in which independence could be observed was through self-reliance. Selfreliance here refers to remaining independent and not accepting help from others, almost to prove to self that they are capable of being independent. It was not voiced by participants but observed and referred to by carers. Both Bret and Theresa demonstrated ways in which self-reliance was so important to them in different but similarly covert ways. Theresa did not voice any concerns about self-reliance, but was quick to deny any need for help or discussion of inability. Bret did not talk about independence himself, his daughter outlined how herself and her siblings could not just take over and do things for him:

"you've got to be really careful with him, you want to do stuff for him and make sure he's all right, but you've got to do it in a way that he doesn't think you're doing it for him" 


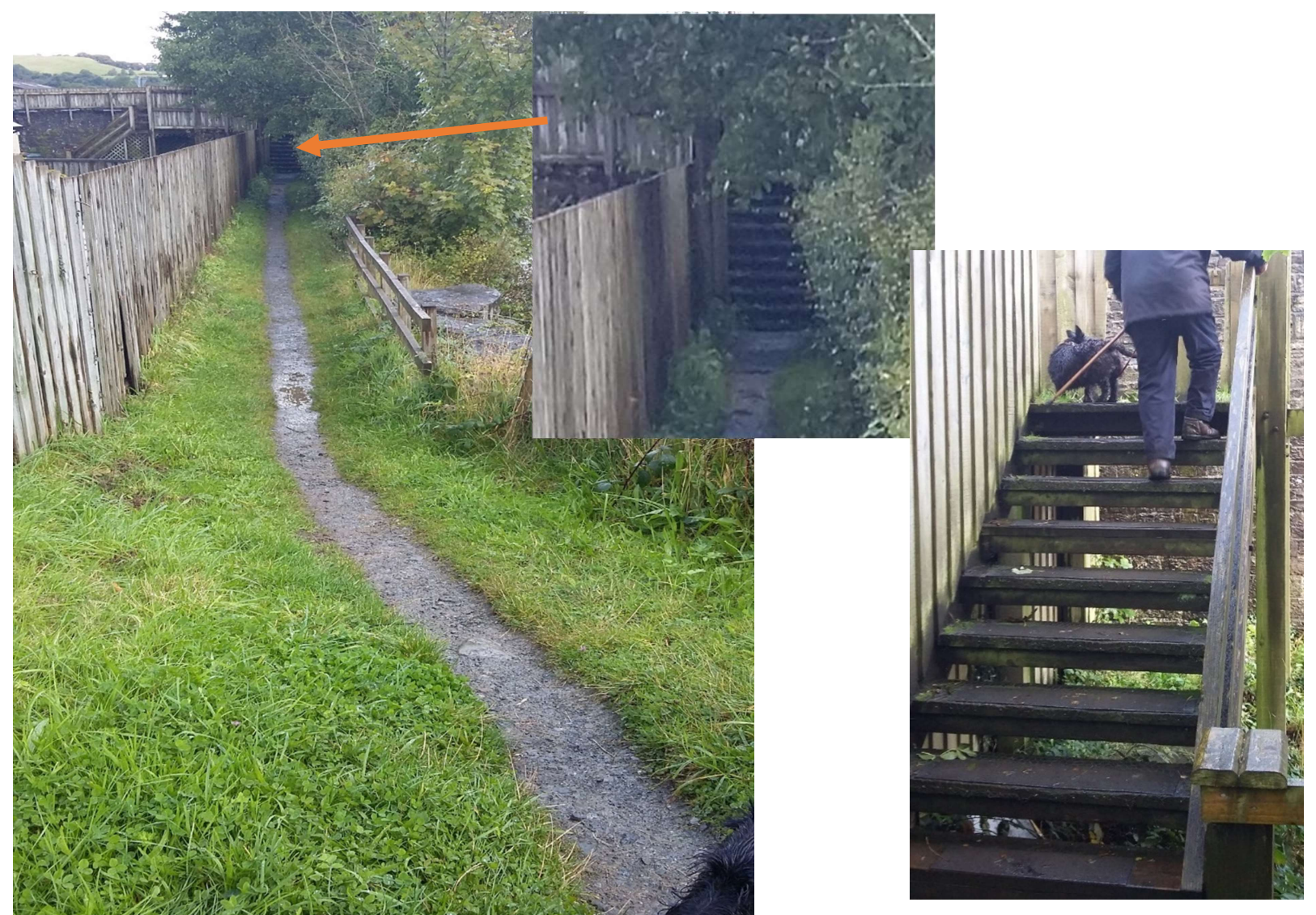

Figure 6.1 Bret at the top of the 'famous steps'.

Figure 6.2 Steep slope with steps in the distance.

Bret did display his independence during the interview, through his determination to walk in places that are not frequented by many people and his failure to admit reduced capability. As we began our walk, Bret decided "I can show you my famous steps where I ( ) usually take a nose-dive", taking me to a location at which he had fallen 3 times. The steps he spoke of lead from a muddy riverbank back to the street, and incur a steep slope downwards before reaching the steps which led from a muddy riverbank (see figures 6.1 and 6.2) . I asked if Bret carried his mobile phone with him in case of emergencies. He replied "Yeah. Well, that was the theory. In practice it's er not always so ( ) I mean er Sean and Jenny played hell with me cos I didn't have a phone with me". Although he did not actually say the words, 
I got the sense that Bret wanted to be left alone and not be contactable on occasion. He also felt it imperative that he could keep active on his own terms, stating "As long as you can keep moving. I dread to think of a time when I couldn't go out".

Theresa and her carer Ivy note that Theresa had been unable to get out before acquiring her mobility scooter. The scooter had enabled Theresa to become more independent in a lot of ways. Ivy was a paid carer for Theresa, the only paid carer within the study, but she was also Theresa's friend, having been her carer for many years. They explained that before obtaining her scooter, Theresa had been almost housebound. Ivy commented on how much Theresa's life has opened up because of her mobility aid because "it does the same job as a little car in as much as it takes you up to town...the bike has really opened Teresa's life...you're not stuck to the house"

Theresa did not like to admit to any difficulties or give any sense of reduced independence either. While Theresa had been housebound at one stage, it was Ivy volunteered this information. This avoidance of showing a lack of independence or need for assistance was apparent during our walking interview also. Theresa made light of any difficulties, making positive of everything she could. As we approached a shop she needed to enter and not accessible to a mobility scooter, Theresa parked her scooter on the pavement outside. The following exchange illustrates the challenging and dichotomous nature of embodied mobility difficulties that accompany ageing in addition to a need for independence.

RES: $\quad$ Dear what have I done with my stick. I'm struggling with my step now.

INT: Oh, do you want it?

RES: No, it's okay.

INT: $\quad$ Let me get it.

RES: No, I can get it, thank you (slight indignance in voice) () Ahh

INT: Do you find that step difficult? (It looked difficult and sounded like she was having difficulty)

RES: No, It's all right. 
Being able to go to the town and get out by her own means, on her own timescale, was very important to Theresa particularly because she lived alone and had no children. Ivy was one of the only people she could call upon but being able to just pop into town every day enabled Theresa to catch up with friends and go to the bakers for her lunch. None of this would have been possible had it not been for her bike (mobility scooter). Theresa herself did not mention her need for independence, but it was apparent in her other comments and actions. As we stopped to enter a shop, Theresa asked where her stick was but would not allow me to pass it to her, neither would she admit to difficulty in climbing the step into the shop despite a visible and audible struggle. This is discussed further in the dignity section as Theresa's comments about her ability to find and retrieve her stick when entering the shop also highlighted the inextricable links between independence and dignity. Theresa's dignity was also aligned to her need to appear independent and able to cope alone. This was not just about being independent, but about making sure other people perceived her to be independent.

Participants such as Bret, Theresa and Dave actively seek to retain independence which very closely linked to dignity via autonomy. Independence is the ability to live and act without dependence, that is be self-sufficient, and within that falls the concept of autonomy, the power and capacity to decide behaviour for oneself and act accordingly. Autonomy and independence, as the capacity to decide and act for self without reliance on others, enables dignity.

\subsection{Dignity}

Kant defined dignity as an intrinsic self-worth that is absolute, incomparable and unconditional (Dillon, 1995). Dignity is intrinsically linked to Identity because identity grows from a distinction between inner self and an external world and dignity emanates from adequate recognition or acknowledgement of the worth of inner self. In modern society, the inner sense of dignity requires recognition, and the sense of self-worth requires public acknowledgement (Fukuyama, 2018). For the purpose of this study, despite the vague and pervasive nature of dignity as described in Chapter 4, the sense that dignity is a value belonging to all human beings, that evaluates self within the values and norms of an external world is the one used. In defining it thus, it also follows that dignity can be violated 
or reduced and promoted or enhanced in interactions or comparisons with others. For the respondents of this research, dignity was most evident in the interface between individuals and social perceptions, that is, the space around how people perceived self and how they believed others perceived them.

'Maintaining identity (manifesting as identity)' (6.2.3) is indicative of the way in which the dignity sub theme began as vanity. Terms and concepts of vanity occurred often, however, I felt that vanity did not do the behaviour justice. Vanity is the result of pride and conceit, whereas the behaviours described and displayed within this context relate to participants needing to maintain image and self-worth by denying stigmatising characteristics. Whether the self-evaluation involved aspects of age or disability (or both), the term vanity does not align to people attempting to maintain social norms. Such behaviours align to dignity, which was apparent in participants actions and interviews to varying degrees and in different ways. The behaviour of making up for any sense of maintaining dignity extended to 'compensating by demonstrating ability' (6.2.3.1) whereby the actions are not expressed only through maintaining social norms, but overtly voicing and enacting abilities that avoid being helped. 'Clothing and style' (6.2.2) was a way dignity materialised, with expression of self through appearance being an important way to enact identity For some, dignity manifested through 'underuse of mobility aid' (6.2.1), need to demonstrate continued independence by either refusing to use mobility aids or failing to use them for their intended use with an added focus on relationships for one participant who used 'leaning on others' (6.2.1.1) as a mobility mechanism rather than using a mobility aid.

\subsubsection{Under use of mobility aid}

Bret took his walking stick on the walking interview, but when asked if he was going to use it to walk with, his reply evaded admitting he needed his stick to aid walking saying, "It's handy for giving him [Bret's dog] tap if he doesn't behave". Bret did concede in the next utterance that "I find that especially walking down the field, that if I haven't got the stick my back starts to ache." Admitting to relying on his stick for some of his daily routine was not something Bret did readily. In fact, the next exchange illustrated Bret's reticence to admit his need for a walking stick for mobility purposes

Interviewer: I was just going to say, what differences does [your stick] make 
to where you go in [town]?

Bret: $\quad$ Not a lot.

When Bret was asked later during the walk if his leg hurt, as was usual for Bret, he responded light heartedly:

RES: $\quad$ Aye. It's hurting. It's getting stiff.

INT Do you just ignore it?

RES Aye. If it falls off, I'll let you know.

I had asked if his leg was painful because our pace had slowed, and his gait had become visibly different. The sense of retaining dignity in Bret's response by minimising any reduced capacity was strengthened when coupled with his daughter's comments. Knowing Bret is in pain but does not use his stick as much as he should, Jenny conceded some recent progress

at least he's using it now. He had a couple of falls and his knee got so bad that he felt he had to. But he doesn't use it as much as he should, but he does tend to take it when he's walking to dog now... it helps to balance him out a bit better. But he should use it more to be honest....

The dignity in Bret's approach to his mobility aid is also underpinned by omitting to tell Jenny when he has fallen, which she found out about a few days later when somebody else told her. These comments could be classed as a need for independence, but there is something more. A need to not make a fuss, not ask for help, not interrupt Jenny's life, and perhaps most importantly not to admit that he cannot do something he used to find easy, to avoid admitting reduced capability. Such reticence to admit a reduction in ability or capacity is often referred to as vanity, yet does not align to conceit or pride. As consistently highlighted throughout the study the behaviour that even participants themselves might call vanity was in fact about retaining dignity.

At 58, Aileen was one of the youngest participants, but her failure to use mobility aids resonated with Bret's underuse of his walking stick. During the pre-walk interview in a café, and then walking around a shopping area with her daughter Lauren, Aileen was very open and honest about being unwilling to use mobility aids because of the impact they have on her identity, and more specifically her dignity. One Aileen's first comments about mobility 
aid use was " ...I would rather people think I'm pissed than going blind and crippled with arthritis, I'd rather them think I'm drunk ... God I don't want people to pity me". The stark and revealing statement highlighted the tangible concern of being visibly different Aileen, with a pointed uneasiness about pity. The extreme descriptors she uses for her feelings about mobility aid use illuminate a dread of standing out as being different. Aileen's dignity is severely dented by her difference, her reduced sense of self-worth and a sense of stigma which was discussed later in the interview

\section{R...So there is this stigma there's this embarrassment about}

L...Oh yeah

\section{A...Because I feel embarrassed ( ) I dunno why I feel embarrassed by it and I don't know why I feel embarrassed ...I I I actually do think sometimes it would be nice to sit in a wheelchair being pushed around I do [laughing] because my legs are crucifying me.}

Aileen received what she called 'blind lessons', which basically entail somebody from the Royal National Institute for the Blind (RNIB) taking her around her locale, teaching her how to use the white stick, what tactile paving can be used for and other devices that are available for blind people. The emotions she feels at being recognised when using her white stick are intense:

.... I'm not gonna lie the only time I use it is when I go out on a blind lesson and then I got the horrors... I got the right horrors in case I see anyone I know .... and then I see someone I know, and I feel like it's Harry Potter's wand Oh! Outrageous.

\subsubsection{Leaning on others}

Much later, while resting for the third time during the walking interview because Aileen's legs and back were very painful, I asked Aileen if she felt vanity stopper her using mobility aids. Asked if she felt vanity was more important than independence, she replied both were important, however she conceded that vanity was winning because she refused to use a 
mobility aid and instead constantly had to rely on people, usually her two daughters, to help her navigate the environment:
A... we're saying not vanity but image I totally agree with that ... those people tend to be selfish people ... I know I'm not a selfish person ... but I do feel like a selfish person with the time I take up

I introduced the word vanity, but Aileen, Lauren and myself established that self-image and identity were more appropriate. Dignity is also an appropriate descriptor for Aileen's behaviour and feelings. Relying on her daughters and her friends to help her brings a sense of selfishness which is slightly undignified, but it is preferable to that reduction in dignity brought on by use of mobility aids. The negative attention they bring to her is something she cannot reconcile with her identity, especially as none of her peers use mobility aids. The reduced status makes her 'embarrassed'. Combining the negativity, embarrassment, and feeling like she stands out as different from her peers, Aileen's feelings can be summarised as indignity; her dignity is removed if she has to resort to using mobility aids.

\subsubsection{Clothing and style}

Aileen's appearance obviously mattered to her. She arrived at our meeting wearing smart and fashionable clothes, with a modern hairstyle and make up and made sure she fixed her lipstick before we left for the walking interview. As highlighted within section 6.2.1 'under use of mobility aid', Aileen failed to properly use mobility aids because of the difference they made to her appearance, not because of vanity but because they mark her out as different from her peers. As discussed above, Aileen's appearance is an important aspect of her identity and she feel use of a mobility would impinge upon her ability to enact her identity through her appearance.

Dignity also manifested in Dave's appearance. At one point during out interview on a December day, I mentioned to Dave that I was cold even though I was wearing a coat and long trousers, while he was not cold and was wearing shorts. He responded "why do I wear? I just find it easier. I dunno,... I put a pair of trousers on if I'm going out. but it doesn't look right (laugh)". The notion that long trousers looked stupid was all pervasive, as Dave had even made special arrangements with his Karate Sensei to allow him to shorten one leg of his Gi (karate suit). Dave felt that his appearance and clothing needed to be appropriate for 
the changes to his embodiment. The tailoring of his clothes was almost symbolic of the way he tailored his life to fit his new body. Unlike other participants, Dave wanted to show his disability as it demonstrated a lack of dependence on others. There was a sense that he wanted to continue to be the same Dave even though his body was slightly altered, but other people would not allow it to happen as shown here:

DAVE: it's been quite a shock over the last year and 14 months or so people perceive, friends included yeah anyone

\section{$[\ldots]$}

when I stand, I purposely stand up when I pay and they 'oh' and they change

INT: as soon as you sit in a wheelchair

DAVE: the attitude changes

Dave felt his identity was impacted through changes to his use of mobility aids, and used his ability to visibly show his disability to mitigate any negative implications. In fact, he took pride in displaying his abilities despite being an amputee.

\subsubsection{Maintaining identity (manifesting as vanity)}

As explained at the beginning of the dignity sub-theme (6.2), I have not used the term vanity as in the true sense of the word, nobody displayed vanity despite Will, at 73, affirming that vanity was part of the reason he did not want to use a mobility aid or progress to use of a more substantial aid. Vanity involves conceit, arrogance and self-importance, whereas the behaviours discussed and observed were aligned to maintaining identity. Circumstances of mobility aid use highlighted the work involved in maintaining identity and dignity which thus manifested as vanity.

Will, a retired bus driving instructor, was the only participant to use the word vanity when he volunteered the information during the pre-walk interview:

Another thing about this is that I'm vain and I don't like to be walking along with my stick because I think it makes me-ages me but that's me as a person so ... but vanity is alright 
In terms of mobility aid use, the word vanity it is perhaps not the most apt because rather than displaying any kind of arrogance or inflated sense of self, Will's use of vanity is to enable him to retain his identity and status. The term vanity itself may be considered Will's tool to maintain dignity, in that it suggests a certain status, which is what he is attempting to maintain. The sense of dignity is not only about how he sees himself but how he feels other people perceive him, although he also saw a positive to using his walking stick because "I do find it ... makes it more obvious to people that, you know l've got a problem".

As the above statement shows, Will did accept that using a stick helped him to navigate the environment better, not just because it helped with balance, but also because of the way it indicated his mobility difficulties to others. Image was very important to Will, especially as his hobby was singing at local venues. Living with Parkinson's disease, Will's singing hobby had become a casualty of his changing embodiment, partly because his throat is affected by the medication he takes, but also because "I can't go on the stage doddering ... with a walking stick". Later in the interview, stigma was brought into the conversation, but Will established that he did not feel stigmatised, "I don't think the stigma side of things it's vanity with me 'oh doddery old bugger coming toward us now".

Will was unabashed in labelling himself as vain mentioning either vain or vanity five times in his pre-walk interview. As previously mentioned, the term vanity has been absorbed by Will almost in a further act of denial that his illness and ageing issues are as confounding to his mobility and lifestyle as they were from (my) observations. In referring to himself as vain, Will was able to make light of the situation and propose a defect for people to see that would disguise those more damaging to his dignity, i.e., his mobility difficulties. Will's walking interview involved another sit-down interview in the coffee-shop of a hardware store and then approximately 50 minutes of walking around the store. I watched him park his car and get out, which was very difficult for him, as was walking around the store. At no point did he ever concede this fact though. I mentioned this at one point:

$$
\text { INT ... you don't really pay much attention to your walking stick ... }
$$

However, you do need it to lean on, I've watched you and you do lean on it and you need it for reaching for bottom shelves and you do stop sometimes and 
WILL: If I dropped it here now it's er I know it's strange but ... It's strange but if I don't use it I have a job to get back up, but I gotta get back up but the pain in my knees but I think I gotta keep playing

\section{INT: Yeah () you'd rather do this than use your mobility scooter}

\section{WILL: Oh yeah, I'd rather do this yeah}

The above exchange demonstrates that Will would rather tolerate some pain than rely on mobility aids more than necessary. He was unwilling to use a more substantial mobility aid, preferring to continue using his walking stick. Will did not walk for a very long time, and when agreeing to take part, seemed to want to walk for the sake of the process, or to impress, but did not outline any usual walking routine. Neither did he deny any walking routine or admit difficulty, all of which also point to an unwillingness to admit a reduced ability, yet this was not any part of the conversation.

Will and Aileen both tolerated pain for the sake of their appearance and identity and both admitted to feeling envious or conscious of the embodied abilities of other people who are of similar age or older than them. Both consider use of a mobility aid to bring pity or decreased worth to their status within the social strata suggesting a reduction in dignity with mobility aid use. While Will was happy to classify himself as vain, Aileen conferred that she would rather people think she was 'pissed' than disabled. Both, then, endure pain and would rather take on other negatively weighted traits or descriptors to avoid being classed as disabled or old. These actions suggest a valuable inner self discordant with societal views or structures that have been absorbed, aligning with the definition of dignity outlined at the start of this sub-theme.

\subsubsection{Compensating by demonstrating abilities}

Dave bucked any dignity trends as the youngest participant, with dignity evident in his adamant resistance to help. While Dave avoided asking for help as discussed in section 6.1.1, there was further depth to his determination to do things for himself and remain independent. The first indicator of Dave's concern came in the first few minutes of our meeting when he had bought a coffee from a snack shop, then was called in for his consultation. The nurse carried his coffee into the consultation room for him, to which Dave responded, "I'm quite capable". As an onlooker, and having just seen Dave's friendly nature 
with staff and other patients, it is important to point out that this was a light-hearted comment, but it was still a statement of ability and dignity. Later in the interview, and previously discussed in the independence section, Dave evidenced several times that he did not want to be helped, and he certainly did not want to be pushed. Dignity, autonomy, and independence are intertwined because of the dignity involved in being able to make decisions for self and act upon them. There is also a sense of inferiority in being unable to do things for oneself. I am not sure that Dave could articulate this, but his dignity seemed to emanate from the fact that he could be self-sufficient. It was about independence, but it was more than that, it was a need to be dignified in his activities and being helped or pushed disallowed that. As discussed in the independence sub-theme, Dave also finds the continuous offers of help to be a little demeaning, evidenced by the sarcastic thoughts he describes at offers of help saying, " how do you think I got out, pushed myself round Asda's [...] do I look like I'm starving?".

Dave wanted to continue with his life as he had before the amputation before he required use of mobility aids. His dignity was dented at any suggestion that he was dependent, or more dependent on others than other people. Dave also felt it would look stupid to pretend he had a leg where he did not, so he tailored his clothes to reflect the changes. His dignity did not diminish because he had lost a leg, only if that loss affected his life in a way he felt he should not allow it to. Dave reflected that he was probably at one of the better stages in his life since the illness which resulted in amputation:

my Mum said to me you're doing a lot more than you ever did before

$$
[\ldots]
$$

my life seems better [...] yeah that's basically what it is, I'm doing things now that I would have never ever have done before or even think of doing [...] and get my independence back which pushed me to do things I would never do before

Dignity for Dave was expressed and felt in his ability to live and express himself as he saw fit. In some way, his disability and mobility aid use allowed him to enhance and promote his dignity by being authentic to himself with his appearance, being independent and self- 
sufficient by not allowing anyone to help him and transcending the difficulties of illness and other people's perception of him (see Chapter 8 for discussion of Jacobson's taxonomy of dignity). Dave almost appeared to need to prove to himself that he was still the same person, or even a better person than he had been before becoming physically impaired and requiring the use of a mobility aid.

\subsection{The contingent nature of dignity means it can be lost or gained through} social interaction. Dignity can be violated, especially for those in a position of inequality or vulnerability (Edlund et al., 2013; Jacobson, 2007, 2009). A reduction in dignity can occur because of a low or reduced social status, brought on by mobility difficulties, age or mobility aids all of which can ultimately lead to stigma, being socially unacceptable because of a certain characteristic. Use of a mobility aid sends signals that one is either old or disabled (or both), giving a clear indication of difference or reduced ability. The impact of perceived or actual judgements of others results in negative consequences to dignity, whereas the visible affectations or physical consequences of ageing and senescence are likely to result in the stigma, a reduced status according to social norms and expectations. Stigma originates from social values, whereas dignity emanates from identity and self-worth compared to those values. It is the phenomena of stigma to which we turn next. Stigma

Stigma is a well-known term describing the disgrace of being unusual, different or of low social status (Goffman, 1963/1990). It has been highlighted in the section above that 'stigma and dignity' (6.3.1) are closely linked. Mobility aids seem to encompass both the disgrace and the (almost) bodily evidence of being unacceptably different. The use of mobility aids is stigmatising because it is a visual sign in and of itself, but it also signifies some other potentially stigmatising categorisation such as being an older person or living with disability. An aspect of stigma that Goffman (1963/1990) explores in his influential book 'Stigma; Notes on the management of spoiled identity', is the role symbols play in controlling 
identity. In opposition to status or prestige symbols, the participants in this study highlighted the notion of 'mobility aids as symbols of stigma' (6.3.2). Some people were reticent to use their mobility aids because of a message sent to other people. The terms vanity, image and intelligence were used when talking about presentation of self, indicating a sense of stigma and dignity involved in mobility aid use.

\subsubsection{Stigma and dignity}

Theresa's use of her walking stick and discussion of lending a stick to her neighbour illustrate the ways in which stigma is entwined with independence and dignity. The interactions between these states and aligning opinions expressed by Theresa demonstrate the complex negotiation of dignity and how stigma can be navigated. Theresa was happy to use her walking stick, despite her fierce independence, or maybe because of it. She did not demonstrate any concerns about stigma, in fact a segment of our conversation turned to a neighbour to whom Theresa had loaned one of her walking sticks

I gave her my stick that I had here and she used it for a couple of day ....and then I said where's the stick "Oh I don't want people to see me"...she falls regularly...I mean what does it mean, I mean people see me walking with a stick it doesn't make any difference does it.

For Theresa, using a stick held no kind of stigma, and it seemed her sense of dignity and independence were entwined with the use of her stick. As Bos et al (2013) highlight stigma involves sense of reduced self-worth, therefore factors that are important to an individual's selfworth will be highly important in the perception of stigma. Independence was important to Theresa's sense of self-worth, and therefore fed into her dignity. Perhaps because of the importance of independence to Theresa's dignity, any sense of stigma that may have arisen was over-ridden.

Deidre's outline of an event whilst using a wheelchair also captures the connection and overlap of stigma with dignity. Deidre was 81 at the time of the interview and I would describe her as a robust lady. A retired nurse, Deidre had campaigned for local services, was an active member of local groups and activities and a confident individual. Even with this sense of confidence, Deidre recounted an anecdote about her own wheelchair use which illustrates how stigma is created by the way other people behave. Having undergone several 
operations, Deidre used a wheelchair for a while, and was being pushed around her locale by her husband. On one occasion she felt "so humiliated ... there were two men by a garden gate, talking, and one said to the other, hmm, she doesn't fancy walking today ...It made me feel really, really small".

Deidre's dignity was impacted by the comments, she felt her dignity and her worth were violated by his comments, but she had also been stigmatised because of her mobility aid use. Her sense of inner worth had been dented, but her status and value externally, to the men commenting, was reduced because of her mobility aid use. The mobility aids Deidre used most were a walking stick, and crutches following another different operation. Diedre also reported being reluctant to use a walking stick when she first needed it, but her physical need and practicality overtook her sense of image: -

RES Years ago,... I thought, I'm not using a walking stick...and then after my knee, I did use a walking stick and it was so much better.

INT What do you think was the crossover point ...

RES I think it's because of the mobility. I could really get going at quite a rate with a stick, where before, I was a bit shuffly ... it gave me a new lease of life really.

Will was also concerned with his physical appearance or how people saw him as part of the self -worth appraisal of dignity. During our discussion, Will described himself as vain and, as highlighted in the Dignity sub-theme, suggested "I can't go on the stage doddering ... with a walking stick" adding "I don't think it's the stigma side of things, it's vanity with me 'oh doddery old bugger coming toward us now'". These comments are interesting in that they indicate concern of being judged as old, relating to the stigma of age rather than that of disability or illness. It is not an absolute rule that performers must walk on stage, he could be on stage at the start of the performance for example, so I am not sure that the walking stick was the only reason he no longer performed. Will also has difficulty swallowing caused by complications of his illness and medications.

While Will's comments suggest using a walking stick inhibits performances, it is not the only reason he does not perform. Coupled with the statement that he feels it is not stigma, it appears that Will does not consider age to be stigmatised. In addition, Will could be called 
vain by his peers, or has been vain all his life. This small snapshot of his life does not establish whether his is vain or not, but it does establish his dislike of being seen as old and mobility aid use increases the visibility of his age. It is not surprising that Will does not think of ageing having a stigma attached because of how socially accepted ageism is (Nelson, 2005). The lack of attention paid in society to the stigma of ageing will be addressed further in Chapter 8.

\subsubsection{Mobility aids as symbols of stigma}

Aileen, perhaps more than any other participant, exemplified the effects of stigma. Refusing to use her mobility aids, Aileen would rather feel extensive pain and end up in tears. Aileen succinctly describes the way a mobility aid singles her out as different from her peers "I' $m$ different than all my friends ... cos none of my friends are like it". Aileen Lauren and I continued this theme a little later in the interview:

A: $\quad$ if the majority of people or they all used them we'd feel perfect, we'd think oh well thank goodness.

L: $\quad$ It's the minority then.

A: $\quad$ How many people do you see walking round town with a white stick L: $\quad$ Not many

A: $\quad$ You see one or two, not many. And then when I( ) do it, when I've got that blooming stick I get so Ooogh ( ) annoyed I think the word is I know it sounds silly when I was out on a blind a lesson a couple of months back I happened () to be walking up here () and I saw a chap looking at me from right close up and I thought oh my god he was one my ex boyfriends... and he was looking at me like Ahhh like a I had the white stick ohhh I wanted to curl up and die. I know it sounds silly

L: $\quad$ It doesn't

A: I was embarrassed I was absolutely...

R: [It doesn't sound silly]

A: $\quad$ Mortified, I thought oh no! 
The quote above demonstrates Aileen's acute embarrassment at encountering an exboyfriend whilst using a mobility aid. She suggests he must have been happy he did not end up with her, a consideration that will also be discussed in the sub-theme of Burden in Chapter 7. The stigmatising characteristic of disability makes her feel different from her peers, reduces her self-worth and makes her feel embarrassed. As with in the dignity subtheme, stigma is closely related to an individual's management of identity, and for Aileen, mobility aid use makes her feel conspicuous, and makes visible her sense of vulnerability and perhaps inferiority:

... it's () visible like with me...this ruddy stick ... I'm not gonna lie the only time I use it is when I go out on a blind lesson and then ... I got the right horrors in case I see anyone I know ... and then I see someone I know, and I feel like it's Harry Potter's wand Oh! Outrageous.

These statements shone a bright spotlight on how visibly and tangibly different Aileen feels. Aileen's identity had been further impacted by some encounters she has had while being instructed on the use of her mobility aid. Whilst attending a 'blind lesson' she encountered an acquaintance who did not know about Aileen's visual impairment. The acquaintance made a comment which prompted her instructor from the RNIB to respond

... she said something like erm what do you use [that for] check if there's puddles of water or something is it? And Lyn turned round, and she said this is a mobility aid ... because Aileen is losing her eyesight and ... let me tell you ... just be thankful it's not you

The response from the RNIB worker is completely understandable feeling a sense of responsibility and even protection for Aileen from what she perhaps felt was a thoughtless comment. Consideration of this response, though, highlights a narrative of pity being instigated by the charity worker. To suggest one must be thankful for not having to use a mobility aid infers that using a mobility aid is something to be unhappy or disaffected by. Such a message could subconsciously reinforce Aileen's feelings of inferiority. Within Aileen's account there are elements of self-perpetuated stigma or low self-esteem which have been exacerbated by the responses other people have, or indeed her perception of the response other people have of her mobility aid use. 
Aileen's complete interview was punctuated with accounts of how embarrassing she finds being physically impaired, and how she refused to bring further attention to herself with a mobility aid. Conversely, but also in juxtaposition with this 'horror' of bringing attention to herself by using mobility aids, Aileen feels guilty about relying on family and friends, or on the fact that she cannot function in the way they do, so for her to be included in social activities they must alter their plans.

Phil had already stated that he felt a walking stick is not as disruptive to daily routines as a wheelchair, especially a powered wheelchair, as a walking stick is easily portable, whereas a powered wheelchair is difficult to transport, and very noticeable. He felt that this large visual apparatus for mobility acts as a suggestion of more than physical impairment " ... many people seem to deem ... that a wheelchair is akin to some sort of mental illness, that you are mentally slow". Further to this, Phil felt that wheelchair use singles people out as being less than human which leads to more than just being different. Phil determined that wheelchair not only defines people as being dysfunctional, but also the continual requirement to justify themselves:

that you wheel rather than walk, that in fact ...you're just not a tenable human being, you just don't function you don't work ...if the average person had to walk round and explain to everybody they met that actually ... I may be wearing this pointed hat but I'm actually a normal person ... because the reality of life is that the ordinary person would take the pointed hat off, but I can't take my wheelchair off.

From this, a sense of attitude to one's mobility status and therefore any use of mobility aid is linked to identity and social status, and a relationship with the mobility aid itself as integral to self-esteem. I would characterise Phil as a robust personality, but the constant influx of negativity and being defined by a mobility aid rather than his actual identity is a daily reality for him. Phil confirmed more than once that wheelchair use completely changed his life. For both Aileen and Phil, mobility aids stood as clear symbols of stigma. 


\subsection{Conclusion}

This chapter has identified the ways in which mobility aid use impacts upon the identity of older people who use mobility aids. Mobility aid use by older people impacted on identity through concerns of independence, dignity and stigma. Each of these sub-themes highlights the additional costs incurred by mobility aid users in terms of the impact mobility aid use has on identity. As a factor of independence, 'asking for help' (6.1.1) in any way interrupted autonomy and reduced independence which changed perceived status and roles of the individual. This leaves the individual with a choice of either making additional effort to complete every-day routines or tasks, or making additional effort to maintain identity. This is also true of navigating outside environment, whereby 'knowing place' (6.1.2.) is important. Regina's experience highlighted that moving to an unknown place can remove some of the independence that comes with intimately knowing one's surroundings, especially when reliant on a mobility aid. Not only does a new place reduce confidence in navigating the environment, which adds to the additional identity work mobility aid users have been shown here to invest, it also negatively influences 'self-reliance as an indicator of independence' (6.1.3),

Dignity is closely related to self-worth, which manifests through independence as highlighted above, but also through factors related to each individual's evaluation of selfworth and factors important to their identity, which were different for each individual. For some, 'under use of mobility aid'(6.2.1), which led one participant to lean on others (6.2.1.1) (quite literally on occasion), helped to maintained dignity by allowing them to remain visually as similar as possible to their cohorts. 'Clothing and style' (6.2.2) were also important to some to enable enactment of identity in a way, not necessarily as they always have done, but in a way they can control. This allowed participants to portray themselves as they wished to be seen. In some ways, this could also lead to 'Maintaining identity manifested as vanity' (6.2.3), however vanity is too strong a word as in has a connotation of arrogance. Older mobility aid users in this research demonstrated behaviours that showed they were compensating for costs to their identity incurred by mobility aid use by managing their identity in visual ways. This included 'compensating by demonstrating abilities' (6.2.3.1), but was deemed necessary to maintain dignity and avoid stigma. 
Changes of status and independence could lead to reduced dignity and feelings of stigma, or enactment of stigma by others could lead to reduced self-esteem thus impacting dignity. These behaviours incur additional costs in terms of identity and social processes to remain on the same level as those who do not use mobility aids. The complexly interconnected nature of identity, independence, dignity and stigma were displayed within segments of data which sometimes included aspects of all these concepts at the same time. Indeed, the section on stigma and dignity (6.3.1) highlights the complex negotiation between stigma, dignity and self-worth which are all derived from an individual's conception of identity. Similarly, identity is interlinked with relationships in ways that cannot be disentangled. Discussion of self and identity is by definition a way of identifying or comparing oneself with others (Abrams, 1998; Deaux, 2000; Stets \& Burke, 2000; Stets \& Serpe, 2016). With dignity and appearance being so important to identity, it is also apparent that mobility aids act as symbols of stigma (6.3.2) and mobility aids negatively affecting dignity in different ways, therefore use of a mobility aid can incur a cost to identity and social interaction for some people. .

In order for such comparisons and impacts on identity to occur, one must conduct relationships, which are integral to social life and interactions. Indeed, the sub-theme of stigma could have been situated within the theme of relationships. Stigma is not possible without interaction with, perceived or actual judgement by and communication with other people. This was exemplified by use of the term 'Does he take sugar?' by both Aileen and Phil, used to highlight the way in which people, strangers, tend to converse with a mobility aid user's companion rather than they themselves. The phrase relates to a BBC radio 4 magazine programme for disabled listeners, but which also succinctly describes the way in which people tend to assume wheelchair users cannot think or speak for themselves. The term demonstrates that while some stigmatising behaviours stem from within an individual through their identity management, they also emanate from external relationships and social structures which in turn feed into social identities and expectations of individuals and social groups.. Social roles are absorbed by individuals, but also assigned, adjudged and assessed by onlookers, meaning identity and its constituent mechanisms of dignity and independence are heavily impacted by stigma. With these complex interrelationships involved in the management of identity and the implications of stigma arising from mobility 
aid use, older mobility aid users had to invest additional effort to maintain identity and social roles.

The role of relationships is pivotal to all aspects of social participation, and it is specifically the role of relationships in mobility aid use we come to next. 


\section{Chapter 7. Relationships}

The very premise of the mobilities paradigm within sociology is that social life cannot exist without movement. Accessing mobility enables social lives and relies on relationships with people, places and things (Urry, 2007). Relationships are formed with people, places and objects, with varying levels of need and intimacy. Perhaps the fundamental starting place for relationships is the one we have with self, then emanating outwards relationships are formed with family and friends, acquaintances and within short snapshots of time and space, with strangers. In amongst the relationships with people are relationships with places and things that play a part in social life, such as the places people meet, the transport methods we choose or have to utilise. All kinds of relationships are informing and affecting each other within everybody's daily routines. When one uses a mobility aid or lives with mobility difficulties different circumstances emerge that do not necessarily exist within relationships for those not reliant on an aid or someone else for their mobility. Delicate and inextricable issues of identity, dignity, stigma and feeling like a burden on loved ones all come together in spoken and unspoken, conscious and subconscious formulations that are difficult to disentangle, not just as a researcher but for those living and breathing the everyday exchanges whilst living with mobility difficulties, chronic illness, senescence and mobility aid use.

One way or another, all aspects of mobility aid use are regulated by relationships in a complex and multi-layered exchange of information, social status, inclusion or exclusion, social acceptance and self-regard. It would not be possible to examine all of the possible relationships within this analysis, as explained in Chapter 5, so the relationships to be focused upon within this section of analysis are those older mobility aid users have with their family members or carers, and with the wider community. Within these relationships are the concerns carers have for their mobility aid using loved ones. The data illustrated a difficult balance to be achieved between the mobility difficulties experienced by the older person, the concern their families and carers might feel for them, and the resulting ways concern might impede the lifestyle of the mobility aid user. Such situations, or failure to completely understand the frustration or limitations of mobility aid use or mobility difficulties can lead to disquiet or ultimately disagreements and discord. Being reliant on both a mobility aid and potentially another person can affect identity, as examined in 
Chapter 5, but can lead to a mobility aid user feeling like a burden on others, which can also impact on relationships. The feeling of being a burden emerged as a big consideration to people, and it is entwined with the sense of independence and stigma relating to identity. Relationships with place are also explored because of how important place can be to identity. The environment is active in shaping human behaviour with identities developed between humans and environment. Identity and place are therefore significant to each other (Belon, Nieuwendyk, Vallianatos, \& Nykiforuk, 2014; Cheshmehzangi, 2014; Hauge, 2007; Johnstone, 2012; Peace et al., 2006; Sheller, 2008; Spinney, 2008, 2016; Spinney et al., 2015).

The ease or difficulty mobility aid users reported having when navigating place, and how included or excluded they felt, could impact upon how often or if they visited places. Being unable to access a place independently can lead to loss of access to a place of comfort, or a hobby. Such losses in turn have the potential to negatively impact identity as has been discussed in Chapter 5, but also the health and well-being of older people. The 'Healthy Lives, Healthy People' White Paper produced by the UK government (Department of Health, 2010), states that being part of a community and social network and remaining active in later life is important to health and well-being. Physical and social accessibility help to enable older people to enjoy healthy lives and contribute to society (Aguiar, \& Macário 2017; Mackett, 2018; Schwanen \& Zeigler, 2011).

Relationships with self and others are impacted by mobility aid use in many ways as Phil explains:-

I think it [mobility aid use] has a marked effect upon all your relationships... my wife has said to me that it doesn't change me as a person, my illness does not change me as person. ... the reality of life is that whilst you are still fundamentally the same person, it does change your outlooks ... and your outlook of yourself within society. ...it does change your outlooks and it-and you and your outlook of yourself within society, your place in society changes for you yourself because you are now the person in the wheelchair, because people see the wheelchair, they don't see the person they see the wheelchair. 
Phil is very articulate here in explaining how his relationships are informed by the presence of his wheelchair and how the wheelchair establishes the way other people, places and things affect his social status. While Phil feels he is 'fundamentally the same person', it is impossible to enact that identity within his relationships because there is a wheelchair in the way. Moreover, use of a mobility aid creates the potential for reliance on family and friends who may need to facilitate use or transportation of an aid.

\subsection{Carers Concerns}

All relationships are complex and multi-layered, but mobility aid use adds a further consideration. One of the reasons for this is the carer is concerned for the welfare of the mobility aid user or would be mobility aid user in some cases. There were two types of concerns emanating from the carers' interviews and interactions. The first, 'concern versus intrusion' (7.1.1) outlines the balance between being loving and helpful to a loved one, compared to intruding on their autonomy and independence. The second concern appears in the form of 'protectiveness' (7.1.2), where the carer feels like they are intruding into the everyday life of the person and their relationships, and protectiveness, where strategies are used by carers or family members to protect the mobility aid user.

\subsubsection{Concern versus intrusion}

The following excerpt illustrates both concern and the lack of mobility aid use.

And it's like where we live it's like a main road to the top and I can't stand if she says I've been up the park ... I always try and make sure I go with her or at least I can see her to come back across the road cos ... you have to cross a main road and then about 15 minutes it's a lot isn't it?

The quote is from the daughter of a 58-year-old lady who has arthritis in her hands and legs, and is registered blind, although she can see a little. The worry is not only due to the walking but also the safe crossing of a road. At the same time, Lauren feels conscious of her Mum's identity and does not want to force her into doing things she does not want to do. During our walking interview, conducted with both mother and daughter present Lauren commented: 
I don't feel like she would be ready to have a wheelchair yet...I wouldn't want to put her in a situation where I think its prematurely kind of putting her in a position that I know she doesn't want to be in.

The pattern was similar in another parent/daughter relationship. Jenny says of her 84-yearold father Bret, "I worry about him all the time". She does not class herself as his carer because Bret is very capable and self-sufficient. Through much of the interview Bret described how his career had taken him away from home for long periods, and later in his life he assumed the role of carer for his late wife who live with dementia and rheumatoid arthritis. Jenny also testified to his ability to care for himself, although she does sometimes worry that he pushes his ability too far. One aspect that worries Jenny is Bret's insistence on walking his dog without taking his mobile phone. The following interchange indicates how Bret wants to be left to make his own decisions about safety, yet his family are worried about the activities he takes on:

Bret: $\quad$ This is a bit tricky

INT: I hope you've always got your phone with you?

Bret: Yeah. Well, that was the theory. In practice it's er not always so () I mean er Sean and Jenny played hell with me cos I didn't have a phone with me ... They said, where do you go? ...Why haven't you got a phone with you? What's the point? It doesn't work up there. There's no signal.

INT: You need a phone when you're down here though.

Bret: Oh, yes.

INT: Just in case you did fall over. I think you could be here quite a long time before anybody realised.

Bret: What's life if you don't get into a bit of trouble....

Jenny concedes that she must try to support him in the best way she can:

you've got to be really careful with him, you want to do stuff for him and make sure he's all right, but you've got to do it in a way that he doesn't think you're doing it for him. 
The relationship dynamic of adult offspring caring for their parent brings some difficulties for relationships. Concerns about the older person's safety can become entangled with the older person's sense of independence as can be seen with Bret and Jenny, although they seem to manage this well, maybe because neither of them considers Jenny to be Bret's carer in any practical sense.

\subsubsection{Protectiveness}

As exemplified by the 'does he take sugar' comment outlined within the stigma sub-theme in chapter 5 , strangers identifying a person by their mobility aid affect more than just the mobility aid user. In a short conversation about this, Aileen's daughter Lauren and I discussed how angry and defensive we can get of our loved ones when they are not shown the respect we believe they deserve.

Aileen: I mean look at you( ) I mean It's that classic thing of em does he take sugar?"

Res: $\quad$ That's exactly the phrase he uses

Aileen: Ohhh It's that classic thing It's a classic thing they speak to someone not (points to self) I'm like excuse me I'm quite intelligent don't you dare don't you dare. I hate it

Res: $\quad$ That is exactly what he says 'does he take sugar'

Lauren: Definitely

Aileen: $\quad$ I hate it I hate it

Lauren: $\quad$ And I do

Res: I'm trying to- (laughing) we have to be very careful when I am out with [him] because he's actually got a powered wheelchair () and not only does he get cross about it, but as you just said, I get cross about it as well.

Conversation between Aileen, F, 58, Lauren, F 28 (Aileen's daughter) and researcher

Caring for someone, or being mindful of the welfare of someone whose mobility is reduced, but who retains the same needs, wishes, pastimes and cares as pre-mobility difficulties, adds a sense of protectiveness that is not necessarily helpful in many situations because it 
adds to the mobility aid users' sense of being a burden or increases the sense that their lives are being taken over. The resulting tension can lead to disagreements, as can tensions from other situations related to mobility aid use as explored in the next subtheme.

\subsection{Disagreement and Discord}

Disagreement and discord are interesting because they seem to arise from frustration. Frustration at not being able to navigate the environment independently, easily, as one used to. 'Discord within close relationships' (7.2.1) including family units, accounts for much of the material discussed in this section, there can also be discord and disagreement within 'wider social relationships' (7.2.1) which arises from conflicts of interest, inadequate provision for disabled people or failure of service providers or the general public to understand the needs of disabled people and mobility aid users.

\subsubsection{Discord in close relationships}

It is difficult to define the exact nature of the discord because it seems to be latent, just under the surface, but it was present in the interviews conducted in dyads (Aileen and Lauren, Theresa and Ivy, Regina and Gayle, Pam and Bernard). Although the discord was not present to the same degree in each dyad, it was evident that in the same way as mobility aid users can no longer navigate the environment as they used to, they can no longer navigate relationships in the same way either. Discord is a sub-theme that interests me as a carer, because when I listened to a recording of myself and my husband attending a hospital appointment (Phil's walking interview), I found my memories of the tension between my husband and myself had increased the actual tension that had existed. While on the recording I was telling him to be calm and stop getting cross, the recording demonstrated that he was not really getting cross, just being practical. The situation occurred as there are never enough disabled parking bays at the hospital. As Phil uses a fully powered wheelchair, it requires a lift to alight from the vehicle, and many of the regular parking spaces are not suitable for operating the lift. This means I have to stop by the entrance to let him leave the vehicle and then go and find a space. On this occasion, as he was leaving the vehicle, a space next to us became available so Phil sat in space (in the rain) so that I could drive the van around the car park to return at a suitable angle to park. 
P: $\quad$ (sounds of the lift moving) nnnn (more lift sounds) Right you'll have to get out of your- come on

A: $\quad$ I'm doing it

P: let's get on with it babes (more lift sounds) that'll do you that'll do, I've got get on there

A: are you putting your coat on?

P: $\quad$ erm I think er no I don't want to cos I don't need it in there do I and it's just... I'm gonna get wet but it's just a huge problem ...(lift sounds) Am I all right behind there?

A: yeah go on

P: $\quad$ I'm not dropping it on to anyone?

A: $\quad$ no, he's just realised he's moved backwards

The discussion was fretful because the situation involved being fast and efficient at what we were doing, and getting in the way of other people, which we never like to do. However, on listening to the recording, I felt that there was not as much stress in Phil's voice as I had felt there to be at the time.

Regina and Gayle, a mother and daughter dyad, were less obvious in their failure to agree, but differing opinions regarding Regina's ability and activities were evident. During the interview at Regina's home that preceded the walking interview, Regina's daughter Gayle appeared very encouraging and supportive of her mother's continuing ability to remain independent and active, although Regina herself indicated a wish to slow down a bit, At almost 90, Regina felt she had lived a very active life but that ageing bodies were a fact of life saying "we've got to be truthful, if the machine that you've got whatever wears out you accept it". She went on to say

R: $\quad$ But really I don't need to go off anyway I don't do things anymore

G: Nah

A: $\quad$ So how do you feel about that do you feel erm

R: $\quad$ Frustrated sometimes but on $I=$ the older I'm getting the more I'm 
getting docile and I quite willing to accept it I mean they take me down to Carmarthen and things and they'd take me for a trip if I wanted to go but no no as I say I will be on $2^{\text {nd }}$ April I'll be 90 years old so it's time that I behave myself is it and gave in (laughing)

Gayle encourages Regina to attend the local friendship club and luncheon club, however when interviewed away from Gayle, Regina suggests she would be happy to stay at home:-

Int: When you say you wouldn't bother coming out if it wasn't for Gayle telling you to, is that-... because it's too much fuss getting here?

R: $\quad$ Oh no no no that's all right ... you know I don't know I don't want to go I don't want to be bothered erm and I think it... I ran Brownies I ran Guides ...I did things but otherwise I was a loner by, I wasn't too much bothered about going out, I used to like reading a lot, but I can't do it anymore Int: So, you don't think you'd get lonely if you stayed at home on your own?

R: $\quad$ (confirms not with shake of head)

Disagreement and misunderstanding can occur because it is difficult for a family member to understand the physical and psychological impact of embodied changes. A very simple and seemingly insignificant exchange between Aileen and her daughter Lauren identifies the potential for a much deeper sense of misunderstanding.

R: $\quad$ Do you still go to all the places that you used to go

A: $\quad$ No and [I'll

L: $\quad$ Yes you do]

A: $\quad$ Well not really becau- not because of my eyesight because of my arthritis isn't it cos I can't walk like I used

L: $\quad$ Yea you probably don't cover as much ground

A: $\quad$ No, I don't $\cos I \operatorname{can}^{\prime} t$

The meaning within what is said here is probably more recognisable by myself as a carer 
because I have uttered similar words in similar circumstances. The significance of this interchange is an assumption on the part of Lauren that Aileen continues uninterrupted in her abilities, her daily routine and perhaps more importantly in her identity. Lauren considers Aileen to be the same person with the same abilities, doing the same things as she has always done. It is not that she is ignoring the mobility difficulty, more that she still perceives her Mum to be the same person. The fact that Aileen does not cover quite so much ground is insignificant to Lauren. Aileen, however, finds even the smallest reduction in her ability to continue with daily routines a major problem and impacts her identity.

A: $\quad$ Sometimes I gotta go down to Savers for my hair dyes it's the only place I can buy the hair dye ...I'm like an old fart you know I sound old...No they'd put me to sleep if I was horse honest to God there's so much wrong with me....Erm no they would they'd put me to sleep. But erm but actually not before I run the 3.45 at Lingfield today [bangs table and laughs]... But no I'm I've noticed that if I'm here now and I'm thinking oh God I've gotta go to Savers my [back's aching

L: $\quad$ A lot] of the time you send me don't you

A: $\quad$ Yeah, but I physically can't, my legs hurt so much and so does my back and that's not my sight that's the arthritis

The humour of Aileen's comments was accompanied by a comment about how old she must seem, highlighting the impact Aileen's mobility difficulties are having upon her identity. The insignificance placed on this by Lauren is in no way an indictment of her ability to empathise with her mother's situation. Lauren understood how mobility aid use would interrupt the continuation of her mother's identity:

(so I know exactly) what she's saying about having a white stick and even having a walking stick ... I don't want her to have that because I see my mother as still what she was like before ... but I think practically and pragmatically speaking I think yes she should because it would improve her quality of life it would make things easier for her she wouldn't probably have to be so reliant on me 
Within all of this there also seems to be a misunderstanding or miscalculation of her mother's abilities. Lauren suggested her mother could do more but is psychologically limiting herself.

L: $\quad$ Maybe it's because, as well do you think it's a perception that you've got now as well cos this is what you're used to doing in just this little area you're sticking to that now

\section{A: $\quad$ No it's not a perception l'd love to do [it]}

As the quoted section shows, Aileen confirms that it is an embodied reality, not a belief. The conflicting beliefs regarding Aileen's abilities and perceptions of her abilities can lead to differences of opinion. There is a depth of discord that can occur with demands on Lauren's time that may be subconsciously intensified by her belief that Aileen could perhaps push herself more, organise herself better or make less demands of Lauren, as demonstrated later in the walking interview:-

L: $\quad$ Don't be long in here though Mam right

A: I won't be long

$\cdots$

L: $\quad$ I've got my work to do and we've been out for two hours

A: $\quad O i$

L: $\quad$ What do you mean oi I got my writing to do

A: I know that

The dialogue became quite animated towards the end of the exchange and was broken when entering a shop. Aileen had previously acknowledged feeling like a burden on her daughters, exacerbated by the loss of confidence felt when Aileen unsuccessfully attempted to use public transport (discussed within the transport theme). With the reliance she feels on her daughters, there is a sense that Aileen feels she needs to get best use of every moment she can of their time. While Lauren understands her mother's wish not to utilise 
mobility aids for her sense of vanity and identity, other demands on Lauren's time led to a sense that Lauren does not have the time to 'wander' around the shops for too long, only to perform the essential needs and requirements. The underlying sense of burden for Aileen and added responsibility to facilitate Aileen's needs and time consumption for Lauren are evident in these simple and seemingly ordinary exchanges. The stress and sense of responsibility for each other within this situation can create an extra strain on their relationship.

\subsubsection{Wider social relationships}

An element of disagreement and discord also occurs outside of close relationships, with the world at large. The discord in wider social relationships seems to occur more in the under 80 male category. Both Will and Dave described incidents in which they have almost come to blows with people regarding the use of parking bays designated for disabled users. For Will, it concerned a space near his home which he admits was not installed for him, but he felt that he needed to ensure non-disabled people did not abuse the space. On one occasion the person who had parked in the bay gesticulated fists at Will for parking too close. On asking, Will discovered this person was in fact disabled. Dave described the arguments he has had because of people parking inappropriately, either in bays designated for disabled people or across dropped kerbs which means wheelchair users cannot get onto or off pavements. Dave also believes older people cannot or will not challenge incorrect use, so he challenges people for them:-

... I tend to get so it's kind of with elderly people who don't open their mouth and have a go at someone erm so ((laughing)) so my mum won't go in a car with me now cos every time they do it I'll stop and I'll say something but the abuse you get back and it's like yeah and you're disabled aren't you. So, like you generally get out of the car and I go 'disabled enough for you' [showing amputation]...

The statements and actions of Dave in this segment identify an interesting anomaly that whilst visibility of disability can be stigmatising, it can also be useful. As highlighted in Chapter 6, Dave is significantly different to other participants in that he likes to demonstrate his independence by showing that his disability does not 
impair his independence, so the visibility of his amputation is not embarrassing for him. This seems to be about the way Dave has contextualised disability in his life.

\subsection{Feeling like a burden}

The impact mobility aid use had on some participants' sense of identity, through the ways their abilities and roles changed or reduced, led to a feeling of being a burden to their loved ones or carers. The sense of burden emanated often from an inability, or a perceived future inability to remain independent. Feeling like a burden is interlinked with identity, dignity and independence and can be experienced simultaneously or in quick succession, as outlined in chapter 5 . Some of the behaviour observed, and the way relationships were discussed, indicated an additional sense of responsibility felt by the carer, which may also have been sensed by the mobility aid user. This section begins with an overview of the subtheme of feeling like a burden, then continues with two aspects of feeling like a burden. 'Compensating' (7.3.1) is a strategy to compensate carers, loved ones or friends for the extra effort the mobility aid user feels they have to make to enable their mobility. 'Impotence' (7.3.2) outlines the implications of always having to ask someone else to facilitate access or activity, always having to rely on someone else for your needs.

Being or feeling like a burden is not necessarily spoken about overtly or with a sense of great importance. Will, a 73-year-old man who lives with chronic illness and uses a walking

stick suggests that without his wife, he would choose to live in sheltered accommodation so that he would not become a 'burden' on his adult children. He then passed this comment off with a joke, without further elaboration. Dave made a statement about returning to life after the operation during which his leg was amputated which illustrates how independence, relationships and burden are closely intertwined, acknowledging that "my first initial goal to get back driving as that was my independence, not relying on my Mum and Dad, or my daughter". The above statement also signals the way in which relationships are affected by changes to mobility, whether psychologically or practically, and implies issues of burden within Dave's thinking, even if he is not voicing it as that. Dave is indeed very independent and very active in a local wheelchair rugby club. He also remains in employment. 
Jenny told me that her father Bret does not necessarily talk of being a burden but will comment that he does not "want to make a fuss" or "oh, I don't want to be a bother". Neither Will not Bret were forthcoming with explanations of feeling burdensome, but it seemed a sense of burden colours their thoughts. It is interesting that Phil, Dave and Aileen, all of whom are under sixty years old and Will at 73, are more articulate about their fears of being a burden than older participants. It could be that some were like Bret and did not like to make a fuss. Indeed, as we began our walking interview, we encountered a neighbour/friend of Bret's. Once out of earshot, Bret confided “He's a moaner. Can't do a thing for himself". This seemingly off the cuff, gossipy comment underlines the importance Bret places on being able to do things for himself.

Regina also demonstrated this need to be autonomous. Gayle, recounted 89-year-old Regina's nightly routine:

she likes to put curtains on the window ... she's got nets, but she then decided she'd put a little curtain on it every night before she goes to bed so she gets the ladder and steps up to do that.

This happens despite Regina herself suggesting “it [age] does disable a little and you've ...got to accept it in that I am 89 ... what do you expec- I don't think pe- so many people lived as old as that years ago". This sense of burden is linked to Regina's relationship with her daughter, but there is a wider sense of role and identity, with Regina's relationship with self and her embodied abilities and also with her home being part of the relational story. Perhaps this happens more for people over 80 as both Regina and Bret display these contradictions. It could be that they embodied changes as a consequence of ageing rather than ill fortune and therefore both accept that their bodies will age, but have not quite psychologically absorbed the reality that their bodies do not do what they did maybe five, ten or twenty years ago. Rather than feeling like a burden, they just do not remember, or want to admit that they cannot do what they used to. This is not as easy for someone with a chronic illness to do because of the pain or symptomatic consequences. It does seem that feelings of burden seem to come with severity of incapacitation or wheelchair use. The four people who voiced concerns about being a burden either lived with serious chronic illness, Phil, Aileen and Will or had experienced a life changing event, as with Dave an amputee. 
The case could be made that those over 80 may have been more reticent to admit to feeling like a burden, or may feel the need to display their independence because of discourses that stigmatise older people, again indicating an inextricable link between relationships and identity, independence, autonomy and feeling like a burden, with an added impetus of stigma. Retaining health and agency are factors of social status for all of us as we age. Maintaining health proactively produces a positive third age status, whereas dependency on medical intervention indicates entry into dependency and decline, therefore reduced social status (Gilleard \& Higgs, 2010; Higgs, Leontowitsch, Stevenson, \& Jones, 2009). Being active and healthy, which aligns to healthy and successful ageing tropes, may feed into the requirement to continue independent activity without help from others. Accessing social events and the environment are important factors to identity, so feeling like a burden is a complex situation. Not only is the situation associated with decline and dependency, but also choice, agency and autonomy become compromised which further impacts social relationships and relationships with place.

\subsubsection{Compensating}

The notion of feeling like a burden on others was discussed by most of the participants in one way or another. The sense of burden impacts both relationships and identity. As we saw in exchanges in section 7.2, there are additional strains put on relationships because of the demands and responsibility of caring. Compensating for feeling like a burden is a strategy Aileen used to make up for the extra time and effort she felt she cost her daughters. The sense of burden felt by Aileen makes her feel beholden to her family so she tries to make amends in the ways she feels she can, compensating for her loved ones' time with money:

Between you and me I think I'm a hell of a burden on them and I know I am ... I try and give them money every now and again because they like clothes or whatever but in a way because l've given them money it makes me feel better it's about the only thing I can do for them you know.

The situation is a very concerning and difficult one for Aileen. As mentioned in chapter 6 , Aileen described being mortified because an ex-boyfriend had seen her with a white stick, but also felt she should find a new partner to take some of the burden away from her daughters. These expressions of thought demonstrate that Aileen feels she is a burden on 
those around her. Aileen appears to be absorbing her mobility needs as a personal failing that other people are having to make up for. The following exchange is a good example of the difficult, dichotomous, complex, interlinking aspects of mobility difficulties and how the sense of burden affects lives and relationships.

A: $\quad$ it would be better if I met a partner and maybe the responsibility would go onto to him ... it would take a lot of pressure off you two wouldn't it ... I feel absolutely horrendous that sometimes thinking I'm ruining their lives

L: $\quad$ You're not ruining our lives

As we have seen in the discord and disagreement theme, the time-consuming aspect of caring for someone with mobility needs can spill out into discord, stress and misunderstanding. It could be that this feeling of burden is intensified by the heated exchange with Lauren regarding use of Lauren's time. It could be that Aileen is constantly feeling defensive because she is aware of feeling like a burden. The possibilities were not discussed in depth, but the complexity of relationships, identity and feeling like a burden seemed intrinsically linked. What was evident was that the cost of using a mobility was more than Aileen's her identity could bear so it was borne out on her relationships with her daughters, even though it made her feel like an extreme burden.

\subsubsection{Impotence}

Phil also outlined the sense of burden he feels being reliant on someone else ...although somebody else may be perfectly happy for you to be reliant upon them ... you still feel that you are constantly having to interject ... can you just get this for me, or could you get that for me or could you get this or move that or push this or whatever...

Phil acknowledges that his wife/carer (the researcher) may see the situation differently to him, but his discussion gives the sense of feeling like a burden, a nuisance and there is also a sense of impotence in his discussion. During his walking interview Phil encountered an older gentleman who asked about Phil's powered wheelchair. In explaining the situation Phil recounted that the man said, "'I'm slow and you just feel like a burden' and all the usual, all the usual, so he's now getting one of these foldable scooters". For Phil, it seems from this 
statement, that feeling like a burden is usual when having mobility difficulties or using a mobility aid. This is difficult for his relationship with his wife and carer and his sense of identity.

The more requirement you have for a bigger mobility aid, the more your life is removed from you...I think it has a marked effect upon all your relationships ... but the reality of life is that whilst you are still fundamentally the same person, it does change your outlooks and ... your outlook of yourself within society, your place in society changes

The sense of burden for Phil and Aileen is connected to both identity and relationships in ways that are too complex to extract one from the other. That is not surprising considering way in which one's sense of identity impacts on the way we relate to others.

\subsection{Relationships with place}

The places people frequent are an important factor in the way they interact with and participate in society. The relational aspects of mobility aid use are more than interpersonal as space and place help to shape identity and relationships. Places were identified as important to daily routines and activities for different reasons to different people. 'Knowledge of place' (7.4.2) is a factor that informs the ability to navigate spaces independently, allowing those with mobility difficulties and mobility aids to navigate their environment. Knowing a place highlights the interaction of independence with identity, mobility aid use, the built environment, however, even with knowledge, mobility aid use usually involves 'extra effort to access familiar places', which is discussed in the next section.

\subsubsection{Extra effort to access familiar places}

Some people continue with the same patterns and behaviours as they always have done. This does, however, afford extra effort when using a mobility aid. The walking interview with Bret illustrated his relationship with places he has known and used for many years even though his experience has changed slightly because of his body's changing abilities. At the start of the interview Bret offered to take me to the "famous steps where I usually take a nosedive". As the quote indicates, Bret takes the situation lightly, and does not want to feel he is vulnerable or in need of any assistance. Bret has fallen at the 'famous steps' he refers 
to in the quote 3 times, and once in a field where someone found him prone on the ground. The only response Bret has for continuing to use the same routes is that his dog needs the exercise. An interpretation of this could be that Bret would like to continue to use the same places he always has. Bret has reduced the length of his dog walks, but not the places, suggesting the places are important and his attachment to place seems to indicate to Bret his ability to remain his own person. He likes to visit a seaside town he used to spend a lot of time in with his late wife. "Went down for the day. I like going down there, thing is I feel very close to her down there. Cos we spent so much time down there". Bret is only able to manage his trips to reminisce because he drives his own vehicle. His independence means he can take these trips whenever he wants to.

Ron, an 84-year-old retired head teacher who uses walking sticks and a mobility scooter regularly attends rugby matches. Mary his daughter and carer, described the relationship they have with the rugby club that enables Ron to continue to attend weekly matches. Ron was previously the club chairman, so he knows the place and the people well. He stores an old scooter at the ground which a groundsman gets out for him on match days. Attending away games is a more complicated process. Mary commented that she enlists the help of "two men to lift it [the scooter] out of the boot of the car" when she needs to do so. For the purpose of attending away games, some grounds are very accommodating, but some are more difficult to access:

There are some grounds that aren't very accommodating for disabled people like for example he doesn't like to go to Ground A, you've got to park on the street there that's down near S but S itself I think the ground is very accommodating.

Ron's attendance at away games is very much dictated by the accessibility of the host ground and his knowledge of them.

\subsubsection{Knowledge of place}

Knowledge of place is concerned with familiarity that comes with knowing one's surroundings, and how that knowledge informs mobility or immobility. In some cases, knowing a place arms the mobility aid user with information to plan routes and feel safer or more comfortable in their environment, allowing accessibility and feeding into relationships 
with both places and people. Knowing place also facilitates accommodation of the extra effort, an additional amount of mobility work involved in navigating the environment when using a mobility aid.

Regina's account of relocating to a more manageable residence highlighted the importance of knowing place and its role in mobility and accessibility. Being familiar with the surroundings of a town she had lived in for many decades had afforded her the ability to navigate the environment confidently with her white stick, using the mental map and local knowledge she had stored. Living in this new town, Regina has a greatly reduced familiarity with her new surroundings, which has reduced her ability to navigate the locale and thus move around independently. She does not know the layout of the houses and streets, and the main A-road through the middle of town carries two-way traffic, something she had not encountered in the much larger town. Regina was daunted by the speed and direction of the traffic which led to reduced mobility and some loss of independence.

Regina admitted that "If you told me two years ago that l'd be living here l'd say don't be so silly, cos I'm an independent person". Later, Regina reiterated the point, musing that she is not sure why she moved

\section{Well, I never would have thought I would be living in Land Town, because I} was quite independent, I was quite contented, in fact I think now, why the devil did I come here? What happened? I don't know.

While the living space is more manageable for Regina where she now resides, the external environment has become more problematic to her because she does not know it very well, and her visual impairment and mobility aid use are impacted by this. Regina did go out alone, but always avoided the larger roads. The walking interview we undertook involved us walking to the luncheon club which she had walked to alone on previous occasions. The luncheon club was $127 \mathrm{~m}$ from her front door and involved no major roads, in fact the only road to be crossed was the cul-de-sac in which she lives.

People with bigger mobility aids, or who need more support from their aids are also very reliant on their knowledge of a place. Using a mobility aid, particularly when it is a wheeled aid, requires knowledge of dropped kerbs, hills, pavement types and accessibility. Pam 
stated that "knowing your town, that's important... you know ( ) because I know the shops I can get in to, can't get in to “.

In the pre walk interview, Pam and Bernard had discussed how difficult it can be to navigate their town,

because if you go on the pavement past the butchers it looks fine when you start out but by the time you get opposite the hairdressers the pavement's gone like that (uses hands to demonstrate how narrow the pavement is)

During the walking interview itself, we visited the place they described, and once again they commented on the difficulty they because "that pavement there starts wide at the top and by the time you get down here ... you can't get on it, it's not wide enough".

Even with that knowledge, our navigation of the road was punctuated with other difficulties. An articulated lorry attempted to manoeuvre down the very narrow street and negotiate with a motor home at the other end. Pam noted "I'm sure he's come the wrong way". We had to tuck ourselves close to a wall around a corner to avoid being collected by the lorry. As we moved further along the street, Bernard indicated another obstacle that concerned him

here we can get up [onto the pavement] by the butchers () these are what I don't like, these are drains, there's some down by the Swan and they are really deep

Pam and Bernard are happy to live in this town, having moved here because their previous home was a little too difficult for Pam's mobility after she had surgery. They do feel that knowing the town is key to remaining active; "You just have to learn where's the best to go, I think we've got it worked out now I can go to the [arts centre] but I can't get in through the front door".

Knowing the town for Pam and Bernard also means knowing the people and the shops. Building and maintaining relationships with people is the way they carry out their daily lives regardless of mobility aid. It seems that this helps to lubricate their ability to establish accessible ways to manage their needs we shop locally ... and we know everybody in the shops ...if they see me 
coming, they open all the doors (laughs) and they let me tak- bring things home because I can't try things on in the shop.

Overall, Pam and Bernard feel they manage to stay socially active, and their needs are generally met because of their ability to establish relationships with people and understand places.

Conversely, Diedre discussed how difficult visiting unknown places can be when mobility is difficult, and one uses a mobility aid. Diedre found this out during a visit to a tourist attraction with a group from U3A (University of the $3^{\text {rd }}$ Age, an organisation that facilitates learning for those for whom full time work has ceased, usually retirees).

We went in the museum. There was one toilet in the corner for all and everybody. No way could anybody, it was difficult for me, with a walking stick, to get in it and it was low for children as well, ... we went around with the, what are they called, curator and then he took everybody downstairs, really steep stairs, to show some artefacts and I said, oh, is there a lift? No, I'm sorry. ... So, I sat for an hour because I couldn't do the stairs and other people went down, but it was a hazard for them anyway.

Diedre was shocked that she had to sit out part of the tour. She felt the museum should have had some way of getting her down the stairs to conduct the tour with her counterparts. This is the kind of situation that can impact on identity, cause people to be disheartened and lonely, and compound stigma. The ultimate consequence of Diedre being unable to conduct the tour in with the other attendees was that she was singled out as different.

During the same excursion, the group went to another attraction

Then we went along the road, and they hadn't been open that long, ... thinking there'll be toilets there and they hadn't even got toilets there. They hadn't got a ramp, they hadn't got anything and that hasn't been open that many years. Absolutely, nothing for disabled. Just as if disabled didn't happen. It amazed me and that, I suppose, is council run. 
Diedre has campaigned to ensure bus services continue to run and was a nurse before she retired, so in some ways, she is more understanding of both the needs of chronically ill patients, and the machinations of policy and planning. She did not elucidate on how she felt, only how she overcame the situation by "going up the road a bit and across to a hotel, ... there were lovely clean toilets, so we had a coffee there".

Moving on to a hotel to have coffee for the ultimate purpose of using the toilet is an option open only to those who are of a certain economic status. For some members, the excursion itself may have been as much as they could afford and have no extra funds to pay for coffee at other premises. As with the other excerpts in the relationships with place sub-theme, Deidre's excursion experience shows how much place and the facilities available within places and spaces affect the actions and movements, experiences and opportunities people have to engage with space, place and social activities.

\subsection{Conclusion}

Relationships are inextricably linked to identity and place. Places have identities that are shaped by the people who use them, and those people have relationships with and in places. Identity of place is not formed by physical characteristics alone but also socially constructed by the people who use and identify with it, a phenomenon well documented in the disciplines of geography, psychology and sociology (Cheshmehzangi, 2014; Johnstone, 2012). If a certain group of people are excluded by obstacles, they therefore do not get to help shape the identity of that place, and it increasingly forms an identity that excludes them. As demonstrated in the relationship with place sub-theme, exclusion of mobility aid users in space leads to 'extra processes to access familiar places' being encountered by older mobility aid users, who for the reasons of their reduced mobility in the first place, can less afford this extra cost of effort, and sometimes monetary cost to access the same social interactions as their more mobile counterparts. 'Knowledge of place' (7.4.2) assists in planning journeys and manoeuvring around unexpected obstacles, but this it is not always possible to know places in advance, or the alternative cost is to avoid or miss out on visiting unknown places.

The processes involved in planning journeys and knowing places impact on both older mobility aid users and their loved ones/carers as the more assistance is needed, the more 
identity is impacted with the mobility aid user feeling like a burden. To begin with, participants who do not require assistance discuss the notion of burden in a way that suggests they are already guarding against feeling this way. Those who feel like a burden either find ways of 'compensating' (7.3.1) for this sense of negative impact on other people's lives or feel 'growing impotence' (7.3.2) in their ability to function in everyday routines. The ultimate cost is to feel like a burden, or become more and more isolated. Carers become more concerned for their mobility aid using or mobility compromised loved one because of increased difficulties navigating both places and relationships, which can in turn lead to disagreement and discord. The exclusion thus impacts not only mobility aid users but their friends and family who either accompany them to places, or do not go out any more because of the hassle involved(as will be demonstrated in chapter 8) which can lead to discord in close relationships (7.2.1). New, acquaintance and potential relationships are also negatively affected because mobility aid users are not able to navigate the environment in the same way as their non-mobility aid using counterparts, resulting in less outings and lost encounters with people. Moreover, discord and disagreement occur in 'wider social relationships' (7.2.2) because tensions between those with disabilities who bear so many additional costs to afford similar social relationships are either not understood or not seen by people who do not have or do not understand disability. This connects to the extra layer of protectiveness (7.1.2) that can be evident in carers and loved ones who have witnessed the extra costs incurred by mobility aid use. However, there is a balance of 'concern versus intrusion' (7.1.1) that can be brought about within relationships of older people who use mobility aids when the mobility aid user feels their independence or autonomy might be encroached upon.

It is again evident how inextricably linked and intermeshed these the different aspects of life, presented here as themes, are experienced through daily routines. Relationships could be considered the link that connects everything, as the diagram in figure 7-1 illustrates. 


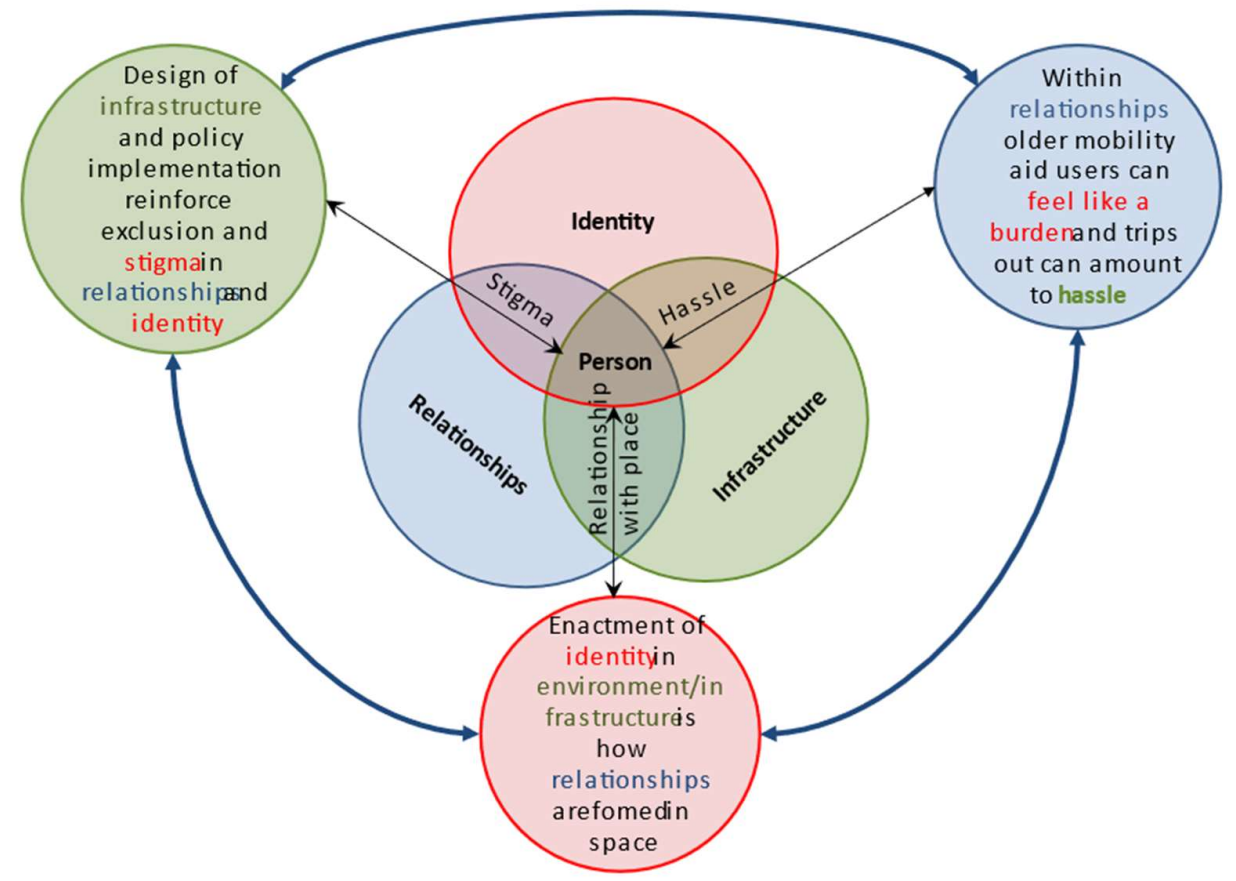

Figure 7.1 Relationships are important to all themes

Relationships are integral to human life, with individuals connecting to others at different levels of interaction for social, psychological and emotional well-being. If interaction is physically, psychologically, emotionally or socially impacted in some way because of different embodiment, or the use of a tool (i.e., a mobility aid) to navigate the environment, it is shown here that relationships can be negatively impacted. The design and planning of spaces, especially the built environment of public spaces, along with the infrastructure of systems that support them are imperative to ensuring all citizens are included and active in their community and environment. Even relatively inconsequential aspects or small inconsistencies can have large impacts. As noted at the start of this chapter, concern about crossing a road, the additional complications when trying to park for a hospital appointment or just having to ask for additional help makes people feel burdensome. As Pam and Bernard pointed out, people with mobility aids and mobility difficulties feel they need to know a place in order to use it, which excludes them from a great deal of places they would or could have utilised before use of aid. Relationship with self, others and place are all affected 
because without the ability to potentially go where one would like to go, a huge amount of personal potential is removed.

Relationships are essential to health and well-being. The inability to participate in normal relationships available to majority of society results in social exclusion and affects the quality of life (e.g., Lucas, 2011). When people cannot move without difficulty, and their mobility is not supported by the infrastructure and systems of the built environment, relationships become interrupted or even broken. Systems such as transport and health systems are necessary and taken for granted in modern day Wales. However, access is not as easy or seamless for mobility aid users as it is for those without mobility difficulties, as illustrated next in chapter 8. 


\section{Chapter 8. Infrastructure and Interlinking Systems}

The mobilities paradigm (e.g., Urry, 2007, Sheller and Urry, 2006) theorises that all human life requires movement. It follows, then, that aspects of policy, planning and the built environment such as housing, transport and mobility, municipal services and healthcare provision must work in conjunction with each other to support social engagement and civic participation. Transport and planning or human geography disciplines might not group infrastructure with customer service for example, but within this theme (and research project as a whole), the important factor is how the environment is experienced, not how it is categorised by planners or academics. Moreover, it is the systems and staff members of the governments, authorities and companies providing services who are the gatekeepers to use of infrastructure and systems. This chapter is set out into sub-themes which capture the nature of the different types and ways infrastructure affects mobility aid use.

Pavements and road were the subject of many participant's and carer's concerns for various reasons as outlined below. The general planning and design of places and spaces such as hospitals, car parks and in particular disabled parking bays, transportation and customer service are the subjects of the planning, design and service provision sub-theme. The final sub-theme is perhaps one of the less obvious but striking nonetheless, in that people who do not use mobility aids or experience mobility difficulties may not have considered the hassle involved in navigating daily routines when the infrastructure is designed without enough consideration of those who are not able bodied.

The congruence and consistency of infrastructure, it's interlinking systems and services is paramount to social participation for people who use mobility aids. Before delving into each of the sub-themes in detail, Dave's recollection of the various requirements, adjustments and adaptations to adjust his life to his changed embodiment adequately illustrates the ways in which infrastructure and the systems and services it supports can impact upon life experience and mobility aid use. One aspect Dave highlighted was the inconsistent approach to adaptations to his home. After helping to fund adaptations to his home, Dave's local authority would not install a dropped kerb outside his drive so that he could get in or out of his home in his adapted vehicle. He described a conversation with a customer service operative at the authority 
I said look I got a problem I said I've got no dropped kerb. And she said oh, this year the end of the budget, we haven't got the funding to do it. She said we've used all the funding, it's about $£ 1,000$ for them to do it. I said well how am I supposed to use a house that you spent $£ 40,000$ how am I going to get to it?

This exchange was the best one to illustrate the significant problems that can be caused by lack of policy salience or consistency. Dave had been awarded a lot of money to adapt his home by his local authority, yet they could not finish the adaptations because the money was spent only on the inside, and the budget for the outside was controlled elsewhere. Planning and design inside his home was not coherent with the infrastructure of pavements and roads outside his home, or the ability to have these adapted. Not only does this situation illustrate the way that infrastructure, systems and services of social and economic networks in a community or country need to be congruent and consistent, it also demonstrates the reasoning behind the construction of the theme and sub-themes in Chapter 7. The hassle Dave incurred when constantly trying get his local authority to install a dropped kerb is indicative of the types of combative interactions, to a greater or lesser degree, people described about their interactions with infrastructure and the systems and services it supports. Dave's account also highlights again the complex interconnectivity of all themes and sub-themes. A telephone conversation with an operative, whether fleeting or over time, incurs some kind of relationship. Dave's changed embodiment also meant a changed relationship with his car and the space outside of his home. Of all participants, Dave was the most positive and energetic, himself admitting that his amputation may have given him a new lease of life, but his identity has been affected by the changes to his body, the way he navigates the world, and the different expectations of him and his abilities. Many others live with chronic illness or senescence that makes facing these difficulties much harder and can result in less social activity, as discussed in Chapter 8.

\subsection{Pavements and Roads}

One of the most common themes within the comments of mobility aid users, and sometimes their carers, was the design, planning and maintenance of pavements and roads, which would fall under the remit of transport and mobility. The two aspects of pavements 
and road that were prominent in discussion were 'overcoming inaccessibility' (8.1.1) in the form of road and pavement layout, and the 'safety concerns and additional physical costs' (8.1.2) that arise from navigating environments that are not easily accessible for some mobility aid users.

\subsubsection{Overcoming inaccessibility}

Dropped kerbs were mentioned by many participants as problematic, either because of a lack of them, or because they are inappropriately sited. Theresa, like Dave, commented on how she had to ask a friend to arrange for the local authority to insert a dropped kerb at the end of the pavement on which her bungalow is situated;-

when I got my bike, a friend of mine ... got one of these pink things (meaning a dropped kerb) there and the other side of the road for me ...But they are very few and far between.

As the photograph in figure 8.2 shows, without the dropped kerb that was installed, Theresa would not have access to the garage that is attached to ownership of her property. The garage is not actually 'attached' to her property (see map at figure 8.1). The red arrow points to Theresa's scooter which is barely visible behind the local authority's road sign and a van parked on the pavement. The scooter is parked next to Theresa's gate and front door. The yellow line delineates the path to Theresa's garage door, just over $58 \mathrm{~m}$ from the entrance to her home. This line represents the walk Theresa takes every morning to collect her mobility scooter to carry out her daily routines. The blistered light-coloured square of paving under the longer black arrow represents the 'pink thing', the dropped kerb Theresa had to arrange installation of, and without which access between her garage and her home would not be possible with her scooter. The rear door of her property is above street level and enclosed by a brick wall with no gate. At the time of this picture, the already narrow became even narrower because of road works in the area. The signs for oncoming traffic took precedence over needs for any users of the pavement. As Theresa had pointed out, there is no other route from that pavement for a mobility aid user so crossing the road to avoid the signs was not an option.

Planning and subsequent building of these two bungalows does not seem to have accounted for older (or even younger) occupants requiring the use of mobility aids or to access the 
built environment. For a mobility aid user such as Theresa, and surely anyone for whom a bungalow is the ideal occupant, there has been an addition cost in effort to have the dropped kerb installed, which may have also had some financial implication to her (we did not discuss that element). Moreover, because of the design of the home and the pavement, just being able to go to the local shops or visit friends comes at a higher physical effort to fetch her scooter every day that would be necessary for a person without mobility difficulties.

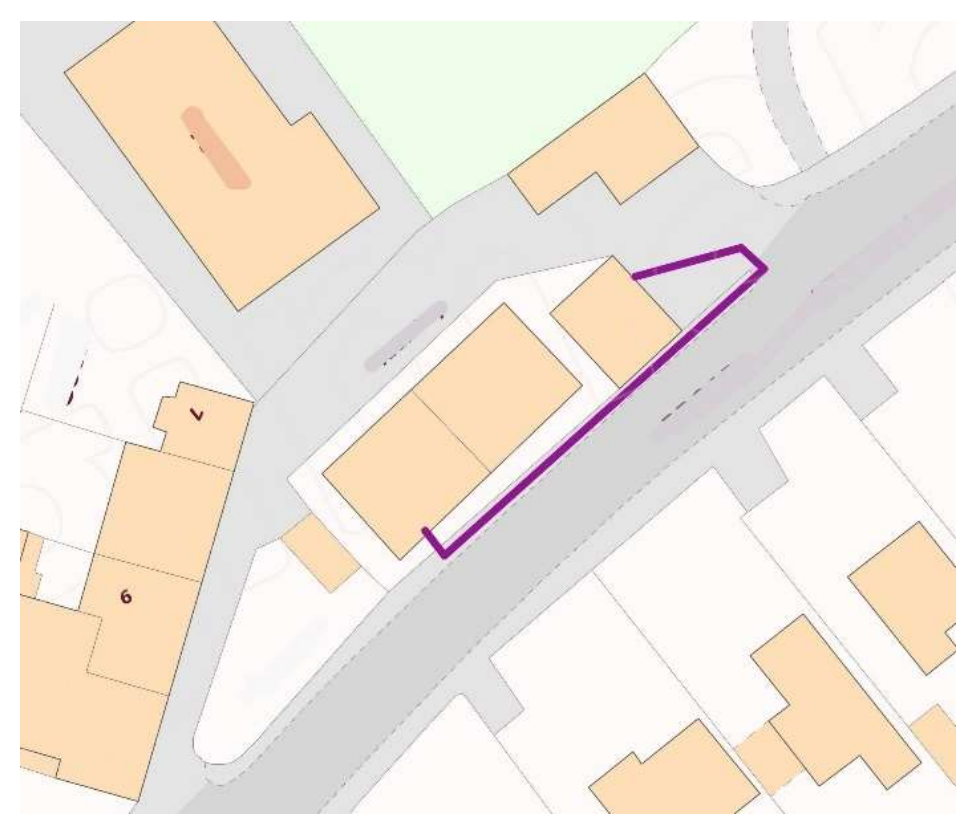

Figure 8.1 Map showing walk from Theresa's front door to garage door

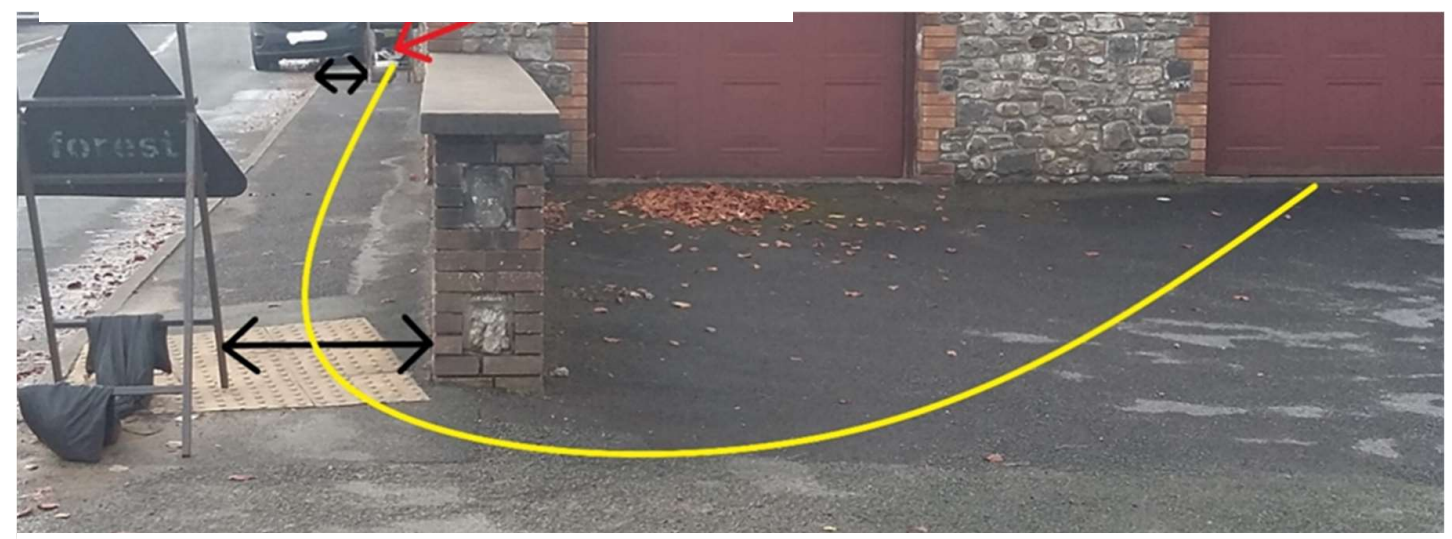

Figure 8.2 Photo of pavement layout outside Theresa's garage door 
Theresa's daily routine of walking from her bungalow to the garage to fetch her mobility scooter and park it outside her home because it cannot be stored in her home highlights links between transport and mobility, housing, social participation and municipal services. The planning and building of the home were originated by a company and sanctioned by a local authority service of some kind. There cannot have been either suitable legislation or regulations ensuring those with mobility aids could access the property easily, or adherence to such regulations. As Theresa points out:-

Because I can't put it... [in the house] ... well, I can bring it in through the gate but... if it gets very wet, the battery gets wet and that's why the brakes get stuck.

There is a confluence of housing, built environment, transport and mobility and social participation all within the experience of Theresa being able (or not) to easily store and access her mobility scooter. At the time of this photograph, signs for road works (indicated by the yellow arrows) had been placed directly on the path taking up a large proportion of the pavement and creating an obstacle for all pedestrians. The pavement is very slim (figure 8.3) particularly those using mobility

Dropped kerbs, such as the one Theresa needed to be installed, were mentioned by other mobility aid users as being problematic in a variety of ways. Phil finds getting around in towns difficult because the placement of dropped kerbs can be random, inconsistent and often inadequate. As Phil pointed out "There's no drop off points, I can't get off the pavement, I can't get on to the pavement you know it just completely impractical." Diedre, a lady who used crutches at the time of the interview following a leg injury and subsequent operation outlined the difficulty she has with dropped kerbs being difficult to navigate.

The drop kerb, there. So, that is quite difficult. Now, why is it difficult? I think it's the differing camber. The camber of the pavement and then the camber of the road It sort of goes a bit to a hump and it's not easy with crutches at all.

The lack of dropped kerbs requires mobility aid users to find different routes and sometimes go quite long distances out of their way to reach their destination. Again, this incurs addition physical effort, a cost borne by the mobility aid user and their carer if applicable, 
and imparts a psychological notion of being unwelcome. The next section outlines more examples of this and how lack of planning for mobility aid users affects their safety in the built environment.

\subsubsection{Safety concerns and additional physical cost}

Pam and Bernard were concerned at the ratio of pavements to roads, along with the size of vehicles and speed at which they pass through the old town in which they live. The measures taken to limit and protect the features and historic landscape are also limiting for pedestrians and mobility aid users.

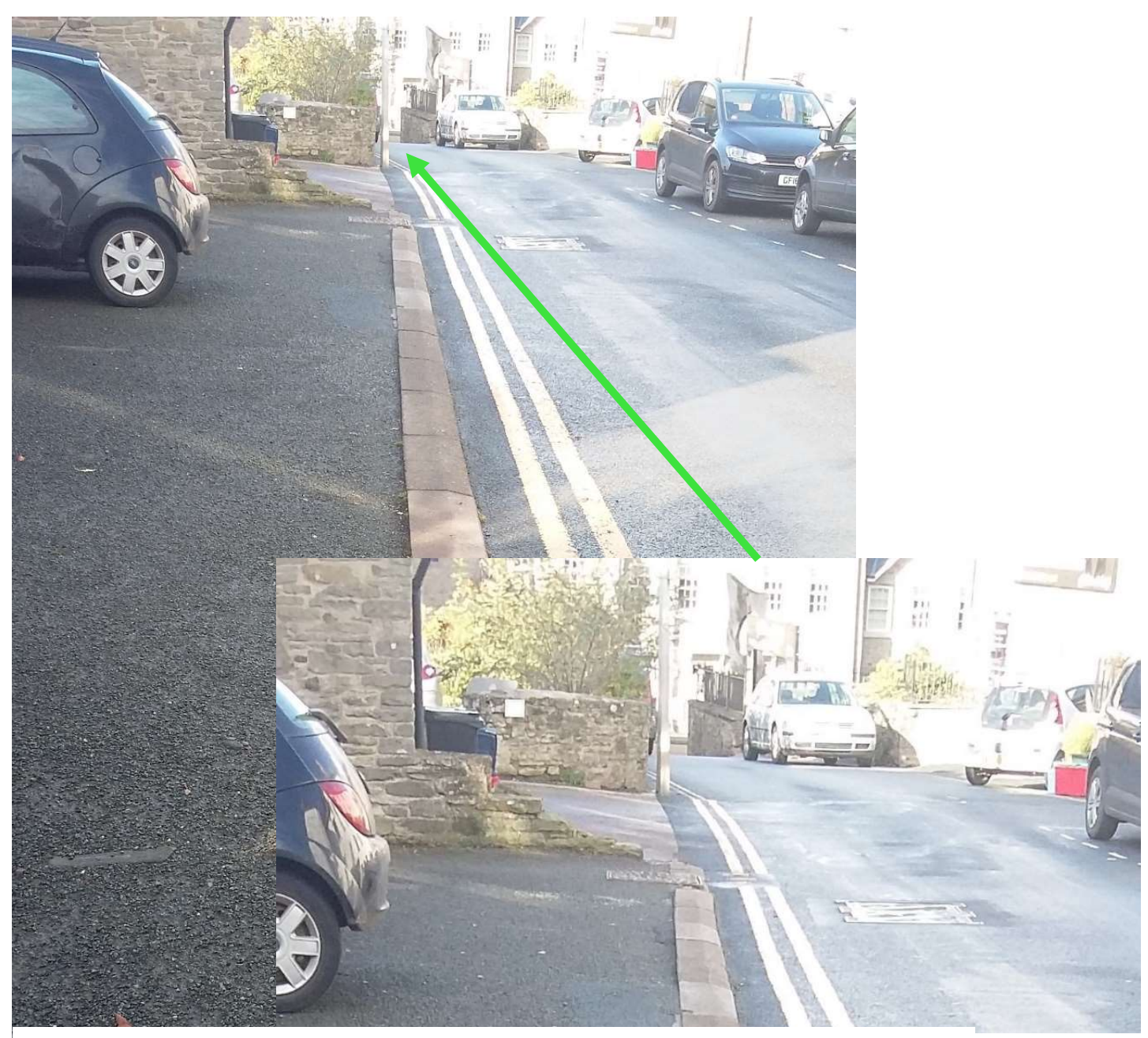

Figure 8.3 Pavement ends 


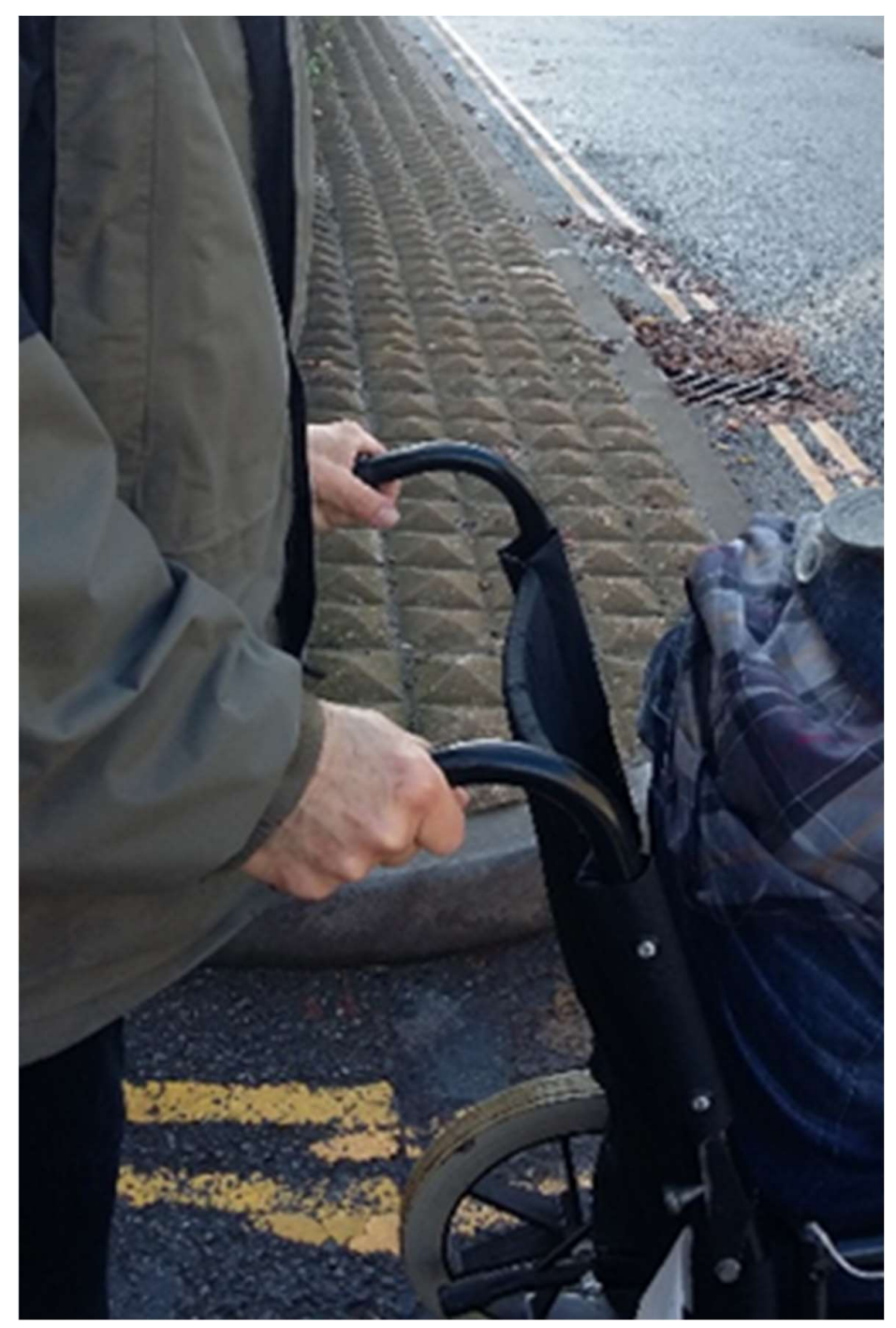

Figure 8.4 Traffic calming measures

As we walked our conversation became stilted because the pavement ended, as seen in figure 8.4. It became difficult to navigate the space and talk with a narrow road, traffic, 2 pedestrians walking unaided and another in a wheelchair - even though I am probably more aware of the space needed for the wheelchair. This is partly because it is not only about manoeuvring the wheelchair but trying to angle each person so that we could all hear each other, which is not easy when one person is lower and in front of the others. Even as someone used to walking alongside a wheelchair, I found myself unable to gauge where to walk and still talk. In fact, it became impossible, and I commented "I keep taking up your space". What is not necessarily visible in this photo is that there is a pavement on the 
opposite side of this road up to the point where the silver car is parked in the distance that could be used, but there is no accessible dropped kerb to get over to use it. We arrived at a corner (pictured at figure 8.4) with no pavement and a kerb beside which there are angled bricks designed to disallow wheels of any kind to get near the wall. This leaves the wheelchair inhabitant and the pusher in a very precarious position trying to cross the road. There is really no alternative to navigate this junction. The green arrow indicates the junction at which Pam and Bernard are pictured in figures 8.4 trying to cross the road The pavement configuration, the traffic calming measures, the lack of dropped kerbs and the priority given to motor vehicles leads to a difficult and somewhat dangerous journey into this part of town. Bernard explains:-

you see years ago this was hardly ever used as a through road, but people have discovered now it's a rat run, and they can miss the high street and come zooming down here, and they don't, there's no speed limits () and no signs.

As the photograph in figure 8.4 illustrates, the traffic measures, presumably to stop people driving or parking on the corners and protect walls, result in Pam and Bernard having to stand in the path of traffic on a reasonably busy road. The alternative route involves a very narrow road with narrow pavements that barely accommodate a wheelchair. The route also includes facing traffic in similar ways to this, parking on pavements and Bernard having to push Pam uphill.

Phil echoed the difficulties of getting around some towns with a substantial mobility device "because the pathways are barely wide enough for a person let alone my wheelchair".

Theresa explained that she rides her scooter through a car park to avoid narrow pavements with obstacles and people sitting outside cafes, in order to reach the local Post office. To get to a café she likes, one that we visited together, it was necessary for Theresa to leave her scooter outside a shop and walk the remaining 46 metres with the aid of a walking stick along a very narrow cracked pathway (figures 8.5 and 8.6). 

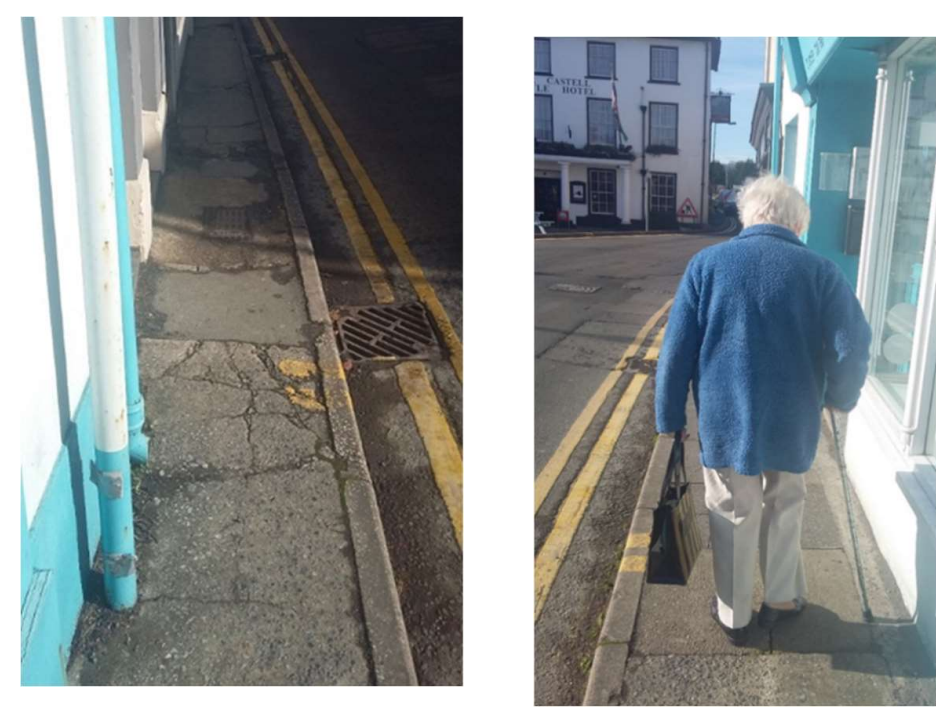

Figure 8.5 Cracked pavement \& Theresa walking on the cracked pavement

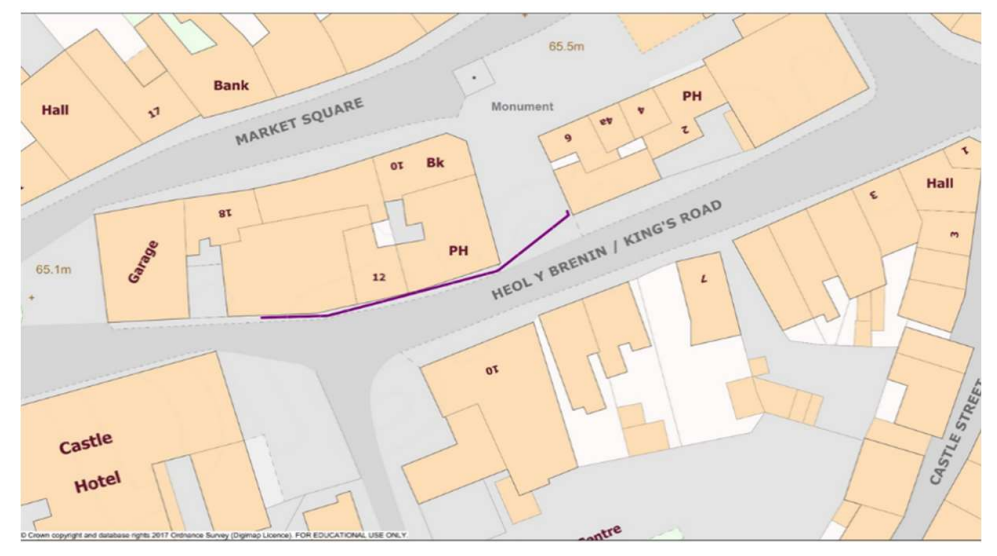

Figure 8.6 Distance walked by Theresa along cracked pavement as shown in photo

The maintenance of pavements was discussed by participants who used other types of mobility aids. Stuart points to the fact that new paving slabs have been put in "but they weren't terribly good because they are not level" and "when they do get loose, they don't do it straight away and very often when they redo it, they leave a hump or a lump or a hole".

Will, a dapper gentleman who lives with Parkinson's disease uses a walking stick but suggests an obstacle to mobility is that:-

pavements are obvious obstacles what isn't obvious is uneven pavement I mean the uneven... flag flagstones ... They can really give you a nasty fall because ... you are not expecting these ... uneven and erm undulating 
pavements.

Eight of the eleven mobility aid users participating in the study mentioned obstacles or difficulty with pavements, and three of six carers voiced concerns about their loved ones' safety on the pavements and roads in their locale. The difficulties and extra precaution involved in remaining safe, including having to take longer routes, walk more slowly, or indeed fall echo the extra processes and costs incurred by mobility aid use outlined in Chapter 7 sections 7.4.1 and 7.4.2. The result for all mobility aid users is that pavements that cause concern, difficulty or even inability to access places indicates how important pavements, roads and planning are to the mobility of all older pedestrians, particularly those with mobility difficulties and those who use mobility aids. Accessing the built environment incurs additional costs for mobility aid users which are currently borne by the mobility aid user themselves, although arguably the cost is ultimately borne by society and the tax payer in costs or care and health costs when mobility aid users physical and psychological well-being is reduced due to social exclusion. As highlighted in Chapter 2, while lack of access to community participation and transport are considered significant factors in social exclusion(Lucas, 2011; Newton et al., 2010; Raymond et al., 2014), there is a failure take a holistic view of mobility in relation to the health and well-being of older people (Aguiar \& Macário 2017)

\subsection{Planning, design and service provision}

Planning and design incorporate the issues discussed in pavements and roads along with many other aspects of infrastructure. Infrastructure refers to the core elements of practical, social, legal, economic and political structure that support a society. Planning and design are influenced by all these factors in producing a built environment and social structure within which we all live. One story demonstrates inconsistent provision between housing and transport whereby older people's housing solutions are not well supported by transport systems, highlighting the 'lack of co-ordinated services' ( 8.2.3) for either older people or mobility aid users. One of the difficulties involves hospital parking, and subsequent accessibility difficulties within the hospital, an institution in which accessibility should be of paramount importance.. Planning is supported by public and private services, from healthcare to transport, legal advice and local authority provision to accessing the local 
shops, all of which are staffed. The potential for 'insensitive staff' (8.2.2) can have a highly negative consequences for older mobility aid users. People do not live in sectioned off lives, but one life in which transport is linked to health, health linked to housing, housing to transport and so on. Participants also described ways in which the planned built environments could make social lives or accessing health care difficult because of some

highly contentious and impractical situations involving parking bays for people with disabilities as discussed in 'inadequate provision of parking and access' (8.2.1). This subtheme uncovers the interlinking nature of different areas of planning, design and service provision, and how they can fail to provide a cohesive accessibility for those who are ageing and use mobility aids.

\subsubsection{Inadequate provision of parking and access}

Inadequate provision of parking and access refers to the amount of parking bays and accessibility provided to public spaces and buildings, including hospitals, shops and on some occasions, homes. Disabled parking bays are a contentious issue, and the following incidents could have been included within either the disagreement and discord or relationship with space sub-themes, indicating the wider sense of relationships running through all aspects of all mobility aid use. The decision to include parking bay incidents in planning and design under the wider heading of Infrastructure and interlinking systems was two-fold. First, disability parking bays are provided as part of the infrastructure of the built environment, and a provision to enable the social, economic, educational and civic participation of people with mobility difficulties. The second reason is that it aligns with the mobilities notion of infrastructure not only being created by people and societies, but also orchestrating their behaviour. Public judgement and criticism have become an aspect of disabled parking and blue badge use (a blue badge being the required document to allow parking in a disabled bay). There are news stories of negative public opinions about blue badge use and often onlookers make judgements about whether a blue badge holder should have been allocated the document. This is exacerbated by a lack of disabled bays in most places, even in rural Wales.

Both Will and Dave described incidents in which they have almost come to blows with people regarding the use of parking bays designated for disabled users. For Will, it 
concerned a space near his home which he admits was not installed for him, but he felt that he needed to ensure non-disabled people did not abuse the space. On one occasion the person who had parked in the bay gesticulated fists at Will for parking too close. On asking, Will discovered this person was in fact disabled. Dave described the arguments he has had because of people parking inappropriately, either in bays designated for disabled people or across dropped kerbs which means wheelchair users cannot get onto or off pavements. Dave also believes older people cannot or will not challenge incorrect use, so he challenges people for them:

I tend to get so it's kind of with elderly people who don't open their mouth and have a go at someone erm so ((laughing)) so my mum won't go in a car with me now cos every time they do it I'll stop and I'll say something but the abuse you get back and it's like yeah and you're disabled aren't you. So, like you generally get out of the car and I go 'disabled enough for you' [showing amputation].

Dave recounted a disabled parking incident which highlights how policy and practice can uphold negative stereotypes rather than support mobility aid users to go about their business as non-mobility aid users are able to. An avid wheelchair rugby player and organiser, Dave was host to a player from Denmark to attend a conference for the sport in Stoke on Trent. In order to allow for both to alight into their wheelchairs from Dave's car, he had to park between bays as the bays only had yellow hatched markings on one side and butted next to another parking space the other side (see picture).

he had to get into his wheelchair and I had to get into mine ... if I parked normally there wouldn't have been enough room for him to get out and me to get out and the guy next to me was a wheelchair user saying oh no fine I'm ok I can use mine, I said ok you're ok, we did it and I got a ticket for it.

The design of the spaces therefore does not allow for two wheelchair users to travel in a car together and park in these bays. This suggests either the local authority concerned does not believe wheelchair users might have other wheelchair users as friends, partners or family members, or they have just not considered the situation at all.

Disabled parking bays were problematic during Phil's walking interview, which took the 
form of an appointment at the hospital. Even as we arrived at the car park entrance an hour before the appointment, because we know how difficult parking can be, we discussed the lack of disabled bays. As we made our way around the enforced circuit of the car park, Phil stated that he knew he would have to alight on his wheelchair lift at the doors while I found a parking space. This caused some stress:

R: $\quad$ are you putting your coat on?

P: $\quad$ erm I think er no I don't want to cos I don't need it in there do I... I'm gonna get wet but it's just a huge problem ... (lift sounds) Am I alright behind there?

R: $\quad$ yeah go on

P: $\quad$ I'm not dropping it on to anyone

R: $\quad$ no, he's just realised he's moved backwards ... (wheelchair sounds) You going to drop me down ... hold on, don't touch it till I'm on. Go on then. Waaah (too soon). Go on then.

Conversation between Phil, M 57 and researcher (also Phil's wife and carer)

We had been confronted with the same situation on many occasions and managed in the same way. One of the difficulties on this site is that it is not flat, but all disabled bays are on flat terrain. Trying to use a wheelchair lift when the vehicle is not level is frightening at best, impossible at worst. On this occasion, as Phil was lowering on the lift, a space became available next to us. The vehicle was situated too far forward for me to manoeuvre it into the space, which meant driving all the way round the one-way system again. Even regular spaces are difficult to find at that hospital, so while I drove around the car park, Phil sat in his wheelchair in the space, getting wet in the rain, until I returned to park the van in it. With an hour to waste, Phil recovered with a take-away cup of coffee from the café in the foyer; his wheelchair does not fit into the café unless the table nearest the entrance is free. Hospitals, like many other infrastructural buildings, have often evolved from older buildings. As we continued further into the building to access the appointment, we encountered a corridor wide enough for his wheelchair, but with items of hospital equipment stored to one side: 
so, they've got a wide corridor, but they just fill it with stuff cos they've got nowhere else to put the stuff, which sort of seems to be somewhat defeating the object. But again, you know like with this hospital it's not planned is it... it's just expanding ... like some nuclear riddled, nuclear riddled baby isn't it? it just keeps growing the next arm ( ), but it's not planned really, it's just jammed on wherever they can find a space.

Even with planning for disability needs, other needs seem to supersede the requirement for mobility needs and human behaviour outweighs plans. Whether it is hospital policy to keep such items in the corridor is difficult to tell, but they make even walking past people coming in the opposite direction difficult. Mobility aid users are more disadvantaged because they need more room to manoeuvre.

\subsubsection{Insensitive Staff}

Staff are the interface of infrastructural systems providing for the needs of communities, the people who staff services, manage and implement policy and thus are the gatekeepers of accessibility. Insensitive staff or those oblivious to the needs or difficulties of mobility aid use can be an aspect of inaccessibility. Although this could have been included within the relationships sub-theme relationship with place, or relationships with strangers or service providers. The situation described above by Phil inspects the consideration or perhaps understanding of the movement patterns and requirements of mobility aid users. This could be a fundamental of the planning, or an issue of lack of storage or belief that the corridor provides enough access with the line of apparatus stored there. Aileen's account of transport service staff demonstrates how much difference staff training and/or consideration can make. Considerate staff members representing service providers can increase or decrease the sense of accessibility of a service. An inaccessible service becomes obstructive rather than enabling, as Aileen's experience of bus use highlights. Aileen reported that she does not use her mobility aids because of the negative attention she perceives they bring to her. The shame and negativity she felt the first time she attempted to use the free bus pass she is afforded because of her visual impairment, disincentivised her to use a bus ever again. Not knowing how a free bus pass worked, Aileen showed her 
card to the driver and then sat down, but the driver did not explain the protocol in a very sympathetic way: $a$

He said "excuse me he said, and I said oh yes he said you don't just march off" he said to me you've gotta show your card to this machine and get a ticket or something I dunno and I didn't know what I was doing, and I thought I'm not using this again cos I felt a right nutter. Stupid isn't it. Now I've got a free bus pass and I won't use it.

Stating that she would not use a bus again, Aileen concluded "how stupid am I...?" The question is not about stupidity, rather the attention brought to Aileen. Even with the lengths taken and pain endured by Aileen to guard her identity, never using mobility aids as she feels they reflect negatively on her persona. The driver's lack of training or lack of time which led to insensitive treatment of Aileen, brought the attention to her disability that she takes great effort to conceal. Aileen has borne a cost in terms of her identity and also because of the time and extra effort she takes to make journeys because she is now unwilling to use public transport.

The customer service Aileen received from the bus driver, or lack thereof, added to her feelings of insecurity and drew the attention to her that she hates. It is possible there are two things happening here, firstly that the bus driver was rude and could have been much more sympathetic to a new bus pass user. Secondly, the stigma that Aileen feels daily could constantly reinforce her sense of humiliation. The event may not have been as dramatic or visible as Aileen felt it to be, but the shame and indignity involved were enough for her to cease using the service. The incident was another form of hassle, an unwanted negative interchange that altered Aileen's experience of her day, the bus service and her ability to navigate the environment. Again, this segment could have been coded in the stigma or dignity sub-themes within identity, or disagreement and discord in the relationships theme, but the sense of hassle is overwhelming. Service provision is the aspect highlighted at this point, but it is a good example of the ways in which all the themes interact and relate to each other. Aileen's identity is impacted by her relationship with the bus driver, which is in turn influenced by bus timetables, bus driver training, bus pass provision and maybe even the information provided with the bus pass. Interlinking systems which interact with and influence relationships with people within those systems and ultimately impact upon 
Aileen's self-esteem and identity. Each aspect has a pre-existing history, but the interaction demonstrates how all the themes in the study affect each other and are difficult to study without the context of the others.

\subsubsection{Lack of co-ordinated services}

Context and lived experience are not necessarily included or well represented in policy and planning, perhaps because they involve two or more services that potentially are planned and governed separately, but in the daily lives and routines of older people, are experienced simultaneously. Gayle and Regina discussed an example of such a situation. After moving about 25 miles to a new town, Regina has considered inviting friends from her previous hometown to visit. However, the nearest bus stop (for any bus) is $825 \mathrm{~m}$ from the entrance to the road Regina lives in . Figure 8.7 indicates the road Regina lives in marked in dark red with a yellow star at the end, the bus stop in marked by the other star, and a dotted line denotes the route. As the road has been constructed with properties purpose built for older people or those with disabilities, it seems to be an oversight that one needs the

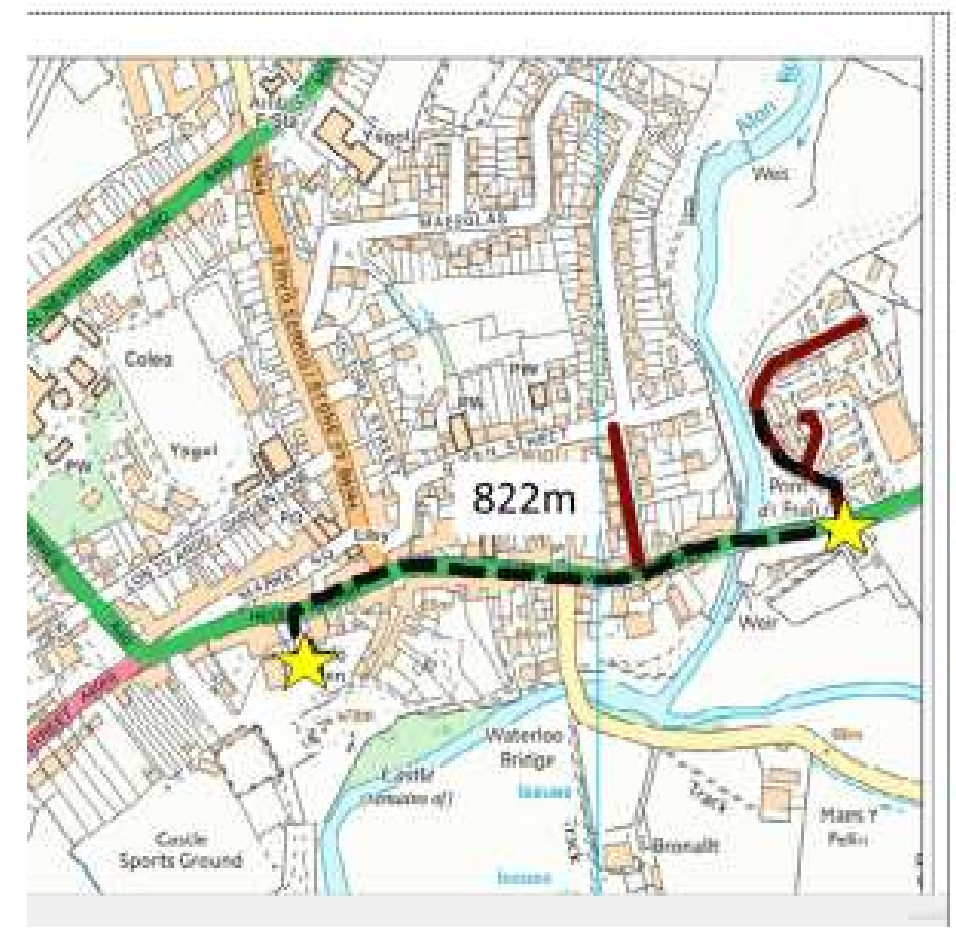

Figure 8.7 The walk from Regina's home to the bus stop

functionality to walk $825 \mathrm{~m}$, drive or get a taxi to the nearest bus stop. Gayle has offered to collect Regina's friends from the bus stop should they chose to visit, but it is not an option 
for everyone. The bus journey from the town in question takes about 2 hours, so an extra consideration at the end of the journey makes travelling more onerous and less possible. It also removes the option for Regina to arrange for friends to visit without involving someone else, Gayle or anyone else. While the various levels of planning and communication required for a simple visit from a neighbouring town could qualify this sequence of events for the hassle sub-theme, it is included here because of the local authority level planning involved in creating such a situation. make routines and daily life difficult to continue or achieve.

A lack of policy cohesion from planning and policy around housing and transport for older people, as one example, may be due to a historical reflection of medical conceptualisations of old age which focus on dependency rather than social considerations. Design of housing for older people often fails to incorporate the social or emotional needs and views of older people themselves. Added to this, social policies of ageing have not traditionally included housing as a key concept of sustained community care, so notions of ageing in place and retaining independence can be at odds with housing policies (Phillips et al., 2010). Infrastructures both construct and constrict daily lives with the support and services provided, as only behaviour supported by systems and structures is possible. Human life is thus arranged and organised by infrastructure and systems (Gaete-Reyes, 2015; Hamraie, 2017; Hodgson, 2011; Merriman et al., 2013; Sheller \& Urry, 2006, 2008; Spinney et al., 2015; Urry, 2007). For mobility aid users, it is the terms of accessibility offered by infrastructures, systems and services that impact upon their daily lives. Consideration of both internal and external environments are necessary to enable social participation. Put simply, older people's housing that has no access to public transport does not offer suitable social or physical accessibility.

The experiences of planning, design and service provision represented here are a consensus of those experiences relayed by all participants. From Phil's inability to park to attend a hospital appointment to Aileen's difficult experience with a bus driver, accessing services or using infrastructure can become much more difficult when using a mobility aid. As Regina's situation demonstrates, the lack of congruence between housing policy and transport provision has completely removed avenues for social activity in her life and forced her to become more dependent, linking back again to the independence sub-theme within the theme of identity. Accessing participation to social, political or economic activity becomes 
more difficult with each obstacle or oversight, aggravating attempts to navigate life and the environment. The word that sums up the catalogue of successive obstacles best is hassle.

\subsection{Hassle}

Obstacles to participation or social inclusion are not always necessarily insurmountable. There are some obstacles which are difficult to overcome such as steps and kerbs which are especially difficult to navigate with wheeled mobility aids. Hassle describes the litany of obstacles that, if faced in isolation would be surmountable, but when these obstacles are faced in succession during each excursion, going out begins to be perceived as an insurmountable and onerous task. Planning and services tend to create hassle through illconceived planning processes or policies which can often be attached to aspects of life and daily routines that might seem inconsequential, but cause mobility aid users' considerable effort to overcome.

The term hassle and sub-theme of hassle is discussed as an overall concept followed by four sub-sections which discuss the types of hassle outlined by participants. Mobility aid users are faced with 'not fitting in' (8.3.1) to spaces, which also translates to not fitting in to the social structure. 'Advanced planning' (8.3.2) is a strategy used to navigate environments, even those that are known well by the mobility aid user. Problems can especially occur when with 'rearranged town furniture' (8.3.3) which interrupts or suspends plans and can prevent the completion of daily tasks. The overarching message is that daily lives and routines are littered with catalogues of surmountable obstacles which can be potentially overcome, but when faced in succession, the 'accumulation of obstacles' (8.3.4) become too much to face. The multi-layered nature of hassle in the built environment and access to mobility results in journey avoidance, potentially leading to social exclusion. The themes that emanated from the discussions in this study were incredibly complex and interconnected and hassle exemplifies this.

Hassle was a term Phil used to explain why he is happier to stay at home than venture out "you just think to yourself you know what, I'm not going to do that because I can't take the hassle". As explained in the introduction, Phil is very intimately related to me. The depth of isolation, exclusion, stigma and disruption to life described within his analogy of how much the hassle of constant obstacles to social participation is profound: 
if I just said to the average person ... every time you leave the house you have to wear a gas mask but you've only got a canister for five minutes and out there somewhere in the locale in the town there are other canisters dotted about ...so to go into town to buy food you have to put your gas mask on and then you have to walk to town and then you have to make sure that you find a canister or you die and its exactly the same with a wheelchair ... because those cannisters are actually pavement drop off points those cannisters are doorways you cannot get in those cannisters are ramps that do not exist, they are all of those things. You cannot function in that town because those things do not exist, so yeah it just absolutely completely changes your life.

Even as Phil's wife, I found this analogy shocking. It gave an insight into the depth of disruption to Phil's identity, independence, his ability to carry out what another person might consider a simple, even mindless task. To relate the lack of accessibility to the ability to live or die gives an insight into the extreme of isolation and exclusion Phil feels at being so compromised by the lack of accessibility in the built environment. He would rather stay at home than face these isses continually and consistently.

\subsubsection{Not fitting in}

An incident occurred during Phil's walking interview at a hospital appointment. The appointment required waiting in a waiting room that was part of a treatment suite. The room contained 10 armchair size chairs. There were no other patients there on our arrival but even with the waiting room empty other than furniture, Phil's wheelchair did not fit easily into the room as it is a large, battery-powered wheelchair (figure 8.8). We decided to sit in the corridor as there was a smaller desk type chair out there and he could park his wheelchair next to it. While I was absent for a few minutes the recording captured this:-

(- nurse comes to check if Phil is alright) I'm alright love I'm fine, I'll just hit it at speed. (Parks himself in the hallway - another member of staff approaches to tell him he can go in the waiting room, it's alright) No I'm happy here thank you (told he should move in case there is an emergency - Phil points to a chair in the hallway) yeah, well I'm no bigger than that am I, and if there is an emergency l'll move. (ok, as long as you are happy). 


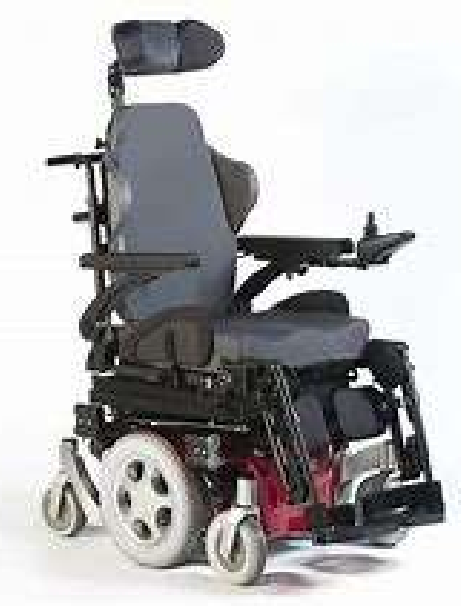

Figure 8.8 Phil's wheelchair

It is interesting that the first member of staff is happy to leave Phil where he is if he is alright, but the second member of staff wants to use fire regulations as an authority mechanism to encourage him to move. There were notes trolleys and other chairs as big as his wheelchair along the hallway. Because of my close relationship with Phil, I know that this is not a one-off occurrence, and I also know that he feels uncomfortable not fitting into a space and hates fuss.

He described his unease with an anecdote about a restaurant visit...

You know you go, you phone a pub up, we went to a public house that was doing a Sunday lunch thing and we said is it wheelchair accessible, yes yes it's wheelchair accessible so we turn up, I turn up in my wheelchair we go in and they plonk me in my wheelchair absolutely in the direct path of the door so that nobody else can get into the damn dining room without me moving I mean it is just absolutely ludicrous, I makes me feel awful, I feel like I'm getting in everybody's way and it shows how much thought these businesses give to their clients which is none at all. I mean it was incredibly stupid.

Phil summed up his thoughts about mobility aid use in the following way if you need a wheelchair you need a wheelchair, walking stick, Zimmer frame whatever it may be, if you need it you need it, but they are they are a deep disadvantage to your life. 
One observation that could be made here is that Phil uses that largest mobility aid within the study. It is a powered wheelchair of some proportion and does take up quite a lot of space both to stand and to manoeuvre, but any wheeled aid would face the same accessibility issues as Phil's wheelchair. These anecdotes do illustrate the connectedness of infrastructure with identity and relationships. Phil does not like to bring attention to himself, but ultimately has to in some way whenever he leaves his home because there will inevitably be an obstacle that he cannot navigate without the involvement of a staff member or other stranger to move something. His identity becomes impacted, as demonstrated by the profound statement he makes about the lack of accessibility he experiences that removes his autonomy. Moreover, whether the intentions are good or not, he ends up having to feel 'special' or different, as in the restaurant story. Relationships are impacted too, because he has to communicate his feelings or needs to staff, and often he is expected to be grateful that there are solutions, even if they are not fit for purpose. The complex interplay of infrastructure, identity and relationships are fully interactive informing Phil's perception of self and sense of being a burden because he is often made to feel like an obstruction.

\subsubsection{Advanced planning}

The town in which Pam and Bernard live is made up of narrow streets on hills with twisting paths and shops with big steps and narrow doorways. As is usual in historic towns, nothing is uniform, which makes for a quaint and pleasant place, but does create accessibility problems. It means that Pam and Bernard need to pre-plan their routes and have prior knowledge of accessibility. Pam was generally positive about her local town and how inclusive the shop staff, local residents and the town council tried to be, however there were occasions that could create hassle causing her and her husband to cease an activity:

Bernard: $\quad$ Well they give up as well because there's one particular shop ... they've got a ramp and they will get it out for you and they eh quite happily get it out for you but it's such a steep one ... it's such a steep one, it's heavy for them to manipulate, I don't know where they've got it from, but anyway we just don't bother anymore, we just don't go there anymore, or if the weathers alright and you can leave the chair outside an walk in its not so bad 
Pam: But I feel so rotten sometimes because people are so pleased when they've got a ramp and then you say, I'm not going on that at that angle, oh dear.

Pam was feeling bad because she negates the positive feelings people display when providing what they believe is the appropriate access is indicative of Pam absorbing the cost of society's failure to ensure accessibility as an expected norm. Although Pam feels not all attempts and efforts at accessibility are successful, I got the sense was that all could be overcome with good local relationships and reasonable expectations. There was the concept of hassle with the narrow path, the difficult doorways and unrealistic ramps, but they seemed to feel that overall, the hassle was worth the effort.

\subsubsection{Rearranged town furniture}

The sense of hassle, again, comes with aspects of planning and design that might seem insignificant. Pam commented "the worse thing that's happened in town recently is a letter box". Relocation of a post box moved to accommodate a cash dispensing machine was subsequently discussed by Pam and Bernard. With a replacement situated in the middle of a hill (figures 8.9 and 8.10 ), any person experiencing mobility difficulties might find this difficult to access and for Pam and Bernard, the incline/decline is too much for Bernard to manage to push Pam. To post a letter he must go alone or leave Pam somewhere while he posts the letter. There is an inference that someone who self-propels in a wheelchair would find this post box difficult to access, as would anyone with walking difficulties. While the relocation of the post box might not appear to be a matter of great consequence, in the daily routine of Pam and Bernard or others who use mobility aids it has created a difficulty, a hassle. 


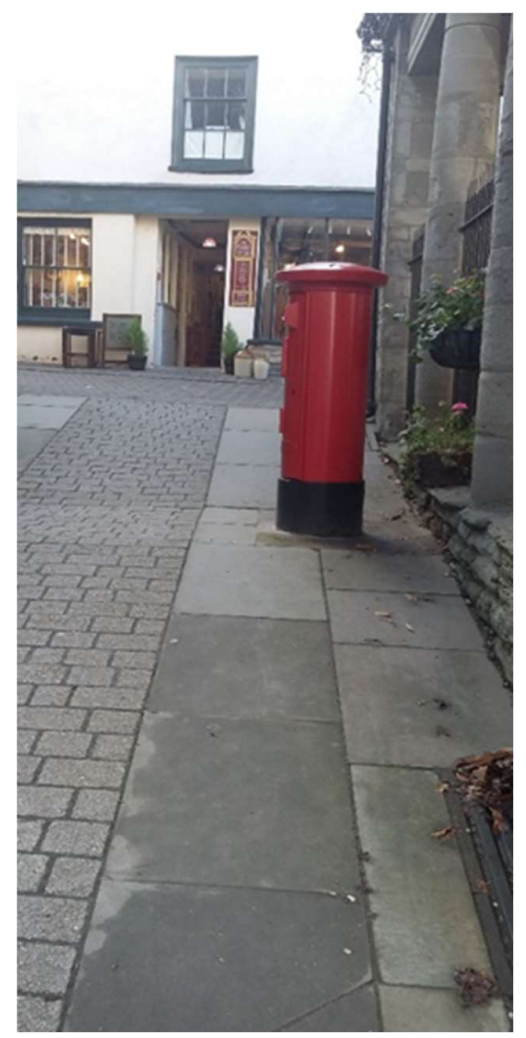

Figure 8.9 Looking up the incline to the post box

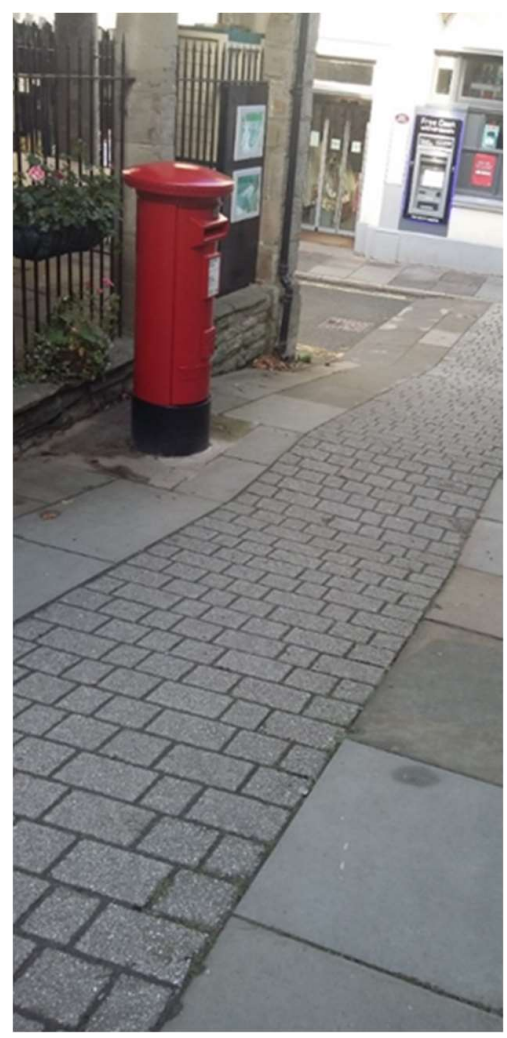

Figure 8.10 Looking down the hill to the ATM that displaced the post box

The post box, of all the issues Pam and Bernard discussed, seemed to be of more irritation than some of the larger obstacles such as unusable ramps and steps into shops. Perhaps because the post box did not need to be placed where it was. Perhaps because of the interruption to a routine task. With each of these incidents comes and added hassle, more manoeuvring, time and effort taken up. Each incident might seem small, but they add up to large amounts of time and effort, always remembering that older mobility aid users are often accompanied and cared for by their partner who is also older. The physical, social and psychological toll of the additional seeming insurmountable obstacles add up to an insurmountable collection of obstacles. 


\subsubsection{Accumulation of obstacles}

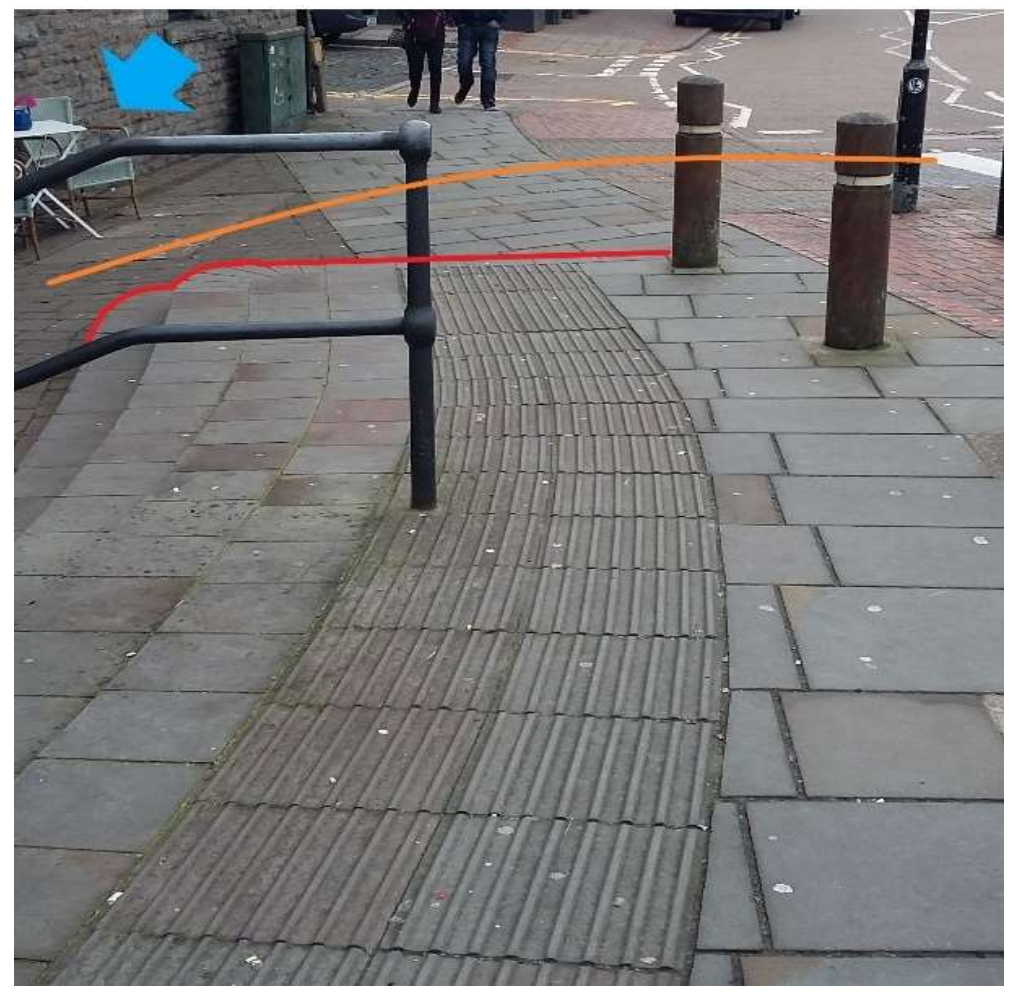

Figure 8.11 Pavement terrain after crossing the road from the car park

The first section of Dave's walking interview gives some context to the way in which obstacles can mount up. As we entered the city centre, we had already encountered a difficult junction as we exited the car park. Figure 8-11 illustrates the pavement opposite the pedestrian exit of the car park, showing the route from the zebra crossing to the city centre, via a pathway strewn with street furniture, signs and signals that impeded Dave's progress in his wheelchair. The orange line shows the only route possible with a wheeled device. Having been vigilant crossing the road, as being in a wheelchair makes one feel less visible and more vulnerable when crossing the road in a wheelchair, Dave was faced with the Belisha beacon pole and uneven paving. The blue arrow indicates a table and chairs outside of a café, making the gap narrower, especially when viewed along with the red line which indicates the edge of the steps. Add to this many people trying to avoid each other, the street furniture and not necessarily seeing Dave, it makes for a difficult junction to navigate in a wheelchair. To recap, Dave had to manoeuvre through narrow gaps between a Belisha 
beacon and two other poles, tactile paving, other pedestrians, some awkwardly placed café furniture and steps just to cross a road and reach safely to the other pavement. All of this while being less visible, and less able to react to people and things because of the way a wheelchair moves. Even if we do not count the other pedestrians there are 6 obstacles that could have been not placed or better placed in Daves path.

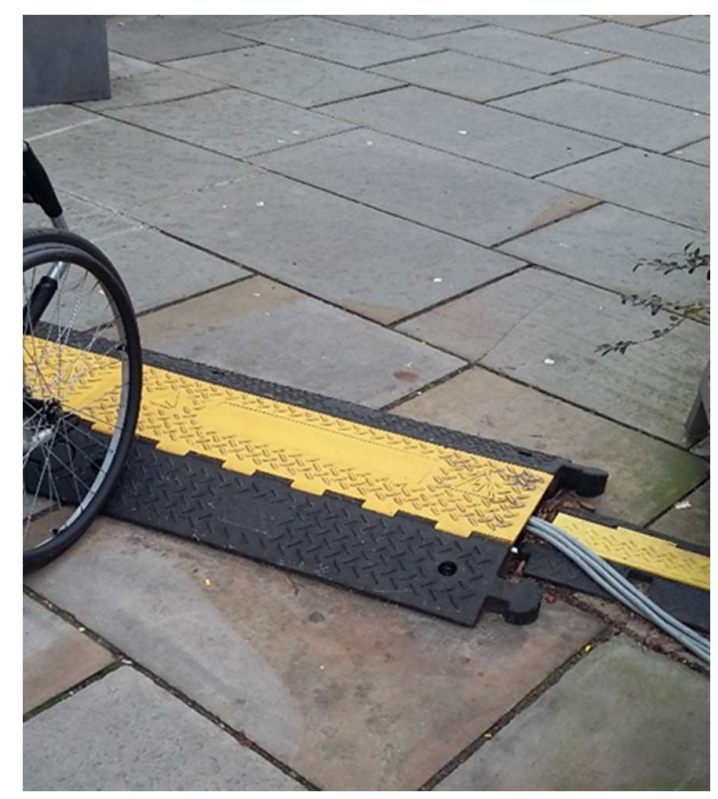

Figure 8.12 Cable ramp

Having manoeuvred through that difficult terrain, we were faced by buildings on our left and steps to the right. We continue straight forward talking as we went, until the discussion was broken. Dave commented "this is what, things like this... do you notice I'm going to do it backwards" as we encountered an obstacle. Work being carried out in one of the buildings to our left incurred cables being placed across the pavement. For safety purposes, the cables had been covered with a cable cover in the form of a ramp, but as seen in figure 8.12, the ramp was lumpy and difficult to traverse when self-propelling a wheelchair. Comparing the ramp covering the cables to the wheel of the wheelchair indicates the size and ratio of the obstacle this presents. Within a few minutes of arriving in the city centre, we had already faced many obstacles despite the terrain being designed for mobility aid users. The list of issues we faced in this short time could potentially have been included within the pavements and roads sub-theme, or even planning, design and services provision, because the design of the pavement and roads were the main obstacles. However, there was an additional table and chairs outside a café, and the ultimate obstacle which broke the flow of 
conversation which was the cable ramp. Each obstacle originated from a different aspect of infrastructure, systems or planning, each obstacle might seem separate and easy to overcome, but when faced in short succession, these obsstacles can become overwhelming.

To contextualise how overwhelming and intrusive relatively minor but hassle generating obstacles can be, the obstacles described above occurred within the first ten minutes of being in the city centre. In between facing a road that was really difficult to cross with many barriers on the opposite pavement (figure 8.11), which become even more difficult to navigate when other pedestrians were using the pavement as there were at the time, and encountering the cables as pictured in figure (8.12) we tried a route Dave had used before to find it cordened off, so we had to find an alternative that did not incur steps. Within the first ten minutes of the visit we had faced three sets of obstacles, and then when entering a shop front a few minutes later, the door was heavy and had to be pulled. It is not easy to pull a door and enter when using a wheelchair. When faced with these obstacles in most places, all day every day, they take up so much time and effort that people simply do not want to keep facing them. The person with mobility difficulties feels like a burden and stigmatised because they cannot move freely around without having to draw attention to themselves, ask for help or act autonomously.

Daily life and routines when using a mobility aid use can incur additional hassle through inaccessible provision of what can often seem insignificant items and services. Obstacles are not always or necessarily completely prohibitive, but when considered alongside mobility aid use with progressive senescence, comorbities and chronic illness, these obstacles that afford hassle become more and more prohibitive to social participation. As Phil pointed out, non-existent aspects of infrastructure that exclude mobility aid users, like insufficient dropped kerbs or inaccessible shops lead to non-participation, and non-participation ends up with failure to function like other people. In some circumstances. Attitudes of staff members and service providers can increase the sense of hassle with insensitivity at best, rudeness at worst, along with inefficient or ineffective mobility solutions increasing rather than decreasing stress. Hassle is a factor that is not scientific or readily quantifiable, but it is very real in the experiences of mobilty aid users and acts as a barrier in it's own way. 


\subsection{Conclusion}

This chapter has examined the ways in which lived experiences of people who use mobility aids intersect with infrastructure and interlinking systems, and how these systems presented obstacles to daily routines. Healthcare, civic and social participation and housing are all aspects of infrastructure that require interaction within day to day lived experience. Movement to access the different aspects of life is usually taken for granted (Urry, 2007), but when movement and mobility become limited, such issues are emphasised. As outlined at the beginning of the chapter infrastructure is important to achieving an age friendly environment, with WHO Age Friendly Communities guidance, outlining factors such as outdoor environments, housing, municipal services and transport and mobility as being vital to inclusivity. Although the guidance suggests each of these domains should work in cohesion with the others, this does not seem to have been achieved.

The pavements and roads sub-theme presented participants experiences of badly maintained and narrow pavements, lack of dropped kerbs and traffic calming measures all impairing the ability to get out and about. Extra processes in accessibility (8.1.1) were outlined with provision for mobility aid use being secondary to basic or fundamental provision. There was also a sense that vehicle user needs were dominant over the needs of pedestrians. Transport and travel disciplines which inform the planning and policy of street environment tend to discuss transport as an abstract concept of movement from a to $b$, divorced of any social context so the needs of users can be overlooked (Musselwhite, 2018). This leads to safety concerns (8.1.2) whereby traffic calming measures, road camber and installation of sufficient drop kerbs to provide visible and safe navigation has been shown to be problematic.

The sub-theme demonstrated how planning, design and service provision does not adequately incorporate the needs of older mobility aid users. Inadequate provision of parking and access (8.2.1) can be highly stressful for mobility aid users which not only affects physical accessibility, but psychological and emotional aspects of daily life. Parking was highlighted as an obstacle in the accounts of three participants, in situations which required work on relationships to maintain a social status quo. Insensitive staff (8.2.2) also influenced mobility aid users' experience of navigating the environment. Thoughtlessness to 
the needs of those using mobility aids, social and psychological as well as physical, and can impact not only that experience but their whole outlook on going out and about, as demonstrated by both Phil and Aileen. A lack of co-ordinated services (8.2.3), demonstrated here by a large distance between older people's purpose-built housing and the nearest access to public transport negatively impacts the health and well-being of older people with mobility difficulties. The cost incurred by older people with mobility difficulties is physical, emotional, social, and psychological (see also Aguiar \& Macario, 2017; Musselwhite, 2018) as they have to spend extra time and effort to access social spaces, and/ or ask for help which is contrary to people's requirements for independence (see chapter 6 ). Work is required to maintain identity despite compromised independence in ways that able bodied people do not encounter.

The final sub-theme reflects the combined cost of all the above infrastructural hurdles presented by pavements and roads, planning, design and services. Hassle outlines the complexities involved in continuing normal life when faced with a combination of what might seem to an some like minor obstacles. Each separate obstacle remains singular, but when faced with one after another, many minor hurdles become just too much hassle and it becomes too difficult to carry on with normality. The hassle does not just build over one day's experience but with every outing involving difficulty, every exchange with inconsiderate staff or public. Not fitting in (8.3.1) with either the structure or staff expectations of environments as Phil outlined, leads to emotional and psychological fatigue which adds to any physical fatigue experienced by the physical obstacles that may be experienced.

The presence of expected physical obstacles prompt older mobility aid users to utilise advanced planning (8.3.2) techniques in an attempt to abate the extra effort incurred in navigating the built environment when using a mobility aid. This becomes especially problematic, however, when planners or other services have rearranged town furniture (8.3.3) which results in interruptions to daily routines that are potentially not considered by those doing the rearranging. Dave's walking interview illustrated well the accumulation of obstacles (8.3.4) and general notion of hassle, highlighting an additional cost and extra mobility work involved in the use of mobility aids (or those with mobility difficulties) gaining access to daily life routines. 
The interlinking nature of not only infrastructures and services, but themes of infrastructure, relationships and identity make experiencing obstacles perhaps not as clear cut or easily categorised as presented here. Difficulty in navigating the built environment and infrastructure can lead to reduced social connections and neglected relationships. The consequences of such reduced interactions are wide reaching as they affect participation in clubs and groups, ad-hoc encounters with known people and strangers. Hassle every time an older mobility aid user leaves the home can lead to social isolation through changes to relationships with place, notions of being a burden and sometimes disagreement and discord as discussed in the relationships chapter. If a person argues with their partner or carer every time they go out because of the difficult terrain or poor service, it is likely to lead to fewer outings. Planning and services that do not meet the needs of older mobility aid users potentially lead to aversion behaviour and ultimately reduction of journey making or journey avoidance.

Infrastructure is created and maintained by people and societies, so power relations become important in terms of whose needs are met. Imrie (2000) highlights a hegemonic world of mobile bodies whereby political assumptions of abled versus disabled leads to strata of worth and power. Rather than enabling people with less abled bodies (which automatically includes older mobility aid users), societal aversion to presence of disabled people means there is a lack of encouragement for disabled people to move around freely. At best, disabled people are required to mimic what is perceived to be normality (Hansen \& Philo, 2007). Such expectations are likely to be internalised and incorporated into identity roles and meanings bringing full circle the feedback loop of identity, relationships, infrastructure and interlinking systems that are so complex and inter-reliant. Older people using mobility aids do not experience their daily lives in sections of identity or relationship, independence or hassle, but as one, indiscriminate, intermeshed experience in which relationships, identity, systems and planning all play out through the lens of their daily practices. Infrastructure and its interlinking systems and services, however, are the base provider of the movement and communication necessary to achieve the social engagement and participation that equate to lived experience. 


\section{Chapter 9. Outliers and demographic considerations}

This discussion is devoted to discussing two important factors of research findings: outliers and demographic considerations. Both sets of information are vital to understanding the findings as a whole. In grounded theory, outlying data is significant because it is different. Said difference could illuminate an important finding in and of itself, as discussed in the outliers section below. Intersectional aspects of the research inform the ways inequalities or social circumstances other than those being inspected may have a bearing on findings.

Gender, race, socio-economic background and age are all factors that categorise people in social strata. To begin with, my role as researcher involved interlinking aspects of privilege and oppression. In many of the social interactions within the research process, I was privileged in one sense, subverted in another. My role as researcher placed me in a perceived role of authority, yet in other ways I could be perceived as a lower status individual, as a woman, and potentially as an outsider because I am from London, not Wales. For example, when interviewing Stuart, he held gender privilege, I held scholarly privilege, race and socio-economic background was equal. Such social forces are a fundamental part of social research and require consideration within study outputs (Hughes \& Pennington, 2017). I have discussed my role and my perception of it through the research process and made every effort to make all interactions as level as possible.

The trends in the study are not necessarily representative of those reported in literature. For example, there is equal representation of male and female mobility aid users in this study, but research consistently demonstrates a higher ratio of female mobility aid users. It is worth reiterating here though, that most of the studies sourced before data collection commenced related to mobility and/or disability. The current study set out to understand mobility aid use, not mobility or disability per se. That is a distinction that needs to be expressed, because some of the focus of my study was the practicality of using a mobility aid, the facilitation of movement or experience of reduced movement due to the practicality of using an aid, rather than the experience of disability. While a large experiential cross over exists, there are differences in focus, for example the fact that Theresa had to store her mobility scooter in a garage that is $46 \mathrm{~m}$ away from any entrance to her home is of more relevance to this study than it might be to a study of the embodied experience of becoming 
disabled. There are of course similarities and ways in which these factors are interlinked and cannot be separated. Some of the responses in this study relate to the experiences of identifying as disabled, likelihood of using a mobility aid and the consequences of mobility aid use combined. However, the focus was on the mobility aid as a tool by which to navigate the environment, rather than the disability or senescence that caused its use. A useful analogy might be a study of shoes to understand how they influence walking - the shoes are the focus, but one must understand walking and how it is influenced to gauge the impact of the shoes.

\subsection{Outliers}

One of the interesting ways to understand the findings in grounded theory is to consider outlying findings, the data that is significant because it is different to other data, it does not fit in. The process of constructed grounded theory research and constant comparative analysis incorporates the notion that concepts illuminated through the research process earn their way into analysis (Charmaz, 2014; Charmaz \& Belgrave, 2019). That does not necessarily mean, however, that only the concepts spoken about most are considered interesting. It is a characteristic of grounded theory to seek understanding of what negative or outlying data suggests in term of the overall findings. What do the outlying findings tell us about the phenomena being researched (Charmaz, 2014) Discussed here are some of the outlying or puzzling data responses that do not fit into the main patterns of data and analysis but are nonetheless noteworthy. A point of consideration here is that because the sample is relatively small, outliers can represent quite a large minority.

\subsubsection{Non-Walking participants}

One of the important factors of data collection to be discussed was participants who did not walk. Stuart, Ron and Deidre all participated in traditional interviews but did not take part in walking interviews, each for different reasons. Deidre was willing and able to walk, but I was unable to contact her following the first stage interview. I followed up the interview with 2 telephone calls, leaving a message but she did not contact me. She had mentioned I could find her at a café she volunteers at, but I could not find her there either, and for the sake of anonymity and confidentiality I did not ask for her. It is a small town and other people know 
who I am and what I do from attendance at the friendship club. I allocated the loss of one participant walking to natural attrition.

Stuart hardly ever walks anywhere. He did not share this information directly and avoided talking about his mobility. Stuart is my neighbour and I only ever see him using his car. When asked during his interview if he stayed at home mostly, or popped out in the car, he shared that:-

Well, I take the car I go into town every day to do the shopping in the morning usually except on a Sunday and then I go into town I have lunch in town because Alice unfortunately cannot cook anymore and I never learnt how to cook. Alice was a brilliant pastry cook... absolutely wonderful and I dutifully ate all her cakes.

Looking back through his interview, Stuart seemed to avoid talking about his own mobility or health issues, only ever discussing his wife's mobility and the increasing difficulty he has coping with it. He would not answer a direct question about it. As the above quote illustrates, his answers were full of information and jokes, but it never related to his mobility, it related to his other capabilities. Stuart is that kind of person who is always making jokes, it is his way of connecting with people and being friendly. For this reason, it is impossible to assume that Stuart was purposefully avoiding discussing his mobility, although he could have been doing it subconsciously. I can only surmise that his responses and reluctance to walk were related to either an inability to walk that he did not want to discuss, or that using the car meant he was not away from his wife Alice for long. Continuing with his usual repartee Stuart did not include such personal information, and always wanted to include Alice in the conversation. Living with mental health issues, I felt it better not to include Alice's response as gaining informed response was a more complex situation that again, Stuart did not want to address. I felt that Stuart coped best by not confronting what might be perceived as negative aspects of their health and well-being head on, which meant that he did not admit to mobility problems, or Alice's mental health issues to their full extent. His coping mechanisms were carrying on regardless, and humour. Stuart also considers himself to be a leader in the community so perhaps he felt admission of mobility difficulties might reduce his status. 
All of the reasons suggested above for Stuart not walking would link to the concepts of both dignity and stigma. Fukuyama's (2018) definition, for example indicates that an inner sense of dignity requires both a sense of self-worth and public acknowledgement. Stuart's avoidance of discussing any problems may have been his way of maintaining dignity. The pattern of behaviour also fits with notions of stigma, which may be related to age, disability or even in this instance notions of gender and masculinity. Stigma occurs within historical and cultural contexts (Parker \& Aggleton, 2003; Pescosolido et al, 2008; Tyler \& Slater, 2016), and Stuart may feel that talking about weakness or even perceiving himself to be complaining were not acceptable to his standing and persona in that situation.

Ron chose to take part because it was convenient to do so on a day that I was giving a presentation that he and his carer (daughter) were attending. I believe they did not agree to a walking interview because, like Stuart, Ron was reticent to admit to any reduced activity, even though it was clear there were some changes to his ability to access places. It was Mary who spoke of Ron's mobility aids whilst Ron only spoke of the solutions, for example, he spoke about an optician attending his home to provide an eye test. Mary, Ron's daughter was also quite shy, so she may have been reluctant to take part in what she might have perceived to be a more in-depth session. It is also worth noting that particularly Mary, but also Ron were the type of participants who were difficult to get talking. They responded in one-word answers to begin with, so I did a lot of talking about the circumstances I find myself in with my husband to try and prompt some conversation. We did find some things to discuss, as Ron and Mary are rugby fans and attend matches at the local club as often as possible. We discussed the actions people at the grounds have taken to accommodate Ron's mobility needs. Ron, like Stuart, talked in terms of mobility not being problematic yet Mary discussed limitations, as discussed in Chapter 7.

Ron and Stuart share a few similarities, in that they are men, of similar age, and both would consider themselves to be middle class. Ron is a former head teacher, Stuart previously a financial services adviser. Both are active within community and club committees so prominent members of the same rural community. Ron will have used his car to commute to work as there will have been no public transport links from his home to the town in which he worked. As a financial services representative, Stuart used his car for many years to visit clients. He also previously lived in an area not easily accessible to public transport. While it 
would be easy to attribute their reluctance to walk or talk about walking to a deep seated need to protect their identities, or avoid accepting issues of ageing, senescence or disability, it is possible that these men have just been used to jumping in the car to carry out their daily activities so using the car is habitual (Daramy-Williams, Anable, \& Grant-Muller, 2019). They may just do it without too much thought.

Within the mobilities literature discussed in Chapter 2 and Chapter 3 section 3.1, there is a consensus that car use dominates the construction of infrastructure and discourses of transport, travel and movement (Daramy-Williams et al., 2019; Green, Steinbach, \& Datta, 2012; Sheller, 2018; Sheller \& Urry, 2008; Stjernborg, Wretstrand, \& Tesfahuney, 2014; Thrift, 2004). Access to public transport for people who use mobility aids can be problematic, which makes accessibility to shops and other facilities more difficult without personal access to a vehicle. Trips to the suburbs and rural areas are more problematic for public transport users than car drivers. If older people do not have access to car use, flexibility of travel and visits to family and friends can become limited (Mackett, 2018; Musselwhite \& Shergold, 2013). Giving up the use of one's own vehicle has been linked to feelings of bereavement and loss, reduced well-being, social isolation and depression and increased risk of mortality (Chihuri et al., 2016; Edwards, Perkins, Ross, \& Reynolds, 2009; Fonda, Wallace, \& Herzog, 2001; Marottoli et al., 2000; Murray \& Musselwhite, 2019; Musselwhite \& Shergold, 2013; Ragland, Satariano, \& MacLeod, 2005).

Driving cessation is related to loss of independence, reduced well-being and social isolation, which is likely to be a factor in the continual car use of Stuart and Ron. In terms of daily experience, the choice not to take part in a walking interview seems to have been taken because these two participants do not walk anywhere. Car use has been a dominant aspect of working and domestic lives, so continued use of private vehicle is not surprising. They may have refused to walk because it is just not a daily routine of theirs, or it may have been for other reasons which they did not wish to discuss relating to identity and dignity as discussed throughout chapter 6 . Whatever the reasons, participants not taking part in a walking interview occurred three times, all in different ways. Diedre had agreed to walk but the meeting could not be arranged and can be considered participant attrition. Stuart and Ron both indicated within their participation that they rarely walked anywhere, using their cars to access shops and social events. 3 participants out of 11 mobility aid users amounts to 
$27 \%$, however with a small sample size, this is not necessarily a generalisable statistic. It is also worth adding that both Stuart and Ron remained positive in their attitudes to life, and both are active in local groups and activities.

\subsubsection{Positivity and new life focus}

Another finding that stood out as different, also reported by two male participants, was the discovery of new pastimes and hobbies as the result of the onset of disability and/or chronic illness. Dave and Will both discussed new hobbies and skills they have discovered as a result of mobility aid use. Both participants are in the younger range of participants, Dave being 52 and Will 73.

Will indicated that he had found a new world with the internet and YouTube that he feels he would not have discovered had he not reduced his outdoor activity so much. He discussed his newfound computer skills had opened a different lifestyle to him. He suggested that in some ways becoming chronically ill had saved his life because it removed the pressures of working life. Will recounted that the time he now has available has allowed him to learn new skills with includes use of the computer and internet which he found enlightening.

Later in the interview Will reiterated a positivity he perceived in his reduced mobility. He felt glad to be able to rest from a strenuous and stressful work life, withdrawing from commuting, and focusing on things he found more interesting and stimulating. Before being chronically ill, Will was a singer and performed locally. Having more time now that he cannot work, Will has learned computing skills, which he described as an "enlightenment". Now that he has the skills and equipment to access YouTube content, he enjoys seeking out videos of singing and opera. Will was forthcoming with his feelings about vanity and feeling restricted by mobility aid use and chronic illness, so his sense of positivity at finding a new skill and outlet for his creativity felt genuinely inspiring. Will loves to spend time researching his hobbies with his newfound computer skills but also comments on a slight envy of people his age and older "bounding about the place" and wondering what his life would be like without "damn Parkinson's".

Will is an affable man who offered his experiences with openness. As is the case with every other participant, he has many other facets to his life and does not want to be defined by his illness and disability. Being quite reflexive, Will's experience of chronic illness and 
mobility aid use outlines negative experiences, concerns about infrastructure and admissions of vanity. However, Will had considered his options, and decided to take up the best options open to him to remain fulfilled in some ways.

Dave also found new activities through the challenges of his illness and amputation, discussing a renewed sense of confidence and independence to the point of doing new things that he would not have considered pre amputation. Dave discussed many aspects of his life that had caused him stress and may have contributed to his attitude to rehabilitation. He had been through a difficult divorce which included difficulties gaining parental access to his daughter when she was growing up, and nearly died of carbon monoxide poisoning a few years before he contracted the illness that led to the amputation of his leg below the knee. Dave could not articulate whether he had made a conscious choice to be positive, suggesting instead that he had no choice. I reminded him at that point that he had almost died three times and that not everyone takes this positive approach to embodied changes, to which he replied that the loss of his limb would remain with him for the rest of his life, so he just had to get used to it. Dave outlined many projects and activities he is involved in and is very positive about his life. At the point we met, he was attempting to manage walking with a prosthesis but had some peripheral health issues. While walking was the goal, Dave was not too perturbed if he could not achieve it as long as he could "do all the things I want to do".

I encountered Dave as a positive and driven individual who had gained a new lease of life through amputation, as odd as that might sound. He had gained a new social life through wheelchair rugby and better control of his working life as he could work specific hours and refuse other hours because of his health needs. Dave felt confident and in control of his life, only considering his actions because I was asking about them. I feel there is a caveat here though, that this interview ( as with all the interviews) was taken at a snapshot of time and his attitude may not always be like this. It is noteworthy that as a researcher it occurred to me that Dave's positivity may be temporary, but that thought did not occur to me with other people's responses. That may be because other people's responses were not as strong as Dave's, or it could be that as a researcher I am more wary of something that sits outside of the trend. I did not feel that Dave was insincere, far from it, but his attitude was by far 
the most positive. He was also the youngest participant and potentially the most mobile in that he was able to manoeuvre himself adeptly and quickly more than any other participant. Literature often posits disablement or loss of mobility as a negative event, a loss or impact as discussed within the medical and social models of disability. Medical model conceptualisations situate impairments within individuals, within biomedical definitions of normal and abnormal (Davis, 2017; Owens, 2015; Paterson \& Hughes, 2000; Tregaskis, 2002) extending as far as being harmed and in a position rationality would dictate was unwanted (Harris, 2000, p97). While there is great debate regarding impairment versus disability and whether disability is situated in the person or the environment, these examples show that the emergence of chronic illness and disability in older people is not always perceived totally negatively by the individual. People who incur a disabling condition do not necessarily 'survive', but can achieve higher levels of purpose, pursuit of goals, self and spiritual awareness that surpasses their previous levels. (Elliott et al., 2005). From as early as the 1940s and 1950s researchers have recognised that a shift of values could increase personal worth for individuals with acquired disability (Dembo, Leviton, \& Wright, 1975; Elliott, Kurylo, \& Rivera, 2005; Lovett, Joiner, \& Goodwin, 1989). Will also demonstrates a shift in his value of movement to non-movement. Rather than requiring hypermobility, as transport and engineering discourses emphasise (Doughty \& Murray, 2014; Musselwhite, 2018), Will has found some happiness in non-movement which contrasts his former working lifestyle.

Both Dave and Will highlight the value of finding a new focus and pursuing adjusted goals to adjust their lifestyles to changed conditions. The story is not one of complete positivity, but it would be false to suggest all was negative either. Both of these accounts of positivity reflect not on medical categorisations or exclusionary infrastructure, which both Will and Dave find problematic, but instead on the ability to adapt to circumstances. In a way, the accounts of positivity oppose the outlying information of non-walking participants, in that Stuart and Ron had continued with their existing lifestyle behaviours, whether through necessity or habit. An interesting factor of the outliers is that they are all male. Ron and Stuart, who did not walk are both over 80 , while Dave and Will are both in the younger age group, which brings us to the next section which examines the potential influence of demographic factors. 


\subsection{Gender, Age and socio-economic factors}

I found it difficult to write this section, partly perhaps because my focus has always been using of the mobility aid, its practicality and function within the lifestyle of the user. I was not looking at or for gender or socio-economic factors unless they were overtly obvious. My own observations within the study were that mobility restrictions are experienced similarly by all, apart from an obvious difference between older older people and younger older people. With encouragement from my supervisors, I analysed the data to seek out gender and socioeconomic facets of the analysis. Through this process I found the concept of the third age, which helps to explain the age differences, and dignity aspects of the findings as well as notions of successful ageing and gender differences within it.

The contexts of age, socio-economic status and gender will be framed here within the economic and historical context of what Peter Laslett (1987) referred to as 'the Third Age'. The emergence of the third age in around the 1950s has been facilitated by health and lifestyle factors which include better access to healthcare, living longer into retirement past the age of 70 and beyond with access to income (Gilleard \& Higgs, 2010, 2011; Gilleard, Higgs, Hyde, Wiggins, \& Blane, 2005; Laslett, 1987). Demographically, the Third Age is defined as a population condition in which both the general expectation of living from 25 to 70 is half or more plus $10 \%$ or more of the population being over 65 years of age. This did not emerge in the UK until the 1950s and did not become a feature of social structure until the 1980s (Laslett, 1987). Represented as a culture of autonomy, self-expression and pleasure in later life, the third age is offset with the stigmatisation of an old age in which the arrival of disability, diminishment and death are represented as the fourth age (Gilleard \& Higgs, 2010). The delineations of entry into the third and fourth age are not biological or calendar age in nature, but instead involve activities, lifestyle and independence versus dependence. It is only when they are no longer able to manage with daily rounds, or at least seen as no longer managing do older people become subjects of fourth age (Gilleard \& Higgs, 2011).

\subsubsection{Socio-economic background}

The first observation to be made about socio-economic background is that probably everybody participating in the study would consider themselves to be middle class, or 
maybe not belonging to a class (Savage, 2015). Class was not something asked about or a concern of the research per se. As previously established this research set out to examine individual experiences of mobility aid use in the built environment. To delineate differences between background I have looked at profession and made education level to establish a ranking. Those ranked low on table 9.1 have a lower skilled job and presumed lower education level. This does not necessarily have a bearing on income, as Stuart does not have a high level of skill or education but would be in the top three according to income and assets. The need to address the socio-economic background arose because there were some definite differences between some people from a lower socio-economic background compared to those higher on the scale. It must be reiterated here that this is a scale which relies only on previous occupation and presumed education status of participants.

\begin{tabular}{|l|l|l|l|}
\hline Name & Age & sex & education level \\
\hline Teresa & $80 \mathrm{~s}$ & $\mathrm{f}$ & mid \\
\hline Bret & $80 \mathrm{~s}$ & $\mathrm{~m}$ & low \\
\hline Regina & $80 \mathrm{~s}$ & $\mathrm{f}$ & mid \\
\hline Ron & $80 \mathrm{~s}$ & $\mathrm{~m}$ & high \\
\hline Aileen & $50 \mathrm{~s}$ & $\mathrm{f}$ & low \\
\hline Stuart & $80 \mathrm{~s}$ & $\mathrm{~m}$ & low \\
\hline Deidre & $80 \mathrm{~s}$ & $\mathrm{f}$ & high \\
\hline Phil & $50 \mathrm{~s}$ & $\mathrm{~m}$ & high \\
\hline Pam & $70 \mathrm{~s}$ & $\mathrm{f}$ & high \\
\hline Will & $70 \mathrm{~s}$ & $\mathrm{~m}$ & mid \\
\hline Dave & $50 \mathrm{~s}$ & $\mathrm{~m}$ & mid \\
\hline
\end{tabular}

Table 9.1 Gender, age and education level

High = graduate level, mid = college or highly skilled, low = secondary school education

More of the focus on differences between participants can be placed on gender and age than socio-economic status alone within this study because the participants lived similar 
types of lifestyles with differences relating to illness or age. Savage (2015) utilises Bourdieu's notion of cultural capital, in which the capacity to succeed in education is inherited from parents, helps to explain new formations of class understandings. Whereas class has historically been linked to occupation and the demarcation between middle and working class, current consideration of class categorisations is more focused on the ways in which different form of inequality intersect. Cultural capital is linked to social capital by way of where on lives and who one mixes with, therefore what kinds of places and people one has access to. Class can form through combinations of access and inequality associated with factors such as age and gender, however, many people are ambivalent about which class they belong to (Savage, 2015). Class systems of yesteryear are no longer identified with in the same way, instead people identify with lifestyles (Gilleard et al, 2005). These notions of changes to class structures would explain the lack of largescale variations in terms of socioeconomic status.

\subsubsection{Gender}

Gender is an important aspect of mobility aid use within the daily routines and social participation to interrogate is that of gender. Gilleard and Higgs (2002) note that the third age lifestyle which has enabled successful ageing, is most accessible to wealthier males. Concrete conclusions are difficult to draw from such a small sample, but trends can be identified and compared. Beginning with carers, of six carers taking part, only one was male. Bernard is Pam's husband and the only participant identified as a male spousal carer. However, to contextualise this, Bret had been a carer for his late wife for many years, Stuart cares for his wife who is a mobility aid user herself but not a participant in the study, and Will also cares for his wife, but her needs do not require use of a mobility aid. These various formulations of care dynamics demonstrate the complexity of spousal relationships, ageing, comorbidity and caring roles. While statistics show a higher ratio of female to male carers, it could be co-incidence in this study.

Apart from one formal carer, all other carers are daughters which does reflect statistics on filial caring situations (e.g. Twigg, 1992) and more time spent caring for adults by women than men (ONS, value of unpaid care work 2016). The specific family situation of this study means few conclusions can be drawn, as only one of the participants has any sons. It is true 
that this participant is 'looked after' by his daughter, but it is also true that his daughter lives closest. It is open to debate whether the daughter stayed closer because of family ties and caring responsibilities, so no real conclusion regarding gender and caring can be drawn from these circumstances within this study.

Gender ratio for mobility aid users was six males to five females. Average age of females was 78 , average age of males 73 , but with a small sample such calculations are not hugely meaningful. It does draw attention to the fact that there were more males, and the extra male was 52, greatly reducing the average age of male participation. Age by age comparison would appear as shown in table 9.2.

\begin{tabular}{|l|l|l|l|}
\hline Teresa & 85 & Ron & 85 \\
\hline Regina & 89 & Stuart & 87 \\
\hline Aileen & 58 & Phil & 57 \\
\hline Diedre & 81 & Bret & 83 \\
\hline Pam & 77 & Will & 73 \\
\hline & & Dave & 52 \\
\hline
\end{tabular}

Table 9.2 Gender and age comparisons

Males were more likely to be car users as all males in the study drove and owned cars, whereas none of the female mobility aid users were able to drive at the time of the study. This is not necessarily because they were never able to, but illness, injury or disability precluded them from driving. Deidre was able to drive, and was indeed the driver of the family since her husband became visually impaired, but had to cease temporarily due to her ankle injury. They continue to own a car and Deidre will take up driving again when her ankle has healed. Regina and Aileen were both able to drive before their vision became compromised but are obviously unable to continue. Theresa also commented that she was no longer able to drive due to medical issues. Driving was not discussed with Pam and Bernard as such, although they mentioned the car they sourced when Pam first started to 
require a wheelchair. Participants who did not want to conduct a walking interview were both male.

\begin{tabular}{|l|l|l|l|}
\hline Name & $\begin{array}{l}\text { Age } \\
\text { group }\end{array}$ & Walked & Mobility aid \\
\hline \multicolumn{5}{|l|}{ Females } \\
\hline Regina & 89 & yes & white stick + walking stick \\
\hline Aileen & 58 & yes & should use white stick + stick \\
\hline Deidre & 81 & no & crutches + walking stick \\
\hline Pam & 77 & yes & wheelchair + frame + stick \\
\hline Males & & & \\
\hline Bret & 83 & yes & walking stick \\
\hline Ron & 85 & no & scooter + stick \\
\hline Stuart & 87 & no & walking stick \\
\hline Phil & 57 & yes & wheelchair + walking stick \\
\hline Will & 73 & yes & $\begin{array}{l}\text { walking stick + occasional mobility } \\
\text { scooter }\end{array}$ \\
\hline Dave & 52 & yes & stick + crutches + callipers \\
\hline
\end{tabular}

Table 9.3 Age, walking and mobility aid data

The equal ratio of male and female represented in the study is not representative of mobility difficulties or functional limitations reported in the literature. Studies have consistently shown a gender gap in mobility difficulties with women more likely to be mobility impaired than men in older age (Ahmed, Vafaei, Auais, Guralnik, \& Zunzunegui, 2016; Leveille, Resnick, \& Balfour, 2014; Mechakra-Tahiri, Freeman, Haddad, Samson, \& Zunzunegui, 2012; Newman \& Brach, 2001; Zunzunegui et al., 2013). One explanation is that women live longer than men, thus spending more years at risk of chronic diseases such as osteoarthritis and other musculoskeletal diseases which are also associated with pain (Ahmed et al., 2016; Merrill, Seeman, Kasl, \& Berkman, 1997; Peterson, Meng, Dobbs, \& Hyer, 2017). The slightly higher age ratio of women to men in this study, then, is indicative of global trends that women are more likely to live longer than men, although as previously stated, with such a small sample it is difficult to make generalisations. Little is known about the role of gender stereotyping in the mobility gender gap (Ahmed et al., 2016). The International Study of Mobility in Aging (IMIAS) examined the mobility people of aged 65-74 
in five locations: Brazil, Colombia, Albania and Quebec and Ontario in Canada. The study found women to have a statistically significant higher chance of experiencing mobility disability at all sites other than Ontario (Ahmed et al., 2016).

A study of walking stick use among people aged 65 and older in the United States, shows that a greater number of women (22\%) used walking sticks compared to men (16\%), which is predictable considering there are greater mobility limitations for women that can be compensated for with walking sticks (Peterson et al., 2017). However, when balancing factors related to disablement and health behaviour, women were found to be less likely than men to use a walking stick. Analysis was conducted on many facets of walking stick use including asking yes or no questions about impairments and capabilities and conducting the Short Physical Performance Battery (SPPB), a battery of lower extremity function tests that is predictive of mobility, disability, and mortality (Peterson et al, 2017). Qualitative research with women has concluded a complex negotiation between cane use and notions of ageing and being vulnerable (Porter, Benson, \& Matsuda, 2011).

Aileen's concerns and behaviours fit into this complex negotiation of mobility aid use balanced with ageing, being vulnerable, and stigma as will be discussed in Chapter 10. Disliking the attention, a mobility aid brings to her, Aileen commented that she would not feel so bad if more of her peers use them. Both Aileen and her daughter Lauren discussed the reduction of 'glamour' and 'attractiveness' involved in uptake of a mobility device. Deidre also discussed her dismissal of mobility aid use, until the pain and mobility difficulty meant she could not avoid using a stick any longer. When she realised the assistance the aid gave her, she wished she had used it when it had first been suggested to her many years before. Theresa and Ivy discussed how much freedom a mobility scooter had afforded Theresa. From these accounts, there seems to be a complex evaluation of mobility aid use in which Theresa, and Deidre as discussed above, consider their appearance balanced with ability to carry out day to day tasks. It seems the balance point of using the aid versus disruption of embodied image is different for each individual. I will return to the notion of disrupted embodied image in Chapter 10. 
A factor in the complex evaluation of mobility aid use and appearance is identifying as disabled and thus an individual's evaluation of identity. Ridolfo and Ward (2012) suggest that being "disabled" makes it difficult for women to enact a feminine gender role because disability counteracts standards of feminine beauty. Disablement process is more complex than the social or medical models of disability suggest and not all individuals with mobility impairments or physical disabilities identify as disabled. Social statuses are influential in identity construction. Using both qualitative and quantitative data in their study of disabled identity, Ridolfo and Ward's research found that women are less likely than men to identify as disabled with quantitative results supporting the qualitative. Moreover, even when women decide to claim a disabled identity it can sometimes be invalidated by others, meaning women were not viewed as disabled even if they self-identified as disabled (Ridolfo \& Ward 2012).

Men's disability identity was also found to be influenced, in that the loss of physical and athletic ability was seen as a sign of disability which could result in claiming of disabled identity (Ridolfo \& Ward 2012). It is also worth noting here that Will as a male also shared concerns of appearance being compromised by mobility aid use. Some studies have shown that older men are not as concerned with their appearance as older women and have higher self-esteem. Others, albeit fewer studies, have shown older men to be more likely to demonstrate decreased self-esteem and sense of attractiveness as they age. It is possible that disfiguring or disabling chronic conditions in later life could play an important role in older men's negative attitudes to their bodies (Hurd Clarke \& Korotchenko, 2011). This would adequately account for Will's concerns about his appearance, especially when taken into consideration with his thoughts about not wanting to be a burden as mentioned in Chapter 7. These two aspects feed into the notions of third and fourth age, whereby being active and able to live independently in the third age retains social value, whereas those in the fourth age are dependent on others and society in general (e.g., Gilleard \& Higgs, 2010). Individuals in the third age are actively warding off the fourth age, which Will could be doing.

There are social and cultural contexts in which men and women absorb expectations and construct identities based on their various roles and responsibilities which affect mobility aid use. Loss of mobility, disability and ageing can all negatively impact social value, and 
therefore identity. Complex negotiations around being vulnerable, feeling stigmatised, and feminine or masculine expectations of character and appearance all impact mobility aid use. It is interesting that within the participants of this study, two stood out has being particularly concerned about their appearance. Bucking gender trends, one is male and one female, but both fall in the younger age bracket, with Will aged 73 and Aileen 58. Age is another factor that has implications for the way people feel about and use mobility aids which is discussed next.

\subsubsection{Age}

Discourses of normal ageing invoke the will to remain healthy by individual means whereas natural ageing accepts physical and mental decline as part of the trajectory of life. Health is therefore central to agency in third age. Through manifestations of a third age and successful ageing, that is, people living longer and healthier lives that allow them to pursue their life goals (Laslett, 1987) a chasm has formed between ideas of normal ageing and natural ageing (Higgs et al., 2009). The distinction between natural and normal ageing means an increasing emphasis on delaying decline (Higgs et al., 2009). Health is a fundamental requirement of agency in third-age lifestyles within which is the need to prevent physical and mental decline. Such declines demarcate the fourth age which is associated with frailty and dependency (Laslett, 1987; Gilleard \& Higgs, 2010; Higgs et al., 2009). With contemporary normative ideas of ageing focused on discourses of being active, and successful and healthy, everyday life experience for individuals involves maintaining identities within family and social networks. Failure to manage daily routines might result in entry into the fourth age which would result in loss of control over their own lives. While there seem to be a variety of markers to demarcate entry into fourth age, it is a public inability to continue to independently carry out daily routines that seems to mark transition into fourth age status (Gilleard \& Higgs, 2010). The observation of individuals within this $\mathrm{PhD}$ could be aligned to the notion of older people currently within the third age trying to avoid fourth age conceptions. The necessity to be seen to be independent was evident in many participants, especially those over 80 .

Independence is closely aligned to autonomy, being the ability to make decisions and act upon them for self (Dillon, 1995; Edlund et al, 2013). Autonomy, required to legislate moral 
behaviour for oneself and act accordingly, is necessary for dignity. Autonomy as a capacity to decide and act for self enables dignity which makes one worthy of respect (Dillon, 1995). As with all other factors discussed in this research project, no singular facet of identity can be separated from others, so a sense of independence is not only driven by ideas autonomy, but a general sense of self-worth and dignity linked to identity. Identity, dignity and independence are thus intrinsically linked and were observed as such within the study, but dignity was observed differently through the age range. Dignity was evident in actions such as making the best of things, not complaining or just not mentioning feelings. With a relatively small sample size and large variation, it is difficult to establish exactly what the boundaries of dignity were determined by for each person (neither is it what I set out to do), and it is likely be different for each individual. The participants taking part in the study were most likely not consciously thinking through all the stages or voicing their concern for dignity, but it would be a realistic conclusion that dignity was manifested through their actions and comments.

A sense of dignity requires public recognition and acknowledgement (Fukuyama, 2018), perhaps another reason for it being more observable than some of the other traits. Dignity appears to be strongly related to what one values in identity and self-worth, therefore if independence is most important, dignity will be intertwined with a sense of independence. It is potentially more pertinent to state that how one thinks others perceive one's independence is most important, an aspect that appeared in the older old - those over 80. The sense of dignity seems to be linked strongly to appearing independent and not asking for help. Bret displayed this sense of independence by insisting on continuing with his daily routine despite mobility concerns. It seemed that Bret did not want to admit to himself that he might be losing some mobility and could not continue to do everything as he used to. His many falls, some of which his daughter Jenny told me about, and which she had been informed of through a third party, his uneven gait but non-admission of needing to use a stick, some stories of getting stuck in the bath all lead me to the conclusion that his mobility and abilities have reduced a little, but he either does not want to admit this or has not realised it.

Regina displayed a pattern of independence, discussing the body as a machine, stating that ageing with its wear and tear is to be expected and must be accepted, yet she also 
continued to hang curtains at her window every night by climbing a step ladder whilst visually impaired and needing a stick to walk. She also stated how independent she is, with an air of how important that independence is to her. Theresa did not state her need for independence but when I offered her help (which I should not have done because had I not been studying her routine, she would have got it for herself anyway, but politeness overrode my role as researcher in that moment) she was indignant in her refusal. The sense of dignity and independence for both Regina and Theresa seem to come not necessarily from what others can see them achieve, but what they know in themselves they can continue to achieve. There was a sense with all of the octogenarians, Bret, Regina and Theresa, and also Stuart, Deidre and Ron, that they wanted to continue to be autonomous, independent and remain in control of their lives with what I sensed to be an undercurrent of not wanting to 'make a fuss'.

The fourth age represents not a life stage but a kind of destination which is stripped of social and cultural capital, thus disallows articulation of choice, autonomy, self-expression and pleasure in later life (Gilleard \& Higgs 2010). Gilleard and Higgs (2010, 2011) metaphor of the fourth age as a black hole of ageing allows conceptualisation of the sense that there is no return from it, and no real sense of communication seems to emerge from those who have entered it. This may explain why the stigma of it cannot be reduced and its status is not normalised Gilleard and Higgs (2010). While participants may not know this status as 'the fourth age', older people like Bret, Regina and Theresa want to be seen to be independent because the discourse of dependency and being referred to in the third person is not a point they wish to arrive at. It makes senses that older old people within the study are at pains to demonstrate their independence.

As mentioned above, Will's behaviour and attitudes toward his appearance and not wanting to be a burden could be in line with discourses that emphasise health as being imperative to independence and dignity. Aileen displayed similar tendencies to dignity as being highly important to her status, although this manifested as being unwilling to use mobility aids. For Aileen and Will, loss of function and/or visible use of mobility aid seemed to equate to loss of dignity. Appearance was an important indicator of status and dignity. Aileen refused to use mobility aids at all because of the attention they bring to her, and Will only uses a walking stick even though a mobility scooter or wheelchair might serve him better on 
occasions. Their concern with dignity was very closely linked to the stigma of ageing, and both participants were concerned with their appearance more than any other participants. Aileen's refusal to use mobility aids to the point of pain and tears was linked to her appearance and ultimately fitting in, as illustrated by her comment that 'if others my age were like it. Will's determination to avoid negative stereotypes aligns more with a fear of being categorised as doddery and old. For these two participants, the mobility aid constituted a negativity that may be linked to body image. Body image, defined "as a person's perceptions thoughts and feelings about his or her body" (Grogan, 2007) p.3) has been the focus of much research, which tends to focus on young and middle aged women and is focused on youth in media and advertising representations (Hurd Clarke \& Korotchenko, 2011).

Notwithstanding, a useful review of work in this area by Marika Tiggemann (2004) highlighted that women's body dissatisfaction does not differ with age until later old age. Women over 60 were found to have increased body satisfaction with more focus on body function and health, and similar levels of body satisfaction to men in this age group. Work by feminist writers has suggested that older women become less visible to the point of invisibility which would decrease the importance of body size and weight for this older age group. As this can lead to reductions in self-objectification, body monitoring, and appearance anxiety, a freedom from self-consciousness and pressure to aspire to the slender, youthful, sexually available ideal becomes available (Grogan, 2008). Aileen, at 58 falls below the 60-year age threshold mentioned regarding body satisfaction, and it is certainly true that body monitoring and appearance anxiety are still functions of her identity and dignity.

Theresa and Deidre, both over 80 , alluded to the evaluation of appearance in use of mobility aids, but they both came to a decision that their independence and mobility were more important to their dignity and lifestyles. This reflects the notion that women over 60 include function and health in evaluations of body satisfaction as suggested by Tiggeman (2004). That said, it is not possible with such a small sample to differentiate whether these age differences are solely related to the theories mentioned so far, or are associated with cohort differences. 
The cohort of older people who are now 80 or older would have been born around 1940 or before. The ideas of ageing, body- image and independence are likely to differ from those born in the 1950 s or 1960s. Regina, the oldest participant mused that she remembered life without a National Health Service and explained that old people now are not like they were when she was a child:
R: $\quad$ See so I mean ( ) what do you expec- I don't think pe-so many people lived as old as that years ago ... When I was I child I can't remember that they did ... No, I don't think they did [and in any case
G: Yeah no] ( ) you said your own grandparents lived until their 90s
R: $\quad$ Oh yes, they did too you quite- pops but
G: $\quad$ But they as you said just sat in a chair and everybody waited on them... and they didn't do anything

Witnessing a social normality in which old age meant sitting without any purpose or access to healthcare will have given Regina and potentially those around her age a different perspective on old age. All participants over 80 were at least in late adolescence when NHS services were introduced, with Regina being around 20 years old. In contrast, younger participants were either children who may not remember the consequences of the introduction of a free health service such as Pam who was around 7 and Will 3 . The younger participants were not born until after its introduction with Aileen Dave and Phil born 12, 13 and 18 years after its establishment. Changes in health behaviours and attitudes are likely to be apparent in the attitudes of these different cohorts to ageing, mobility, health, bodyimage and independence.

In 1941, the United States (US) Health Service National Institute of Health set up a new unit for research into gerontology which focused on the normal processes of late maturity, namely the years between 40 and 60 years of age (Gilleard \& Higgs, 2010). The sense that an age range of 40 to 60 was considered late maturity seems out of date in contemporary thinking which would evaluate this range as middle aged in most modern Western contexts. The social experience of understanding and witnessing old age in others has thus been 
different for different age groups which cannot be eliminated from the differences in attitudes to mobility aid use, independence, and dignity. Those over 80 witnessed old age being treated as a reduced social and physical status, whereas younger participants have had more exposure to an old age in which one can access healthcare and technology to evade and diminish decline (Gilleard \& Higgs, 2010)

This chapter has outlined the ways in which age, gender and socio-economic background have factored into the findings. There are no comments relating to race because all taking part were white British, and religion was not asked. Socio-economic background did not present as much of a differentiator as gender and age. Gender roles were apparent nonwalking participants who were both male, both still using their cars. Slightly bucking the trends outlined in literature in terms of concern for appearance, though, were a female and a male discussing failure to use the correct level of mobility aid because it impacts their appearance. This seemed in both cases to be interconnected with concerns about ageing and the stigma of ageing as much as that of disability. Age differences were the biggest influence in terms of differences to approach to mobility aid use. The issues aligning to age differences link closely with independence, dignity, and the stigma of ageism. Those under 80 displayed issues of dignity and concern for independence in slightly different ways to those over 80 as outlined above. It is difficult to understand from these findings alone whether this is indeed a cohort difference, in that those over 80 have experienced different attitudes and behaviours towards ageing than their younger counterparts. Their experience of the emergence of the National Health Service is a good example of how life experience may influence their understanding and concerns of ageing. Alternatively, it could be an issue of age itself, and as the younger participants in this study reach 80 and their evaluations of mobility and mobility aid use focus more towards independence than appearance as a factor of dignity. 


\section{Chapter 10. Discussion:}

\subsection{Methodological reflection}

The literature of ethnographic grounded theory, and particularly walking interviews, promised contextually situated insights that were prompted by the social and physical environment. Walking interviews provided a useful method for evaluating the mobility of older people who use mobility aids as the method facilitates access to routine daily movements within the context of daily routines. As the methods reduced as much as possible the enforcement of preconceived ideas on participants, with each participant leading their own interview, data was as true to their experience as possible. Applying the mobilities paradigm using walking or go-along interviews provided interesting highlights that would have gone undiscovered via alternative methods. The opportunity to lead gave participants a sense of control, although sometimes this did begin with some apprehension. The process of carrying out a risk assessment and a pre-walk interview offered a chance to discuss the role of the participant in shaping the research. It gave me an opportunity to emphasise the importance of their insights, and the highs and lows of moving around the environment in their daily routine, in their own opinion.

Participants were empowered to lead me through their locale, which enabled them to speak freely and also helped to balance any social power imbalances they may have perceived. I was able to observe physical mobility as well as discuss perceptions of the environment, and assess notions of potential mobility, immobility or inaccessibility. At times, people made a statement, but I witnessed something different (see section 6.2.1 regarding Bret's use of walking stick for an example). This underlines the value of walking interviews in gaining insights into unspoken or unacknowledged aspects of mobility. I valued the ability of walking interviews to highlight both what is said, and what is not said. People navigating their environment, encountering people and places as they talk, enables researcher to listen to conversation, observe activity and observe facial expression which can betray pain, consternation, mistrust or annoyance.

During the first few sessions, I felt that I was not gaining information, but that changed when I realised I needed to tap into this unspoken aspect of the data. I heard Bret's uneven 
gait, I realised that participants who did not want to walk or discuss why they did not want to walk was information in and of itself. Kusenbach (2003) discusses authenticity in go-along interviews as requiring familiar routines and environment. If people do not usually walk, that is authentic for them, and informative. Walking interviews fulfilled the promise of gaining information in the environmental context, gaining insights and information that a static interview would not have done. In fact, just raising the prospect of a walking interview gave valuable insights into older people's thoughts on walking in the locale. Most happily agreed, some were coaxed by relatives and others refused. No-one gave reasons, but all of these responses highlighted different levels of confidence and ability to navigate the environment. While a couple of people did not walk, they did discuss what happens when they do go out. This approach does not offer those same insights as walking interviews did, it still provides comparative information, especially regarding the more unspoken aspects of mobility aid use that tend to be around identity.

\subsection{Bringing themes together}

The aim of this chapter is to indicate the ways in which the findings of this research are implicated in policy and practice settings. The chapter begins with a summary of the aims of the research regarding policy and planning, followed by the proposal of a feedback loop of obstacles to mobility aid use. The feedback loop demonstrates the ways that policy, practice, planning and design are implicated in mundane routine practices, affecting the identities and relationships of people within everyday environments. I use stigma as an established concept to highlight the flow of social, emotional and psychological factors through the various levels of interaction with the environment. This demonstrates the complex interrelated nature of built environments, and the reason infrastructure must be considered as more than perfunctory or practical. The feedback loop of obstacles leads to interrupted embodiment, my own variation of disrupted biography which highlights the changes to identity and relationships which occur with the assimilation of a mobility device. The chapter continues by outlining the ways my proposal of a critical Geromobilitie approach supports the appraisal of infrastructural practices to provide for a more AgeFriendly Wales. 
At the outset, the research in this PhD was designed to investigate the use of mobility aids by older people in the context of ageing populations, to describe and understand any obstacles faced by this quite substantial portion of society, especially in Wales. Within this wider context are the experiences of older mobility aid users, and the ways policy and planning decisions impact upon their routines. The ultimate reason for this type of investigation is to move towards the best situation for both mobility aid users, and society. If people who use mobility aids are enabled to have optimum access to the environment, this in turn allows each individual optimum ability to contribute to society.

One of the aims discussed in Chapter 1 was to identify how policy and planning are implicated in the use of mobility aids by older people represented in this proportion of society. This part of the discussion will examine the implications of the findings for policy and planning, discussing how the experiences of older people who use mobility aids uncovered in this study translate into implications for policy and practice relating to the mobility of older people, and particularly those who require the use of aids or tools to navigate the environment. Funding of this PhD fell under the remit of 'Age and Dementia Friendly Wales', so, as discussed in Chapter 2, the approach to Ageing Well in Wales and Age Friendly Cities and Communities formed part of the social and political underpinnings of the research. The analysis of data uncovered the ways mobility aids intercept the ability to conduct relationships. Relationships are not only fundamental to social behaviours, but are inseparably linked to identity and to infrastructure in complicated ways that are not necessarily obvious during daily life. The term relationships as referred to here does not only mean with other people, but with self, with places, with systems of control, power, production and provision that amount to infrastructure. The term relationships refers to two-way interactions between the individual and other people, along with policies, practices and procedures emanating from these which also impact upon relationships with others, with places and with service providers. Another form of relationship is that between the citizen and society in general, so participation in community and political affairs also applies to this sense of relationships being the basis of all social interactions and in all levels of participation. 


\subsubsection{Relationships as the basis of participation}

The nature of human social relations is complex and occurs at various levels. Participation in events and activities, communities and citizenship are reliant on both movement and relationships (Bigonnesse, 2018; Gaete-Reyes, 2015; Green et al., 2014; Spinney et al., 2015; Tuvikene, 2018). Figure 10.1 shows a diagram of the intersection of relationships in the results of this research, indicating the overlapping nature of personal relationships, identity and infrastructure, the themes visited throughout. The triangular shape at the centre of the Venn diagram indicates the person who interacts with all three, that is self, other people and the built environment, at the same time usually when navigating the built environment.

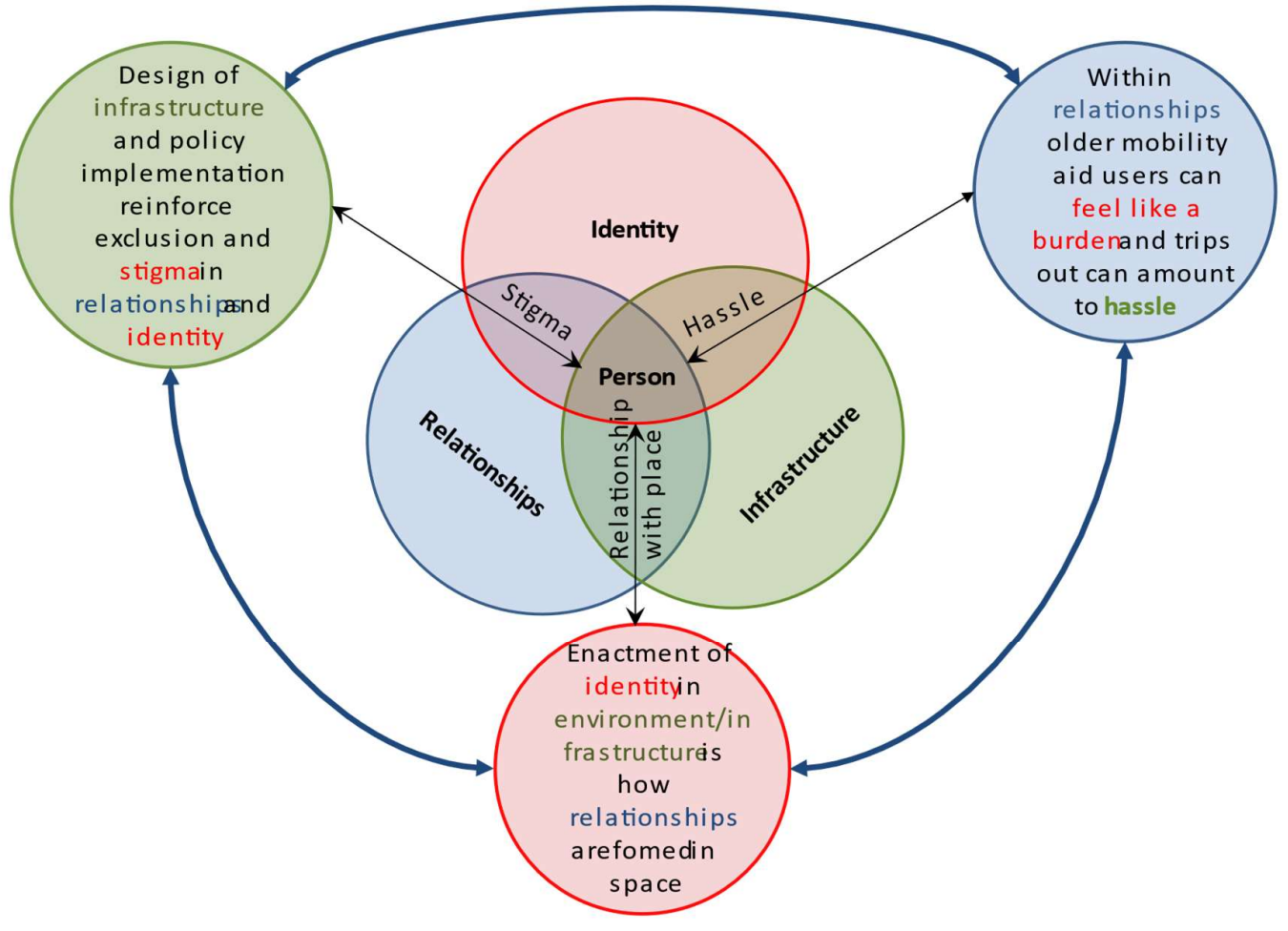

Figure 10.1 The intersection of relationships in analysis and results

Chapter 5 introduced these themes with diagrams related to the one above, explaining the themes and how intricately intermeshed they are. The subject matter of the themes, separated for the purposes of examination and explanation, and for further research, are 
not experienced in such easily distinguishable ways by individuals. Chapters 6 to 8 defined each theme in more detail, going on to explain how, for example, stigma is presented as part of identity within this research, but is readily explainable as an aspect of relationships. The diagram at Figure 10.1 highlights even more complexity in that the crossover of interpersonal relationships with personal identity labels the crossover as the place where stigma occurs. The interaction between identity and infrastructure represents the reality of identity interacting in space for mobility aid users. Where place becomes inaccessible because it is not mobility aid friendly, it can lead to what I have called in this study 'hassle'. As discussed in chapter 8 , hassle is not just one or two occasions of inaccessibility, but a litany of smaller but consistently obstructive and obtrusive events and obstacles that make going out an onerous task. Where day to day relationships overlap with infrastructure and systems relationships with place are manifested. Chapter 7 outlined the importance of place in maintaining identity and relationship with self, alongside relationships with others; partner or spouse, family, loved ones, friends and attendance at social events in terms at social gatherings.

The central triangular shape highlights the crossover of all themes, which is effectively the lived experience of older mobility aid users. It is difficult to over stress, or describe how inextricable identity, relationships and infrastructure are in daily experience and are not often separated in the thoughts of people as they carry out their routines. The outer circles of the diagram at 10.1 illustrate the interactions occurring within this triangle. The lower red circle indicates that enactment of identity in the environment enables relationships to be formed in space. As space and place impact identity, the nature of infrastructure in place informs identity and therefore relationships. The green circle to the left indicates that the design of infrastructure and policy implementation can reinforce exclusion and stigma which impacts up relationships and identity. Policy and implementation of services, even with the goal of supporting older people, can exclude some of the target population.

Reticence to make fuss or be a burden in relationships because of the extra difficulty of accessing the environment with a mobility aid equates to avoiding hassle. Burden and hassle impact upon identity as indicated in the blue circle to the right. These complex individual, social and infrastructural transactions are occurring through everyday life experiences and may not always be noticed consciously. However, because these factors are so 
interdependent, they form a feedback loop (figure 10.2) in which the built environment plays a part in identity formation and impacts on relationships. Relationships are therefore reliant on both the identity of the people involved, and the environment in which relationships are formed and maintained. Likewise, infrastructure, policy and planning are important factors in identity and relationships. It is imperative that policy, planning and services are envisaged and implemented not only in terms of the practical issue at hand, but the social, emotional and psychological factors impacted by the issue. Relationships are impacted by what might seem to be minor accessibility issues. As accounts outlined in sections 6.2 Dignity, 6.3 Stigma, 7.3 Feeling like a burden, 7.4 Relationships with place, 8.2 Planning design and service provision and 8.3 Hassle demonstrate, relationships are integral to experience, and therefore to experiencing use of a mobility aid. In addition, the fact that these issues are outlined in all three themes of this research shows how fundamental relationships are to experience and therefore how much attention needs to be paid to the ability to maintain relationships within policy and planning domains

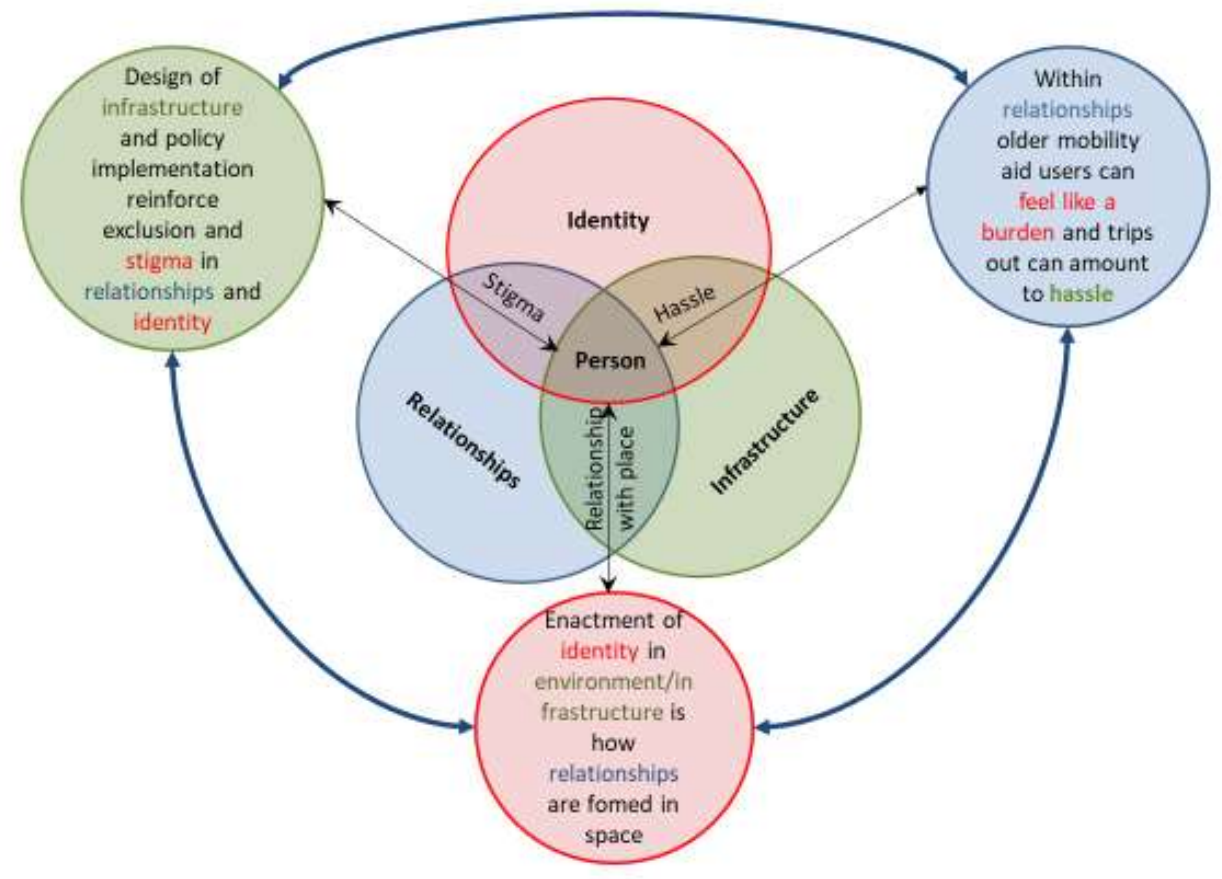

Figure 10.2 Feedback loop of obstacles to mobility aid use 
The feedback loop of consequences relating to mobility aid use can be seen more clearly in figure 10.3. By removing the Venn diagram that illustrated the intersecting themes emerging from data collection, it is easier to see the feedback loop. It is a simplified version that nonetheless reflects the accounts of participants. Design of space and place through infrastructural norms, systems such as health, housing and transport and implementation of policies for ageing, disability and mobility are all pertinent to the use of mobility aids by older people. The ability to navigate the environment and therefore participate socially, involves historically and socially situated norms of ageing and embodiment (for reasons outlined in Chapter 9). Inaccessibility and inability to participate also affect the identity of places, which are shaped by both infrastructure and those who use places (Cheshmehzangi, 2014; Imrie, 2000, 2012; Imrie \& Edwards, 2007; Johnstone, 2012; Spinney, 2016; Spinney et al., 2015). Both actual ability and the perception of ability to get around and carry out necessary daily routines independently affects identity and relationships. People do not want to be seen as different from others (Aileen), to be seen as old and doddery (Will), or to be perceived as not coping (Bret, Theresa). Such factors impact on identity because they make people feel embarrassed, inadequate or even unwelcome. Moreover, the need to call on loved ones for help or service providers to adjust for accessibility can make daily routines more time consuming and onerous and make the mobility aid user feel like a burden. Hassle, feeling like a burden and the difficulties created by inaccessible places and services serve to reinforce stigma.

The diagram in figure 10.3 could be further simplified as described in figure 10.4 , with an individual existing within relationships which are situated in the environments made up of social structures and infrastructure. This image makes clear the reasons for aspects of each section of obstacle being so connected to the others, that is, all are aspects of human experience and participation in life. 


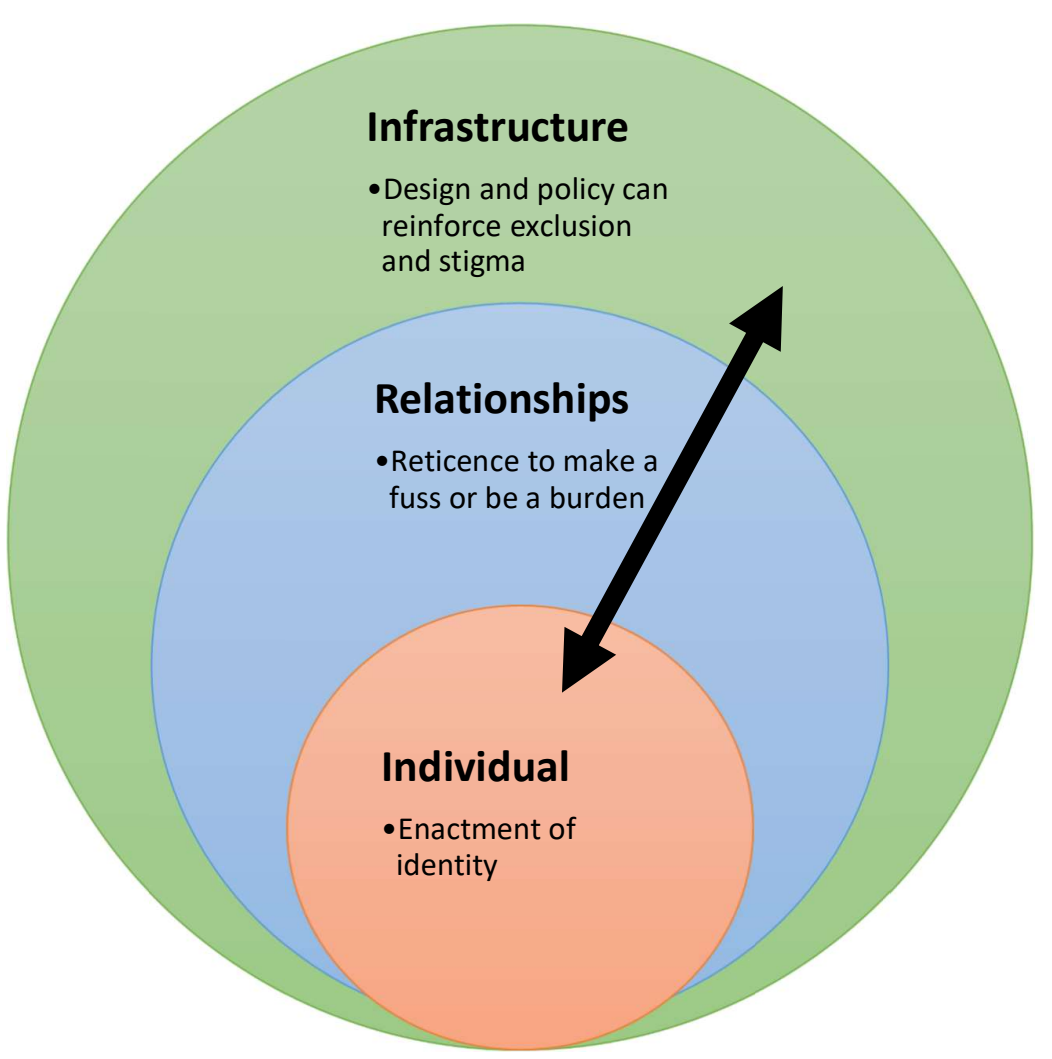

Figure 10.3 Situated reality of feedback loop consequences for individual

\subsubsection{Stigma, relationships and systems}

Wherever relationships can occur, so too can stigma. Goffman, when defining stigma, writes that stigma is used to refer to an attribute that discredits an individual, but that "a language of relationships, not attributes, is really needed" (Goffman, 1963/1990, p. 13.). Social relationships and social identity are where stigma is configured, personal identity control is the mechanism involved with stigma management in the individual, and what Goffman refers to as the ego identity (Goffman, 1963/1990, p. 130) manages the individuals feeling about stigma. People who are stigmatised simultaneously consider themselves to be normal, but also attend management of the stigmatising characteristic to appear normal to others. While knowing the disability or mobility aid (within the interest of this project) does not change the identity felt by the individual, they are also aware that it changes the way they are viewed by others. Aileen felt embarrassed by her disability and especially her mobility aid, so she did not use it as discussed in chapter 6 . Phil also noted that his illness and mobility aid changed how he sees himself as well as how he perceives other see him. 
The examples presented in chapter 6 above are in line with the concept of ego identity as a biography, presented by Goffman (1963/1990), whereby an individual perceives a continued sense of self that is added to throughout the life course. Goffman (1963/1990) notes that the idea of personal biography may be sustained in the minds of individuals but also some form of biography will be recorded in official documentation. While the former is subject to the memory and to some degree choice of the individual, the latter is not. Systems of official records and documentation create a further interaction between systems and identity. This personal identity biography can be compared to and contrasted this with the identity of state necessity. The mechanisms by which nation states and their legal systems utilise personal identification for economic, legal and welfare purposes are outlined by Goffman (1963/1990) as domains of regulation and power that enable and control social relationships. Using stigma as an analytic device for examining power relations, a mechanism by which certain people are dehumanised and devalued, Imogen Tyler (2020) uncovers stigma as a punitive apparatus. Tyler suggests the mechanisms of stigma are processes through which bodies are subjugated. Rather than relying on approaches that seek to ameliorate stigma, questions regarding the social causes, political function and understanding who profits from stigma are needed.

Neoliberal outlooks have brought about a culture which does not value older people for the wisdom, tradition and memory that ageing brings (Katz, 2015). When ageing is not valued as a natural process and becomes distorted by political and economic ideology, the poverty and disability that is often associated with ageing also fail to be recognised; individuals are blamed for failing to participate in their own empowerment with no account for the practical or social factors that may have been involved (Katz, 2015). As outlined in the examples from Aileen and Phil above, it is likely that only those affected by stigmatising traits will be aware of the discourses and impacts of stigma. The most striking thing about stigma for me in researching older people using mobility aids is the extent to which stigma still exists. Equality legislation has been in place for many years now, with the Equality Act 2010 preceded by the Disability Discrimination Act 1995, theoretically meaning an increasingly disability aware public, yet stigma and ableist views are still evident in the social structures and infrastructures of society as well as its citizens (Chasteen \& Cary, 2015; Dobbs et al., 2008; Hatzenbuehler, 2016; Tyler, 2020). Although policies and legislation are 
not necessarily concurrent with popular opinion, it is notable that infrastructure does not support legislation. This means to people who become chronically ill or have to use mobility aids because of ageing bodies find their identities and biographies altered through their changed embodied experience .

\subsubsection{Interrupted embodiment}

Interrupted embodiment is my conceptualisation of Bury's (1982) and Engman's (2019) notions of biographical disruption. This section highlights the ways in which mobility aids present interruptions to embodied experience which subsequently impact upon identity, which can in turn change the ways people interact with others and the environment. This section highlights the altered ability of individuals to interact with the environment, which has profound implications for the individual and planning and policy arenas. Chapters 6 and 7 highlight that the confluence of age and mobility aid use result in consequences for both identity and relationships for those who use any type of mobility aid. Whilst the section on Age Friendly Wales highlights the implications for policy and planning, this section demonstrates the individual perspective.

Changes to embodiment interrupt the flows of daily routines. In part, this study was inspired by the changes to daily routines, habits and attitudes occurring in my life with my husband and the subsequent changes to my own social rounds. The changes to circumstances that accompany mobility aid use ripple out through relationships to loved ones and beyond. Although assimilating the use of a walking stick or rollator may not incur the biographical disruption of chronic illness (Bury, 1982) as outlined below, it still changes the flow of movement and routines, which thus interrupts embodiment which in turn affects relationships and identity.

I completely identify with the participant in Giesbrecht and colleagues' (2015) study of older people transitioning to wheelchair use who spoke of buying sandwiches in the deli.

"You go to a deli - 'what kind of sandwich would you like?' - because he can't get in the door - so then you gotta remember the type of sandwich ... it's like 'with fries, without fries' - you have to kind of guess what [he] would like" ( Giesbrecht et al 2015 p7). 
For me, this kind of decision speaks of a much deeper sense of responsibility for myself, and loss of independence for my husband. It is all too much fuss to fight his way into a deli or bakers or other small shop, which means if we want to purchase something I either have to go inside to check the choices and then relate them back to him, or just make decisions for him. The whole situation stresses him so much that cross words are often shared, one of the only times we raise our voices at each other. It is a situation that illustrates what seems like a very small detail in a daily routine, but it represents the impotence he feels, as his quote from the feeling like a burden sub-theme highlights "The more requirement you have for a bigger mobility aid, the more your life is removed from you". Changes to Phil's embodied self have changed his ability to conduct mundane tasks and make commonplace decisions that feed into a feeling of his own life ebbing away. Such changes to self were observed in other participants, sometimes admitted, sometimes not, but changes to embodiment alter perceptions of self. Changes to embodiment interrupt the biography of self and the biography of records and categorisations if one becomes considered dependent or disabled. Interruptions to the sense of self because of changes to health status have been identified as biographical disruption, introduced as a concept by Michael Bury (1982) within a study of rheumatoid arthritis. Bury noted the additional effort it takes just to lead a normal social life when faced with a debilitating chronic illness and the attention brought to previously unattended actions and behaviours. The additional effort and attention incur a disruption to the biography of self. The fundamental rethinking of biography, self-concept and the need to re-examine resources are all facets of biographical disruption. Daily routines become an exercise in planning and deliberate action rather than the automatic actions they were before chronic illness. Activities that were once mundane and habitual in nature take more effort and planning to the point that they become not worth the extra effort (Bury, 1982). Rather than a focus on mobility difficulties, however, biographical disruption indicates this additional effort and disruption occur with the onset of illness. Biographical disruption is not produced by illness alone, but the effects of illness on ability to continue with pre-conscious, spontaneous activities in familiar ways. The nature of change was not experienced through the direct onset or experience of symptoms, but the way those symptoms encroached on daily routines and activities (Bury, 1982). 
Investigating the occurrence of biographical disruption using semi-structured interviews of organ transplant recipients in Canada, Engman (2019) highlights the important role of embodiment in biographical disruption. The physical body relating to the world forms the foundation of experiencing of the world. Rather than just experiencing our bodies, our bodies form the basis of our experience of the world. The term embodiment encapsulates Merleau-Ponty's (1968) concept of the body as a "vehicle" for being in the world, engaging with the environment through bodily experience (p84). The body provides perceptual and motor capabilities which develop continually through experience. Ultimately these capabilities and experiences lead to autonomy and decision-making abilities as experiences are incorporated into knowledge, understanding and the ability to take action (Engman, 2019). Both Bury's (1982) and Engman's (2019) studies found that biographical disruption starts with disruption to daily life. Embodied changes invite attention to physical limitations and cause consideration of subsequent limitations to the trajectory of that individual's life course. When people are unable to enact the roles they associate with their identities, identity becomes threatened. The experience of limitation and therefore biographical disruption posed by illness is relative to a person's embodiment (Engman, 2019).

While the findings herein support both Bury's and Engman's notion that disruption to daily life instigates changes to experiencing the world which threaten identity, the changes can occur through embodiment itself, or having to incorporate tools to assist embodied practices rather than necessarily through illness. Mobility aid use, no matter the illness or mobility issue, seems to create a difficulty because it disrupts or interrupts embodied experience, which seems to be the crux of biographical disruption. Mobility aids must be incorporated into embodiment to support or enable movement. It is an interruption rather than disruption because in some ways, the mobility aid enables continuation of activity, but it also interrupts previous flow, ways of conducting routines that must alter with mobility aid use. Interrupted embodiment is different to disrupted biography because it does not necessarily involve illness. Chronic illness and senescence, when creating the necessity for mobility aid use can be conceptualised as interrupted embodiment. As we age, we continue to add to our biography of lifestyle, history and identity, yet embodied changes interrupt these narratives with issues that require changes of course. These changes can be physical, structural or to relationships. Physical because of the need to incorporate a mobility aid into 
movement and mobility, structural because of the implications to altered ways of moving, different ways of traversing environmental elements such as dropped kerbs or steps.

Relationships can be interrupted by the change in pace and flow of movement and mobility, along with the changes to identity that can be absorbed by individuals when accessibility reduces.

Aileen and Phil, for example, consider(ed) themselves to be regular members of society, working, having relationships with family members and friends, meeting people, participating in social, economic and political proceedings as they saw fit, until their bodies made it impossible for them to continue working in the same ways. For Phil, chronic illness made employment impossible for him from the age of 49 . Aileen continues to be employed at the age of 58 , but requires specialist equipment provided by a charity. Dave continues to work following his amputation, but with contingent changes including reduced hours and alterations to hygiene facilities - work that he carried out himself. The changes in the ways their bodies work, and consequential use of assistive technology have interrupted the embodiment, and as a result the lifestyles and lifestyle choices of Phil, Aileen and Dave. Pam and Bernard highlighted the extra effort taken to reach a mailbox that is situated half way up a rise, meaning posting a letter for them becomes a more onerous task than for people who do not have to consider mobility aids or mobility difficulties for that matter. For other mobility aid users, they can be put off using an aid, as Will described with his mobility scooter when he knocked over a mannequin in a shop because the scooter is not permitted in shops, and he did not know. Moreover, there is not enough space to manoeuvre scooters or wheelchairs in shops or even hospitals, as recounted by Phil.

Engman (2019) asserts age mitigates biographic disruption because ruptures in identity are expected with retirement and older age, but any aspect of change to identity automatically alters relationships both intimate and more formal. The evidence presented here indicates interrupted embodiment also applies to the whole age range included in the study, that is 50 and over. Those over eighty also demonstrated aspects of wanting to retain biographical narratives. Bret wanted to keep his identity intact and did not want to be seen as unable to continue walking his dog, so did not tell his daughter about his falls. In fact, he talked about falling in a very nonchalant, offhand manner. Regina continued to hang her curtains nightly, not only wanting to be seen to be independent, but to feel independent and continue their 
identity and biography without interruption. To ask someone else to put them up and take them down would be to admit that she could not continue her routine as she always would have done. Retirement and ageing do not lead to disengagement from life and society, as some theories of the mid- $20^{\text {th }}$ century have purported according to the evidence presented here. People continue with the wish to add to a life course biography, spending time as spouse, parent, grandparent, friend and many other roles they may carry out, continuing their story with their identity intact. The use of a mobility aid interrupts a previous flow of life that continued almost without thought, in a pre-conscious manner before an aid was necessary.

The factors of interrupted embodiment demonstrate the ways in which policy and practice infiltrate all aspects of life. Changes to mobility and using a mobility aid can incur some level of change, or perceived change, to the ability to enact autonomy. Being able to continue with as much autonomy in life as safely as possible is paramount to maintaining identity. Although I have critiqued successful ageing for their own poverty or disability, thus disenfranchising them, it is true that Rowe and Kahn (1987) indicate autonomy as relevant to understanding successful ageing. As autonomy is shaped to a significant degree by the opportunities and constraints in the environment of the individual, social support is a psychological factor of well-being. Care needs to be taken that support provided enhances autonomy, as reducing autonomy can have negative effects on function (Rowe \& Kahn, 1987).

\subsubsection{Interrupted embodiment in the built environment}

The research presented here shows how embodied changes and the ways these changes interact with environmental factors enforce behaviour to be altered. Practices that were once automatic or not thought about too deeply actions become more difficult, time consuming and frustrating. Mobility aid use interrupts embodied experience, making mundane, previously pre-conscious actions require planning and effort. An appendage, be it a walking stick or wheelchair, brings an embodied alteration and the added association of stigma which impact upon identity, relationships and the ability to participate. In addition, people are wont to enact the old age portrayed by government, media and social discourse which is therefore expected by society. The old age in which they are to be active and 
successful, responsible for their own lives and activities within them. Chapter 2 illustrated the ambiguity involved in active and successful ageing tropes. Within academia, there is a difficulty for successful ageing theories in defining and measuring success. In evaluating successful ageing measures, Havighurst (1961) concludes that personality is a factor in which type of ageing is successful and no single simple theory of successful ageing is likely to account for all people. Nevertheless, the concepts of successful and active ageing have been incorporated into government rhetoric, policy and practice. While the term successful ageing may not be used overtly, ideas of ageing successfully and actively influence policy (Bowling \& Dieppe, 2005; Foster \& Walker, 2015; Katz \& Calasanti, 2015; Moulaert \& Biggs, 2013; Pfaller \& Schweda, 2019).

Literature reviewed in Chapter 2 highlighted notions of successful and active ageing evident in contemporary ageing theory and policy. The concept of successful ageing idealises a continuation of the activities, values and practices from middle age through into an individual's later ages as a way to maintain health and well-being (Aguiar \& Macario, 2017; Katz \& Calasanti, 2015; Schwanen \& Ziegler, 2011). This ideology may have informed lay attitudes because participants in this study all preferred to be seen to be independently active. As discussed above, independently is the important word in that phrase, as without independence, the activity seemed redundant in their approaches. Embodied changes during the ageing process can result in reduced bodily mobility, but the wish to remain independent remains. Being unable to continue independent life or becoming interdependent, whereby one has to ask for help or rely on others when an action could previously have been carried out alone without thought, anonymously, is felt as detrimental to identity (Crocker \& Major, 1989; Schwanen et al., 2012). Independence was a factor that emerged as highly important to identity with many participants either talking about independence or rejecting help.

None of the mobility aid users in the study mentioned active ageing, or policies about ageing or older people in their accounts, but some of the carers commented of a lack of salience in policies and planning for older people. Chapter 8 highlights the ways in which policy and planning can affect the daily lives of mobility aid users with inconsistency in approach, and by upholding negative stereotypes albeit possibly unknowingly. However, when given a remit of providing for all needs and people, local authorities have a duty of 
care to promote understanding and reduce rather than duplicate negative understandings of ageing, disability or mobility aids. Both as the researcher analysing responses and observing environments and situations of participants, and as the carer of a mobility aid user, I recognised the failure of policies to meet the needs of people who have mobility difficulties, illustrated by Dave's struggle with his local authority to establish the correct adaptations to his home both inside and outside, or residents of my town who have a friendship club, but those with accessibility issues cannot attend because transport has been stopped. Moreover, people living in the purpose-built housing need to catch a lift or taxi to the bus stop. To understand how local planning and policy addresses the needs of older people who use mobility aids, it is necessary to examine policy designed to provide for this population.

\subsection{Critical Geromobilities}

Thinking through a mobilities lens can provide a distinctive social science to formulate different theories, methods, questions and solutions (Urry, 2007 p. 18).

Mobilities research seeks to expose ways in which mobilities are produced and productive of social relations and constructions. Life is not static and cannot be researched as such (Urry, 2007). The movement of people revises borders and thus attitudes surrounding what is inside or outside of those borders, meaning that omission or non-inclusion of a group can lead to excluding the group through physical and metaphorical means (Smith \& Hall, 2016). If the circulations of people can be responsible for generating politics of power and inequality, policy and planning are responsible for ensuring accessibility in all its senses, otherwise they reinforce accepted norms that can reproduce politics of inequality and stigma. Dominant discourses of mobility within policy dialogues establish accepted truths emanating from travel, transport and communication which do not necessarily reflect social lives. The concept of mobility justice proposed by Doughty and Murray (2014) seeks to explore economic regimes of truth which maintain inequality, and are upheld by institutional and systemic practices as reflected in Ageing Well policies. Doughty and Murray's depiction of regimes of truth, which set the parameters of both understanding and practising socially, spatially and politically situated mobility, are reminiscent of Kafka's 'The 
Penal Colony' (2002[1941]) which inspired Imogen Tyler to conceptualise stigma as a machine. Tyler does not discuss mobilities, but the idea of machines of power echoes the corollary within much mobilities research that systems influence behaviour and thus people. Recursive relationships between space and place, personal and collective identities are influential in how included or excluded people feel. While much of mobility research focuses on the governing of mobilities, societies are often governed through mobilities (Doughty \& Murray, 2014; Kaiser, 2009; Newton et al., 2010).

It has been emphasised throughout this $\mathrm{PhD}$ that all aspects of [social] life for participants rely on some form of movement and accessibility. Obstacles to participation are physical and metaphorical, systemic and psychological. Utilising a combination of mobilities and critical gerontology as critical geromobilities is conceptualised to examine all aspects of the mobility of older people, as highlighted in Chapter 3. Critical gerontology questions an assumed ontological interpretation, suggesting a multidisciplinary approach is required to understand how much choice the individual has within the parameters of their life and social situations, socio-economic status, family and religious background (Luborsky \& Sankar, 1993). Combining this critical approach with the mobilities insight that movement is necessary for life to proceed allows examination of access to movement and mobility to be factored into gerontology as a discipline. Perhaps more importantly for the lives of older people who use mobility aids, critical geromobilities would be applied to policy and practice, to ensure the movement and mobility aspects of older people's lives are accounted for. Routine and perpetual work are needed to sustain day to day contact and social activity. Social synchronicity, the practices of understanding and building relationships with others, is achieved only by proximity and communication (Hodgson, 2011). Without the ability to maintain social contacts, social synchronicity is lost, and subsequently contacts diminish. To maintain mobility for those with mobility aids/assistive technology, or who live with interrupted embodiment or disability, it is imperative to ensure the mobility of all users is considered through all stages of design within planning and policy. End users, care-givers and health professionals should be at the centre of design innovations (Tsang, 2012), but mobility justice is regarded as peripheral, and more problematic for equality, subordinate to normative rhetoric of mobility (Doughty \& Murray, 2014; Hodgson, 2011). The concept of mobility justice seeks to level out inequality of mobility. Mobility capital (as discussed by 
Musselwhite and Scott 2019) is proposed as a method to understand the ability for older people to mobilise and understand the impact of mobility on health and well-being. Critical geromobilities is proposed as a sub-paradigm of the mobilities turn, an approach to researching the mobility of older people, and also to address mobility in all aspects of gerontology. Büscher et al (2016) note many mobility intersections to enrich and deepen conversation about the breadth of mobilities research, however gerontology or ageing research is not mentioned. In light of the ageing population, understanding the ways age and movement are approached, constructed and intersect in society is imperative. Critical Geromobilities could and should coordinate the unheard voices of social groups into a set of coherent ideas.

\subsection{Age Friendly Wales}

The final section of chapter 2 outlined the drive by the Welsh Assembly Government and Older People's Commissioner for Wales to make Wales an Age Friendly Nation. As many as $46 \%$ of those over 50 in Wales reported a mobility restricting disability in the 2011 census (ONS, 2018), meaning mobility restrictions should be reflected in ageing policy and Ageing Well in Wales documentation. However, the Ageing Well in Wales document produced by the Welsh Assembly, and the Dublin Declaration on which the ageing well framework in Wales is based, both omit to mention mobility or mobility aids at all. There is consideration of accessibility, but it does not appear to have been translated effectively through implementation of policy and practice in day-to-day terms. This part of the discussion considers mobility issues being misunderstood, misrepresented or disconnected from other service provision within policy and planning within local authorities. The potential reasons for and consequences of not linking systems to make different services synchronise is outlined here to underline the implications for mobility aid users.

One of the reasons the theme in Chapter 8 is called Infrastructure and interlinking systems is to highlight the lack of salience in policy, planning and systems intended to support older citizens, but do not work in a coherent way to provide consistent service. The icons of age friendly cities are a good way to discuss this. The icon at figure 10.4 displaying singular petals was the one displayed on the Age Friendly Wales website when I first accessed it in 2017 and later in 2018. It displays the eight domains of age friendliness all held together by 
the central pistil of the flower. Each section is stand alone and seemingly not related to the others other than they all need to be age friendly.

When first researching the Ageing Well in Wales initiative, the icon shown in figure 10.5 was the one showing on the Ageing Well in Wales website (OPC. It shows a similar flower, this

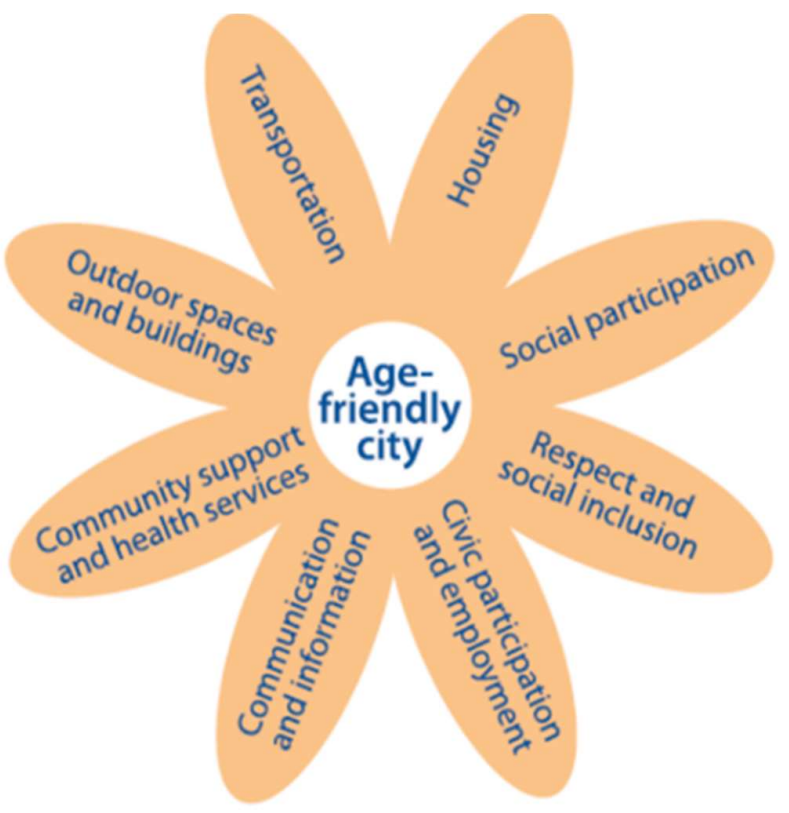

Figure 10.4 Who Age Friendly Topic areas (2007) as per Age Friendly Wales website Nov.2018

time with some of the inner petals grouped together by external petals. The names of the eight domains are slightly different and the order has changed. The first three in the newer icon, 1. Outdoor environments, 2. Transport and mobility and 3. Housing are grouped within a 'Physical Environment' petal, highlighting the relationship between the three domains as interrelated. The social environment is grouped together in another external petal, enscapsulating 4. Social participation, 5. Social inclusion and non-discrimination, and 6. Civic engagement and employment. Social inclusion, social participation and civic engagement are all related. They may be contested or debated terms in academic or political arenas, but in the lives and day to day experience of older people, these domains represent taking part and being valued as equals with all other age groups. The final grouping is Municipal services which incorporates 7. Communication and information and 8. Community and health services. The three external petals, that is physical environment, social environment 
and municipal services all appear to overlap slightly. The WHO Age Friendly Environments Europe Icon shown in figure 10.6 better illustrates the overlapping, inter-reliant and interlinking nature of the domains of age-friendliness or policy, practice and planning that seek to implement age friendy approaches. While these are all slightly different aspects of the built and social and environment, they all make up what would be defined as

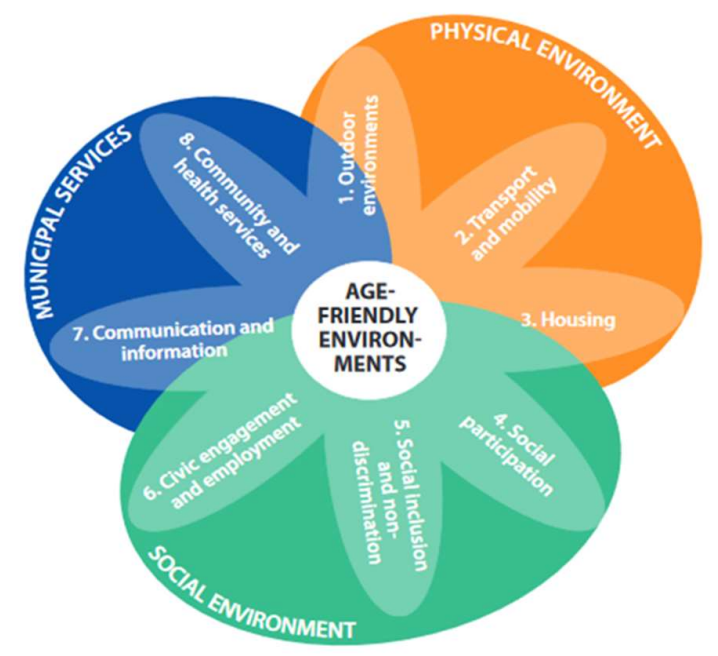

Figure 10.6 WHO Age Friendly Environments Europe Icon

infrastructure.

The latest iteration of an age friendly icon used on the Ageing Well in Wales website is shown in figures 10.7 and 10.8 . In figure 10.7, whilst the petals are overlapping, they do not

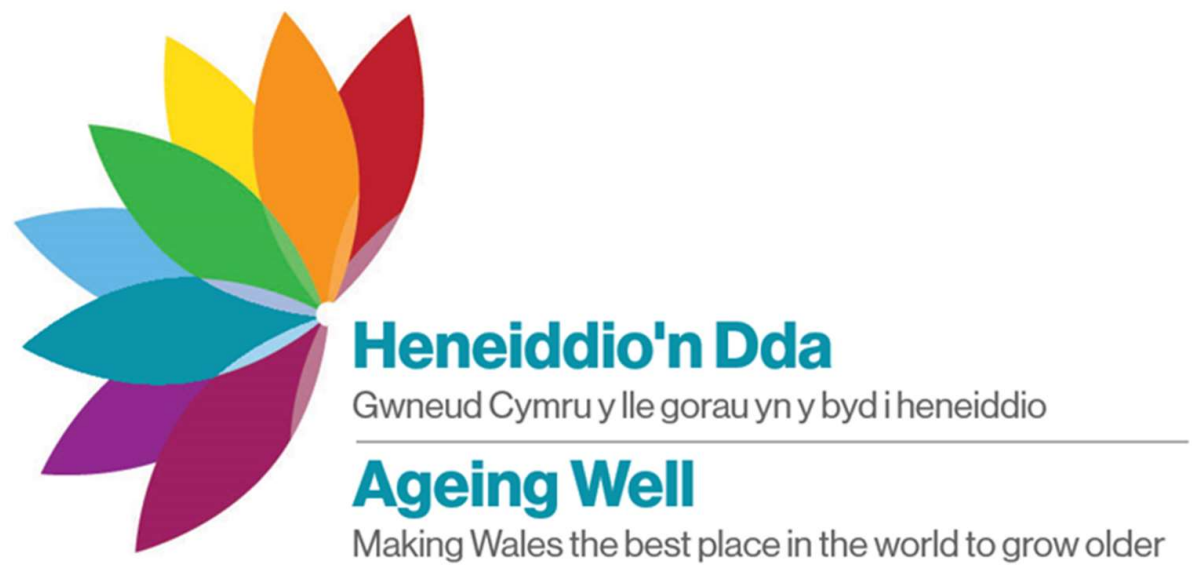

Figure 10.5 OPC Ageing Well Icon

name the domains and as such are redundant in signifying any meaning of domains being 
interlinked or overlapping. The pictoral representations of age-friendliness also erode the message and values provided in WHO literature outlined in section 2.7.2 which multifaceted and inter-reliant nature of age-friendliness policies.

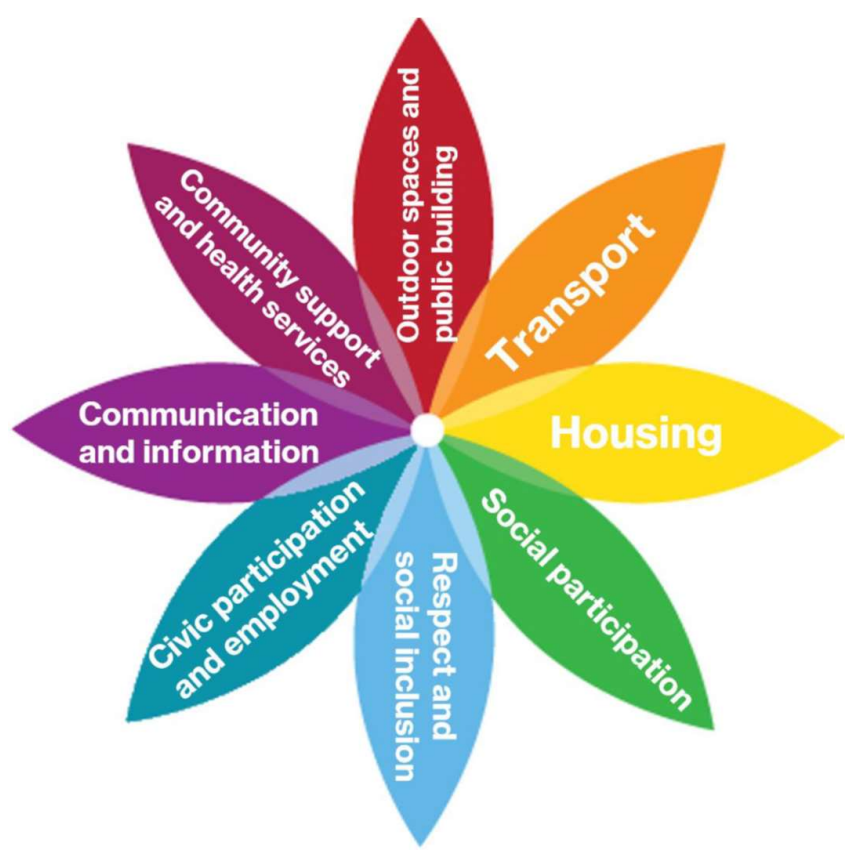

Figure 10.7 Current online version of 'Making Wales a nation of age friendly communities'

Whilst introducing the of theme of Infrastructure and interlinking systems in Chapter 5, I highlighted the necessity for outdoor environments and transport and mobility to work be synchronised in order to provide accessibility for all, thus providing opportunities for social participation:-

This theme explores how the external environment presents obstacles to mobility aid users, incorporating planning and policy because these regulate all aspects of the built environment in some way. Outdoor environments, social participation and transport and mobility are three of the eight domains or age friendly cities or communities, and potentially not only have to be age friendly in and of themselves, but also work coherently with each other and the remaining domains to provide accessible environments for all.

There is a need for different domains within infrastructure and the systems and services linked to it to be synchronised and work together to provide consistent access to social 
participation and citizenship. As discussed by participants, housing, transport and outdoor environments are inter-reliant in daily routines. Access to bus routes is needed for older people to access shopping, family and friends or social events. While in the interrupted embodiement section of this chapter outlined the difficulty of incorporating interrupted embodiment into daily routines, the examples given in this section and within chapter 8 highllight the added obstacles presented by inconsistnet policy, service provision and outdoor environment. Dave's difficulty obtaining a dropped kerb to gain wheelchair access at his residence, Regina noting the distance to the nearest bus stop from her purpose built older persons' bungalow. In fact the complex, inter woven nature of all of these aspects of daily life are outlined throughout chapter 5 whereby the overlapping, fluid and to some extent inseparable properties of the social, physical, psychological/individual and infrastructural environments were outlined.

Housing, transport, health care and even disability provisions are theoretically meant to support and enable the lifestyle and mobility of people with mobility difficulties (among others). The reality is a stream of not so well thought out, added on provisions that do not necessarily meet the needs of the people they purport to support. One reason for this could be that governance holds assumptions that those who live with embodied mobility difficulties cannot live without the support of other people (Kaiser, 2009), thus help from other people tends to be assumed with accessibility solutions. Another is the lack of interdepartmental co-ordination or interdisciplinarity with mobility and accessibility requirements failing to be incorporated with all other needs at planning stages (Aguiar \& Macário, 2017). Musselwhite and Scott (2019) found that infrastructural mobility capital, the provision for mobility needs by infrastructural means such as public transport and wellmaintained pavements, was the most important factor in facilitating mobility. Their study highlights that the difficulties older people have accessing transport and mobility are with the systems rather than there being a 'problem' with older people. When a mobility aid is included, more accessibility solutions can be required, but governance and planning assumptions or oversights disallow mobility aid users from easily accessing infrastructural provision.

The Infrastructural aspects of daily life are provided and managed by municipal services, with the needs of society intended to be met by those in authority. Laws, government and 
local strategies, policies and initiatives are impleted at local levels by municipal services, meaning implementation is dependent on both interpretation and local conditions. This could also leave the best intended aims open to misinterpretation, ineffective implementation and inability to manifest plans into services especially in times of austerity. Further examination of the ageing well plans presented by unitary authorities throughout Wales, discussed in chapter 2, highight this. None of the ageing well plans, nor Ageing Well in Wales documents, WHO or European Union Ageing Well literature mention mobility aids. This is problematic because from the point of inception, Ageing well plans assume able bodied mobility. The language used in literature, not only of policy documentation but also the general approach to health, well-being and service provision is important. Language can be preloaded with values and false dichotomies (Pfeiffer, 2000) so the absence of mobility being mentioned implied it to be abnormal and not incorporated as a normal factor of ageing. Meanwhile, $46 \%$ of people 50 and over, $54.5 \%$ of people 60 and over identify as having a restriction to their mobility. Around half the target population meaning mobility changes are not abnormal in older people.

The dichotomy of normal and abnormal subsequently reinforced by omissions are values placed on variations of biological function funcion according to Pfeiffer (2000) who notes that these variations are deemed acceptable or unacceptable with value judgements that justify advantage or disadvantage. This notion reflects the value judgements used in successful ageing theories and active ageing discourses and policies whereby judgements regarding the physical mobility, and mobility aid use of older people revolves around valued judgement of policy makers and the public. Being members of the public as well as mobility aid users, general attitudes and opinions of the day influence evaluation of self so identity is impacted by policy discourse and rhetoric regarding ageing, mobility, and mobility aids. As a case in point, Gwynedd Council's (n.d.) Ageing Well literature presented a fictional lady widowed and lonely. The solutions offered in the plan suggest relocation to nearer family and or closer to an Age Well Center, yet did not provide any suggestion of provision for this. There is an element of responsibilisation to this, in that each older person is responsible for getting near to a services, rather than services being provided within reasonable distance of all older people. Swansea's plan was presented on an extremely difficult to read document that did not appear to be designed for public reading, yet the information indicated the 
inclusion of older people's views. As pointed out in the literature review, it is difficult to ascertain how physically accessible or publicised the forums were. Carmarthenshire highlighted inadequate funding, which is no doubt an issue for all local authorities.

The issues highlighted above indicate inconsistency in both interpretation and implementation of policy. There appears to be incosistency and a lack of understanding of the extent or impact of embodied changes as people age. A large section of people over 50 report activity restricting disabilities yet this is not incorporated into planning, policy or service provision in any meaningful way. While not all of those with mobilty restrictions need mobility aids, embodied differences and altered mobility must be a priority in addressing ageing in the population. While there is a focus on accessibility within the Dublin Declaration (2013) and WHO literature, local plans do not seem to have carried this idea forward and addressed what accessibility actually means for older people. Embodied capacity is spatial and environmental (Doughty \& Murray, 2014; Finlay, 2003; MerleauPonty, 1968; Schwanen \& Ziegler, 2011). Thus policy and planning, as documented by ageing well plans and strategies for older people, can only be useful when they are meaningfully implemented and positively impact on daily life.

10.4.1. Role of policy and planning in the Feedback loop

The two main aspects of policy and planning highlighted in this discussion of findings, the synchronisation of different areas of policy and planning, and the acknowledgement of mobility aids and mobility difficulties in planning and policy discourse (or lack thereof) have a meaningful impact on the daily lives and routines of people 50 or over who use mobility aids or live with mobility difficulties. Discussion around the interpretation and implementation of Ageing Well Plans, and within them age friendly cities and communities' initiatives, highlights the ways policies need to be consistent and work in conjunction with each other to be effective. While not all of mobility restrictions represented in census statistics will necessitate use of a mobility device, failing to consider mobility aids directly fails to address an important fact of ageing, and also reinforces a reticence to discuss mobility aids and mobility difficulties. This can lead to further marginalisation because lack of representation is compounded by social and economic concerns of the public. Disability, especially when manifested as significant bodily differences has historically been identified 
as a threat to society. Not a physical threat but a threat to social, economic and corporeal stability (Burghardt, 2013; Kristeva \& Lechte, 1982). Moreover, promotion of accessibility for the mobility of people who have mobility difficulties is seen as an add on to infrastructure that already exists which represents additional cost and burden on public funds for a minority (Aguiar \& Macario, 2017; Hansen \& Philo, 2007 ). Approaches to infrastructure and social structures that consider the emotional, psychological and relational aspects of the interaction in and with the environment would enable better synthesis of people with all types of embodied abilities.

Successful ageing does not incorporate notions of choice and lifestyle or intersecting inequalities (Katz \& Calasanti, 2015). This is reflected in the absence of mobility and accessibility discourse in both policy and planning which translates into less physical presence of people who use mobility aids. Without accessibility, people tend to frequent places less. Regina, Theresa, Bret, Will, Pam, Stuart, Aileen and Ron all reported a reduction in activity of some kind. Regina could not walk as much because she did not feel safe and did not know the environment well since relocating; Ron could not get into the bank, so bank staff came out to his car; Aileen felt completely vulnerable and refused to use buses, Will had a bad experience in his mobility scooter so refused to use it, and refused to go on stage being 'doddery'. Stuart does not go out with his wife as much because both of their mobility needs have become more complex, although they did not express it this way. Phil has become a practical recluse because of the way using a wheelchair makes him feel, not just physically, but the attention it brings socially. Some of these issues have no solution and are personal choice, some are not. Sometimes, particularly for those in the 'older' older people category, generally aged 80 and above, there is a reticence to admit the need for help, even to oneself, because it activates thoughts of reduced autonomy, enforced care and decision-making rights being removed, as identified by Gilleard and Higgs (2011).

The consequences of older people with mobility aids being unable to carry out activities of their choice returns us to the feedback loop. Just as identity and relationships are affected by policy and planning, all areas of policy and planning pervade issues of identity and relationships, particularly for those with interrupted embodiment. Without the correct policy and planning in place, people who use mobility aids become excluded both physically and socially, in a spiral of feedback that both informs and is informed by social practice 
which influences policy. Places are defined and made by use, with infrastructural and social structures playing a part in constructing the rules determining who belongs and who does not. Much research from a variety of disciplines has found that social and spatial interactions define inclusion or exclusion (Burghardt, 2013; Cheshmehzangi 2014; Imrie 2000; Johnstone, 2012; Korotchenko \& Hurd Clarke, 2016; Milner \& Kelly, 2009; Peace 2013; Spinney et al, 2015). The ways people interact with each other, and how the environment encapsulates these behaviours can highlight how issues of personal identity, embodiment and sociability can make the environment more or less enabling and inform social participation.

The concept of successful ageing as interpreted within policy and practice does not incorporate notions of choice and lifestyle or intersecting inequalities (e.g. Katz \& Calasanti, 2015). What does address inequality and accessibility is the supporting pledge listed on the Dublin Declaration on Age Friendly Cities and Communities in Europe 2013 as signed by all unitary authorities in Wales. The pledge states that by signing the declaration, the authority is taking responsibility for highlighting the needs of older people as shown in the section quoted below:

\section{Supporting Pledge}

11. The undersigning cities and communities also pledge, where it is within their area of responsibility and economically feasible, to further the following specific actions, and to incorporate them into key planning instruments to strengthen long term sustainability:

- Promote among the general public awareness of older people, their rights, needs, and potentials, and highlight the positive social, economic and cultural contribution they make.

Raising public awareness and education regarding older people's mobility and use of mobility aids, along with the contribution older people make to society would help to address the issue of mobility difficulty as a normal part of the ageing process. More complex is addressing the needs of older people, because of the inter-reliant, complex and multifaceted nature of those needs which are also highly interrelated to identity and personal relationships. There are older people who refuse help and are determined to demonstrate 
their independence, as illustrated by the narratives of Bret, Regina and Theresa. As discussed through the independence sub-theme in chapters 5 and 6, and in explanations above regarding interrupted embodiment, independence is closely aligned to autonomy and the ability to apply one's own will to daily life and decision making.

In addition to questions of independence and autonomy with diminishing mobility is discourse of disability. Research seeking to understand representation of disability indicates the echoes of historic marginalisation and disempowerment that has been suffered historically. The categorisation of disabled remains a state considered diminished as a human being (Korotchenko \& Hurd Clarke, 2016), or at the very least ableist assumptions dictate normality (Hammel et al., 2013; Hamraie, 2017; Milner \& Kelly, 2009; Pfeiffer, 2002; Serlin, 2012; Woods \& Watson, 2004). If people are having to arrange installation of dropped kerbs so that they may navigate the environment in wheelchairs, scooters and with rollators as two of the participants in this small sample had to, the society we live in can only be termed ableist. Ableism of environments is linked to the everyday stigmatisation, patronising and demeaning social acceptance of differently abled people not just through discourse, but also design and planning (e.g., Hammel et al., 2013; Hamraie, 2017; Hansen \& Philo, 2007; Imrie, 2000, 2012; Milner \& Kelly, 2007).

Policy and planning, along with the research that informs it, must incorporate the movement necessary for each aspect of human life. The value of mobility and movement is evident throughout this research in the narrative and analysis of participants accounts of their daily experience and routines. Relationships require some aspect of movement and mobility to be enacted. The absence of movement has a negative effect on both identity and the ability to navigate the infrastructure of the built environment. Without the same access to mobility and movement, people who require mobility aids are not able to conduct relationships, or utilise elements of infrastructure in the same way as those with more able bodies. As demonstrated throughout this research, these factors can negatively affect identity. Other research also indicates a link between lack of mobility and detriment to health and well-being (Aguiar \& Macario, 2017; Lucas, 2011; Mackett, 2018; Musselwhite, 2018; 2017; Schwanen \& Ziegler, 2011). The importance of access to movement evident in the lives of older people who use mobility aids is highlighted by the mobilities paradigm. Movement is accessed or blocked, constructed or constricted according to social, spatial and 
environmental structures (Büscher et al., 2016; Dorow et al., 2017; Doughty \& Murray, 2014; Lucas, 2011; Murray, 2015; Sheller \& Urry, 2006, 2008; Smith \& Hall, 2016; Urry, 2007). Lack of access to any of these structures is tantamount to an obstacle to participation.

Mobility aid use has been shown here to interrupt embodied experience of self. This can be magnified by environmental factors in policy, practice and infrastructure. Omittance of mobility aids or their management in plans or literature regarding ageing adds to stigmatising processes which impact upon both identity and relationships, as demonstrated by the feedback loop in figure 10.2. If mobility aid use and users were more visible, their use would be less stigmatising to use mobility aids and loss of dignity may be reduced. Losses and frustrations can both be caused by the complex interactions between individual, relational and infrastructural elements of everyday mundane experiences Frustrations that may occur from loss of independence, autonomy and dignity impact upon relationships. Relationships with family and friend are affected because of the complicated evaluations we all make about our independence, requirements and needs. While policy and planning cannot provide all the answers to these personal and relationship complications, the hassle involved in navigating environments that do not cater for the needs, or do not understand the needs of mobility aid users could be resolved. To better maintain pavements and provide training that involves understanding of how mobility aids work, and perhaps more pertinently, how people who use them might feel, would go some way to improving the stigma, frustration, sense of burden and hassle involved in mobility aid use. Infrastructure infiltrates relationships and identity, as do planning and policy as human aspects of infrastructure.

This chapter has indicated the interconnected nature of human experience that can be splintered and disjointed through policy approaches. Relationships and relational aspects of human life are implicated both in and alongside mobility practices. The individual navigates the environment through relationships, the environment is sustained and maintained through policy, planning and practice. The feedback loop of obstacles to participation demonstrates the ways in which the themes identified in this research, that is 'Identity', 'Relationships' and 'Infrastructure and interlinking systems' are inextricably linked. Policy and planning is therefore highly implicated in the identity and relationships of citizens, with 
the potential to reinforce stigma (as noted by Pescosolido and colleagues (2008) regarding approaches to mental health policies). When policies are aimed at older people, the particular issues relating to that population need to be accounted for. Mobility is a consideration that passes through all eight domains of age-friendliness, so more care in understanding, communicating and implementing the need for synthesis of policy and service provision is a goal for an Age Friendly Wales. 


\section{Chapter 11. Conclusions}

The conclusions outlined in this chapter refer to the research questions that I set out to answer, the themes that emerged as data collection and analysis occurred, and the way I have conceptualise the obstacles to participation uncovered. The implications of the findings within this research are then discussed in relation to both older people's mobility aid use, and the Age Friendly Cites and Communities framework. The chapter, and indeed the thesis ends with a summation of the contribution to knowledge, opportunities for future research and recommendations. To begin with, I reflect on my role as a researcher.

\subsection{Reflexivity and the research process}

It has been an important facet of this research project to be reflexive, not only because it is an important factor of the methodology, but also because it provides a transparent account of the research decisions and role of the researcher. On a personal and professional level (holding an MSc in Social Research Methods) transparency is of paramount importance because it allows the reader to understand the reasoning and context of the research. It has occurred to me through this process of achieving a PhD that the thesis is not a report of research per se, but a story of the process of learning how to conduct research. In that way, it has been important to indicate the thoughts and reasoning behind decisions in much the same way as the workings are as important as the answer in a mathematics exam.

In Chapter 4.2 I outlined my understanding of my role and position within the context of this research. Through the process I have given insight to the challenges of the research. In 4.1.4, for example, I indicated the challenge of not finding what I thought would be present when interviewing participants. Later, in section $8.3 \mathrm{I}$ recount the shock I felt at my own husband's analogical account of feeling so stripped of basic need when accessibility is not available. It has been my intention to be transparent and professional with these reflexive accounts, but also to allow readers to evaluate my personal and theoretical perspectives within my account and analysis.

Utilising the mobilities paradigm using walking or go-along interviews has highlighted the way that this method of interviewing provides interesting insights. Firstly, because walking around a neighbourhood being led by the participant gives some control of the interview to 
them and away from the research, levelling the social status, and allowing the participant to feel more at ease. The person leading gets to choose where to go and can take charge. This research highlights the value of that social levelling effect when approaching potentially socially unacceptable or stigmatising issues such as ageing and disability. There is another great advantage with the go-along interview, in that it provides insight into what is not said. I have demonstrated in this research, either through the people who chose not to walk, those who said something but acted slightly differently, or those who did not do something but did not offer a reason, that sometimes, the interesting data is what is unspoken. These are contributions to knowledge regarding the application of walking interviews

At the outset of this research, I expected to find mixed feelings about mobility aid use; in a broadly general way, positivity about enabling movement and negativity about disabling environments and impractical mobility aids. As outlined throughout, I have in depth knowledge of mobility aid use as the wife of a walking stick and powered wheelchair user. My understanding of mobility aid use (more than ageing) is a much social and personal as it is academic I viewed mobility aids as tools for navigating the environment and set out to understand them as such, with underlying knowledge that there would be obstacles to participation given the existing literature regarding accessibility and participation of older people with mobility difficulties. While understanding mobility aids as tools of navigation remained the aim throughout, it is not necessarily how those using mobility aids viewed them, with social and emotional aspects over-riding practical considerations. A complicated and consistently intermeshed set of circumstances and experiential concerns have been uncovered to provide insight into the complexity of understanding mobility aid use by older people.

\subsection{Overview of findings}

The predominant aim noted at the start of this project was to describe the routines and activities of mobility aid use by older people in Wales. This was not necessarily achieved in the way I hoped it might have been because of some limitations in the research. I was able to describe the activities I witnessed with participants, and many also discussed their experiences with me. The daily routine experiences discussed ranged from weekly blood test appointments attended at the local hospital to daily dog walks, having to hire a taxi to 
attend a doctor's appointment to a financial advisor from the bank coming to the car to give advice when accessing the bank was impossible. However, people seemed either reticent to discuss their daily routines, or as is just as likely, routines are so automated that they cannot be easily recounted. Some people do not find their daily activities to be centred around their mobility issues, chronic illness or disability especially if the mobility is not greatly limited (Elliott et al., 2005). The routines were only part of the information of interest, as some of the material recounted in the themes demonstrates. The disparity between what was being said and what was occurring was very interesting. In terms of describing routines and activities, this goal was only achieved in small part, but then it was an ambitious goal. The accomplishment here was in establishing the ways people who use mobility aids do or do not continue with daily routines, and how they change their lifestyles and routines to accommodate the changes to their mobility and mobility aid use.

The research objectives were as follows:-

- To describe older people's mobility aid use within daily routines and activities.

- To explore the meanings older people attach to mobility aid use.

- To explore any changes to participation that have occurred since each participant began to use their mobility aid.

- To identify and critically discuss the individual, relational and structural factors involved when older people use mobility aids, including any obstacles and barriers faced.

- To evaluate age friendly policy on national and local authority levels to understand how mobility aid use is assimilated into policy, planning and services

The project has described well some older people's mobility aid use in both rural and urban locations in Wales. Although collecting data regarding mundane daily activities presented difficulties during participation as by my presence, I was interrupting routines , the research produced a rich set of data outlining mundane but highly important ways in which mobility aids shape the routines and movements of older people who use them. The sample set was relatively small, and a larger sample size with a wider variety 
of settings and types of mobility aid would have given a more comprehensive description. However, the smaller sample size allowed for deeper exploration of meaning attached to mobility aids by users. As reflected in the sections below, meanings were complex and impacted identity and relationships in the infrastructure of the built environment. Stemming from this were aims to understand any obstacles or barriers to participation that may be present with mobility aid use, as discussed in previous literature (Bromley \& Matthews, 2007; Bromley et al., 2007; Hansen \& Philo, 2007; Imrie, 2012; Mazurik et al., 2014; Oliver, 2009, 2013; Williams et al., 2001). Individual, relational and infrastructural obstacles to participation were identified and highlighted as interrelated via the feedback loop model of obstacles to mobility aid use as shown in figure 11.1.

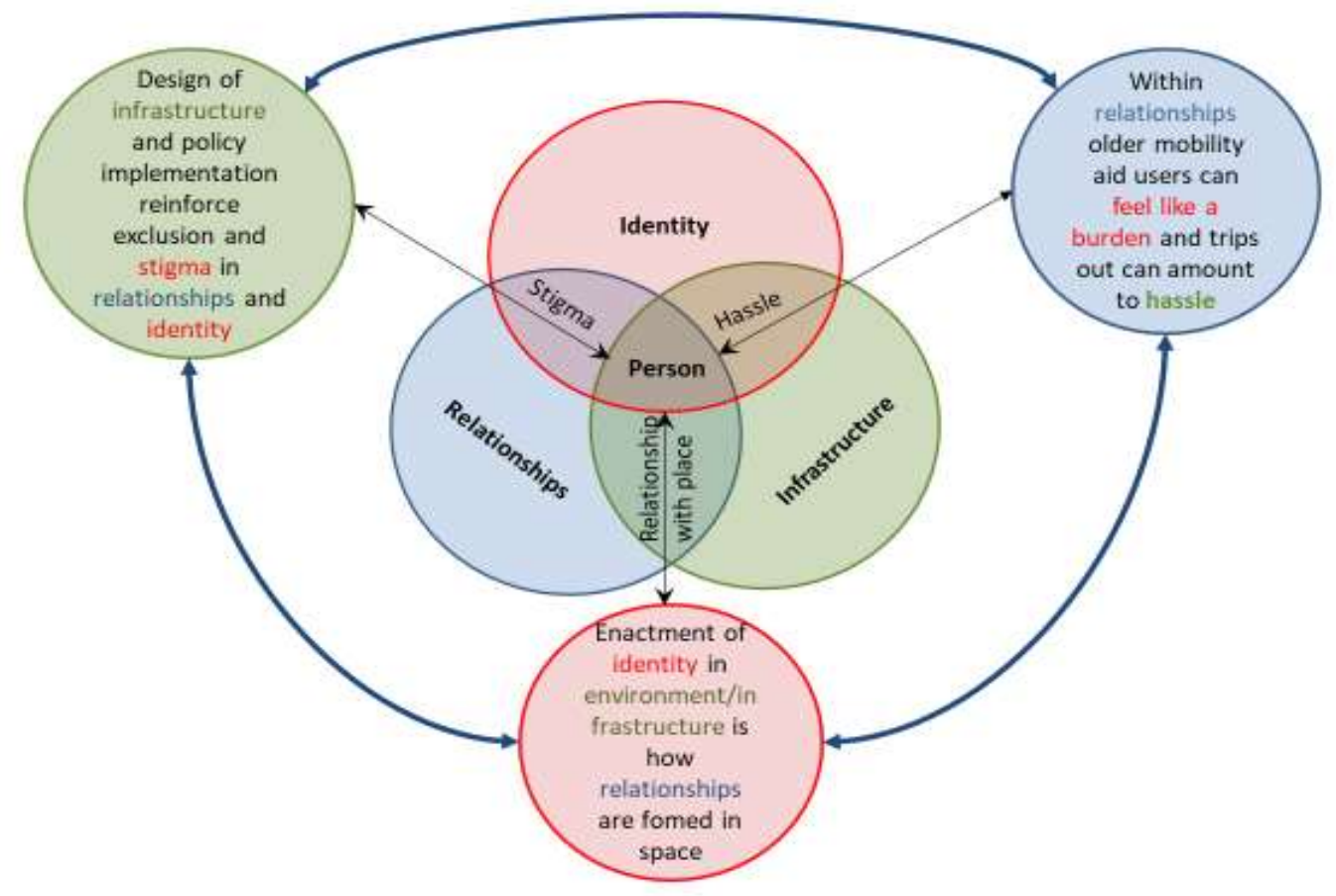

Figure 11.1 Feedback loop of obstacles to mobility aid use

Aims also included exploring the use of mobility aids within the built environment to examine perceptions of planning, policy and services. This has been achieved through evaluation of international, national and local policy interpretation and implementation of Age Friendly Communities initiatives as discussed in chapters 2 and 10. 


\subsubsection{Research Questions}

The overarching research question of 'How do older people relate to mobility aids and how useful are mobility aids in navigating and enabling everyday routines and scenarios?' included the sub-questions:

a. How do older people relate to their own mobility aids?

b. How do older people feel about or consider mobility aids in general?

c. How do older people feel about themselves when needing to use a mobility aid?

d. How do carers feel about their loved one's use of a mobility aid?

e. How do older people feel the built environment incorporates the need for mobility aids?

f. How well do older people feel their mobility aids enable them to continue with their everyday routines and lifestyles?

g. If there are obstacles to participation as previous literature suggests, what are the obstacles to participation?

h. How effective are age friendly policies in addressing the needs of older mobility aid users?

The first three sub questions ( $a, b$, and $c)$ are answered mainly by the section on identity. Older people's thoughts about their own use of mobility aids, consideration of mobility aids in general and feelings about themselves when needing to use a mobility aid are closely linked to identity and social roles. As discussed throughout this study, aspects of identity, relationships with others and the external environment are interlinked and impact upon each other. The way an older person feels about self when needing to use a mobility aid (c) is also closely linked to how carers feel about their loved one's use of mobility aids (d) and how older mobility aid users feel the environment incorporates the need for mobility aid use (e). These three subsections are addressed in the relationships subsection, highlighting both the inter-relationship between identity and the subsequent changes to relationships with other people, and also how changed interactions with place can affect perceptions of self and ultimately the identity of places, leading to lack of accessibility and social exclusion. These consequences are discussed in the section on infrastructure which outlines the 
changes to participation (g) and obstacles to participation found in this study. The effectiveness of age friendly policies $(h)$ is addressed in the section on obstacles to participation, which also underlines the intricate complexity of the inter-relating nature of physical, psychological, social and infrastructural obstacles.

11.2.2. Overall, the question 'How do older people relate to mobility aids and how useful are mobility aids in navigating and enabling everyday routines and scenarios?' has been answered, however, the practical factors of mobility aid use I expected were superseded by more psychological and social factors. It is highlighting physical, social, and psychological costs to older mobility aid users in gaining accessibility and mobility, and indeed the complex interaction of these factors, that forms an original contribution to knowledge within this research. Identity Chapter 6 identified the ways in which mobility aid use is implicated in the continued process of identifying self with groups and roles (e.g., Carter, 2013). Taking up use of a mobility aid led the participants of this study to question their roles or adapt to new ways of thinking about self. Roles are impacted by an individual's ability to be independent, which in turn affects dignity. Dignity is important in light of an ability to be autonomous, but also plays a part in feeling stigmatised. Stigma bridges a gap between identity and relationships because it impacts an individual's identity but exists within social constructs and relationships.

\subsubsection{Relationships}

Chapter 7 highlights the integral nature of relationships to personal experience in social worlds. Loved ones and carers highlighted the difficult terrain of relationships around mobility aids use, as the mobility aid user themselves are often navigating complex feelings of wanting to remain independent, continue with life needs, goals and requirements, yet at the same time feeling like a burden, which can lead to disagreement and discord.

Relationships also includes relationships with place, as the identity of people and place are co-constructive. Relationships are impacted by elements of infrastructure that lead to extra attention, pain, effort or exclusion of older people who use mobility aids. 


\subsubsection{Infrastructure}

Chapter 8 uncovers the ways in which infrastructure is instrumental in older people's use of mobility aid in the built environment, as infrastructure basically constructs the built environment, through design, planning and provision of services. It is worth reiterating here that the code 'Infrastructure and interlinking systems' recorded the second highest number of references, second only to mobility aids themselves. As demonstrated through accounts of difficulty navigating pavements and roads, and the inconsistency of planning, design and service provision, design and policy can reinforce exclusion and stigma. This is often not because of a particular policy, but that lack of cohesion through related policies which authorities may not understand as related (such as housing and transport). The resulting hassle in accessing provision and participation can be a major factor in exclusion. Not only does this impact on the individual, but on social views that older people who use mobility aids do not want to or cannot take part, whereas it is as likely that they just cannot access events due to transport difficulties.

\subsubsection{Obstacles to participation}

The findings of this research provide descriptions of mobility aids use by older people and obstacles to participation. At the start of the research, I expected physical and some social or emotional obstacles. What has been uncovered in this examination of mobility aid use is a mix of physical and infrastructural, emotional, psychological and social obstacles. More than this, though, is the complexity and interconnectivity of these different types of obstacles.

The feedback loop in figure 11.1 demonstrates the ways in which experience from each of the physical, social and psychological elements interact with and affect the others. The interlinking and entangled physical, social, and psychological aspects of mobility aid use result in additional costs to older people who use, or who would be physically aided by using mobility aids. This finding is an original contribution to knowledge, highlighting that the nature of obstacles is not only opaque and unclear, but also lays far deeper than surface level of either the individual or within infrastructure. Rather than specific, obvious obstacles, the barriers to participation and inclusion exist within a constant stream of additional effort to afford accessibility and mobility. Mobility aid use by older people is incorporated in the 
continued process of identifying self with groups and roles, a process which is exacerbated by concerns of changing roles related to ageing. Relationships are integral to personal experience in social worlds and intricately connected to identity. Infrastructure impacts on the individual via accessibility, and also views that older people who use mobility aids do not want to or cannot take part. Additional cost and effort in taking part also makes older mobility aid users feel excluded, resulting in a loop of exclusion partly fed by infrastructure, planning and policy.

Any obstacle in one aspect of mobility aid use by an older person can feed into other areas of experience. Experience of inaccessible infrastructure can lead to feelings of reduced dignity or being stigmatised. Relationships with places and people are often changed because of embodied changes which alter self-perception. The hassle of facing a multitude of seemingly insignificant obstacles can lead to a person feeling like a burden, which again ultimately impacts on identity. These are just some of the ways each type of obstacle impacts upon or feeds into other obstacles in the experience of older people who use mobility aids. This is illustrated in the feedback loop in figure 11.1, demonstrating a further contribution to knowledge that policy and planning can exacerbate the obstacles to participation and increase social exclusion by failing to successfully plan, interpret or implement cohesive policies to promote accessibility and mobility. 


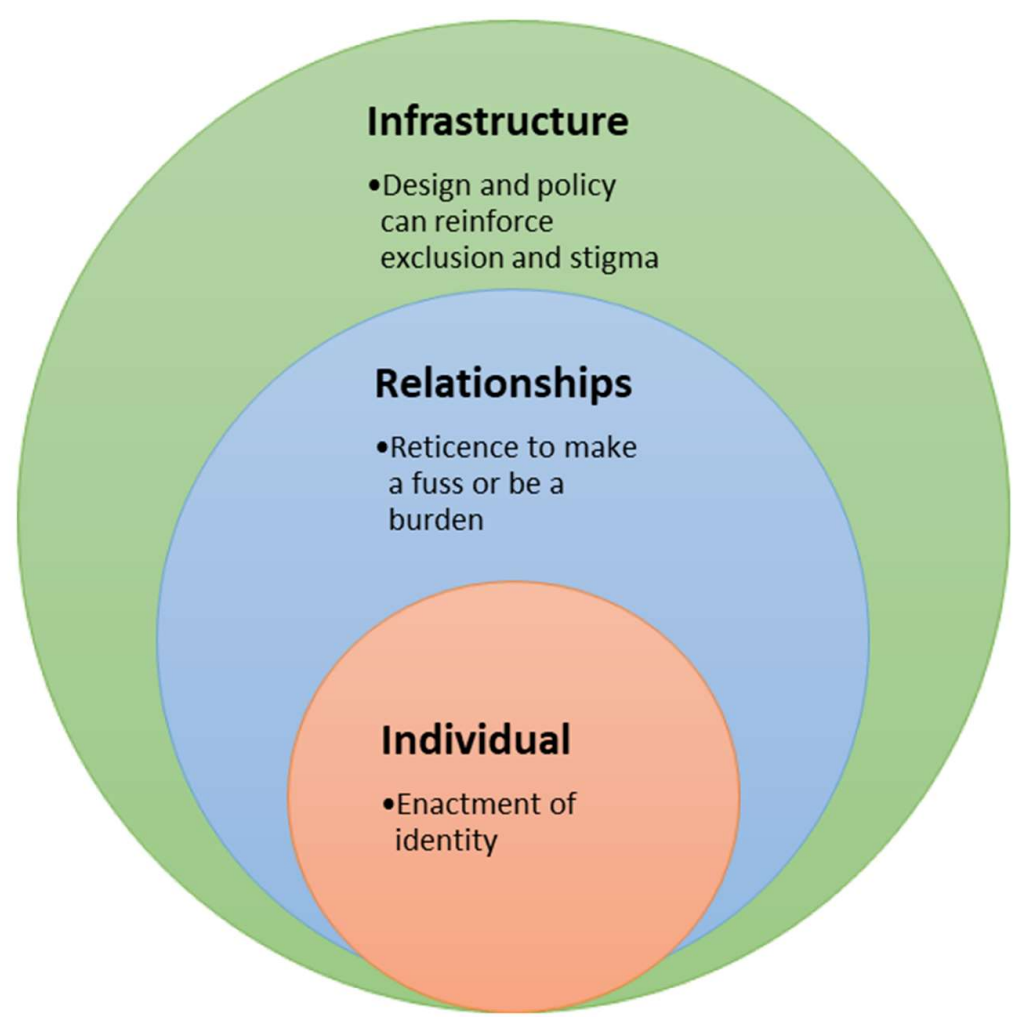

Figure 11.2 Situational reality of older people using mobility aids.

The nature of obstacles to participation take individual, relational and infrastructural forms. Figure 11.2 highlights a different way to conceptualise the reasons for interconnections between an individual's experience of self, relationships and infrastructure, as discussed in Chapter 10. The person experiences self within relationships and both self and relationships within the infrastructure of wider society, policies, planning and built environments. Indeed, infrastructure is implicated throughout this research concerned with all eight domains of WHO age friendly communities. Policy, practice and planning not only design and implement services, but also influence and maintain the behaviour of people within the infrastructure. Identity, relationships and infrastructure intricately interlinked, so they are difficult to disentangle in the experience of individuals and their identities. If people are already concerned about impacts to their identity of using a mobility aid, relationships will be negatively affected. The inability to continue with mapping methodology to understand the movements of people using mobility aids signifies both the reduced ability to participate, and a reduction that is difficult to measure because it is both insidious and further adds to individual concerns regarding their dignity, independence and identity. 
A second factor that runs throughout the themes is that each obstacle is not always significantly large and insurmountable. The insurmountable aspect arises from many smaller obstacles that arise on a daily basis when navigating the environment. Badly maintained structural elements such as pavements to inconsistent provision of transport, accessing places for mundane requirements, such as food shops, banks and chemists, become difficult. Added to the physical difficulties are the strain on relationships because of extra time and effort incurred, which again, were not reported as problematic or creating dysfunction, instead creating discord and disagreement. Not potentially a huge obstacle but not something an individual wants to continue with each time they carry out necessary routines. The obstacles outlined throughout the thesis outline the physical, social, emotional and psychological costs incurred by using a mobility aid as an older person. While much policy and planning addresses both older people and disability, it can present or fail to remove obstacles which has practical, emotional and psychological consequences and therefore costs for older mobility aid users and the rest of society. The nature of obstacles being not necessarily insurmountable in a singular form, but existing in a quantity that makes them insurmountable, is also a contribution to knowledge. Existing literature tends to consider policies and planning in singular terms, that is a transport policy or a social policy, whereas this research highlights the need for policies, and obstacles, to be considered in conjunction with others. The obstacles are small in comparison, but in the quantity and pattern in which they are faced, the barriers are huge.

\subsection{Implications of research}

The research outlined here has many implications for the description, knowledge and understanding of older people who use mobility aids. It also has wider implications for policy and planning, the disciplines of disability studies and gerontology.

\subsubsection{Older people's Mobility aid use}

The finding of this research highlights the complex, interdisciplinary nature of combinations of embodied changes with ageing, mobility aid use, and coming to terms with ageing and senescence itself. Understanding mobility aid use cannot be limited to medical interventions 
and rehabilitation. There are complex psychological, social, medical and infrastructural factors involved in the uptake of mobility aid use for older people, and all of these aspects must be considered, especially in light of safety and social isolation agendas of local and national governments. The needs and requirements of older people who use mobility aids need to be addressed, with care taken to understand the role of social and psychological factors such as stigma, dignity and independence.

\subsubsection{Age Friendly Cities and Communities Initiative}

The undertaking to understand Age Friendly Cities and Communities initiatives provided insight into the ways in which policy and planning intentions are communicated to governments from global organisations, and then rolled out across local authorities, being interpreted and implemented in different ways. Official WHO publications explaining Age Friendly approaches to city and community design does include direction that all policies must be connected and work in unison. This message seems to have been lost along the way, however, as most age friendly policies within Wales do not incorporate this notion of ensuring all policies complement each other. The findings of this research inform policy, planning, research design and methodology that obstacles are not discrete and disconnected entities. Moreover, the experience of older people who use mobility aids is fundamental to construction of policy and planning that meets their needs because of this complexity. The major finding in this area is that obstacles are not always largescale or insurmountable, but a litany of smaller obstacles that are faced in succession. These obstacles build into the experience of hassle, which makes the mobility aid user feel like a burden and thus impacts upon their relationships with family and friends, and also negatively affects their identity.

\subsection{Contribution to knowledge}

A social and experiential approach to the use of mobility aids by older people in the context of daily routines within lived environments has rarely been taken before, so the aim has been as much descriptive as explanatory, setting the ground for future research. The contribution to knowledge of this research is outlining the complexity and cross-sectional nature of obstacles to participation for older people who use mobility aids. There are 
different voices in this research representing different age groups of older people, types of mobility aid and levels of mobility. The combined voice is one of identity that is altered by both ageing and disability, further impacted by relational and infrastructural factors. The effect of infrastructure and policy on the identity of older mobility aid users has been clearly demonstrated here.

A major contribution within this research is the rich case study of the ways in which objects, in the form of mobility aids, both construct and constrict movement within the formation of planned environments, social structures, emotional and psychological settings. The findings outlined here have demonstrated social, emotional, psychological and physical processes that incur costs over and above those incurred by people who do not use mobility aids.

The Feedback loop of obstacles to participation provides a visual and descriptive explanation of the ways policy and practice can intercept individual, emotional and psychological aspects of mobility aid use. This model could be used as a powerful tool to educate anyone involved in policy, planning, health, transport and care provision of older people. As this is an inclusive measure it should be aimed at all older people particularly because some do not want to admit mobility difficulties. This model could be used in future research and dissemination of findings to help create understanding of the social and psychological factors of mobility which incorporate notions of potential and discretionary mobility discussed in section 3.2, rather than the mobility for health care purposes that seems the sole focus currently. Ideas of potential mobility are also involved in the interrupted embodiment, a notion that works in reflection of and combination with to disrupted biography. Interrupted embodiment is a specific term that relates to changes in embodied ability that in affect daily routine behaviours and disrupt biographies. Lack of spontaneity in the realm of mobility, as highlighted by the concepts of potential and discretionary mobility, can be detrimental to well-being and health. Further examination and research are needed to better understand how these concepts of accessibility and mobility translate into social inclusion and participation and well-being for older people who use mobility aids. There is a credible case for examining the role of potential and discretionary travel in the identity, relationships and infrastructural aspects of mobility aid use as discussed in this research. 
In order to address the older people's mobility needs and requirements, critical Geromobilities paradigm, as discussed in Chapters 3 and 10, would address the different kinds of mobility needs of all older people in the form of mobility capital. As stated previously, older people with activity restricting disabilities makes up somewhere between 8 and $17 \%$ of the population in Wales, so addressing their needs is a necessary endeavour for social and economic purposes. To do so will assist in reducing falls and healthcare needs which links back to the goals of the Older People's Commissioner for Wales, and health and wellbeing foci (OPC, 2015). Conceptualisation of a critical geromobilities framework to address the mobility capital of older people is a further contribution to knowledge.

\subsection{Opportunities for Future Research}

In light of the largely exploratory and unique nature of this research, there are a number of opportunities for future research from this study. It would be pertinent to conduct an extension of this study across a wider sample throughout Wales to provide a more comprehensive picture of older people's mobility aid use in the built environment. It would be pertinent to continue with ethnographic grounded theory research, to confirm and clarify these findings, or indicate other phenomena involved. As discussed above, the rich descriptions and accounts are valuable, but more are required to provide a complete picture. This should include different age groups of older people in light of the findings that people over 80 have some different responses and emotions to those under 80 . It would be useful to conduct a longitudinal study to understand whether these differences are related to age or cohort difference. We need to understand whether people reach the age of around 80 and begin to change emotionally, physically and psychologically, or whether the people who are 80 and over at this present time have had different life experiences which make them respond in certain ways. If it is the latter, then we could expect people who are 60 now to behave differently when they reach 80 and require the use of mobility aids. This is an important question to investigate as it pertains to health and well-being policy and practice.

Connected to ideas of a country-wide project to examine the needs of older people who use mobility aids is the need to make closer examination of Age Friendly Cities and Communities policy frameworks. This work should address the mobility needs of older people with ideas 
of mobility capital explored through the proposed Geromobilities framework to examine the ways older people not only mobilise, but are unable to mobilise and internalise social and institutional attitudes and approaches to mobility. Furthermore, from nation and local authority perspectives, the ways in which policies impact upon and affect each other requires more attention. The initial proposals from WHO establish the need to integrate different areas of policy and age friendly domains to provide a seamless, age-friendly experience for all people in society as discussed in Chapter 2. The findings discussed throughout this research reinforce the context of interconnectivity and perhaps more importantly inextricable aspects of individual, relational and infrastructural factors of environment and therefore obstacles to participation. It is imperative, then, that authorities understand what these are to find the best way to tackle consistent inconsistencies in policy and practice. Not only would it provide a better environment for all, but potentially save money in terms of overall costs of health and well-being. This includes the cost of caring for older people who use mobility aids (and those who do not for that matter).

Carers also formed an integral part of this research because they opened up an alternative view into the mobility of the mobility aid user. The carer relationship also helped to uncover the wider aspects of relationships and social accessibility both demonstrated and experienced by the participant. In a perfect world, it would have been preferable to interview more of each type of mobility aid user and carer dyad, for example spousal carers, filial carers, different ages of carer (for example Lauren as a filial carer was 27, Mary 52 and Jenny 57). These differing caring dynamics have the potential to offer different insights into both the caring role for an older adult who uses a mobility aid, and mobility aid use itself, as carers have a different perspective on the practicalities and changes in role of the user. It could also illuminate any increased load on carers and loved ones that arises from mobility aid use, which in turn could help to manage support and assistance for both mobility aid user and carer. The ultimate goal of this research and resulting intervention would be to improve the health and well-being of carers alongside older mobility aid users. 


\subsection{Limitations of research}

The research presented here has a number of strengths in its originality and focus on the daily, routine experiences of older people who use mobility aids, however there are some limitations with this research which will be addressed here.

The first limitation that could be argued is the relatively small sample size. 17 participants in all, 6 of whom were carers, is not a large sample. However, qualitative research methods favour smaller samples which focus on depth of experience, rather than breadth of findings which are more generalisable but not as contextualised. The in-depth accounts within this study provide a snapshot of the experiences of older people who find themselves using mobility aids in Wales. The combinations of different types of people, different relationships of carers and different mobility aids gives rise to understanding some of the themes that occur across daily mobility aid use in general.

Including my husband as a participant could also be considered a limitation. I have been as reflexive and transparent as possible throughout the research process, including full disclosure of the involvement of my husband in the research. My vicarious experience of mobility aids through his use of them will have undoubtedly coloured this research. Although this potentially makes the research nonreplicable, it is in line with much qualitative research which reflects subjective experiences which are not necessarily replicable but remain important because of the rich descriptions and accounts uncovered. Each experience is unique, and as such, nonreplicable. In light of the sensitive nature of aspects such as stigma, dignity, feeling like a burden and hassle, I believe my experience of mobility aid use allowed access to recognition and understanding that may not have been available to the awareness or understanding of other researchers. Moreover, in terms of interviewing my husband, the experience proved enlightening and uncovered a depth of feeling that I was not aware of. His analogous descriptions of barriers surprised me in their depth of exclusion. Without our joint experience, with myself as his carer, neither his account or many of the other observations and accounts would have been accessed.

The ways in which combinations of different types of carers and mobility aid users were interviewed could have been more uniform. Where there was a carer willing to be interviewed, they were not necessarily interviewed uniformly because of diary and working 
commitments. For example, Aileen was not interviewed in a setting away from Lauren, conducting both pre-interview discussion and walking interview in dyad. Bret was interviewed completely separately from his daughter Jenny, while Rosena was interviewed with Gayle first, and then the walking interview was conducted without Gayle. It is likely that these differing interview patterns gave rise to different responses and observations. Whilst it would have been preferable to have the same opportunity of feedback for all, that is, interview together, interview separately then walking interview with the mobility aid user, that would not necessarily be possible. The methods of interview reflected the ways these caring relationships play out. Aileen needs the help of Lauren (or another family member or friend) to navigate a walk, whilst Bret is very independent and only requires Jenny to be notionally available. Rosena is quite independent and encouraged to be so by Gayle, but does need some help from time to time and some encouragement. As a researcher, I would like a uniformity to the interview methods, but lifestyles and abilities may dictate the ability to conduct interviews in such ways.

Another factor that could be considered a limitation is the inability to expand to a wider sample base across Wales. The study aims focus on Wales; however, it was very difficult to gain access to participants beyond South and West Wales. As discussed in the recruitment section of Chapter 4, recruitment did not reach beyond extended contacts. This research is therefore not necessarily representative of North or East Wales. Although this is disappointing, in view of the fact that Wales is a small nation with devolved responsibility for its older people in many ways, and a countrywide Older People's Commissioner, it is unlikely that everyday life is very different for older people in different areas of Wales, other than the ways their local authority interprets and implements policy. There are representations of both rural and city life within the study, however, which addresses different types of built environment.

\subsection{Recommendations}

Directives for Age Friendly Policies from the Older People's Commissioner for Wales, and the Welsh Assembly Government should include creation of an Older People's Advocate within each local authority. The sole role of such an advocate would be to ensure all policies work in cohesion to provide a holistic age friendly framework. The resulting policy 
framework should thus provide a cross-sectional, consistent approach to age-friendliness that works from the perspective of older people's experience, rather than budget silos or departmental decisions, or in piece meal fashion. The role should be one that holds the power to over-rule decisions that harm or do not consider the mobility needs of older people. This role would also involve liaison with external agencies such as transport companies and care providers to ensure all aspects of older people's mobility has been considered. An Older People's Advocate within each local authority would be able to facilitate the interpretation and implementation of age friendly policies and goals at ground level, retaining the context, principles and values of Ageing Well and Age Friendliness championed by the government and the OPC.

A further recommendation would be that any person involved in planning, policy or care of older people should take part in training which involves experiencing the barriers involved in reduced mobility and mobility aid use. This would help to alleviate some of the barriers encountered by participants in this study, such as inaccessible hospital corridors and waiting rooms or impractical disabled parking provision. Mirroring the example set in car manufacturing, people who design car parks for example, might designs spaces with greater consideration if they parked in a space and tried to alight the vehicle manoeuvring a mobility aid in the space provided. Whilst there is economic consideration, this should be given wider scope of thought as social isolation costs impact the cost of care for local authorities. To mobilise older people giving access to social participation may incur an initial outlay, it would be offset in reduced care, health and well-being costs. 


\section{Chapter 12. Bibliography}

Abrams, D. (1998). Social Identifications A Social Psychology of Intergroup Relations and Group Processes: Hoboken : Taylor and Francis.

Abrams, D., Hogg, M. A., \& Marques, J. M. (2005). The social psychology of inclusion and exclusion. New York: New York : Psychology Press.

Adey, P. (2010). Mobility. London; New York: London; New York : Routledge.

Adey, P., Bissell, D., Hannam, K., Merriman, P., \& Sheller, M. (2014). The Routledge handbook of mobilities: London : Routledge.

Adler, P. A., \& Adler, P. (2010). The Past and the Future of Ethnography. SAGE Qualitative Research Methods, 16(1), 4-24. doi: 10.1177/0891241687161001

Aguiar, B., \& Macário, R. (2017). The need for an Elderly centred mobility policy. Transportation Research Procedia, 25, 4355-4369. doi: 10.1016/j.trpro.2017.05.309

Ahmed, J. (2004). The Body as a Site of Contestation. In H. Thomas \& J. Ahmed (Eds.), Cultural bodies : ethnography and theory / edited by Helen Thomas and Jamilah Ahmed. Malden, MA: Malden, MA : Blackwell Pub.

Ahmed, T., Vafaei, A., Auais, M., Guralnik, J., \& Zunzunegui, M. V. (2016). Gender Roles and Physical Function in Older Adults: Cross-Sectional Analysis of the International Mobility in Aging Study (IMIAS). PloS one, 11(6), e0156828-e0156828. doi: 10.1371/journal.pone.0156828

Baltes, M. M., \& Carstensen, L. L. (1996). The Process of Successful Ageing. Ageing and Society, 16(4), 397-422. doi: 10.1017/S0144686X00003603

Baltes, P. B., \& Baltes, M. M. (1990). Psychological perspectives on successful aging: The model of selective optimization with compensation. In M. M. Baltes \& P. B. Baltes (Eds.), Successful Aging: Perspectives from the Behavioral Sciences (pp. 1-34). Cambridge: Cambridge University Press.

Barlew, L., Secrest, J., Guo, Z., Fell, N., \& Haban, G. (2013). The Experience of Being Grounded: A Phenomenological Study of Living with a Wheelchair. Rehabilitation Nursing Journal, 38(4), 193-201. doi: 10.1002/rnj.96

Barnes, C. (2002). Introduction: Disability, policy and politics. Policy \& Politics, 30(3), 311-318. doi: $10.1332 / 030557302760094685$

Beaudry, J.-S. (2016). Beyond ( Models of) Disability? Journal of Medicine and Philosophy, 41(2), 210228. doi: $10.1093 / \mathrm{jmp} / \mathrm{jhv} 063$

Belon, A. P., Nieuwendyk, L. M., Vallianatos, H., \& Nykiforuk, C. I. J. (2014). How community environment shapes physical activity: Perceptions revealed through the PhotoVoice method. Social science \& medicine (1982), 116, 10-21. doi: 10.1016/j.socscimed.2014.06.027

Benson, D., \& Keyes, S. (2014). Challenging Barriers and Enabling Inclusion: The Role of Families. In J. Swain, S. French, C. Barnes \& C. Thomas (Eds.), Disabling Barriers - Enabling Environments (pp. 149-156): Sage Publications.

Bigonnesse, C. (2018). The role of neighborhood physical environment on mobility and social participation among people using mobility assistive technology. Disability \& Society, 33(6), 866-894. doi: 10.1080/09687599.2018.1453783

Blaenau Gwent Local Service Board. (n.d.). Blaenau Gwent Strategy for Older People 2015-2017. Retrieved from https://www.blaenau- 
gwent.gov.uk/fileadmin/documents/Resident/Health and Social Care/Blaenau Gwent Str ategy for Older People 2015 - 2017.pdf.

Boerema, S. T., Van Velsen, L., Vollenbroek-Hutten, M. M. R., \& Hermens, H. J. (2017). Value-based design for the elderly: An application in the field of mobility aids. Assistive Technology, 29(2), 76-84. doi: 10.1080/10400435.2016.1208303

Bos, A. E. R., Pryor, J. B., Reeder, G. D., \& Stutterheim, S. E. (2013). Stigma: Advances in Theory and Research. Basic and applied social psychology, 35(1), 1-9. doi: 10.1080/01973533.2012.746147

Bowling, A., \& Dieppe, P. (2005). What Is Successful Ageing And Who Should Define It? BMJ: British Medical Journal, 331(7531), 1548-1551.

Boyer, K., \& Spinney, J. (2016). Motherhood, mobility and materiality: Material entanglements, journey-making and the process of 'becoming mother'. Environment and planning. $D$, Society \& space, 34(6), 1113-1131. doi: 10.1177/0263775815622209

Brach, J. S., Studenski, S. A., Perera, S., VanSwearingen, J. M., \& Newman, A. B. (2007). Gait Variability and the Risk of Incident Mobility Disability in Community-Dwelling Older Adults. The Journals of Gerontology Series A: Biological Sciences and Medical Sciences, 62(9), 983988. doi: 10.1093/gerona/62.9.983

Bromley, R. D., \& Matthews, D. L. (2007). Reducing Consumer Disadvantage: Reassessing Access in the Retail Environment. The International Review of Retail, Distribution and Consumer Research, 17(5), 483-501. doi: 10.1080/09593960701631904

Bromley, R. D., Matthews, D. L., \& Thomas, C. J. (2007). City centre accessibility for wheelchair users: The consumer perspective and the planning implications. Cities, 24(3), 229-241. doi: 10.1016/j.cities.2007.01.009

Brown, D. M., Gertig, P., Gillman, M., Anderson, J., Clarke, C., \& Powell, S. (2014). Accessing Social and Leisure Activities: Barriers to Inclusion Experienced by Visually Impaired People. In J. Swain, S. French, C. Barnes \& C. Thomas (Eds.), Disabling Barriers - Enabling Environments (pp. 214-221): Sage Publishing.

Brown, R. L. (2015). Perceived Stigma Among People With Chronic Health Conditions: The Influence of Age, Stressor Exposure, and Psychosocial Resources. Research on Aging, 37(4), 335-360. doi: $10.1177 / 0164027514533133$

Brown, W. (2016). Sacrificial Citizenship: Neoliberalism, Human Capital, and Austerity Politics. Constellations (Oxford, England), 23(1), 3-14. doi: 10.1111/1467-8675.12166

Bryant, A., \& Charmaz, K. (2007). Grounded Theory in Historical Perspective: An Epistemological Account. In A. Bryant \& K. Charmaz (Eds.), The SAGE handbook of grounded theory edited by Antony Bryant, Kathy Charmaz. Los Angeles, [Calif.] ; London: Los Angeles, Calif. ; London : SAGE.

Buffel, T., Phillipson, C., \& Scharf, T. (2012). Ageing in urban environments: Developing 'age-friendly' cities. Critical social policy, 32(4), 597-617. doi: 10.1177/0261018311430457

Burghardt, M. (2013). Common frailty, constructed oppression: tensions and debates on the subject of vulnerability. Disability \& Society, 28(4), 556-568. doi: 10.1080/09687599.2012.711244

Bury, M. (1982). Chronic illness as biographical disruption. Sociology of Health \& IIIness, 4(2), 167182. doi: 10.1111/1467-9566.ep11339939

Butler, M. (2016). Equality and anti-discrimination law : the Equality Act 2010 and other antidiscrimination protections: London, England : Spiramus Press. 
Bytheway, B. (2005). Ageism and Age Categorization. Journal of social issues, 61(2), 361-374. doi: 10.1111/j.1540-4560.2005.00410.x

Bülow, M. H., \& Söderqvist, T. (2014). Successful ageing: A historical overview and critical analysis of a successful concept. Journal of Aging Studies, 31, 139-149. doi: 10.1016/j.jaging.2014.08.009

Büscher, M., Sheller, M., \& Tyfield, D. (2016). Mobility intersections: social research, social futures. Mobilities, 11(4), 485-497. doi: 10.1080/17450101.2016.1211818

Caerphilly County Borough. (2015). Strategy for Older People in Wales

Phase 3: LIVING LONGER AGEING WELL

Caerphilly County Borough Delivery Plan. Retrieved from

https://www.caerphilly.gov.uk/CaerphillyDocs/Adults-and-older-people/50-

Positve Action delivery plan.aspx.

Campbell, A. J., Reinken, J., Allan, B. C., \& Martinez, G. S. (1981). Falls in old age: a study of frequency and related clinical factors. Age and ageing, 10(4), 264. doi: 10.1093/ageing/10.4.264

Campbell, C., \& Deacon, H. (2006). Unravelling the contexts of stigma: from internalisation to resistance to change. Journal of community \& applied social psychology, 16(6), 411-417. doi: 10.1002/casp.901

Carel, H. (2008). Illness : the cry of the flesh. Stocksfield: Stocksfield : Acumen.

Carmarthenshire County Council. (2015). Ageing Well Plan

(Incorporating Strategy for Older People)

2015-2018. Retrieved from https://www.carmarthenshire.gov.wales/media/3350/ageing-planv2.pdf.

Carpiano, R. M. (2009). Come take a walk with me: The "Go-Along" interview as a novel method for studying the implications of place for health and well-being. Health \& place, 15(1), 263-272. doi: 10.1016/j.healthplace.2008.05.003

Carter, M. J. (2013). Advancing Identity Theory: Examining the Relationship between Activated Identities and Behavior in Different Social Contexts. Social Psychology Quarterly, 76(3), 203223. doi: 10.1177/0190272513493095

Castrodale, M. A. (2018). Mobilizing Dis/Ability Research: A Critical Discussion of Qualitative GoAlong Interviews in Practice. Qualitative inquiry, 24(1), 45-55. doi: 10.1177/1077800417727765

Chambers, R. (2006). Vulnerability, Coping and Policy (Editorial Introduction. IDS Bulletin, 37(4), 3340. doi: 10.1111/j.1759-5436.2006.tb00284.x

Chaney, P. (2013). Electoral Competition, Issue Salience and Public Policy for Older People: The Case of the Westminster and Regional UK Elections 1945-2011. The British Journal of Politics and International Relations, 15(3), 456-475. doi: 10.1111/j.1467-856X.2011.00488.x

Charmaz, K. (2014). Constructing grounded theory (2nd ed. ed.). London ; Thousand Oaks, Calif.: London; Thousand Oaks, Calif. : Sage.

Charmaz, K. (2017). The Power of Constructivist Grounded Theory for Critical Inquiry. Qualitative Inquiry, 23(1), 34-45. doi: 10.1177/1077800416657105

Charmaz, K., \& Belgrave, L. L. (2019). Thinking About Data With Grounded Theory. Qualitative Inquiry, 25(8), 743-753. doi: 10.1177/1077800418809455 
Chasteen, A., \& Cary, L. (2015). Age Stereotypes and Age Stigma: Connections to Research on Subjective Aging. Annual Review of Gerontology \& Geriatrics, 35(1), 99-IX. doi: 10.1891/0198-8794.35.99

Cherry, F. E. (1995). The 'stubborn particulars' of social psychology : essays on the research process / Frances Cherry. London: London : Routledge.

Cheshmehzangi, A. (2014). Spatial syntagma and identity of a place: Sensing, relating to, and knowing a place. Journal of Human Behavior in the Social Environment, 24(7), 799-811. doi: 10.1080/10911359.2013.876377

Chihuri, S., Mielenz, T. J., DiMaggio, C. J., Betz, M. E., DiGuiseppi, C., Jones, V. C., \& Li, G. (2016). Driving Cessation and Health Outcomes in Older Adults. Journal of the American Geriatrics Society (JAGS), 64(2), 332-341. doi: 10.1111/jgs.13931

Christensen, K., Doblhammer, G., Rau, R., \& Vaupel, J. W. (2009). Ageing populations: the challenges ahead. Lancet (London, England), 374(9696), 1196-1208. doi: 10.1016/S01406736(09)61460-4

City and County of Swansea. (2015). Swansea Local Service Board \& Healthy City Board Ageing Well and Strategy for

Older People Action Plan. Retrieved from

file:///C:/Users/Allys/Downloads/English_Language_Swansea_Ageing_Well_Plan_Final.pdf.

Clandinin, D. J., \& Connelly, F. M. (1998). Personal experience methods. In N. K. Denzin \& Y. S. Lincoln (Eds.), The landscape of qualitative research : theories and issues (pp. 150-178). Thousand Oaks, Calif.: Thousand Oaks, Calif. : SAGE.

Costamagna, E., Thies, S. B., Kenney, L. P. J., Howard, D., Liu, A., \& Ogden, D. (2017). A generalisable methodology for stability assessment of walking aid users. Medical Engineering and Physics, 47, 167-175. doi: 10.1016/j.medengphy.2017.06.013

Council, G. (n.d.). Gwynedd

Ageing Well Plan. Retrieved from

https://democracy.gwynedd.Ilyw.cymru/documents/s9052/Item\%206\%20-\%20Appendix\%201\%20\%20Aging\%20Well\%20Action\%20Plan.pdf.

Cresswell, T. (2010). Mobilities I: Catching up. Progress in human geography, 35(4), 550-558. doi: 10.1177/0309132510383348

Creswell, J. W. (2014). Research design : qualitative, quantitative, and mixed methods approaches / John W. Creswell (Fourth edition, international student edition. ed.): Los Angeles, Calif. : SAGE.

Crocker, J., \& Major, B. (1989). Social Stigma and Self-Esteem: The Self-Protective Properties of Stigma. Psychological review, 96(4), 608-630. doi: 10.1037/0033-295X.96.4.608

Csordas, T. J. (2004). Evidence of and for what? Anthropological Theory, 4(4), 473-480. doi: $10.1177 / 1463499604047922$

Daramy-Williams, E., Anable, J., \& Grant-Muller, S. (2019). Car Use: Intentional, Habitual, or Both? Insights from Anscombe and the Mobility Biography Literature. Sustainability (Basel, Switzerland), 11(24), 7122. doi: 10.3390/su11247122

Davies, M., \& Hughes, N. (2014). Doing a successful research project : using qualitative or quantitative methods (Second edition. ed.): Basingstoke : Palgrave Macmillan.

Davis, L. J. (2017). The disability studies reader (Fifth edition. ed.): New York, NY : Routledge, Taylor \& Francis Group. 
Day, E. (2002). Me, Myself and I: Personal and Professional Re-Constructions in Ethnographic Research. Forum, qualitative social research, 3(3)

Deaux, K. (2000). Models, Meanings and Motivations. In D. Capozza \& R. Brown (Eds.), Social identity processes trends in theory and research (pp. 15-26). London: London : SAGE.

Dembo, T., Leviton, G. L., \& Wright, B. A. (1975). Adjustment to misfortune: A problem of socialpsychological rehabilitation. Rehabilitation Psychology, 22(1), i-iv, 1-100. doi: 10.1037/h0090832

Denzin, N. K., \& Lincoln, Y. S. (1998). The landscape of qualitative research : theories and issues / edited by Norman K. Denzin and Yvonna S. Lincoln. Volume 1. Thousand Oaks, Calif.: Thousand Oaks, Calif. : SAGE.

Department of Transport, \& ONS. (2017). Transport Statistics for Great Britain 2017.

Dillon, R. S. (1995). Dignity, character and self-respect / edited and with an introduction by Robin S. Dillon. New York ; London: New York ; London : Routledge.

Disabled World. (2018). Information on Mobility Aids and Ambulation Devices. Retrieved 21 May, 2019, from https://www.disabled-world.com/assistivedevices/mobility/

Dobbs, D., Eckert, J. K., Rubinstein, B., Keimig, L., Clark, L., Frankowski, A. C., \& Zimmerman, S. (2008). An Ethnographic Study of Stigma and Ageism in Residential Care or Assisted Living (Vol. 48, pp. 517-526).

Donati, P. (2011). Relational Sociology: A New Paradigm for the Social Sciences. London: London: Routledge.

Dorow, S., Roseman, S. R., \& Cresswell, T. (2017). Re-working mobilities: Emergent geographies of employment-related mobility. Geography Compass, 11(12), n/a-n/a. doi:

10.1111/gec3.12350

Doughty, K., \& Murray, L. (2014). Discourses of Mobility: Institutions, Everyday Lives and Embodiment. Mobilities, 1-20. doi: 10.1080/17450101.2014.941257

Dumbaugh, E. (2008). Designing Communities to Enhance the Safety and Mobility of Older Adults. Journal of Planning Literature, 23(1), 17-36. doi: 10.1177/0885412208318559

Edlund, M., Lindwall, L., Post, I. V., \& Lindström, U. Å. (2013). Concept determination of human dignity. Nursing Ethics, 20(8), 851-860. doi: 10.1177/0969733013487193

Edwards, J. D., Perkins, M., Ross, L. A., \& Reynolds, S. L. (2009). Driving Status and Three-Year Mortality Among Community-Dwelling Older Adults. The journals of gerontology. Series $A$, Biological sciences and medical sciences, 64A(2), 300-305. doi: 10.1093/gerona/gIn019

Elliott, A. (2010). Mobile lives self, excess and nature. New York, NY: New York, NY : Routledge.

Elliott, T. R., Kurylo, M., \& Rivera, P. (2005). Positive Growth Following Acquired Physical Disability. In C. R. Snyder \& S. J. Lopez (Eds.), Handbook of Positive Psychology. Cary, UNITED STATES: Oxford University Press, Incorporated.

Emirbayer, M. (1997). Manifesto for a Relational Sociology. The American journal of sociology, 103(2), 281-317. doi: 10.1086/231209

Engman, A. (2019). Embodiment and the foundation of biographical disruption. Social science \& medicine (1982), 225, 120-127. doi: 10.1016/j.socscimed.2019.02.019

Evans, J., \& Jones, P. (2011). The walking interview: Methodology, mobility and place. Applied Geography, 31(2), 849-858. doi: 10.1016/j.apgeog.2010.09.005 
Finkelstein, V. (2001). The social model of disability repossessed. Manchester Coalition of Disabled People(1), 1-5.

Finlay, L. (2003). The intertwining of body, self and world: A phenomenological study of living with recently-diagnosed multiple sclerosis. Journal of Phenomenological Psychology, 34(2), 157178. doi: $10.1163 / 156916203322847128$

Flick, U. (2002). An introduction to qualitative research / Uwe Flick (2nd ed. ed.). London: London : SAGE.

Flintshire County Council. (2016). Ageing Well in Flintshire

Action Plan 2016-2020 Summary. Retrieved from https://www.flintshire.gov.uk/en/PDFFiles/SocialServices/Ageing-well-in-Flintshire.pdf.

Foley, M. P., Prax, B., Crowell, R., \& Boone, T. (1996). Effects of Assistive Devices on Cardiorespiratory Demands in Older Adults. Physical therapy, 76(12), 1313-1319. doi: 10.1093/ptj/76.12.1313

Fonda, S. J., Wallace, R. B., \& Herzog, A. R. (2001). Changes in Driving Patterns and Worsening Depressive Symptoms Among Older Adults. The Journals of Gerontology: Series B, 56(6), S343-S351. doi: 10.1093/geronb/56.6.S343

Fontana, A., \& Frey, J. H. (1998). Interviewing: The Art of Science. In N. K. Denzin \& Y. S. Lincoln (Eds.), Collecting and interpreting qualitative materials: Thousand Oaks, CA : SAGE Publications, Inc.

Foster, L., \& Walker, A. (2015). Active and Successful Aging: A European Policy Perspective. The Gerontologist, 55(1), 83-90. doi: 10.1093/geront/gnu028

Foucault, M. (1977). Discipline and punish : the birth of the prison. London: London : Allen Lane.

Foucault, M. (1982). The Subject and Power. Critical Inquiry, 8(4), 777-795. doi: 10.1086/448181

Fukuyama, F. (2018). Identity : contemporary identity politics and the struggle for recognition / Francis Fukuyama: London : Profile Books Ltd.

Gaete-Reyes, M. (2015). Citizenship and the embodied practice of wheelchair use. Geoforum, 64, 351-361. doi: 10.1016/j.geoforum.2014.09.010

Gagliardi, C. (2007). The outdoor mobility and leisure activities of older people in five European countries. Ageing \& Society, 27(5), 683-701. doi: 10.1017/S0144686X07006198

Gell, N., Wallace, R., Lacroix, A., Mroz, T. M., \& Patel, K. (2015). Mobility Device Use in Older Adults and Incidence of Falls and Worry About Falling: Findings from the 2011-2012 National Health and Aging Trends Study. J. Am. Geriatr. Soc., 63(5), 853-859. doi: 10.1111/jgs.13393

Gendron, T. L., Welleford, E. A., Inker, J., \& White, J. T. (2016). The Language of Ageism: Why We Need to Use Words Carefully. The Gerontologist, 56(6), 997-1006. doi: 10.1093/geront/gnv066

Giesbrecht, E. M., Miller, W. C., \& Woodgate, R. L. (2015). Navigating uncharted territory: a qualitative study of the experience of transitioning to wheelchair use among older adults and their care providers. Bmc Geriatrics, 15 doi: 10.1186/s12877-015-0092-2

Giles, H., \& Reid, S. A. (2005). Ageism Across the Lifespan: Towards a Self-Categorization Model of Ageing. Journal of social issues, 61(2), 389-404. doi: 10.1111/j.1540-4560.2005.00412.x

Gilleard, C., \& Higgs, P. (2002). The third age: class, cohort or generation? Ageing and Society, 22(3), 369-382. doi: 10.1017/S0144686X0200870X 
Gilleard, C., \& Higgs, P. (2010). Aging without agency: Theorizing the fourth age. Aging \& Mental Health, 14(2), 121-128. doi: 10.1080/13607860903228762

Gilleard, C., \& Higgs, P. (2011). Ageing abjection and embodiment in the fourth age. Journal of Aging Studies, 25(2), 135-142. doi: 10.1016/j.jaging.2010.08.018

Gilleard, C., Higgs, P., Hyde, M., Wiggins, R., \& Blane, D. (2005). Class, cohort, and consumption: the British experience of the third age. The journals of gerontology. Series B, Psychological sciences and social sciences, 60(6), S305-S310. doi: 10.1093/geronb/60.6.S305

Goffman, E. (1990). Stigma : notes on the management of spoiled identity: London : Penguin Books.

Green, J., Jones, A., \& Roberts, H. (2014). More than A to B: the role of free bus travel for the mobility and wellbeing of older citizens in London. Ageing Soc

Green, J., Steinbach, R., \& Datta, J. (2012). The travelling citizen: rmergent discourses of moral mobility in a study of cycling in London.(Author abstract). Sociology, 46(2), 272-289.

Grogan, S. (2007). Body Image: Understanding Body Dissatisfaction in Men, Women and Children (2nd Ed.. ed.). London

S.I: London ; S.I. : Routledge.

Gwynedd Council. (n.d.). Gwynedd

Ageing Well Plan. Retrieved from

https://democracy.gwynedd.llyw.cymru/documents/s9052/Item\%206\%20-\%20Appendix\%201\%20\%20Aging\%20Well\%20Action\%20Plan.pdf.

Hammel, J., Southall, K., Jutai, J., Finlayson, M., Kashindi, G., \& Fok, D. (2013). Evaluating use and outcomes of mobility technology: a multiple stakeholder analysis. Disability and rehabilitation. Assistive technology, 8(4), 294. doi: 10.3109/17483107.2012.735745

Hamraie, A. (2017). Building access : universal design and the politics of disability / Aimi Hamraie: Minneapolis : University of Minnesota Press.

Hansen, N., \& Philo, C. (2007). THE NORMALITY OF DOING THINGS DIFFERENTLY: BODIES, SPACES AND DISABILITY GEOGRAPHY. Tijdschrift voor economische en sociale geografie, 98(4), 493506. doi: 10.1111/j.1467-9663.2007.00417.x

Harris, J. (2000). Is there a coherent social conception of disability? Journal of Medical Ethics, 26(2), 95-100. doi: 10.1136/jme.26.2.95

Hastings, J., \& Thomas, H. (2005). Accessing the Nation: Disability, Political Inclusion and Built Form. Urban Studies, 42(3), 527-544.

Hatzenbuehler, M. L. (2016). Structural stigma: Research evidence and implications for psychological science. The American psychologist, 71(8), 742-751. doi: 10.1037/amp0000068

Hauge, Å. L. (2007). Identity and Place: A Critical Comparison of Three Identity Theories. Architectural Science Review, 50(1), 44-51. doi: 10.3763/asre.2007.5007

Havighurst, R. (1961). Successful Aging. The Gerontologist, 1(1), 8-13.

Hebl, M. R., \& Dovidio, J. F. (2016). Promoting the "Social" in the Examination of Social Stigmas. Personality and social psychology review, 9(2), 156-182. doi: 10.1207/s15327957pspr0902_4

Higgs, P., Leontowitsch, M., Stevenson, F., \& Jones, I. R. (2009). Not just old and sick - The 'will to health' in later life. Ageing and Society, 29(5), 687-707. doi: 10.1017/S0144686X08008271

Hillcoat-Nallétamby, S., \& Phillips J. E. (2011). Sociological Ambivalence Revisited. Sociology (Oxford), 45(2), 202-217. doi: 10.1177/0038038510394018 
Hodgson, F. (2011). Structures of Encouterability: Space, Place, Paths and Identities. In M. Grieco \& J. Urry (Eds.), Mobilities : new perspectives on transport and society (pp. 41-64). Farnham: Farnham : Ashgate.

Holstein, M. (2011). Ethics, aging, and society the critical turn. S.I.]: S.I. : Springer Publishing Company.

Holstein, M., \& Minkler, M. (2007). Critical Gerontology: reflections for the 21st century. In M. Bernard \& T. Scharf (Eds.), Critical Perspectives on Ageing Societies (pp. 13-26). Bristol: The Policy Press.

Hughes, B. (2015). Disabled people as counterfeit citizens: the politics of resentment past and present. Disability \& Society, 30(7), 1-14. doi: 10.1080/09687599.2015.1066664

Hughes, R. (2010). The social model of disability. British Journal of Healthcare Assistants, 4(10), 508511. doi: 10.12968/bjha.2010.4.10.79078

Hughes, S. A., \& Pennington, J. L. (2017). Autoethnography : process, product, and possibility for critical social research / Sherick A. Hughes, Julie L. Pennington: Los Angeles, CA : SAGE Publications, Inc.

Hurd Clarke, L., \& Korotchenko, A. (2011). Aging and the Body: A Review. Canadian journal on aging $=$ La revue canadienne du vieillissement, 30(3), 495.

lezzoni, L. I. (2003). When walking fails mobility problems of adults with chronic conditions / Lisa I. lezzoni. Berkeley : New York: Berkeley : University of California Press ; New York : Millbank Memorial Fund.

Imrie, R. (2000). Disability and discourses of mobility and movement. Environment and Planning. A, 32(9), 1641. doi: 10.1068/a331

Imrie, R. (2012). Auto- disabilities: the case of shared space environments. Environment \& Planning A, 44(9), 2260-2277.

Imrie, R. (2016). Barriered and Bounded Places and the Spatialities of Disability. Urban studies (Edinburgh, Scotland), 38(2), 231-237. doi: 10.1080/00420980124639

Imrie, R., \& Edwards, C. (2007). The Geographies of Disability: Reflections on the Development of a Sub-Discipline. Geography Compass, 1(3), 623-640. doi: 10.1111/j.1749-8198.2007.00032.x

Isle of Anglesey County Council. (2014). A Strategy for Older People

Ageing Well in Anglesey.

Jacobson, N. (2007). Dignity and health: A review. Social Science \& Medicine, 64(2), 292-302. doi: 10.1016/j.socscimed.2006.08.039

Jacobson, N. (2009). A taxonomy of dignity: a grounded theory study. BMC international health and human rights, 9(1), 3-3. doi: 10.1186/1472-698X-9-3

Jepsson Grassman, E., \& Whitaker, A. (2013). Ageing with disability: An introduction. In E. Jepsson Grassman \& A. Whitaker (Eds.), Ageing with disability: A lifecoure perspective (pp. 1-16). Bristol: Policy Press.

Jette, A., \& Haley, S. (2005). Contemporary measurement techniques for rehabilitation outcomes assessment. Journal of Rehabilitation Medicine, 37(6), 339-345. doi: 10.1080/16501970500302793

Jirón, P. (2011). On becoming 'la sombre/the shadow'. In M. Büscher, J. Urry \& K. Witchger (Eds.), Mobile methods. Abingdon, Oxon ; New York: Routledge. 
Johnson, K. J., \& Mutchler, J. E. (2014). The emergence of a positive gerontology: from disengagement to social involvement. The Gerontologist, 54(1), 93-100. doi: 10.1093/geront/gnt099

Johnstone, M.-L. (2012). The servicescape: The social dimensions of place. Journal of Marketing Management, 28(11-12), 1399-1419. doi: 10.1080/0267257X.2012.694370

Jones, P., Bunce, G., Evans, J., Gibbs, H., \& Hein, J. R. (2008). Exploring space and place with walking interviews. Journal of research practice, 4(2), 1-9.

Jönson, H., \& Taghizadeh Larsson, A. (2009). The exclusion of older people in disability activism and policies - A case of inadvertent ageism? Journal of Aging Studies, 23(1), 69-77. doi: 10.1016/j.jaging.2007.09.001

Kader, M., Jonasson, S. B., Iwarsson, S., Odin, P., \& Nilsson, M. H. (2018). Mobility device use in people with Parkinson's disease: A 3-year follow-up study. Acta neurologica Scandinavica, 138(1), 70-77. doi: 10.1111/ane.12942

Kafka, F. (2002). In the penal colony [KnowledgeNotes student guides]. Cambridge: Cambridge : ProQuest Information and Learning.

Kahn, R. L. (2002). On "Successful aging and well-being: self-rated compared with Rowe and Kahn". The Gerontologist, 42(6), 725.

Kaiser, H. J. (2009). Mobility in old age: Beyond the transportation perspective. Journal of Applied Gerontology, 28(4), 411-419. doi: 10.1177/0733464808329121

Kastenbaum, R. (2016). Theories of Ageing. In D. Harris, L. Branch \& E. B. Palmore (Eds.), Encyclopedia of Ageism (pp. 318-327): Taylor and Francis.

Katz, J., Holland, C., \& Peace, S. (2013). Hearing the voices of people with high support needs. Journal of Aging Studies, 27(1), 52-60. doi: 10.1016/j.jaging.2012.11.003

Katz, S. (2015). Five eye- openers in my life of critical gerontology. IJAL, 1O(1), 21-34. doi: 10.3384/ijal.1652-8670.15276

Katz, S., \& Calasanti, T. (2015). Critical Perspectives on Successful Aging: Does It "Appeal More Than It Illuminates"? The Gerontologist, 55(1), 26-33. doi: 10.1093/geront/gnu027

Kaufmann, V. (2016 [2002]). Re-thinking mobility : contemporary sociology: London, England ; New York, New York : Routledge.

Kaufmann, V., Bergman, M. M., \& Joye, D. (2004). Motility: mobility as capital. International journal of urban and regional research, 28(4), 745-756. doi: 10.1111/j.0309-1317.2004.00549.x

Kendall, N., \& Thangaraj, M. (2012). Ethnography. In A. A. Trainor \& E. Graue (Eds.), Reviewing Qualitative Research in the Social Sciences : A Guide for Researchers and Reviewers. London, UNITED KINGDOM: Taylor \& Francis Group. Retrieved from http://ebookcentral.proquest.com/lib/swansea-ebooks/detail.action?doclD=1143680.

Korotchenko, A., \& Hurd Clarke, L. (2016). Canadian power mobility device users' experiences of ageing with mobility impairments. 36(6), 1238-1253. doi: 10.1017/S0144686X15000288

Krantz, O., \& Egard, H. (2016). Use of active wheelchairs in everyday life: experiences among experienced users of active ultra lightweight rigid frame wheelchairs. Disability and Rehabilitation: Assistive Technology, 1-8. doi: 10.3109/17483107.2015.1065514

Kristeva, J., \& Lechte, J. (1982). Approaching Abjection. Oxford Literary Review, 5(1\2), 125-149. doi: 10.3366/olr.1982.009 
Kusenbach, M. (2003). Street phenomenology: The go-along as ethnographic research tool. Ethnography, 4(3), 455-485. doi: 10.1177/146613810343007

Kusow, A. M. (2004). Contesting Stigma: On Goffman's Assumptions of Normative Order. Symbolic Interaction, 27(2), 179-197. doi: 10.1525/si.2004.27.2.179

Larkin, B. (2013). The Politics and Poetics of Infrastructure. Annual Review of Anthropology, 42(1), 327-343. doi: 10.1146/annurev-anthro-092412-155522

Laslett, P. (1987). The Emergence of the Third Age †. Ageing and Society, 7(2), 133-160. doi: $10.1017 /$ S0144686X00012538

Leonard, J. (2017). Medical News Today. Medical News Today Retrieved July 1, 2019, from https://www.medicalnewstoday.com/articles/318463

Leveille, S. G., Resnick, H. E., \& Balfour, J. (2014). Gender differences in disability: Evidence and underlying reasons. Aging clinical and experimental research, 12(2), 106-112. doi: 10.1007/bf03339897

Levine, J. M. (2012). Group Processes. London, UNITED KINGDOM: Taylor \& Francis Group.

Link, B. G., \& Phelan, J. C. (2001). Conceptualizing Stigma. Annual review of sociology, 27(1), 363-385. doi: 10.1146/annurev.soc.27.1.363

Lovett, P. S., Joiner, J. G., \& Goodwin, L. K. (1989). Positive assertion and acceptance among persons with disabilities. The Journal of rehabilitation, 55(3), 22.

Luborsky, M. R., \& Sankar, A. (1993). Extending the critical gerontology perspective: cultural dimensions. Introduction. Gerontologist, 33(4), 440-444.

Lucas, K. (2011). Transport and social exclusion: Where are we now? In M. Grieco \& J. Urry (Eds.), Mobilities : new perspectives on transport and society (pp. 207-222). Farnham: Farnham : Ashgate.

Lyall, J. (2007). Implementing strategy for older people in wales: Joanna Lyall says that a plan to improve services for older people in Wales has only had limited success, three years since its implementation. Nursing older people, 19(5), 7-8. doi: 10.7748/nop.19.5.7.s10

Mackett, R. (2018). Older People's Travel and its relationship to their health and wellbeing. In C. Musselwhite (Ed.), Transport, Travel and Later Life (pp. 15-36). Bingley: Emerald Publishing.

Mann, J. (1998). Dignity and Health: The UDHR's Revolutionary First Article. Health and human rights, 3(2), 30. doi: 10.2307/4065297

Marottoli, R. A., de Leon, C. F. M., Glass, T. A., Williams, C. S., Cooney, L. M., Jr., \& Berkman, L. F. (2000). Consequences of Driving Cessation: Decreased Out-of-Home Activity Levels. The Journals of Gerontology: Series B, 55(6), S334-S340. doi: 10.1093/geronb/55.6.S334

Martens, K. (2012). Justice in transport as justice in accessibility: applying Walzer's 'Spheres of Justice' to the transport sector. Transportation (Dordrecht), 39(6), 1035-1053. doi: $10.1007 / \mathrm{s} 11116-012-9388-7$

Martens, K. (2017). Transport justice : designing fair transportation systems: New York ; London, England : Routledge.

Martens, K. (2018). Ageing, impairments and travel: Priority setting for an inclusive transport system. Transport policy, 63, 122-130. doi: 10.1016/j.tranpol.2017.12.001

Martin, D. J., \& Gillen, L. L. (2014). Revisiting Gerontology's Scrapbook: From Metchnikoff to the Spectrum Model of Aging. The Gerontologist, 54(1), 51-58. doi: 10.1093/geront/gnt073 
Martin, P., Kelly, N., Kahana, B., Kahana, E., Willcox, B. J., Willcox, D. C., \& Poon, L. W. (2015). Defining Successful Aging: A Tangible or Elusive Concept? The Gerontologist, 55(1), 14-25. doi: 10.1093/geront/gnu044

Martins, A., Pinheiro, J., Farias, B., \& Jutai, J. (2016). Psychosocial Impact of Assistive Technologies for Mobility and Their Implications for Active Ageing. Technologies, 4(3), 28. doi: 10.3390/technologies4030028

Martinson, M., \& Berridge, C. (2015). Successful Aging and Its Discontents: A Systematic Review of the Social Gerontology Literature. The Gerontologist, 55(1), 58-69. doi: 10.1093/geront/gnu037

Mazurik, K., Desjardins, M., de Grosbois, È., Poldma, T., \& Gelech, J. (2014). Individual stakes and collective ideology in tension: Looking at physical and spatial obstacles from an experiential perspective. Alter - European Journal of Disability research, Revue européen de recherche sur le handicap, 8(3), 194-205. doi: 10.1016/j.alter.2014.02.008

McLean, K. C., Syed, M., \& Hammack, P. L. (2015). Theoretical Foundations of Identity (1 ed.): Oxford University Press.

Mechakra-Tahiri, S., Freeman, E., Haddad, S., Samson, E., \& Zunzunegui, M. (2012). The gender gap in mobility: A global cross-sectional study. BMC Public Health, 12, 598. doi: 10.1186/14712458-12-598

Medicine Plus. (2016). Mobility Aids. from https://www.medlineplus.gov/mobilityaids.html

Menec, V. H., Means, R., Keating, N. C., Parkhurst, G., \& Eales, J. (2011). Conceptualizing Age-Friendly Communities. Canadian journal on aging, 30(3), 479-493. doi: 10.1017/\$0714980811000237

Merleau-Ponty, M. (1968). The visible and the invisible : followed by working notes / [by] Maurice Merleau-Ponty; edited by Claude Lefort; translated by Alphonso Lingis. Evanston, III: Evanston, III : Northwestern University Press.

Merrill, S. S., Seeman, T. E., Kasl, S. V., \& Berkman, L. F. (1997). Gender differences in the comparison of self-reported disability and performance measures. J Gerontol A Biol Sci Med Sci, 52(1), M19-26. doi: 10.1093/gerona/52a.1.m19

Merriman, P., Jones, R., Cresswell, T., Divall, C., Mom, G., Sheller, M., \& Urry, J. (2013). Mobility: Geographies, Histories, Sociologies. Transfers, 3(1), 147-165. doi: 10.3167/tranS.2013.030111

Merthyr Tydfil County Borough Council, County, R. C. T., \& Borough Council. (n. d.). Cwm Taf

Ageing Well in Wales Plan. Retrieved from

https://www.rctcbc.gov.uk/EN/Resident/AdultsandOlderPeople/AdultSocialServicesCareandSupport Relateddocuments/CwmTafAgeingWellinWalesPlan.pdf.

Meyers, A. R., Anderson, J. J., Miller, D. R., Shipp, K., \& Hoenig, H. (2002). Barriers, facilitators, and access for wheelchair users: substantive and methodologic lessons from a pilot study of environmental effects. [Article]. Social Science \& Medicine, 55(8), 1435-1446. doi: 10.1016/s0277-9536(01)00269-6

Milner, P., \& Kelly, B. (2009). Community Participation and Inclusion: People with Disabilities Defining Their Place. Disability \& Society, 24(1), 47-62. doi: 10.1080/09687590802535410

Minkler, M. (1996). Critical Perspectives on Ageing: New Challenges for Gerontology. Ageing and Society, 16(4), 467-487. doi: 10.1017/S0144686X00003639

Minkler, M., \& Fadem, P. (2002). "Successful Aging": A Disability Perspective. Journal of Disability Policy Studies, 12(4), 229-235. doi: 10.1177/104420730201200402 
Moulaert, T., \& Biggs, S. (2013). International and European policy on work and retirement: Reinventing critical perspectives on active ageing and mature subjectivity. Human Relations, 66(1), 23-43. doi: 10.1177/0018726711435180

Muir-Hunter, S. W., \& Montero-Odasso, M. (2016). Gait Cost of Using a Mobility Aid in Older Adults with Alzheimer's Disease. Journal of the American Geriatrics Society, 64(2), 437-438. doi: 10.1111 /jgs.13973

Murray, A., \& Musselwhite, C. (2019). Older peoples' experiences of informal support after giving up driving. Research in Transportation Business and Management, 30 doi: 10.1016/j.rtbm.2019.100367

Murray, L. (2009). Looking at and looking back: Visualization in mobile research. Qualitative Research, 9(4), 469-489. doi: 10.1177/1468794109337879

Murray, L. (2015). Age- friendly mobilities: A transdisciplinary and intergenerational perspective. Journal of Transport \& Health, 2(2), 302-307. doi: 10.1016/j.jth.2015.02.004

Murray, L., \& Robertson, S. L. (2017). Intergenerational mobilities : relationality, age and lifecourse: Abingdon, Oxon ; New York, NY : Routledge.

Musselwhite, C. (2010). Mobility, accessibility and quality of later life.

Musselwhite, C. (2015). Further examinations of mobility in later life and improving health and wellbeing. Journal of transport \& health, 2(2), 99-100. doi: 10.1016/j.jth.2015.04.002

Musselwhite, C. (2017). Exploring the importance of discretionary mobility in later life. Working with Older People, 21(1), 49-58. doi: 10.1108/WWOP-12-2016-0038

Musselwhite, C. (2018). Transport, travel and later life. Bingley: Bingley : Emerald Publishing Limited.

Musselwhite, C., \& Haddad, H. (2018). Older people's travel and mobility needs: A reflection of a hierarchical model 10 years on. Quality in Ageing and Older Adults, 19(2), 87-105. doi: 10.1108/QAOA-12-2017-0054

Musselwhite, C., Holland, C., \& Walker, I. (2015). The role of transport and mobility in the health of older people (Vol. 2, pp. 1-4).

Musselwhite, C., \& Shergold, I. (2013). Examining the process of driving cessation in later life. European Journal of Ageing, 10(2), 89-100. doi: 10.1007/s10433-012-0252-6

Navaratnarajah, A., \& Jackson, S. H. D. (2017). The physiology of ageing. Medicine, 45(1), 6-10. doi: 10.1016/j.mpmed.2016.10.008

Nelson, T. (2005). Ageism: Prejudice against our feared future self. J. Soc. Issues, 61(2), 207-221.

Newman, A. B., \& Brach, J. S. (2001). Gender gap in longevity and disability in older persons. Epidemiol Rev, 23(2), 343-350. doi: 10.1093/oxfordjournals.epirev.a000810

Newton, R., Ormerod, M., Burton, E., Mitchell, L., \& Ward-Thompson, C. (2010). Increasing Independence for Older People through Good Street Design. Journal of Integrated Care, 18(3), 24-29. doi: 10.5042/jic.2010.0246

NHS. (2018). Walking aids, wheelchairs and mobility scooters. Retrieved 1 August, 2020, from https://www.nhs.uk/conditions/social-care-and-support-guide/care-services-equipmentand-care-homes/walking-aids-wheelchairs-and-mobility-scooters/

Nordbakke, S., \& Schwanen, T. (2014). Well- being and Mobility: A Theoretical Framework and Literature Review Focusing on Older People. Mobilities, 9(1), 104-129. doi: 10.1080/17450101.2013.784542 
Novoa, A. (2015). Mobile ethnography: emergence, techniques and its importance to geography. Human Geographies, 9(1), 97-107. doi: 10.5719/hgeo.2015.91.7

Oliver, M. (1984). The politics of disability. Critical Social Policy, 4(11), 21-32. doi: 10.1177/026101838400401103

Oliver, M. (1996). Defining Impairment and Disability: Issues at Stake. In C. Barnes \& G. Mercer (Eds.), Exploring the divide : illness and disability. Leeds: Leeds : The Disability Press.

Oliver, M. (2009). Understanding disability : from theory to practice (2nd edition. ed.). Basingstoke [England]: Basingstoke England: Palgrave Macmillan.

Oliver, M. (2013). The social model of disability: thirty years on. Disability \& Society, 1-3. doi: 10.1080/09687599.2013.818773

ONS. (2018). 2011 Census: Aggregate Data. In U. D. Service (Ed.).

ONS. (2019). Population estimates for the UK, England and Wales, Scotland and Northern Ireland: mid 2019. 2020, from https://www.ons.gov.uk/peoplepopulationandcommunity/populationandmigration/populati onestimates/bulletins/annualmidyearpopulationestimates/mid2019estimates

OPC. (2015). Ageing Well in Wales 2014-2019.

OPC. (n.d.-a). Ageing Well in Wales. 2020, from https://ageingwellinwales.com/en/home

OPC. (n.d.-b). Making wales a nation of age friendly communities. 2020, from http://www.olderpeoplewales.com/Libraries/Uploads/AFC guide eng - v2 FINAL.sflb.ashx

Ormerod, M., Newton, R., Philips, J., Musselwhite, C., McGee, S., \& Russell, R. (2015). How can transport provision and associated built environment infrastructure be enhanced and developed to support the mobility needs of individuals as they age? Future of an ageing population: Evidence review. London: Foresight. Retrieved from https://www.gov.uk/government/publications/future-of-ageing-transport-and-mobility.

Owens, J. (2015). Exploring the critiques of the social model of disability: the transformative possibility of Arendt's notion of power. Sociology of Health \& IIIness, 37(3), 385-403. doi: 10.1111/1467-9566.12199

Owton, H., \& Allen-Collinson, J. (2014). Close But Not Too Close: Friendship as Method(ology) in Ethnographic Research Encounters. Journal of Contemporary Ethnography, 43(3), 283-305. doi: $10.1177 / 0891241613495410$

Parent, L. (2016). The wheeling interview: mobile methods and disability. Mobilities, 11(4), 521-532. doi: 10.1080/17450101.2016.1211820

Parker, R., \& Aggleton, P. (2003). HIV and AIDS-related stigma and discrimination: a conceptual framework and implications for action. Social Science \& Medicine, 57(1), 13-24. doi: 10.1016/S0277-9536(02)00304-0

Paterson, K., \& Hughes, B. (2000). Disabled bodies. In P. Hancock, B. Hughes, E. Jagger, K. Paterson, R. Russell, E. Tulle-Winton \& M. Tyler (Eds.), The body, culture and society : an introduction (pp. 29-44). Buckingham: Buckingham : Open University Press.

Peace, S. (2013). Social Interactions in Public Spaces and Places: A Conceptual Overview. In G. D. Rowles \& M. Bernard (Eds.), Environmental gerontology making meaningful places in old age (pp. 25-49). New York: Springer.

Peace, S., Holland, C., \& Kellaher, L. A. (2006). Placing the self Environment and identity in later life. Maidenhead: Maidenhead: Open University Press. 
Pembrokeshire County Council. (2016). AGEING WELL

IN PEMBROKESHIRE. Retrieved from https://www.pembrokeshire.gov.uk/social-care-whatsavailable/full-of-life-at-50-plus.

Pescosolido, B. A., Martin, J. K., Lang, A., \& Olafsdottir, S. (2008). Rethinking theoretical approaches to stigma: a Framework Integrating Normative Influences on Stigma (FINIS). Social science \& medicine (1982), 67(3), 431. doi: 10.1016/j.socscimed.2008.03.018

Peterson, L. J., Meng, H., Dobbs, D., \& Hyer, K. (2017). Gender Differences in Mobility Device Use Among U.S. Older Adults. Journals of Gerontology Series B: Psychological Sciences and Social Sciences, 72(5), 827-835. doi: 10.1093/geronb/gbw081

Pfaller, L., \& Schweda, M. (2019). Excluded from the Good Life? An Ethical Approach to Conceptions of Active Ageing. Social Inclusion, 7(3), 44-53. doi: 10.17645/si.v7i3.1918

Pfeiffer, D. (2002). The Philosophical Foundations of Disability Studies. Disability Studies Quarterly, 22(2) doi: 10.18061/dsq.v22i2.341

Phillips, J., Ajrouch, K., \& Hillcoat-Nallétamby, S. (2010). Key concepts in social gerontology. Los Angeles: Los Angeles : SAGE.

Phillipson, C. (2008). Authoring aging: personal and social constructions.(Author abstract)(Report). Journal of Aging Studies, 22(2), 163.

Pink, S. (2006). Doing Visual Ethnography. London, GBR: London, GBR SAGE Publications Ltd.

Porter, E. J., Benson, J. J., \& Matsuda, S. (2011). Older Homebound Women: Negotiating Reliance on a Cane or Walker. Qualitative Health Research, 21(4), 534-548. doi:

10.1177/1049732310385822

Pryor, J. B., Reeder, G. D., \& Landau, S. (1999). A Social-Psychological Analysis of HIV-Related Stigma: A Two-Factor Theory. The American behavioral scientist (Beverly Hills), 42(7), 1212-1228. doi: $10.1177 / 00027649921954723$

Ragland, D. R., Satariano, W. A., \& MacLeod, K. E. (2005). Driving Cessation and Increased Depressive Symptoms. The journals of gerontology. Series A, Biological sciences and medical sciences, 60(3), 399-403. doi: 10.1093/gerona/60.3.399

Raymond, É., Grenier, A., \& Hanley, J. (2014). Community Participation of Older Adults with Disabilities. Journal of Community \& Applied Social Psychology, 24(1), 50-62. doi: $10.1002 /$ casp. 2173

Reeder, G. D., \& Pryor, J. B. (2008). Dual psychological processes underlying public stigma and the implications for reducing stigma. Mens sana monographs, 6(1), 175-186. doi: 10.4103/09731229.36546

Reeve, D. (2014). Counselling and Disabled People: Help or Hindrance? In J. Swain, S. French, C. Barnes \& C. Thomas (Eds.), Disabling Barriers - Enabling Environments (pp. 255-261): Sage Publishing.

Reeves, S., Kuper, A., \& Hodges, B. D. (2008). Qualitative research methodologies: ethnography. $B M J, 337$ (aug07 3), a1020-a1020. doi: 10.1136/bmj.a1020

Resnik, L., Allen, S., Isenstadt, D., Wasserman, M., \& lezzoni, L. (2009). Perspectives on use of mobility aids in a diverse population of seniors: Implications for intervention. Disability and health journal, 2(2), 77-85. doi: 10.1016/j.dhjo.2008.12.002

Ridolfo, H., \& Ward, B. W. (2012). Mobility Impairment and the Construction of Identity. Boulder, CO, UNITED STATES: Lynne Rienner Publishers. 
Rowe, J., \& Kahn, R. (1987). Human aging: usual and successful. Science (American Association for the Advancement of Science), 237(4811), 143-149. doi: 10.1126/science.3299702

Rowles, G. D. (1978). Prisoners of space?: Exploring the geographical experience of older people: Westview Press.

Rowles, G. D., \& Bernard, M. (2013). Environmental gerontology making meaningful places in old age. New York, UNITED STATES: S.I. : Springer Publishing Company.

Rémillard-Boilard, S. (2018). The UK Network of Age-friendly Communities: a general review. Working with Older People, 22(1), 30-38. doi: 10.1108/WWOP-12-2017-0034

Savage, M. C. N. D. F. F. S. L. D. M. L. M. A. S. H. T. M. W. P. (2015). Social Class in the 21st Century: Pelican, London.

Schwanen, T., Banister, D., \& Bowling, A. (2012). Independence and mobility in later life. Geoforum, 43(6), 1313-1322. doi: 10.1016/j.geoforum.2012.04.001

Schwanen, T., \& Páez, A. (2010). The mobility of older people - an introduction (Vol. 18, pp. 591595).

Schwanen, T., \& Ziegler, F. (2011). Wellbeing, independence and mobility: an introduction. Ageing and Society, 31(5), 719-733. doi: 10.1017/S0144686X10001467

Sendi, R. (2014). A social innovation for combating discrimination against persons with disabilities in the built environment. Urbani izziv, 25(2), 119-129. doi: 10.5379/urbani-izziv-en-2014-25-02004

Serlin, D. (2012). On walkers and wheelchairs disabling the narratives of urban modernity. Radical History Review, 2012(114), 19. doi: 10.1215/01636545-1597988

Shaffir, W. (1999). Doing ethnography. Journal of Contemporary Ethnography, 28(6), 676-686. doi: 10.1177/089124199028006009

Shakespeare, T. (2013). The Social Model of Disability. In L. J. Davis (Ed.), The Disability Studies Reader (pp. 214-221). Florence, UNITED STATES: Taylor \& Francis Group.

Sheller, M. (2008). Mobility, Freedom and Public Space. In S. Bergmann \& T. Sager (Eds.), The Ethics of Mobilities: Rethinking Place, Exclusion, Freedom and Environment. Abingdon: Abingdon: Routledge.

Sheller, M. (2018). Theorising mobility justice. Tempo social : revista de sociologia da USP, 30(2), 1734. doi: 10.11606/0103-2070.ts.2018.142763

Sheller, M., \& Urry, J. (2006). The New Mobilities Paradigm. Environment and Planning A, 38(2), 207226. doi: $10.1068 / a 37268$

Sheller, M., \& Urry, J. (2008). The City and the Car. International journal of urban and regional research, 24(4), 737-757. doi: 10.1111/1468-2427.00276

Shergold, I., Lyons, G., \& Hubers, C. (2015). Future mobility in an ageing society - Where are we heading? Journal of transport \& health, 2(1), 86-94. doi: 10.1016/j.jth.2014.10.005

Shergold, I., Parkhurst, G., \& Musselwhite, C. (2012). Rural car dependence: an emerging barrier to community activity for older people. Transportation planning and technology, 35(1), 69-85. doi: 10.1080/03081060.2012.635417

Shilling, C. (2005). The body in culture, technology and society Chris Shilling. London: London : SAGE.

Smith, R. J., \& Hall, T. (2016). Pedestrian circulations: urban ethnography, the mobilities paradigm and outreach work. Mobilities, 11(4), 498-508. doi: 10.1080/17450101.2016.1211819 
Smith, S. J., \& Easterlow, D. (2005). The strange geography of health inequalities. Transactions of the Institute of British Geographers, 30(2), 173-190. doi: 10.1111/j.1475-5661.2005.00159.x

Sonenblum, S. E., Sprigle, S., \& Lopez, R. A. (2012). Manual wheelchair use: bouts of mobility in everyday life. Rehabilitation research and practice, 2012, 753165-753165. doi: 10.1155/2012/753165

Spinney, J. (2008). Cycling between the traffic: mobility, identity and space.

Spinney, J. (2009). Cycling the City: Movement, Meaning and Method. Geography compass, 3(2), 817-835. doi: 10.1111/j.1749-8198.2008.00211.x

Spinney, J. (2016). A Place of Sense: A Kinaesthetic Ethnography of Cyclists on Mont Ventoux. Environment and planning. D, Society \& space, 24(5), 709-732. doi: 10.1068/d66j

Spinney, J., Aldred, R., \& Brown, K. (2015). Geographies of citizenship and everyday (im)mobility. Geoforum, 64, 325.

Star, S. L. (2007). Living Grounded Theory: Cognitive and Emotional Forms of PragmatismThe SAGE Handbook of Grounded Theory (pp. pages 75-93): SAGE Publications Ltd. Retrieved from https://methods.sagepub.com/book/the-sage-handbook-of-grounded-theory. doi: $10.4135 / 9781848607941$

Stets, J., \& Burke, P. (2000). Identity theory and social identity theory. Social Psychology Quarterly, 63(3), 224-237. doi: 10.2307/2695870

Stets, J., \& Serpe, R. T. (2016). New directions in identity theory and research: New York, NY : Oxford University Press.

Stjernborg, V., Wretstrand, A., \& Tesfahuney, M. (2014). Everyday Life Mobilities of Older Persons A Case Study of Ageing in a Suburban Landscape in Sweden. Mobilities, 10(3), 383-401. doi: 10.1080/17450101.2013.874836

Stowe, J. D., \& Cooney, T. M. (2015). Examining Rowe and Kahn's Concept of Successful Aging: Importance of Taking a Life Course Perspective. The Gerontologist, 55(1), 43-50. doi: 10.1093/geront/gnu055

Stryker, S., \& Burke, P. J. (2000). The Past, Present, and Future of an Identity Theory. Social psychology quarterly, 63(4), 284-297. doi: 10.2307/2695840

Sunderland, N., Bristed, H., Gudes, O., Boddy, J., \& Da Silva, M. (2012). What does it feel like to live here? Exploring sensory ethnography as a collaborative methodology for investigating social determinants of health in place. Health and Place, 18(5), 1056-1067. doi: 10.1016/j.healthplace.2012.05.007

Taghizadeh Larsson, A. (2013). Is it possible to 'age successfully' with extensive physical impairments? In E. Jepsson Grassman \& A. Whitaker (Eds.), Ageing with Disability: a lifecourse perspective (pp. 55-72). Bristol: Policy Press.

The City of Cardiff Council. (n.d.). Ageing Well in Cardiff. Retrieved from https://www.cardiff.gov.uk/ENG/Your-Council/Strategies-plans-and-policies/Equalitydiversity-and-cohesion/Strategic-Equality-Plan-andPolicies/Documents/strategic\%20equality\%20plan/Ageing\%20Well\%20In\%20Cardiff\%20Loca \%20Delivery\%20Plan.pdf.

Thomas, C. (2014). Disability and Impairment. In J. Swain, S. French, C. Barnes \& C. Thomas (Eds.), Disabling barriers - enabling environments (Third edition. ed., pp. 9-16): Los Angeles : SAGE.

Thrift, N. (2004). Driving in the City. Theory, Culture \& Society, 21(4-5), 41-59. doi: $10.1177 / 0263276404046060$ 
Tiggemann, M. (2004). Body image across the adult life span: stability and change. Body image, 1(1), 29-41. doi: 10.1016/S1740-1445(03)00002-0

Timmermans, S., \& Tavory, I. (2007). Advancing Ethnographic Theory through Grounded Theory Practice. In A. Bryant \& K. Charmaz (Eds.), The SAGE Handbook of Grounded Theory: SAGE Publications Ltd. Retrieved from https://methods.sagepub.com/book/the-sage-handbookof-grounded-theory. doi: 10.4135/9781848607941

Timonen, V. (2016). Beyond successful and active ageing: A theory of model ageing (1 ed. Vol. 57734). Bristol: Bristol: Policy Press.

Torfaen Public Service Board. (2015). STRATEGY FOR OLDER PEOPLE

PHASE 3: TORFAEN DELIVERY PLAN (2015 to 2023). Retrieved from

http://www.torfaenpublicservicesboard.co.uk/en/Documents/Torfaen-Delivery-Plan-Strategy-ForOlder-People-Phase-3.pdf.

Tregaskis, C. (2002). Social Model Theory: The story so far. Disability \& Society, 17(4), 457-470. doi: 10.1080/09687590220140377

Tremain, S. (2006). Foucault and the Government of Disability. Scandinavian journal of disability research : SJDR, 8(1), 75-77. doi: 10.1080/15017410600570972

Tsang, M. (2012). Connecting and caring: innovations for healthy ageing. Bulletin Of The World Health Organization, 90(3), 162. doi: 10.2471/BLT.12.020312

Tulle-Winton, E. (2000). Old bodies. In P. Hancock, B. Hughes, E. Jagger, K. Paterson, R. Russel, E. Tulle-Winton \& M. Tyler (Eds.), The body, culture and society : an introduction (pp. 64-83). Buckingham: Buckingham : Open University Press.

Turner, T. (1994). Bodies and anti-bodies: flash and fetish in contemporary social theory. In T. J. Csordas (Ed.), Embodiment and experience : the existential ground of culture and self. Cambridge ; New York: Cambridge ; New York : Cambridge University Press.

Tuvikene, T. (2018). Post-socialist (auto)mobilities: Modernity, freedom and citizenship. Geography compass, 12(3), e12362-n/a. doi: 10.1111/gec3.12362

Twigg, J. (1992). Carers : research and practice / edited by Julia Twigg. London: London : H.M.S.O.

Twigg, J., \& Martin, W. (2015). The Challenge of Cultural Gerontology (Vol. 55, pp. 353-359).

Tyler, I. (2020). Stigma: The machinery of inequality. London: Zed Books.

Tyler, I., \& Slater, T. (2018). Rethinking the sociology of stigma. The Sociological Review, 66(4), 721743. doi: $10.1177 / 0038026118777425$

United Nations. (1948). Universal Declaration of Human Rights. Retrieved from https://www.un.org/en/universal-declaration-human-rights/.

Urry, J. (2000). Sociology beyond societies mobilities for the twenty-first century. London ; New York: London; New York : Routledge.

Urry, J. (2007). Mobilities. Cambridge: Polity.

van Dyk, S. (2014). The appraisal of difference: Critical gerontology and the active-ageing-paradigm. Journal of Aging Studies, 31, 93-103. doi: 10.1016/j.jaging.2014.08.008

Vannini, P. (2014). Slowness and Deceleration. In P. Adey, D. Bissell, K. Hanham, P. Merriman \& M. Sheller (Eds.), The Routledge Handbook of Mobilities (pp. 116-124). London: Routledge. 
Velho, R., Holloway, C., Symonds, A., \& Balmer, B. (2016). The Effect of Transport Accessibility on the Social Inclusion of Wheelchair Users: A Mixed Method Analysis. Social Inclusion, 4(3) doi: 10.17645/si.v4i3.484

Verlinghieri, E., \& Schwanen, T. (2020). Transport and mobility justice: Evolving discussions. Journal of transport geography, 87, 102798-102798. doi: 10.1016/j.jtrangeo.2020.102798

Welsh Government. (2013). Strategy for Older People in Wales 2013-2023 Cardiff.

Wenger, G. C., Davies, R., Shahtahmasebi, S., \& Scott, A. (1996). Social Isolation and Loneliness in Old Age: Review and Model Refinement. Ageing and Society, 16(3), 333-358. doi: $10.1017 /$ S0144686X00003457

Werner, P., Stein-Shvachman, I., \& Heinik, J. (2009). Perceptions of self-stigma and its correlates among older adults with depression: a preliminary study. Int. Psychogeriatr., 21(6), 11801189. doi: $10.1017 / \mathrm{S} 1041610209990470$

WHO. (2007). Global Age-friendly Cities: A guide Geneva: World Health Organisation.

WHO. (2014). Age Friendly World: Dublin Declaration on Age-Friendly Cites and Communities in Europe 2013. Retrieved 20th January, 2017, from https://extranet.who.int/agefriendlyworld/the-dublin-declaration-on-age-friendly-citiesand-communities-in-europe-2013/

WHO. (2016). Creating age friendly environments in Europe: a tool for local policy-makers and planners Copenhagen. Retrieved from https://www.euro.who.int/en/health-topics/Lifestages/healthy-ageing/publications/2016/creating-age-friendly-environments-in-europe.-atool-for-local-policy-makers-and-planners-2016.

WHO. (2017). 10 Priorities Toward a Decade on Healthy Ageing.

WHO. (2018). Age Friendly Environments in Europe: Indicators, monitoring and assessments Copenhagen: World Health Organisation. Retrieved from https://www.euro.who.int/en/health-topics/Life-stages/healthyageing/publications/2018/age-friendly-environments-in-europe-indicators,-monitoring-andassessments-2018.

WHO. (n.d.). Ageing and Lifecourse. 2020, from www.who.int/ageing/en/

Williams, P., Hubbard, P., Clark, D., \& Berkeley, N. (2001). Consumption, exclusion and emotion: The social geographies of shopping. Social \& Cultural Geography, 2(2), 203-220. doi: $10.1080 / 14649360122365$

Wood, C., Littleton, K., \& Oates, J. (2007). Lifespan Development. In T. Cooper \& I. Roth (Eds.), Challenging Psychological Issues (pp. 1-64). Milton Keynes: Open University.

Woods, B., \& Watson, N. (2004). The social and technological history of wheelchairs. International journal of therapy and rehabilitation, 11(9), 407-410. doi: 10.12968/ijtr.2004.11.9.19587

Zunzunegui, M., Phillips, S., Karna, G., Tu, M., Qirjako, G., Guerra, R., \& Gomez, J. (2013). THE MOBILITY GAP BETWEEN OLDER MEN AND WOMEN FROM DIVERSE POPULATIONS: THE EMBODIMENT OF GENDER. Gerontologist, 53, 610-610.

Öberg, P. (1996). The absent body: a social gerontolo-gical paradox. Ageing \& Society, 16(6), 701719. 


\section{Chapter 13. Appendices}

\subsection{Appendix 1 Literature searches}

- Wherever a term has an asterisk, all possible alternatives for the term were used in the search, i.e.

- $\quad$ *mobility aids $=($ mobility aids or mobility device or walking aid or walking devices)

- $\quad$ older people $=$ (older people or older adults or elderly)

- $\quad$ *ageing $=$ (ageing or aging or older adults or older persons or elderly $)$

- $\quad$ carers $=$ (carers or caregivers or family members or relatives or informal carers )

- Wherever a term is in inverted commas the term was searched in inverted commas to keep the term as a phrase in the search.

- As highlighted in the main text of the PhD (chapter 2), referencing literature was not always carried out in a linear way. Within the Infrastructure theme even more than the other two themes and sub-themes the theme emerged because of literature I had already read. There is therefore no diagram for this theme 


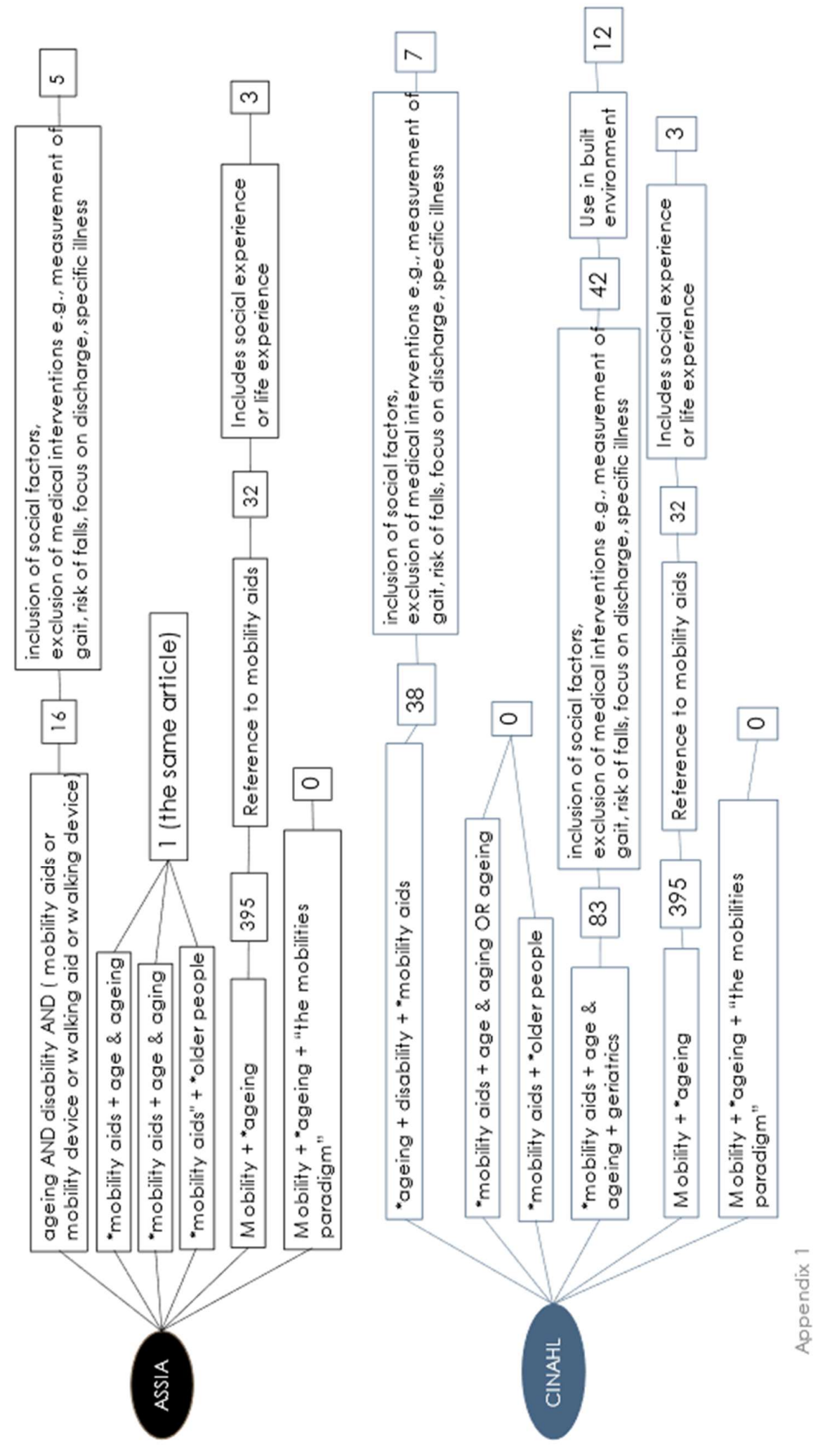




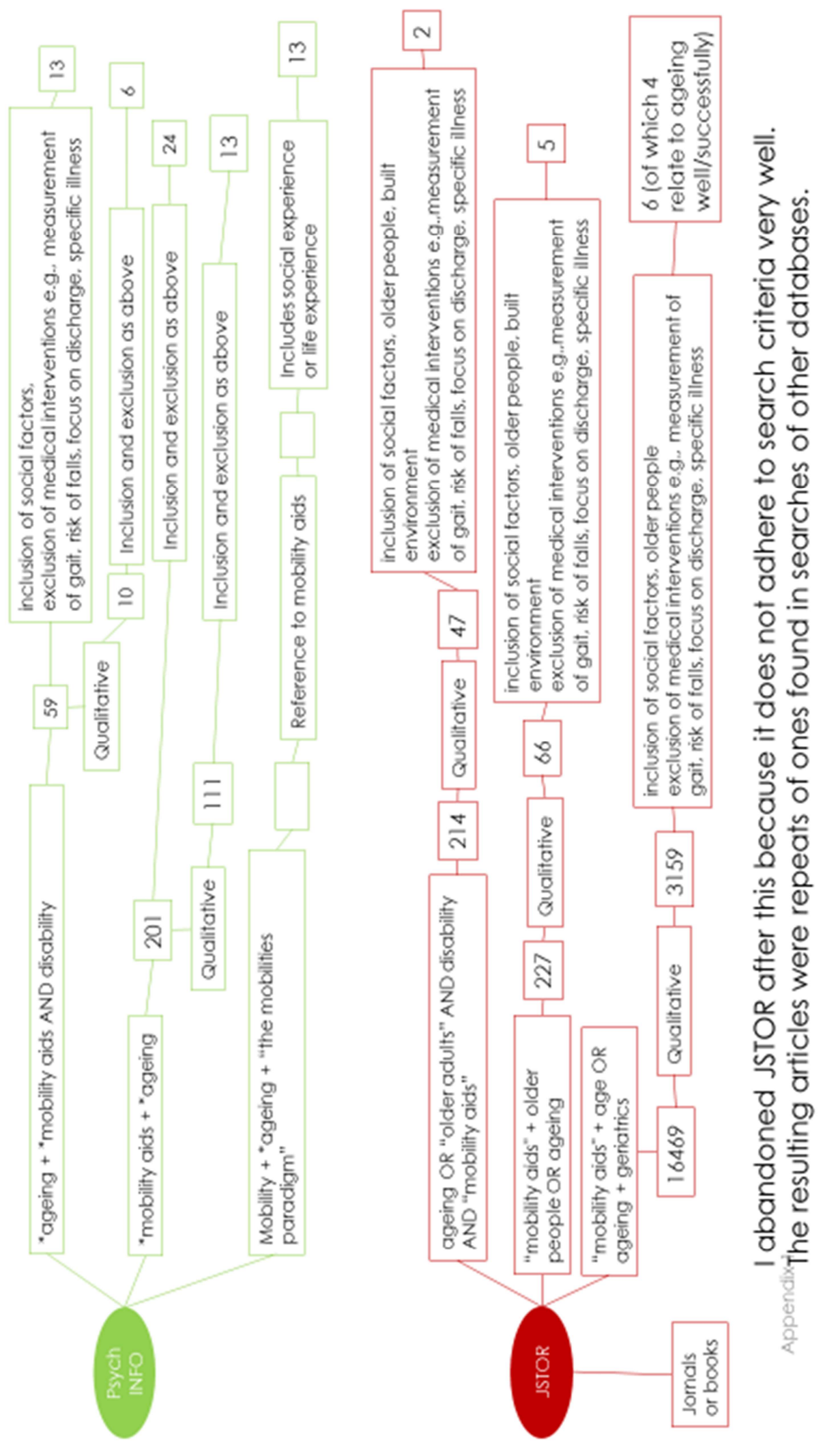




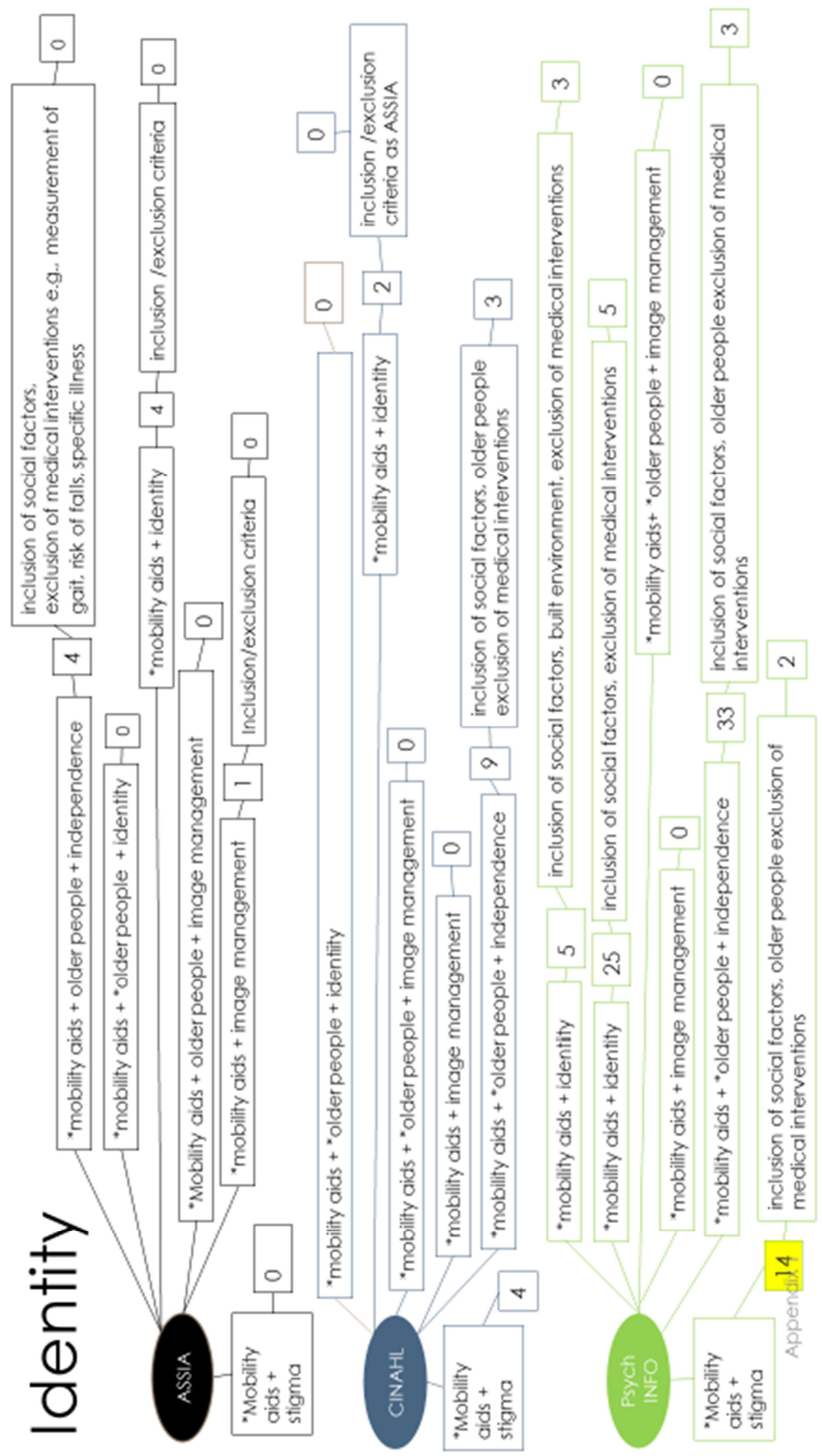




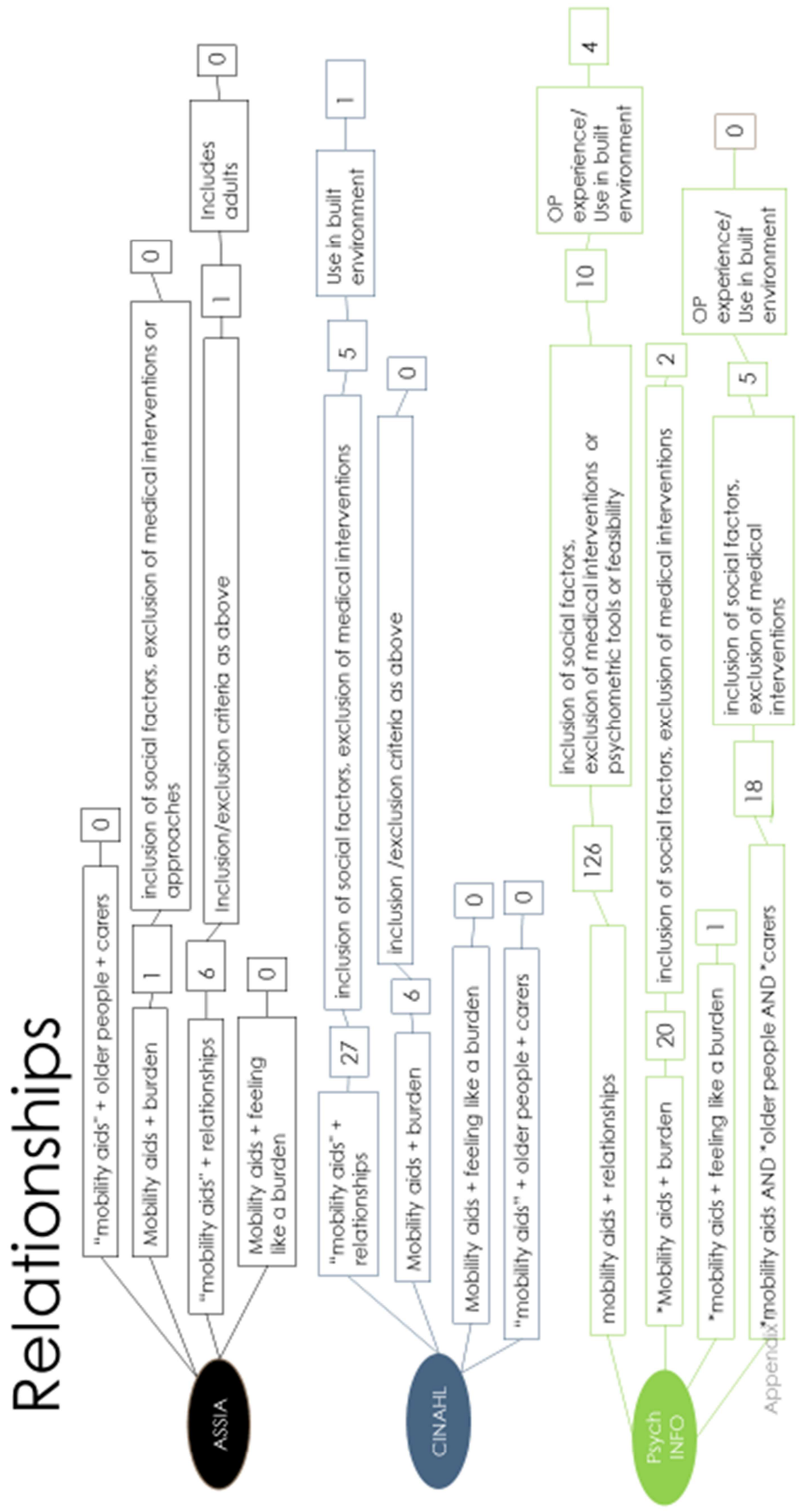




\subsection{Appendix 2 Ageing Well in Wales Evaluation}

The tables below provide an overview of information accessed and available through the Ageing Well in Wales Website up until January 2020,

https://ageingwellinwales.com/en/themes/age-friendly-communities.

After this date, Ageing Well became part of the core work of the Older People's

Commissioner for Wales.

The tables outline information available and accessed through the Ageing Well in Wales Website (https://ageingwellinwales.com/en/themes/age-friendly-communities). The website provides access to portals of each of the 22 local authorities in Wales's Ageing Well in Wales policy approach. The table assesses each authority's approach by indicating which policy strand the approach is address within or if there is a stand-alone Ageing Well policy, when that policy was last updated, and whether the policy explains the approach via the World Health Authority domains of age friendliness (listed below. Presence of the 5 Ageing Well in Wales (AWW) themes (listed below) is also assessed as age friendly communities (AFC) is one of these themes. Other factors assessed were explanation of age friendly communities, why they matter and what the demographic of the area to underline the importance of age friendliness. Ease of use/reading, mention of disability, mobility, transport, the built environment and mobility aids was also evaluated.

\begin{tabular}{|c|l|l|}
\hline \multirow{2}{*}{$\begin{array}{c}\text { Ageing Well in Wales priority } \\
\text { themes }\end{array}$} & Age-friendly communities & \\
\hline & $\begin{array}{l}\text { Falls prevention } \\
\text { Dementia supportive communities }\end{array}$ & \\
\hline & $\begin{array}{l}\text { Opportunities for learning and employment } \\
\text { Loneliness and isolation }\end{array}$ & \\
\hline
\end{tabular}

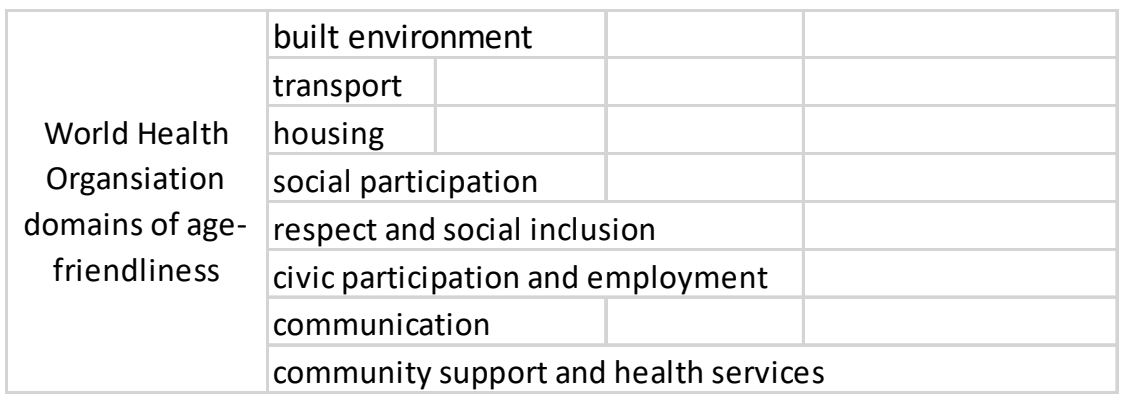




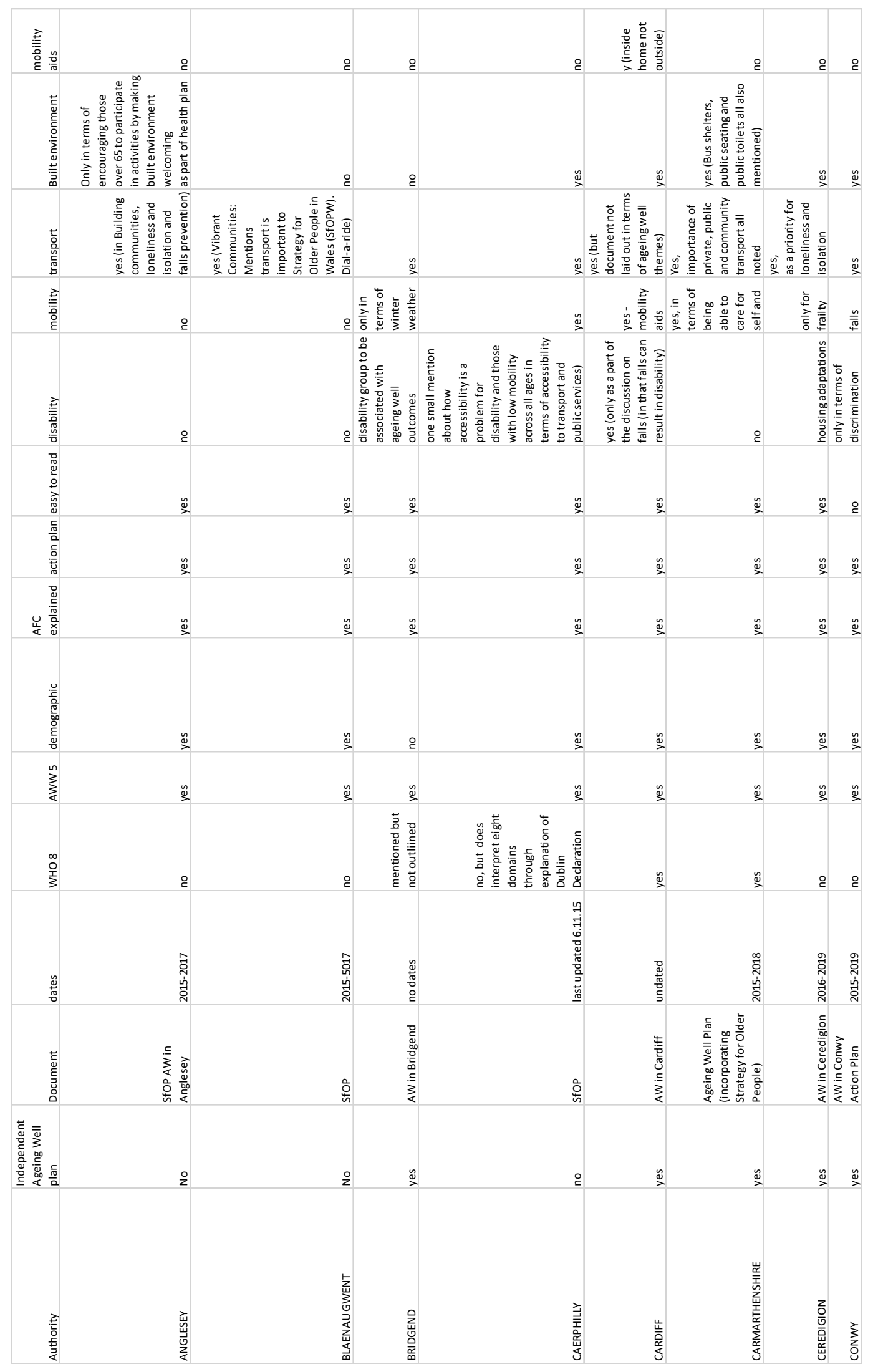

324 


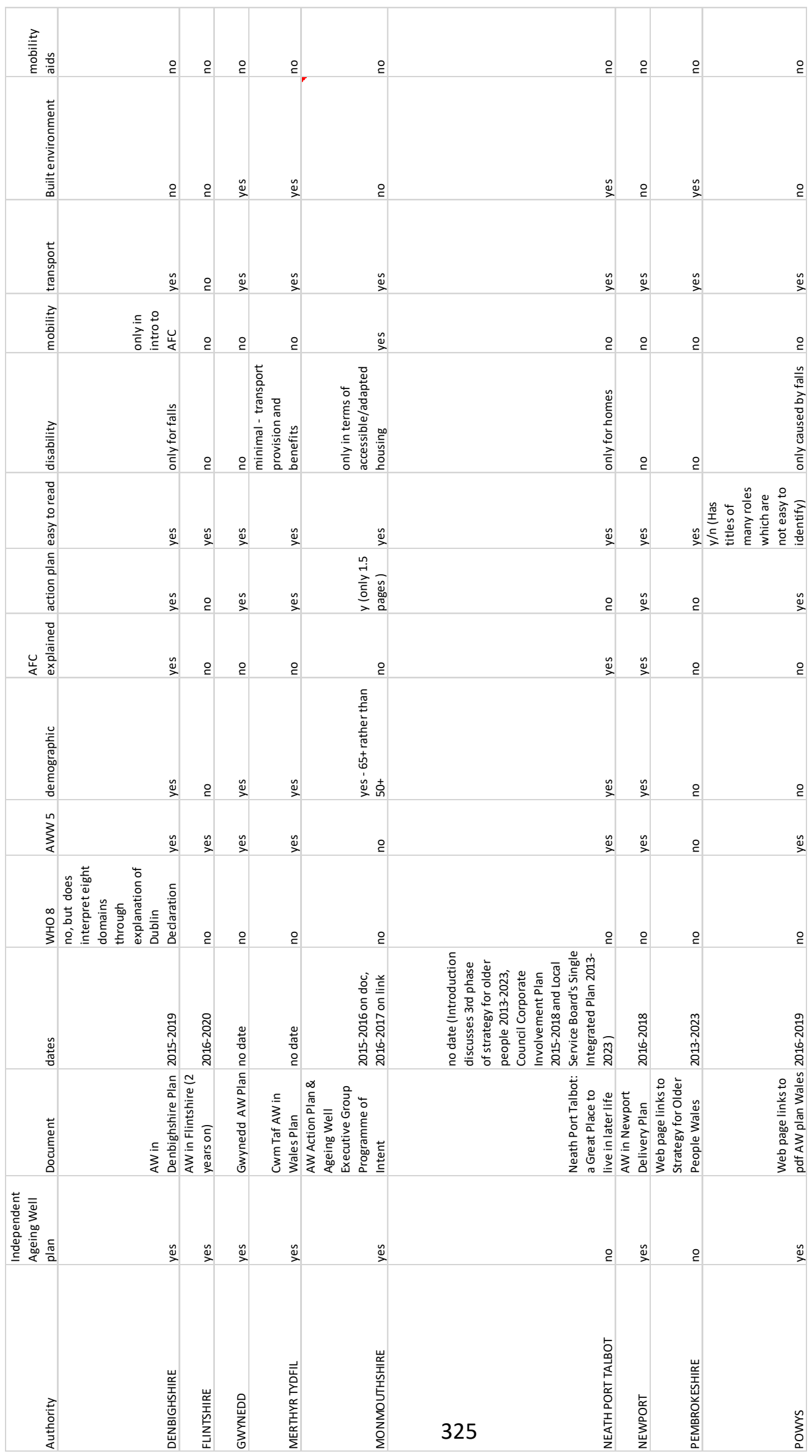




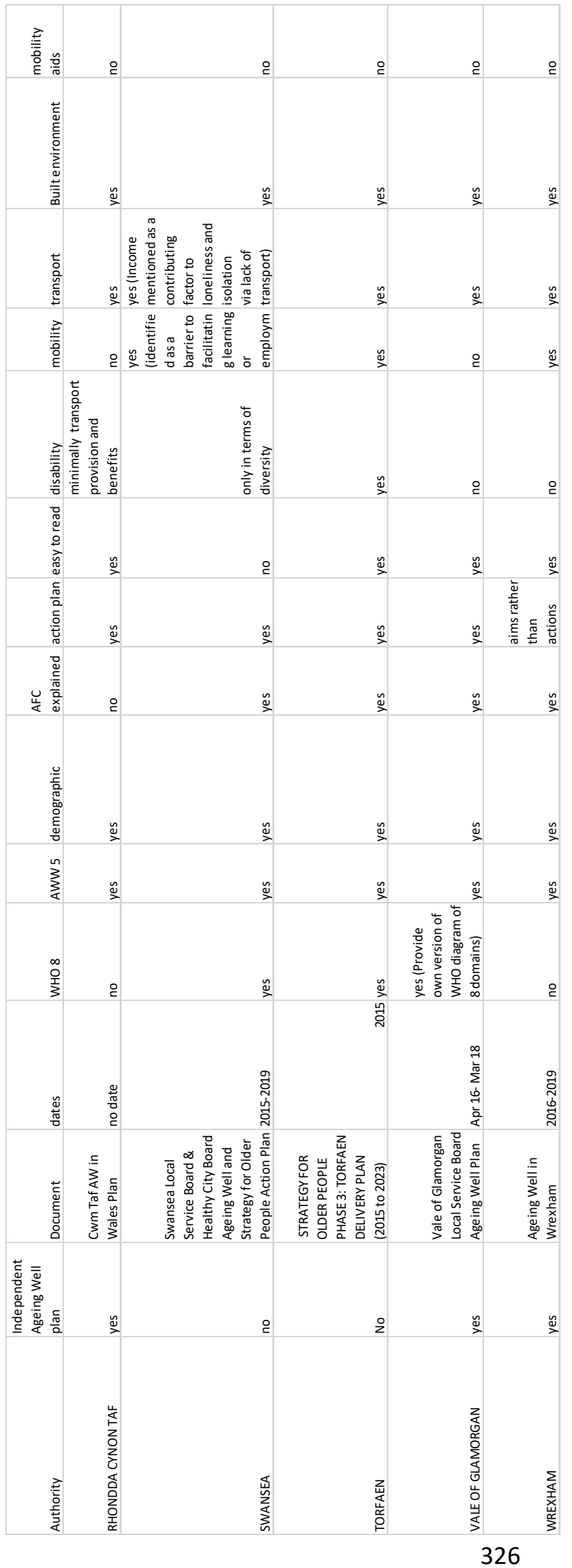




\begin{tabular}{|c|c|}
\hline Authority & Summary of Ageing Well Plans \\
\hline ANGLESEY & $\begin{array}{l}\text { Kind of traffic light structured diagram to indicate programme } \\
\text { structure. Easy to read document. Links Older Peoples Plan to } \\
\text { Social Services and Well-being Wales Act, Well-being of Future } \\
\text { Generations Act, Declaration of Rights for Older People Wales, } \\
\text { Ageing Well in Wales and the National Public Health } \\
\text { Framework. Inverts the triangle of care model. Talks of } \\
\text { appointing an age friendly communities champion. Loneliness } \\
\text { and isolation action plan includes accessible transport and } \\
\text { inclusion of transport costs in all funding applications. }\end{array}$ \\
\hline BLAENAU GWWENT & $\begin{array}{l}\text { Document is about Local implementation of the Older People's } \\
\text { Strategy for Wales. Document very difficult to read but } \\
\text { identifies that its general aims and plans as a council are age } \\
\text { friendly. No specific age friendly plan but does mention that } \\
\text { there is a need to be mindful of transport }\end{array}$ \\
\hline BRIDGEND & $\begin{array}{l}\text { Delivers against outcomes of Strategy for Older People } 2013 \\
\text { (2013-2023). Links to Single Integrated Plan and Corporate } \\
\text { Plan }\end{array}$ \\
\hline CAERPHILLY & $\begin{array}{l}\text { Links to Equalities Act, Social Services and Wellbeing Act, SfOP, } \\
\text { mentions sing up to Dublin Declaration. Links isolation to lack } \\
\text { of accessibility. }\end{array}$ \\
\hline CARDIFF & $\begin{array}{l}\text { Mentions Dublin Declaration, mobility aids as part of } \\
\text { Independent Living requirements (although not as } \\
\text { considerations for planning per se). Good, readable document } \\
\text { with diagrams }\end{array}$ \\
\hline CARMARTHENSHIRE & $\begin{array}{l}\text { Reports public concerns as canvassed by } 50+\text { forum which } \\
\text { includes street lighting, paving bus shelters. Provides solution }\end{array}$ \\
\hline
\end{tabular}




\begin{tabular}{|c|c|}
\hline & $\begin{array}{l}\text { suggestions in light of inadequate funding including influencing } \\
\text { other organisations. }\end{array}$ \\
\hline CEREDIGION & $\begin{array}{l}\text { Gives diagram of plan in Dahlgren and Whitehead climate } \\
\text { change diversity chart. Priorities and focus with target } \\
\text { outcomes and reporting framework (not so clear in other } \\
\text { documents). Partnerships with external organisations } \\
\text { mentioned. Suggests one good practice they have in place is a } \\
\text { park and ride link with the hospital. }\end{array}$ \\
\hline CONWY & $\begin{array}{l}\text { Outline eight key outcomes (not related to WHO domains). } \\
\text { Article } 4.3 .3 \text { encourages active ageing by supporting a healthy } \\
\text { lifestyle which may miss the point. }\end{array}$ \\
\hline DENBIGHSHIRE & $\begin{array}{l}\text { Links ageing well plan to well-being plan. States that ageing } \\
\text { well programme (not sure if they mean their own or the } \\
\text { Central Wales one) is to ensure the improvement of well-being } \\
\text { of people 50+. Support and champion a positive attitude } \\
\text { towards ageing. Seems to be very 'responsibilised' version of } \\
\text { ageing well. Does demonstrate goals and ways to implement } \\
\text { but does not really implement age friendliness. Does } \\
\text { encourage synergy between Ageing Well, Well-Being and } \\
\text { County Council Corporate Plans }\end{array}$ \\
\hline FLINTSHIRE & $\begin{array}{l}\text { Postcards from the future' with scenarios of desired outcomes. } \\
\text { No actual plans for how to achieve these or how ageing well } \\
\text { plans might integrate with transport, planning or policy. }\end{array}$ \\
\hline GWYNEDD & $\begin{array}{l}\text { Reflects essential principles of social services and wellbeing } \\
\text { act. Links housing and transport needs to isolation and } \\
\text { loneliness. Highlights good practice and plans for future }\end{array}$ \\
\hline
\end{tabular}




\begin{tabular}{|c|c|}
\hline MERTHYR TYDFIL & $\begin{array}{l}\text { CWM TAF joint plan with Rhondda Cynon Taf. Explains Age } \\
\text { Friendly Communities concept and incorporates Authorities' } \\
\text { own Well Being Plan. In Merthyr, 53\% of carers are 50+. Age } \\
\text { friendly champions to be provided/accredited. One downside } \\
\text { that an outcome of Loneliness and isolation outcome is that } \\
1.3 \text { states 'Empower older people to be aware of risks of } \\
\text { loneliness and isolation to their wellbeing'. Not sure their } \\
\text { knowledge of it is the problem. On the plus side special } \\
\text { transport for communities now well served by public transport } \\
\text { or those with disabilities. }\end{array}$ \\
\hline MONMOUTHSHIRE & $\begin{array}{l}\text { 2015-16 action plan and an Ageing Well Executive Group } \\
\text { Programme of Intent 2015-2017. Sarah Rochira quote as with } \\
\text { Denbighshire- uses first half about people being responsible } \\
\text { for self but ignores the rest that she says about infrastructure. } \\
\text { Incorporates older population into single integrated plan. } \\
\text { Tendency to look at 65+ rather than 50+. Only mention of built } \\
\text { environment is in connection with RNIB. Vision for sustainable } \\
\text { and resilient future states 'Enable older people to be fully } \\
\text { independent, maintaining good mobility, health and wellbeing, } \\
\text { confidence and dignity and plan for their future'. }\end{array}$ \\
\hline NEATH PORT TALBOT & $\begin{array}{l}\text { Data partly based on consultation but does not say who was } \\
\text { consulted. Pats itself on the back for being commended for } \\
\text { providing good data. Provides information about Care homes, } \\
\text { corporate improvement plan, local service board integrated } \\
\text { plan, health and social care programme, health board, } \\
\text { voluntary service, 3rd sector. No real plan and talks in circles. }\end{array}$ \\
\hline NEWPORT & $\begin{array}{l}\text { bare bones document with little information more than the } \\
\text { basic policy and how it relates to existing documents }\end{array}$ \\
\hline
\end{tabular}




\begin{tabular}{|c|c|}
\hline PEMBROKESHIRE & $\begin{array}{l}\text { Web page lists } 13 \text { desired outcomes of strategy for older } \\
\text { people including diversity, access to information, shared } \\
\text { spaces and housing as well as the } 5 \text { themes. }\end{array}$ \\
\hline POWYS & $\begin{array}{l}\text { The whole focus of Age Friendly Communities is health and } \\
\text { social care. There is no suggestion of social needs. Transport is } \\
\text { only a provision consideration for isolated communities. There } \\
\text { is no mention of mobility difficulties or mobility aids and the } \\
\text { solution to people unable to get to medical consultations is } \\
\text { video conferencing. }\end{array}$ \\
\hline RHONDDA CYNON TAF & See Merthyr \\
\hline SWANSEA & $\begin{array}{l}\text { Minute scale writing. Incorporates older people views: this } \\
\text { entails } 3 \text { people at LSB Older People Strategy Partnership } \\
\text { Group (members of } 50+\text { group) } 60 \text { people at a drop in event } \\
\text { (accessible?) } 73 \text { respondents online survey, } 35 \text { people in } 2 \\
\text { forums, } 20 \text { people in another forum. Identifies work and gaps } \\
\text { in AFC and recognises importance of transport. Recognises } \\
\text { contribution older people make. Action plan includes } \\
\text { measuring outcomes against WHO AFC indicators as adapted } \\
\text { to Welsh context }\end{array}$ \\
\hline TORFAEN & $\begin{array}{l}\text { Links to Dublin Declaration. Includes recognition that people in } \\
50 \text { s outrages by being included in plans for ageing. Notes that } \\
\text { older people are not a homogeneous group. Planning journeys } \\
\text { for all ages especially with pushchairs, babies, children, } \\
\text { disability and poor mobility. }\end{array}$ \\
\hline VALE OF GLAMORGAN & $\begin{array}{l}\text { Fairly sparse document which sets out feedback from older } \\
\text { people and aims. Does demonstrate interpretation of WHO } \\
\text { domains and role of transport and built environment in } \\
\text { outcomes. }\end{array}$ \\
\hline
\end{tabular}




\begin{tabular}{|l|l|}
\hline WREXHAM & $\begin{array}{l}\text { Connects ageing well plan to community strategy plan. } \\
\text { Includes transport in isolation and loneliness. Recognises that } \\
\text { some barriers to participation are attitudinal (how we treat } \\
\text { older people and think about ageing) or physical (transport and } \\
\text { building design). Under 'What we have now' sections lists only } \\
\text { what it is working towards. Transport is a theme throughout } \\
\text { with mentions of accessibility and affordability. Shopmobility is } \\
\text { mentioned. Public transport linked to loneliness and isolation. }\end{array}$ \\
\hline
\end{tabular}




\subsection{Appendix A Health and disability statistics}

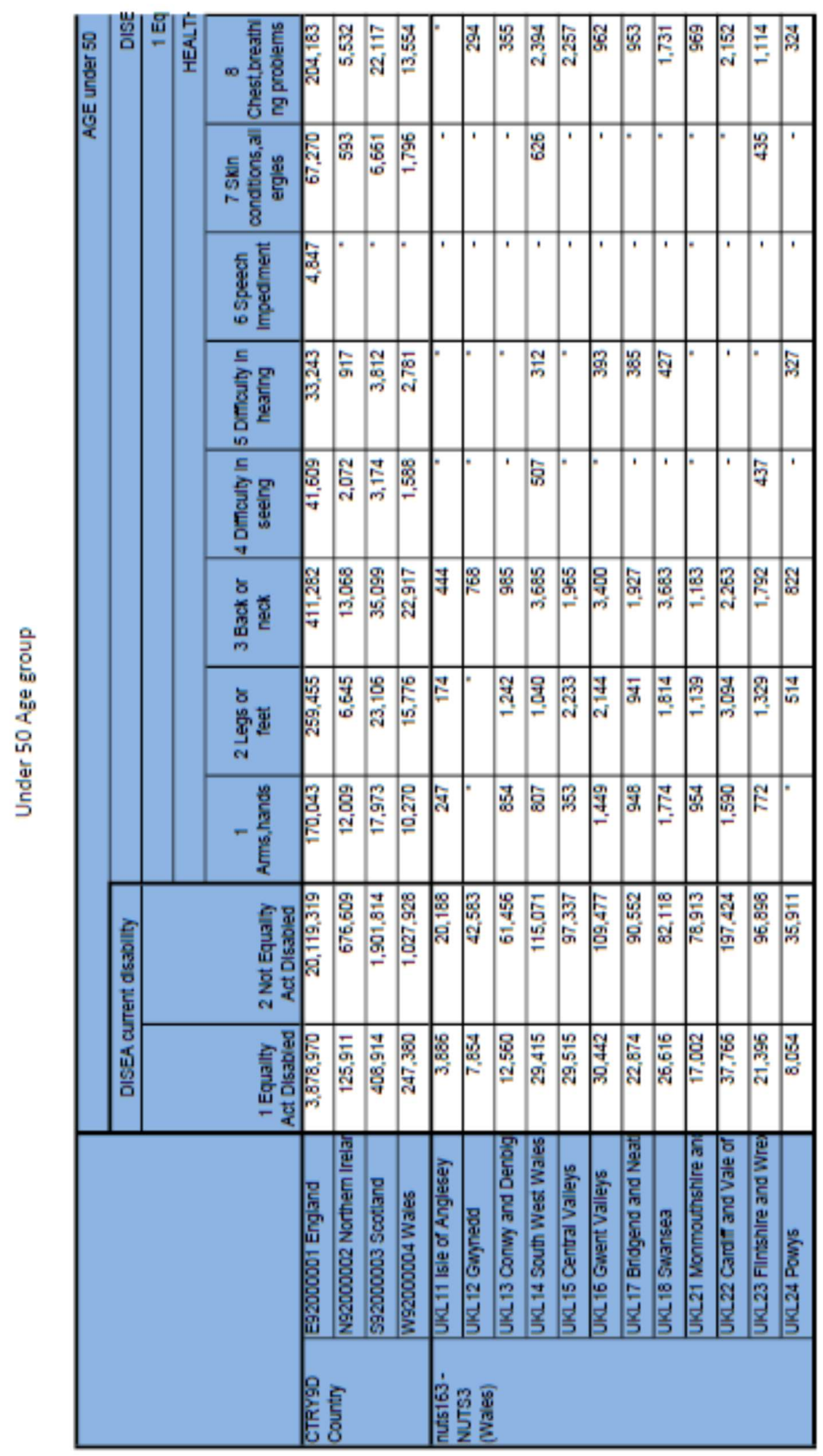




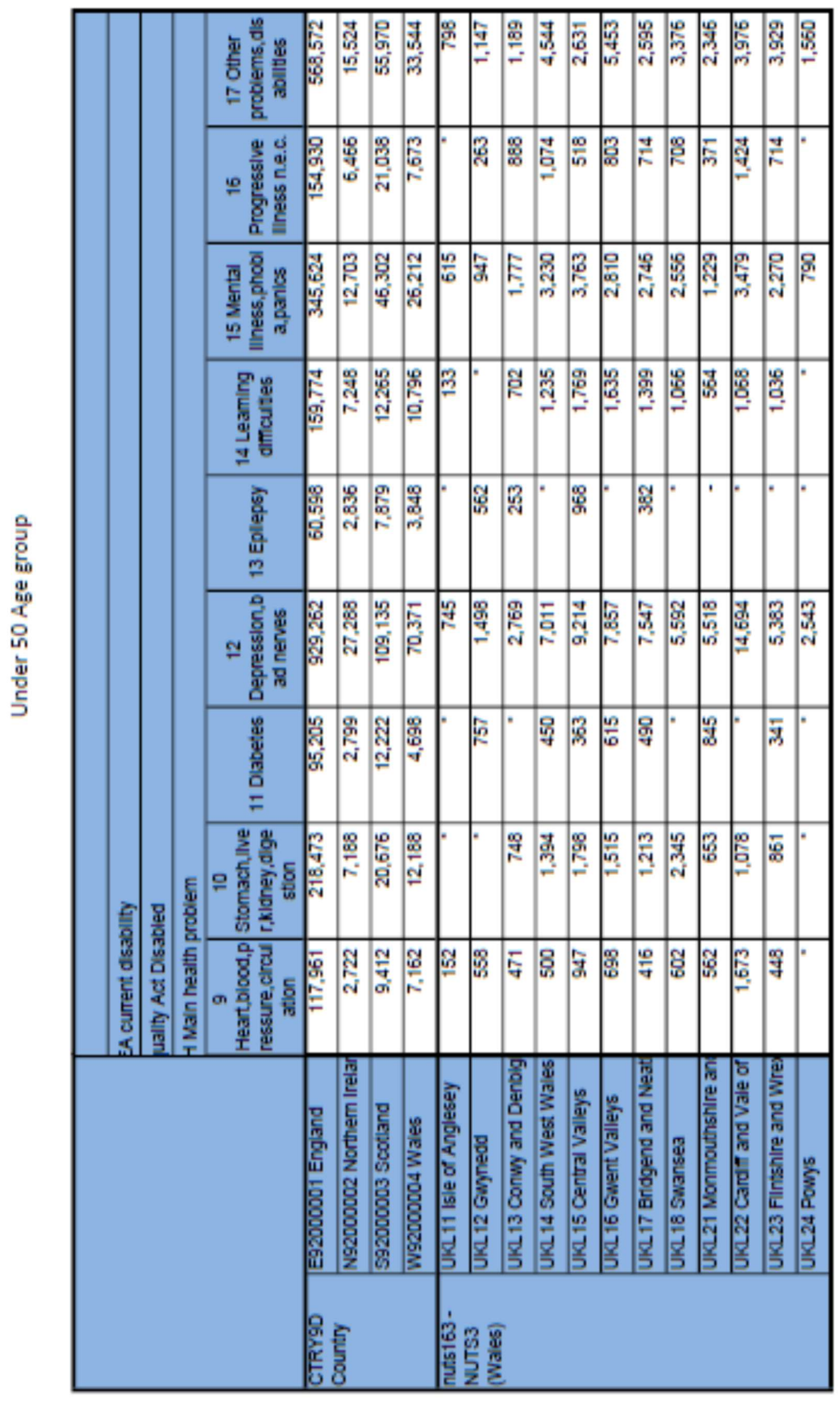




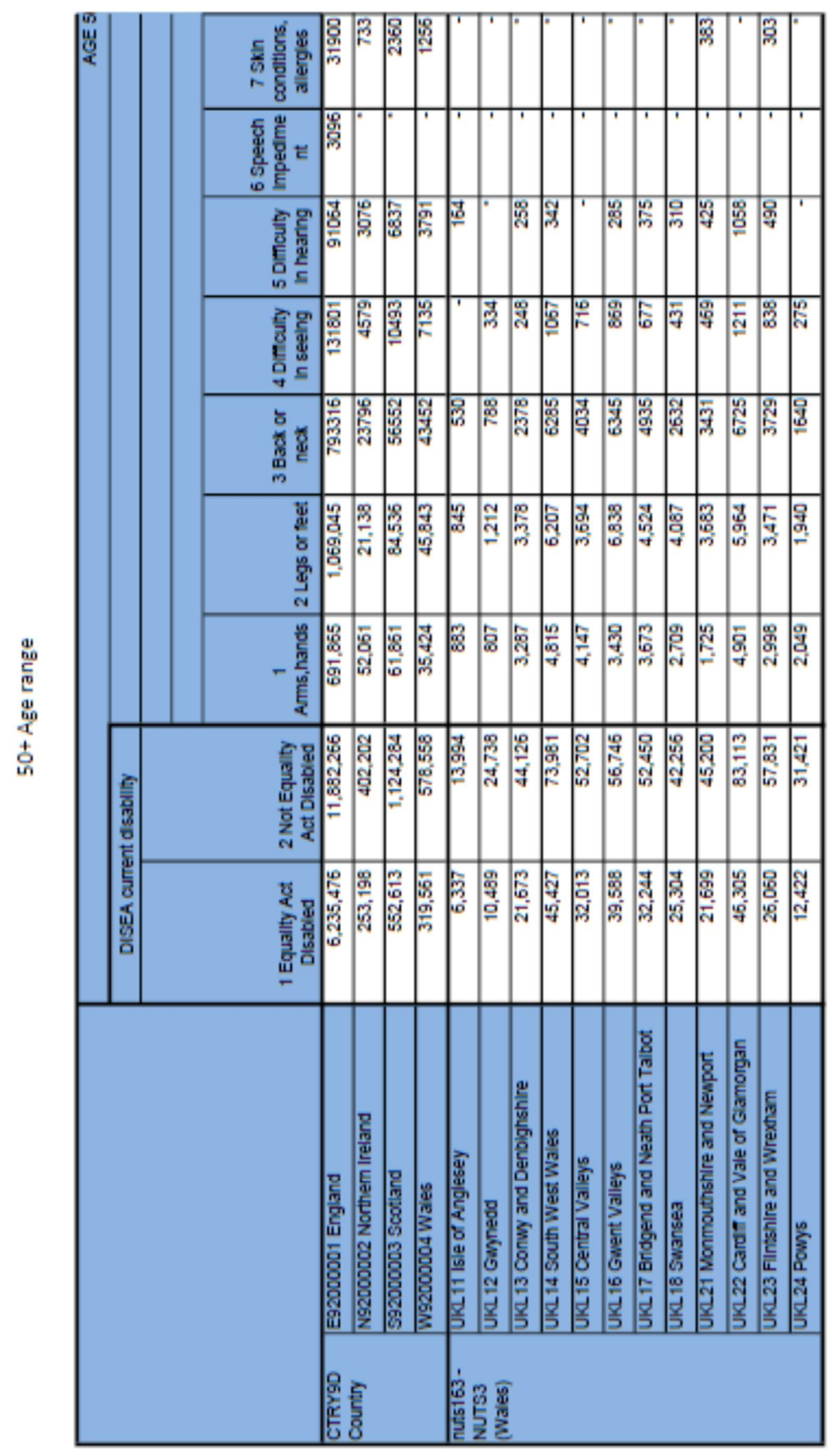




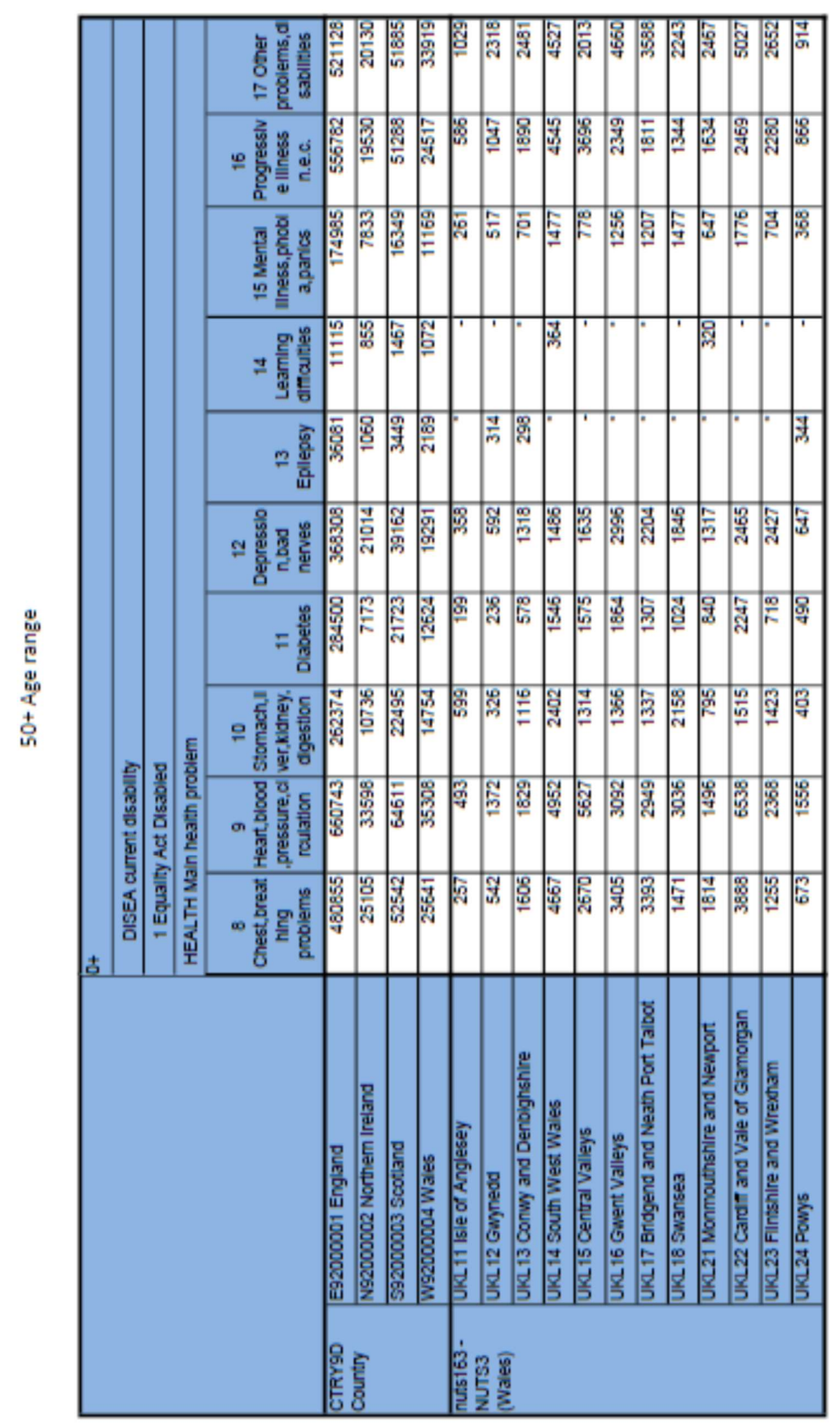




\subsection{Appendix B Literature search}

Date of Search: $\quad 10.10 .17$

Search terms: "mobility aid" and identity

No. of results: 540

Relevant to social science: 98

Relevant to study: $\quad 3$

Gardner, P. (2017). MAPx (Mobility Aid Personalization): Examining why older adults "pimp their ride" and the impact of doing so. Disability and Rehabilitation: Assistive Technology, 12(5), 512-518.

Description: We all do this. We personalize things. We buy leopard-printed seat covers and fuzzy dice for our cars, and display action figures and photographs in our offices. Studying older adults who have extended this process of personalization to their mobility devices, the purpose of the mobility aid personalization (MAPx) project is to examine MAPx and its impact on the health and mobility of older adults. Using a qualitative research design, field observations and interviews were conducted with 72 older adults to gain an in-depth understanding of device customization from an emic (insider's) perspective. Findings illustrate that older adults personalize their devices for reasons of fun, function and fashion. MAPx - the process of purposefully selecting or modifying a mobility device to suit individual needs and preferences - was also found to promote health and mobility by encouraging device acceptance, increasing social participation, enhancing joy and preserving identity. MAPx makes an important contribution to our understanding of the complex relationship between older adults and assistive devices and provides a new approach to some old problems including falls, inactivity and social isolation. Encouraging MAPx is a promising rehabilitation strategy for promoting health and community mobility among the older adult population. Implications for Rehabilitation Personalizing an assistive device facilitates device acceptance, promotes health and well-being and should be supported and encouraged in rehabilitative care. Choice, variety and access are critical aspects of assistive devices; vendors, manufacturers and practitioners should work together to provide clients with a 
greater range of affordable options for new devices. Function is more than mechanical or physical; social factors including social identity, stigma and social roles must be adequately considered and explicit in rehabilitative practice.

$\bullet 0$ Language: ENG

Identifier: ISSN: 17483115

Lindenberg, J, \& Westendorp, R.G.J. (2015). Overcoming Old in Age-Friendliness. Journal of Social Work Practice, 29(1), 85-98.

In this article, we explore views on an age-friendly space in the Netherlands by analysing the responses of older individuals $(N=54)$ in focus groups and by examining the perspectives around an age-friendly zone in the Netherlands, Parkstad Limburg. We found that a central issue in the wishes for living at a later age are adjustments to envisioned physical limitations that come with the ageing process; this includes adjustments to ensure safety, accessibility and mobility, in order to facilitate older individuals' efforts to stay engaged with the world around them. In their wishes, the older participants constructed ideal dwelling places that closely resembled a senior home, but at the same time they rejected wishing to live in a place that was identified as a senior home. We explain this paradox by the representation of such a space as being for old people, i.e. needy older individuals, which was not how the older participants wished to be identified. We conclude that the conception of age-friendly environments will have to face the difficult challenge of overcoming the association with old age, while simultaneously considering adjustments that signify and relate to the ageing process and that seem inescapably tied to oldness.

\section{Rush, K L, \& Ouellet, L L. (1997). Mobility aids and the elderly client. Journal of Gerontological Nursing, 23(1), 7-9.}

As life expectancy increases, nurses working with elderly clients will continue to be challenged to maintain mobility, particularly in clients with chronic illness. Because of this, it is important to have research-based strategies to assist clients. Currently there is a dearth of nursing related research in the area of mobility and mobility aids. The findings 
from this study may provide the impetus for re-examining the concept of mobility, and its underlying assumptions as foundational to nursing practice. 


\subsection{Appendix C Ethical Approval}

Application for Standard Ethical Approval (V.06/15)

\section{CHECKLIST}

Please note that we are able to review an application only when all documentation is submitted alongside this application form. Should any necessary appendices not be attached, this could delay the submission until the following month. Please use this checklist below to ensure that the application is complete. Many thanks.

\begin{tabular}{|l|l|l|}
\hline & $\begin{array}{l}\text { Attached } \\
\text { Yes / No / }\end{array}$ & Comment \\
N/A & No & \\
\hline Recruitment advertisement or email(s) & Yes & \\
\hline Participant information sheet(s) & Yes & \\
\hline Consent form(s) & Yes & \\
\hline Debrief sheet(s) & N/A & \\
\hline Questionnaire(s) & No & \\
\hline Interview or Focus Group schedule(s)/questions & N/A & \\
\hline Workshop schedule(s)/questions & N/A & \\
\hline Written consent from public or private body & Yes & \\
\hline Supervisor signature & & \\
\hline
\end{tabular}


PLEASE COMPLETE THE FORM USING TYPESCRIPT

(hand-written applications will not be considered)

\begin{tabular}{|c|c|c|}
\hline $\begin{array}{l}\text { Principal } \\
\text { Investigator }\end{array}$ & Allyson Rogers & \\
\hline Date & $12 / 06 / 17$ & \\
\hline School & $\begin{array}{l}\text { Centre for Innovative Ageing, College of Human and } \\
\text { Health Sciences }\end{array}$ & \\
\hline $\begin{array}{l}\text { E-mai } \\
\text { I address }\end{array}$ & 843126@swa" I address & 843126@swansea.ac.uk \\
\hline $\begin{array}{l}\text { Title of Proposed } \\
\text { Research }\end{array}$ & $\begin{array}{l}\text { Age Friendly Communities for people with Mobility } \\
\text { Difficulties }\end{array}$ & \\
\hline $\begin{array}{l}\text { Type of } \\
\text { Researcher } \\
\text { (please tick) }\end{array}$ & $\begin{array}{l}\text { Undergraduate student } \\
\text { Postgraduate student } \\
\text { Member of staff } \\
\text { Other, please state: }\end{array}$ & \\
\hline $\begin{array}{l}\text { Name of course \& } \\
\text { supervisor }\end{array}$ & $\begin{array}{l}\text { PhD Gerontology and Ageing Studies, Charles } \\
\text { Musselwhite }\end{array}$ & \\
\hline $\begin{array}{l}\text { Supervisor e-mail } \\
\text { address }\end{array}$ & C.B.A.Musselwhite@Swansea.ac.uk & \\
\hline $\begin{array}{l}\text { Qualifications and } \\
\text { professional } \\
\text { background }\end{array}$ & $\begin{array}{l}\text { BSc Criminology and Psychology } \\
\text { MSc Social Research Methods }\end{array}$ & \\
\hline
\end{tabular}


1. Briefly describe the rationale and the main aims of the research you wish to undertake, including a statement of the intended benefits of the research. Please use non-technical language wherever possible.

The world population is ageing, by both number and share of older people (WHO 2013), leading to ageing issues being increasingly discussed within transport and mobility fields (Murray 2016; Schwanen \& Paez, 2010). As those over 70 are the group most likely to have mobility and accessibility issues, there is a need to look at the wider relationship between mobility and ageing in relation to health and well-being (Musselwhite 2016). The social model of disability and resulting accessibility legislation has heralded a great deal of improvement for the inclusion of those with disabilities, but there seems to be little attention paid to emotional or psychological needs of people with physical impairments (Oliver, 2013). From the perspective of mobility aid provision, outcomes are measured in terms of independence for user, without considering the impact of this functionality on mobility aid user or carer, family or social life (Hammel et al, 2013).

- The main aim of this study is to understand how older people feel about using mobility aids as tools to assist everyday living. The research centres around how older people relate to mobility aids, and how they feel planning, services and the environment cater for their needs. Carers are also to be explored by the research as an overall investigation into how well mobility aid users and those who care for them feel socially and practically included (or excluded) by the planned environment and society. Much literature has concluded that people with embodied differences who use mobility aids feel socially excluded (e.g. Barlew et al, 2013; Mazurik et al, 2014) but has yet to understand what the barriers to inclusion are. Research into the everyday lives of those with disabilities has lacked contextualisation of obstacles or interpretation of the experience of social exclusion in the lived world (Hansen \& Philo, 2007; Mazurik et al, 2014). 
- This study aims to understand in some way what the barriers to inclusion might be for those using mobility aids within the wider context of ageing

- To understand the experience of using a mobility aid around the locale

- To understand if the use of a mobility aid is perceived to/does constrict movement

- To understand if a person's use of a mobility aid increases the workload of carers or family members.

- To assess any differences in the answers to the above questions depending on what type of mobility aid is used.

2. Briefly describe the overall design of the project including dates and/or the proposed period of investigation

The study is taking an ethnographic grounded theory approach using mapping, observation, sound recording, drawing (participant and researcher), photography, storytelling and discussion as methods for data collection. Recruitment will take the form of snowballing, commencing with people already known to the researcher who live in her home town. Constant comparative analysis through coding and revisiting the field on an iterative basis will continue until the research has reached a saturation point. Data collection will commence in July/August 2017 and continue for approximately eight to ten months.

3. Briefly describe the methods of data collection and analysis. Please describe all measures to be employed. If questionnaire or interviews are to be used, please provide the questionnaire / interview questions and schedule.

Participants will be provided with maps to understand their usual movement around their locale. The researcher will converse with participants, or observe actions and interactions to understand 
how, when and why people move around their environment, and how they feel about their movements, locations in their locale and any changes to the environment that may have affected participants positively or negatively.

1. Identify participant (see participant information in boxes 5 and 6 below)

2. Seek informed consent (appendix A), explaining the aims and methods of research as per the participant information sheet (appendix B). (Information given in Box 10 gives a more detailed outline of the briefing process.)

3. Agree a convenient time to accompany each participant individually. Participation is expected to take one or two hours. Participation may take place on more than one occasion.

4. Maps will be the starting point of research collection with each participant, followed by a combination of interviews and walking interviews depending on the schedule, wishes and physical abilities of each individual participant.

4.1. Each participant will be shown a map of the locale and asked to discuss their movements in relation to the map. Individuals will be asked if there are particular places they like, dislike, hold memories or evoke feelings of exclusion.

4.2. Researcher and participant will conduct walking interview as possible and safe for each individual, going to places, encountering the environment and people, observing actions and interactions in walking style interviews as outlined by Evans \& Jones (2011).

4.3. Researcher will take notes and record conversations en route where and when safe to do so. Audio recordings may take place at destinations such as the home and in the local environment. Researcher may also photograph or sketch environments, especially if a place or item is outlined as important in some way.

5. Participants will also be asked to describe and converse about important locations or items. They will be asked to photograph them (or researcher may photograph them), share memorabilia, draw representations if they are willing and able. Any photographs used for research purposes will only include consenting participants. It is intended that photographs will not contain people.

6. Writing detailed notes, coding and analysing data after each participation, comparing all new data with any existing data in constant comparative analysis. Analysis of the first participant's 
data will take place before collecting data from the next participant, whose data will be analysed along with the first set of data before collecting the next set, and so on until the point of saturation. If saturation is not reached, data collection will cease at 40 participants.

7. Analysis cannot be fully substantiated until the researcher has some acquaintance with the setting and participants

8. Using the outputs noted above (e.g., recordings of conversations, fieldnotes, sketches any memorabilia the participant might choose to share) the researcher will identify plausible patterns and themes or conceptual groupings and connections.

9. Once these themes or patterns have been identified within the data of the first participant, substantiation and support for the theme(s) or pattern(s) will be sought in the next data set.

10. Data will be continually analysed, compared within the data set, and referring to external preexisting concepts and theories.

11. Through this continual process of comparing and contrasting, regrouping variables as and when necessary, and differentiation of variables, themes or patterns a coherent chain of evidence will be sought to ultimately form a theory or concept

12. This iterative process is designed to identify regularities in the physical and social worlds, so in this particular case, understand the physical and social reality of living with mobility aid use.

Refs

Evans, \& Jones. (2011). The walking interview: Methodology, mobility, and place. Applied Geography, 31(2), 849-858.

Huberman A. and Miles, M. (1998) 'Data Management and Analysis Methods' in Collecting and interpreting qualitative materials / edited by. Denzin N. and Lincoln Y. Thousand Oaks, Calif.: SAGE.

This research is guided by grounded theory so only information relating to the research question and guided by participants will be collected and used. 
Visual data will be used as a prompt to discuss issues around accessibility and ability to inhabit the locale. Any visual data used in the research will be anonymised.

Age and gender differences in attitude to mobility aid use will also be analysed.

NVivo will be used as a collation and analysis tool

4. Location of the proposed research (i.e., Departmental labs, schools, etc)

The local environment as inhabited by participants. This could include, but is not limited to, their homes, cars, public areas such as cafés, or other areas of social interest usually attended in the course of participants' daily activities. Participants will be fully informed that part of the research will include speaking to the researcher while walking in the local area, if that is the usual practice for that participant. Any private conversation will be stopped if there is a chance it is being overheard. All participants will be reminded that they can withdraw at any point without the need to give a reason.

Any space that makes either the participant or researcher uncomfortable will be avoided or moved on from. The places visited will be driven by the interests and life of the participant. Instructions will include both written and verbal instructions that participation can be continued at a later time or cease without reason or prejudice. The researcher feels a duty to adjust to participant needs, but if the researcher feels unsafe at any time the research, or with a particular action, it will be ceased as per safety protocols. Please also see box 8 for participant safety and box 9 for researcher safety.

5. Describe the participants: give the age range, gender, inclusion and exclusion criteria, and any particular characteristics pertinent to the research project.

Participants will be people over the age of 50 as per the Welsh Government's Strategy for Older People in Wales (2013). 
As the study has been inspired and informed by the researcher's role as carer to her chronically ill, mobility aid user spouse, conversations/interviews with him will hold key information.

Men and women are of equal interest and comparisons made between genders to understand if there are any differences. The inclusion criterion is use of mobility aid or being the carer or loved one of a mobility aid user. This can mean anything from a walking stick, to a walking frame, an aid for vision impairment or a wheelchair. The important aspect is the need to use an item for navigation that perceived 'normal' or 'fit' people do not require in normal circumstances. People do not, however, need to use mobility aids permanently or constantly. It is of interest to the study why people choose not to use their mobility aids on some occasions.

Comparisons made within the analysis will include gender and age or cohort differences.

\begin{tabular}{||l|l|}
\hline Inclusion Criteria & Exclusion Criteria \\
\hline Over 50 & Under 50 \\
\hline $\begin{array}{l}\text { Uses or cares for someone who } \\
\text { uses a mobility aid }\end{array}$ & $\begin{array}{l}\text { Does not use or care for } \\
\text { someone who uses a mobility } \\
\text { aid. }\end{array}$ \\
\hline $\begin{array}{l}\text { Should use, or cares for } \\
\text { someone who should use, a } \\
\text { mobility aid but refuses to do } \\
\text { so. (Need for use decided by } \\
\text { medical intervention, physical } \\
\text { difficulty or family and friends' } \\
\text { observation) }\end{array}$ & Has no need for a mobility aid \\
\hline
\end{tabular}




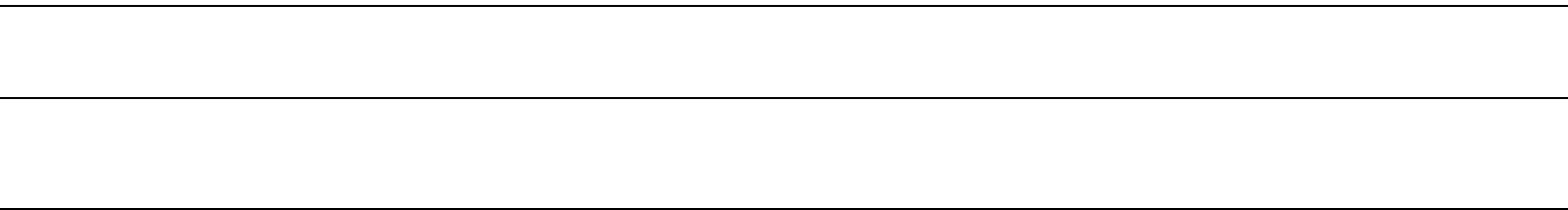

6. How will the participants be selected and recruited? Please describe in detail the process of recruitment, including how and by whom initial contact is made with participants (e.g. advertisement, e-mail).

Participants will be recruited through word of mouth and snowballing methods using the researchers own informal personal network to make suggestions about who might meet the inclusion criteria. The individuals suggested will be contacted by the researcher, but this action will be kept confidential. These individuals will also be asked to suggest people and so on. No disclosure will be made from one person to the next whether any suggested people actually took part.

In order to make explicit the researcher's experience and understanding of mobility aid use by a person over 50, the decision was made to interview her spouse. This gives insight into her understanding of mobility aid use, and offers key information to the inspiration, insights and grounding of the researcher's pre-existing knowledge of mobility aids and caring for someone through transition to mobility aid use in middle age. The researcher's spouse has been in no way coerced to take part and is willing to share his experience and knowledge.

People who take part in the research will be advised that there is a chance they may be identified by other people who know of the research if they are seen talking to the researcher in public as part of the informed consent process. They will be informed there will be no comparisons between participants or relationships between people discussed. The people, places and any visual data used will be anonymised and not identifiable. Anonymisation is not completely achievable with my husband but every attempt to obscure his identity will be made.

The researcher was informed of a local friendship club by a neighbour and has been along to give a presentation about her research. Following this short presentation, some attendees asked if they could participate and club organisers asked if I would keep them informed of my progress. They were informed that the research needs to gain ethical approval before it can actually begin 
data collection, but that the researcher would return on receipt of this. Before commencing research, representative of the club be asked to sign consent to research being communicated through the club (Appendix F)

If any of these individuals are recruited, the fact that there will be no prejudice if they decide not to withdraw and have nothing to gain personally will be reinforced.

Participation is not limited to the researchers' home town or the surrounding rural areas as some participants may be drawn from personal networks in Swansea and/or Carmarthen.

A saturation technique will be utilised in terms of participant numbers, but there will be a maximum of 40 participants.

7. What procedures (e.g., interviews, computer-based learning tasks, etc.) will be used to gather information from participants?

Observation, map data, ethnographic discussion and observation, interviews, audio recording of daily activities, conversations and stories told by participants to the researcher. Sketching and drawing of the locale and/or photographs of street architecture or places. Nothing within the photos or drawings will identify or name places.

The design of the research follows ethnographic principles and methods from the above list will be used as they evolve with each participant.

8. What potential risks to the participants do you foresee and how do you propose to ameliorate/deal with potential risks? Declare any relationship with the participants.

BPS Code of Human Research Ethics (2014) states that researchers must respect the dignity and rights of participants in research. It is an important basic principle to start from this basis of 
respect and dignity when approaching research design to ensure that 'dignity, rights and welfare are protected'(p5).

The researcher is likely to know some of the participants as neighbours but not so well that any of them will feel coerced into participating. None of the researchers' direct neighbours are mobility aid users, so do not fall within the inclusion criteria. As previously stated, individuals will be told during the consent process that they are not under any obligation to take part, and nobody will think any different of them whether they take part or not, that it is completely their own decision. They will also be told that to withdraw at any point during the process is both acceptable and normal.

The researcher's husband is fully aware of the basis, inspiration, potential outputs and consequences of taking part in the research, most. He is subject to informed consent and right to withdraw protocols in the same way as any other participant. However, as my husband his anonymity and confidentiality cannot be protected in the same way as other participants, which we discussed at length. He fully acknowledges and endorses use of his information as it is inextricable from my own experience which forms the basis of the research.

As this research will be conducted whilst carrying out normal routines and practices, the risks will mainly be the same faced in day-to-day life i.e. risks of falls, issues of road safety. To avoid detracting attention conversations taking place whilst in transit will be audio recorded. To ameliorate the risk of researcher's presence potentially altering perception and concentration, a risk assessment will be carried out beforehand (appendix E). This will cover how safety will be managed and that participation can take place over a number of occasions or cease at any time.

The issues will be those brought to the fore by participants themselves, and as such will already be on their minds. This somewhat ameliorates the potential for introducing self-doubt. Whilst it is not predicted that the experience will evoke any excessive emotional or psychological reactions, 
the researcher will advise any distressed participants that they may cease participation at any time. The researcher will have helpline and local medical advice lines numbers available (also included on the Debrief sheet).

There is a chance that stigma is likely to discussed, but that is about the stigma of using a mobility tool which is already used, and will only be brought up by the participant. If they wish to suggest a conversation in private that will of course be arranged, however any confidential or contentious issues that might arise should have been addressed during initial conversations. Participants will be informed beforehand that any conversation they feel is being overhead can be stopped, and the researcher will stop for the same reason. Any discussions whilst in public would be only those expected to be held in public anyway. The chance that a participant might be seen chatting to me is a risk that will be discussed in the consent process. Recording devices will be inconspicuous and no note taking will occur during walking interviews, so a conversation could potentially be about anything.

An excessive psychological or emotional reaction cannot be ruled out, but this is true of any social research. If anyone does show distress the participant will be offered a break and to cease participation if this is appropriate. The researcher will guide the individual to their GP and/or support groups in the longer term, as per numbers on the debrief sheet

Participants will be told that conversations can be stopped at any time if they feel private conversations can be overheard. Also, any participation can be carried out over a number of occasions in a way that suits each individual. Participants will be informed that the information given will be confidential and anonymised, so that in the research places, people and things will not be named or identifiable in any way so information cannot be linked to anyone.

For confidentiality of data also see item 16. 
The research will be conducted with integrity, respecting the needs and wishes or participants whilst intending to contribute to the development of research and understanding. It is also hoped some elements of this research will contribute directly to the community with visits to local community groups to both gain and provide understanding at the beginning and end of the research process with an introductory presentation and presentation of results for participants to comment on the findings if they choose (member validation). It will be made clear to participants that the research cannot directly influence local decision-making processes, how the knowledge gained within this process will add to wider understanding of how design needs to accommodate people who use mobility aids.

9. What potential risks to the interests of the researchers do you foresee and how will you ameliorate/deal with potential risks?

I will follow the Centre for Innovative Ageing's 'Personal safety guidelines for researcher and interviewers' by making a family member and first or second supervisor always knows when I am actively collecting data and where I am by telephoning or texting on arrival and cessation of research practice/interview/observation. As discussed for participant safety, discussions whilst in transit will be recorded to avoid distraction from health and safety issues of traffic or falls. Any discussion with passers-by inadvertently recorded will not be used in the research.

The idea of ethnographic grounded theory research is that normal routines are observed, studied and discussed. Therefore, walking interviews are to be carried out with people who usually walk (and walking can mean using a wheelchair or any other mobility aid for the purposes of this statement) as part of their everyday routine. If the routine involves driving a car everywhere, that participant would not be asked to walk. The useful information would be about where they drive to, how often and why. Anyone who is housebound would remain housebound, as per the aim of the study - to examine the lifestyle of the person and to understand if it has changed as a 
consequence of using the mobility aid, of chronic illness or ageing issues, or a combination of issues.

10. How will you brief and debrief participants? (Please attach copy of participant information sheets and relevant debrief information)

Participants will receive a participant information sheet as part of an informed consent process. Debrief will take the form of a thank you leaflet again outlining the aims of the research, providing contact details of the researcher and supervisor and helpline numbers.

Each new potential participant will be contacted via a telephone call, email or letter (appendix D) depending on the information provided by the participant making the referral.

This first contact will be to determine whether the person is interested in participating in the study by providing a brief explanation of the purpose and methods used. Following the initial contact, a letter, email or visit (depending on individual preference and circumstances) will brief participants (with Participant Information Sheet, Appendix B) about what participation involves. This will outline what participants might expect from taking part in the study, the possible benefits and any possible risks. Right to withdraw, data protection and anonymity will also be explained at this point. The researcher will ensure potential participants understand what they will be expected to do before continuing with participation, at which point a date will be made for participation to take place.

Participants will have as long as they need to decide to take part, up until data collection ceases. If a potential participant wants to think about participation, the researcher will either set a date to make another contact, or leave it to the individual to make the next contact, depending on the wishes and needs of the individual in question. 
Before commencing participation, researcher will again go through the participant information sheet. Once both researcher and participant are happy that informed consent has been gained, participation will take place.

Following participation, individuals will be given a debrief sheet (appendix $\mathrm{C}$ ) thanking them for taking part and reiterating the aims and use of the research.

A Member validation process is intended, whereby some or all participants (depending on availability of the participants) will get the chance to comment on the main findings of the study, which will be included in the final report. Participants will also be informed that they may opt to receive a summary of the final report/PhD or copy of the full PhD if they wish.

\begin{tabular}{|l|l|l|}
\hline $\begin{array}{l}\text { 11. Will informed consent be sought from } \\
\text { participants? }\end{array}$ & $\begin{array}{l}\text { Yes (Please attach a copy of } \\
\text { the consent form and } \\
\text { participant information sheet) }\end{array}$ & $\mathrm{V}$ \\
\cline { 2 - 3 } & & $\begin{array}{l}\text { See } \\
\text { appendices } \\
\text { Af nond B please explain below: }\end{array}$ \\
\hline
\end{tabular}

12. If there are doubts about participants' abilities to give informed consent, what steps have you taken to ensure that they are willing/competent to participate? 
Participants must be able to give full informed consent. If the researcher has any doubt regarding a participant's capacity to provide such consent, the participant will be excluded from the research.

13. If participants are under 18 years of age, please describe how you will seek informed consent.

Participants will be over 18

14. How will consent be recorded?

Consent will be recorded in the form of signed documentation and kept at the Centre for Innovative Ageing in a locked cabinet.

15. Will participants be informed of the right to withdraw from your study without penalty? If no, please explain why.

Yes. Right to withdraw is explained in Participant Information Sheet (Appendix B)

16. How do you propose to ensure participants' confidentiality and anonymity?

Other than consent forms, no documentation will record real names. All data collected will be identified with a pseudonym. The researcher will be careful to ameliorate the risk of any information that might identify participants inadvertently, such as the use of a particularly unusual mobility aid in a specific place. As previously pointed out, this is not completely possible for my husband but where possible, the connection between his data and his status as my husband is avoided. 
All consent forms will be kept in a locked cabinet in the Centre for Innovative Ageing.

No place names or personalisable information (such as profession) will be used. Photographs will be used for description and to exemplify situations or architectural details (e.g., cobbles) and will not be published if they are potentially identifiable.

17. Please describe the arrangements for storing and disposal of data:

Please explain, for each of the above, the arrangements you will make for the security of the data Written consent forms will be kept in a locked cabinet as outlined above.

Audio recordings and photo hard copies will be kept in a locked desk at the home of the researcher, and computerised copies will be password protected/encrypted and stored on a hard drive which is kept in a locked cabinet when not in use.

18. Does your research require the written consent of a public or private body, e.g., school, local authority or company? If so, please attach letter of consent.

Consent form for Club. Please see explanation of relationship to club.

19. If your proposed research is with 'vulnerable' groups (e.g., children, people with a disability etc.), has an up-to-date Disclosure and Barring Service (DBS) check (previously CRB check) if UK, or equivalent non-UK clearance been requested and/or obtained for all researchers? 
N/A - however I have applied for a DBS check anyway

20. Does your research involve the collection of Yes

Human Tissue? E.g., saliva, urine

\begin{tabular}{|l|l|}
\hline Yes & \\
\hline No & $\mathrm{v}$ \\
\hline
\end{tabular}

Applicant's signature:

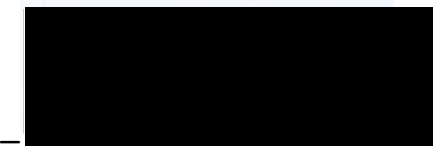

Date: $13 / 06 / 2017$

Supervisor's signature:

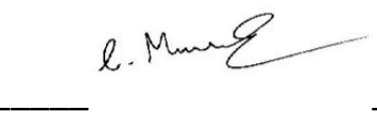

Date:13/06/17

Upon completion, please forward an electronic copy (as a single document, Word or PDF) by e-mail to Sarah Jones C.S.Jones@swansea.ac.uk

Sarah Jones 
Administrative Support

Research Ethics Committee,

College of Human \& Health Sciences

Swansea University

Singleton Park, Swansea, SA2 8PP.

Sherrill Snelgrove

Research Ethics Committee,

College of Human \& Health Sciences

Swansea University

Singleton Park, Swansea, SA2 8PP.

Email:

Chairperson

CHHSREC

**RESEARCH MAY ONLY COMMENCE ONCE ETHICAL

APPROVAL HAS BEEN OBTAINED**

Ethical Approval

Ethics Committee Use Only 


\begin{tabular}{|l|l|}
\hline Principal & \\
Investigator & \\
\hline Title of Proposed & \\
Research & \\
\hline
\end{tabular}

\begin{tabular}{|c|c|c|}
\hline Application approved & Yes & No \\
\hline Date & & \\
\hline Name & & \\
\hline Signature & & \\
\hline $\begin{array}{l}\text { Position } \\
\text { (please state if a member or Chair of } \\
\text { ethics committee and name of } \\
\text { committee) }\end{array}$ & & \\
\hline
\end{tabular}

FW: Amended Ethical Approval Form

Snelgrove S.R.

Sent: 03 August 2017 16:20

To: ROGERS A. (843126) 
Cc: Jones C.S.; Musselwhite C.B.A.

Attachments: CHHS App Standard Ethical 1.docx (92 KB)

Dear Allyson,

Thank you for your amendments to the ethics application. I can now give ethical approval.

All the best

with your study

Kind Regards

Sherrill Snelgrove (Chair) 


\section{RE: Retrospecitve Ethical Consideration.}

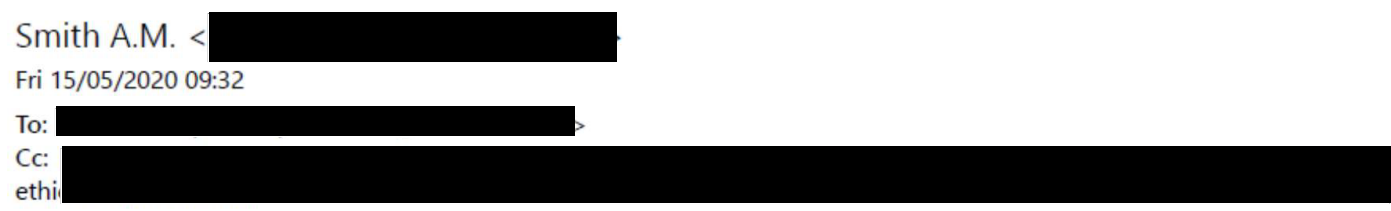

Dear Allyson,

Ethics application Reference 050717

Many thanks for your amended application. As you are aware, this is not usually something that would be approved, but I am satisfied from your application that this did not involve coercion and that the risks were minimal. Accordingly, and on the basis that this issue is referred to and reflected upon within your thesis that this amendment is approved.

I wish you well with the final stages of your study.

Best wishes,

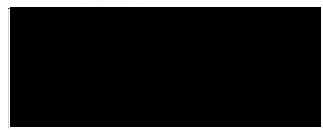

Angela Smith

Senior Lecturer in Health Care Law and Ethics | Uwch Ddarlithydd mewn Cyfraith Gofal lechyd a Moeseg

Chair, CHHS Ethics Committee

https://www.swansea.ac.uk/humanandhealthsciences/research-at-the-college-of-human-and-health /research-ethics-committee/

College of Human and Health Sciences | Coleg y Gwyddorau Dynol ac lechyd

Singleton Park | Parc Singleton

Swansea | Abertawe

Wales | Cymru

SA2 8PP 
13.6. Appendix D Risk Assessment

\section{Risk assessment}

Name:

Emergency

contact name:

Any emergency medication

carried: Emergency

contact relationship:

Emergency contact tel. no:

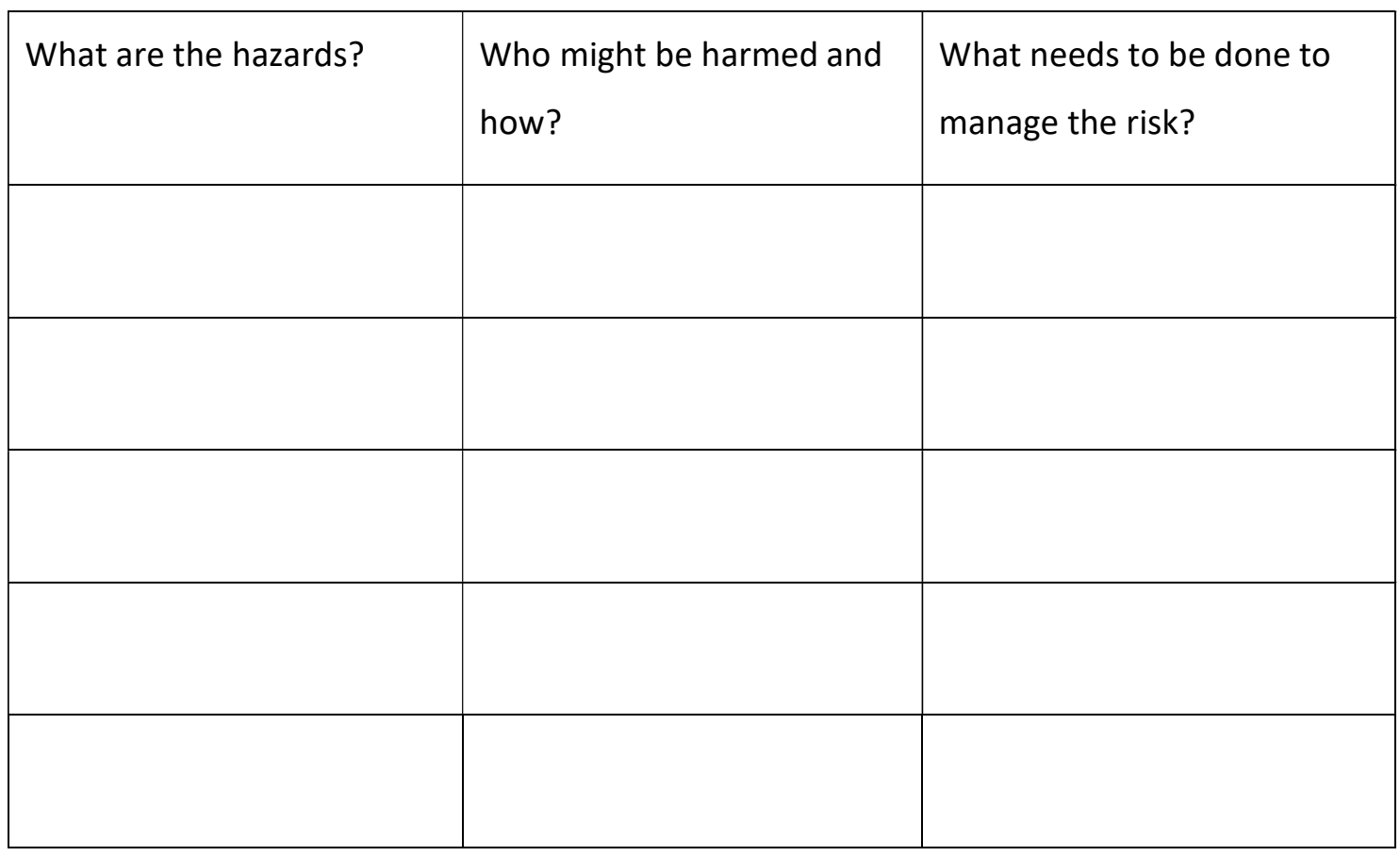




\subsection{Appendix E Consent Form}

\section{Ageing in Wales}

\section{Consent Form}

Name:

Email: (Optional)

Age:

Male/Female

I agree to take part in the above research. I have read the Participant Information Sheet, which is attached to this form. ? (Please tick)

I understand what my role will be in this research, and all my questions have been answered to my satisfaction. ?

I understand that I am free to withdraw from the research at any time, for any reason and without prejudice. ? (Please tick)

I have been informed that the confidentiality of the information I provide will be safeguarded.

? (Please tick)

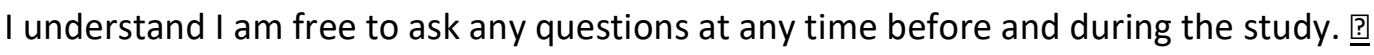
(Please initial)

I have been provided with a copy of this form and the Participant Information Sheet. ? (Please initial) 
Data Protection: I agree to the University processing personal data that I have supplied. I agree to the processing of such data for any purposes connected with the Research Project as outlined to me.

Name of participant

(print) ............................... Signed.........................................

Witnessed by:

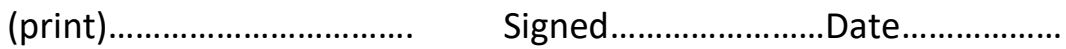




\subsection{Appendix F Debrief Sheet}

\section{Age Friendly Wales and Mobility Aids}

\section{Debrief Sheet}

Thank you for volunteering to take part in a Research Study. This sheet tells you more about the research you have taken part in. Please ask if there is anything that is not clear to you, or if you would like more information.

\section{The aims of the research}

The research aims to get a good description and understanding of people over 50 using mobility aids. The best way to know how easy or difficult it is to get around in everyday life is to ask people, which is what this research does.

We hope that you have found the experience interesting and rewarding.

\section{Will my information be kept confidential?}

Yes. All the information gained will be kept strictly confidential. Within the research, once you have taken part, you will be given a pseudonym and any information that might single you out will be treated with great care or omitted.

Your name and address are kept only for checking purposes.

Your personal details will only be seen by the main researcher on the project, Allyson Rogers, and her supervisor Dr. Charles Musselwhite. As you were informed before taking part, procedures for handling, processing, storage, and destruction of your data has complied with the Data Protection Act 1998. All data will be destroyed at the end of September 2020 which will be a year after the research is completed.

\section{What Happens Next?}

The researcher (Allyson) will be presenting a summary of her findings when she has finished processing the information she has collected. This will give you a chance to give her feedback on what she has found out. You can tell her if you agree with it or not, whether you are surprised or if it is what you expected. A summary of the findings can also be sent to you in written form for you to give feedback. It is an important part of the research process that you feel the findings represent your lifestyle. 
You can still choose to withdraw up until April 2018. You may also receive a copy of the PhD Thesis if you would like to. Please let Allyson know if you would like either of these options. The research is being conducted by Allyson Rogers as part of her PhD (Doctorate) in Gerontology. Her supervisor is Dr. Charles Musselwhite who is an Associate Professor in Gerontology at the Centre for Innovative Ageing (CIA) at Swansea University. Contact details are at the end of this form.

\section{What will happen to the results of the research study?}

The results will form the basis of a PhD thesis at Swansea University. The information collected from people who use mobility aids and those who care for people who use mobility aids will be used to add to the growing body of knowledge relating to social inclusion, ageing and disability. Often, the people who design areas or make policy decisions do not ask people who use mobility aids, or understand the day-to-day use of them, so make decisions that do not necessarily favour mobility

aid users. This impacts carers too, as there is evidence to show that as the mobility aid users stop going to certain places. Your day-to-day routine will help us understand whether your type of mobility aid is considered properly in the way the local area is planned and set out.

Thank you for taking the time to read this information and choosing to participate. If you have any further queries please contact Allyson, who will do her best to answer any questions you may have.

Her contact details are:

\section{Allyson Rogers}

PhD Researcher

Swansea University

Email:

For any additional information, please contact Allyson's supervisor:

Dr. Charles Musselwhite, Associate Professor of Gerontology, Swansea University, Singleton Park, Swansea SA2 8PP. 


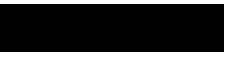

\section{Useful Numbers}

$\underline{\text { Age Cymru }}$

Age Cymru advice line800 223444.

Age Cymru Sir Gar 01554784080 email: info@agecymrusirgar.org.uk

http://www.ageuk.org.uk/cymru/sirgar/information--advice/

\section{$\underline{\text { Disability Wales }}$}

Tel: $\quad 02920887325$

Email: info@disabilitywales.org 


\subsection{Appendix G Participant Information Sheet}

\section{Participant Information Sheet}

\section{Age Friendly Wales and mobility aids}

Thank you for volunteering to take part in a Research Study. This sheet tells you more about the research you have agreed to take part in. Please ask if there is anything that is not clear to you, or if you would like more information.

\section{What is the purpose of the study? What are we looking at?}

The research is being conducted by Allyson Rogers as part of her PhD (Doctorate) in Gerontology. Her supervisor is Dr. Charles Musselwhite who is an Associate Professor in Gerontology at the Centre for Innovative Ageing (CIA) at Swansea University. Contact details are at the end of this form.

\section{Why have I been chosen to take part?}

This research is seeking to understand the everyday experience of anybody over 50 years of age who uses a mobility aid sometimes or all the time. You may also be asked to take part if you are the partner or carer of someone over 50 who uses a mobility aid.

\section{What will be required of me if I take part?}

You will be asked to look at a map of the local area and discuss the places on that map that you go to regularly or any that you might avoid. This will help to create a picture of the places different people go to and whether there are places people who use mobility aids avoid. Your name and identity will not be associated with the information you provide for the map.

Allyson, the researcher, would then like to spend 4-6 hours with you as you go about your normal day. This could be at home, or going out and about, to meet people, to a club, whatever you do on the chosen day. She will observe your activities, recording the conversation or the sounds of places, take photographs or sketch things. You will also be invited to comment on things, take pictures or sketch things if you would like to.

What are the possible benefits, disadvantages and risks of taking part? 
The possible benefits of taking part in this research are that you will get to put forward your point of view. Planning departments and local authorities do not usually ask people what is like to use a mobility aid, so you will get to have a say in what day to day life is really like. There is a risk that the research may make you think about things in a way you have not thought of them before. This could be good or bad, but there is a possibility it could be distressing for you. There could also be a risk that my presence could increase your risk of falling, but we will assess this before we start to minimise this risk.

You may consider the inconvenience of giving up some of your time, and having a researcher observing you to be a disadvantage. However, we hope that participants will find the experience interesting and rewarding.

\section{Do I have to take part?}

No. Taking part in this study is completely voluntary. You can decide not to take part at any time. it is normal for some people to withdraw without giving any reason and without prejudice. You can take part over a number of occasions if that is easier for you.

You can change your mind at any time up to the start of data analysis (one month after your interview has been recorded) and withdraw without giving us a reason. If you withdraw, we would destroy any information (data) that you have given us. To withdraw contact one of the named people at the end of this form.

You will be given this information sheet to keep and will be asked to sign a consent form on the day you take part.

On the day, you can opt not to answer any particular question, ask to take a break or reschedule your participation.

If you choose to withdraw from the study, we can destroy all the information collected and will not use it, unless you are happy for us to retain it.

\section{Will my taking part in this study be kept confidential?}

Yes. All the information gained through the research will be kept strictly confidential. Your personal details will be accessible to the main researcher on the project, Allyson Rogers, and her supervisor Charles Musselwhite only. As soon as your information is analysed, it will be anonymised so your name will not be associated with the information you have given. No 
town will be named in the research, and neither will any places of interest such as shops, streets etc.

Electronic data held on computers will be password protected. Procedures for handling, processing, storage and destruction of your data will be compliant with the Data Protection Act 1998. All data will be destroyed at the end of September 2020 which will be a year after the research is completed.

\section{What will happen to the results of the research study?}

The results will form the basis of an PhD Thesis at Swansea University.

We hope that the research findings will add to the growing body of knowledge relating to social inclusion and disability, and help to inform policy makers.

Thank you for taking the time to read this information. If you have any further queries please contact Allyson, who will do her best to answer any questions you may have.

Her contact details are:

Allyson Rogers

PhD Gerontology and Ageing Studies

Swansea University

Email:

For any additional information, please contact Allyson's supervisor:

Dr Charles Musselwhite, Associate Professor of Gerontology,

Swansea University, Singleton Park, Swansea SA2 8PP. 


\subsection{Appendix H Friendship Club Consent}

\section{Llandovery Friendship Club Consent}

I/we, as representative(s) of Llandovery Friendship Club, agree to allow Allyson Rogers to communicate her PhD research through the Club.

We have asked to be fully informed of her progress and updated with any findings.

Any members who wish to take part in the research are free to do so of their own free will and take the decision individually.

Signed. Position

Date...................

Signed.

Position

Date. 


\subsection{Appendix J Phil Interview transcript}

INT: Ok so l'd like to talk to you today about your mobility aid and erm what difference it makes to your life if it makes a difference to your life either positively or negatively

RES: Ok

INT: So firstly, what l'd like you to talk about is your movement around town and whether that's changed since you had your mobility aid

RES: Yeah it it its changed totally erm (intake of breath) because now you have to because I use a wheelchair and it's a large wheelchair electric wheelchair erm so I have to number one always consider a pathway to getting to where I want to go to and and, I don't necessarily mean a pathway I mean the the route an accessible route whereas if you're walking you just walk there erm but when you're in a wheelchair then you have to think about an accessible route. Can I get there and if so how do I get there.

INT: $\quad$ So erm do you use your wheelchair indoors or do you only use it outside?

RES: I w..I only really ever use my wheelchair outside because inside of the house erm then I can move around the little bit that I move around with a walking stick.

INT: Ok that was my next question. Do you use any other...?

RES: $\quad$ Mm yeah, I use a walking stick

INT: Erm so does that mean that you are in pain when you use your walking stick? Or how helpful is your walking stick?

RES: If I stand up, then my feet hurt. A great deal because I have two types of arthritis and my feet are- II have it all over my body and my feet are very bad 
erm so if I stand up at all it instantaneously hurts a huge amount erm but it is easier to take half a dozen steps in the house with a walking stick than to have this vast electric wheelchair trying to circumnavigate the house and I can't use a manual chair because I can't move a manual chair because of my arthritis in my arms and my shoulders and my back and everywhere else ( ) so the the functional answer is to just use a walking stick in the house

INT: Ok so how much do you go out and about in the town?

RES: Very little very little, erm in fact I would say next to zero in the actual town itself erm I go to the eh Co-op superstore or supermarket I should say rather which is at the other end of the town from from where I live so I drive down there in my hugely adapted van with my wheelchair in the back and then because it's all fully automatic I come out of the back of the van, and I can go into the shop, buy what I want, come back out, back into the van and back home. I don't actually go into town hardly at all because, because the pavements are not conducive to a large wheelchair. They're not conducive to any wheelchair but they are extremely not conducive to my large, powered wheelchair, they are too narrow. They are too lumpy; they are too bumpy they are too everything.

INT: Have you ever been in a position where you have walked around in a town with your walking stick?

RES: No because I can't walk. I can't walk like that. I can walk in the house from my bedroom to the lounge or from the lounge to the toilet or from the lounge to the kitchen you know I live in a bungalow obviously which has been specially adapted, and walls knocked down, doorways widened and all the rest of it, but I can't walk walk. I can't go out and walk

INT: No

RES: Erm because a) because of the pain as soon as I stand up and b) once l've taken about 10 or 15 steps my feet start to swell very badly so it's just not possible 
INT: How long have you lived in Llandovery?

RES: $\quad$ Err erm 5 years, 6 years

INT: Why did you move here?

RES: We moved to Llandovery because we used to live up in the mountains, literally up in the mountains about 25 miles away erm, but the property that we lived in was not negotiable by wheelchair at all ( ) and there was nothing there ( ) if you wanted to buy anything then it was a 22 mile return round trip to buy a pint of milk or a loaf bread or anything else cos there was just nothing there at all so we, we moved I moved down to Llandovery erm because it is flat and there's shops

INT: So, you really moved here for erm the ability to move around for your disability purposes

RES: Well yeah for for ease of living

INT: Yeah

RES: Yeah for ease of living and for cost of living as well, I mean to do to do you know 20 odd miles return every time you want a pint of milk is really not tenable when you live on benefits

INT: And you live on benefits because of your disability

RES: Yeah absolutely I can't work

INT: No

RES: I can't work I have very bad functionality of my body and I actually have really in reality have very bad functionality in my mind, I have a vast plethora of of medication that I take and that means that I'm often foggy and without focus 
and confused and all sorts of things that you you couldn't work, if I could work I would but I can't possibly work because it's just not practically possible

INT: No ( ) so one of the reasons I'm doing this research is that I want to understandthere's a common perception that mobility aids are just helpful they just enable you to get around and there's not really anything negative about them. Would you agree with that perception?

RES: No not all, I think that they're, they're as a life option there is nothing else but a mobility aid, you know if you need a wheelchair you need a wheelchair, walking stick, Zimmer frame whatever it may be, if you need it you need it, but they are they are a deep disadvantage to your life. They get in the way of everything that you do everything that you want to do, you know III have a van that that is hugely adapted and that vehicle's probably, probably cost I don't know, f50,000 $£ 60,000$ you know and what l've got for that is a highly adapted van, I haven't got a lovely sports car or or or a lovely saloon car, l've got a van! You know, and that's because I have this huge wheelchair and I have to have the van to move the huge wheelchair ( ) so everywhere you go you have to think about what you are doing so, just very rapidly for argument's sake if I think about going out I will always automatically think about going to places like Tesco's or Wilkinson's or Marks and Spencer's or somewhere where I perceive it's big and it's flat and I can move about. But I would not consider going to to a town like Llandeilo because the pathways are barely wide enough for a person let alone my wheelchair.

INT: $\quad \mathrm{Mm}$

RES: There's no drop off points, I can't get off the pavement, I can't get on to the pavement you know it just completely impractical.

INT: So, would you say that since you've had your wheelchair you've gone out less?

RES: Oh God yes, absolutely well I mean I mean my you know your, your illness I mean I'm chronically ill and you know my illness came on literally overnight I 
was as if I'd been hit by a train erm, I had some viral attack from something I'd scratched my back on and and this seemed to instantly trigger rheumatoid arthritis all across my body in a very very bad attack a very bad way erm so I I I went from from running a large business with my wife to being unable to work, from being a person who was always active and and who rode motorbikes and could drive articulated trucks and worked on cars and and and and also had worked in the financial services industry high up in the City of London and all of these things and then I'm taken from that to (contempt in voice) can barely remember my own name some days unable to move about, so yeah I mean it's a complete and utter change of life and you don't go out because you do feel ill and because if you don't feel ill then you are fully aware that everywhere you go you have to think about and that just wears you out it it you just what's you know what is the point you know if I if I just said to the average person you know every time you leave the house you have to wear a gas mask but you've only got a canister for five minutes and out there somewhere in the locale in the town there are other canisters dotted about and so to go into town to buy food you have to put your gas mask on and then you have to walk to town and then you have to make sure that you find a canister or you die and its exactly the same with a wheelchair. Because that is the same because those cannisters are actually pavement drop off points those cannisters are doorways you cannot get in those cannisters are ramps that do not exist, they are all of those things. You cannot function in that town because those things do not exist, so yeah it just absolutely completely changes your life.

INT: That's quite a strong analogy, in terms of you're telling the analogy is that if you can't find your cannisters you'll die. Is that how you feel if you can't move around, do you feel that it kills your ( ) identity in a way

RES: YES, you're no longer the- you cannot possibly be the person you once were. You know I I I've spent all my life in business, I've spent all my life dealing with people, I've spent all my life moving around different countries different places you know when I was taken ill I was living in Europe, you know we weren't living 
in the UK at all, so so it is a fundamental change. My illness, other people may be different from me. If if you only have to use a walking stick say, then I'm sure that is very different to having to use an electric wheelchair you know, because I can fit a walking stick into an ordinary car, I can walk down a pavement with a walking stick, I can do all those things with a walking stick I can climb a small step with a walking stick. What I cannot do is that in a wheelchair. So, it absolutely fundamentally changes your life. The more requirement you have for a bigger mobility aid, the more your life is removed from you.

INT: $\quad$ Mm ( ) Do you think it's stopped you enjoying even your life with your family

RES: I think it has a marked effect upon all your relationships. It it undoubtedly it has taken me you know, my wife has said to me that it doesn't change me as a person, my illness does not change me as person. You have all these people who are forever running around shouting about you know I'm not identified by my illness and all the rest of it but the reality of life is that whilst you are still fundamentally the same person, it does change your outlooks and it- and you and your outlook of yourself within society, your place in society changes for you yourself because you are now the person in the wheelchair, because people see the wheelchair, they don't see the person they see the wheelchair, and and there are, it appears, many pre-conceived ideas of why people are in wheelchairs. Er many people seem to deem that it is- that a wheelchair is akin to some sort of mental illness, that you are mentally slow, that you are, that you are ehhh, you are not a normal person at all. In any way, not that you wheel rather than walk, that in fact you're you know you're just not a tenable human being you just don't function you don't work and and and you know again if the average person had to walk round and explain to everybody they met that actually "Do you know what I may be wearing this pointed hat but I'm actually a normal person"

\section{INT: $\quad \mathrm{Mm}$}


RES: "Even though I'm wearing a pointed hat I'm still just a normal person" because the reality of life is that the ordinary person would take the pointed hat off, but I can't take my wheelchair off. I'm stuck with it. So yes, I mean if affects it affects everything and it must do, and it can't not. It has to affect everything, and it affects your life deeply it really does, not being able to move about without relative ease is very difficult and it is life changing it is absolutely life changing.

INT: I've had some professional people suggest that people who use wheelchairs people who use mobility aids, but specifically people who use wheelchairs, tend to- can ( ) incorporate the wheelchair as part of their identity. What would you say to that? Because what I'm hearing at the moment is that it though it is a tool that can be used to get from $A$ to $B$, that is the only positive thing about it

RES: It seems to me, in my own personal view obviously, that if you were born to a wheelchair then a wheelchair is just part of who you are

INT: $\quad \mathrm{Mm}$

RES: And it's what you do and it's just who you are it it's no different to there's your legs. I mean that wheelchair is you and that makes perfect sense to me. When like myself you come to a wheelchair at the age of 48 or 49 when you can you can no longer do all the things that you used to do, you can't ride your motorbikes, you can't even drive a normal car because you can't use the controls and you you certainly you know can't drive and articulated truck cos you'd never get into it for a start or anything else you know or all of these things you can't do and and you are used to being able to walk into a shop or walk into here or walk into there or walk up there or take those steps or whatever it may be and then all of a sudden you can't do any of that then yeah, that's a negative impact on your life, and the only good thing about a wheelchair is that it allows me to go on flat surfaces to places that I could not walk to.

INT: $\quad \mathrm{Mm}$ 
RES: And that's it, there's nothing else good about it, there's nothing good about a wheelchair, it's not part of my life, it's not part of who I am, it's not ( ) what I choose as part of my identity. I don't choose that. I have to have it that's a different thing I have to have it I am forced to have it because I have no option

INT: How does it affect your independence?

RES: $\quad$ Erm well it affects it a great deal really you see because because er I am very fortunate that I have my my adapted van ( ) and my adapted van allows me to wholly independently get in it and get out of it and drive it so so so that gives me independence. However, first of all because you feel ill you don't go out very much so so that doesn't really help and then from the point of view of independence you know I, I because my mind doesn't work as regularly as it used to then it you know my mind is fine and it works but it it it comes and goes and I have very little patience I have really next to no patience at all with people I can't deal with the vagaries of of life the things that are thrown up, we all know that life is not perfect and we all know that there are endless problems and to that extent, II don't engage. My wife very fortunately my incredible wife, she deals with all these things even to the extent that I do not have a bank account, I do not have money I have not carried money for the last 10 or 12 years or whatever it is the last 10 years, I don't carry money. I just don't carry money, I have no money no cards no nothing erm and whilst yes I can say that my wife would give me fifty quid or whatever I don't want to go out on my own because it is very difficult to negotiate things on your own because sometimes that door needs opening or or or whatever it is and also because I feel huuuh I feel that my wife is an integral part of myself and she always has been but it is now more so because because I actually have a need for things that she does for me. Rather than we just wanted to be together, I actually now have needs because of my disability and illness and that makes it, that makes it you don't want to be independent, you're not looking to be independent

INT: $\quad \mathrm{Mm}$ 
RES: I'm not looking to be independent. Some people are but I'm not

INT: No indeed. What ( ) some people have talked to me about how erm they don't use the town because there's nothing left anyway and you've hit upon the things like erm there's no dropdowns and you can access anything, that pavements are too small, erm do you do social things in the town like erm I know you've got/t a dog, do you walk the dog, do you go out for meals? Do you do any of that sort of stuff?

RES: $\quad$ Erm no not really. Erm I occasionally will follow my wife when she's walking the dog I won't walk the dog with the wheelchair in case the dog gets, our dog is very small, erm a little jack Russell and I am scared to death that she will somehow get caught under the wheel of my wheelchair and and so I would never walk her plus I have all the issues of if she erm makes a mess then I have to clear it up and that means that I somehow have to get out of the wheelchair

INT: $\quad \mathrm{Mm}$

RES: And negotiate picking that up and keeping hold of her and all of that so I would never her on my own any way. I very occasionally go out with my wife. If the weather is ok I will follow her. And that's fine that's good. Erm going out to eat, well no you are again faced with all the same issues about access. About the only place we really go to eat erm would be either somewhere like Debenhams or Tesco's in Carmarthen because they are accessible. You know I know if I go to a Tesco's, even if I've never been to a Tesco before, that the café will be accessible. I know that for a fact. There is nowhere else that's like that

\section{INT: $\quad \mathrm{Mm}$}

RES: You know you go, you phone a pub up, we went to a public house that was doing a Sunday lunch thing and we said is it wheelchair accessible, yes yes it's wheelchair accessible so we turn up, I turn up in my wheelchair we go in and they plonk me in my wheelchair absolutely in the direct path of the door so that 
nobody else can get into the damn dining room without me moving I mean it is just absolutely ludicrous, I makes me feel awful, I feel like I'm getting in everybody's way and it shows how much thought these businesses give to their clients which is none at all. I mean it was incredibly stupid

INT: $\quad \mathrm{Mm}$

RES: But that's what you're up against so you just think to yourself you know what, I'm not going to do that because I can't take the hassle and the aggravation, I just can't be bothered. I'm not well I don't feel well I'm not interested, and I don't want all this aggravation in my life, I just want to be left alone so actually I'm just happiest at home.

INT: Mm. ( ) Do you think erm it could lead to social isolation

RES: Well absolutely I am socially isolated, but then you know huh you know that er in some ways you have to say that I was always socially isolated all my life I don't like people I don't get on with people I would I mean business that is a a er a display a personification of what they need to see, they need to see certain things when I'm in business and they will see those things. That doesn't necessarily mean I want to socialise with people cos it doesn't I'm not interested in friends or all the rest of it I'm a very, I'm a very singular sort of person the only person I really get on with is my wife, that's the only person I really have ever actually got on with in my whole life is my wife and outside of that I'm not that interested. If I wanted to be social I think it would make it extremely difficult extremely difficult

\section{INT: Yeah}

RES: You know how how how you know how could you possibly go to a friend's house unless they happen to live in a warehouse because otherwise you're never going to get into a normal house. You're not going to negotiate the door. You're just not going to get in there, you're not going to negotiate the furniture, you're not going to do any of those things and if you come out of your 
wheelchair there will not you know I sit in a chair which is either very, which is high like a big high office chair which means I'm already half way standing up or I sit in a riser recliner which when needs be throws me up on my feet. If I go to a friend's house, some supposed friend's house and I sit in their DFS sofa, I'll probably never get out again

INT: Huh mm

RES: You know these are the things that people don't think about. And once you're in the wheelchair this is what you have to think about all the time, and I find it very hard I will just say I find it very hard to imagine why people cannot imagine how difficult it is being in a wheelchair. I don't understand how they cannot just put themselves mentally into that chair and think right, I've got to get that chair now everywhere

INT: Yeah

RES: Yeah and and then look at everything and think well I couldn't get up there could I, I couldn't get through there, I couldn't get into that shop or if I want to you know, if I want to go into the post office I have to ring the bell, which I can't reach from my wheelchair so then I have to get somebody to ring the bell. And then somebody has to come out from behind the counter in a shop full of people and put down a special ramp for me to get into the building, so they can all turn round and watch me doing that

INT: $\quad \mathrm{Mm}$

RES: II don't need that, I don't want to be the centre of everybody's attention thank you very much. You know I'm a private person I don't want that. So there we go.

INT: () What do you think () What do you think about the fact that your wife is a carer?

RES: In what way? 
INT: $\quad E R$

RES: Can you formulate the question differently please

INT: Yeah yeah erm, well there's lots of aspects to that. What do you feel about having a carer? What do you feel about them being called a carer?

RES: Well, ( ) I feel that having to have a carer is a pain because you are once again reliant on somebody else and although somebody else may be perfectly happy for you to be reliant upon them you are still reliant upon somebody else and you still feel that you are constantly having to interject with them erm about can you just get this for me or could you get that for me or could you get this or move that or push this or whatever it so that's not particularly good. Erm what do I think about them being called a carer I don't think anything about them being called a carer they can call them Fred Bloggs for all I care makes no difference to me at all. What do I think about my wife being my carer well on the one hand it's it's very heartening that she wants to be my carer and look after me and on the other hand I find it deeply irritating that I have to put her into that position and that I feel that because she's in that position that she misses out on things in life and my being stuck in a wheelchair means that she misses out on things and my being ill means she's missing out on things in life you know we used to go on holiday four or five times a year but we don't go on holiday now because I'm just not well enough you know I find that very difficult and I'm sure she finds it extremely difficult.

INT: Is it purely because you're not well enough or does it have anything to do with the fact that you need mobility aids and that is just a lot of faff as well

RES: If I, first of all I would say it is just because I'm not well, that is the basic bottom line

INT: $\quad \mathrm{Mm}$ 
RES: $\quad$ But then if it came round to well you are well enough but now what are we going to do, then yes obviously the wheelchair would come into it and the only answer would would would be that we would have to go somewhere that we could drive to in the van because I can't my wheelchair onto an airplane and I certainly can't er you know I suppose if you went to somewhere like Benidorm then you could, you could, you could erm you could hobble off of the plane, you could use some sort of erm I know at Malaga airport of that you know they have golf carts that they move people about in erm I suppose you can do that you get into a taxi they could take you down to one of those places that rent out mobility scooters you could rent one of them for the week and and move around there or you would or you would have to erm take the van with your wheelchair. But then you know straight away can you get to the hotel? Can you get into the hotel? Where can you park the van? You know it it it presents endless problems

\section{INT: $\quad \mathrm{mm}$}

RES: And then as soon as you go to somewhere which says oh you know cottage, disability friendly, which may or may not be the case anyway, you will also find that the price is at least $60 \%$ higher than if it didn't have the word disability attached to it. So, you know there is a great deal that holds you back, but the main reason is I don't go because I'm ill and I never know when I'm going to be well, and well is a relative term. My my day feeling well is you know is your day taking two weeks off of work with the flu. That's me feeling well.

INT: Do you think, do you think mobility aids could be designed better or do you think the environment should be designed better or both?

RES: I think that, I think that wheelchair really hasn't changed much in a very long time I mean you know knobs and whistles but in the same way that you know if you think about it a motor car hasn't fundamentally changed very much in the last 100 years. I mean the electronics are more and whatever but you know we are still using reciprocating engines and we are still using diesel or petrol. I mean 
yes we're starting now to get electric cars but then you know 1905, 1910 New York everything, nearly everything in New York was an electric vehicle so there's nothing new, there's nothing new under the sun. these sort of hybrid things we've got coming around now all all they seem to do is cost more and break easier but but the reality is that cars really haven't moved forward very much and wheelchair have not moved forward very much there really is not a lot of incisive and and and for want of the awful term blue sky thinking about wheelchiars

INT: No

RES: There just isn't. yes the environment is is is also a major difficulty but you know in these days of wonder how diffi- you know often I come to somewhere and it's a small step or a pavement. It's a pavement it's a small step it's a pavement. Is it really beyond the behest of man, I've got a six-wheel wheelchair, I've got two drive wheels in the middle which are bigger and four smaller wheels front and back, is it beyond the behest of man at this point in humanity for a computer to recognise a drop, push the wheels down, put the wheels down, and then we go across and then it lowers itself down. I mean really is that beyond our scope? No of course it's not beyond our scope, but we are not doing it.

INT: No

RES: But that's because out of 7 billion plus people in the world fff you know a couple of hundred million of us sit in a wheelchair and the rest don't.

INT: $\quad \mathrm{Mm}$

RES: If everybody needed it it would happen, or the majority needed it it would happen, or there was a profit in it it would happen

INT: It would happen yeah

RES: But there ain't and there isn't so it doesn't 
INT: Yeah. ( ) So ( ) The tool itself is what you really think is the biggest thing that could change rather than the environment

RES: I think that to I think to expect the environment to change is, is, unrealistic. It is just unrealistic. So yes, I think you need a tool which adapts better to the environment as it stands.

INT: $\quad \mathrm{Mm}$

RES: You know there will always be a step, there will always be a pavement there will always be you know cobblestones, there will always be something. There will always be, so you just need something that actually you know, works better.

INT: $\quad \mathrm{Mm}$

RES: You know.

INT: As you know, I did a study about people in wheelchairs who have never used wheelchairs before, and I found that they all thought if affected their relationships with people they knew and people they didn't know and close relationships. Erm how do you think it affects your relationships? Particularly with your wife because you talk a lot about your wife being

RES: Yeah no I mean II to be honest with you l've now been in the damn thing for so long, it's sort of hard to remember not being in it

INT: Yeah

RES: So, it is what it is. Do we get to hold hands as much? No. Do we do we have the same interaction in shops? No, no because because half the time my wife will walk into an area that I actually cannot access because the- everything's too close together and I can't move and all the rest of it erm so that that has an effect. The fact that I'm not well has an effect. I mean it has to. You can't but help that your tolerance for things and people and ever- is is lower. You just you can't help that I mean its its part of who I am, it's part of what she loves about 
me, but but also the reality is that you can't change that. So, it it does have an effect, of course it has an effect, it has an effect in lots of ways both socially with my wife and privately with my wife it has all sorts of repercussions. Erm both the illness and and the waking aid whatever it may be, wheelchair or that you know has a difference. As for people in general, well as I sort of already mentioned, they view you in a completely different way.

\section{INT: $\quad \mathrm{Mm}$}

RES: They see the wheelchair, if they see you at all but they discount you, quite obviously discount you. They see the wheelchair and you are instantly discounted; you are instantly a lower form of life. Absolutely without doubt. The other thing that you get is you will get, you know, I'm I'm a large man and I don't always look particularly friendly because I'm not particularly friendly in all honesty, and yet you will have women walk past you and they will smile at you, and they are smiling at you because it is an act of 'oh dear I'm sorry'. Cos if I was standing up they wouldn't smile at me, they would absolutely not smile at me.

INT: Do you think it's pity?

RES: Yeah, I do think it's pity, yeah. I think if I was standing up I think I would I would scare half of them to death, just the very fact that I was standing there, but when I am sat down in a wheelchair it's it's is an act of pity.

INT: $\quad \mathrm{Mm}$

RES: $\quad$ And that's irritating

INT: $\quad \mathrm{Mm}$

RES: That irritates me cos I don't want anything from these people let alone pity because I don't find myself pitiable

INT: No 
RES: So, there we go, yeah it has a dramatic effect on everything you do absolutely, absolutely. Illness plus wheelchair totally changes your existence, just totally. And and anyone who says it doesn't err err I I'm much of the opinion that they are putting some sort of emotion based brave face upon their situation. That they cannot, they cannot deal with not having a hope of change or deal with a hope of of of their situation

INT: For this study I haven't spoken to any other wheelchair users, well one other

RES: $\quad \mathrm{Mm}$

INT: Erm and I think the fact that it's a wheelchair makes a difference, but I was interested in you saying that you think the walking stick in some ways makes a difference.

RES: $\quad$ Did I? Well, you said indoors maybe

INT:

RES: Well, no I don't think a walking stick makes, I actually said I don't think a walking stick makes much difference at all, I think I can walk down the pavement as a person I can walk down the pavement

INT: About your interaction with me

RES: No, I don't think so

INT: You don't think so

RES: No, I don't think so really, I think the only problem it does create is that that you you're missing a hand cos you

INT: Yeah

RES: Cos you got a hand on a walking stick so so you know previously if I was- if I'd got the dog on a lead I could hold my wife's hand with the other hand or l'd got 
shopping in my hand I could hold my wife's hand with the other hand or you know so forth, but when you've got a walking stick then you're down to one hand

INT: $\quad \mathrm{Mm}$

RES: $\quad$ But I don't think a walking stick has very much effect on your life at all that really, really

INT: No

RES: I don't think people I don't think people really notice a walking stick. If you're particularly young and you're using one, then they might think oh he must have damaged his foot or something playing football or whatever,

INT: Yeah

RES: or if you're that bit older they think oh well he must have a bit of a bad leg or something, but I am personally convinced that the bigger the walking aid the bigger the issue

INT: Yeah

RES: So, if you're using a Zimmer, if you're using a walker those sort of wheeled walkers you know

INT: $\quad \mathrm{Mm}$

RES: $\quad$ They're ten times worse than a walking stick but only half as bad as a wheelchair

INT: Yeah, yeah so the bigger the aid, the more stigmatising

RES: Absolutely. Absolutely

INT: And the more difficult they are to manoeuvre and 
RES: Absolutely, you can't manoeuvre, you can't get about and the more readily people see them. You know they barely see a walking stick, they'll see a walker,

INT: Yeah

RES: But when they're faced with a six-wheel wheelchair coming down the street towards them they notice that, it's a big old thing you know

INT: Well thank you very much for that

RES: Pas probleme

Walking interview at Hospital appointment

INT: Driving to hospital appointment for interviewer/wife of participant

RES: I hate this weather

INT: I know darling

RES: I hate this weather in a wheelchair because it is wet and it is cold and it is miserable. Unfortunately you are going to have to drop me off by the door as I said cos I can't go scooting around the car park in this rain

INT: no, well we might be lucky enough to get that car parking space

RES: $\quad$ well you never know but as I say that's like winning the lottery twice in a row isn't it

INT: it is yeah

RES: $\quad$ and I was saying to the chap in mcdonalds just now that because they are working at the top, 
INT: yeah, it reduces

RES: they have lost more [disabled space]

INT: [disabled spaces]

RES: yeah ( ) and I think the reality is that as limited as their parking is as much problems with the parking as they have they need to

INT: yeah

RES: really expand the number of disabled spaces

INT: well especially in a hospital

RES: it is a hospital for God's sakes so you know the the ones that are disabled now in front of of A\&E fine but you know they need make the first whole row across in the car park disabled

INT: yeah

RES: you know ( ) or even change the routing system and make and make a change so that the road actually comes down and instead of going where it goes now actually goes through what is the car park and then move those car parking spaces on to what is presently the road

INT: yep

RES: $\quad$ you know, it's a very bad use of space that they've got on there

INT: it is yeah

RES: ( ) but you know when you have to go to the hospital really at least an hour before your appointment to find a car park space you know its its pretty crazy

INT: yeah 
RES: but there we are. (We both talk to and about the dog who is travelling with us for a little while)

INT: (Turn into hospital car park) Not looking very good at the moment is it

RES: not at the moment no, it's not looking very bright anywhere at the moment. Just an absolute gash of a place. It really is awful. Just awful. Well I think all we can really do is I'Il have to get out and then you'll have to innit

INT: yeah - well we'll wait for this white car to go and then you can get out, then I'll have to go and find somewhere to park

RES: well yeah its just crazy. It is just crazy ( ) absolutely insane isn't it - there you go. Arrgggh dear (sounds of the lift moving) nnnn (more lift sounds) Right you'll have to get out of your come on

INT: I'm doing it

RES: let's get on with it babes ( more lift sounds) that'll do you that'll do, I've got to get on there

INT: are you putting your coat on?

RES: erm I think er no I don't want to cos I don't need it in there do I and it's just

INT: no alright

RES: no I know but I mean I'm gonna get wet but it's just a huge problem (tells dog to get out of way) it's just a huge problem (lift sounds) Am I alright behind there?

INT: yeah go on

RES: $\quad 1^{\prime} \mathrm{m}$ not dropping it on to anyone

INT: no he's just realised he's moved backwards 
RES: (wheelchair sounds) You going to drop me down? Yeah hold on, don't touch it till I'm on. Go on then. Waaah (too soon). Go on then. (Wheelchair sounds - as Phil alights from the lift a space becomes available so he sits in it in his wheelchair in the rain without a coat so that I can manoeuvre the van into the space) What a way (under breath) (many van sounds, footsteps and voices) what a bloody day

INT: poor darling

RES: there we are created our own miracle - Got a bit damp in the process

INT you did bless you

(Greet a staff member we know quite well)

INT: $\quad$ I'm going to find out where the A suite is

RES: I thought it was up in the new building, but you can get into there from here can't you?

INT: yeah you can (find out directions) This wah

RES: ooohhh, much dampness. (In Welsh accent!) (Big pause) must charge this (talking about wheelchair)

INT: Hello, we're back again (talking to administrator in hospital who knows us quite well)

RES: hello, yeah we love it here, we're going to book for a fortnight next year

INT: (After finished admin job) thank you very much, ok thank you

RES: thank you ( ) thank you babes (for me holding door) corridors are very tight, they are very tight, yeah corridors are tight and nobody nobody's looking, nobody's watching and I mean like there 
INT: The chairs are so wide

RES: yeah absolutely and I mean like those people that nurse there had no idea what I'm seeing. Which then belies the question you know it's like all this stuff here, all these wheelchairs and beds and all the rest of it. Allright madam (let someone pass through the gap) you know they've obviously got no storage space

INT: no

RES: $\quad$ and have no storage space planned when they refitted the place

INT: no

RES: So, they've got a wide corridor but they just fill it with stuff cos they've got nowhere else to put the stuff, which sort of seems to be somewhat defeating the object. But again, you know like with this hospital it's not planned is it

INT: no

RES: you know it's it's just you know ( ) it's just ex- it's just expanding like some like some nuclear riddled, nuclear riddled baby isn't it? it just keeps growing the next arm ( ) but it's not planned really it's just jammed on wherever they can find a space ( ) but I'm not sure what that is on the floor there

INT: I just saw that

RES: $\quad-$ I am not sure wat that was (in a jokey voice) (remarking on a suspicious yellowish puddle on the floor) I didn't get it on my wheels I can tell you that

INT: laughs I got my tissue out to wipe you down

RES: oh yeah yeah ( ) it is all a bit like that

INT: It's all covered in rain 
RES: I'm alright - I was all covered in rain

INT: I know you were Shall I shake this coat out?

RES: no no no I was waiting for some idiot to start an argument

INT: yeah

RES: but they wouldn't have got far with that

INT: I'm trying to get round the car park fast but I had so many drivers

RES: yeah I know I saw it all going on ( ) it's just a nightmare, the whole place is a nightmare

INT: it is a nightmare - Don't say we've got here and the lift's not working

RES: no, it seems to be working for a change

INT: it is yeah thank you (to people getting out of lift)

RES: (sings)

INT: oops

RES: Jesus that was a sudden stop then

INT: that's a jolting lift

RES: oh we've stopped then. You know even though this wheelchair is - where we going -

INT: I don't know she said up there

RES: ask that lady there the one who's just walking away- sorry sorry (nearly bumped by someone) Please sir, please (little annoyance in voice) please please please please (says thank you) No problem - right 
INT: you are here

RES: $\quad$ so that is there somewhere here ( ) sorry folks (other people trying to see board) there we are.

INT: We're very early

RES: $\quad$ well we'll go and get a cup of coffee We'll go and get a cup of coffee

INT: There's a lady behind you

RES: $\quad$ There is always a lady behind me - I shall turn round, and we'll come back later

INT: yeah we here, but my appointments 10.20

RES: we're really early so we're going to go and get coffee

INT: yeah

Yeah if you're really early you might as well

RES: yeah well we're going to, but we have to arrive on Monday to get a car park space

staff I know

RES I've had to sit in the rain for like 5 minutes protecting a parking space

staff oh grief

RES So she can come round to get into it I have to

INT: I dropped him off at the door

RES: I have to

INT: and then a parking space became available

RES: because I have to park on the flat otherwise my vehicle doesn't work 
INT: anyway

RES: $\quad$ have a nice day, see you in a bit (sings)

INT: We gonna go back down again

RES: $\quad$ yeah, just go and get a coffee, yeah we'll just go and get a coffee. It's er 9.40. by the time we go and get a coffee, have a bit of a slurp, come back

INT: bit of a slurp?

RES: bit of a slurp come back bish bosh bing it's a happy one we're in. I wouldn't put that on your report

INT: bish bosh bing?

RES: bish bosh bing booby bird

INT: there are bits I will miss out

RES: laughs - that's ok no problem (to people in lift) oh those wheelchairs are useful for something

INT: (Whistle in lift) oop no that wasn't the right button, that was the alarm

RES: oh well done

INT: ha ha

RES: $\quad$ Stopping (abrupt stopping lift again) Jesus, I was saying even though this thing has got

INT: Oop sorry (lots of people on alighting from the lift)

RES: even though this thing has got suspension

INT: yeah 
RES: it's not the softest suspension in the world it thumps and bangs all the timedon't step in the poodle, whatever the poodle is

INT: the poodle?

RES: please. ()

INT: This lady is lost - can we help what are you looking for? ( We help her find A\&E)

RES: Keep moving fast (someone coughing in background and Phil worries about his compromised immunity)

INT: Tells you something when you know where everything is

RES: $\quad$ yeah, I don't like being here it's full of sick people

INT: I know

RES: that's why I wanted you to move fast

INT: yeah

RES: I was thinking, when I'm bad in the houses, we have got that zimmer frame haven't we?

INT: yeah

RES: $\quad$ should bring that in, it'll be a lot better for me

INT: yeah it would

RES: I was thinking that the other day, when I'm rough

INT: yeah

RES: and falling about, we should have the zimmer frame in there

INT: yeah 
RES: it makes perfect sense

INT: one of the issues with all these things is storage space

RES: yes yes absolutely I know you have to put it somewhere and I'm not disagreeing, but I'm just saying. Well yeah but I mean there's nothing to stop you keeping it in the shed but bring it in when I'm ill

INT: no I'm thinking that. It's at the back of the shed at the moment but I could bring it to the front or

RES: $\quad$ hang it up from the ceiling or something near the front, you know

INT: What do you want my darling?

RES: Oh I'll just have a small cappuccino

INT: Can you just hold that for me

RES: held (talking to people in coffee shop) yeah I'd rather have legs that worked (laughs) These are great (wheelchair) these are great, the thing you have to the thing you have to really remember is that you need something to move it

ah yeah

I have an $£ 80,000$ van that moves it

yeah, oh right

it's like a full hospital lift lockdown everything yeah

INT: yeah

RES: and that's the problem, they're fine, but you have to move them.

INT: yeah

RES: I'm actually getting rid of this erm nest year I'm going to get rid this and I'm going to go to a scooter because I want to get rid of this van, because it is just 
huge and it is just impractical you know ....yes well just about every major town and shopping centre hires scooters anyway, I mean they change your life, they absolutely change your life I mean without it you're you're you know ( ) but it you know for your wife is incredibly difficult and will cause damage to her if you are not very careful, it's an incredible amount of pressure, you know my wife's 8 years younger than me but I have an electric chair because she can't push me and she's fit, so you have to be very careful of that ...you can get, what you can get are small electric wheelchairs which are like a little transit chair, like half the size of this

\section{INT: $\quad$ Right where we going}

RES: I'm following you just move somewhere, God sakes just move somewhere babe, don't keep farting about move otherwise I'm going to take the back of your legs straight out and I don't want to do that. See now he was just saying he's getting a erm he's just getting a foldy wheel, er a foldy erm scooter

INT: right

RES: because it's been that erm -Jesus that's hot-

INT: it is hot

RES: because he's been he's been I need a bottle of water as well,

INT: I'll get you one in a minute

RES: $\quad$ yeah, because he's been, Jesus that's dangerous, that is just dangerous (hot tea with lid not fitting well) erm he er he's got to the stage for the last year or so or whatever that erm you know they've said oh we're going to go our or whatever you know the family and he's said oh no you go , you know, l'll stay home because he's you know he said I'm slow and you just feel like a burden and all the usual, all the usual, so he's now getting one of these foldable scooters that can be chucked into the boot of a car but and then he was saying oh well they're going get a manual one or whatever because it's just easier but then I said but 
he said oh well then I'll get, I said you don't want a wheelchair with the big wheels because I said you can't move that because you're heavy. Oh well my wife you know, I said she can't push you man. I said because you will end up damaging your wife...you've got to be powered so if you get a chair you want to be powered and even if you're not powered you want a transit chair because at least it's light and it's smaller and easier to move. There's no point having a wheelchair cos you can't wheel it. There's a There's a aaaahhh lack of practicality. That thing I was watching last night that video I was watching last I said to you, you know erm that they're showing this folding scooter and they're showing this guy on this folding scooter who's this sort of 75-year-old bloke and then they're showing this 75-year-old bloke picking it up and putting it in the boot of his car and it's just absolute garbage. You know, I'm not even sure it was the same guy per se cos you only saw him from the back, I have a horrible feeling it was somebody dressed much the same ( ) you know? But it wasn't, it just wasn't it wasn't practical it was - ooh my good god-awful top - it was (laughs) it was er an impractical suggestion that would put thoughts into people minds which are not reasonable. I didn't think it was reason- I thought it was an unreasonable advert,

INT: yeah

RES: I thought it was silly of the people to advertise like that, to produce expectations in people which they could not possibly

INT: yeah

RES: you know? ( ) Where's he going to run to?

Drinking coffee and irrelevant chitchat - we make our way to appointment.

INT:

RES: $\quad$ huh fat arse bitch (under breath)

INT: Did you get lost (Phil lags behind as we make our way down a corridor) 
RES: $\quad$ No some fat arsed bitch sticking her arse in my face

INT: I'Il transcribe that, I've just remembered you're being recorded

RES: ahh no no fat bitch was the word because she was a bitch. Jesus, some woman just stuck her backside straight in my face nearly pushing me under the table where that woman was

INT: right, yeah I know people don't think to see you

RES: no no people don't see me at all no no ( ) no I just kept veering, veering veering veering and in the end I had to stop. No they don't see you in the wheelchair they don't care you're in the wheelchair, they don't give a monkeys about anything, they're too busy with their own heads in the clouds doing what they're doing ( ) well it's interesting that, I don't know what it is, but it doesn't dry up (going past same puddle)

INT: No it's still there

RES: Wow

INT: I don't think it's suspicious, it looks too clear for that

RES: $\quad 000$ I don't know

INT: (laughs)

RES: but it's not drying up, that's suspicious. ( ) Aww the world's most brutal lift going up ooh god we've stopped

INT: do you want a paper or something

RES: $\quad$ no no we're not going to be here long

INT: oh we are because they're going to do an investigation, they're going to ...

RES: oh! 
INT: that way

RES: right ( ) I'm coming hahaha that's the couple we saw downstairs in the lift, down in the hallway, they're now stood there

INT: oh hahaha

RES: looking at another map aww that's funny I know they're doing an investigation, but how long does it take?! mean is it all day or what

INT: no no no no no

RES: no well exactly so we ain't gonna be here long - that's the right reception

INT: aha () hiya, I've got an appointment (discuss details with receptionist)

RES: Right you give me the letter and go to the loo (chatter in the background and sound of chairs moving because there is not enough space for his wheelchair nurse comes to check if Phil is alright) I'm alright love I'm fine, I'll just hit it at speed. (Parks himself in the hallway - another member of staff approaches) to tell him he can go in the waiting room, it's alright) No I'm happy here thank you (told he should move in case there is an emergency - points to a chair in the hallway) yeah, well I'm no bigger than that am I, and if there is an emergency I'll move. (Ok as long as you are happy) I'm absolutely delighted to be here thank you

INT: (Returns from bathroom) indecipherable

RES: No there is room baby but she's moved their chair so I'm sitting here, I've already had this discussion. ( How you doing)

INT: good

RES: danger of fake medicines

INT: pardon 
RES: danger of fake medicines flooding Britain (reading headline of another person's newspaper)

INT: $\quad \mathrm{mm}$ (cynically)

RES: oh I see, well the lady who came out of there said ooh I can xxx more chairs for you so you can sit in there but because I am so antisocial I do not wish to sit in there so I am happy sitting here. So she said well (clears throat) she said there may be an emergency coming through I said well you've got to move that chair and I can move faster than that chair. So I'm sitting here.

INT: fair enough

RES: $\quad$ Cos I don't want to sit in there.

INT: Well the room doesn't really very well accommodate one wheelchair let alone two

RES: I doesn't accommodate, it doesn't accommodate wheelchairs in it at all, it's got too many chairs in it, and I strongly doubt it requires that many chairs in the first place

INT: no indeed, well there's (counts) 9 chairs in there not being used at the moment

RES: hm, plus the one you're sat on

INT: well it would be being used if it were in there

RES: well yeah true. Well no it's er yeah no mm. they've just got a load of chairs and they don't know what else to do with them so they've stuck them in there

INT: there is the possibility that they could be busier at times but I doubt it

RES: I very much doubt that there would be 9 people sat in there at any point, not in such a specialised unit. 
INT: no indeed

RES: but you know look at it, look at the corridor, there's stuff jammed in the corridors all over the place

INT: $\quad \mathrm{mm}$

RES: you know you've got a you've got an antenatal wheeled box there, you've got whatever it is down there, that she's the paperwork rolling filing cabinet or something, there's some other rubbish chute bin thing down the bottom end there

INT: They're cleaning down there ( ) we got past the builders downstairs didn't we, but they were very good at getting out of the way

RES: no the builders were fine. ( ) It just seems to be a general lack of space ( ) again it's not planned is it

INT: No well and for a hospital, there's a lack of

RES: everything,

INT: it's the fact that er I mean if you look at this corridor, there corridor's wide enough but the doorways aren't really wide enough, there's not enough space to manoeuvre wheelchairs, and when wheelchairs are be3ing used here all the time, you would think that would be a major consideration

RES: well yeah, and also of course they have got the world's most stupid wheelchairs here, with these with these fixed front wheels and sol- and moving rear wheels which is just ri- is just ludicrous

INT: $\quad \mathrm{mm}$ 
RES: absolutely ridiculous, they are the most unwieldy un-natural idea I've ever seen. People naturally try to pull at the- push them whereas really they need to pull them

INT: yeah

RES: I'm going to have to take this shirt off because it is so hot in here and airless ( ) that is a sight for er something eyes ( ) innit

INT: certainly is

RES: (rustling of getting shirt off) Thank you. Sorry madam. (more rustling changing microphone position) clip it in the...on the side a bit so it's not in my neck thank you is that still running alright that's still running

INT: $\quad$ yeah - we could probably turn it off now until we go out again.

RES: Why don't you just leave it, it's got plenty of time hasn't it?

INT: it has yeah

RES: in case you forget, unless you want to check it is recording

INT: (Long interval as procedure carried out)

RES: Hello kid how you doing?

INT: I'm ok

RES: Is it all good?

INT: yeah (Nurse to Phil: good driver)

RES: yeah I used to drive big trucks artics (...) how you doing?

INT: (...)

RES: Ok but you are ok. 
INT: they've taken a biopsy but everything looks perfectly fine

RES: right

INT: everything looks absolutely normal

RES: well that's good then 